\title{
VALIDATION OF HYGROTHERMAL MATERIAL MODELLING UNDER CONSIDERATION OF THE HYSTERESIS OF MOISTURE STORAGE
}

\section{VALIDIERUNG HYGROTHERMISCHER MATERIALMODELLIERUNG UNTER BERÜCKSICHTIGUNG DER HYSTERESE DER FEUCHTESPEICHERUNG}

\author{
For achievement of the academic degree \\ Doctor of Engineering (Dr.-Ing.) \\ at the Faculty of Civil Engineering \\ at Dresden University of Technology \\ accepted thesis submitted by

\begin{abstract}
Dipl.-Ing. Gregor Albrecht Scheffler
born at May $11^{\text {th }} 1978$ in Dresden, Germany

Supervisors: $\quad$ Prof. Dr.-Ing. habil. Peter Häupl

Prof. Dr. habil. Henryk Sobczuk

Prof. Dr. Carsten Rode
\end{abstract}

Date of defence: February $12^{\text {th }} 2008$

This thesis has been supported by the National German Foundation CUSANUSWERK. 



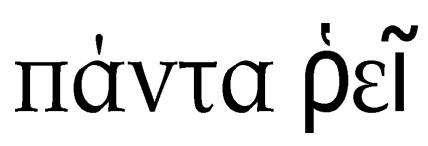

Heraklit of Ephesos (ca. 540 - 475 BC)

for Rudi and Katharina 



\section{PREFACE \& ACKNOWLEDGEMENT}

The work reported in this thesis has been accomplished during my research studies at the Institute of Building Climatology, Dresden University of Technology in the years of 2003 to 2007. Once introduced to hygrothermal building component simulation, it became one of my main objectives to connect the two powerful research fields of material investigation and hygrothermal transport modelling. I am convinced that here, sophisticated and sustainable research is only possible when it is based on a fundamental understanding of both, experimental material investigation and hygrothermal transport modelling.

The actual connection between these fields is the material modelling. Limited by both, the available input data as well as the current state of transport modelling, the material model is frequently blamed to be responsible for insufficient precision of simulation results. It is often difficult to clearly assign an observed effect to its actual cause. Therefore, the presented study aims on a fundamental material model validation combining experimental material investigation with hygrothermal transport as well as material modelling. The access to it requires though a broad review on each of these fields. The presented thesis starts therefore on a broad theory as well as experimental basis from which the accomplished developments and investigations are conducted.

The achievements of this broad study finally justify both, the chosen approach and the particular procedure. It is hence with both, pride and satisfaction to be able to submit this thesis now and present it to the interested scientific public. However, such a work is hardly achievable without mentors, promoters and friends. I have had the pleasure of having several of them whom I would like to express my gratitude for their various support. Moreover, I received financial support from different bodies which I want to be gratefully acknowledged, too. 
In the years 2004 to 2006, I was awarded with a Ph.D. scholarship by the Cusanuswerk, the German national foundation for gifted catholic students. Besides the financial support of my research, I am very grateful for the various opportunities to participate in graduate seminars, symposia and other educational events I have been offered. The encouragement and motivation I gained during encountering so many open minded, enthusiastic and talented people contributed significantly to the success of my work.

Out of the people who supported and promoted me, I wish to thank Prof. Peter Häupl first for leaving the field of material model validation to me as the subject of my research. Almost ten years ago, it was he who sparked my interest in Building Physics, and especially in modelling and understanding heat and moisture transport. With his supportive frankness, the many lively discussions and his appreciation of scientific tenacity, he benefited both, my conducted research and my own development, for which I am very grateful.

Prof. Henryk Sobczuk and Prof. Carsten Rode were unreservedly willing to be the other supervisors of my thesis. I would like to thank them for their advises and comments concerning my work as well as their encouragement during the last year of completion.

The person who encouraged me most and who was probably most closely involved in the whole growing process of my work is Dr. Rudolf Plagge, the laboratory chief at the Institute of Building Climatology. I am both, proud and grateful of having him as a teacher, a mentor and a friend. There are many ways in which he shaped me and my work, and which I am grateful for. For instance our conversations and our plenty discussions, the nights we spent in the laboratory installing and calibrating the TDR technology, the different perspectives on our field of research he opened, the frankness in which he introduced me to the scientific community and, last but not least, the kind and friendly atmosphere in the laboratory.

The laboratory would though have been rather empty without another person who I wish to thank for his continuous and smooth support. Frank Meißner accompanied most of the conducted experiments, helped during the sensor calibration and installation, and contributed sustainably to the laboratory spirit.

For their aid in the laboratory, I also wish to thank Irene Heuchler and Peter Schmieder who made many devices to become brilliant reality which I had never dared to dream of. Moreover, I thank Sören Klose and Markus Beug for their steady assistance.

My fellow Ph.D. students Andreas Nicolai and Max Funk I want to thank for the many illuminative discussions on the theory of heat and mass transfer and for the time they spent with me during my stays in Zurich and Syracuse. Moreover, I wish to acknowledge the generous financial support I received from Prof. Jianshun Zhang and the Department of Mechanical, Aerospace, and Manufacturing Engineering at Syracuse University (NY) which enabled my research stay in Syracuse. Furthermore, I wish to thank Andreas Nicolai for the well structured introduction into $\mathrm{C}++$ programming which provided the basis for my own program developments.

At the Institute of Building Climatology, I want to thank Heiko Fechner for answering many programming questions, supporting me with his own software developments and being a constant and authentic dialogue partner. Furthermore I thank Prof. John Grunewald for allowing me to participate in the national salt project and for supporting my studies in many discussions. Ultimately, my gratitude may be acknowledged 
to all the other colleagues at the Institute of Building Climatology for the warm and welcome atmosphere there.

Besides this wide and distinct financial and professional support, my research as well as my thesis could not have developed the way they did without my family and my friends. Most important was and is my wife Katharina who helped me carrying the load of set backs, consistently encouraged me and patiently read and reviewed the whole thesis. My parents and my sister and brother gave me support whenever I needed it and ensured me being part of a great family.

There are many more people I am grateful for, as Claudia Nikol who significantly helped to improve my English, Daniel Münch who has drawn the future in the brightest colours for me whenever I was down and Kornél Magvas, Jakob Polak, Janek Neubauer and Hans Janssen who encouraged me in many long conversations.

Gregor A. Scheffler,

Dresden \& Meißen, $12^{\text {th }}$ of February 2008. 


\section{Abstract}

The achievable accuracy of hygrothermal building component simulation is significantly dependent on the applied material functions. These functions are determined by the material modelling marking the connection between the basic storage and transport parameters which are obtained from basic measurements, and the storage and transport coefficients which are defined within the balance and flow equations. It is the aim of the present study to develop a flexible and widely applicable material model which is not restricted to the current level of the transport theory. Furthermore, limits and options of this model are to be validated by means of four building materials on the basis of special transient moisture profile measurements.

The study's starting point is a comprehensive investigation of both, the different existing modelling approaches and the available experimental methods to determine basic hygrothermal material parameters. On this basis, the material modelling is set into the context of the heat and moisture transport theory derived from thermodynamics. The involved limits and restrictions are highlighted and options as well as requirements for further developments are pointed out. The developments this study focuses on comprise three fields: experiments for basic property determination, material modelling, and experiments for material model validation.

The set of basic material investigation methods has been extended by the drying experiment under defined conditions. The different influences on the drying as well as its application to hygrothermal material model calibration are pointed out and appraised. On this basis, a drying apparatus is designed, built and applied. Ultimately, standardisation criteria and the derivation of a single-value drying coefficient are evaluated. Appropriate extensions are indicated.

Based on the bundle of tubes approach, an own material model is developed. It is coupled with a mechanistical approach accounting for serial and parallel structured mois- 
ture transport phenomena. The derived liquid water conductivity is adjusted by the help of measured conductivity data close to saturation as well as within the hygroscopic moisture range. Subsequently, two internal modelling parameters are calibrated which is done by numerical simulation of the water uptake and the drying experiment under consideration of the hysteresis of moisture storage.

Facilitating its application to the obtained laboratory data, the material model has been implemented into a computer program. It is applied to the four building materials brick, lime-sand brick, aerated concrete and calcium silicate. The adjusted material functions are shown and discussed. In all four cases, the calibration provides an excellent agreement between measured and calculated material behaviour.

As experimental basis of the material model validation, the instantaneous profile measurement technique (IPM) has been extended to be applied in Building Physics. Special equipment is developed and measurement procedures are designed. Different models to derive the water content from dielectric data obtained by Time Domain Reflectometry (TDR) measurements are evaluated and implemented. Ultimately, an extensive program of transient moisture profile measurements within the hygroscopic and the overhygroscopic moisture content range is conducted and evaluated.

Within the frame of validation, the developments on the experimental as well as on the modelling fields are combined. The IPM experiments are recalculated on the basis of the measured initial and boundary conditions applying the adjusted and calibrated material functions. The comparison of measured and calculated data reveals the power of the developed material modelling just as the consequences of the simplifications made on the transport theory level. The distinct influences of the hysteresis of moisture storage consisting of effects depending on the process history and effects depending on the process dynamics, are proven.

By the presented study, the material modelling has been decisively further developed, the set of basic measurement methods has been extended by a substantial experiment and the instantaneous profile measurement technique has been made applicable to Building Physics. Moreover, the influences of the process history and the process dynamics on the moisture transport and the resulting moisture profiles could be shown and proven. By that, not only a material model is now available which perfectly applies to the requirements of flexibility, applicability and extendability. The obtained data provides also a powerful basis for further research and development. 


\section{KURZFASSUNG}

Die Genauigkeit hygrothermischer Bauteilsimulation hängt maßgeblich von den verwendeten Materialfunktionen $\mathrm{ab}$. Sie werden durch die Materialmodellierung bestimmt, welche die Verbindung zwischen den aus Basisexperimenten gewonnenen Speicher- und Transportparametern sowie den innerhalb der Bilanz- und Flussgleichungen definierten Speicher- und Transportkoeffizienten herstellt. Ziel der vorliegenden Arbeit ist zum einen die Entwicklung eines flexiblen, breit anwendbaren und gleichzeitig nicht auf den gegenwärtigen Stand der Transporttheorie beschränkten Materialmodells. Dessen Grenzen und Möglichkeiten sollen zum anderen auf der Grundlage spezieller instationärer Feuchteprofilmessungen anhand von vier Baustoffen untersucht und aufgezeigt werden.

Ausgangspunkt der Arbeit ist eine ausführliche Beleuchtung sowohl der vorhandenen Modellansätze als auch der zur Verfügung stehenden experimentellen Methoden zur Bestimmung hygrothermischer Basisparameter. Auf dieser Grundlage wird die Materialmodellierung in den Kontext der aus der Thermodynamik abgeleiteten Wärmeund Feuchtetransporttheorie eingeordnet. Die damit verbundenen Grenzen und Einschränkungen werden hervorgehoben und Entwicklungsmöglichkeiten sowie weiterer Entwicklungsbedarf aufgezeigt. Dieser umfasst drei Bereiche: die Experimente zur Bestimmung von Basisparametern, die Materialmodellierung, sowie Experimente zur Modellvalidierung.

Die Reihe der Basisexperimente wird um den Trocknungsversuch unter definierten Bedingungen erweitert. Die verschiedenen Einflüsse auf die Trocknung und deren Anwendung in der Kalibrierung hygrothermischer Materialmodellierung werden herausgestellt und bewertet. Darauf aufbauend wird eine Apparatur entworfen, gebaut und angewendet. Schließlich werden Kriterien zur Standardisierung und Ableitung eines Einzahlenkennwertes evaluiert. Sinnvolle Erweiterungen werden aufgezeigt. 
Es wird ein eigenes Materialmodell auf der Grundlage eines Porenbündelansatzes hergeleitet, welches mit einem mechanistischen Ansatz gekoppelt wird, der den Feuchtetransport in seriell und parallel strukturierte Bereiche untergliedert. Die abgeleitete Flüssigwasserleitfähigkeit wird anhand von Leitfähigkeitsmessdaten im nahe gesättigten sowie im hygroskopischen Feuchtebereich justiert. Zwei interne Modellparameter werden anschließend unter Berücksichtigung der Hysterese der Feuchtespeicherung anhand des Aufsaug- und des Trocknungsversuches kalibriert.

Das Materialmodell ist zur Erleichterung der Anwendung in ein Computerprogramm zur Anpassung an die Labordaten implementiert worden. Das Programm wird auf die vier Baustoffe Ziegel, Kalksandstein, Porenbeton und Calciumsilikat angewendet. Die entsprechend angepassten Materialfunktionen werden gezeigt und diskutiert. Im Rahmen der Kalibrierung wird eine hervorragende Übereinstimmung zwischen gemessenem und berechnetem Materialverhalten erreicht.

Zur Modellvalidierung wird die Augenblicksprofilmethode (IPM) für die bauphysikalische Anwendung erweitert. Spezielle Apparaturen werden entwickelt und Versuchsabläufe entworfen. Modelle zur Ableitung des Wassergehaltes aus mit Hilfe der Time Domain Reflectometry (TDR) gewonnenen Dielektrizitätsmessdaten werden evaluiert und implementiert. Schließlich wird ein umfangreiches Programm an Feuchteprofilmessungen im hygroskopischen und überhygroskopischen Feuchtebereich umgesetzt und ausgewertet.

Im Rahmen der Validierung werden die Entwicklungen auf experimenteller sowie auf Modellierungsebene zusammengeführt. Die IPM Experimente werden anhand der gemessenen Anfangs- und Randbedingungen und auf der Grundlage der angepassten und kalibrierten Materialfunktionen nachgerechnet. Der Vergleich zwischen Messung und Rechnung offenbart die Stärke der entwickelten Materialmodellierung ebenso, wie den Einfluss der auf Ebene der Transporttheorie getroffenen Vereinfachungen. Ein deutlicher Einfluss der sich aus der Prozessgeschichte sowie der Prozessdynamik zusammensetzenden Hysterese der Feuchtespeicherung kann nachgewiesen werden.

Mit der vorliegenden Arbeit ist somit nicht nur die Materialmodellierung entscheidend weiterentwickelt, die Reihe der einfachen Basisexperimente um einen wesentlichen Versuch erweitert und die Augenblicksprofilmethode für bauphysikalische Belange anwendbar gemacht worden, es wurden auch die Einflüsse der Prozessgeschichte, und erstmals auch der Prozessdynamik, auf den Feuchtetransport sowie die sich einstellenden Feuchteprofile deutlich aufgezeigt und nachgewiesen. Es ist demnach nicht nur ein Materialmodell, welches den gestellten Anforderungen an Flexibilität, breite Anwendbarkeit und Erweiterbarkeit genügt, entwickelt worden, es wird mit den gewonnenen Messdaten auch die Grundlage weiterer Forschung zur Verfügung gestellt. 


\section{TABLE OF CONTENTS}

Preface \& Acknowledgement $\ldots \ldots \ldots \ldots \ldots \ldots \ldots \ldots \ldots \ldots \ldots$

Abstract $\ldots \ldots \ldots \ldots \ldots \ldots \ldots \ldots \ldots \ldots \ldots \ldots \ldots \ldots \ldots \ldots$

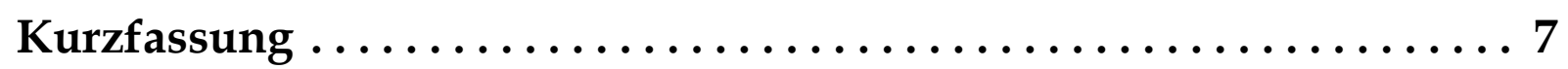

Table of Contents $\ldots \ldots \ldots \ldots \ldots \ldots \ldots \ldots \ldots \ldots \ldots$

1 Introduction and Overview $\ldots \ldots \ldots \ldots \ldots \ldots \ldots \ldots \ldots$

2 Theory of material modelling $\ldots \ldots \ldots \ldots \ldots \ldots \ldots \ldots \ldots \ldots$

2.1 Heat and mass transport in porous media $\ldots \ldots \ldots \ldots \ldots \ldots \ldots$

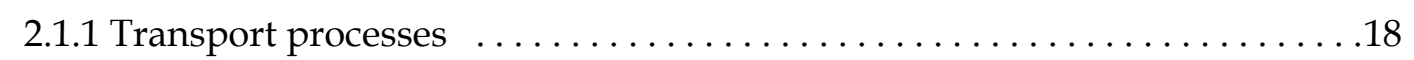

2.1.2 Balance equations of heat, air and moisture $\ldots \ldots \ldots \ldots \ldots \ldots \ldots \ldots$

2.1.3 Constitutive equations for heat, air and moisture $\ldots \ldots \ldots \ldots \ldots \ldots .21$

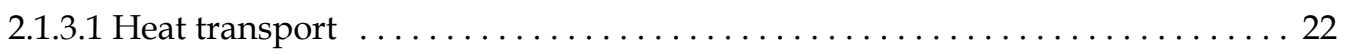

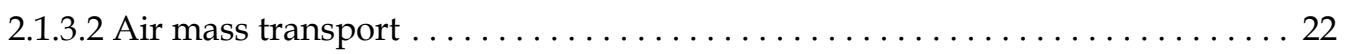

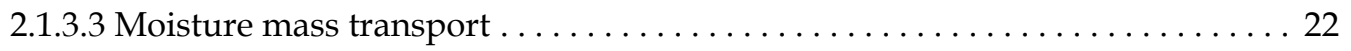

2.1.4 Remarks on heat, air and moisture transport $\ldots \ldots \ldots \ldots \ldots \ldots \ldots$

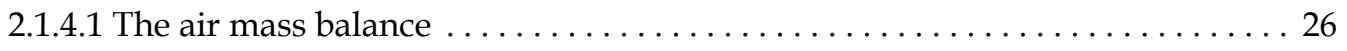

2.1.4.2 Hygroscopic and overhygroscopic moisture range $\ldots \ldots \ldots \ldots \ldots \ldots \ldots$ 


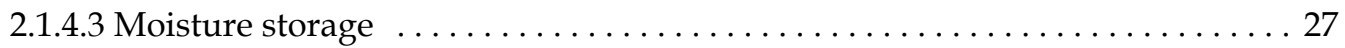

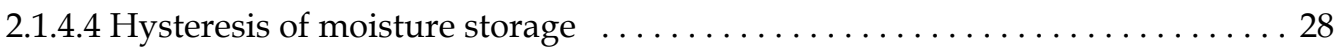

2.2 Material Modelling in Building Physics .................... 31

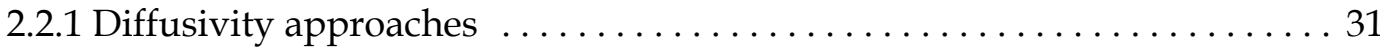

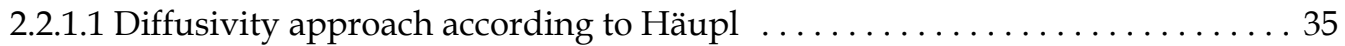

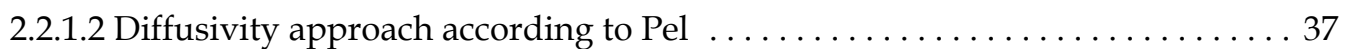

2.2.1.3 Diffusivity approach according to Kießl and Künzel $\ldots \ldots \ldots \ldots \ldots$

2.2.1.4 Diffusivity approach according to Krus and Holm ................ 39

2.2.1.5 Diffusivity approach according to Carmeliet and Janssen $\ldots \ldots \ldots \ldots \ldots 41$

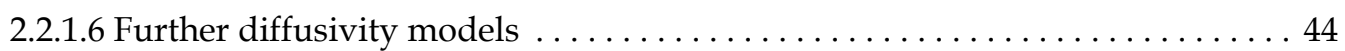

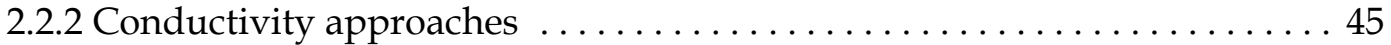

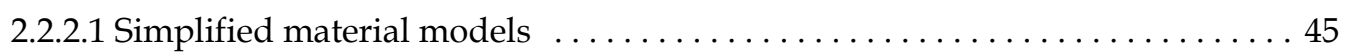

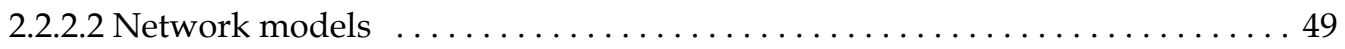

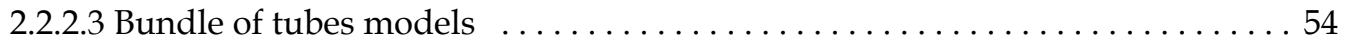

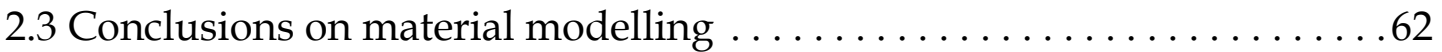

3 Experimental Methods $\ldots \ldots \ldots \ldots \ldots \ldots \ldots \ldots \ldots \ldots \ldots \ldots$

3.1 Basic hygrothermal material properties $\ldots \ldots \ldots \ldots \ldots \ldots \ldots \ldots$

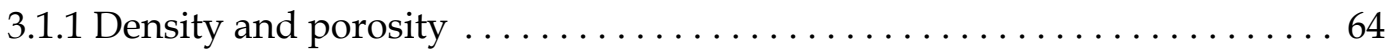

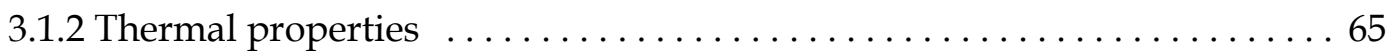

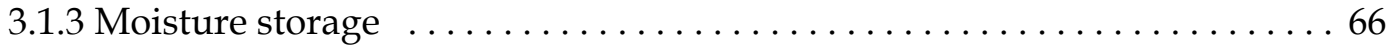

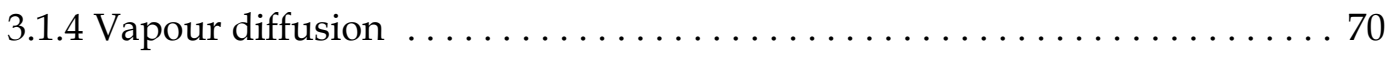

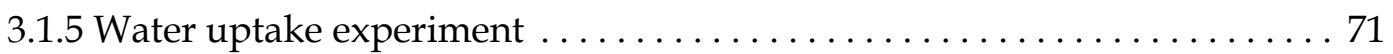

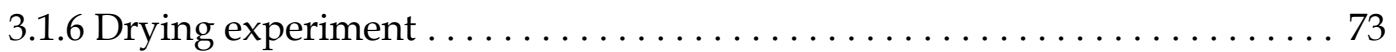

3.1.7 Saturated and unsaturated flow experiments $\ldots \ldots \ldots \ldots \ldots \ldots \ldots$

3.1.8 Concluding basic experimental methods $\ldots \ldots \ldots \ldots \ldots \ldots \ldots \ldots$

3.2 Instantaneous profile measurements $\ldots \ldots \ldots \ldots \ldots \ldots \ldots \ldots 77$

3.2.1 Principle of instantaneous profile methods $\ldots \ldots \ldots \ldots \ldots \ldots \ldots \ldots 77$

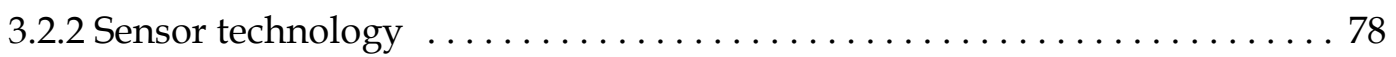

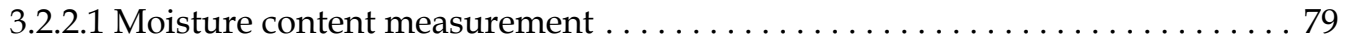

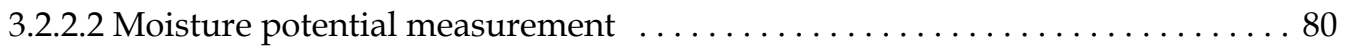

3.2.3 Concluding instantaneous profile measurements $\ldots \ldots \ldots \ldots \ldots \ldots 2$

3.3 Conclusions on Experimental Methods $\ldots . \ldots \ldots \ldots . \ldots . \ldots . \ldots 2$

4 Investigated Material $\ldots \ldots \ldots \ldots \ldots \ldots \ldots \ldots \ldots \ldots \ldots$

5 Development and Utilisation $\ldots \ldots \ldots \ldots \ldots \ldots \ldots \ldots \ldots \ldots$

5.1 Developments in Material Modelling . . . . . . . . . . . . . 87

5.1 .1 Moisture storage and pore structure $\ldots \ldots \ldots \ldots \ldots \ldots \ldots \ldots$

5.1 .2 Liquid water and water vapour transport $\ldots \ldots \ldots \ldots \ldots$ 
5.1.2.1 Moisture content regions $\ldots \ldots \ldots \ldots \ldots \ldots \ldots \ldots \ldots \ldots \ldots \ldots \ldots \ldots$

5.1.2.2 Mechanistical approach: serial-parallel model . . . . . . . . . . . . 93

5.1.2.3 Calibration of liquid and vapour transport coefficients $\ldots \ldots \ldots \ldots 6$

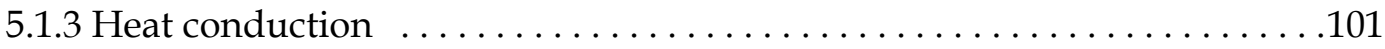

5.1.4 Concluding the developments in material modelling ............101

5.2 Methodological Developments for the Drying Experiment . . . . . . . 102

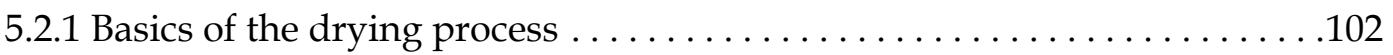

5.2.1.1 Vapour transfer - Influence of boundary conditions . . . . . . . . . . 103

5.2.1.2 Moisture inside the material - Influence of transport properties . . . . . . . 104

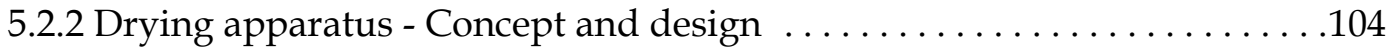

5.2.2.1 Influences and considerations . . . . . . . . . . . . . . . . 104

5.2 .2 .2 Development of a drying apparatus . . . . . . . . . . . . . . 106

5.2 .2 .3 Realisation of the drying apparatus $\ldots \ldots \ldots \ldots \ldots \ldots \ldots \ldots \ldots$

5.2.3 Derivation of a drying coefficient . . . . . . . . . . . . . . . . . 108

5.2 .3 .1 Attempts to standardize drying data $\ldots \ldots \ldots \ldots \ldots \ldots \ldots \ldots$

5.2 .3 .2 Discussion on the proposed drying coefficient $\ldots \ldots \ldots \ldots \ldots \ldots \ldots 11$

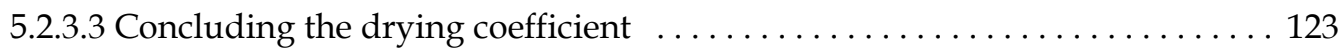

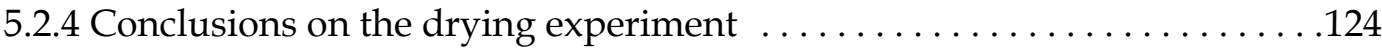

5.3 Instantaneous profile measurements . . . . . . . . . . . . . . 125

5.3.1 Measurement set-up for the hygroscopic moisture range $\ldots \ldots \ldots \ldots .125$

5.3.2 Measurement set-up for the overhygroscopic moisture range $\ldots \ldots \ldots .127$

5.3.3 TDR calibration and data evaluation ........................... . . . . . . . .

5.3.3.1 TDR system configuration and calibration $\ldots \ldots \ldots \ldots \ldots \ldots \ldots \ldots \ldots \ldots$

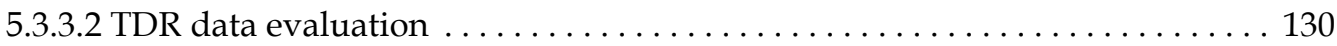

5.3.4 Conclusions on instantaneous profile measurements ............136

5.4 Concluding Developments and Utilisation . . . . . . . . . . 137

6 Implementation and Application $\ldots \ldots \ldots \ldots \ldots \ldots \ldots \ldots \ldots$

6.1 Implementation of the material model . . . . . . . . . . . . . . . . . 139

6.1.1 Program components ... . . . . . . . . . . . . . . . . . . . . . . . . . . . . . . 140

6.1.2 Adjustment and calibration procedure ....................143

6.2 Application of the material model . . . . . . . . . . . . . . 145

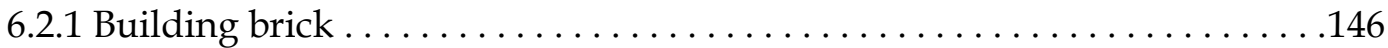

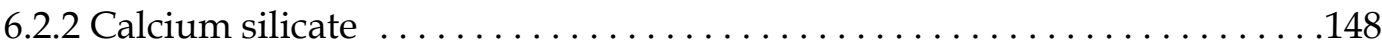

6.2.3 Aerated concrete . . . . . . . . . . . . . . . . . . . . . . . . . . 150

6.2.4 Lime-sand brick . . . . . . . . . . . . . . . . . . . . . . . . . . . . . . . . . . 152

6.3 Implementation of TDR data evaluation $\ldots \ldots \ldots \ldots \ldots \ldots \ldots \ldots$

6.4 Application of the TDR models . . . . . . . . . . . . . . . . 156

6.5 Conclusions . . . . . . . . . . . . . . . . . . . . . . . . . . . . . . . 159 
7 Simulation and Validation $\ldots \ldots \ldots \ldots \ldots \ldots \ldots \ldots \ldots \ldots \ldots \ldots \ldots \ldots$

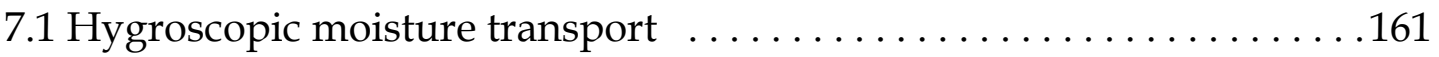

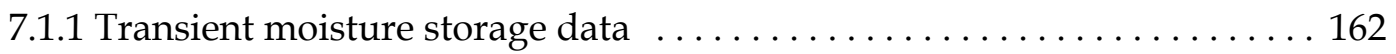

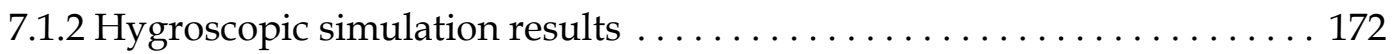

7.1 .3 Concluding hygroscopic assessment $\ldots \ldots \ldots \ldots \ldots \ldots \ldots \ldots \ldots \ldots$

7.2 Overhygroscopic moisture transport ..................... 190

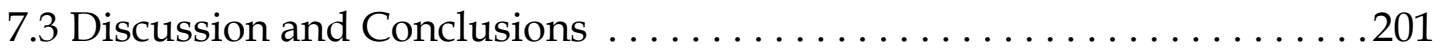

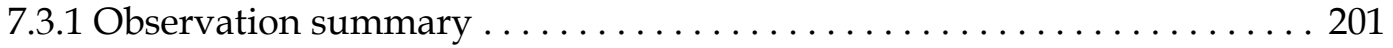

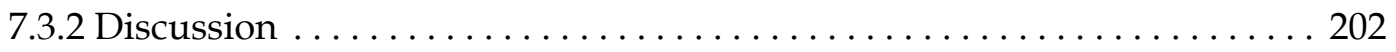

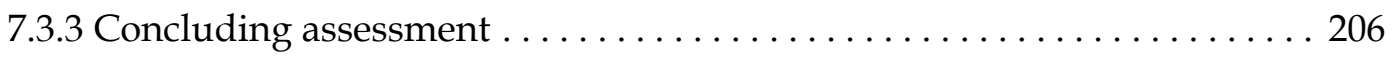

8 Summary, Conclusions and Future Prospects ............ 209

8.1 Content summary .................................209

8.2 Results and conclusions ............................211

8.3 Future prospects ................................213

List of Symbols and Abbreviations ................... 215

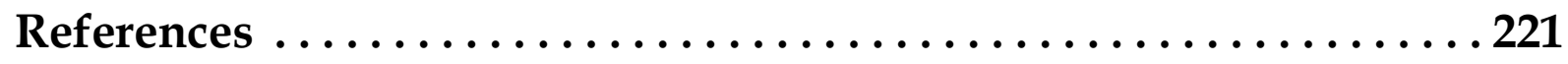




\section{CHAPTER 1}

\section{INTRODUCTION AND OVERVIEW}

Building component simulation has become a very important design tool. Building physical teaching, research and design can not be imagined without such numerical simulation programs anymore. Not only the large number of developed simulation codes, as e.g. presented by Pedersen (1990), Galbraith (1992), Künzel (1994), Grunewald (1997), Roels (2000), Janssen (2002), Sasic Kalagasidis (2004) and Nicolai (2006), but also the increasing number of research topics being evaluated on a numerical simulation basis indicate their increasing importance. New areas of research and application are explored, as the coupling of building component with whole building simulation (Rode et al. (2002), Grau E Rode (2005), Fechner (2007)) or with air flow and CFD modules (Grunewald E Nicolai (2007), Gnoth et al. (2007)).

By such broad application, the demands on quality and precision of simulation results have increased, too. The conclusions of the European HAMSTAD ${ }^{1}$ project indicate, that the numerical methods of different simulation programs deliver the same results in case the same initial and boundary conditions as well as the same material data are applied (Roels et al. (2004)). The simulation quality hence depends on the reliability of initial and boundary conditions, and especially on the available hygrothermal material data in combination with the applied material model.

It is consequently desired to rely on a material modelling which is generally applicable and flexible by means of adjustability and extendability. In addition, for the assessment and evaluation of hygrothermal building component simulation results, it is very important to know the limits of the employed modelling approach. The present study

1. The HAMSTAD project was initiated within the 5 th framework programme of the EU in the year 2000 to provide a platform of benchmarking heat, air and moisture transport simulation programs. By this, the quality of such programs has been standardized and assured. 
therefore aims at development and validation of a material model which is generally applicable to hydrophilic building materials, which is adjustable to a varying amount of basic input data and which is extendible by means of process dependency as well as solute transport modelling. It is furthermore aimed to challenge this material model by comparing data of instantaneous profile measurements with corresponding simulation results. On this basis, limits and options of the developed material modelling are supposed to be derived and concluded.

Material modelling associates the basic material property determination with the material coefficients defined in balance and constitutive equations of heat and moisture transport. These equations define the required moisture physical relationships the material model has to provide whereas the available basic material data mark the scope of input information to adjust the model. The material model is hence dependent on both of them. To develop an advanced, general and flexible material model and to investigate its limits and options within the frame of heat and moisture transport modelling, a wide view on basic material property determination as well as on hygrothermal material and transport modelling is required. In addition, an experimental investigation method is necessary providing whole range water content data for different building materials and under varying but defined boundary conditions. The instantaneous profile measurement technique is such a method which needs to be adjusted, applied and evaluated. The outlined objectives lead therefore to an enlarged methodological basis. This is reflected in the structure of the present work which is introduced as follows.

Chapter 2 starts with a brief introduction into the general heat, air and moisture transport modelling. The balance and constitutive equations are specified and concluded by a number of remarks on the scope of the whole study. The main part of this chapter forms a comprehensive review on material modelling approaches either based on Fick's first law which are called diffusivity approaches, or based on the Richards-equation called conductivity approaches. A general assessment of these models with regard to the objectives concludes this chapter.

Chapter 3 provides a review on experimental methods for both, basic material property determination and instantaneous profile measurement. The basic storage and transport properties of heat and moisture are specified and the different methods for their experimental determination are introduced. Existing problems are discussed and demands for further developments derived. Finally, the principle of the instantaneous profile measurement technique is presented, and moisture content measurement by time domain reflectometry (TDR) and different moisture potential measurement technologies are sketched.

Chapter 4 presents the four investigated building materials: a building brick, an aerated concrete, a lime-sand brick and a calcium silicate inside insulation board. Besides a brief introduction to each material, the basic hygrothermal properties are specified and some illustrating pictures are provided.

Chapter 5 is the development and utilisation chapter. First, the material model is developed based on the pore structure of the material. A bundle of tubes model as the most suitable approach fulfilling the requirements on flexibility, applicability and extendability is coupled with a mechanistical model. It accounts for serial and parallel structured moisture transport providing two modelling parameters. These are adjusted by the aid of numerical simulation of the water uptake and the drying experiment. In a 
second part of this chapter, developments on the drying experiment and its utilisation for material model calibration are accomplished. A comprehensive investigation regarding the influences on the drying and its data evaluation is elaborated and concluded. Finally, first attempts to standardize drying data are evaluated and discussed before a reliable solution is indicated. The third part of this chapter ultimately introduces the developed instantaneous profile measurement devices and the designed measurement schedules. The TDR sensor calibration is illustrated and the water content derivation from measured TDR data by different dielectric approaches is compiled.

Chapter 6 comprises the implementation and application of the developed material modelling as well as the TDR evaluation. Initially, the implementation of the developed material model into a computer program is outlined facilitating its application to basic material data. The calibration procedure to determine the internal modelling parameters on the basis of water uptake and drying simulation is specified. Subsequently, the adjusted material functions are given for the four investigated building materials. Within the second part of this chapter, the implementation of the TDR data evaluation models into a second computer program is sketched. Its application is exemplarily shown for one material.

Chapter 7 finally combines the results of material modelling with the results of the instantaneous profile measurements enabling a fundamental material model validation. At the beginning, transient moisture storage data is compared with data determined by static methods already indicating significant dynamic effects. Thereafter, the measured and calculated water content profiles are compared. A number of interesting effects is observed and attributed to either the material model or the general moisture transport modelling. Limits and options of both of them are clearly pointed out, implications of further developments are discussed and a final assessment résumé is given.

Chapter 8 concludes the whole conducted research and development. The obtained achievements are specified and the deduced results are summarized. A prospect on future research topics derived from the presented work completes this chapter. 
CHAPTER 2

\section{THEORY OF MATERIAL MODELLING}

Hygrothermal material modelling is embedded into the theory of heat and moisture transport in porous media. For a better identification of their fields of duty, it is very important to clarify the terms in use. Hence, a distinction is made between the transport theory and material modelling first. The transport theory in porous media, on the one hand, comprises the whole range of microscopic and macroscopic treatment based on thermodynamics. On the microscopic scale, states of energy and transport mechanisms for heat and mass are studied leading to the formulation of balance equations and the connection of cause and effect of the transport processes. On the macroscopic scale a phenomenological treatment of these transport processes leads to the formulation of constitutive equations that associate the fluxes to their driving forces defining the transport coefficients.

The storage and transport coefficients are normally non-linear functions of different dependencies. Their definition and identification on the basis of measured material properties is, on the other hand, the task of material modelling. Therefore, material modelling is dependent on the transport theory which derives and defines the material coefficients. A scheme of this distinction is given in Fig. 2.1.

The first part of this chapter is an introduction to the theory of heat and mass transport in porous media which leads to the definition of the balance equations for heat, air and moisture. The required transition from a microscopic treatment to the macroscopic scale is marked by the constitutive equations given afterwards. They define the transport coefficients required for numerical simulation. Different approaches exist here, leading to different approaches in material modelling, too, which is outlined subsequently and completed by some remarks on heat, air and moisture transport. 


\section{Transport Theory}

microscopic scale

derivation of balance equations based on thermodynamics

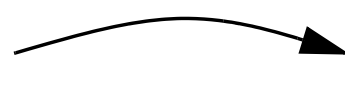

phenomenological treatment derivation of constitutive equations

\section{Material Modelling}

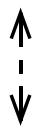

measured material parameters observed material behaviour

coefficients in constitutive equations

functions depending on state variables

Figure 2.1: Distinction between transport theory and material modelling.

The main part of this chapter is the literature review of existing material models describing the underlaying assumptions and the adjustment procedures to measured material data. A distinction is made there for different coefficients required by different driving potentials.

\subsection{Heat and mass transport in porous media}

Heat and mass transport in porous media has been investigated for many years. Some authors as Krischer (1940) and Krischer (1942), Philip \& De Vries (1957) and De Vries (1958), Whitaker (1969) and Whitaker (1977), Bouma (1977), Dullien (1979), Kießl (1983), De Vries (1987), Bear \& Bachmat (1991) and Hassanizadeh E Gray (1997) may be named deputizing to the large number of publications on this topic.

Most authors in Building Physics, however, start with their modelling on the basis of derived balance equations. Only few go into detail of the thermodynamic principles lying behind them. The following paragraph gives a survey on the topic. It refers to the theory specified by Grunewald (1997) which was further developed in Grunewald et al. (2003), Grunewald \& Häupl (2003) and Funk E Grunewald (2005).

\subsubsection{Transport processes}

Grunewald formulated the fundamental heat and mass transport theory on the basis of the continuum model, i.e. the assumption, "the real matter can be treated as a continuously distributed substance whose behaviour can be described by a set of steady and differentiable functions of time and space" (Grunewald (1997), p.35; see also Bear E Bachmat (1991), p.14). Most advantageously, this approach does not require the description of the exact configuration of the porous medium which, in most cases, is extremely difficult or even impossible. Instead, the microscopic examination can be done on the basis of Representative Elementary Volumes (REV, see also Whitaker (1969) and Bear $\mathcal{E}$ Bachmat (1991)) containing all components of the porous medium in a representative configuration. According to their state of aggregation the components $v$ are combined to phases $\alpha$, in particular solid $(m)$, liquid $(l)$ and gaseous $(g)$. 


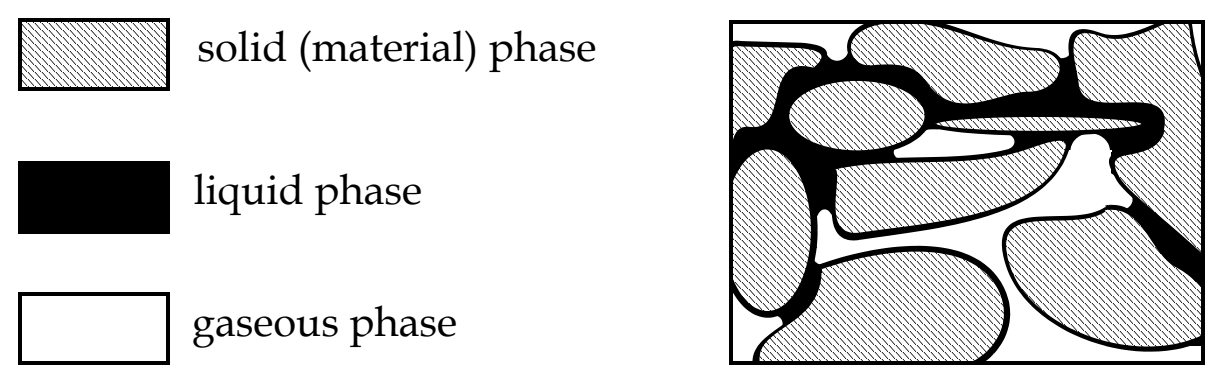

Figure 2.2: $\quad$ Section of a Representative Elementary Volume (REV) containing 3 phases.

The system state can be described by a set of independent state variables $\vec{Y}_{\alpha}$ for each phase $\alpha$, which is generally given with

$$
\vec{Y}_{\alpha}=\left(M_{\alpha}, U_{\alpha}, S_{\alpha}, I_{\alpha}\right)
$$

Within a phase of the REV the extensive state variables mass $M_{\alpha}$, inner energy $U_{\alpha}$, entropy $S_{\alpha}$ and momentum $I_{\alpha}$ can be related to the volume leading to intensive state variables or property densities. As the change of position in time, the velocities for each of those properties can also be defined. Combined they result in the total fluxes as the product of density and velocity containing both, advective and diffusive shares. Chemical reactions, phase changes and external volume forces can cause changes of mass, energy and momentum or the production of entropy which is accounted for by source production rates.

The modelling assumptions accompanied by the above sketched approach are listed below in the way they were summarized by Funk \& Grunewald (2005):

- No storage of kinetic energy in the mass of the moving phases is considered (no inertial forces, no free convection).

- No particle or energy storage in surface terms is considered. Surface effects are only taken into account at the calculation of the capillary pressure.

- Distortions of the solid material matrix are neglected (no changes of the size of the REV are considered).

- $\quad$ The spatial heat and mass flows are all calculated via a linear approach, i.e. force multiplied with transport coefficient. This is equivalent to the assumption of local thermodynamic equilibrium. Deviations from that equilibrium are infinitely small.

- $\quad$ The driving forces derived from thermodynamics of particle mixtures are also valid in the more complex system of the REV.

- The advective flow is a laminar flow.

- $\quad$ Minor flows according to the Dufour-effect (heat transport due to a concentration gradient, see Dufour (1873) and Clusius $\mathcal{E}$ Waldmann (1942)), or the Ludwig-Soret-effect (thermodiffusion meaning a particle 
diffusion due to a temperature gradient, see also Ludwig (1856), Soret (1880) and Soret (1881)), and pressure diffusion are neglected.

- Dissipation of kinetic energy by friction or compression and associated generation of inner energy is neglected.

- The only volumetric force is gravity.

- $\quad$ Far range interactions between different regions of the system are neglected.

Based on the set of state variables, the fluxes and the source production rates, general balance equations can be set up as follows.

\subsubsection{Balance equations of heat, air and moisture}

The balance equations can be derived in different ways depending on the point of view of observation. The most common way - which will be used here as well - is to use a locally fix REV as the basis (local approach). Other approaches choose a control volume which is moved with the mean reference velocity (advective approach) or with the mean mass velocity (substantial approach). These formulations are useful to distinguish between advective and diffusive flows which will, with regard to Grunewald (1997), not given further emphasis here.

To be balanced, a quantity has to be a conserved quantity, as which the inner energy and the masses of air and moisture are regarded to. Then, the change in time of the conserved quantity within a REV must equal the sum of all in- and outgoing fluxes together with the sum of all produced and dissipated quantities accounted for by source production rates.

\section{Moisture mass balance}

The moisture mass balance (2.2) relates the time change of the volume-specific total moisture mass (moisture mass density $m_{w+v}$ ) to the sum of advective and diffusive flows of liquid water and water vapour. A source term accounts for moisture sources and sinks within the reference volume.

$$
\bar{\partial}_{\partial t} w+v=-\sum_{k}\left\{\frac{\partial}{\partial x_{k}}\left[m_{w} \cdot v_{k}^{M_{l}}+m_{v} \cdot v_{k}^{M_{g}}+j_{k, \text { diff }}^{M_{v}}\right]\right\}+\sigma_{l}^{M_{w+v}}
$$

\section{Air mass balance}

The air mass balance (2.3) relates the time change of the volume specific air mass to the sum of advective and diffusive air flows. The diffusive air flow corresponds to the negative diffusive vapour flow because the transported vapour needs to be replaced by dry air. 


$$
\bar{\partial}_{\partial t} a=-\sum_{k}\left\{\frac{\partial}{\partial x_{k}}\left[m_{a} \cdot v_{k}^{M_{g}}-j_{k, d i f f}\right]\right\}
$$

\section{Inner energy balance}

In eq. (2.4), the specific inner energy time change is related to the advective, diffusive and reduced heat flows due to heat conduction, advective liquid and vapour transport and vapour diffusion. The production of energy due to phase changes occurs only for a component or phase specific formulation. For the whole system they must vanish as the specific phase change enthalpy is considered within the specific inner energy. $\sigma_{u}^{Q}$ therefore accounts for external energy sources, for instance due to chemical reactions, which are not directly included into the system of balance equations.

$$
\frac{\partial u}{\partial t}=-\sum_{k}\left\{\frac{\partial}{\partial x_{k}}\left[j_{k, \text { diff }}^{Q}+c_{l}^{H_{l}} m_{l} v_{k}^{M_{l}}+c_{g}^{H_{g}} m_{g} v_{k}^{M_{g}}+c_{v}{ }_{v} j_{k, d i f f}^{M_{v}}\right]\right\}+\sigma_{u}^{Q}
$$

The nomenclature used here follows the definition in Funk $\mathcal{E}$ Grunewald (2005). Hence specific state variables use small, but similar letters as the corresponding extensive quantities. The volume-specific mass therefore reads $m_{v}$ coming from $m_{v}=M_{v} / V_{R E V}$ and not $\rho_{v}$ as one might expect for a density. For a detailed explanation of the used symbols, please refer to the List of Symbols and Abbreviations.

\subsubsection{Constitutive equations for heat, air and moisture}

The constitutive equations specify the fluxes used within the balance equations. They represent the assignment of transport coefficients to their driving forces. According to Funk \& Ghazi Wakili (2007), the different driving forces can be used equivalently as long as they are derived from independent state variables. The difference, however, whether choosing the actual thermodynamic potential or not, occurs in the number of fluxes which have to be taken into account and in the ability to include further phenomena as effects of hysteresis and the transport of salt or volatile organic compounds (VOC), see e.g. Funk et al. (2003) and Nicolai \& Zhang (2006). Using a driving force not being the actual driving potential may cause artificial flows or emphasize the influence of side effects which in that case can not be neglected anymore. Moreover, the extension to effects as the hysteresis of moisture storage may become more difficult as other driving forces assign them additionally to the transport coefficients and not only to the water content - water potential relation.

From the above given balance equations one can see the number of fluxes in need to be specified. In particular, there are the reduced heat flow, the advective air mass flow, the advective water mass flow and the vapour diffusion. 


\subsubsection{Heat transport}

The elements in curly braces within eq. (2.4) mark the different flows contributing to the total heat flow. All except one depend on mass flows which also transport energy. The only heat flow independent of mass flows is the conductive or reduced heat flow $j, Q$, diff due to heat conduction. It is proportional to the temperature gradient and can be expressed by Fourier's law:

$$
j_{k, \text { diff }}^{Q}=-\lambda \cdot \frac{\partial T}{\partial x_{k}}
$$

The thermal conductivity $\lambda$ is dependent on the material, its structure and on the moisture content.

\subsubsection{Air mass transport}

The air mass balance of eq. (2.3) consists of the advective and the diffusive air mass flow. The latter corresponds with the vapour diffusion, since if vapour diffuses into one direction, air must diffuse in the opposite one.

The advective air mass flow is related to the gas pressure gradient and to gravity in direction of $k . K_{g}$ is the advection coefficient of the gaseous phase (Grunewald (1997) and Funk E Grunewald (2005)).

$$
\dot{j}_{a d v, k}^{M_{a}}=m_{a} v_{k}^{M_{g}}=\frac{m_{a}}{m_{g}} \cdot K_{g} \cdot\left[\frac{\partial p_{g}}{\partial x_{k}}+m_{g} g_{k}\right]
$$

\subsubsection{Moisture mass transport}

In literature different approaches exist assigning the moisture flow to a driving force which causes a number of different approaches to define the corresponding material parameters, i.e. material models. According to eq. (2.2), the total moisture flux contains an advective liquid flow, an advective vapour flow and a diffusive vapour flow. As the advective water vapour transport is associated with the advective air flow, it can be derived from eq. (2.6).

$$
m_{v} v_{k}^{M_{g}}=\frac{m_{v}}{m_{a}} \cdot j_{a d v, k}^{M_{a}}=\frac{m_{v}}{m_{g}} \cdot K_{g} \cdot\left[\frac{\partial p_{g}}{\partial x_{k}}+m_{g} g_{k}\right]
$$

Due to its minor contribution to the whole moisture current, this flow is usually neglected, as further explicated in paragraph 2.1.4 Remarks on heat, air and moisture transport below. As follows, the advective liquid water flow and the diffusive vapour flow will be examined in detail. Both of them follow different transport mechanisms. Hence, a distinction between water vapour transport and liquid water transport is appropriate. 


\section{Water vapour transport}

Without advective contributions, vapour transport reduces to vapour diffusion. According to Fick (1855), diffusion can be described as a transport process proportional to a concentration gradient.

$$
\begin{aligned}
& M_{v} \\
& j_{k, \text { diff }}
\end{aligned}=-D_{v} \cdot \frac{\partial c_{v}}{\partial x_{k}} \quad \text { Fick's first law }
$$

$D_{v}$ is called the diffusion coefficient of component $v$ and is dependent on the kind of diffusing particles as well as on the medium in which diffusion occurs. Regarding air as an ideal gas allows the use of the ideal gas equation.

$$
p V=m R T \text { rearranged to vapour yielding } \quad c_{v}=\frac{m_{v}}{V}=\frac{p_{v}}{R_{v} \cdot T}
$$

Applying eq. (2.9) to eq. (2.8) replaces the concentration gradient by the vapour pressure gradient. As the diffusive component is vapour, the $v$ might be replaced by $v$.

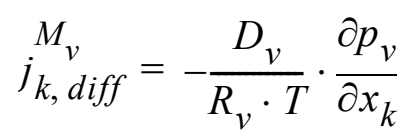

The vapour pressure is a function of relative humidity and temperature according to the following relation: $p_{v}=\varphi \cdot p_{v s}(T)$. The saturation vapour pressure $p_{v s}$ is temperature dependent. A functional description can be found e.g. in DIN 4108-3.

Vapour diffusion within porous materials is influenced by the vapour diffusivity in air, by the material's pore structure, and its water filling level. Therefore $D_{v, \text { mat }}$ is normally expressed by the diffusion coefficient of vapour in air reduced by a so called vapour diffusion resistance factor $\mu$. A number of empirical relations exist for the vapour diffusion coefficient in air. The most common one was found by Schirmer (1938), which is also given in DIN EN ISO 12572:

$$
D_{v, \text { air }}=0.083 \cdot \frac{p_{0}}{p} \cdot\left(\frac{T}{273.15}\right)^{1.81} \quad \text { vapour diffusion coefficient in air }
$$

In eq. (2.11), $p_{0}$ is the reference gas pressure of $101323 \mathrm{~Pa}$. A good overview on relations found by other authors is given by Galbraith (1992).

The vapour diffusion resistance of a material is expressed in form of a vapour diffusion resistance factor $\mu$ indicating how many times less vapour can diffuse through the material in the same time than through still air. The vapour diffusion coefficient of a material can be expressed by eq. (2.12).

$$
D_{v, \text { mat }}=\frac{D_{v, \text { air }}}{\mu}
$$


For material modelling, the vapour diffusion coefficient will normally be a function of the volumetric moisture content $\theta_{l}$. There has been a long discussion whether the vapour diffusion coefficient is dependent on the moisture content or not. This topic is not going to be stretched here anymore, although a general dependency on the pore filling level appears rather conclusive, as liquid water also reduces the pore space available for vapour diffusion. Further opinions on that will be discussed in the frame of the particular material models.

The following equation specifies the vapour diffusion flow within porous materials and defines the respective coefficients. Sometimes the temperature $T$ (as reference temperature) and the gas constant of vapour $R_{v}$ are integrated into the diffusion coefficient, in which case it will also be accentuated within the particular material model.

$$
\underset{j_{k, \text { diff }}}{M_{v}}=-\frac{D_{v, \text { mat }}\left(\theta_{l}, T\right)}{R_{v} \cdot T} \cdot \frac{\partial p_{v}}{\partial x_{k}}
$$

Some authors, as Künzel (1994), Krus (1995) and Klopfer in Lutz et al. (2002), further distinguish the molecular processes of diffusion. But these processes are difficult to be identified during measurement, which is the reason why they finally still apply to Fick's First Law in the form of eq. (2.13).

\section{Liquid water transport}

Advective liquid water transport, in eq. (2.2) regarded to as the product of liquid water mass density multiplied with the mean liquid mass velocity, $m_{w} \cdot v_{k}{ }_{l}$, can be expressed in different ways. As mentioned above, different approaches based on independent state variables can be used equivalently, although applying another than the actual driving potential might cause additional problems (Funk \& Ghazi Wakili (2007)). Most
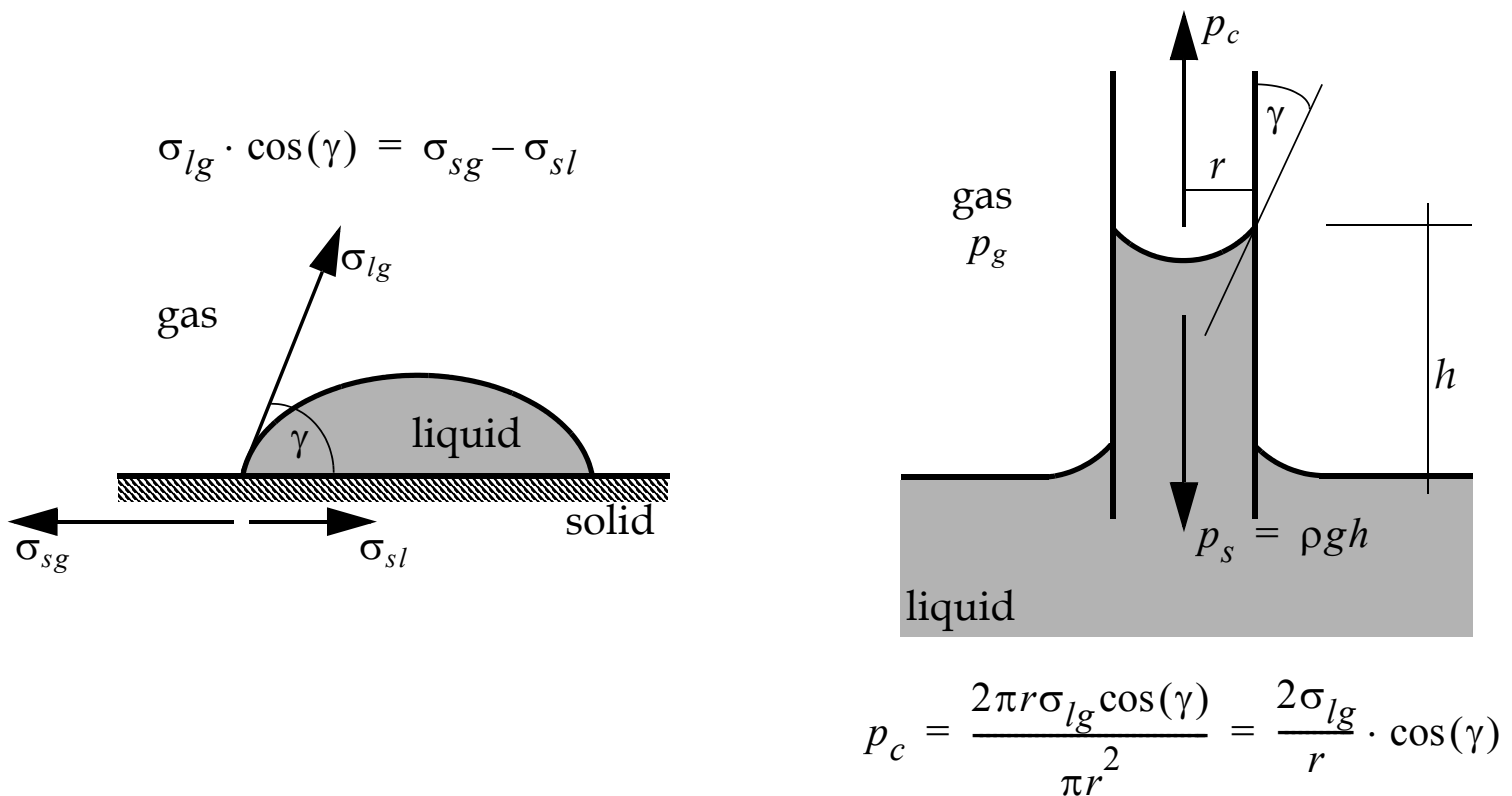

Figure 2.3: Definition of capillary pressure in the porous system following Krischer (1956), Dullien (1979), Descamps (1997), Roels (2000) and Häupl (2006). 
common are descriptions depending on the liquid/capillary pressure gradient and those which apply to Fick's First Law by the aid of the diffusion equation (2.8).

The actual cause of liquid water movement in porous media is the liquid pressure gradient or the capillary pressure gradient, respectively (see e.g. Dullien (1979) and Bear $\mathcal{E}$ Bachmat (1991)). Due to cohesion and adhesion forces, the interface of liquid and gaseous phases stays under tension. It is called the surface tension $\sigma_{l g}$ and is dependent to both fluids. At the contact surface between liquid and solid phase, a defined angle $\gamma$ called contact angle forms depending on the liquid, the solid material, the surface roughness and others, which is shown in Fig. 2.3 at the left. The capillary pressure $p_{c}$ within the pore system of a porous medium, defined as the difference of liquid pressure $p_{l}$ and gas pressure $p_{g}$, can then be derived in dependence to the surface tension $\sigma_{l g}$, the contact angle $\gamma$ and the pore radius $r$, as shown in Fig. 2.3 at the right and given in eq. (2.14). This equation is known as Laplace equation.

$$
p_{c}=p_{l}-p_{g}=\frac{2 \sigma_{l g}}{r} \cdot \cos (\gamma)
$$

Laplace equation

The dependence of the capillary pressure on the contact angle plays an important role for the modelling of moisture transport in porous media. Thus, big efforts have been undertaken to determine the contact angle for different solid-liquid-air compositions, as can be seen e.g. in Dullien (1979) and Atkins $\mathcal{E}$ de Paula (2006). In Building Physics, the recent work of Adolphs et al. (2002) concerns this topic.

Another phenomenon called contact angle hysteresis additionally complicates the capillary pressure relation. For advancing liquids, the contact angle $\gamma$ is often found to be significantly larger than for receding ones, see Fig. 2.4. This one and other effects influence the relationship between state variable and potential i.e moisture content and moisture potential. As this problem is part of the transport theory, see Fig. 2.1, it will not be further emphasized here. A common simplification is to directly measure the capillary pressure moisture content relation and set $\gamma=0^{\circ}$ leading to $\cos (\gamma)=1$ which repeals that dependence. Some additional remarks will be given below in paragraph 2.1.4.3 Moisture storage and paragraph 2.1.4.4 Hysteresis of moisture storage.

The constitutive equation for advective liquid water using the capillary pressure gradient as the driving potential is given in eq. (2.15). It represents an adaptation of Darcy's Law (e.g. Whitaker (1986) and Galbraith (1992)). The liquid water conductivity $K_{l}\left(\theta_{l}\right)$ with unit [sec] is dependent on the porous medium and is a function of the moisture content. Insertion of eq. (2.15) into the balance equation (2.2) yields the differential equation of liquid water transport which is often referred to as Richards-equation.

$$
\underset{j_{k, a d v}}{M_{l}}=m_{w} \cdot v_{k}^{M_{l}}=K_{l}\left(\theta_{l}\right) \cdot\left[\frac{\partial p_{c}}{\partial x_{k}}+\rho_{l} \cdot g_{k}\right]
$$


The other common way to describe the advective liquid water flow is to apply Fick's First Law (2.8) assuming the liquid water movement to follow the water concentration gradient (Philip \& De Vries (1957), De Vries (1958), Häupl (1987), Künzel (1994), Pel (1995) and others). It is given in eq. (2.16) as follows.

$$
\underset{j_{k, a d v}}{M_{l}}=m_{w} \cdot v_{k}^{M_{l}}=\rho_{l} \cdot D_{l}\left(\theta_{l}\right) \cdot \frac{\partial \theta_{l}}{\partial x_{k}}
$$

The liquid water diffusivity $D_{l}\left(\theta_{l}\right)$ has the unit $\left[\mathrm{m}^{2} / \mathrm{s}\right]$ and depends on the porous medium as well as on the moisture content. Under some assumptions as isothermal conditions and the validity of the Boltzmann-transformation, $D_{l}\left(\theta_{l}\right)$ can be derived from measured moisture profiles; this is one reason why this approach is widely applied although it has very limited expandability (Rawlins \& Gardner (1963)).

\subsubsection{Remarks on heat, air and moisture transport}

It is not the intention of this work to point out the theory of heat and mass transfer in porous media most precisely. But since this theory is the basis material modelling stands upon, a rough overview of its substantial principles and assumptions was meant to be given. Within a general but preferably straight survey, some issues could not be highlighted the way they might require, which is the reason why, concluding this introductory chapter to heat, air and moisture transport, some additional remarks shall be passed.

\subsubsection{The air mass balance}

The above given theory concerns heat, air and moisture transport. Under the assumptions listed in paragraph 2.1.1 Transport processes, the air mass balance is though very limited. It is thus neglected in most cases this theory is applied to, assuming immediate gas pressure equilibrium with the environment.

In case of moisture transport in a totally sealed specimen, this assumption may cause significant errors, as proved e.g. by Wilson $\mathcal{E}$ Luthin (1963), Youngs $\mathcal{E}$ Peck (1964), Peck (1965a), Peck (1965b), Touma E Vauclin (1986) and Descamps (1997). Therefore for both, numerical and experimental investigations, the problem of gas pressure equilibrium has to be taken into account. The sealing of specimen, on the one hand, may be pierced a couple of times to allow pressure equilibrium. Numerical simulation of moisture transport in a totally sealed (unpierced) specimen is, on the other hand, under this assumption subject to errors.

For hygrothermal material modelling, immediate gas pressure equilibrium is normally assumed and the air mass balance and the air mass permeability are neglected. To be consistent, the following assumption should be added to the list in paragraph 2.1.1 Transport processes:

- Gas pressure changes equalize immediately. The advective air flow velocity is so small that the air mass balance and its contribution to vapour transport can be neglected. 
Hygrothermal material modelling generally disregards the air mass balance if not specified differently within the corresponding paragraphs.

\subsubsection{Hygroscopic and overhygroscopic moisture range}

The range of moisture contents from totally dry to saturation is often subdivided in two: the hygroscopic and the overhygroscopic moisture range (Broken (1998) and Scheffler et al. (2007-a)). The borderline between them is not clearly to be drawn since they pass over into each other. Its definition depends on the certain material properties.

The hygroscopic moisture range is defined between $0 \%$ and $95 \%$ to $98 \%$ of relative humidity. It describes the range, where water molecules are absorbed at the inner material surface with an increasing number of molecular layers but where no continuous liquid phase exists. The vapour transport dominates the liquid water transport or both are of the same order of magnitude, respectively.

The overhygroscopic moisture range is defined from above $95 \%$ to $98 \%$ relative humidity up to saturation. The absorbed water starts to form a continuous liquid phase and capillary condensation occurs. The liquid water transport dominates the transport by vapour diffusion. Normally, moisture content and relative humidity at the point where the sorption isotherm starts to have a strong increase are taken as the transition between both moisture ranges.

\subsubsection{Moisture storage}

Porous media possess the ability to store moisture under a given moisture potential. As moisture potential, either the relative humidity $\varphi$ or the capillary pressure $p_{c}$ can be regarded. For pure water, both can be converted into each other applying the Kelvin equation (2.17). The moisture storage function can hence either be expressed in dependence on the relative humidity $\theta_{l}(\varphi)$ or in dependence on the capillary pressure $\theta_{l}\left(p_{c}\right)$ as shown in Fig. 2.5.

$$
p_{c}(\varphi)=\rho_{l} \cdot R_{v} \cdot T \cdot \ln \varphi \quad \text { Kelvin equation }
$$

Different processes of moisture absorption occur within the different moisture ranges. Starting with a completely dry porous medium, at low relative humidities water molecules are bound at the inner surfaces. Due to the binding forces, first a molecular monolayer establishes. With increasing relative humidity the number of molecular water layers increases until capillary condensation occurs inside the small pores. A further increase of moisture potential causes that also the larger pores fill up and capillary absorption becomes the important mechanism of moisture storage up to saturation.

Strictly speaking, the Kelvin equation is not valid for the whole moisture range since a continuous liquid phase, at least inside the small pores, and thus more than a few molecular water layers at the inner surface is required. However, it offers a good illustration of the moisture storage function within the different moisture ranges, as the effects of water sorption and capillary condensation become apparent in the sorption isotherm and the effect of capillary absorption in the moisture retention characteristic, see Fig. 2.5. 

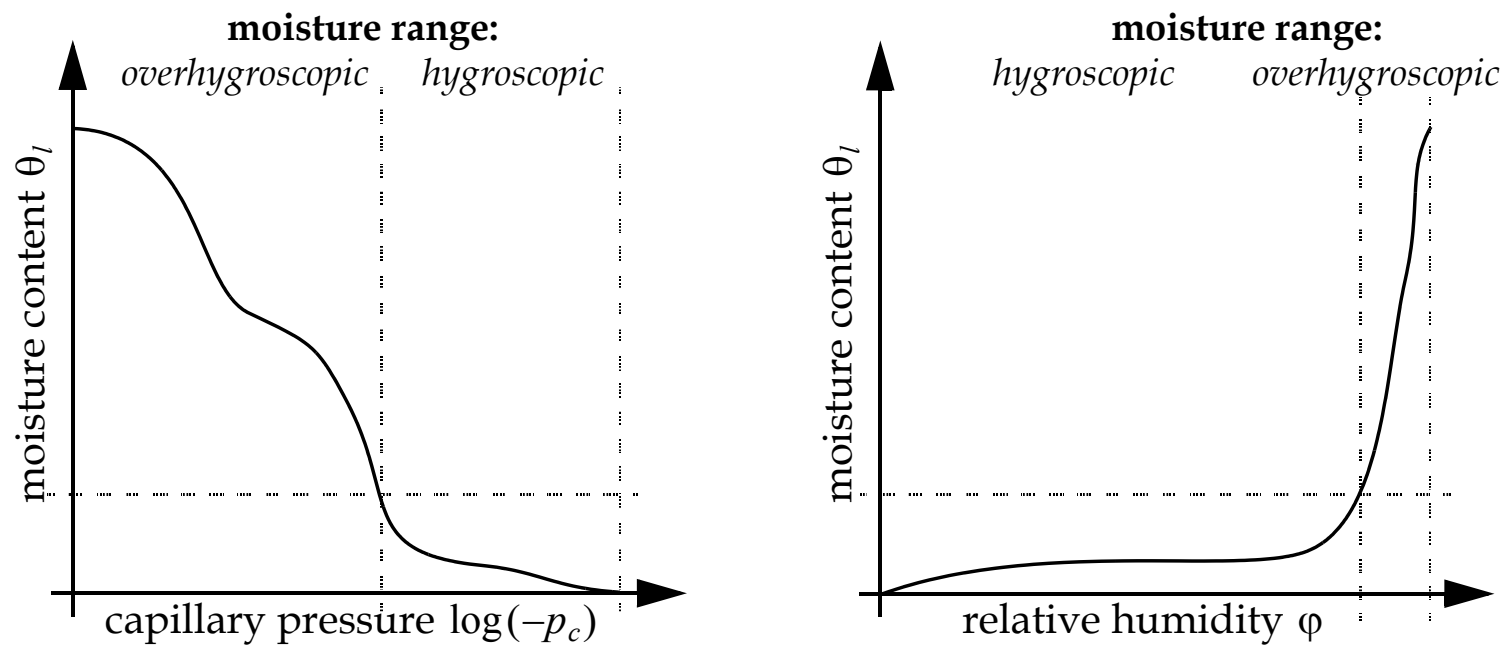

Figure 2.5: $\quad$ Moisture storage function as moisture retention characteristic in dependence to capillary pressure (left) and as sorption isotherm in dependence to relative humidity (right).

The processes of sorption have been studied for a long time. Starting with Langmuir (1918) describing monolayer adsorption, and Brunauer et al. (1938) and Everett (1950) who described the processes of multilayer adsorption, many thermodynamical approaches concerning physisorption have been derived. A well structured overview can be found in Adolphs (1994). Moreover, Adolphs (1994) and Adolphs \& Setzer (1996) derived a new method based on the Gibbs adsorption equation whose validity could be enlarged into the range of capillary condensation. A general description of the processes of moisture storage with respect to Building Physics can be found on behalf of the vast number of publications in Häupl (1987), Pedersen (1990), Galbraith (1992), Krus (1995), Broken (1998) and Espinosa (2004).

The moisture storage function marks equilibrium stages. It is an important material property for material modelling describing the relation between state variable (moisture content) and potential (relative humidity or capillary pressure). Therefore, it represents the connection between the balance and the constitutive equations. Moreover, due to the relation given in eq. (2.14) and the simplification of vanishing dependence to the contact angle, a number of material models use the moisture storage function to derive the liquid water conductivity applying different pore models.

\subsubsection{Hysteresis of moisture storage}

As indicated in Fig. 2.4, the moisture storage function is not a unique but process dependent relation. Although describing stages of equilibrium, the function belonging to a desorption process takes higher values than one following an adsorption process, which is schematically illustrated in Fig. 2.6.

Responsible for this behaviour are a number of effects as

- the ink bottle effect, i.e. big pores are connected to the pore system only via small pores and thus stay filled until the small pores are emptied, 
- $\quad$ entrapped air inside the water filled pore system can not leave, which is an inversion of the ink bottle effect,

- $\quad$ changing contact angles for advancing and receding liquid in a capillary and

- $\quad$ physical or chemical changes of the pore system due to water (i.e. shrinkage and swelling, chemical reactions etc.)

\section{Hysteresis in literature}

A selection of literature available on measurement and modelling approaches to the hysteresis are briefly outlined in the following section. The effect of hysteresis in the relation of moisture content and moisture potential was first reported by Haines (1930).

In Everett \& Whitton (1952) a general discussion of the hysteresis can be found, giving a unified approach to a general modelling of hysteretic effects which is not only limited to adsorption processes. It is called the independent domain model and was further developed by Everett \& Smith (1954), Everett (1954), Everett (1955), Enderby (1955) and Enderby

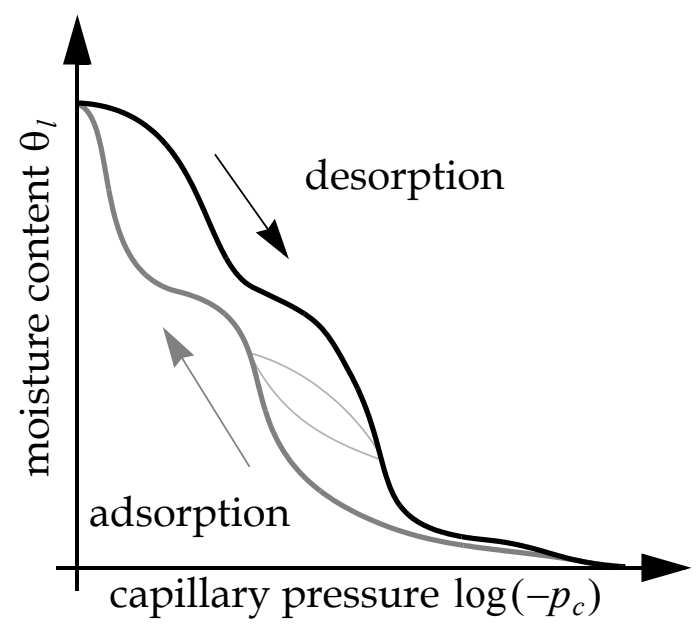

Figure 2.6: Hysteresis of moisture storage. (1956). Poulovassilis carried out experiments with columns of mixed sand fractions to prove the model of independent domains (Poulovassilis (1962) and Poulovassilis (1969)) and investigated the effect of air entrapment in Poulovassilis (1970). The computational model was further simplified by Mualem (1974) and Mualem (1976b) implying that the geometrical relationship of the contact angle is independent of the pore size. The model allowed to predict the moisture content - moisture potential relation only in dependence to the main curves of wetting and drying.

The existence of hysteresis also in the capillary conductivity - water content relation was long disputed. Poulovassilis $\mathcal{E}$ Tzimas (1975) and Tzimas (1979) could prove experimentally a significant hysteretic behaviour for the relation of moisture content and moisture potential and also for the relation of capillary conductivity and moisture potential but found that no hysteresis exists for the relationship of capillary conductivity and moisture content.

Jaynes (1985) investigated four different hysteresis models with emphasis on the pumping effect, indicating that a linear scanning curve would be the algorithm of choice. In Jaynes (1992) a profound discussion on the different existing approaches to model hysteresis is given.

A numerical study on the effect of air entrapment, either applying a hysteresis model or using the main wetting or the main drying curve only, is reported by Kaluarachchi $\mathcal{E}$ Parker (1987). They concluded that even if no profound data is available, the use of a 
simple and low parametric hysteresis model instead of no hysteresis model is to be preferred. Moreover, their simulations showed that the use of the main drying curve produced much larger deviations than the use of the main wetting curve.

Viaene et al. (1994) tested six hysteresis models, the models of Mualem (1974) and Jaynes (1985) among them, and compared their simulation results with own measurements and with data reported in literature. Emphasis was placed on models requiring only input data of either one or both main curves (drying and wetting). The investigation revealed that the conceptual models by Mualem (1974) and Mualem (1984) using the main wetting and the main drying curve as input data delivered the best results. However, the importance of considering the domain interdependency accounted for by Mualem (1984) could not be shown. Of the two examined empirical models, the one pronounced by Jaynes (1985) delivered acceptable results, which could be improved if the modelling parameters were not set fixed but adjusted using measured scanning curve data. Finally, Viaene et al. (1994) concluded that hysteresis models using only one branch do not lead to significantly better results than disregarding hysteresis. In such a case, they suggest to use the model by Parlange (1976) to generate the other branch and then use one of the conceptual 2-branch models.

More recent works on hysteresis are reported e.g. by Simunek et al. (1999) and Beliaev $\mathcal{E}$ Hassanizadeh (2001). Simunek et al. (1999) used cone permeameter measurements to determine the soil moisture retention and the hydraulic conductivity from infiltration. Analysis of the infiltration and redistribution phase allowed to estimate both, wetting and drying curves. They used a simple empirical model for their simulations delivering good agreement. However, only the pressure head characteristics were evaluated, since no water contents were measured. Beliaev $\mathcal{E}$ Hassanizadeh (2001) distinguished between hysteretic effects and dynamic effects. They derived the capillary pressure from thermodynamic considerations to be dependent not only on the pressures of the fluid phases, but also on the temporal rate of change of saturation. Two models are introduced that incorporate two different, rather simple scanning curve relations but account for both, hysteretic and dynamic effects.

For measurement of soil hysteretic behaviour, the instantaneous profile measurement technique was introduced by Plagge (1991) and Sobczuk et al. (1992). The method applies Time Domain Reflectometry (TDR) sensors to measure moisture content and tensiometers to measure moisture potential versus time. Sensors are installed at different sample positions delivering instantaneous profiles of the moisture content - moisture potential relation in time. An error analysis for the derivation of the hydraulic conductivity from these measurements has recently been done by Slawinski et al. (2006).

In Building Physics, hysteresis is mostly neglected. Pedersen (1990) applied a simple hysteresis model in his simulation program but used very simplified material functions. Espinosa (2004) developed a hysteresis model for hardened cement paste and mortar. Unfortunately, this model is valid only in the hygroscopic moisture region.

Plagge et al. (2006b) and Scheffler et al. (2006) applied the instantaneous profile measurement technique also to building materials indicating dependencies of both, hysteresis of moisture storage and an influence of the process dynamics. A summary of mechanisms of sorption and approaches to hysteresis was given by Funk (2006). 


\section{Implications of hysteresis on material modelling}

Although the modelling of hysteretic behaviour is not the task of this work, the hysteresis of moisture storage influences material modelling. As mentioned above, only the relation of moisture content and moisture potential (capillary pressure or relative humidity) is hysteretic but not the relation of capillary conductivity and moisture content. Material models describing the advective liquid water flow in dependence to the capillary pressure according to eq. (2.15) will thus have a transport function which is independent to hysteresis. There, only the moisture storage function contains hysteretic effects. For material models that use Fick's law to describe the advective liquid water flow according to eq. (2.16), however, also the transport function becomes hysteretic, see eq. (2.22).

To avoid the necessity to change the material model - including all adjusted material functions - in case a hysteresis model is included into the transport theory, material modelling should account for the requirements of established hysteresis models. These models normally necessitate at least one main branch, better both, the main wetting and the main drying branch of the moisture storage function. Therefore, material models using the conductivity approach and providing an adsorption and a desorption moisture storage function would be immediately compatible. Material models using the diffusivity approach, on the other hand, would require further modelling since also the liquid transport function is hysteretic.

\subsection{Material Modelling in Building Physics}

With respect to the different approaches to describe liquid water transport, material modelling in Building Physics is divided into diffusivity and conductivity approaches. A literature review on both will be given in the following subsections. The nomenclature of the different approaches is adopted to the general rules of nomenclature which are used here. Therefore the symbols of coefficients and variables might look different of what they were given by the corresponding authors.

\subsubsection{Diffusivity approaches}

Based on the works of Krischer (see e.g. Krischer (1940), Krischer (1942) and Krischer (1956)), the diffusion equation (2.8) was often applied in Building Physics, not only for vapour but also for liquid moisture transport according to eq. (2.16). Before numerical simulation was possible the way it is today, analytical solutions were required for both, heat and mass transfer. One big advantage of the diffusion equation is that under isothermal conditions the transport equations for moisture can be solved analytically, as e.g. shown by Häupl (1987). The moisture profiles can be described by a functional approach where the diffusivity function can be derived from. The basic ideas of this method are briefly described before reviewing on functional approaches for the liquid water diffusivity found in literature. 


\section{Diffusivity approach in general}

Diffusivity approaches are normally derived from moisture profiles of water uptake or drying experiments under isothermal conditions, see e.g. Häupl (1987), Pel et al. (1996), Hansen $\mathcal{E}$ Hansen (2002) and Carmeliet et al. (2004). Inserting the diffusion equation (2.16) into the balance equation (2.2) yields eq. (2.18) under negligence of source production rates and the advective and diffusive vapour transport. In this equation, the density of the liquid phase $\rho_{l}$ is reduced, leading to the volumetric water content $\theta_{l}$.

$$
\frac{\partial \theta_{l}}{\partial t}=\frac{\partial}{\partial x}\left[D_{l}\left(\theta_{l}\right) \cdot \frac{\partial \theta_{l}}{\partial x}\right]
$$

By the help of the Boltzmann transformation, the spatial and the time variable can be transformed into one new variable, commonly called $\lambda$, according to eq. (2.19).

$$
\lambda=\frac{x}{\sqrt{t}}
$$

When determining both derivatives and applying it to eq. (2.18), the diffusion equation can be written as follows. By that, this partial differential equation reduces to an ordinary differential equation depending only on one variable.

$$
2 \frac{\mathrm{d}}{\mathrm{d} \lambda}\left[D_{l}\left(\theta_{l}\right) \cdot \frac{\mathrm{d} \theta_{l}}{\mathrm{~d} \lambda}\right]=\lambda \frac{\mathrm{d} \theta_{l}}{\mathrm{~d} \lambda}
$$

Now, eq. (2.20) can be transformed into eq. (2.21), where the diffusivity can be directly calculated from measured moisture profiles. Typically, all moisture profiles fall together forming only one curve in the $\theta_{l}(\lambda)$ scale. The area under that curve is the liquid water diffusivity.

$$
D_{l}\left(\theta_{l}\right)=\frac{1}{2} \cdot \frac{\mathrm{d} \lambda}{\mathrm{d} \theta_{l}} \int_{\theta_{0}}^{\theta_{l}} \lambda d \theta_{l}
$$

As indicated above, this derivation is valid only under isothermal conditions and within a moisture content range in which the diffusivity function can be defined. Therefore, the validity range is restricted to the moisture contents which occur within the measured moisture profiles. The lower integration boundary is normally set to $\theta_{0}=0$, while the upper integration boundary is set to the saturation water content $\theta_{s}$.

The maximum water content reached during water uptake experiments is often called the capillary saturation water content $\theta_{\text {cap }}$. In its strict sense it is the highest water content occurring within the moisture profiles, which is typically reached at the imbibition surface. However, since often moisture profiles are not available, the water content at the end of the water uptake experiment or at that time, where the absorbed water mass deviates from the linear increase in a square root of time scale, are considered as $\theta_{\text {cap }}$. Looking at measured moisture profiles reveals, that this is only an approximation 
where especially the simplification of taking the water content at the bend of water uptake might lead to an underestimation of this water content. For the maximum water content of the diffusivity approaches, the denotation $\theta_{s}$ is used here due to the different possibilities of its determination and inconsistent definition found in literature.

The water diffusivity function can be calculated from measured moisture content profiles according to eq. (2.21). On that basis, numerous approaches have been developed to describe the found shape of the diffusivity in general. Those predefined functions typically contain free parameters that can be adjusted either using measured moisture profiles or measured integral water uptake curves. Depending on the particular approach, the lower integration boundary is often set to a value $\theta_{0}>0$ and $\theta_{s}$ is determined by one of the possible ways discussed.

Calculating liquid transport with the aid of the diffusion equation (2.16) or (2.18) does not require the application of the moisture storage characteristic to determine the moisture potential. Instead, the gradient of water content itself is regarded to be the driving force of liquid transport. Thus, the moisture storage function is not as an important material property for diffusivity approaches as it is in case the liquid water conductivity is used. An explicit function or description of the moisture storage characteristic is therefore rarely given in literature when dealing with water diffusivity.

\section{Connecting Diffusivity and Conductivity}

In case of thermodynamic equilibrium, both transport functions, the liquid diffusivity $D_{l}\left(\theta_{l}\right)$ and the liquid conductivity $K_{l}\left(\theta_{l}\right)$, can be converted into each other via the derivative of the inverse moisture storage function $p_{c}\left(\theta_{l}\right)$ as given in eq. (2.22).

$$
D_{l}\left(\theta_{l}\right)=\frac{K_{l}\left(\theta_{l}\right)}{\rho_{l}} \cdot \frac{\mathrm{d}}{\mathrm{d} \theta_{l}} p_{c}\left(\theta_{l}\right)
$$

Apparently, the hysteresis of the moisture storage function would lead to different diffusivities depending on the branch used for this calculation. As discussed above, the liquid water diffusivity would have to be also process dependent if the hysteresis of moisture storage is to be included into a diffusivity approach.

For a dispartment of vapour and liquid transport, diffusivity and conductivity values are often calculated from vapour diffusion measurements. The vapour diffusivity can be calculated according to equations (2.11) and (2.12) in dependence to the vapour diffusion resistance factor $\mu$. Determined from dry cup measurements (refer to DIN EN ISO 12572), the vapour diffusion resistance factor $\mu_{d r y}$ is normally used to determine the vapour diffusivity. Cup measurements at higher relative humidities, called wet cup measurements, deliver $\mu$-values depending on both, liquid and vapour transport. Thus, from the different transport rates of wet cup and dry cup measurements, the liquid transport at the corresponding relative humidities of the wet cup measurements can be derived.

$$
D_{v}(\mu)=D_{v, \text { air }} \cdot \frac{1}{\mu}
$$




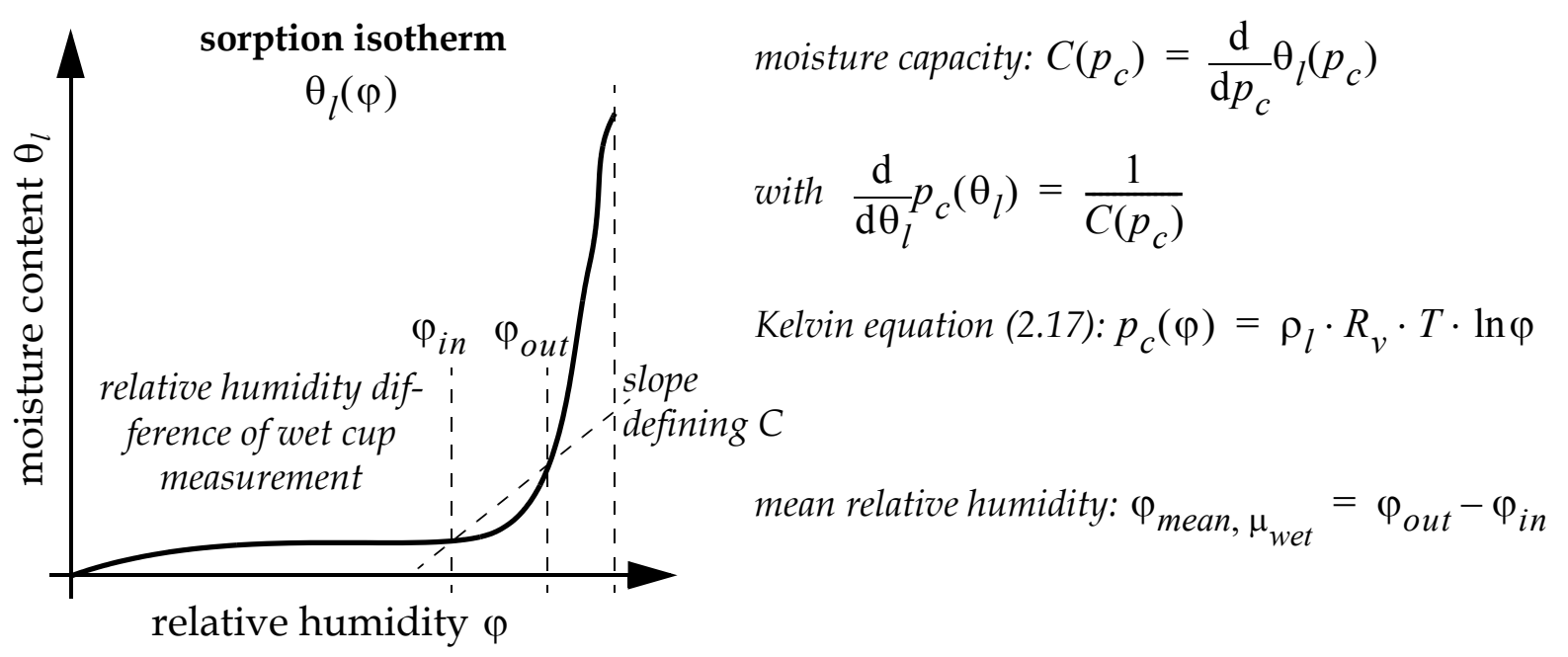

Figure 2.7: Definition of quantities required for calculation of liquid water conductivity and diffusivity in dependence to wet cup vapour diffusion measurements.

The vapour diffusivity according to eq. (2.23) is associated with the vapour pressure gradient. Relating it to the capillary pressure requires the multiplication with the ratio of vapour and water density $\rho_{v} / \rho_{l}$, leading to the vapour conductivity $K_{v}$ specified in eq. (2.24), where $p_{v, s}(T)$ is the saturation vapour pressure depending on temperature.

$$
K_{v}(\mu)=\frac{D_{v}(\mu)}{R_{v} T} \cdot \frac{1}{\rho_{l}} \cdot \frac{\varphi_{\text {mean }, \mu} \cdot p_{v, s}(T)}{R_{v} T}
$$

At the common conditions of dry cup measurements, moisture transport arises only as vapour diffusion. For $\mu_{d r y}$ and the corresponding mean relative humidity and temperature, eq. (2.24) thus delivers the vapour conductivity. For wet cup $\mu$-values however, inserting the temperature and mean relative humidity of the corresponding measurements, eq. (2.24) gives the moisture conductivity $K_{l+v}$. The difference of both, moisture and vapour conductivity, yields the liquid water conductivity at the mean moisture potential chosen for the wet cup measurements according to eq. (2.25).

$$
K_{l}\left(\varphi_{\text {mean }, \mu_{w e t}}\right)=K_{l+v}\left(\mu_{w e t}\right)-K_{v}\left(\mu_{d r y}\right)
$$

Using eq. (2.22), the liquid water diffusivity can be calculated from this conductivity value according to eq. (2.26). The slope of the inverse moisture storage function $1 / C$ in the corresponding relative humidity range is required for this calculation. An illustration of the required quantities is given in Fig. 2.7.

$$
D_{l}\left(\varphi_{\text {mean } \left., \mu_{w e t}\right)}\right)=\frac{K_{l}\left(\varphi_{\text {mean }, \mu_{\text {wet }}}\right)}{\rho_{l}} \cdot \frac{\mathrm{d}}{\mathrm{d} \theta_{l}} p_{c}\left(\theta_{l}\left(\varphi_{\text {mean }, \mu_{\text {wet }}}\right)\right)
$$




\subsubsection{Diffusivity approach according to Häupl}

In previous publications, Häupl dealt with analytic solutions for the differential equation of isothermal moisture transport, as it is given in eq. (2.18). In Häupl (1987), besides a number of more simplified approaches for special cases, he introduced the so called standard diffusivity function which is given in eq. (2.27) and in Fig. 2.8 at the left. For this standard diffusivity function, as well as for the second diffusivity approach according to Häupl \& Fechner (2003a), it is possible to determine an analytic solution of the water uptake problem.

$$
D_{l}\left(\theta_{l}\right)=D_{s} \cdot\left[(k+1) \cdot\left(\frac{\theta_{l}}{\theta_{s}}\right)^{\frac{1}{k}}-k \cdot\left(\frac{\theta_{l}}{\theta_{s}}\right)^{\frac{2}{k}}\right] \quad \text { Häupl's standard diffusivity }
$$

There, the parameter $k$ is a shape factor moving the strong slope of the diffusivity function to lower water contents for $k \rightarrow 1$ and to higher water contents for $k \rightarrow 0.1$. If $k$ takes values $k>1$, the diffusivity function will have a maximum moving in direction of $\theta_{l}=0$ for increasing values of $k$. The parameter $D_{s}$ marks the diffusivity value at saturation where $\theta_{l}=\theta_{s}$. In Häupl et al. (1999), relation (2.28) is given allowing to calculate $D_{s}$ in dependence to $k$ and to the water uptake coefficient $A_{w}$.

$$
D_{s}=\left(\frac{A_{w}}{\rho_{l} \cdot \theta_{s}}\right)^{2} \cdot \frac{k+1}{2 k}
$$

A second diffusivity approach reported by Häupl $\mathcal{E}$ Fechner (2003a) is given in eq. (2.29) and in Fig. 2.8 at the right. As an additional parameter, a fixed water content $\theta_{l}\left(\varphi_{0}\right)$ is introduced at which liquid water transport inside the material begins. It is specified to be the sorption moisture content of $\varphi_{0}$, taking values between $35 \%$ and $80 \%$ relative humidity. It is not fixed but has to be either adjusted or chosen depending on available material data. For $D_{s}$ a slightly different relation is given with eq. (2.30) depending on the water uptake coefficient $A_{w}$, the parameter $k$ and on $\theta_{l}\left(\varphi_{0}\right)$. Häupl gives a relation for the parameter $k$ depending on the water penetration coefficient $B=x_{E} /(\sqrt{t})$. But as the influence is rather small and the penetration depth of the water front $x_{E}$ also requires to measure moisture profiles, Häupl $\mathcal{E}$ Fechner (2003a) propose to set $k$ fixed to $k=3$.

$$
\begin{aligned}
& D_{l}\left(\theta_{l}\right)=D_{s} \cdot\left[\left[1-\frac{\theta_{l}-\theta_{l}\left(\varphi_{0}\right)}{\theta_{s}-\theta_{l}\left(\varphi_{0}\right)}\right]^{\frac{1}{k}-1}-\left[1-\frac{\theta_{l}-\theta_{l}\left(\varphi_{0}\right)}{\theta_{s}-\theta_{l}\left(\varphi_{0}\right)}\right]^{\frac{2}{k}}\right] \\
& D_{s}=\left(\frac{A_{w}}{\rho_{l} \cdot\left(\theta_{s}-\theta_{l}\left(\varphi_{0}\right)\right)}\right)^{2} \cdot \frac{k+1}{2 k}
\end{aligned}
$$



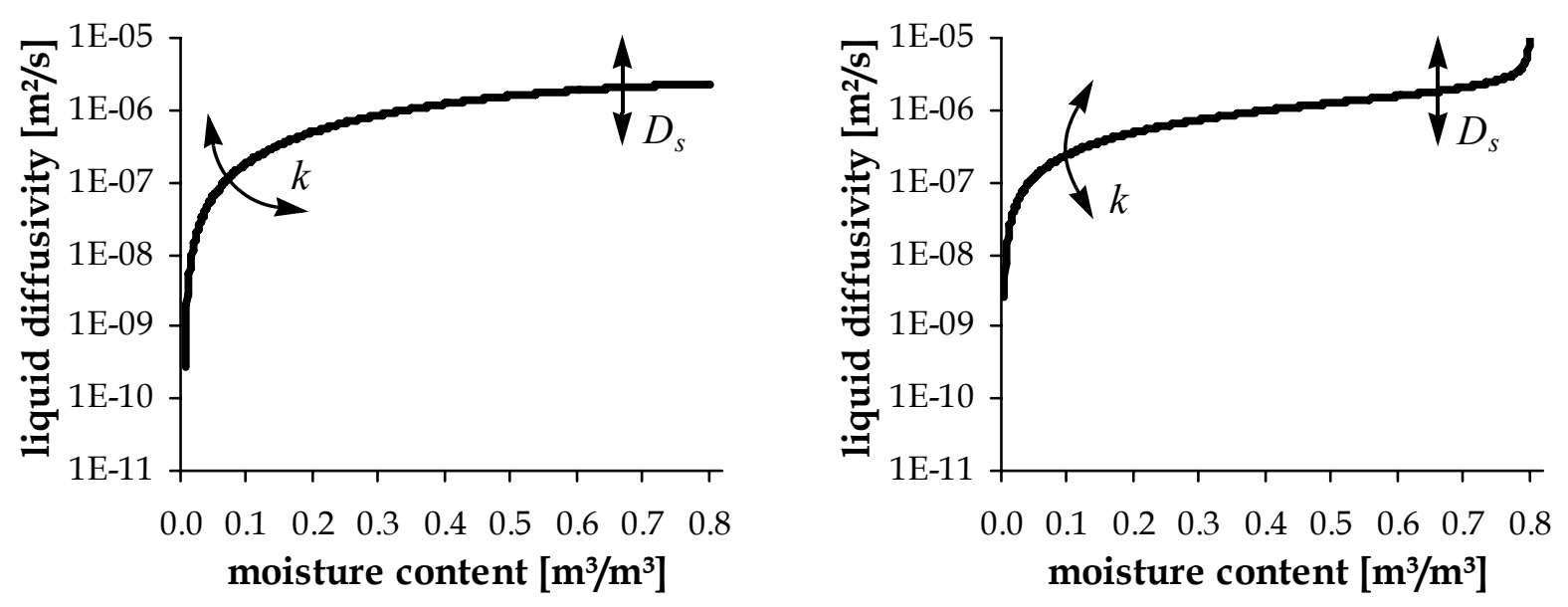

Figure 2.8: $\quad$ Standard water diffusivity according to Häupl (1987) at the left and liquid water diffusivity according to Häupl E Fechner (2003a) at the right.

The standard diffusivity function (2.27) is very flexible and, thus, suitable for most building materials. Measured data of a water uptake experiment is required to determine $D_{s}$. The parameter $k$ can be adjusted by simulating the experiment or by the aid of measured moisture profiles. Typical values of $k$ for a number of materials are specified in Häupl (1987) and Häupl et al. (1999). However, parameter identification is difficult if no numerical simulation of experiments due to lack of data and no "qualified guessing" is applied. In addition, the standard diffusivity function tends to overestimate liquid water transport in the lower moisture range.

The modified diffusivity function (2.29) prevalently holds with higher diffusivity values at lower water contents tightening the problem of overestimation in that moisture range. In general, this function applies well to the physical definition of a diffusivity, i.e. comprising a codomain from zero to infinity. It therefore follows the typical increase of a diffusivity at saturation, as it was proved by measured data e.g. by Pel (1995), Pel et al. (1996) and Krus (1995), much better than other models. However, with regard to the lower moisture content range, the approach is suitable only for a limited selection of materials. The parameters of this function can be derived from basic standard experiments which are usually available. If, as proposed, $k$ is set to $k=3$, only the water uptake coefficient $A_{w}$ and the moisture content $\theta_{l}\left(\varphi_{0}\right)$ remain, where the choice of $\varphi_{0}$ is of lower influence and can be set to a sorption value between $35 \%$ and $80 \%$ relative humidity. The fixing of $k$ leads to less flexibility though.

For the description of vapour transport, Häupl uses the reduction of vapour diffusion in air by the vapour diffusion resistance factor $\mu$ as given in eq. (2.12) and eq. (2.13). A lucid discussion on the dependency of $\mu$ on the one hand and the vapour diffusivity $D_{v, \text { mat }}$ on the other hand to the moisture content can be found in Häupl (1987). However, a vapour transport function depending on the moisture content is not specified there.

Since the moisture storage function does not play the central role within the diffusivity approaches, a functional description, given in eq. (2.31), can only be found in Häupl $\mathcal{E}$ Fechner (2003a) where it is also used for a conductivity approach. The function is based on a sum of distribution functions. The integral of the pore volume distribution function is considered as the moisture storage function. 


$$
\theta_{l}\left(p_{c}\right)=\sum_{j=1}^{N}\left(1-\left(1+\left(\frac{2 \sigma}{p_{c} \cdot R_{j}}\right)^{2}\right)^{1-n_{j}}\right) \cdot \theta_{n j} \quad \text { moisture storage function }
$$

Here, the parameter $N$ is the modality which equals the number of maxima in the pore radii distribution. $R_{j}$ are the pore radii at the maximum positions, $\theta_{n j}$ are the volumetric moisture contents corresponding to those maxima and $n_{j}$ are shape parameters specifying the steepness in the moisture storage function. All parameters are to be adjusted to measured sorption and retention data.

\subsubsection{Diffusivity approach according to Pel}

In Pel (1995), the description of liquid water and water vapour transport mechanisms is outlined phase separated. Effects as hysteresis are discussed as well as the influence of surface diffusion and liquid water islands on vapour transport. Pel specifies a vapour diffusivity function with several parameters considering an increase due to the mentioned effects. However, neither this function, nor the phase separated approach in general is used. Instead, he defines a moisture diffusivity $D_{l+v}$ accounting for both, liquid and vapour transport. Therefore, he introduced an exponential function containing two adjustable parameters. It is given in eq. (2.32) in its normalized form.

$$
D_{l+v}\left(\theta_{l}\right)=D_{0} \cdot \exp \left[\beta \cdot \theta_{s} \cdot \frac{\theta_{l}}{\theta_{s}}\right]
$$

The parameter $D_{0}$ is the minimum diffusivity at $\theta_{0}$. The parameter $\beta$ delivers the slope of the function. Pel uses both parameters to adjust the diffusivity function to measured moisture profiles. In Pel (1995) examples of the parameter values are given for a couple of materials.

Although restricted to the exponential function, the approach has a good flexibility. However, it requires either the measurement of moisture profiles as given by $\mathrm{Pel}$ (1995) and Pel et al. (1996) or a combination of measured data and numerical simulation to adjust the parameters correctly. Especially for $D_{0}$ this might be difficult, if no vapour diffusion or drying data is available. Thus, the approach is not suitable for simplified simulation where only few parameters are known. An explicit description of the moisture storage function is not given by $\mathrm{Pel}$ (1995). 


\subsubsection{Diffusivity approach according to Kießl and Künzel}

On the basis of the theory by Philip \& De Vries (1957), De Vries (1958) and other authors, heat and moisture transport has been modelled phase separated by Kießl (1983). He defined several transport coefficients for liquid water and water vapour transport. However, their practical determination is not elaborated. Instead he only refers to publications concerning moisture property measurement.

Following Kießl (1983), the material coefficients were simplified with regard to their practical determinability by Künzel (1994). For the moisture storage function, a simple rational approach containing two parameters is given in eq. (2.33). One is the moisture content at free water saturation $\theta_{s}$. The other parameter, called $b$, has to be adjusted to measured sorption and retention data.

$$
\theta_{l}(\varphi)=\theta_{s} \frac{(b-1) \varphi}{b-\varphi}
$$

The hysteresis of moisture storage is briefly discussed by Künzel. Based on measured moisture storage data in the hygroscopic moisture range and on conclusions drawn by Pedersen (1990), he regards the hysteresis in the moisture storage function to be negligible. However, inconsistency is to be stated in the discussion of Künzel (1994).

The investigations reported by Pedersen were done with the conductivity, not with the diffusivity approach. He used very simplified material functions and performed simulations for different structural applications. In these special cases, the influence of the hysteresis was relatively small, which is the reason for the conclusions in Pedersen (1990), who did not perform a sensitivity study on hysteresis.

On the other hand, Künzel (1994) proposes to consider the impact of the hysteresis, which in case of the diffusivity approach is also assigned to the transport function, see discussion above in section Connecting Diffusivity and Conductivity. He discusses the necessity to define two diffusivity functions, one for suction (adsorption) and one for further distribution, when no water contact is applied to the material (desorption).

For the liquid water transport coefficient in general, Künzel refers for the hygroscopic moisture range to a relation calculating the liquid water diffusivity from vapour diffusion measurements at higher relative humidities (wet cup measurements) and for the capillary moisture range to the determination from measured moisture profiles. Unfortunately it is not specified how to combine them. However, a simplified approach in dependence to the water uptake coefficient $A_{w}$ and the saturation moisture content $\theta_{s}$ is given according to eq. (2.34). $D_{l, s}$ describes the liquid water diffusivity for suction which is valid, if water contact is applied.

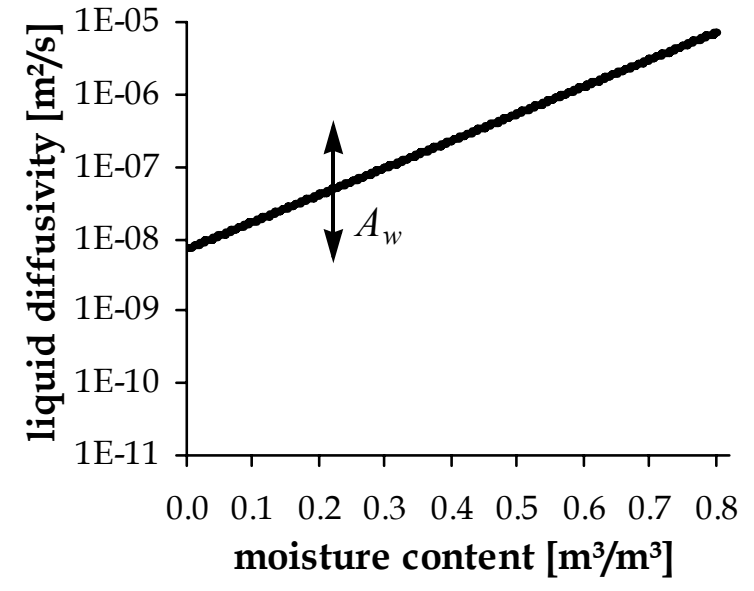

Figure 2.10: $\quad$ Liquid water diffusivity according to Künzel (1994). 


$$
D_{l, s}\left(\theta_{l}\right)=3.8 \cdot\left(\frac{A_{w}}{\rho_{l} \theta_{s}}\right)^{2} \cdot 1000^{\frac{\theta_{l}}{\theta_{s}}-1}
$$

A diffusivity function for further distribution $D_{l, w}$, as discussed by Künzel (1994), is not given explicitly. A remark states that for $\theta_{l}=\theta_{s} \quad D_{l, w}$ would be approximately one order of magnitude lower than $D_{l, s}$.

In contrast to Kießl (1983), who defined a moisture content dependent vapour diffusion coefficient with a distinct maximum due to short-cuts at capillary water islands (an argumentation introduced by Philip \& De Vries (1957)), Künzel (1994) denies this dependency. Instead he uses a constant vapour diffusion coefficient according to eq. (2.12) which does not depend on the moisture content. Although his discussion concerning an increasing vapour diffusion coefficient as pronounced by Kießl (1983) is conclusive, the reduction of available cross section due to an increase of moisture content is not considered by Künzel (1994).

\subsubsection{Diffusivity approach according to Krus and Holm}

Based on the developed numerical modelling of heat and moisture transport by Künzel (1994) and the experimental methods for determination of material properties according to Krus (1995), a more flexible diffusivity approach was introduced by Krus \& Holm (1999). They apply an exponential formulation, which is given in eq. (2.35).

$$
D_{l}\left(\theta_{l}\right)=D_{0} \cdot \exp \left(\frac{\theta_{l}}{\theta_{s}} \cdot \ln \frac{D_{s}}{D_{0}}\right)
$$

An example of this approach is shown in Fig. 2.11. In logarithmic scale, eq. (2.35) forms a linear function between the two points $D_{0}$ and $D_{s}$. According to the specifications in Krus $\mathcal{E}$ Holm (1999), $D_{0}$ can be determined from the difference in moisture flux between dry cup and wet cup experiments as it was explained above in section Connecting Diffusivity and Conductivity. An evaluation of these experiments for different building materials, reported in Krus (1995), revealed that $D_{0}$ often takes values between $1.1 \cdot 10^{-10}$ and $3.5 \cdot 10^{-10}$. For this reason, as an approximation, Krus and Holm proposed to generally set $D_{0}$ to $D_{0}=2.0 \cdot 10^{-10}$. This diffusivity value is actually associated with the sorption moisture content at the mean relative humidity of the wet cup experiment used to determine $D_{0}$. But, since this moisture

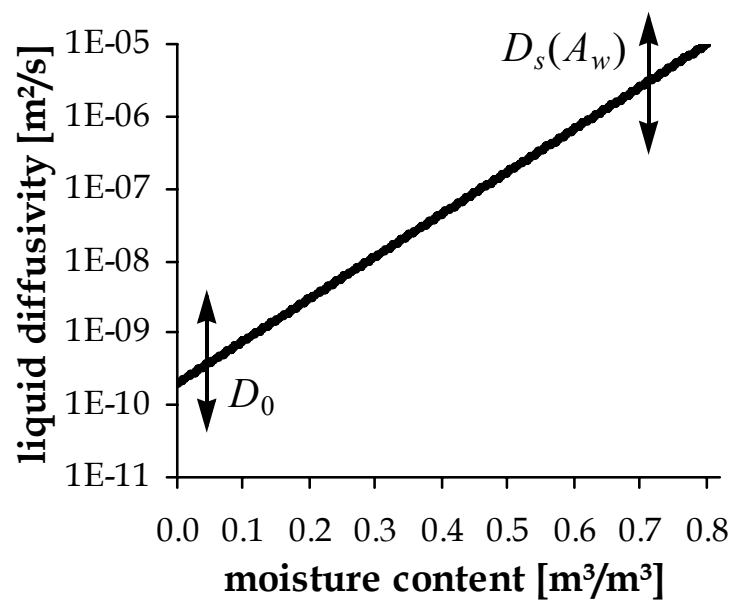

Figure 2.11: Liquid water diffusivity according to Krus \& Holm (1999). 
content might often not be available, it is recommended to regard it to the moisture content at $80 \%$ relative humidity.

For the determination of the diffusivity value at saturation $D_{s}$, Krus $\mathcal{E}$ Holm (1999) give an implicit formulation according to eq. (2.36).

$$
\begin{gathered}
D_{s}=\frac{K \cdot \pi \cdot A_{w}^{2} \cdot \ln \frac{D_{s}}{D_{0}}}{4 \cdot \theta_{s} \cdot\left[\theta_{s}-\theta_{l}(\varphi=0.8)\right] \cdot \rho_{l}^{2}}+D_{0} \\
\text { with } K=0.025 \cdot 10\left(-0.4 \cdot\left[\log \frac{D_{s}}{D_{0}}-3\right]\right)+0.61
\end{gathered}
$$

Equation (2.36) can be rearranged to eq. (2.37) separating the known material parameters to the right hand side and the unknown parameter $D_{s}$ to the left hand side. The known material parameters are the water uptake coefficient $A_{w}$ and the moisture contents $\theta_{l}(\varphi=0.8)$ at $80 \%$ relative humidity and $\theta_{s}$ at saturation. The evaluation of eq. (2.37) can either be done by iteration or by graphical evaluation. A diagram for graphical evaluation can be found in Krus \& Holm (1999).

$$
\frac{4 \cdot\left(D_{s}-D_{0}\right)}{K \cdot \pi \cdot \ln \frac{D_{s}}{D_{0}}}=\frac{A_{w}^{2}}{\theta_{s} \cdot\left[\theta_{s}-\theta_{l}(\varphi=0.8)\right] \cdot \rho_{l}^{2}}
$$

As already done by Künzel (1994), the authors also propose the use of two diffusivity functions, one for capillary suction under water contact and one for liquid water transport without water contact (further distribution), see also Fig. 2.12. Both diffusivity functions are calculated according to eq. (2.35) applying different values of saturation diffusivity $D_{s}$.

For capillary suction, the diffusivity at saturation $D_{s, s}$ is calculated following eq. (2.36) and eq. (2.37) as discussed above. The saturated diffusivity for further distribution $D_{s, w}$ is to be adjusted by numerical simulation based on drying experiments.

This diffusivity approach was also applied and described by Holm (2001). He adopted most of the reasoning from Künzel (1994) and Krus (1995), especially concerning the hysteresis of moisture storage (see discussion under paragraph 2.2.1.3 Diffusivity

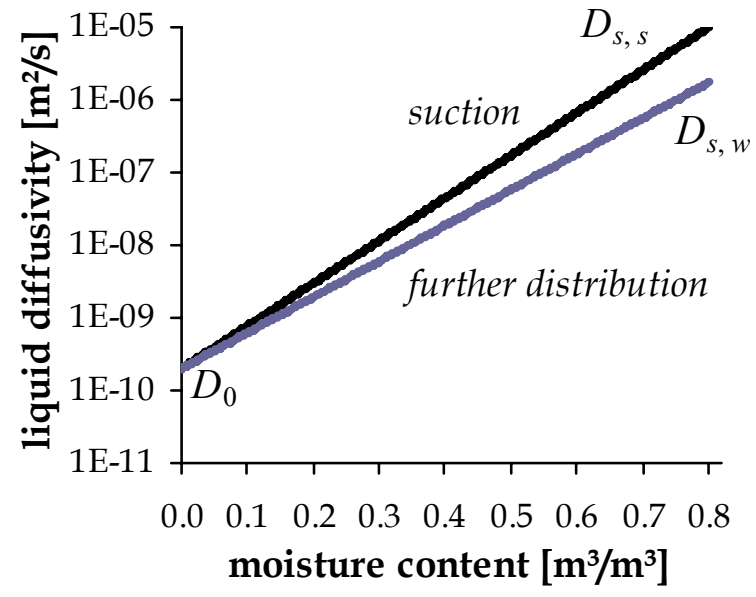

Figure 2.12: Liquid water diffusivities for suction and further distribution according to Krus \& Holm (1999). 
approach according to Kießl and Künzel). For vapour diffusion, Holm utilised the constant description from Künzel (1994). The discussion of moisture storage was also adopted. However, an empirical relation containing two adjustable parameters was introduced. It is dependent to the capillary pressure and given in eq. (2.38). The parameters $p_{k 1}$ and $p_{k 2}$ are to be adjusted by the aid of measured sorption moisture contents at $80 \%$ and $95 \%$ relative humidity. Instead of the value at $95 \%$ also another value at a preferably high relative humidity can be chosen.

$$
\theta_{l}\left(p_{c}\right)=\frac{\theta_{s}}{1+\left(\frac{p_{c}}{p_{k 1}}\right)^{p_{k 2}}}
$$

The approach for the diffusivity is bound to the exponential function. At low moisture contents, the adjustment of $D_{0}$ by the aid of vapour diffusion measurements can be done properly. The recommendation to set $D_{0}=2.0 \cdot 10^{-10}$ gives a general approximation in case no measured data exists, although larger deviations can occur for some materials. Calculations with own material data revealed a variation of $D_{0}$ between $D_{0}=4.0 \cdot 10^{-11}$ (for a lime-sand brick) and $D_{0}=3.2 \cdot 10^{-08}$ (for a calcium silicate). The diffusivity value at saturation $D_{s}$ can be determined rather easily for suction. For further distribution, however, numerical simulation has to be applied in combination with measured drying data. Moreover, the jump between two transport functions is not a sophisticated solution of the hysteresis problem requiring some kind of empirical transfer criterion. For simplified calculations where only few material data is available, as it occasionally occurs with literature data, the approach is expedient.

\subsubsection{Diffusivity approach according to Carmeliet and Janssen}

Two further diffusivity approaches were reported by a consortium of authors in Carmeliet et al. (2004). One is a general exponential function which is comparable to the one published by $\mathrm{Pel}$ (1995). The other is a sum of a decaying function in the lower moisture range and an increasing exponential one in the higher moisture range. An evaluation of those approaches is already given by Scheffler et al. (2007-a). Since both of them were further improved by Carmeliet $\mathcal{E}$ Janssen (2007), only these ones will be treated here.

The general or, as it is called by Carmeliet $\mathcal{E}$ Janssen (2007), simplified diffusivity approach accounts for the liquid water diffusivity and is defined in eq. (2.39). An example chart is given in Fig. 2.13.

$$
D_{l}\left(\theta_{l}\right)=D_{s} \cdot \exp \left[F \cdot\left(\frac{\theta_{l}-\theta_{s}}{\theta_{s}}\right)\right]
$$

The diffusivity function contains two parameters, the diffusivity value at saturation $D_{s}$ giving the maximum value at $\theta_{s}$ and the parameter $F$ which influences the slope. For $D_{s}$ a relation is given in eq. (2.40) depending on $F$ and the water uptake coefficient $A_{w}$. 


$$
D_{s}=f(F) \cdot\left(\frac{A_{w}}{\rho_{l} \cdot \theta_{s}}\right)^{2}
$$

The function $f(F)$ can be calculated according to eq. (2.41).

$$
f(F)=\frac{F^{2}}{2 F-\left(F-1+\left(\frac{3}{2}-\frac{4}{\pi}\right) \cdot F^{2} \cdot \exp F\right)}
$$

Due to relation (2.40) together with eq. (2.41), the approach consists of only one adjustable parameter. The authors recommend to adjust $F$ by the aid of measured moisture profiles, water uptake or drying data. Moreover, in case no further data is available, $F$ can be set to $F=7.5$ which often gives good results.

The approach is restricted to the exponential slope specified by $F$. It does not require much input data, especially if $F$ is estimated. Thus, it is suitable as a simplified approach with the same limitations as discussed already in the previous paragraphs.

The improved diffusivity model of Carmeliet $\mathcal{E}$ Janssen (2007) is defined as the moisture diffusivity in logarithmic scale. It consists of two functions $L D_{I}$ and $L D_{I I}$ given in equations (2.42) and (2.43). $L D_{I}$ describes the course of the diffusivity from dry state up to a limiting moisture content $\theta_{\text {lim }}$, whereas $L D_{I I}$ accounts for the wet moisture

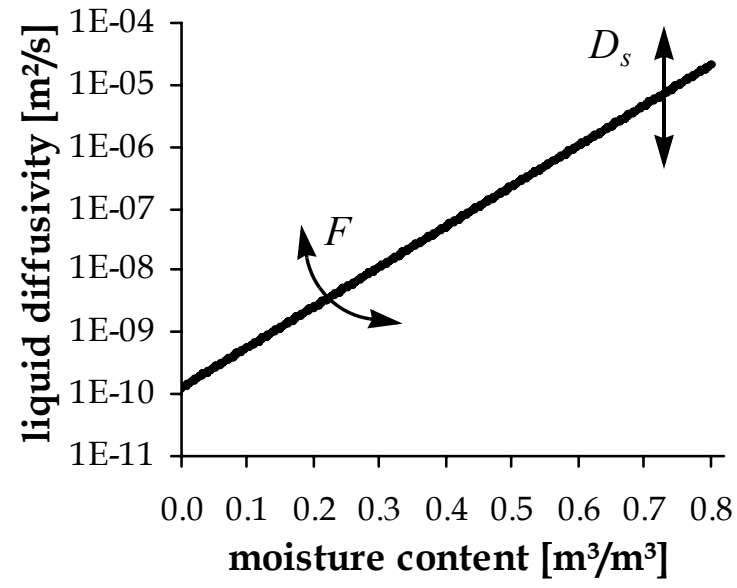

Figure 2.13: Simplified diffusivity according to Carmeliet $\mathcal{E}$ Janssen (2007). range from $\theta_{\text {lim }}$ up to saturation $\theta_{s}$.

$$
\begin{aligned}
& L D_{I}\left(\theta_{l}\right)=a+\left[L D_{0}-a\right] \cdot \exp \left(-b \cdot \rho_{l} \cdot \theta_{l}\right)+c \cdot \rho_{l} \cdot \theta_{l} \quad \text { for } \quad \theta_{l}<\theta_{\text {lim }} \\
& L D_{I I}\left(\theta_{l}\right)=L D_{s}+F \cdot\left(\frac{\theta_{l}-\theta_{s}}{\theta_{s}}\right) \quad \text { for } \quad \theta_{l} \geq \theta_{l i m}
\end{aligned}
$$

The final moisture diffusivity can be calculated according to eq. (2.44) where the functions of equations (2.42) and (2.43) have to be inserted according to the respective moisture content $\theta_{l}$ for $L D\left(\theta_{l}\right)$.

$$
D_{l+v}\left(\theta_{l}\right)=10^{L D\left(\theta_{l}\right)}
$$

The approach contains a total of eight parameters. The diffusivity value at saturation $L D_{s}=\log D_{s}$ can be determined by the aid of the water uptake coefficient $A_{w}$, the sat- 
uration moisture content $\theta_{s}$ and in dependence to $F$ from eq. (2.40). Due to the demand of continuity at $\theta_{l}=\theta_{\text {lim }}, L D_{I}$ and $L D_{I I}$ can be equated allowing to express $c$ in dependence to the other parameters according to eq. (2.45).

$$
c=\frac{1}{\rho_{l} \theta_{l i m}} \cdot\left(L D_{s}+F \cdot\left(\frac{\theta_{l i m}-\theta_{s}}{\theta_{s}}\right)-a-\left[L D_{0}-a\right] \cdot \exp \left(-b \rho_{l} \theta_{l i m}\right)\right)
$$

This leaves the five parameters $a, b, \theta_{\text {lim }}, L D_{0}$ and $F$ to be determined. Fig. 2.14 shows the improved diffusivity function and indicates the influence of its parameters. The authors propose to use values of three vapour diffusion measurements to adjust $L D_{0}$, $a$ and $b$. The parameter $F$ is preferably adjusted by the aid of measured moisture profiles during a water uptake experiment. The remaining limiting moisture content $\theta_{\text {lim }}$ is to be derived from the drying experiment by numerical simulation, which is only possible, if the diffusivity in the high moisture range was already adjusted correctly.

Carmeliet $\mathcal{E}$ Janssen (2007) compared their improved diffusivity approach with the diffusivity function derived from a moisture conductivity ${ }^{1}$ which was regarded to as the actual moisture transport function. From a stepwise parameter identification according to different levels of available data, they concluded that a general application of this diffusivity function, e.g. to calculate hygroscopic loading, a capillary absorption or a drying, is only reliable if its parameters were adjusted to the whole list of material data given above.

The advantage of this approach is the high flexibility, especially in the lower

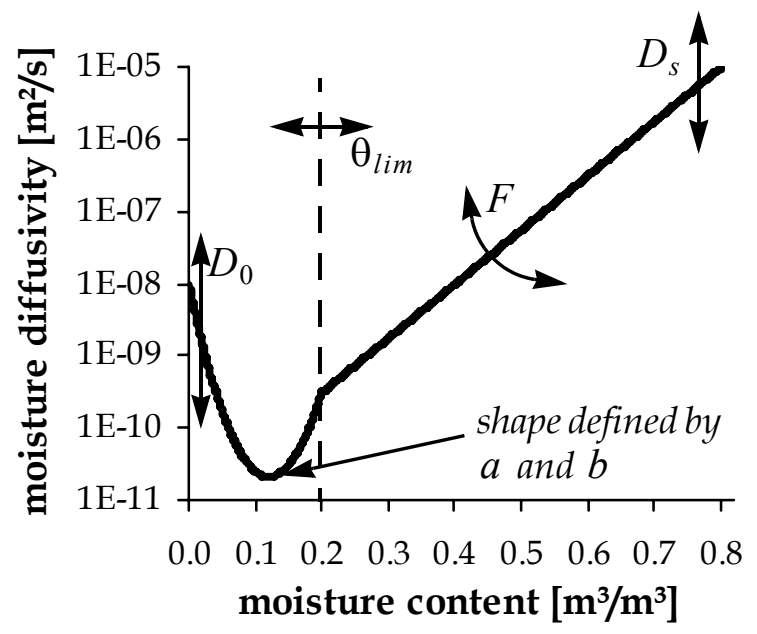

Figure 2.14: Improved moisture diffusivity approach according to Carmeliet E Janssen (2007). adjust the content range. It is possible to number of different applications and, thus, receive a functional shape that agrees well with the ones derived from measured moisture profiles, as reported e.g. by Krus (1995), Pel (1995) or Pel et al. (1996).

Disadvantageous are the many parameters to be adjusted. It is difficult to have as much measured data as is required for a unique parameter identification. Especially measured moisture profiles will be available only for a very limited number of materials.

In addition, the comparison of Carmeliet $\mathcal{E}$ Janssen (2007) was basically done with data from another model, not with really measured data. The hysteresis of moisture storage was neither addressed nor was its influence mentioned. The reference diffusivity func-

1. Carmeliet and Janssen use the term permeability for the transport coefficient defined in eq. (2.15) which is generally called conductivity here. It has the unit $[s]$ and is associated with a pressure gradient. 
tion was derived from the conductivity by the aid of the moisture storage function, but it was not stated whether the adsorption or the desorption moisture storage function was used. As shown by Scheffler et al. (2007-a), these items have a significant influence on the resulting diffusivity function.

For the calculation of hysteretical behaviour, the improved moisture diffusivity is not applicable. In case the required input data is available, the application of a more sophisticated conductivity approach is recommended, since the consideration of hysteresis as well as further dependencies is possible and the typically larger effort for parameter identification is comparable to the requirements of this improved diffusivity approach. However, for demonstration and teaching purposes, the approach is particularly suitable.

\subsubsection{Further diffusivity models}

A number of further diffusivity models from different authors exists. However, the basic ideas behind them resemble often, and no further improvement is given. Taking this into account, only the important approaches with regard to Building Physics were part of this review. Further publications may be mentioned only briefly in the following.

One further diffusivity approach was reported by Bednar (2000) who reproduced most of the contents reported by Künzel (1994) and Krus (1995) poorly structured. Another approach for the diffusivity function containing two parameters is given. However, this function is hardly explained and its parameters can only be adjusted by the aid of numerical simulation of experiments. The influence of hysteresis is not discussed. For vapour diffusion, a moisture dependent vapour diffusion resistance factor is applied. The moisture storage function is proposed to be adjusted by a two-modal function of the type introduced by Van Genuchten (1980) or by another unexplained 5-parametric function. Both are given in dependence to the capillary radius.

Bednar mixes the different physical properties, as there are pore radius, capillary pressure, relative humidity, water content, vapour diffusivity, water diffusivity, moisture diffusivity and also conductivity without clarification. The material functions are given in dependence each to a different variable, whereas a consistent approach of material modelling can not be found.

An evaluation of moisture profile measurements on spruce for determination of the moisture diffusivity by different international laboratories in the frame of IEA Annex XXIV was summarized by Kumaran (1999). He based his very simplified, constant approach for the diffusivity, depending only on the water uptake coefficient and the saturation moisture content, on the large deviations the different measurements comprised. However, for a fundamental material characterisation such approaches are not suitable.

One further approach to be mentioned here was reported by de Wit $\mathcal{E}$ van Schindel (1993). They use an exponential approach containing one adjustable parameter together with the water uptake coefficient and the saturation water content. As this approach does not display the lower moisture range correctly and part of it was improved and included into the approach published by Carmeliet et al. (2004) which was discussed above, it will not be further emphasised here. 


\subsubsection{Conductivity approaches}

Approaches using eq. (2.15) to calculate the advective liquid water flow and the moisture retention characteristic to assign the moisture content to the moisture potential are called conductivity approaches, as $K_{l}\left(\theta_{l}\right)$ is called the liquid water conductivity function. The main advantage of these approaches is the use of the actual driving potential and thus the ability to account for further dependencies, as discussed before.

Two main approaches to determine the liquid water conductivity function can be distinguished, as there are network and bundle of tube models. While network models approximate the actual pore structure by a lattice of tubes and bonds, the bundle of tube models assume a much more simplified pore structure of parallel tubes to derive the liquid flow conditions. Both approaches are based on pore size distribution data and are commonly used in Building Physics. Besides these sophisticated pore structure based material models, the following review contains two further and more simplified approaches.

\subsubsection{Simplified material models}

Two material models that generally attribute the liquid water transport to the liquid pressure gradient will be presented here. Since they do not employ models of pore structure or tortuous water flow, they are regarded as simplified models. One is the model used by Pedersen (1990) in his simulation code which consists of analytical functions due to limited computational capability at that time. The other is the model introduced by Galbraith (1992). Developed from a typical Building Physical perspective, his model is defined in dependence on the relative humidity using analytical functions, too.

\section{Material model according to Rode Pedersen ${ }^{1}$}

Starting from the balance equations given by Philip \& De Vries (1957), the transport theory for heat and moisture transport was implemented into a computer code called Match by Pedersen (1989). In his model (Pedersen (1990)), he describes the vapour transport as a diffusion process according to eq. (2.13) with a moisture dependent diffusivity and the liquid water transport related to the capillary pressure according to the conductivity approach with eq. (2.15) disregarding the gravity. For all functional relations, simple analytic relations are introduced ensuring good simulation performance under strongly limited computational capability.

As a simple hysteresis model is included, moisture storage is described by both, main wetting and main drying curves. Adsorption and desorption isotherms follow eq. (2.46) according to Hansen (1986), where the parameters $\theta_{h}, A$ and $n$ are fitted to measured data.

1. The author's name is Carsten Rode Pedersen. His early works are published under the name Pedersen, while more recent ones can be found under the name Rode. 


$$
\theta_{l}(\varphi)=\theta_{h} \cdot\left(1-\frac{\ln \varphi}{A}\right)^{-\frac{1}{n}}
$$

sorption isotherm

In the overhygroscopic moisture range, different expressions are used for wetting and drying. The adsorption curve given in eq. (2.47) slowly approaches the capillary moisture content $\theta_{\text {cap }}$, see Fig. 2.15. The parameters $A_{\text {wet }}$ and $B_{\text {wet }}$ have to be adjusted so that the curve passes smoothly into the hygroscopic one at a capillary pressure corresponding to $98 \%$ relative humidity.

$$
\theta_{l}\left(p_{c}\right)=\theta_{c a p}-A_{\text {wet }} \cdot \ln \left(p_{c}\right)^{B_{\text {wet }}} \quad \text { adsorption curve }
$$

The desorption curve above relative humidities of $98 \%$ is described by three hyperbolic functions passing smoothly into each other with the maximum moisture content being the vacuum saturation $\theta_{v a c}$.

$$
\begin{array}{ll}
\theta_{l}\left(p_{c}\right)=A_{i p t}-\left[\left(B_{i p t} \cdot \ln p_{c}\right)^{C}+\left(A_{i p t}-\theta_{v a c}\right)^{C^{\frac{1}{C}}}\right. & 1^{\text {st }} \text { hyperbola } \\
\theta_{l}\left(p_{c}\right)=\theta_{0}+A_{d r y} \cdot\left(\ln p_{c}-\ln p_{0}\right)^{B} d r y & 2^{\text {nd }} \text { hyperbola } \\
\theta_{l}\left(p_{c}\right)=A_{d e s}-\left[\left(B_{d e s} \cdot \ln p_{c}\right)^{C}+\left(A_{d e s}-\theta_{c a p}\right)^{C^{\frac{1}{C}}}\right. & 3^{\text {rd }} \text { hyperbola }
\end{array}
$$

The parameters in eq. (2.48) have to be fitted in such a way that a smooth transition from one function into the other is obtained and possibly available material data is approximated. The exponent $C$ has to be chosen large enough to enable fast convergence for which reason Pedersen (1990) uses a fixed value of $C=30$. At saturation $\theta_{v a c}$, a linear function is used to connect the hyperbola to the ordinate. A schematic illustration of this model is given in Fig. 2.15.

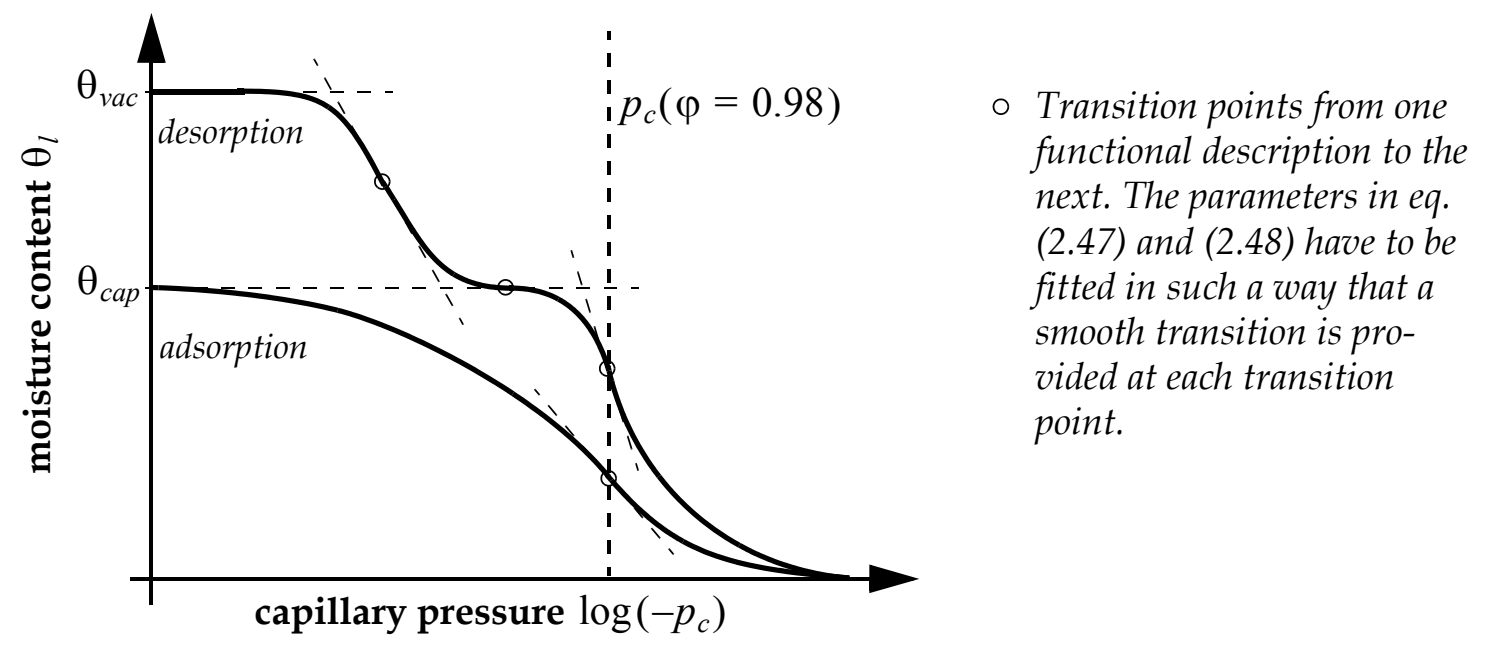

Figure 2.15: Moisture storage functions for ad-and desorption according to Pedersen (1990). 
For the vapour diffusivity $D_{v, \text { mat }}\left(\theta_{l}\right)$, three moisture ranges with different expressions are distinguished according to eq. (2.49). For relative humidities up to $60 \%$, Pedersen uses a constant value following eq. (2.12) with the dry cup value $\mu_{d r y}$. Between $60 \%$ and $98 \%$ relative humidity, a linear function connects the diffusivity values $D_{v, d r y}$ due to $\mu_{d r y}$ and $D_{v, w e t}$ due to $\mu_{w e t}$. Thirdly, a linear function in dependence to the water content is used between $D_{v, \text { wet }}$ at $\theta_{98}$ and 0 at $\theta_{v a c}$.

$$
\begin{aligned}
& D_{v, m a t}\left(\theta_{l}\right)=D_{v, d r y} \\
& \text { for } \varphi<0.6 \\
& D_{v, \text { mat }}\left(\theta_{l}\right)=D_{v, d r y}+\frac{\varphi-0.6}{0.98-\varphi} \cdot\left(D_{v, w e t}-D_{v, d r y}\right) \quad \text { for } \quad 0.6 \leq \varphi<0.98 \\
& D_{v, \text { mat }}\left(\theta_{l}\right)=\frac{\theta_{v a c}-\theta_{l}}{\theta_{v a c}-\theta_{98}} \cdot D_{v, \text { wet }} \quad \text { for } \varphi \geq 0.98
\end{aligned}
$$

The liquid water conductivity is approximated by an exponential function depending on two parameters. It is defined between a critical moisture content $\theta_{\text {crit }}$ and the capillary moisture content $\theta_{\text {cap }}$. Below $\theta_{\text {crit }}$ liquid water transport is regarded to be negligible, above $\theta_{\text {cap }}$ the conductivity value at $\theta_{\text {cap }}$ is applied. The analytic expression is given in eq. (2.50). Fig. 2.16 shows both, the vapour and the liquid transport function.

$$
\begin{array}{lr}
K_{l}\left(\theta_{l}\right)=\text { negligible } & \text { for } \theta_{l}<\theta_{\text {crit }} \\
K_{l}\left(\theta_{l}\right)=A_{k} \cdot \exp \left(B_{k} \cdot \theta_{l}\right) & \text { for } \theta_{\text {crit }} \leq \theta_{l}<\theta_{\text {cap }} \\
K_{l}\left(\theta_{l}\right)=A_{k} \cdot \exp \left(B_{k} \cdot \theta_{\text {cap }}\right) & \text { for } \theta_{l} \geq \theta_{\text {cap }}
\end{array}
$$

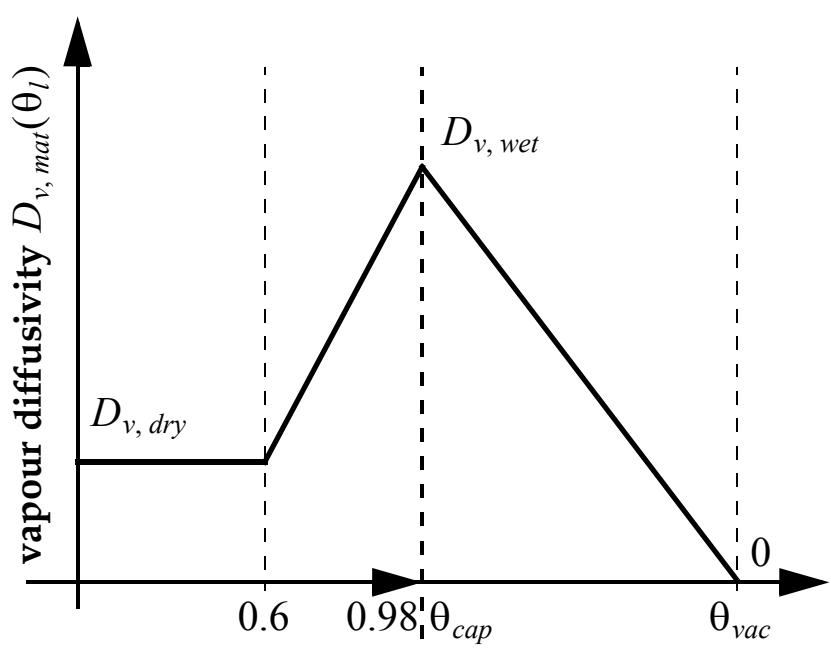

relative humidity $\varphi_{1}^{\prime}$ moisture content $\theta_{l}$

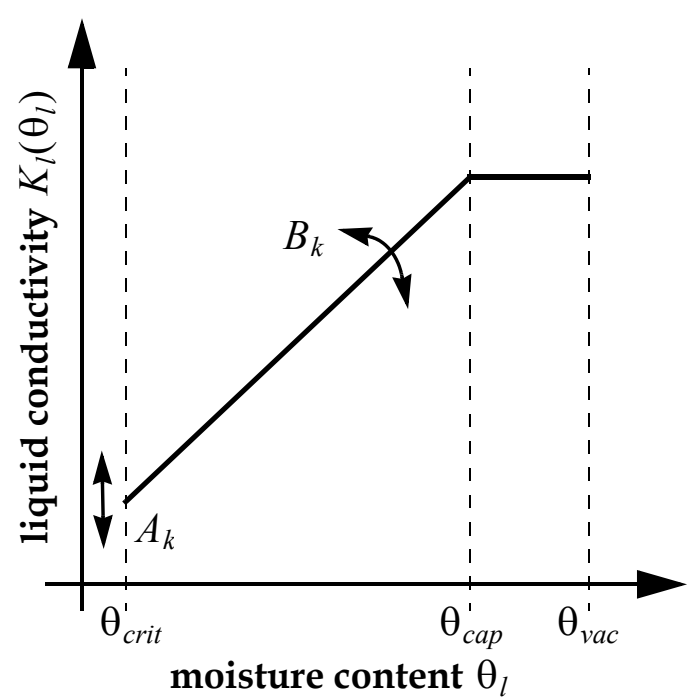

Figure 2.16: Hygric transport functions according to Pedersen (1990): vapour diffusivity (left) and liquid water conductivity (right). 
Unfortunately, Pedersen (1990) gives neither a definition of the critical moisture content $\theta_{\text {crit }}$ nor an advice how to adjust the parameters $A_{k}$ and $B_{k}$. The whole material model displays more or less an attempt to describe the hygric material properties by a set of analytical functions which are easy to be implemented into simulation code. No emphasis is placed on a close representation of experimental data and hardly any instruction is given how to adjust the numerous parameters with respect to this data. The model, although a simple hysteresis model was already included, is - from the authors point of view - not applicable under current engineering requirements on quality and precision. Moreover, the drawn conclusions concerning hysteresis based on this material model are very questionable and should not be generalized (see also the discussion in paragraph 2.2.1.3 Diffusivity approach according to Kießl and Künzel).

\section{Material model according to Galbraith}

A typical Building Physics approach to describe heat and moisture transport was presented by Galbraith (1992). Vapour transport is assigned to the vapour pressure gradient and liquid water transport to the capillary pressure gradient. The latter one is converted to the vapour pressure gradient by the help of the Kelvin equation (2.17), allowing a description of moisture transport only in dependence to the relative humidity.

Moisture transport is then described by modifying eq. (2.13) in such a way that it accounts for liquid water and water vapour transport leading to eq. (2.51). The coefficient $\mu$ contains two additive terms, one for vapour and one for liquid transport.

$$
j_{k, \text { diff }}^{M_{v+l}}=-\mu \cdot \frac{\partial p_{v}}{\partial x_{k}} \quad \text { with } \quad \mu=D_{v}^{*} \cdot \tau+D_{l}^{*} \cdot \varphi^{m}
$$

Fig. 2.17 shows the constituents in dependence to their parameters. The vapour diffusion coefficient can either be chosen independently to the water content with $\tau=1$ or a relation in dependence to the ratio of material and water density can be used. The

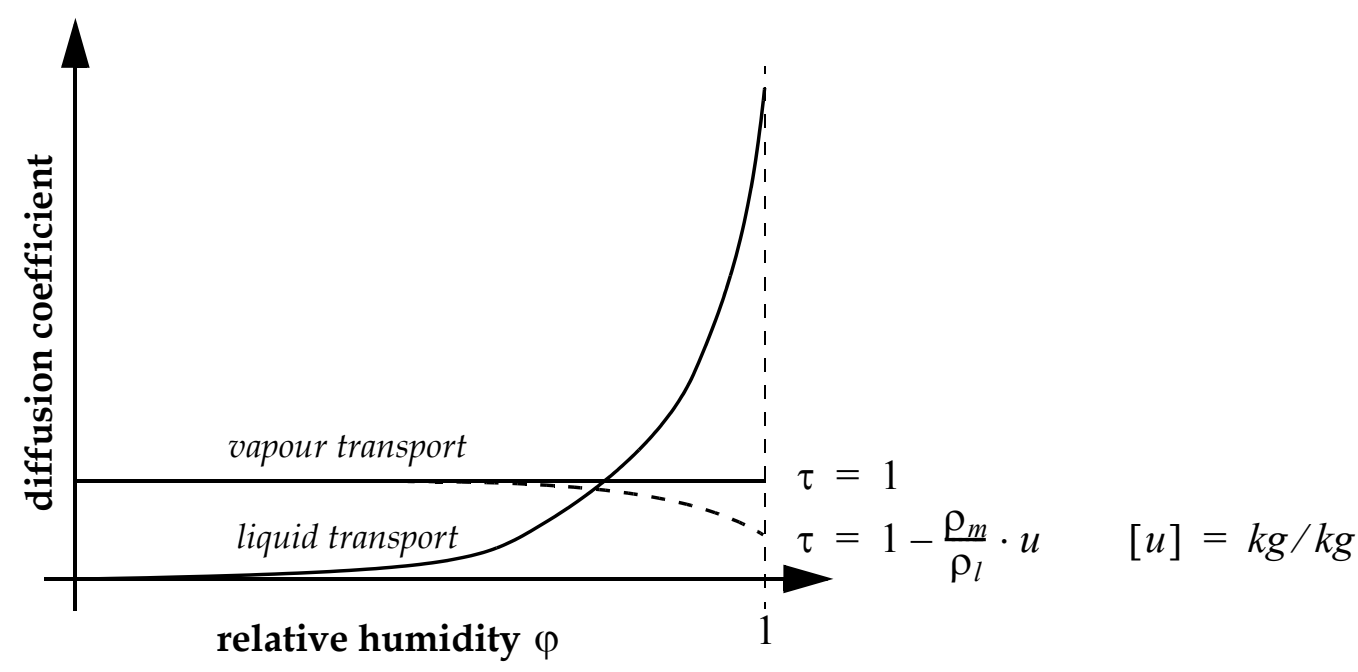

Figure 2.17: Transport functions for vapour and liquid water in dependence to the relative humidity according to Galbraith (1992). 
mass related water content $u$ is employed by Galbraith (1992) in this relation. The comparison of simulation results with either the constant or the moisture dependent vapour diffusion coefficient caused only small differences. As in both cases the parameters $D_{v}^{*}, D_{l}^{*}$ and $m$ have to be determined from vapour diffusion measurements (drycup and wet-cup tests), slightly different values attribute the difference either to liquid or to vapour transport.

Unfortunately the model of Galbraith does not consider the overhygroscopic moisture range in a reasonable way. Moisture storage and transport is accounted for only in relation to the relative humidity. This leads to large errors for the description of both, the moisture storage and the liquid transport function. The model might be applicable with sufficient precision to a number of cases where only hygroscopic moisture transport occurs, but as a general material model which must not be restricted to special application cases, it is not expedient.

\subsubsection{Network models}

The basic idea of network models is to approximate the actual pore structure by a lattice of tubes and bonds on the microscopic scale. There, the tube radii follow, randomly distributed, the measured pore structure data of the material. Simulating the penetration of the network by a fluid, storage and transport functions based on the measured pore volume distribution data can be derived. For that derivation, network models allow to take structural effects as dead end pores, air entrapment and ink bottle effects into account. Fig. 2.18 shows some examples of two-dimensional networks found in literature.

To study the capillary pressure characteristic of a porous medium, network models have first been introduced by Fatt (1956). He used a two-dimensional network to which a distribution of tube radii is assigned according to tables of random numbers. Assuming the network to be initially filled by the wetting fluid (e.g. air) and surrounded by the nonwetting fluid (e.g. mercury), he increased the capillary pressure stepwise. First, the largest tubes in the network having access to the nonwetting fluid were penetrated. With increasing pressure, accessible tubes with decreasing radii were entered until even the narrowest pores with contact to the nonwetting fluid were penetrated. Fatt derived the capillary pressure - saturation relationship by assuming circular tubes and

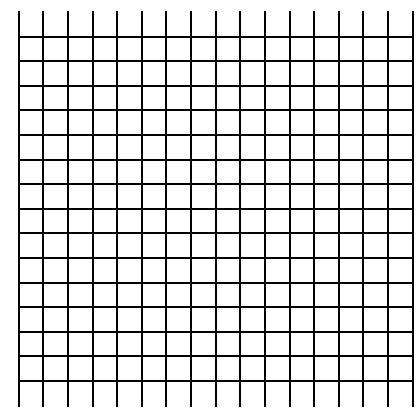

(a)

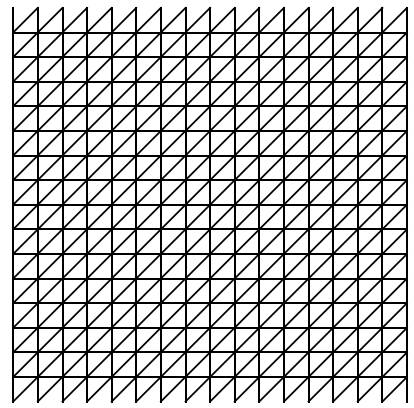

(b)

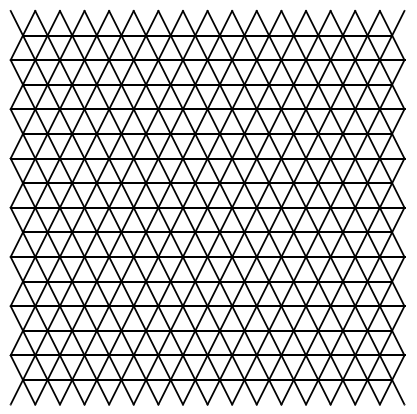

(c)

Figure 2.18: Examples of two-dimensional networks: (a) square lattice, (b) triangular lattice and (c) hexa-triangular lattice. Those and other networks are e.g. given by Dullien (1979) and Descamps (1997). 
calculating the capillary pressure according to the Laplace equation (2.14) with $\gamma=0^{\circ}$. The saturation was then a function of the sum of penetrated pores, the pore radii and the corresponding pore length which he assumed to be dependent to the pore radius.

A good survey of the basic principles of network models and their application for the derivation of capillary pressure - saturation relationships was given by Dullien (1979). Besides a profound investigation of different established networks, general peculiarities of their application are discussed, too. At that time, Dullien had to state, however, that the derivation of the conductivity function by the aid of network models could not be successfully performed (Dullien (1979) p. 181).

More recently, network models have been applied to derive the moisture transport properties of building materials. For that purpose, a pressure gradient of a certain unit is applied and the resulting flow is calculated in dependence to the possible flow path of the lattice. Assuming the geometry of pore shape and length, the transport coefficient can be obtained, normally as a relative function. The relative conductivity can be scaled by the aid of measured conductivity values under saturated conditions (Darcyconductivity) delivering the function to be used in eq. (2.15). Quenard $\mathcal{E}$ Salle (1992) introduced this procedure to model vapour and transport in cement-based materials. As a further development, on behalf of others, the principles of the multiscale network models developed by Xu et al. (1997a) and Carmeliet et al. (1999) are briefly presented here.

\section{Transport properties derived from multiscale networks}

Xu et al. (1997a) developed a multiscale network model to describe the pore structure of a porous medium on the basis of a mercury intrusion curve. They use a three-dimensional cubic lattice on different scales representing different pore classes which are combined by a rescaling technique. A two-dimensional illustration for three pore size classes is given in Fig. 2.19. As the objective was first to represent the pore structure measured by mercury intrusion, the pore system is assumed to be initially evacuated, i.e. neither gas nor liquid is present there. For the invasion of the nonwetting fluid into the pore structure, no rules of tracing or trapping are applied, which corresponds to the assumption that the whole pore space is available for penetration. Thus, air entrapment, the ink-bottle effect and other structural effects are neglected.

Taking the pore system described by the multiscale network model of Xu et al. (1997a) as a basis, also transport properties can be derived. For that purpose, Xu et al. (1997b) introduced a renormalisation procedure. Starting from the smallest pore class, they

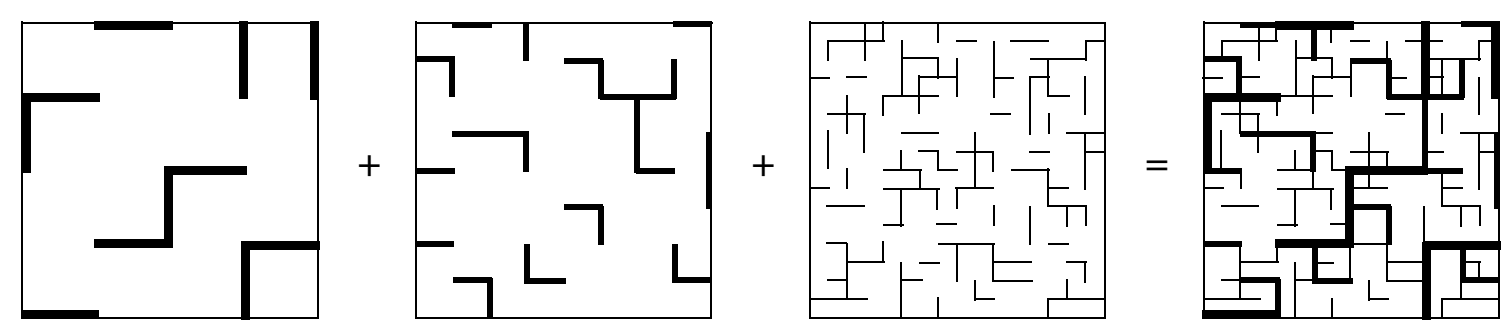

Figure 2.19: Multiscale network, based on a two-dimensional square lattice, as the result of superposition of three pore classes according to Xu et al. (1997a). 
defined the cummulative conductivity at each pore size level and, after renormalisation, incorporated it into an equivalent network of the next larger scale. This procedure is followed for all pore classes delivering the macroscopic conductivity function.

The model introduced by $\mathrm{Xu}$ et $a l$. (1997b) is supposed to be very general deriving a conductivity function for a number of different processes as electrical conduction, molecular diffusion, transport of condensable vapour and hydraulic transport. However, the procedure is very complicated and the derivation of an applicable liquid water conductivity function is not clarified. The model is restricted to the pore size range of mercury intrusion and the given examples show a rather low accuracy. A study reported by Quenard et al. (1998) though proved the model to be able to deliver reasonable results of vapour and air permeabilities for three different building materials on the basis of mercury intrusion and SEM image data.

In contrast, the multiscale network model according to Carmeliet et al. (1999) and Carmeliet $\mathcal{E}$ Roels (2001) uses different levels of magnification, see Fig. 2.20. For each level, a certain porosity and pore size distribution is defined on the basis of measured moisture storage or pore size distribution data. A two-dimensional cross-squared lattice with volumeless intersections is used at each level with no correlation to other levels. The tube radii are selected randomly from the normalised pore radius distribution function by the help of a Monte Carlo simulation.

The pore space not occupied by water is assumed to be filled with a mixture of air and vapour. During wetting, a tube becomes water filled if the following conditions are fulfilled:

- The tube has contact to another water filled tube.

- The tube radius is smaller than the radius corresponding to the capillary pressure $r\left(p_{c}\right)$ following eq. (2.14).

- The air possesses a continuous trace to the atmosphere.

As shown by Descamps (1997), effects as air entrapment and pressure build-up during imbibition processes with sealed boundary can be modelled in that case. For drying, the same rules apply but in the opposite direction.

Moisture migration is calculated by a combination of liquid water flow in a tube, following the Hagen-Poiseuille equation (2.52), and water vapour flow, following the Fickean diffusion equation (2.8) with the vapour diffusivity in air according to Schirmer (1938) and eq. (2.11). Implying a unit pressure gradient at two sides of the network and assigning a non-flow condition to the other two sides, the moisture conductivity can

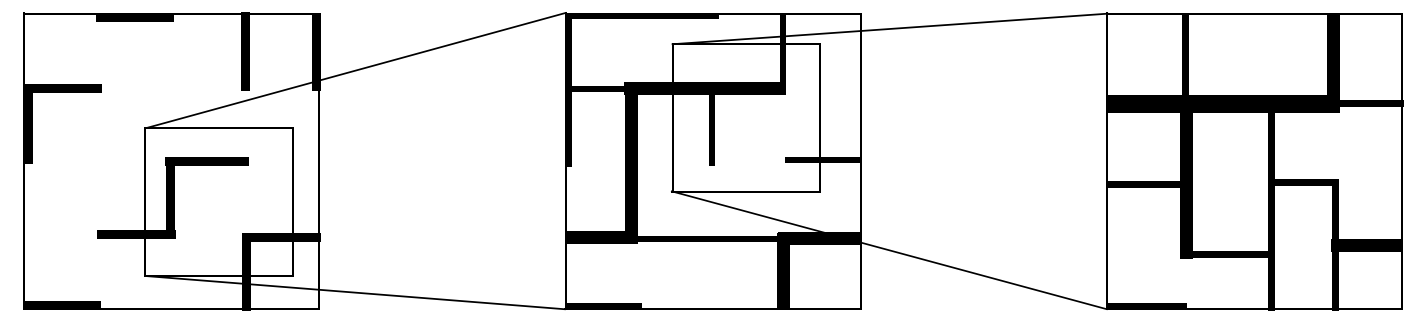

Figure 2.20: Multiscale network, based on a two-dimensional square lattice, at different levels of magnification according to Carmeliet et al. (1999). 
be calculated for a certain moisture distribution in dependence to the capillary pressure. The whole moisture transport function for the corresponding level of magnification is then derived by a stepwise variation of the capillary pressure.

Each conductivity function obtained by that procedure is dependent on the pore length and the so-called cut-off radius $r_{i}$ marking the lower boundary of the level of magnification. It is therefore referred to as relative conductivity. The macroscopic conductivity function of the whole material can be finally obtained by combining the functions from the different levels of magnification using a rescaling technique. Carmeliet et al. (1999) showed that the conductivity from the network model can not be overestimated. Thus, the macroscopic conductivity function can be described as the boundary curve of the rescaled conductivity functions from different levels of magnification. The remaining scaling factor is derived from conductivity measurements at saturated conditions (either directly as Darcy-conductivity or calculated from air permeability measurements).

Influencing parameters of the network model are the network size, the number of network simulations, the connectivity of the network and the pore length. The connectivity value $z$, which specifies the number of tubes connected to a node, is determined by the choice of the network, see also Fig. 2.18. Experimental investigations (e.g. Lin $\mathcal{E}$ Cohen (1982) and Payatakes $\mathcal{E}$ Dias (1984) cited by Carmeliet et al. (1999)) revealed values ranging from $z=2 \ldots 15$. Carmeliet et al. (1999) found this parameter to be less dominant, comparing conductivity functions from a square lattice $(z=4)$, a triangular lattice $(z=6)$ and a cross-square lattice $(z=8)$.

Network size $s$ and simulation number $n$ are parameters of the network simulation procedure and have to be determined in dependence on computational capability and requirements of accuracy. For the pore length $L$ the relation $L(r)=L_{M}\left(r / r_{M}\right)^{\alpha}$ depending on the pore radius is introduced containing two parameters: the pore length $L_{M}$ of the pore with the maximum radius $r_{M}$ and the pore shape ratio $\alpha$. For $\alpha$, different values can be found in literature. Carmeliet et al. (1999) consider $\alpha$ to be a rather sensitive parameter giving the best agreement with measurement results for $\alpha=-1$. Values for $L_{M}$ are not specified by Carmeliet et al. (1999).

The multiscale network model is an appropriate sophisticated approach to derive the liquid water conductivity from pore size distribution data. Hence, it has been used and developed further for scientific investigations, which will be summarized within the next paragraphs. Subsequently, an evaluation of the network modelling approach with regard to the objectives of this work will conclude this survey.

\section{Modelling transport in heterogeneous structures}

To predict the moisture conductivity also for materials with significant microheterogeneities, an extension of the multiscale network model was introduced by Roels (2000). The investigated material was a layered limestone containing distinctive heterogeneities on both, the macroscopic and the mesoscopic scale. Therefore, Roels et al. (2003a) proposed to model moisture transport at the mesoscale and transform the conductivity to the macroscale by the aid of an upscaling technique.

The pore structure was analysed by four different methods, i.e. pressure plate apparatus, sorption measurements, mercury intrusion and micrograph image analysis. Based on data obtained by these techniques, a conceptual hysteresis model was applied. 


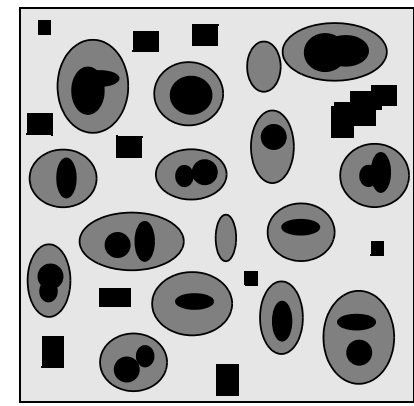

substructure 1

substructure 2
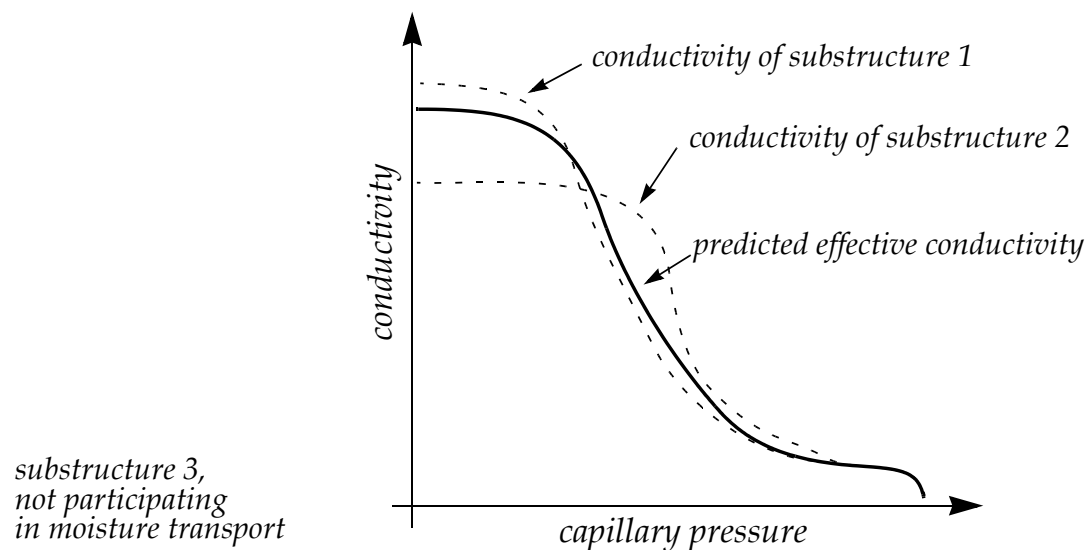

Figure 2.21: Modelling moisture transport of heterogeneous materials on the mesoscopic scale: model of mesoscopic structure (left) and upscaling conductivity (right). Schematic illustration of the approach introduced by Roels (2000).

Defining an accessibility function for the different pore sub-systems being expected to contribute to the ink-bottle effect, the main drainage curve could be predicted from the main wetting curve in very good agreement with measured data (Roels et al. (2001)). By these means, the different pore sub-systems and their connectivity could be identified.

The mesoscopic structures participating in moisture transport were modelled separately using the network approach proposed by Carmeliet et al. (1999). However, simulations on the mesoscale, which showed good agreement between measured and calculated water absorption data (Roels et al. (2000)), are not applicable on the macroscopic scale. Therefore, an equivalent conductivity function for the macroscopic structure had to be derived using an upscaling technique. A review on such methods is given by Renard \& Marsily (1997). Fig. 2.21 illustrates this procedure.

The macroscopic material contained two significant substructures, which are regions of more or less isotropic structure and regions showing a distinctive layering. Water absorption experiments have been carried out for the whole structure parallel and perpendicular to the layering. Then it was cut into small pieces for which absorption experiments were carried out in the same way. The comparison between measured and simulated behaviour shows a good agreement proving the proposed upscaling technique to be suitable (Roels et al. (2003b)).

Further extension of the network model for fractured porous media has been performed by Vandersteen et al. (2003) modelling fracture aperture and aperture variance by a three-dimensional network of parallel plates. Roels et al. (2003c) presented a simplification of this by modelling the fracture as a row of nodes connected by tubes for a two-dimensional water absorption test. The results showed a good agreement with measured X-ray data.

\section{Concluding network approaches}

The liquid water conductivity can be derived by the aid of network models. The multiscale approach introduced by Carmeliet et al. (1999) and Carmeliet $\mathcal{E}$ Roels (2001) was proved to be very suitable, not only for homogeneous, but also for heterogeneous materials (Roels (2000)). The main advantage of the network approaches is the ability 
to appropriately describe the pore structure and to account for structural effects by percolation rules for tracing and accessibility. Air entrapment and ink-bottle effects causing hysteresis of the capillary pressure - saturation relationship can be included into the derivation of the transport properties. Moreover, as indicated above, network models can be extended and applied to other structural compositions as heterogeneous or fractured porous media.

However, employing a network model to derive the conductivity function is laborious. A network has to be chosen and modelled, scales have to be defined, pore sizes have to be assigned by a Monte Carlo simulation in dependence to the pore size distribution, a number of network simulations have to be performed according to chosen criteria for tracing and accessibility and transport properties have to be derived from the achieved flow conditions. In addition, the model depends on a number of parameters at different levels, e.g. network size and connectivity, number of simulations, pore specifying parameters (length, shape) and the chosen percolation rules.

With regard to the objective to define a material model which is applicable for a large number of materials on an advanced engineering level, network models appear to be too extensive and, thus, less appropriate. For advanced research and materials with a complex pore structure which can not be described by less advanced models, network models are a suitable choice to derive the moisture storage and transport properties.

\subsubsection{Bundle of tubes models}

These models are called bundle of tubes models because they follow a simplified representation of the pore structure consisting of a bundle of parallel and interconnected tubes (see e.g. Burdine (1953), Krischer (1956), Dullien (1979)). The laminar flow in a single tube is expressed by the well known Hagen-Poiseuille equation (2.52) assigning the volume flow $\dot{V}$ to the tube radius $r$, the dynamic viscosity of the liquid phase $\eta_{l}$ and the gradient of the capillary pressure.

$$
\dot{V}(r)=-\frac{\pi \cdot r^{4}}{8 \eta_{l}} \cdot\left[\rho_{l} \cdot g_{k}+\frac{\partial p_{c}}{\partial x_{k}}\right] \quad \text { tube flow in a single capillary }
$$

Extending the description of the flow within one tube to a bundle of tubes, eq. (2.52) has to be integrated over the pore radii distribution density $d n / d r$, yielding eq. (2.53).

$$
\dot{V}(r)=-\int_{R} \frac{\pi r^{4}}{8 \eta_{l}} \cdot \frac{\mathrm{d} n}{\mathrm{~d} r} \cdot d r \cdot\left[\rho_{l} g_{k}+\frac{\partial p_{c}}{\partial x_{k}}\right]
$$

To relate the incremental pore radii to the incremental pore volume, length and crosssectional area of the pores have to be known. As all pores are assumed to be of the same unit length $d x$, pore volume distribution density divided by the cross sectional area $\pi r^{2}$ can be set equal to the pore radii distribution density according to eq. (2.54). 


$$
\frac{\mathrm{d} \theta}{\mathrm{d} r} \frac{1}{\pi r^{2}}=\frac{\mathrm{d} n}{\mathrm{~d} r}
$$

Applying eq. (2.53) to eq. (2.54) and multiplying it by the density of the liquid phase $\rho_{l}$ yields the mass flow density $j_{k}^{M_{l}}$ in dependence to the capillary radius, given in $e q$. (2.55). Comparison of eq. (2.55) and eq. (2.15) reveals that the whole term before the brackets is the liquid water conductivity $K_{l}$. If the pore radius is transformed into the capillary pressure following eq. (2.14), the integral contains the inverse of the moisture storage function which can be determined from measured data. For an illustration to this procedure, refer to Fig. 2.22.

$$
\begin{aligned}
& \dot{j}_{k}^{M_{l}}\left(\theta_{l}\right)=-\frac{\rho_{l}}{8 \eta_{l}} \cdot \int_{\theta_{l}} r(\theta)^{2} d \theta \cdot\left[\rho_{l} g_{k}+\frac{\partial p_{c}}{\partial x_{k}}\right] \\
& K_{l}\left(\theta_{l}\right)=-\frac{\rho_{l} \cdot \sigma_{l g}^{2}}{2 \eta_{l}} \cdot \int_{\theta_{l}} \frac{1}{p_{c}(\theta)^{2}} d \theta
\end{aligned}
$$

Since this liquid water conductivity of eq. (2.56) overestimates the liquid transport, Burdine (1953) related it to its value at saturation. Additionally, he introduced a tortuosity factor $\tau$ in the form of $\theta^{\tau}$ accounting for the indirection of flow pathes. For $\tau$ he proposed to use a value of $\tau=2$.

$$
K_{l, r e l}\left(\theta_{l}\right)=\theta^{\tau} \cdot\left[\left(\int_{0}^{\theta_{l}} \frac{1}{p_{c}(\theta)^{2}} d \theta\right) /\left(\int_{0}^{\theta_{s}} \frac{1}{p_{c}(\theta)^{2}} d \theta\right)\right] \quad \text { Burdine model }
$$

The relative conductivity $K_{l, r e l}\left(\theta_{l}\right)$ can then be scaled by a measured conductivity value at saturation $K_{\text {sat }}$ to obtain the absolute conductivity function (see Mualem (1976a), Van Genuchten (1980), Van Genuchten E Leij (1992) and Mualem (1992)). Within the approach (2.57), it is tacitly assumed that all pores of one unit length at $x$ have an ideal connection, i.e. full correlation to the pores of the next unit $x+d x$. Different approaches to incorporate this correlation can be found in literature on which a summary is given by Mualem (1992). One is the provided approach of Burdine (1953) in its different presentations (see e.g. Van Genuchten $\mathcal{E}$ Leij (1992)). Another approach derived by Mualem (1976a) assumes only partly correlation. He employs a correlation factor in the form of $\theta^{\tau}$ to be incorporated into the probability term of connected pore sizes. Analogous derivation leads to the relative conductivity formulation (2.58), which is different from the one given in eq. (2.57). Here, the tortuosity with $\tau=1 / 2$ is frequently used (Mualem (1992)). 

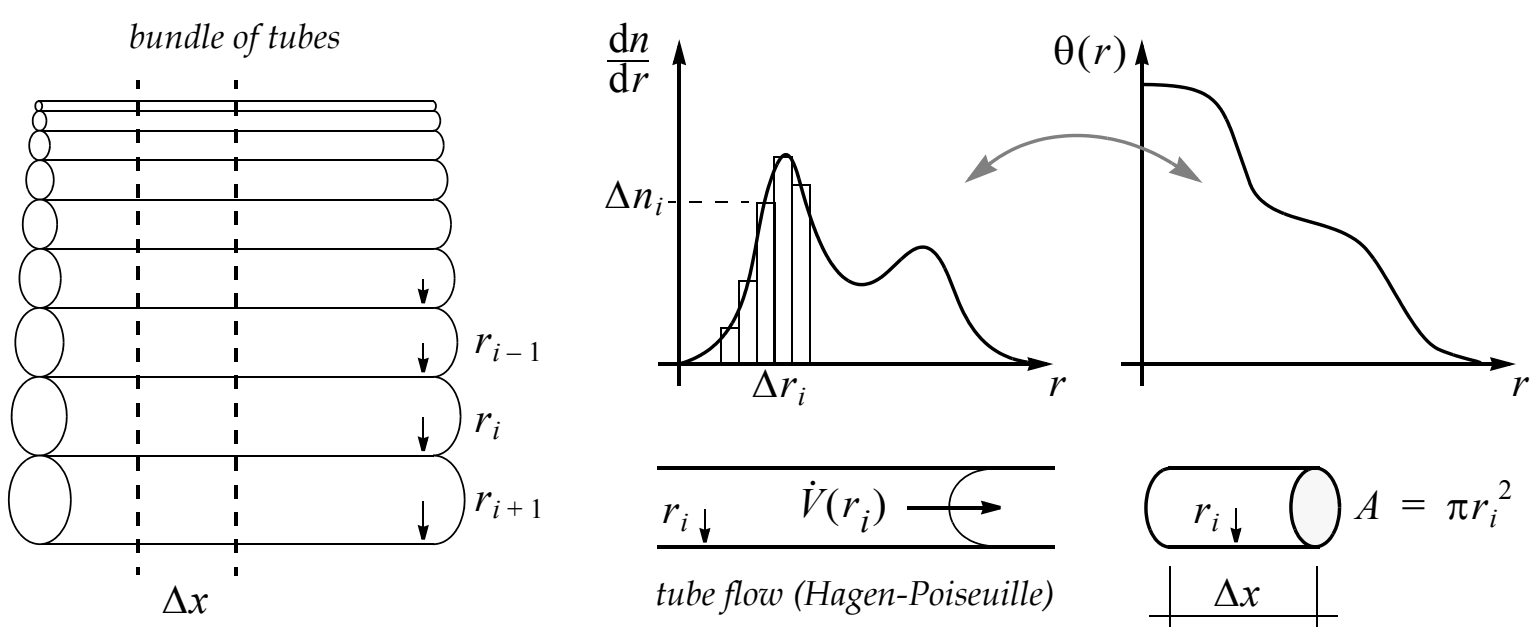

Figure 2.22: Bundle of tubes model to derive the liquid water conductivity from pore size distribution data. Authors as Burdine (1953) worked with a summation of incremental pore classes $\Delta r_{i}$, which is also used here for illustration purposes. Nowadays, the differential operator and integration over steady functions is used to determine the liquid water conductivity.

$$
K_{l, r e l}\left(\theta_{l}\right)=\theta^{\tau} \cdot\left[\left(\int_{0}^{\theta_{l}} \frac{1}{p_{c}(\theta)} d \theta\right) /\left(\int_{0}^{\theta_{s}} \frac{1}{p_{c}(\theta)} d \theta\right)\right]^{2} \quad \text { Mualem model }
$$

The evaluation of both, Burdine model (2.57) and Mualem model (2.58), leads to slightly different conductivity functions. According to Van Genuchten (1980), „Burdine-based equations showed lesser agreement with experimental data than the Mualem-based expressions". This was also concluded by Mualem (1976a). However, both models are commonly used.

Much effort has been paid to develop closed form equations for the moisture storage function in order to gain analytical expressions for both, moisture storage function and capillary conductivity function. Most popular is the expression proposed by Van Genuchten (1980), but also many other approaches exist utilising either one of the given models.

As follows, the model of Van Genuchten and some of its further improvements proposed by other authors will be given. Then, two functional approaches introduced in Building Physics will be explained whereas a discussion on bundle of tubes models will conclude this paragraph.

\section{Closed form equation according to Van Genuchten and Durner}

In Soil Sciences, the capillary pressure $p_{c}$ is normally expressed by the pressure head $h$, i.e. by the height of a water column which exerts the same pressure. At the same elevation and athmospheric pressure, both can be converted into each other by eq. (2.59). For reasons of simplicity, the following approaches will be written in their original form, using the pressure head instead of the capillary pressure. 


$$
h=\frac{p_{c}}{\rho_{l} \cdot g}
$$

For the saturation - pressure head relation, Van Genuchten (1980) introduced eq. (2.60), where $\Theta$ is the relative saturation, $\theta_{s}$ is the saturation moisture content, $\theta_{r}$ is the residual moisture content and $\alpha, m$ and $n$ are adjustable parameters.

$$
\Theta(h)=\left[\frac{1}{1+(\alpha h)^{n}}\right]^{m} \text { with } \Theta=\frac{\theta-\theta_{r}}{\theta_{s}-\theta_{r}} \quad \text { Van Genuchten function }
$$

Calculating the inverse of eq. (2.60), it can be inserted into one of the above given models. Applying some restrictions to the parameters $m$ and $n$, the integration can be carried out analytically. For the Mualem model (2.58), Van Genuchten derived the capillary conductivity function according to eq. (2.61) by setting $m=1-1 / n$.

$$
K_{l, r e l}(\Theta)=\Theta \cdot\left[1-\left(1-\Theta^{1 / m}\right)^{m}\right]^{2}
$$

The same was done for the Burdine model (2.57), where eq. (2.62) could be derived by setting $m=1-2 / n$.

$$
K_{l, r e l}(\Theta)=\Theta^{2} \cdot\left[1-\left(1-\Theta^{1 / m}\right)^{m}\right]
$$

Applying eq. (2.60) to eq. (2.61) or eq. (2.62), the relative capillary conductivity can be written in dependence to the pressure head as well. Also an expression for the diffusivity function was given by Van Genuchten (1980). Based on the comparison of both models and their conclusions mentioned above, further evaluation and discussion on parameter determination were conducted for the Mualem-based equations only.

The residual moisture content $\theta_{r}$ is defined as the lower boundary of the moisture content range. However, Van Genuchten (1980) already gave a brief discussion on $\theta_{r}$, indicating that it should be regarded to more as a fitting parameter of the model instead of a soil physical parameter. Durner (1992) showed, that $\theta_{r}$ indirectly influences the capillary conductivity function. Since in Soil Sciences, this function is required to be precise mainly in the wet range close to saturation, $\theta_{r}$ might be used as a fitting parameter to obtain a better approximation of measured retention data for the wet range, even if some data in the dry range may be disregarded.

For an improved approximation of retention data, Durner (1994) proposed to use a weighted sum of Van Genuchten type functions (2.63). There, the residual moisture content is omitted. Instead, by choosing an appropriate number of modalities, the whole moisture range can be described properly by that approach. In eq. (2.63), $N$ is the number of modalities and $w_{i}$ are the weighting factors with $\sum_{i} w_{i}=1$.

$$
\theta(h)=\theta_{s} \sum_{i=1}^{N} w_{i} \cdot\left[\frac{1}{1+\left(\alpha_{i} \cdot h\right)^{n_{i}}}\right]^{m_{i}}
$$


It was shown by Durner (1994) for different soils that a close representation of measured data leads to a better agreement of the conductivity function with experimental data. Evaluation of the pore model (Durner applied the Mualem model) was done numerically, which is common practice today. Therefore, closed form equations are not as important anymore as they were 20 years ago. Moreover, the number of modalities sometimes exceeds $N=2$ to enable a better fit to measured moisture storage data.

In Building Physics, the multimodal Van Genuchten type function (2.63) is occasionally applied (Roels (2000), Carmeliet $\mathcal{E}$ Roels (2002), Plagge et al. (2002)). Here, a good approximation is required for both, the hygroscopic and the overhygroscopic moisture range, since most processes take place in the transient area. Some further approaches exist applying the same pore structure models which shall be introduced as well. One is a closed form rational expression in dependence to the pore radius proposed by Häupl $\mathcal{E}$ Fechner (2003a). The other is based on Gauss distribution functions to describe the logarithmic pore volume distribution and was introduced by Grunewald $\mathcal{E}$ Bomberg (2003).

\section{Closed form equation according to Häupl and Fechner}

A closed form equation for moisture storage and moisture transport in dependence to the capillary radius was proposed by Häupl et al. (2001) and further developed by Häupl E Fechner (2003a), Häupl E Fechner (2003b) and finally Häupl (2007b). The model is based on the pore size distribution function $f(r)$ which an analytical expression as a sum of weighted functions is given in eq. (2.64) for.

$$
\begin{aligned}
& f(r)=\frac{\mathrm{d} \theta_{l}}{\mathrm{~d}(\log r)}=\sum_{i}^{N}\left[\theta_{i} \cdot \frac{1}{2 \cdot 0.4343} \cdot \frac{\left(\frac{R_{i}}{r \cdot n_{i}}\right)^{3} \cdot\left[n_{i} \cdot\left(n_{i}-1\right) \cdot\left(n_{i}-2\right)\right]}{\left(1+\frac{R_{i}}{r \cdot n_{i}}\right)^{n_{i}+1}}\right] \\
& \theta_{l}(r)=\sum_{i}^{N}\left[\theta_{i} \cdot \frac{\left.1+\frac{R_{i}}{r}+\left(\frac{R_{i}}{r \cdot n_{i}}\right)^{2} \cdot \frac{n_{i} \cdot\left(n_{i}-1\right)}{2}\right]}{\left(1+\frac{R_{i}}{r \cdot n_{i}}\right)^{n_{i}}}\right]
\end{aligned}
$$

Integration of (2.64) yields the moisture storage function $\theta_{l}(r)$ in dependence to the capillary radius. If the Laplace equation (2.14) is applied, pore size distribution (2.64) and moisture storage function (2.65) can be expressed in dependence to the capillary pressure $p_{c}$, too. There, the contact angle $\gamma$ is assumed to be $\gamma=0$. Moreover, if the Kelvin equation (2.17) is applied, both functions can also be expressed in terms of relative humidity $\varphi$.

The parameters both equations contain are the partial pore volume $\theta_{i}$, the corresponding main radius $R_{i}$ and the shape parameter $n_{i}$ for each modality $i$. The number of modalities $N$ characterizes the number of main pore classes. All parameters have to be 
adjusted to experimental data, e.g. pore structure data from mercury intrusion experiments or moisture storage data from sorption and retention experiments.

Häupl derives the capillary conductivity function $K_{l}\left(\theta_{l}\right)$ according to the bundle of tubes model (see Fig. 2.22). Starting from a single tube, the volume flow is integrated over the whole pore space following the pore size distribution data as shown by Krischer (1956). By relating the obtained function to its value at saturation (i.e. at the maximum radius $r_{0}$ ), a relative function is obtained which is then scaled by a measured conductivity value at saturation $K_{s}$. This is more or less equivalent to the model proposed by Burdine (1953). However, no tortuosity factor is introduced, implying the relative conductivity function is correctly derived by the bundle of tubes model and has to be scaled to a proper value at saturation only. The analytical expression of the derived conductivity function $K_{l}(r)$ in dependence to the capillary radius $r$ is given in eq. (2.66).

$$
\frac{\sum_{i}^{N}\left[R_{i}^{2} \cdot \theta_{i} \cdot \frac{n_{i}^{2}-3 n_{i}+2}{n_{i}^{2}} \cdot\left(\frac{1}{1+\frac{R_{i}}{r \cdot n_{i}}}\right)^{n_{i}}\right]}{\sum_{i}^{N}\left[R_{i}^{2} \cdot \theta_{i} \cdot \frac{n_{i}^{2}-3 n_{i}+2}{n_{i}^{2}}\right]}
$$

Unfortunately, as already stated by Häupl $\mathcal{E}$ Fechner (2003a), the conductivity function (2.66) can not be directly expressed in dependence to the moisture content $\theta_{l}$. This can only be done implicitly by numerically rearranging eq. (2.65). The model therefore provides a closed form only with respect to the capillary radius (or capillary pressure), but not with respect to the moisture content. Since the basis of numerical simulation is the moisture mass balance (2.2) requiring the inverse moisture storage function, this is rather a drawback of the approach. It hence can not be implemented in analytical form.

For vapour transport, Grunewald \& Häupl (2002) and Grunewald \& Häupl (2003) introduced a mechanistical approach dividing the porous material into parallel and serial structured areas. The pore system is partially filled with liquid water and water vapour and follows in its structure the pore volume distribution function. While in parallel structured areas, in dependence to the pore filling level, either liquid or vapour transport proceeds, both processes occur at the same time in serial structured areas, see Fig. 2.23. By assuming both structured forms of infinitesimal size and randomly distributed, a scal- parallel structured pore space

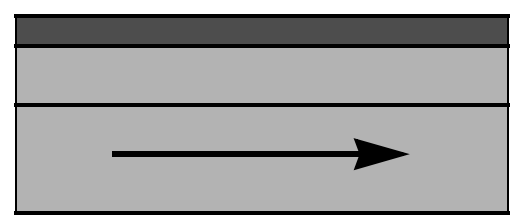

serial structured pore space

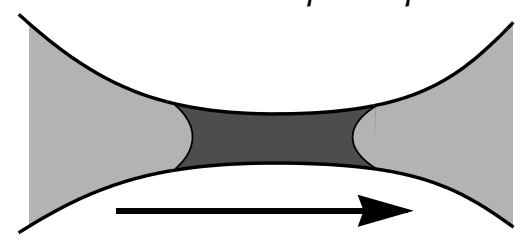

water vapour

liquid water

Figure 2.23: Model image of serial and parallel structured areas of pore space. 
ing function for vapour transport $f_{v}(\Theta, p)$ can be derived according to eq. (2.68) in dependence to the relative moisture content $\Theta$ and the ratio $p$ of serial and parallel structures. The relative moisture content $\Theta$ is here defined according to eq. (2.67).

$$
\begin{aligned}
& \Theta=\frac{\theta_{l}-\theta_{d r y}}{\theta_{p o r}-\theta_{d r y}} \\
& f_{v}(\Theta, p)=\frac{1-\Theta}{p+(1-\Theta)^{2} \cdot(1-p)}
\end{aligned}
$$

To obtain the whole vapour diffusivity function, the vapour transport equation (2.13) has to be multiplied with $f_{v}(\Theta, p)$. For parameter $p$ a relation in dependence to the transition moisture content $\theta_{\text {tran }}$ is proposed according to eq. (2.69).

$$
p=\frac{\left(1-\theta_{r, \text { tran }}\right)^{2}}{1+\left(1-\theta_{r, \text { tran }}\right)^{2}} \quad \text { with } \quad \theta_{r, \text { tran }}=\frac{\theta_{\text {tran }}-\theta_{d r y}}{\theta_{\text {por }}-\theta_{d r y}}
$$

In the hygroscopic moisture range, vapour transport dominates the whole moisture transport. However, with increasing moisture content, the liquid water transport reaches the same order of magnitude as vapour transport while a further increase in moisture content would lead to a (strong) domination of the liquid transport processes. The transition point is characterized by equality of both, liquid and vapour transport, and the corresponding moisture content is called transition moisture content $\theta_{\text {tran }}$. It hence depends on both transport functions and has to be determined by iteration. Further details of this serial - parallel model are given by Grunewald \& Häupl (2003).

The resulting vapour transport function holds a maximum at the transition moisture content and decreases to zero when the moisture content reaches porosity $\theta_{\text {por }}$. The maximum is explained by the short-cut in vapour transport due to liquid islands (see Fig. 2.23) and thus follows the explanation already introduced by Philip $\mathcal{E}$ De Vries (1957).

The model of Häupl E Fechner (2003b) and Häupl (2007b) provides a flexible function for the pore volume distribution and the moisture storage function, respectively. Especially for illustration purposes, the model is very suitable, since the influence of any model parameter can be directly seen in the pore volume distribution, the moisture storage and the liquid water conductivity function. However, the assumption the pore model would represent the relative conductivity function correctly and, thus, only requires scaling by a saturated conductivity value $K_{s}$, is seen problematic. For some materials, good agreement with measured data may be observed, but for a general derivation of the conductivity function this appears to be too inflexible. Admittedly, as a basis for further improvement, the model is suitable.

\section{Engineering model according to Grunewald and Bomberg}

The material model, introduced by Grunewald $\mathcal{E}$ Bomberg (2003) as an Engineering Model, is another bundle of tubes model which is relatively similar to the one of Häupl $\mathcal{E}$ Fechner. Following a similar concept from Soil Sciences, a logarithmic variable 
$p C=\log -p_{c}$ is introduced. Then, the pore volume distribution is described by the aid of a weighted sum of $N$ Gauss distribution functions given in eq. (2.70).

$$
\frac{\partial \theta_{l}}{\partial p C}(p C)=-\sum_{i=1}^{N}\left[\frac{\theta_{i}}{\sqrt{2 \pi} \cdot S_{i}} \cdot \exp \left(-\frac{\left(p C-p C_{i}\right)^{2}}{2 S_{i}^{2}}\right)\right]
$$

Integration yields the moisture storage function (2.71). Both equations contain three parameters. The $p C_{i}$ specify the positions of pore maxima, i.e. positions where the pore volume distribution has its maxima and where the moisture storage function has its steep slopes. The $\theta_{i}$ are the weighting factors for each modality. In the pore volume distribution function, the $\theta_{i}$ determine the height of a maximum. In the moisture storage function they define the plateau level between two slopes. The third parameter vector contains the standard deviations $S_{i}$ which influence the width of a pore maximum and, thus, the steepness of the slope of the moisture storage function.

$$
\theta_{l}(p C)=\sum_{i=1}^{N}\left[\frac{\theta_{i}}{\sqrt{2}} \cdot\left(1+\operatorname{erf}\left(\frac{p C_{i}-p C}{\sqrt{2} S_{i}}\right)\right)\right]
$$

The conductivity function is numerically derived, applying the pore model of Burdine (2.57). The relative conductivity function is scaled with the measured value at saturation $K_{s}$, while the tortuosity factor is neglected. For vapour transport, the same serialparallel model of Grunewald \& Häupl (2003) is applied as given above.

The Engineering Model is a flexible, multi-modal conductivity approach with validity for the whole moisture range. Its advantage is the high flexibility of the moisture storage function which is much better than those of the models of Durner (1994) and Häupl $\mathcal{E}$ Fechner (2003b). Moreover, the contained parameters have a particular meaning and can thus be easily determined from measured pore volume distribution or moisture storage data. However, the restrictions of no flexibility in the conductivity function apply here, too. The relative course of conductivity, scaled to one measured value at saturation, is not suitable to predict liquid water transport in the low moisture range correctly.

\section{Concluding bundle of tube models}

Describing the structure of a porous medium by a system of a connected bundle of tubes with tube radii following the pore radii distribution is a very simplified approach. Therefore, material functions derived by that approach will always require further calibration (e.g. scaling, tortuosity). With calibration, material functions are able to describe homogeneous and isotropic porous media. For less restrictive conditions, e.g. fractured and structured porous media, the approach is not expedient (see Gerke E Van Genuchten (1993), Tuller E Or (2002), Vandersteen et al. (2003)).

However, due to its simplicity the approach appears to be suitable for a broader application. Compared with more complex conductivity approaches, the effort to adjust 
material functions is relatively small. Required further calibration can be applied, either by use of the tortuosity, which has been shown by Ippisch et al. (2006) to be still very sensitive in being subjected to errors, or by coupling with other mechanistical approaches. When choosing conductivity models, an extension to hysteresis or salt and solute transport is possible without major changes. Therefore, the bundle of tubes approach offers the ability of a rather sophisticated but still practically applicable material model.

All models presented here give a functional description of the pore volume distribution which, by integration, yields the moisture storage function. By application of a pore model, the relative conductivity function is derived. The most flexible of these models is the one based on Gauss distribution functions. Hence, this approach will be further employed for an improved conductivity approach developed in paragraph 5.1 Developments in Material Modelling.

\subsection{Conclusions on material modelling}

At the beginning of this chapter, a classification of the transport theory in relation to material modelling was given. It is followed by a brief introduction into the principles of the transport theory defining the balance and constitutive equations with their required transport coefficients. Terms used in the context of heat and moisture transport modelling have been clarified and remaining challenges as the hysteresis of moisture storage have been discussed in the frame of existing literature.

Regarding hygrothermal calculations, material modelling is subdivided with respect to the potentials used as the driving force for moisture transport, which are the moisture content gradient requiring a moisture diffusivity and the capillary pressure gradient requiring a moisture conductivity. Based on the extent of literature available in Building Physics and Soil Sciences, different diffusivity and conductivity approaches were presented and evaluated, concluded by a discussion on their options and limits for a general applicable material description.

This chapter comprised the basis of transport theory and material modelling to which the following chapter will add the experimental methods required for both, basic material investigation providing input data for the material models and additional experimental studies possessing the potential to serve for a profound model validation. 


\section{CHAPTER 3}

\section{EXPERIMENTAL METHODS}

This chapter comprises an introduction of and a discussion on the laboratory methods applied for material investigation. Here, material investigation is subdivided into basic material property determination, which is considered to cover the methods of deriving material data to be used for parameter identification in material modelling, and advanced examination of hygrothermal processes containing the potential for both, material model validation and gaining further insight into the transport processes.

There exists a large number of methods for hygrothermal material investigation which are reported in literature. Thus, a complete survey on all these methods will not be given here. Instead, a selection of basic laboratory experiments is introduced and discussed under the criteria of being reasonable and applicable from a Building Physics point of view. Within the second part of this chapter, the instantaneous profile measurement technique, originally developed in Soil Physics, will be introduced and explained in its principle elements.

\subsection{Basic hygrothermal material properties}

Material models require input material data for the adjustment of their parameters. This material data has to be determined by measurement from different experiments and is regarded as the basis of material modelling. Therefore, these material properties are called basic material properties and the corresponding methods for their determination are regarded to as basic experiments. An overview of the basic material properties and the experiments they are determined with is given in Tab. 3.1. 
The different material models imply different requirements on material data, compare Chapter 2, especially paragraph 2.2 Material Modelling in Building Physics. Therefore, within this chapter, the experiments the author considers to be both, applicable and appropriate for a general but advanced material characterization are briefly introduced. This comprises customary procedures for density, porosity and thermal property determination, different techniques for the measurement of moisture storage data with respect to the different moisture content ranges as well as experiments to investigate the moisture transport properties for both, vapour and liquid water. While most of the methods are to be outlined in principle, there will be placed some more emphasis on two transport experiments. These are the water absorption and the drying experiment, which are important due to their exposed position within material characterization and due to the developments carried out.

Table 3.1: List of basic experiments and derived material properties.

\begin{tabular}{lcc}
\hline Name of the material property / experiment & Symbol & Unit \\
\hline Bulk (raw) density & $\rho_{b}$ & $\mathrm{~kg} / \mathrm{m}^{3}$ \\
Matrix (particle) density / helium pycnometry & $\rho_{m}$ & $\mathrm{~kg} / \mathrm{m}^{3}$ \\
Porosity & $\theta_{p o r}$ & $\mathrm{~m}^{3} / \mathrm{m}^{3}$ \\
\hline Thermal conductivity / heat flux measurement & $\lambda_{d r y}$ & $\mathrm{~W} /(\mathrm{mK})$ \\
Heat capacity / calorimeter experiment & $c$ & $\mathrm{~J} /(\mathrm{kgK})$ \\
\hline Sorption moisture content & $\theta_{l}(\varphi)$ & $\mathrm{m}^{3} / \mathrm{m}^{3}$ \\
Retention moisture content & $\theta_{l}\left(p_{c}\right)$ & $\mathrm{m}^{3} / \mathrm{m}^{3}$ \\
Effective saturation moisture content & $\theta_{e f f}$ & $\mathrm{~m}^{3} / \mathrm{m}^{3}$ \\
\hline Dry-cup vapour diffusion & $\mu_{d r y}$ & --- \\
Wet-cup vapour diffusion & $\mu_{w e t}$ & --- \\
\hline Water absorption data / water uptake experiment & $\theta_{l}(t)$ & $\mathrm{m}^{3} / \mathrm{m}^{3}$ \\
Water uptake coefficient & $A_{w}$ & $\mathrm{~kg} /\left(\mathrm{m}^{2} \sqrt{\mathrm{s}}\right)$ \\
Capillary saturation moisture content & $\theta_{\text {cap }}$ & $\mathrm{m}^{3} / \mathrm{m}^{3}$ \\
\hline Drying data / drying experiment & $\theta_{l}(t)$ & $\mathrm{m}^{3} / \mathrm{m}^{3}$ \\
\hline Conductivity at effective saturation / Darcy flow measurement & $K_{l}\left(\theta_{e f f}\right)$ & $\mathrm{s}$ \\
Unsaturated conductivity data / infiltrometer experiment & $K_{l}\left(\theta_{l}\right)$ & $\mathrm{s}$ \\
\hline
\end{tabular}

\subsubsection{Density and porosity}

There exist two kinds of density which have to be distinguished: The bulk and the matrix density. The bulk density $\rho_{b}$ characterizes the density of the material as it is. The volume of both, material matrix and pores, is comprised. The bulk density is defined as the ratio of the dry mass and the volume of the dry specimen following eq. (3.1). 


$$
\rho_{b}=\frac{M_{d r y}}{V_{b u l k}}
$$

The matrix density $\rho_{m}$ specifies the density of the material matrix without the pore space. It can be determined by helium pycnometry. There, the crushed dry material is put into a vessel of defined volume. Then, a measured amount of helium is given into the vessel until a certain pressure is reached. Since helium is the smallest element, any space not occupied by another element will be entered. From the difference in the amount of helium which could be given to the empty vessel under the same pressure, the volume $V_{\text {matrix }}$ of the crushed material can be determined. The matrix density is then determined as the ratio of the dry material mass and the determined volume, following eq. (3.2). For natural stones, DIN EN 1936 proposes a similar procedure using a pycnometer with deionized water. However, the use of helium offers a higher degree of precision.

$$
\rho_{m}=\frac{M_{d r y}}{V_{\text {matrix }}}
$$

From both densities, the total porosity of the material can be derived. It is defined in eq. (3.3) as the volume fraction which is not occupied by the material matrix.

$$
\theta_{\text {por }}=1-\frac{\rho_{m}}{\rho_{b}}
$$

Sometimes, the open porosity $\theta_{v a c}$ is used as a material parameter, too. It gives a measure for the pore volume which is connected to the pore system and can thus be entered by water. The open porosity can be determined by saturation under vacuum conditions. The primary desorption function starts at open porosity. However, as these conditions are hardly reached in practice, this parameter is not considered within the frame of this work.

\subsubsection{Thermal properties}

The thermal properties of interest are the heat capacity $c$ and the thermal conductivity $\lambda_{d r y}$. The heat capacity can be measured by the calorimeter experiment where a heated specimen of defined temperature is placed into water with another (lower) defined temperature. Knowing the heat capacity of water, the heat capacity of the material specimen can be derived, if the final equilibrium temperature, which the water and the specimen have reached, is measured.

The thermal conductivity can be determined by the help of heat flow experiments. Most common - since applicable for most building materials - is the plate apparatus. Different regulations and standards exist on that, e.g. ISO 8302 (1991), DIN EN ISO 12664 and WärmeleitRechenWRL (1999), on which no further emphasis is placed here. The principle is to apply a temperature difference versus the thickness of the dry specimen and measure both, the temperature on either side and the heat flux through the specimen. From that, the thermal conductivity of the dry material can be derived. 
Another possibility deriving both properties at the same time is the use of a device that is placed on a specimen applying a dynamic circle of heating and cooling. The advantage of such a device is, that the measurement result can be obtained much faster, since no steady state conditions are reached, both thermal properties can be determined at the same time and - which is most important - small specimen can be used, as it is often the case for materials taken from the building stock. The disadvantages comprise limited accuracy especially for unisotropic materials and the dynamic conditions, which sometimes might represent a different behaviour than steady state conditions do.

\subsubsection{Moisture storage}

Moisture storage data in general comprises the hygroscopic and the overhygroscopic moisture content range. Since different procedures and measurement technologies are applied in both moisture regions, a further distinction is made for moisture sorption and moisture retention. For each, a general introduction is given, while the focus is then placed on the measurement techniques applied for the investigated building materials.

Johannesson $\mathcal{E}$ Janz (2002) examined and compared four different methods to determine moisture storage data, including those applied here. However, this study can not be seen as a general evaluation since only two material were investigated of whom one was a building material (sandstone) and one was porous glass, where to some extent very small and crushed samples were used. Moreover, the drawn conclusions on hysteresis, on the limits of pressure plate measurements and the explanation why this technique is hardly being used for adsorption measurements are questionable from the authors point of view.

\subsubsection{Moisture sorption}

The measurement of moisture storage within the hygroscopic moisture content range is widely done by exposing a material specimen to a defined relative humidity, waiting until equilibrium (constant mass) is reached and gravimetrically determining the corresponding moisture content.

Either a complete device, as the IGASORP, a micro balance system (as e.g. used by Johannesson $\mathcal{E}$ Janz (2002)) or a sorption calorimeter (see e.g. Adan (1994) and Broken (1998)), can be applied adjusting the relative humidity and performing the gravimetric measurements automatically, or the conventional desiccators with saturated aqueous salt solutions, see Fig. 3.1, are employed (as reported e.g. by Burch et al. (1992) and Broken (1998)). Here, it is referred to the latter one, which is standardized in DIN EN ISO 12571.

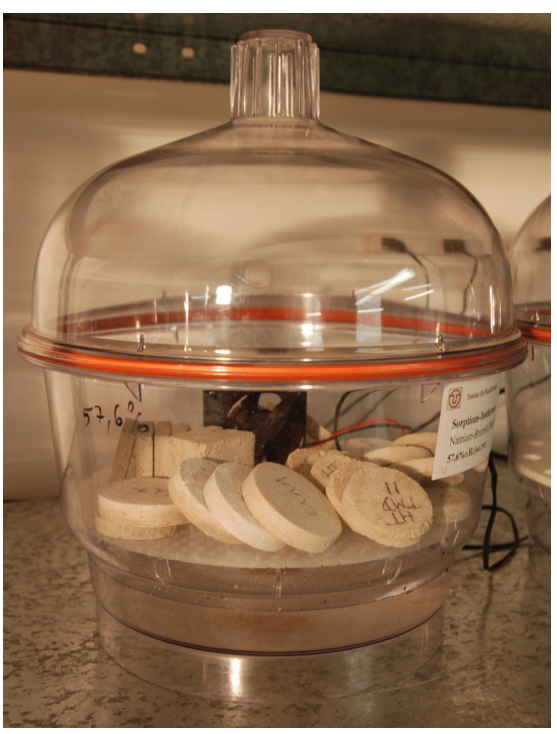

Figure 3.1: Desiccator with salt solution for moisture sorption measurements. 
To adjust a defined relative humidity inside a desiccator, saturated aqueous salt solutions are used. A fan inside the desiccator causes quick equilibration of the humidity conditions. To keep the temperature constant, either a water bath for the desiccators, or a temperature controlled room can be used. The material specimens are placed into the desiccator and left there for a certain time, normally two to four weeks, until equilibrium is reached. The employed criterion for the equilibrium is the one defined in DIN EN ISO 12571: The mass change of the specimen of three subsequent measurements within 48 hours has to be less than $0.1 \%$ of the dry mass of the specimen. Once the equilibrium is reached, the material specimen is weighed. From the ratio to the dry weight, the moisture content can be derived.

\subsubsection{Moisture retention}

The moisture storage within the overhygroscopic moisture range is frequently called moisture retention due to the most common measurement techniques used for its determination. The relative humidity can not be used to adjust the moisture potential in that moisture range. Hence, a pressure is applied to an initially saturated specimen and the retained water mass is measured after equilibrium is reached. Two main principles are distinguished: methods applying an overpressure and methods applying a suction pressure to the material specimen. Both methods are briefly presented as follows.

\section{Pressure plate apparatus}

The pressure plate apparatus consists of a pressure chamber to which an overpressure can be applied, a set of ceramic plates and a gas pressure supply with a regulation system, see Fig. 3.3. The ceramic plate holding a certain air entry value, i.e. a certain resistance to the pressing air to penetrate the plate, is placed inside the pressure chamber. At the left hand side of Fig. 3.2, a ceramic plate with an air entry value of $0.1 \mathrm{MPa}$ is shown. Upon the ceramic plate, saturated specimens are set providing hydraulic contact by a special kaolin-silt mixture. To prevent the specimens from drying out by evaporation, it is recommended to cover them with a wet towel (Klute (1986)). The bottom side of the ceramic plate is connected to atmospheric pressure via an outlet in the pressure chamber, where the water pressed out of the specimens is allowed to leave. By applying a controlled overpressure, a certain amount of pores will be drained
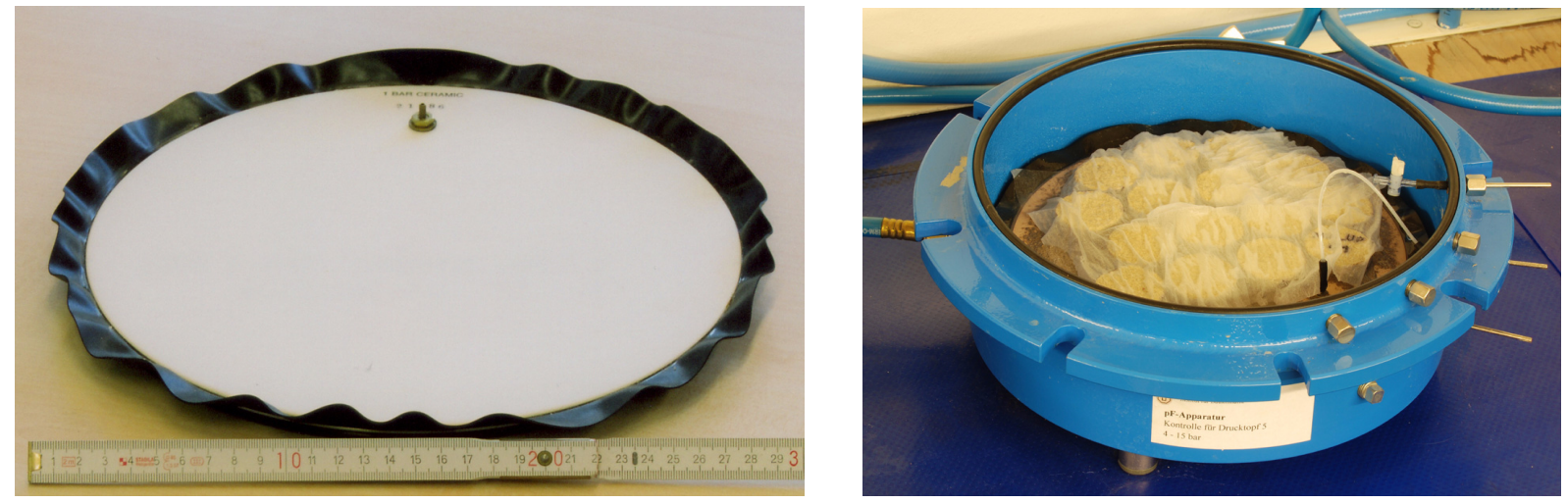

Figure 3.2: New ceramic plate (left) and pressure chamber with specimens on ceramic plate, covered with wet tissue and connected to the outlet (right). 


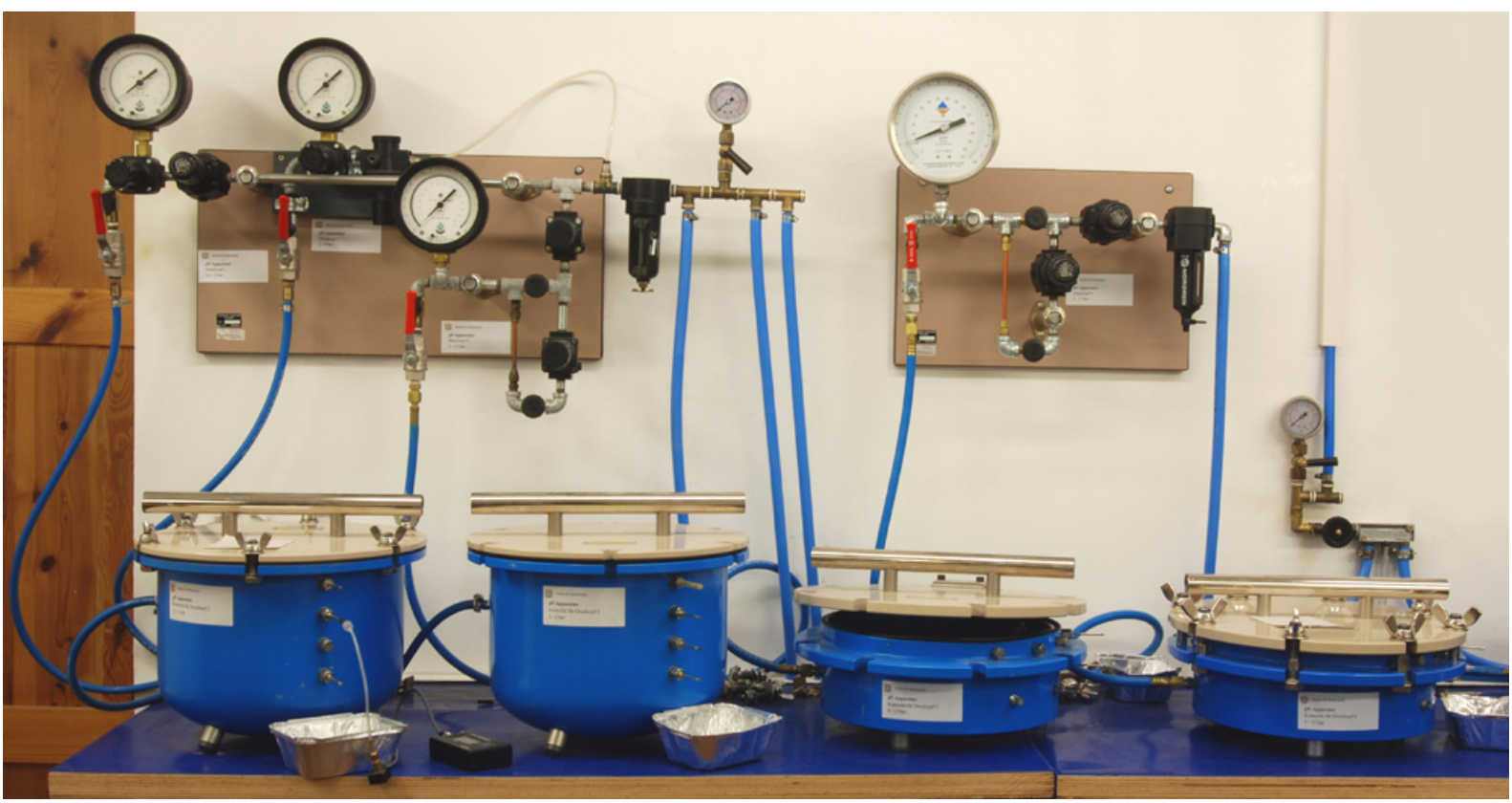

Figure 3.3: $\quad$ Pressure plate apparatus: Pressure chambers for pressures up to 0.5 MPa (left) and up to $1.5 \mathrm{MPa}$ (right), together with the control valves.

according to their size (see Laplace equation (2.14)). When equilibrium is reached, the specimens are removed and their moisture content is determined gravimetrically, before the next pressure step is started.

The criterion for equilibrium according to DIN ISO 11274 is, that the mass change between measurements at two subsequent days is less than $0.02 \%$ of the sample mass. This is slightly different than the criterion given above, which is due to the different disciplines (Soil Science, Building Physics) where the standards were developed in. The time required for equilibration generally depends on the investigated material and the height of the specimens. However, experience from a large number of measurements revealed that for most building materials equilibrium is reached after three to four weeks, for materials with a very low liquid water conductivity (as e.g. concrete) six weeks are sufficient (for a sample height of $1 \mathrm{~cm}$ ). For different applied pressures, different pressure chambers and different ceramic plates according to their air entry value are used, where it is recommended to use always the plate with the smallest possible resistance (Klute (1972), Klute (1986)). The accessible pressure range of the measurements reported here is shown in Tab. 3.2.

Table 3.2: Pressure steps investigated with the pressure plate apparatus.

\begin{tabular}{lccccccccc}
\hline Applied pressure in [MPa] & 0.003 & 0.01 & 0.03 & 0.06 & 0.1 & 0.2 & 0.5 & 0.8 & 1.4 \\
\hline Used ceramic plate in [MPa] & 0.05 & 0.05 & 0.05 & 0.1 & 0.2 & 0.3 & 0.5 & 1.5 & 1.5 \\
\hline
\end{tabular}

Normally, only the desorption characteristic is measured by this method due to the difficulty to ensure perfect water contact at the bottom side of the ceramic plate. With specially designed and flushable ceramics, this would be possible, though very laborious. The idea of the pressure plate apparatus goes back to Richards $\mathcal{E}$ Fireman (1943) who first build such a device and compared their measurement results with those from suc- 
tion measurements obtaining good agreement. An overview on the pressure plate method was given by Klute (1986). A general instruction for both, suction and overpressure methods, can be found in DIN ISO 11274.

\section{Hanging water column}

There exist different possibilities to apply a suction, i.e. a hanging water column to a material specimen depending on the range of suction pressures to be investigated. For very low suction values (used here between 0 to $2 \mathrm{kPa}$, higher suction pressures are possible), a burette device can be used allowing to adjust the suction value very precisely. Higher suctions up to $50 \mathrm{kPa}$ are adjustable with suction tables in combination with different filling materials.

The burette device consists of a glass cylinder funnel which is connected to a burette, see Fig. 3.4. Inside the cylinder, a small, highly permeable ceramic is sealed on which the material specimen is placed. Below the specimen, water contact is provided. After the system is closed again, the height of the hanging water column can be adjusted by the help of the burettes.

The suction table method consists of a bowl filled with a sand or kaolin mixture on which the material specimens are placed. At the bottom, a water supply and a vacuum pump are attached allowing to
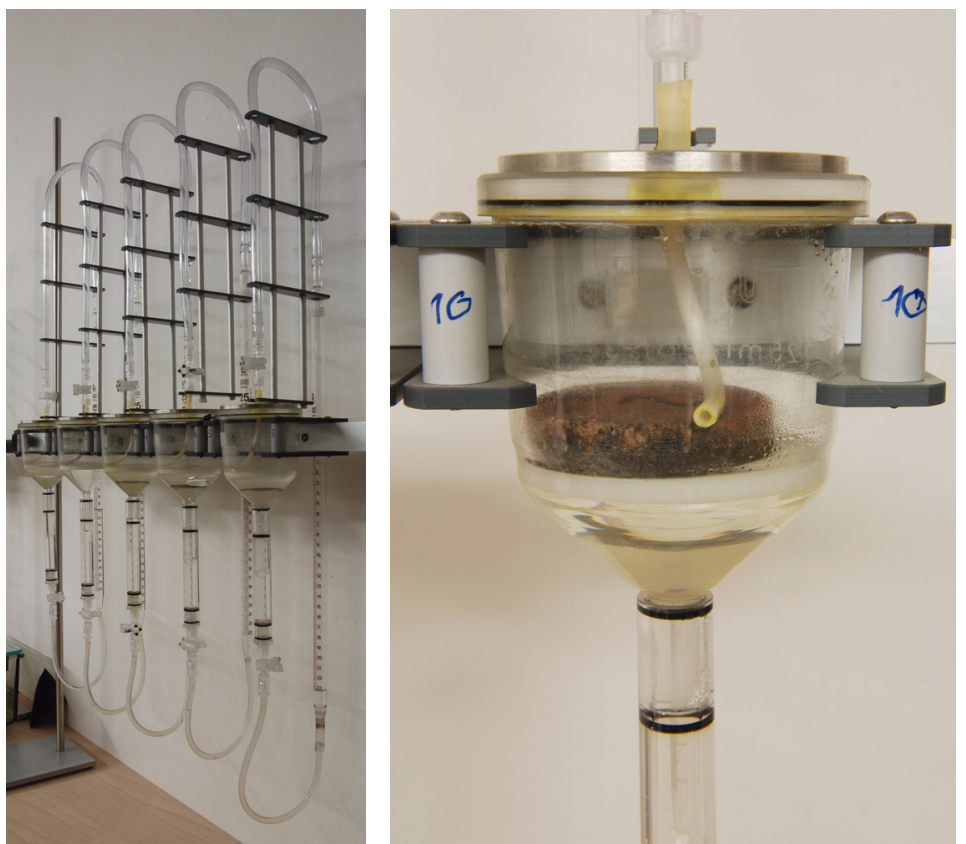

Figure 3.4: $\quad$ Perspective view on the burette device (left) and detail of the funnel cell with ceramic and material sample (right). once the air has been removed. Depending on the filling material, different suction values can be applied.

When the material specimens are brought into hydraulic contact, equilibrium has to be awaited before the corresponding moisture content is determined gravimetrically. The hanging water column methods can be used to investigate both, adsorption and desorption characteristics. Especially for low suction values they are advantageous as they allow a rather precise adjustment of the applied potential. However, special attention has to be paid for the hydraulic contact. 


\subsubsection{Vapour diffusion}

The principle of measuring vapour transport through a building material is based on steady state flow conditions which develop under a constant potential gradient. The potential, i.e. the vapour pressure, can be adjusted by controlling the relative humidity and keeping the temperature constant. The relative humidity can either be adjusted by saturated aqueous salt solutions or by a climatic chamber. If the flow rate through the material is measured, the transport properties can be derived for the corresponding potential gradient.

For vapour diffusion measurements, so called cups are used, see Fig. 3.5. Inside the cup, the relative humidity is adjusted whether with a drying agent or with a salt solution. A material specimen is sealed into the lid of the cup providing one-dimensional transport. The cup is closed with the lid allowing vapour to enter (or leave) only through the investigated material. The whole cup is placed either into a climatic chamber or into a desiccator, where another relative humidity is adjusted. Depending on the vapour pressure gradient, vapour will enter or leave the cup. From gravimetric measurements, the flow rate can be obtained and, after a steady state flow has been developed, the vapour diffusion coefficient can be derived.

With the choice of different relative humidity conditions, the vapour diffusion coefficient can be determined for different potential gradients and correspondingly for different moisture contents. With this regard, the experiment is often called dry-cup or wet-cup experiment. For higher relative humidities, not only vapour diffusion proceeds, but also liquid conduction. In combination with an assumption on up to which relative humidity does only vapour transport proceed, the liquid transport rate and, thus, the liquid water conductivity can be determined from the wet-cup measurements, too.

To achieve reliable measurement results, from the authors point of view it is recommended to keep the

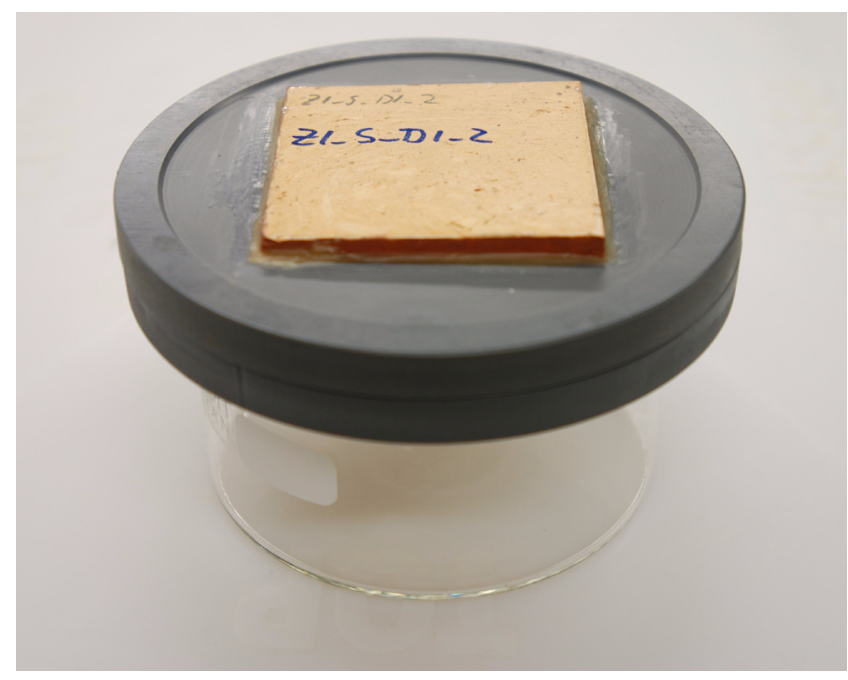

Figure 3.5: Cup for measurement of vapour diffusion. The material specimen is sealed into the removable lid enabling preconditioning and easy change of boundary humidity conditions. relative humidity difference rather small (i.e. $10 \%$ to $20 \% \mathrm{RH}$ ). By preconditioning the material specimens at a mean relative humidity of the planned experiment, the time the steady state flow conditions require to develop can be significantly reduced. This is the reason for the special cup design shown in Fig. 3.5, where the lid can be easily removed for that purpose. Moreover, the mean moisture content of the specimen can be determined at the beginning and at the end of the experiment which is important if no assumption is to be implied whether the adsorption or the desorption storage characteristic is to be used to assign the transport coefficient to a moisture content. The cup design was developed by Plagge (2001). 
The basic principle of the vapour diffusion measurement procedure is standardized in DIN EN ISO 12572. Different measurement devices have been introduced in literature, e.g. by Richards et al. (1992), Galbraith (1992), Galbraith et al. (1997), Galbraith et al. (1998), Time (1998), Hansen et al. (2001), Padfield et al. (2002), Galbraith et al. (2003) and Plagge et al. (2003).

In contrast, Arfidson $\mathcal{E}$ Cunningham (2000) introduced a transient technique to determine the moisture diffusion coefficients in the hygroscopic region, where they applied a stepwise increase in relative humidity to a material specimen and recorded the mass increase in time. With the knowledge of the sorption characteristic of the material, the hygroscopic moisture diffusivity could be derived. The method itself provides a significant gain in time which is necessary for the measurement. However, the sorption curve is required for its evaluation implying the problem of hysteresis and limiting the overall time gain, since the sorption measurement itself is rather time consuming.

\subsubsection{Water uptake experiment}

The water absorption or water uptake experiment measures the mass increase during a one-dimensional imbibition of a material specimen. It is specified in DIN EN ISO 15148. To provide one-dimensional transport, the lateral sides of the specimen have to be sealed. After preconditioning at normal laboratory conditions (between $18^{\circ} \mathrm{C}$ and $28^{\circ} \mathrm{C}, 40 \%$ to $60 \%$ relative humidity), the specimen is placed into water with its bottom side. With increasing time intervals, the weight of the specimen is determined. For this purpose, the specimen is taken out of the water, its bottom side is dabbed off with a towel and it is placed on a balance. This has to be repeated several times to obtain a number of measurement points forming a characteristic water-uptake versus time curve. According to DIN EN ISO 15148, the experiment is completed after $24 \mathrm{~h}$.

For homogeneous and isotropic materials, the water absorption experiment consists of two distinct phases, see Fig. 3.6, curve (a). The first one is characterized by a linear mass increase versus the square root of time. The transition from the first to the second phase proceeds when the moisture front reaches the top side of the specimen. A further mass increase is due to redistribution processes involving dissolution of entrapped air and typically proceeds very slowly.

From this experiment, the water uptake coefficient and the capillary moisture content can be derived. The water uptake coefficient $A_{w}$ in $\left[\mathrm{kg} /\left(\mathrm{m}^{2} \sqrt{\mathrm{s}}\right)\right]$ is determined as the slope of the mass increase versus square root of time during the first phase of the experiment. The capillary moisture content $\theta_{\text {cap }}$ in $\left[\mathrm{m}^{3} / \mathrm{m}^{3}\right]$ is usually taken as the moisture content which is reached when the slope of the mass increase deviates from the linear behaviour in the square root of time scale or as the moisture content at the end of the experiment. This is, however, only a rather rough approximation, as will be further discussed also in section Capillary moisture content range in Chapter 5. 


\section{Remarks on the water uptake experiment}

As it is the typical case for standards, DIN EN ISO 15148 can be seen as a specification of minimum requirements, only. From the authors point of view, a number of details have to be taken into account with more care. These are:

- duration of the whole experiment and amount of data points collected during measurement,

- $\quad$ sealing of the upper side to prevent evaporation / moisture absorption due to boundary conditions,

- $\quad$ dipping / water contact at the bottom surface of the specimen

The duration of the experiment is seen most problematic. Since the time the moisture front requires to reach the top of the specimen is dependent on both, the transport properties of the material and the height of the specimen, it is much too general to stop the experiment after 24 hours, see illustration in Fig. 3.6, curve (b). Therefore, the material behaviour should be taken as the criterion when to stop the experiment. For the interpretation, especially with regard to the calibration of material functions, the whole water absorption curve is of interest. A single coefficient, as $A_{w}$ is useful for a rough approximation. For a profound adjustment of the hygric transport functions, however, $A_{w}$

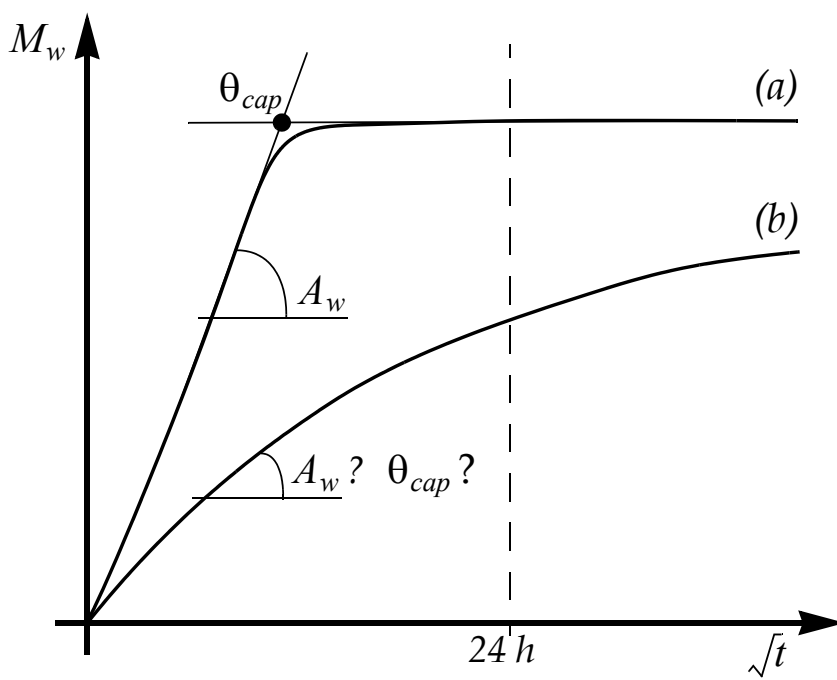

Figure 3.6: Water uptake curves for a homogeneous material (a) and an inhomogeneous material (b). alone is not expedient, especially in case the material does not behave as ideal as curve (a) shown in Fig. 3.6.

To overcome this problem, Häupl et al. (1989) and later Plagge et al. (2005) developed and introduced an automatic device to measure the water uptake behaviour of building materials, of which a picture is given in Fig. 3.7. Here, the mass increase is continuously measured by an automatic balance delivering the whole curve. The duration of the experiment can be adjusted exactly to the material behaviour by evaluating the logged data. Moreover, the water absorption behaviour of building materials which do not follow the ideal square root of time course, as e.g. curve (b) in Fig. 3.6, can be observed and evaluated, too.

To prevent a material specimen from drying by evaporation through the upper surface of the specimen, it is recommended to cover the upper surface, too. However, as already discussed under paragraph 2.1.4 Remarks on heat, air and moisture transport, following Wilson E Luthin (1963), Descamps (1997) and others, the cover must enable air pressure equilibration with the atmosphere. Therefore, the top sealing should be pierced or some gaps should be left for the air pressure to equilibrate. 
For the interpretation of the experiment by numerical simulation, it is important to precisely know the boundary conditions. When a conductivity model is employed, a pressure condition can be applied, otherwise the boundary moisture content must be known. Typically, the pressure condition can be easily determined while the boundary moisture content has to be estimated by one or the other method the capillary moisture content is derived with. As the moisture storage function is normally defined only in the capillary pressure range, i.e. for suction pressures, but not for overpressures, it is recommended to perform the experiment under conditions where no overpressure is applied. Ideally, a zero pressure condition is applied, which means that the bottom surface of the speci-

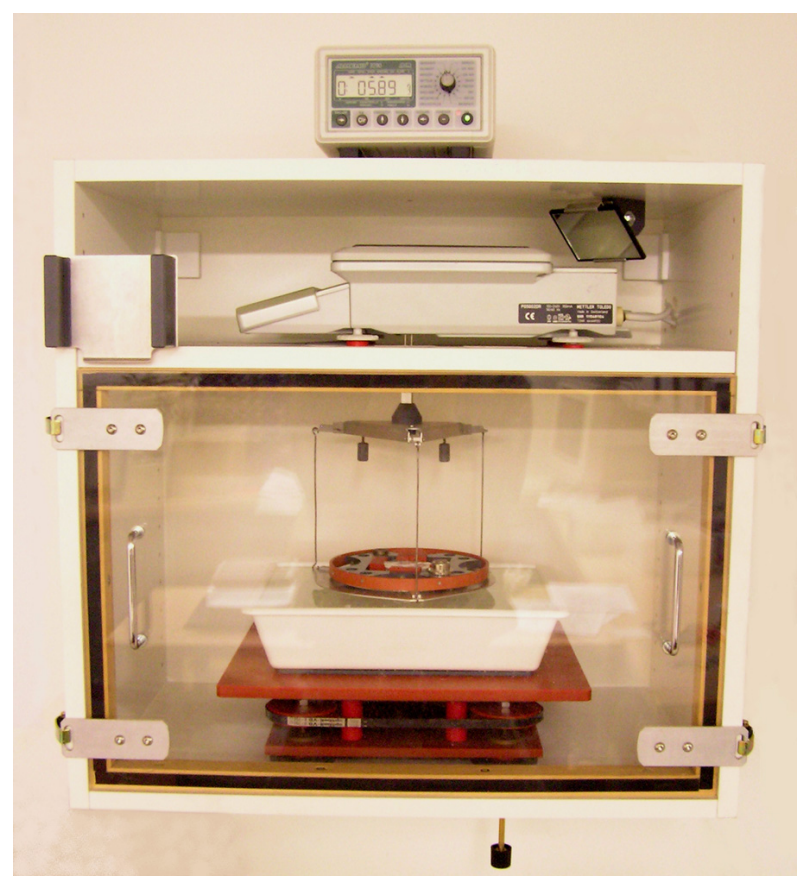

Figure 3.7: $\quad$ Device for automatic water uptake measurement developed by Plagge et al. (2005). men is equal to the water surface and neither a small water column is hanging nor a small overpressure due to the dipping is applied. However, achieving such conditions is rather difficult. Thus, from the authors point of view, a small hanging water column at the bottom surface is to be preferred. However, in contrast to this, the standard recommends a small overpressure, mainly due to practical reasons, which slightly complicates the interpretation of measured data.

In literature, explanations concerning the water absorption experiment are mainly given in the context of moisture profile measurement techniques as nuclear magnetic resonance (NMR) or $\gamma$-ray, e.g. by Pel (1995), Krus (1995), Descamps (1997) and Broken (1998). The experiment in general is briefly summarized, among others, by Roels (2000), Mukhopadhyaya et al. (2002) who investigated the influence of water temperature on the absorption coefficient, Bomberg et al. (2005) and Janssen et al. (2007), while Fitz E Krus (2004) discuss the clutter resulting from different standards which regulate the same experimental method from different points of view. However, a clear and comprehensive examination of the experiment can only be found in the works of Häupl et al. (1989) and Plagge et al. (2005).

\subsubsection{Drying experiment}

The drying behaviour of building materials reveals information about the moisture transport properties in the lower overhygroscopic moisture content range. Thus, data from drying experiments were occasionally used to identify the transport parameters required in simulation models. With this respect, Pel (1995) and Broken (1998) performed a series of drying experiments recording moisture profiles by the 
help of NMR, whereas drying experiments were also mentioned in that context by Künzel (1994) and Krus \& Holm (1999). On the other hand, drying plays an important role for outer wall structures, as indicated by Ojanen et al. (2002), who investigated the drying efficiency of such structures under different thermal conditions.

The general principle of a drying experiment is rather simple. A laterally sealed, saturated material specimen is exposed to certain boundary conditions while its weight change is measured versus time. However, the drying behaviour is strongly dependent on these boundary conditions, especially on the transfer conditions, which is probably the main reason why neither any kind of regulation on drying experiments exists, nor a wider application or explanation of them can be found in Building Physical literature.

A first attempt was made by Bednar (2002) who investigated the influence of surface cooling due to evaporation on the drying behaviour using thermally insulated specimens of aerated concrete. Another study, reported by Bednar E Dreyer (2003), concerns the varying transfer conditions within a room due to ventilation which was performed on the basis of drying experiments, too. Finally, Moropoulou et al. (2005) reported an investigation on the drying behaviour of different bricks, stones and plasters under different boundary conditions. Unfortunately, neither a clear description of the measurement setup, nor an evaluation of the transfer conditions was given significantly complicating the data evaluation with respect to material modelling.

It is to be stated, that a systematic analysis of the process and a development of instructions that should be followed for a reasonable performance of drying experiments could not be found. Hence, a special need for both, analysis and development is noticed here. Therefore, and due to the high level of importance in which the author refers to drying experiments with regard to hygric material modelling, a systematic analysis of the drying experiment leading to the development of a new drying device was performed. This has already been published by Scheffler E Plagge (2005a), whereas a detailed elaboration is given in paragraph 5.2 Methodological Developments for the Drying Experiment.

\subsubsection{Saturated and unsaturated flow experiments}

Within the capillary pressure range close to saturation, steady state flow measurements at different pressure gradients can be performed to determine capillary conductivity data. In principle, the experimental setup is based on the same idea as introduced for vapour diffusion measurements. A potential gradient is applied to a laterally sealed, saturated material specimen and the development of steady state flow conditions is awaited. From this steady state flow rate the transport coefficient under the corresponding potential gradient can be derived and related to the additionally measured state variable, i.e. the water content.

Most of the methods for the hydraulic conductivity measurement were developed in the field of Soil Science. A comprehensive review on different steady state and unsteady state laboratory methods was given by Klute (1972) and Klute E Dirksen (1986), whereas a sound and tight assessment can be found in Plagge (1991). In Building Physics, Descamps (1997) and Roels (2000) used saturated conductivity data. However, they performed air permeability measurements for their materials and recalculated the 
water conductivity in dependence on the ratio of the kinematic viscosities of air and water. The application of a direct water conductivity measurement device called head permeameter was first reported by Plagge et al. (2003). More recently, another device was developed based on tension infiltrometry which allows to measure conductivity data also under unsaturated conditions. The principle ideas of both methods are briefly explained as follows, whereas an elaborate introduction of both devices can be found by Plagge et al. (2007).

For the head permeameter device, a material specimen is sealed with epoxy into a perspex ring, see Fig. 3.8 (b). The ring fits into the permeameter head where it is tightly fixed by a rubber pressure membrane (Fig. 3.8 (c)). One side of the specimen is now accessible to boundary conditions. The other side is, together with the head, connected to a glass container and a vacuum pump. After the permeameter head with the specimen is placed into a water bath, suction is applied to the system connected to the other side causing a water flow through the material sample. The applied suction pressure, the water temperature and the water flow rate are continuously measured enabling the liquid water conductivity to be derived once steady state flow conditions have developed. A view on the whole device is shown in Fig. 3.8 (a).

The second device, called tension infiltrometer, uses a flushed ceramic in combination with a vacuum pump to apply a suction value at the bottom side. A material specimen is set on the ceramic providing capillary contact by the help of a kaolin-silt mixture as shown in Fig. 3.9 (c). At the top side of the specimen, another suction value is adjusted by the help of a closed water reservoir with a small capillary allowing air to enter in case the pressure falls under a certain value (Fig. 3.9 (b)). By that, the water from above stays under a certain tension which is dependent on the size of the small capillary, while a higher suction value is applied to the bottom side of the material sample. The rest of the principle is the same as before: steady state flow is awaited, the flow rate is measured and the corresponding conductivity value is derived in dependence to both applied suction values. The general setup can be seen in Fig. 3.9 (a).
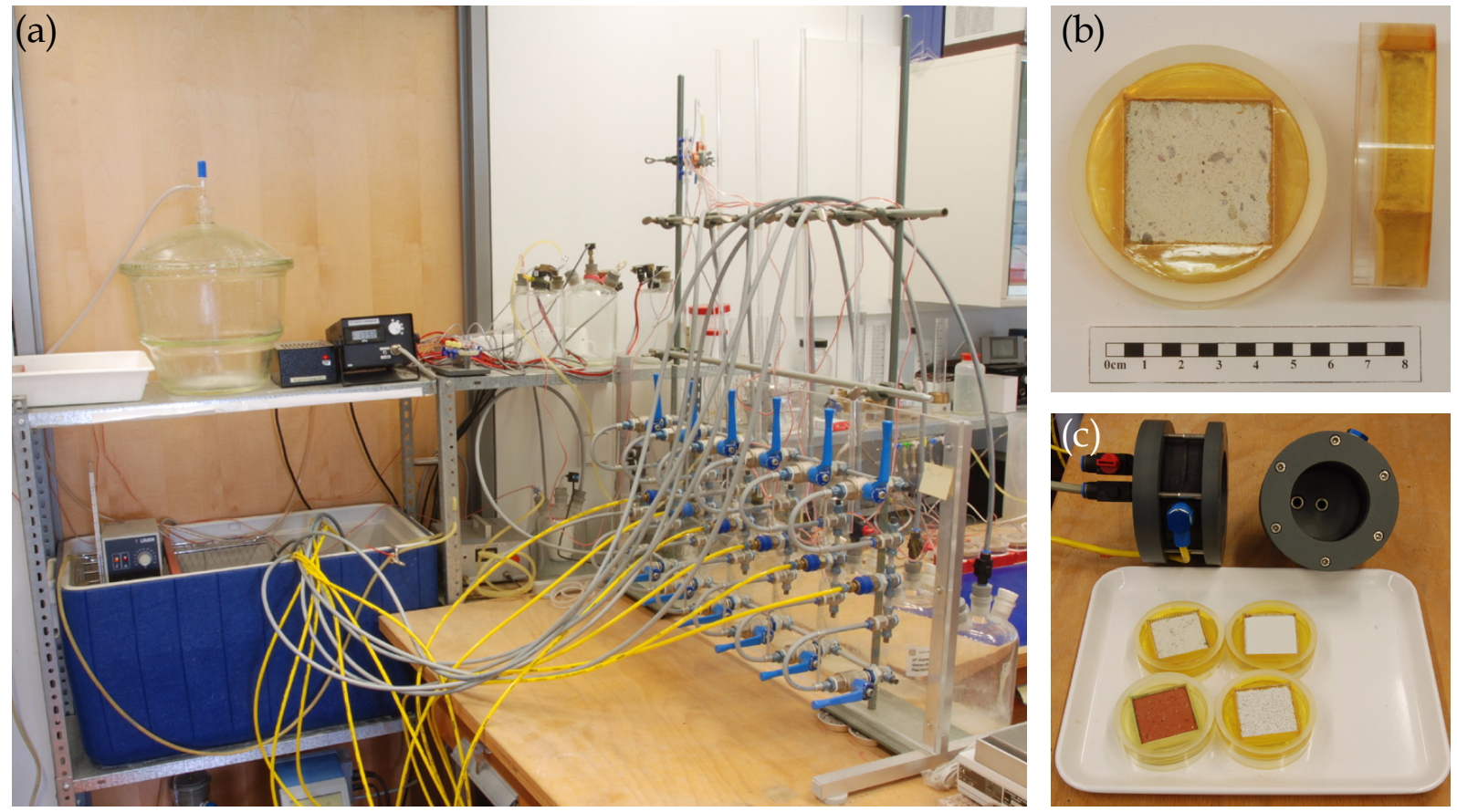

Figure 3.8: Head permeameter: complete device (a), material sample sealed into a perspex ring $(b)$ and permeameter head with material specimens (c). 

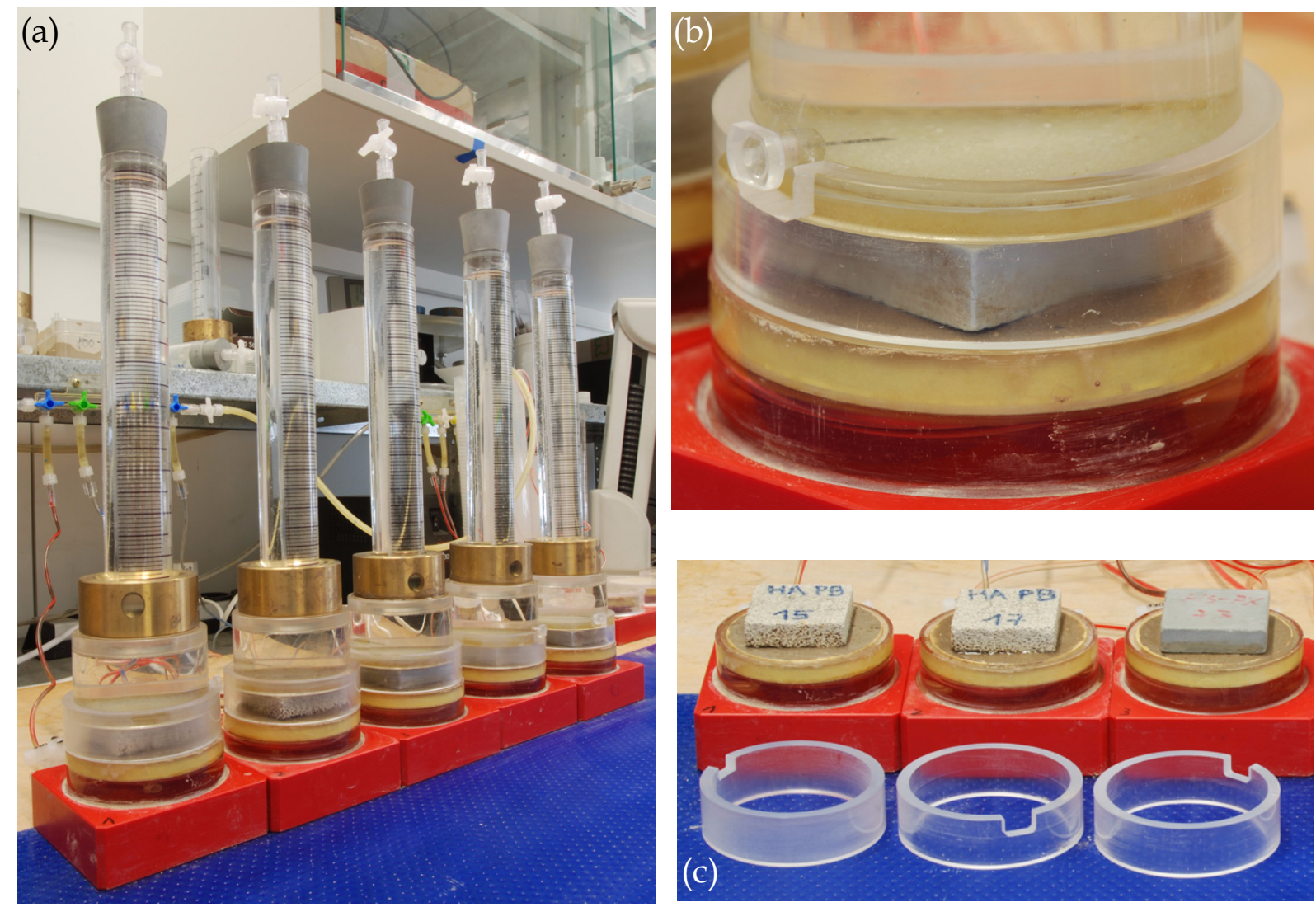

Figure 3.9: View on the tension infiltrometer device (a) with connection detail (b) and placing material samples on the flushed ceramic with a silt-kaolin mixture (c).

\subsubsection{Concluding basic experimental methods}

The set of basic static, steady state and unsteady state experiments for hygrothermal material characterization was introduced on the basis of both, literature and the authors own experience. However, not all these methods are exhaustively investigated and described, yet. Instead, several detail problems can occur which have to be carefully accounted for. Moreover, experimental methods often require minor modifications in dependence on the investigated material. Thus, there exists still a need for further developments in the field of experimental methods and procedures for hygrothermal material investigation, as indicated not only, but with most emphasis, for the drying.

On the other hand, the experimental methods introduced above comprise an expedient methodological foundation for an advanced but still relatively simply applicable hygrothermal material characterization. This thus serves as the basis for material data determination as input for material modelling. For model validation, however, experimental data is required which is not used as model input. Therefore, another unsteady state method was applied, providing moisture profiles in space and time during instantaneous conditions, which will be introduced as follows. 


\subsection{Instantaneous profile measurements}

Validation of hygrothermal material modelling requires experimental data which is, on the one hand, not used as model input and, on the other hand, provides a broad range of states which might occur also under transient and practical conditions. Hence, most suitable are unsteady state methods where both, moisture flow rates and moisture profiles are measured. The instantaneous profile measurement technique (IPM) is such a method and was applied in both, the hygroscopic and the overhygroscopic moisture content range for the investigated building materials. As follows, the principle of the instantaneous profile method will be explained, whereas subsequently, the different sensor types to measure the moisture content and the moisture potential are introduced.

\subsubsection{Principle of instantaneous profile methods}

The instantaneous profile method is based on the knowledge of the moisture distribution inside a material column under transient conditions. From both, the known moisture contents at different positions and times and defined boundary conditions, the moisture flow rates can be derived. When, in addition, the moisture potential distribution is known, the hydraulic conductivity can be directly derived from the flow equation.

There exist several variations in the method due to the different techniques used to determine moisture content or moisture potential and, since the method was developed in Soil Science, due to field or laboratory application. As field methods are not of interest here, the reader may be referred to the corresponding literature, e.g. Klute (1972) and Bouma (1977). The laboratory methods vary in the kind of flow experiment, which could be an infiltration or a drainage, in the kind of controlled boundary conditions, as e.g. a no-flow condition or a no-pressure condition, and in the quantities which are measured. For the latter one, the following combinations are possible (Klute (1972), Plagge (1991)):

- Moisture content profile is measured, moisture potential profile is derived based on measured moisture storage characteristics.

- Moisture potential profile is measured, moisture content profile is derived based on measured moisture storage characteristics.

- $\quad$ Both, moisture content and moisture potential profiles are measured.

Variation in the kind of flow experiment carried out is very appropriate to investigate adsorption and desorption processes and normally also influences the kind of boundary condition controlling. For the quantities to be measured it is certainly the best to determine both, moisture content and moisture potential. An illustration of the principle of instantaneous profile measurements is shown in Fig. 3.10.

Probably one of the first applications of one kind of the instantaneous profile method was reported by Richards $\mathcal{E}$ Weeks (1953) and Weeks $\mathcal{E}$ Richards (1967), who measured the drainage rate of a horizontal column of soil while the moisture potential was controlled by tensiometers. The water content was inferred from the desorption moisture storage characteristic. Other authors followed, either using the same combination of 


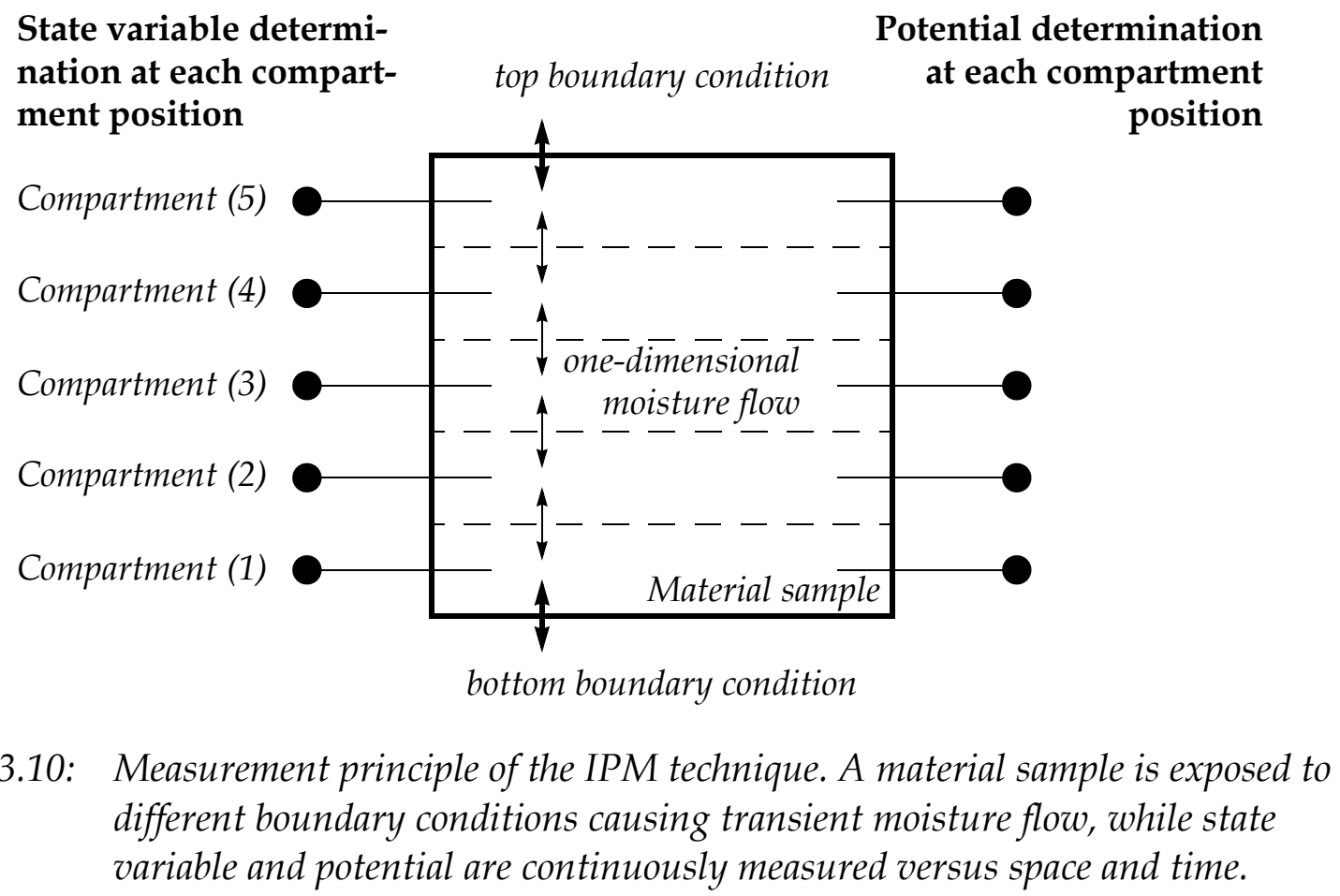

tensiometer sensors and moisture content inference or applying $\gamma$-ray attenuation for the moisture content measurement, as e.g. Gurr (1962). A well structured and comprehensive overview on the early field and laboratory methods developed in Soil Science was given by Klute (1972).

Non-destructive moisture content measurement was much simplified by the development of miniaturized time domain reflectometry (TDR) sensors. With the TDR technique, the material sample is divided into different compartments, where both quantities could be measured in a high temporal resolution. This kind of instantaneous profile method was first reported by Plagge (1991) and Malicki et al. (1992), who applied miniaturized TDR sensors for the investigation of different soils under laboratory conditions. The scheme shown in Fig. 3.10 follows this method.

The IPM technique developed by Plagge (1991) forms the basis of the methodology the validation experiments are carried out with. This is elaborated under paragraph 5.2 Methodological Developments for the Drying Experiment in more detail, whereas the sensors that can be used for a setup according to this principle are introduced within the subsequent sections.

\subsubsection{Sensor technology}

The desired quantities to be monitored are the moisture content and the moisture potential. To measure the moisture content, time domain reflectometry (TDR) sensors are used, of which the measurement principle will be briefly explained as follows. As already indicated in Chapter 2, the relative humidity is typically used as the moisture potential within the hygroscopic moisture content range, whereas the capillary pressure is used within the overhygroscopic range. Therefore, different sensor types have to be used for each and the intermediate moisture content range, that will be introduced here. 


\subsubsection{Moisture content measurement}

The time domain reflectometry (TDR) was originally developed to investigate electric cables with respect to short-circuits or cable brake. During the 1970s, TDR was also discovered by other disciplines and recognized as a rather simple, non-destructive method to measure the moisture content and the electric conductivity of soils (Sobczuk $\mathcal{E}$ Plagge (2007)) $)^{1}$. The measurement principle, as illustrated by Roth et al. (1989), is based on the propagation velocity $c$ of an electromagnetic pulse inside a material, which is only dependent on its apparent dielectric constant $\varepsilon_{a}$ and its magnetic permeability $\mu$ according to eq. (3.4), where $c_{0}$ corresponds with the speed of light in vacuum.

$$
c=\frac{c_{0}}{\sqrt{\varepsilon_{a} \cdot \mu}}
$$

For non-magnetic materials, the magnetic permeability can be set to $\mu=1$ in general (Sobczuk E Plagge (2007)). Thus, the apparent dielectric constant $\varepsilon_{a}$ of the medium can be derived, if the propagation velocity $c$ is known. With the TDR sensor, an electromagnetic pulse is sent and its propagation time $t_{p}$ is measured. Hence, depending on the path length of the pulse, which is normally twice the length of the probe $l_{T D R}$ (there and back), $c$ can be determined following eq. (3.5) enabling the derivation of $\varepsilon_{a}$.

$$
c=\frac{2 \cdot l_{T D R}}{t_{p}}
$$

The applicability of TDR for moisture content measurement is due to the large difference in the dielectric constants of typical mineral components with $\varepsilon \sim 4$, air with $\varepsilon \sim 1$ and water with $\varepsilon \sim 80$. Since the dielectric constant of the apparent material configuration can be measured by TDR, the moisture content can be derived with the knowledge of the other dielectric properties.

TDR sensors are available in many different types and forms (Kupfer et al. (2004), Sobczuk \& Plagge (2007)). Most suitable for the application in the laboratory are the miniaturized probes developed by Plagge (1991), Malicki et al. (1992) and Sobczuk et al. (1992), of which one is shown in Fig. 3.11. For field measurements, larger probes are available, too.

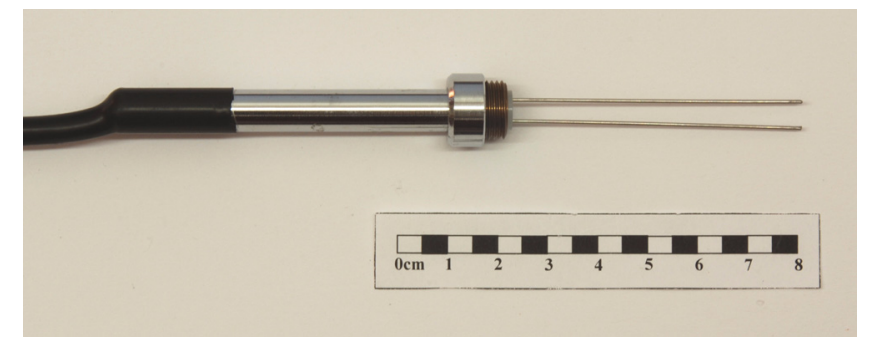

Figure 3.11: Miniaturized TDR probe applied for moisture content measurement.

The moisture content measurement with TDR requires two levels of calibration. The first one, which is the actual calibration, is required for the system configuration. When

1. In addition to the cited literature, an introduction to the history and the measurement principle of the TDR technology can be found at the home page of the TDR sensor producer IMKO, where a brochure "Theorie der Material- und Bodenfeuchtemessung mit der TRIME-Methode" is provided in German and English. The internet address is: www.imko.de, visited at June 8, 2007. 
the electromagnetic pulse is sent, at each impedance change, a part of it is reflected and a part is transmitted. To ensure that the interpreted time interval is the one between entering and leaving the probe, the system configuration has to be calibrated by the help of two media with significantly different dielectric properties.

The second calibration is required for the derivation of the moisture content. In a strict sense, this is not a calibration, but a provision of input data for a model which is used to determine the moisture content from the measured dielectric properties. In Chapter 5, the TDR calibration will be shown exemplarily. In paragraph 5.3.3.2 TDR data evaluation, different models to derive the moisture content will be introduced and discussed in the context of their requirements and their flexibility.

\subsubsection{Moisture potential measurement}

In dependence on the moisture potential range, different sensors are applied for its measurement. In the wet moisture content range, i.e. for capillary pressures up to $0.085 \mathrm{MPa}$, tensiometers can be used to directly measure the suction pressure. For higher capillary pressures, the moisture potential can be measured in form of the relative humidity. Here, two sensor types are employed. One is a capacitive sensor for the whole range from around 5\% to $98 \%$ relative humidity, whereas the other one is a psychrometer sensor, enabling relative humidity measurement at very high relative humidities covering the intermediate range.

\section{Tensiometers}

It is important for the measurement under instantaneous conditions, that the applied sensors are able to observe changes in the adjacent conditions rather quickly. Therefore, micro tensiometers, as described by Plagge (1991), are treated here. They consist of a small porous cell which is connected to a pressure sensor via a pressure tight brass body. The water filled porous cell, which is typically made of a porous ceramic, a sintered metal or another type of porous membrane, is brought in hydraulic contact with the material leading to an equilibration of the suction pressure inside the cell and the surrounding material. Then, by the help of the connected pressure transducer, the pressure inside the material can be

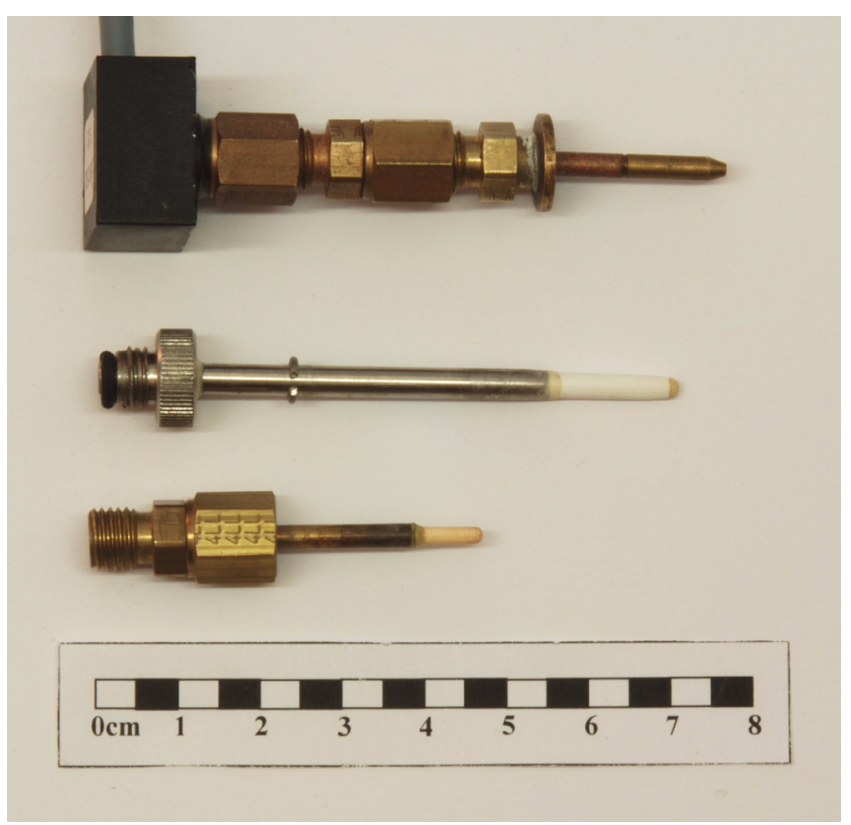

Figure 3.12: Tensiometer sensors to measure the moisture potential. measured.

There exist many different types of tensiometers depending on the application for either field or laboratory investigations. Cassel $\mathcal{E}$ Klute (1986) give an overview on the moisture potential measurement by tensiometers with respect to both, field and labo- 
ratory use. Fig. 3.12 shows an example of a tensiometer with two different ceramic cells. The range of application is limited by the air entry value of the porous cell and by the general measurability of suction pressures. The air entry value of the shown ceramic cells corresponds with a suction pressure of $0.095 \mathrm{MPa}$, enabling the application for the whole possible suction pressure measurement range. The advantages of tensiometers are the ability of moisture potential measurement in the wet moisture range and - for this type of sensor - the quick response on changing conditions. The main disadvantage is, that the sensors fail once the capillary pressure has fallen below the air entry value of the porous cell preventing measurement cycles which scan the whole moisture content range.

\section{Psychrometers}

Psychrometers used for the moisture potential measurement inside of porous materials consist of a miniature thermocouple junction placed inside a small measurement chamber. Due to the Peltier-effect, the junction can be cooled forcing the humidity inside the small chamber to condense water on it. Subsequent evaporation of the condensed water causes a temperature depression inducing a voltage which is measured by a voltmeter. From this voltage, the relative humidity inside the small chamber can be derived.

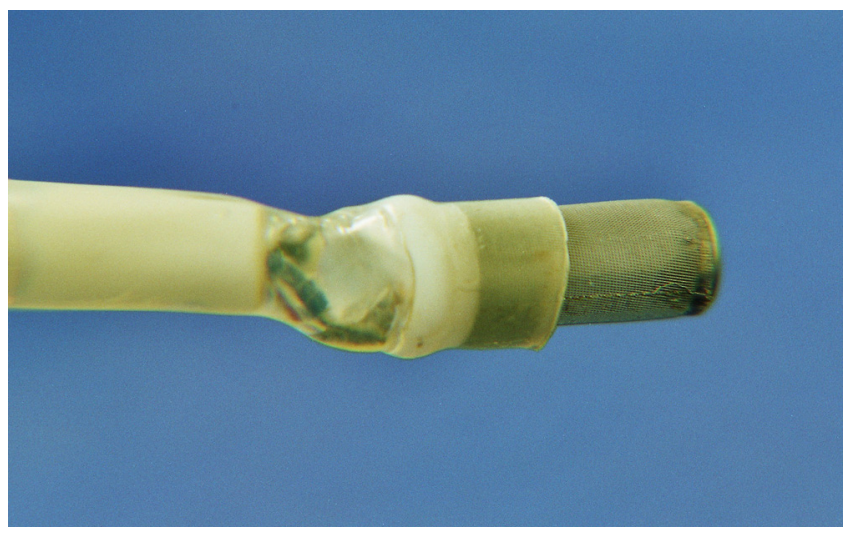

Figure 3.13: Psychrometer probe to measure the moisture potential at high relative humidities close to saturation.

A picture of such a psychrometer is shown in Fig. 3.13, whereas an overview of the measurement principle and different derived sensor types for laboratory and field application is given by Rawlins $\mathcal{E}$ Campbell (1986). The main advantages of psychrometers are the high precision in relative humidity measurement and the applicability at very high relative humidities above $99 \%$. They, hence, represent the moisture potential measurement principle connecting the hygroscopic and the overhygroscopic moisture content range. However, psychrometers are very sensitive to the temperature and, since the measurement procedure requires to disturb the energy state of the system, they can only be recalled in larger time intervals.

\section{Capacitive humidity sensors}

The measurement principle of a capacitive relative humidity sensor is based on the moisture dependent capacity change of an electrical condenser containing a hygroscopic polymeric dielectricum (Freudenberger (1989)). The condenser is used as the frequency determining element of an oscillator. If the moisture content is changing, the capacity of the condenser is changing, too, leading to a frequency reduction. From this, the relative humidity can be derived being inversely proportional to the oscillator's frequency. 
An example sensor, as it was used for the IPM measurements reported here, is shown in Fig. 3.14. Main advantages of such humidity sensors are the wide application range from 5\% to $98 \%$ relative humidity, the high measurement precision of $\pm 2 \%$ for the whole measurement range and the short response time of the sensors. Disadvantages are the long time stability and the longer measurement failures at high relative humidities, once liquid water is condensed inside the measurement cell.

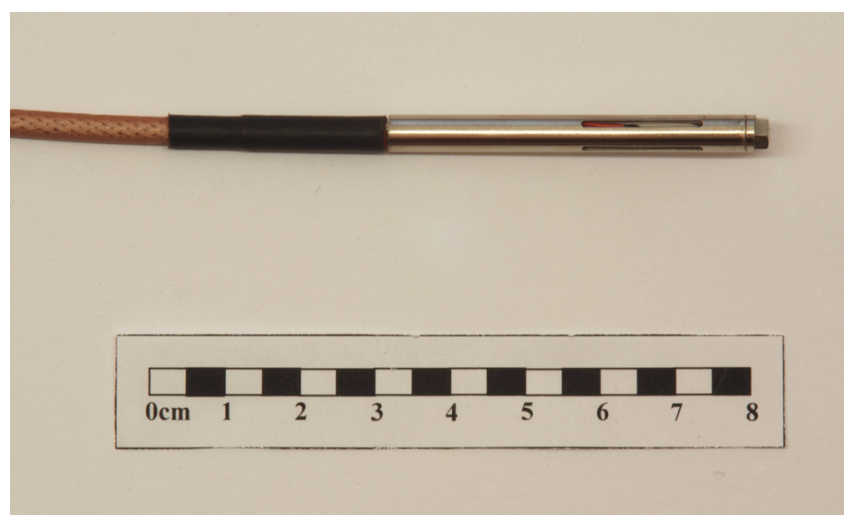

Figure 3.14: Miniaturized temperature and relative humidity sensor.

\subsubsection{Concluding instantaneous profile measurements}

The general method of instantaneous profile measurements was introduced, a brief reference to the context of related literature was given and the measurement principle as particularly applied for the validation experiments was outlined. Subsequently, different sensor types with their prevailing measurement principles were sketched being used to measure instantaneous profiles of moisture content and moisture potential. By that, the methodological basis is provided for an advanced material investigation with respect to both, research and validation purposes.

\subsection{Conclusions on Experimental Methods}

The chapter on experimental methods has been subdivided into basic experimental methods and instantaneous profile measurements. Within the first part, the term basic material properties has been clarified in the frame of the corresponding experiments, which were generally explained and conclusively discussed. As a result, some requirements for further developments were pointed out. The second part gave an outline of the principle of instantaneous profile measurements and the sensor types to be employed for moisture content and moisture potential measurements. There, the dependencies stemming from sensor application for different moisture content ranges were indicated and discussed.

By this, an overview of the experimental methods was given. Whereas the basic methods are considered to be of general applicability for Building Physical laboratories determining the material properties for hygrothermal simulation input, the instantaneous profile method is to be used for further investigation of the moisture transport dependencies indicating limits and options of both, the hygrothermal transport theory and material modelling. The following chapters will bring the different steps of modelling and material investigation together showing new developments in material modelling and experimental methods as well as their implementation and evaluation within the context of hygrothermal material model validation. 


\section{CHAPTER 4}

\section{INVESTIGATED MATERIAL}

A large number of building materials has been investigated with regard to the basic hygrothermal material properties (Plagge et al. (2007)), of which four commonly used materials are selected for the special studies reported below. These materials are a typical building brick, a calcium silicate inside insulation board, an autoclaved aerated concrete and a lime-sand brick.

The selection is made from two different points of view. On the one hand, all materials should be frequently used building materials with a wider range of application. Thus, three chosen materials are building stones representing different structural types, whereas the forth choice is made for an insulation material. On the other hand, the materials should also be representative for the whole range of hygrothermal behaviour, i.e. from light and vapour open to dense and tight, from slow water conducting up to capillary active building materials.

The chosen building brick is a dense but fine-porous material with a relatively high capillary conductivity, a mean vapour diffusion resistance and a low hygroscopic moisture storage characteristic. The calcium silicate is a light and highly porous insulation material with a fine pore structure. It has a very high capillary conductivity, is vapour open, but contains a low hygroscopic moisture capacity. It is used as an inside insulation material, especially for thermal rehabilitation of historical buildings.

The autoclaved aerated concrete represents a light, thermally insulating building stone with a high porosity. It has a wide pore range with several pore maxima and a high hygroscopic moisture capacity. It is a vapour open material with a low capillary conductivity. The lime-sand brick, finally, is a dense and vapour tight building stone. Its pore structure contains several maxima with a distinct moisture capacity already within the hygroscopic moisture range. The liquid transport properties are very low. 


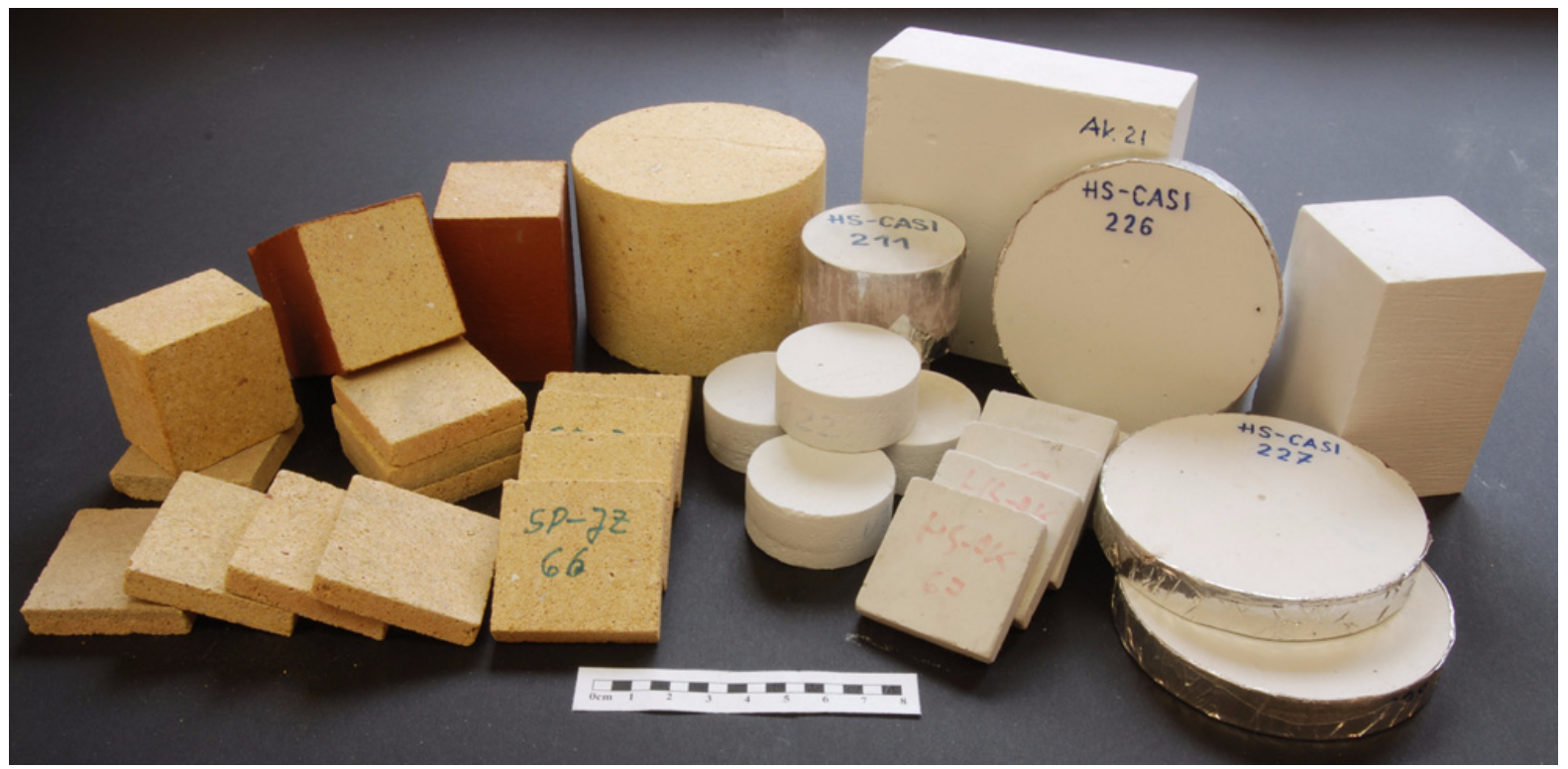

Figure 4.1: $\quad$ Selection of material samples of the building brick (left) and the calcium silicate insulation board (right). The legend shows divisions of $0.5 \mathrm{~cm}$.

According to their moisture transport behaviour, the calcium silicate and the building brick can be classified as well conducting materials, whereas the lime-sand brick and the autoclaved aerated concrete can be regarded as slow conducting materials. A selection of specimens of the first group is shown in Fig. 4.1 and of the second group in Fig. 4.2. The following Tab. 4.1 to Tab. 4.4 contain specific basic material information for each material.

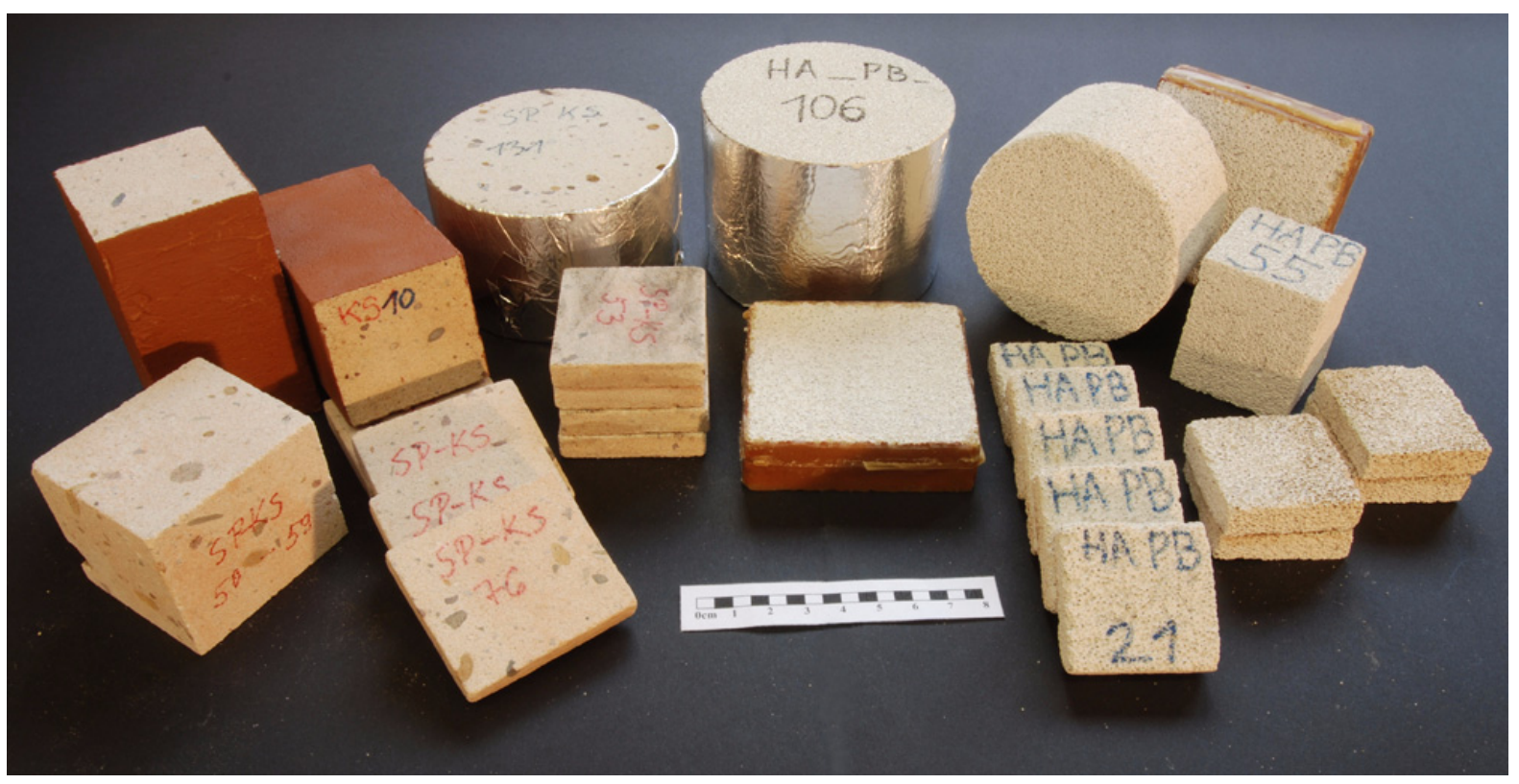

Figure 4.2: $\quad$ Selection of material samples of the lime-sand brick (left) and the autoclaved aerated concrete (right). The legend shows divisions of $0.5 \mathrm{~cm}$. 
Table 4.1: Characteristic material properties of the investigated building brick.

\begin{tabular}{|c|c|c|c|}
\hline Material property & Value & Unit & Miscellaneous \\
\hline$\overline{\text { Dry bulk density } \rho_{b}}$ & 1790 & $\mathrm{~kg} / \mathrm{m}^{3}$ & \multirow{2}{*}{$\begin{array}{l}\text { The brick is a typical building } \\
\text { stone frequently used, especially } \\
\text { in Northern Germany. }\end{array}$} \\
\hline Porosity $\theta_{p o r}$ & 0.353 & $m^{3} / m^{3}$ & \\
\hline Capillary saturation $\theta_{\text {cap }}$ & 0.257 & $m^{3} / m^{3}$ & \\
\hline Moisture content at $85 \%$ RH $\theta_{85}$ & 0.0025 & $m^{3} / m^{3}$ & \\
\hline Water absorption coefficient $A_{w}$ & 0.227 & $\mathrm{~kg} /\left(\mathrm{m}^{2} \sqrt{\mathrm{s}}\right)$ & \\
\hline Dry vapour diffusion resistance $\mu_{d r y}$ & 13.5 & --- & \\
\hline Thermal conductivity $\lambda_{d r y}$ & 0.87 & $W /(m K)$ & $3[\mathrm{~cm}] 4$ \\
\hline
\end{tabular}

Table 4.2: Characteristic material properties of the investigated calcium silicate board.

\begin{tabular}{|c|c|c|c|}
\hline Material property & Value & Unit & Miscellaneous \\
\hline Dry bulk density $\rho_{b}$ & 270 & $\mathrm{~kg} / \mathrm{m}^{3}$ & The calcium silicate is used as a \\
\hline Porosity $\theta_{\text {por }}$ & 0.91 & $m^{3} / m^{3}$ & capillary active inside insulation. \\
\hline Capillary saturation $\theta_{\text {cap }}$ & 0.79 & $m^{3} / m^{3}$ & \\
\hline Moisture content at $85 \% R H \theta_{85}$ & 0.006 & $m^{3} / m^{3}$ & \\
\hline Water absorption coefficient $A_{w}$ & 1.11 & $\mathrm{~kg} /\left(m^{2} \sqrt{s}\right)$ & \\
\hline Dry vapour diffusion resistance $\mu_{d r y}$ & 4.0 & --- & \\
\hline Thermal conductivity $\lambda_{d r y}$ & 0.069 & $W /(m K)$ & יויעי \\
\hline
\end{tabular}

Table 4.3: Characteristic material properties of the investigated autoclaved aerated concrete.

\begin{tabular}{|c|c|c|c|}
\hline Material property & Value & Unit & Miscellaneous \\
\hline$\overline{\text { Dry bulk density } \rho_{b}}$ & 390 & $\mathrm{~kg} / \mathrm{m}^{3}$ & The aerated \\
\hline Porosity $\theta_{\text {por }}$ & 0.87 & $m^{3} / m^{3}$ & Gern \\
\hline Capillary saturation $\theta_{\text {cap }}$ & 0.26 & $m^{3} / m^{3}$ & \\
\hline Moisture content at $85 \% R H \theta_{85}$ & 0.023 & $m^{3} / m^{3}$ & \\
\hline Water absorption coefficient $A_{w}$ & 0.043 & $\mathrm{~kg} /\left(\mathrm{m}^{2} \sqrt{\mathrm{s}}\right)$ & \\
\hline Dry vapour diffusion resistance $\mu_{d r y}$ & 7.5 & --- & \\
\hline Thermal conductivity $\lambda_{d r y}$ & 0.095 & $W /(m K)$ & $3[\mathrm{~cm}] 4$ \\
\hline
\end{tabular}


Table 4.4: Characteristic material properties of the investigated lime-sand brick.

\begin{tabular}{|c|c|c|c|}
\hline Material property & Value & Unit & Miscellaneous \\
\hline Dry bulk density $\rho_{b}$ & 1810 & $\mathrm{~kg} / \mathrm{m}^{3}$ & The investigated lime-sand brick \\
\hline Porosity $\theta_{\text {por }}$ & 0.345 & $m^{3} / m^{3}$ & $\begin{array}{l}\text { is a typical building stone } \\
\text { frequently used in Germany. }\end{array}$ \\
\hline Capillary saturation $\theta_{\text {cap }}$ & 0.218 & $m^{3} / m^{3}$ & \\
\hline Moisture content at $85 \% R H \theta_{85}$ & 0.037 & $m^{3} / m^{3}$ & \\
\hline Water absorption coefficient $A_{w}$ & 0.052 & $\mathrm{~kg} /\left(\mathrm{m}^{2} \sqrt{\mathrm{s}}\right)$ & \\
\hline Dry vapour diffusion resistance $\mu_{d r y}$ & 40.0 & --- & \\
\hline Thermal conductivity $\lambda_{d r y}$ & 1.05 & $W /(m K)$ & $3[\mathrm{~cm}] 4$ \\
\hline
\end{tabular}


CHAPTER 5

\section{DEVELOPMENT AND UTILISATION}

On the basis of the review on both, material modelling and experimental methods for input data determination which was given in the previous chapters, modelling as well as methodological developments were accomplished. These are presented within this chapter where three main contents are distinguished: material modelling, the drying experiment and the instantaneous profile measurement technique.

\subsection{Developments in Material Modelling}

The literature review given in Chapter 2 comprises the large variety in material modelling approaches available in Building Physics. The aspired bridge between sophisticated approaches with a strong physical basis on the one hand and easy to use and widely applicable approaches on the other hand, leads to the following specification of requirements on the material model.

1. The model should be based on the actual driving potential ensuring extendibility for further transport phenomena (hysteresis of moisture storage, salt and solute transport).

2. The general slope of the liquid water conductivity function should be related to the material's pore structure.

3. The liquid water conductivity function should be adjustable within the whole moisture range. 
4. The model should be adjustable by the help of basic hygrothermal material data.

5. The model should be reliably applicable to a broad range of materials with a reasonable degree of precision.

For the reason of 1., the developed approach was chosen to be a conductivity approach which is, due to 2., based on a bundle of tubes model. For the adjustment of the liquid water conductivity function, the simple pore model according to Burdine (1953) in the form introduced by Grunewald $\mathcal{E}$ Häupl (2003) is coupled with a mechanistical approach introduced by Scheffler et al. (2004) which has enough flexibility for both, the lower overhygroscopic and the capillary moisture range.

Finally, the material data required for an appropriate adjustment of the model is to be specified in Chapter 3, while gains and limits of the model are to be elaborated in Chapter 7 , following the objectives of this work.

\subsubsection{Moisture storage and pore structure}

Basis of the model, as it is typical for bundle of tubes models, is the functional description of moisture storage. Due to its high flexibility in adjustment of measured data, the weighted sum of Gauss distribution functions introduced by Grunewald $\mathcal{E}$ Häupl (2003) is chosen here. The moisture storage function is then given by eq. (5.1) and the pore volume distribution as its derivative by eq. (5.2). There, the capillary pressure is expressed by $p C=\log \left(-p_{c}\right)$ in a logarithmic scale.

$$
\begin{aligned}
& \theta_{l}(p C)=\sum_{i=1}^{N}\left[\frac{\theta_{i}}{\sqrt{2}} \cdot\left(1+\operatorname{erf}\left(\frac{p C_{i}-p C}{\sqrt{2} S_{i}}\right)\right)\right] \\
& \frac{\partial \theta_{l}}{\partial p C}(p C)=-\sum_{i=1}^{N}\left[\frac{\theta_{i}}{\sqrt{2 \pi} \cdot S_{i}} \cdot \exp \left(-\frac{\left(p C-p C_{i}\right)^{2}}{2 S_{i}^{2}}\right)\right]
\end{aligned}
$$

Fig. 5.1 gives an illustration of the parameters contained in eq. (5.1) and (5.2) for a modality of $N=3$. The $p C_{i}$ define the positions of pore maxima, i.e. the inflexion points within the moisture storage function. The slope at these inflexion points is influenced by the $S_{i}$. They are the standard deviations in the pore volume distribution function. Finally, the $\theta_{i}$ specify the plateau levels between the different modalities. They represent the weighting factors for each modality. When using relative water contents $\Theta$, the sum of $\Theta_{i}$ must equal 1. Otherwise, the sum of $\theta_{i}$ must result in the moisture content at effective saturation $\theta_{\text {eff }}$.

Alternatively, the functional description (2.63) according to Van Genuchten (1980) and Durner (1994) or any other multi-modal approach could have been chosen. However, besides the good flexibility of eq. (5.1) especially for higher modalities, the direct interpretation of its parameters on the basis of measured data appeared expedient. And 

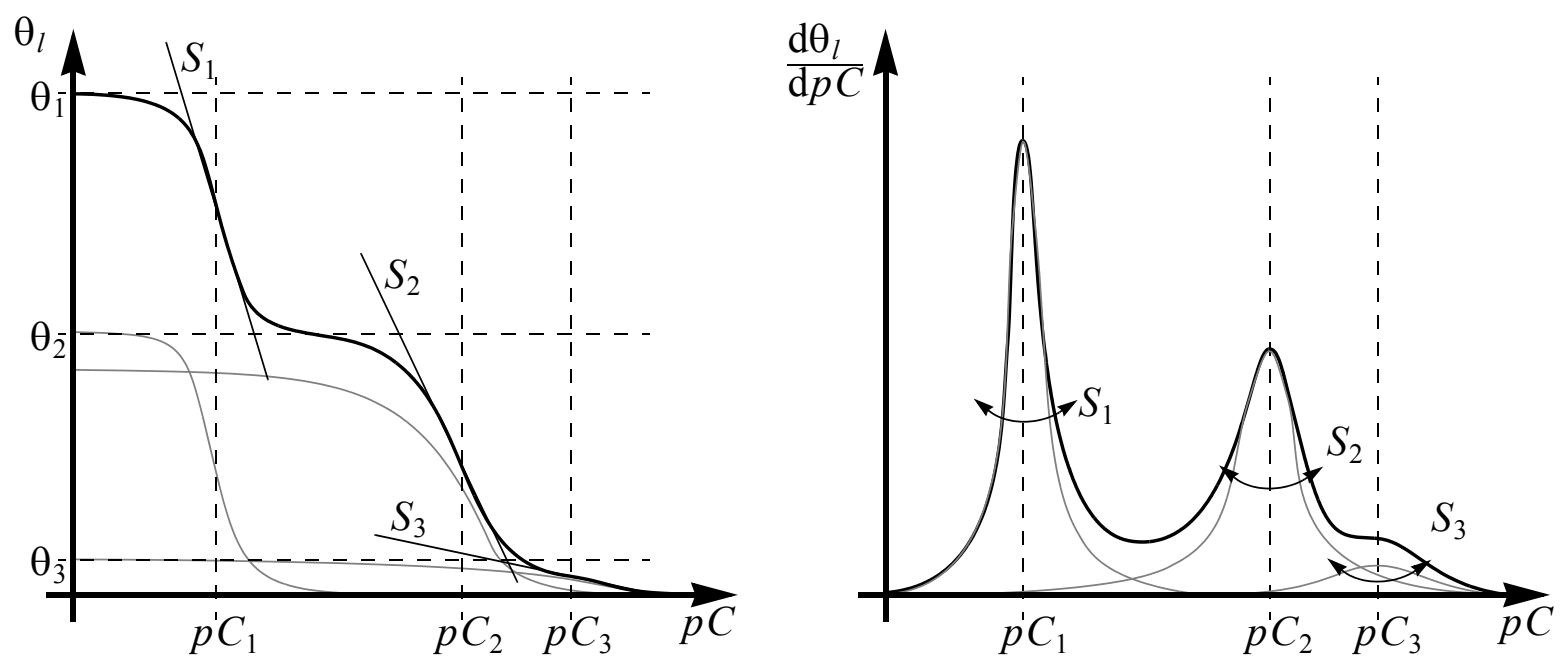

Figure 5.1: 3-modal moisture storage function and its derivation, the pore volume distribution. Parameters possess a mathematical meaning and, thus, can be directly determined from measured moisture storage data.

since computational resources do not strictly limit the numerical evaluation anymore, the previous drawback of higher effort in evaluating the error function is vanishing. Hence, the prevailing advantages vindicate this choice.

Adjusting the moisture storage function (5.1) to measured sorption and retention data, the derived pore volume distribution function (5.2) displays the pore space available to water. In contrast to data from image analysing techniques (e.g. Roels et al. (2001)) and mercury intrusion (see e.g. Adolphs et al. (2002)), this pore structure information is directly related to the quantity of investigation and therefore more reliable for use in pore models. The model introduced as follows is thus based on such data.

\subsubsection{Liquid water and water vapour transport}

The derivation of the liquid water conductivity function follows in principal the pore model according to Burdine (1953) as described in paragraph 2.2.2.3 Bundle of tubes models. The relative conductivity function is numerically determined from the inverse of the moisture storage function $p_{c}\left(\theta_{l}\right)$ according to eq. (5.3).

$$
K_{l, r e l}\left(\theta_{l}\right)=\left(\int_{0}^{\theta_{l}} \frac{1}{p_{c}(\theta)^{2}} d \theta\right) /\left(\int_{0}^{\theta_{\text {eff }}} \frac{1}{p_{c}(\theta)^{2}} d \theta\right) \quad \text { relative conductivity }
$$

The relative conductivity function $K_{l, \text { rel }}\left(\theta_{l}\right)$ contains the relative information of the material's pore structure. However, this function requires further adjustment since the pore structure of the model displays a rather rough approximation (see e.g. Fig. 2.22 in Chapter 2). For that reason, two further steps are introduced. Fist, the relative conductivity function is scaled to a measured conductivity value $K_{\text {eff }}$ at effective saturation. 
And second, since the tortuosity factor $\tau$ introduced by Burdine is omitted, a further coupling with a mechanistical approach is fulfilled and combined with a scaling, allowing to adjust the conductivity function within the whole moisture range. This mechanistical approach will be described in paragraph 5.1.2.2 Mechanistical approach: serial-parallel model below. However, afore a distinction between the different moisture content ranges is required to clarify the terms used in that context.

\subsubsection{Moisture content regions}

At the beginning of this chapter, in paragraph 2.1.4.2 Hygroscopic and overhygroscopic moisture range, a distinction between two moisture regions has already been made. The moisture storage function and the ratio between vapour and liquid water transport were the reference parameter in that case. However, when looking at the liquid water conductivity, a finer distinction within the overhygroscopic moisture region is appropriate.

Certainly, a clear distinction related to a measurable material property is not to be drawn. Nevertheless, different experiments revealing information about the liquid water conductivity within different moisture content ranges exist. It appears reasonable to relate these experiments to their corresponding moisture content range when using them for calibration of the liquid water conductivity function. Fig. 5.2 shows a liquid water conductivity within the different moisture content ranges that are specified as follows.

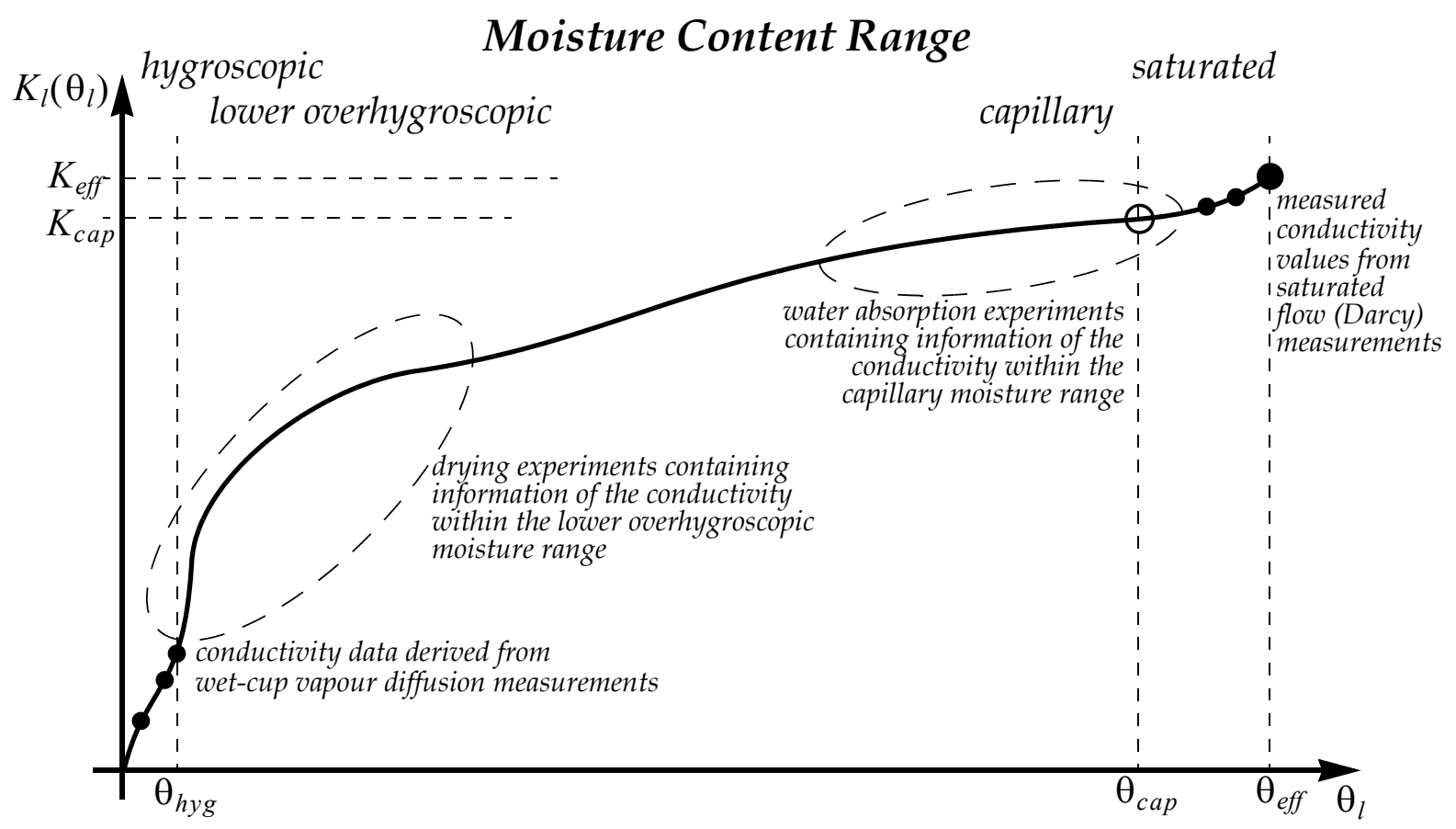

Figure 5.2: $\quad$ Derived liquid water conductivity function in subdivided moisture content ranges together with conductivity data derived from measurements. 


\section{Hygroscopic moisture content range}

The hygroscopic moisture content range has already been characterized above. Moisture transport occurs mainly as vapour transport. With increasing moisture content, also liquid water transport proceeds but remains at lower or the same order of magnitude as vapour transport. Transport properties can be measured by vapour diffusion (dry-cup and wet-cup) experiments, see paragraph 3.1.4 Vapour diffusion. In combination with a model for vapour transport, the liquid transport fraction can be derived as the difference between measured moisture transport (vapour and liquid together) and modelled vapour transport. In Fig. 5.2, the black dots at low moisture contents characterize these data.

\section{Lower overhygroscopic moisture range}

The lower overhygroscopic moisture content range is characterized by highly unsaturated flow conditions. The liquid water flow is the dominant form of transport. The experiment revealing information about the conductivity in that range is the drying described in paragraph 3.1.6 Drying experiment. As building materials will often not be wetted until saturation is reached, for many application cases this moisture content range is most important. A study reported by Scheffler $\mathcal{E}$ Plagge (2005a) displayed the liquid water conductivity's sensitivity in that range. Therefore, calibration of the conductivity function within the lower overhygroscopic moisture content range is very important.

\section{Capillary moisture content range}

With further increasing moisture contents, the lower overhygroscopic passes over into the capillary moisture content range. Flow conditions are unsaturated. The upper boundary of this moisture region is rather clearly defined by the capillary moisture content $\theta_{\text {cap }}$. However, the definition of the capillary moisture content itself varies fre-
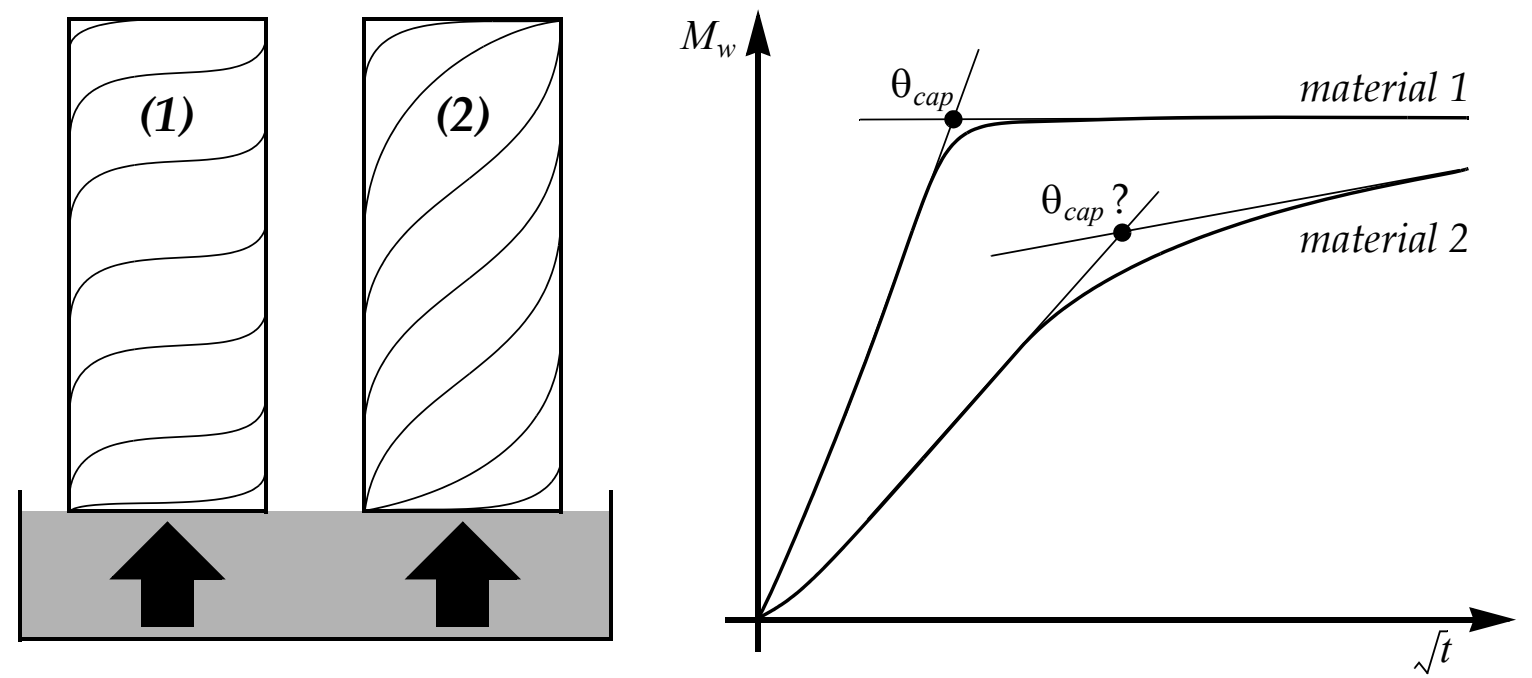

Figure 5.3: Water absorption for a material with a high liquid water conductivity (1) and a material with a low liquid water conductivity (2) and the influence on the moisture profile and the capillary moisture content. 
quently in literature (compare specifications of e.g. Pel (1995), Carmeliet $\mathcal{E}$ Roels (2002) and Grunewald \& Häupl (2003)).

Most definitions have in common that they are related to the water absorption experiment. The derivation of a moisture diffusivity from measured moisture profiles during water imbibition is limited to the moisture content range reached in that experiment, see also paragraph 2.2.1 Diffusivity approaches. The highest moisture content reached within such experiments is therefore often called free water saturation or capillary saturation. Thus, the capillary moisture content is defined as the highest water content reached during a water absorption experiment, which will normally occur at the imbibition surface.

However, this moisture content is difficult to measure, especially if no moisture profiles can be observed. Therefore, it was tried to derive $\theta_{\text {cap }}$ from integral data of water absorption experiments. But since the moisture content at the end of the experiment is strongly dependent on its duration, the (integral) moisture content at the sharp bend was supposed to be taken. Unfortunately this is only suitable for materials which form rather sharp profiles during water absorption. This is illustrated in Fig. 5.3 where for two materials the moisture profiles and corresponding integral water absorption curves are drawn.

It can be stated that the capillary moisture content $\theta_{\text {cap }}$ is a material property which either can be clearly defined but not generally be measured or can be derived from measurement but with a rather uncertain relation to its original definition. For the purpose of relating the moisture regions to different experiments revealing information about the liquid water conductivity, this fact is of lower importance. Instead, the capillary moisture content, derived in one or the other way, characterizes a border range between unsaturated and saturated water flow.

The liquid water conductivity within the capillary moisture content range is mainly responsible for the water absorption behaviour of the material. A scaling of the whole conductivity function between dry and $\theta_{c a p}$ will therefore be introduced which is proposed to be adjusted by water absorption data. For practical application, this moisture range is important when water contact occurs for a longer period of time.

\section{Saturated moisture content range}

Above capillary saturation, the saturated moisture range follows up to effective (or total reached) saturation $\theta_{\text {eff }}$. The connected pore system is filled with water. Transport proceeds therefore under saturated conditions. Some pores which do not contribute to water transport may not be water filled due to air entrapment (Descamps (1997), Carmeliet $\mathcal{E}$ Roels (2001)). Depending on the duration of water contact, different saturation stages are reached, as the air within those pores slowly dissolves in the water, allowing them to become water filled.

In practical Building Physical cases, this moisture range is seldom reached. However, it marks the upper limit of the conductivity function and conductivity values can be measured within that range. This is described in paragraph 3.1.7 Saturated and unsaturated flow experiments. Therefore, the relative conductivity function is scaled to the measured $K_{\text {eff }}$ value at $\theta_{\text {eff }}$ to receive an initial guess of the absolute conductivity function. During further calibration, the conductivity function is truncated at capillary sat- 
uration and measured values within the saturated moisture content range are interpolated in a logarithmic scale.

\subsubsection{Mechanistical approach: serial-parallel model}

The pore model describes the liquid water transport in the pore system in a rather simple way. Among others, a completely interconnected liquid phase is assumed which enables a continuous capillary flow. This concerns mainly the parallel liquid and vapour transport which might apply to the upper capillary and the saturated moisture range. Below the capillary moisture range (hygroscopic and lower overhygroscopic moisture range) the liquid phase exists mainly in form of isolated subdomains which are not or only partly capillary interconnected, i.e. which can mainly interact via the gaseous phase only. This process can be described as serial transport which is - in comparison to the parallel transport - more efficient in the gaseous phase while it is much less powerful in the liquid phase.

It can be expected that the volume fraction participating in parallel transport must increase with the water content and the volume fraction participating in serial transport must decrease, respectively. Therefore, a mechanistical approach is introduced allowing to describe this serial and parallel transport in dependence to the ratio of parallel and serial structured pore domains. The model takes the discussed effects into account and can be adjusted by the help of drying data.

The basic idea of this model is identical to the one introduced by Grunewald \& Häupl (2002). However, a consequent derivation according to the specifications given in Fig.

parallel structured pore space serial structured pore space
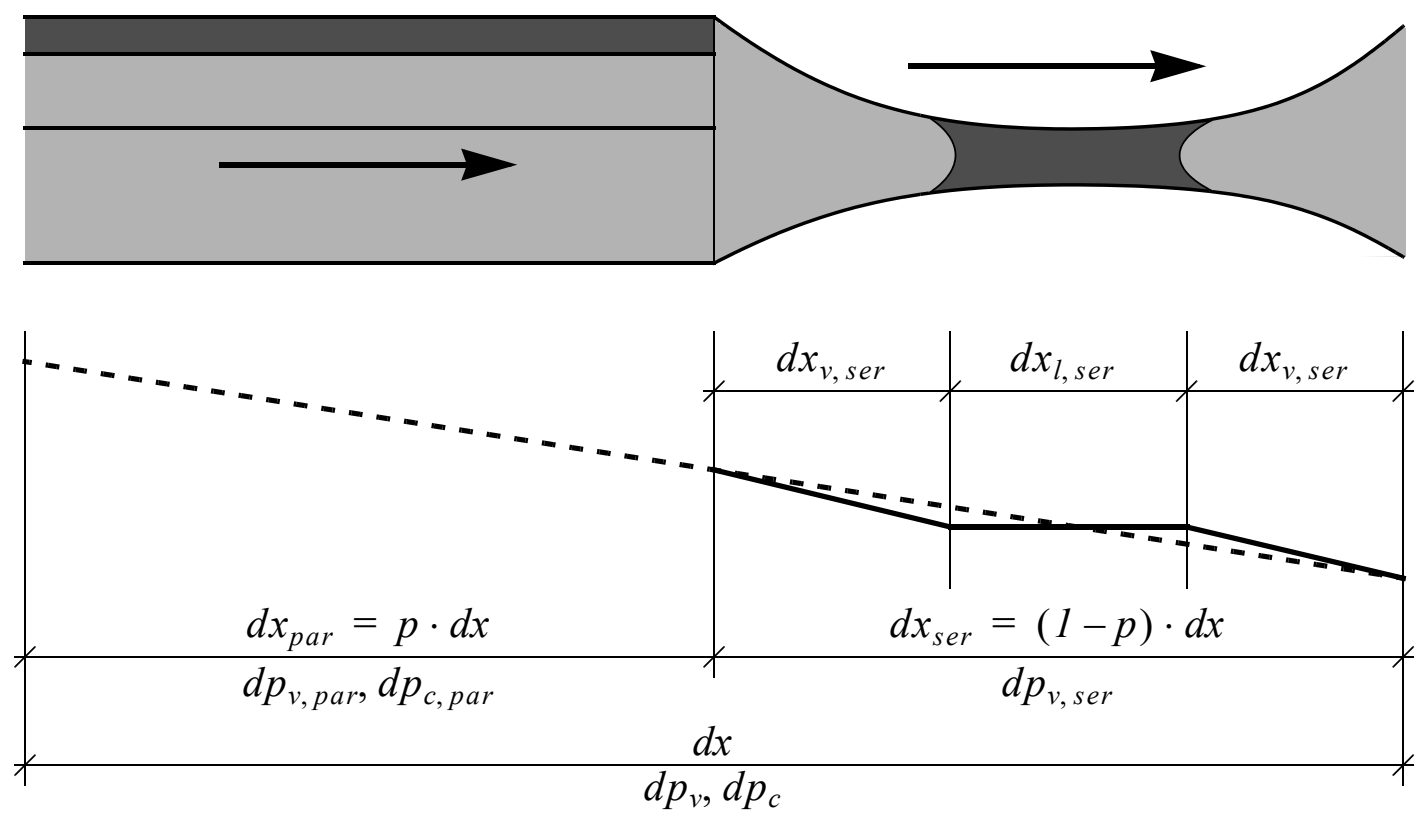

$\longrightarrow p_{v, \text { local }} \quad \ldots-p_{v, \text { mean }} \quad \square$ water vapour $\quad \square$ liquid water

Figure 5.4: $\quad$ Principle of the serial-parallel model following Grunewald \& Häupl (2002): pore domains with either serial or parallel structure lead to different contributions to liquid and vapour transport. 
5.4 results in one term for vapour and one term for liquid water transport. The first one can be found by Grunewald E Häupl (2002) and Grunewald \& Häupl (2003) and is already given in eq. (2.68). The latter one was neglected in the original model. Therefore, the whole serial-parallel model is to be developed as follows.

The model assumes a porous material where serial and parallel liquid water and vapour transport proceed as shown in Fig. 5.4. Both volume fractions contain pores according to the pore size distribution function of the material. The whole material could be imagined to consist of randomly distributed sub-domains of serial and parallel structure according to their ratio expressed by $p$. As independent variable, the relative moisture content $\Theta$ is introduced according to eq. (5.4) as the ratio of volumetric moisture content and porosity.

$$
\Theta=\frac{\theta_{l}}{\theta_{\text {por }}} \quad \text { relative moisture content }
$$

The treated section of the dry porous material is represented by one resulting vapour diffusion coefficient $D_{v}{ }^{\circ}$ for both, the serial and the parallel sub-domains. In the same way, the liquid water conductivity $K_{l}^{\circ}$ is assumed to be given. The ratio of parallel and serial structured areas is introduced by $p$ where $p=1$ if the whole material is only parallel structured. The proportion of serial structured areas is therefore characterized by $1-p$, see also Fig. 5.4.

In pore domains with serial transport, vapour molecules diffuse through the air-filled pore space on both sides of a liquid island in direction from higher to lower vapour pressures. Then, condensation occurs at the capillary meniscus exposed to the higher vapour pressure. At the same time water molecules evaporate from the capillary meniscus at the other side. Between both menisci the water is conducted in the liquid phase. This mechanism was already described by Philip \& De Vries (1957).

Assuming immediate pressure equilibrium within a liquid island, it acts as a bypass by means of vapour diffusion, i.e the vapour pressure at both capillary menisci is equal. Hence, the local vapour pressure gradient within the air-filled pore space at both sides of a liquid island becomes higher. This leads to an increase of the vapour flux density. Under serial flux conditions, the total moisture flux density and the vapour flux density are equal and can be expressed according to eq. (5.5).

$$
\dot{j}_{k, \text { ser }}^{m_{l+v}}=j_{k, \text { ser }}^{m_{v}}=D_{v}^{\circ} \cdot \frac{1}{1-\Theta} \cdot \frac{1}{1-p} \cdot \frac{d p_{v, s e r}}{d x}
$$

In pore domains with parallel transport, the diffusion cross section corresponds to the sum of all air-filled pore sectional areas. The vapour flux density declines with reduced diffusion cross section. Hence it is inversely proportional to the pore filling level, i.e. to the water content. Under parallel flow conditions both, liquid water and water vapour flux densities, contribute to the moisture flux leading to eq. (5.6).

$$
\dot{j}_{k, \text { par }}^{m_{l+v}}=D_{v}^{\circ} \cdot(1-\Theta) \cdot \frac{1}{p} \cdot \frac{d p_{v, \text { par }}}{d x}+K_{l}^{\circ} \cdot \frac{1}{p} \cdot \frac{d p_{c, p a r}}{d x}
$$


The total moisture flux density consists of contributions from serial and parallel structured areas. Therefore, both contributions can be set equal according to eq. (5.7) which is called the condition of continuity.

$$
\stackrel{m_{l+v}}{j_{k, p a r}}=m_{k, \text { ser }}^{m_{l+v}}=\dot{j}_{k, \text { ser }}^{m_{v}}
$$

The sum of the differential serial and parallel vapour pressures must equal the vapour pressure difference over the whole treated area. In addition, the capillary pressure gradient within the serial pore domains is assumed to be negligible. Thus, the capillary pressure gradient within the parallel pore domains can be set equal to the pressure gradient over the whole treated area as written in eq. (5.8).

$$
d p_{v}=d p_{v, \text { ser }}+d p_{v, \text { par }} \quad \text { and } \quad \frac{d p_{c, \text { par }}}{d x_{\text {par }}}=\frac{\mathrm{d} p_{c}}{\mathrm{~d} x}
$$

Applying the condition of continuity (5.7) together with (5.8) and the eq. (5.5) and (5.6) leads, after multiplication with $d x$, to the following expression (5.9). This is rearranged in the next step (5.10) to separate the unknown $d p_{v, \text { ser }}$ which can finally be replaced in eq. (5.5) leading to eq. (5.11).

$$
\begin{aligned}
& D_{v}^{\circ} \cdot \frac{1}{1-\Theta} \frac{1}{(1-p)} \cdot d p_{v, \text { ser }}= \\
& D_{v}{ }^{\circ} \cdot(1-\Theta) \cdot \frac{1}{p} \cdot d p_{v}-D_{v}^{\circ} \cdot(1-\Theta) \cdot \frac{1}{p} \cdot d p_{v, s e r} \cdot K_{l}{ }^{\circ} \cdot d p_{c} \\
& D_{v}^{\circ} \cdot d p_{v, s e r}=\left(D_{v}^{\circ} \cdot \frac{1-\Theta}{p} \cdot d p_{v}+K_{l}^{\circ} \cdot d p_{c}\right) \cdot \frac{1}{\frac{1}{(1-\Theta) \cdot(1-p)}+\frac{1-\Theta}{p}} \\
& \dot{j}_{k}^{m_{l+v}}=D_{v}^{\circ} \cdot\left[\frac{1-\Theta}{p} \cdot \frac{1}{(1-\Theta) \cdot(1-p)} \cdot \frac{1}{\frac{1}{(1-\Theta) \cdot(1-p)}+\frac{1-\Theta}{p}}\right] \cdot \frac{\mathrm{d} p_{v}}{\mathrm{~d} x} \\
& +K_{l}^{\circ} \cdot\left[\frac{1}{(1-\Theta) \cdot(1-p)} \cdot \frac{1}{\frac{1}{(1-\Theta) \cdot(1-p)}+\frac{1-\Theta}{p}}\right] \cdot \frac{\mathrm{d} p_{c}}{\mathrm{~d} x}
\end{aligned}
$$

The expressions in the brackets of eq. (5.11) represent scaling functions for liquid and vapour transport, each in dependence to the relative water content and the fraction of parallel pore domains. Both expressions can be separated and simplified yielding eq. (5.12) and (5.13). 


$$
\begin{array}{ll}
f_{v}(\Theta)=\frac{1-\Theta}{p+(1-\Theta)^{2} \cdot(1-p)} & \text { scaling function for vapour transport } \\
f_{l}(\Theta)=\frac{1}{1+\frac{(1-\Theta)^{2} \cdot(1-p)}{p}} & \text { scaling function for liquid transport }
\end{array}
$$

Following the argumentation above, it does not appear to be reasonable leaving the volumetric fraction of the parallel pore domains, i.e. the variable $p$, a constant. In fact it should be introduced as a function in dependence to the water content. If the water content is low, only few connections may exist between single liquid islands. Parallel transport is not possible, yet. With increasing water content, however, more and more pores will be water filled leading to more and more pore domains where parallel moisture transport is possible. Accounting for this fact, an approach is introduced in eq. (5.14).

$$
p=p\left(\Theta, \eta_{s p}\right)=\Theta^{\eta_{s p}}
$$

Here, a parameter $\eta_{s p}$ is introduced to describe the volumetric fraction of the parallel pore domains $p$ as a function of the relative water content $\Theta$. The parameter $\eta_{s p}$ is material dependent and can be determined by numerical simulation of the drying experiment.

\subsubsection{Calibration of liquid and vapour transport coefficients}

The relative liquid water conductivity $K_{l, r e l}\left(\theta_{l}\right)$ of eq. (5.3) must undergo different steps of calibration. First, an absolute conductivity function is achieved by multiplying $K_{l, r e l}\left(\theta_{l}\right)$ with a measured conductivity value $K_{\text {eff }}$ at effective saturation $\theta_{\text {eff }}$ yielding eq. (5.15).

$$
K_{l, a b s}\left(\theta_{l}\right)=K_{e f f} \cdot K_{l, r e l}\left(\theta_{l}\right)
$$

By doing so, the conductivity function might display reliable values within the saturated moisture content range. However, this does not necessarily mean that the data is feasible for the capillary and the lower overhygroscopic moisture range as well. It is rather probable that the model of an interconnected bundle of tubes overestimates the moisture transport, especially with regard to the lower overhygroscopic moisture range. Therefore, the following procedure is proposed to adjust and calibrate the liquid water conductivity function.

1. Determine the absolute conductivity function according to eq. (5.15) by scaling the relative one to the measured $K_{\text {eff }}$ value.

2. Directly use the conductivity data that can be derived from measurements, i.e. data from cup measurement in the hygroscopic and data from permeameter or infiltrometer measurements in the saturated moisture content range. 
3. Truncate the conductivity function at capillary saturation, introduce a scaling factor $\eta_{\text {cap }}$ at capillary saturation $\theta_{\text {cap }}$ which is to be adjusted by the help of water absorption data and interpolate between the final value $K_{l}\left(\theta_{c a p}\right)$ and the measured $K_{\text {eff }}\left(\theta_{\text {eff }}\right)$ data in a logarithmic scale.

4. Add the scaling function $f_{l}(\Theta)$ according to the serial-parallel model and adjust the contained parameter $\eta_{s p}$ by the help of drying data.

Following these four steps, the liquid water conductivity is to be composed of several terms. The core conductivity is the one derived from the pore structure. It is valid from dry to capillary saturation $\theta_{\text {cap }}$. With the modifications according to the points 3 . and 4. from above, this conductivity function is defined according to eq. (5.16).

$$
K_{l, p m}\left(\theta_{l}\right)=\frac{\eta_{c a p} \cdot K_{e f f}}{1+\frac{\left(1-\frac{\theta_{l}}{\theta_{\text {por }}}\right)^{2} \cdot\left(1-\left(\frac{\theta_{l}}{\theta_{p o r}}\right)^{\eta_{s p}}\right)}{\left(\frac{\theta_{l}}{\theta_{\text {por }}}\right)^{\eta_{s p}}}} \cdot K_{l, r e l}\left(\theta_{l}\right) \quad \text { for } \theta_{l} \leq \theta_{c a p}
$$

The data obtained from dry and wet cup vapour diffusion experiments reveal information about the moisture transport within the corresponding moisture range the experiment is carried out in. The dry cup vapour diffusion resistance factor is assumed to represent vapour transport only. Therefore it is used in eq. (2.12) to define the vapour diffusion coefficient $D_{v, \text { mat }}$. The serial-parallel model provides a term to describe the moisture dependency of the vapour diffusion coefficient. Hence, eq. (2.12) has to be combined with the scaling function $f_{v}(\Theta)$ according to eq. (5.12) yielding eq. (5.17).

$$
D_{v, \text { mat }}\left(\theta_{l}, T\right)=\frac{D_{v, \text { air }}(T)}{\mu_{d r y}} \cdot \frac{1-\frac{\theta_{l}}{\theta_{\text {por }}}}{\left(\frac{\theta_{l}}{\theta_{\text {por }}}\right)^{\eta_{s p}}+\left(1-\frac{\theta_{l}}{\theta_{\text {por }}}\right)^{2} \cdot\left(1-\left(\frac{\theta_{l}}{\theta_{\text {por }}}\right)^{\eta_{s p}}\right)}
$$

Wet-cup vapour diffusion measurements comprise conditions where both, liquid and vapour transport, proceed. The conductivity data derived from these experiments is therefore a moisture conductivity, i.e. liquid and vapour combined. A distinction between these two could either be drawn by the help of a phase dividing function, as introduced by Grunewald (1997), or by the help of a defined function for either liquid or vapour transport. Unfortunately a phase dividing function is difficult and laborious to be measured. However, the serial-parallel model presented above provides a moisture content dependency for the vapour diffusivity which enables to determine the liquid ratio from the measurements.

Even though the function (5.17) might not be the correct vapour diffusivity, it presents a model for vapour transport by which the moisture conductivity can be split up into vapour and liquid contributions. By the help of eq. (2.24) the vapour diffusivity $D_{v}$ can be transformed into a vapour conductivity $K_{v}$. In the same way, the diffusivity values 
which correspond to the measured vapour diffusion resistance factors can be transformed into moisture conductivity values $K_{l+v}\left(\mu_{i}\right)$. From the difference of both, measured moisture conductivity and derived vapour conductivity, the hygroscopic liquid water conductivity can be determined. This procedure is illustrated in Fig. 5.5 for three values from cup measurements. The dry-cup value $\mu_{d r y}$ is regarded to comprising vapour diffusion only. The vapour diffusivity (5.17) is fixed on that point and liquid water transport is supposed to be zero.

Thus, the hygroscopic liquid water conductivity $K_{l, h y g}\left(\theta_{l}\right)$ is defined by logarithmic interpolation of the difference between measured moisture conductivity data derived from wet-cup vapour diffusion experiments and the vapour conductivity according to the serial-parallel model which is adjusted to the dry-cup value. As the derivation according to the pore model is done numerically, the whole conductivity function is provided in form of data points which are to be logarithmically interpolated for use in simulation. Therefore, the hygroscopic liquid water conductivity is simply to be added to the derived data, see eq. (5.18).

$$
K_{l, \text { cap }}\left(\theta_{l}\right)=K_{l, h y g}\left(\theta_{l}\right)+K_{l, p m}\left(\theta_{l}\right) \quad \text { for } \quad \theta_{l} \leq \theta_{\text {cap }}
$$

Above capillary saturation, the measured conductivity data from permeameter or infiltrometer measurements is to be used. Therefore, the derived conductivity function is truncated at capillary saturation $\theta_{c a p}$ and interpolated with the measured values. By this means the saturated conductivity $K_{l, s a t}\left(\theta_{l}\right)$ is determined in the same way as done for the hygroscopic conductivity. Finally, with eq. (5.19) the liquid water conductivity can be defined in dependence to the moisture content consisting of the different conductivities specified above. An illustration of the conductivity function together with the contained calibration options is given in Fig. 5.6.

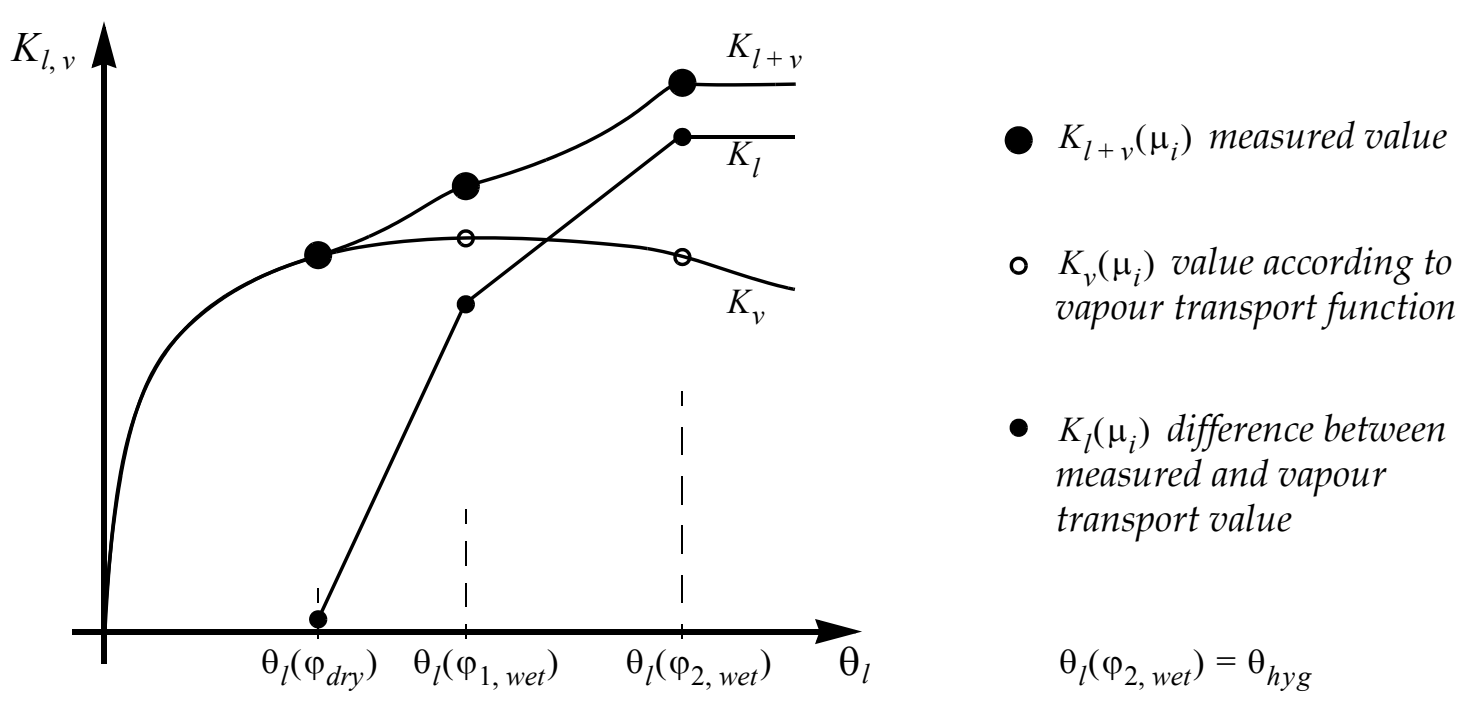

Figure 5.5: Determination of the hygroscopic liquid water conductivity as the difference between derived conductivity data from dry-cup and wet-cup measurements and the vapour transport function according to the serial-parallel model. Note that the conductivity $K_{l, v}$ is plotted in logarithmic scale. 


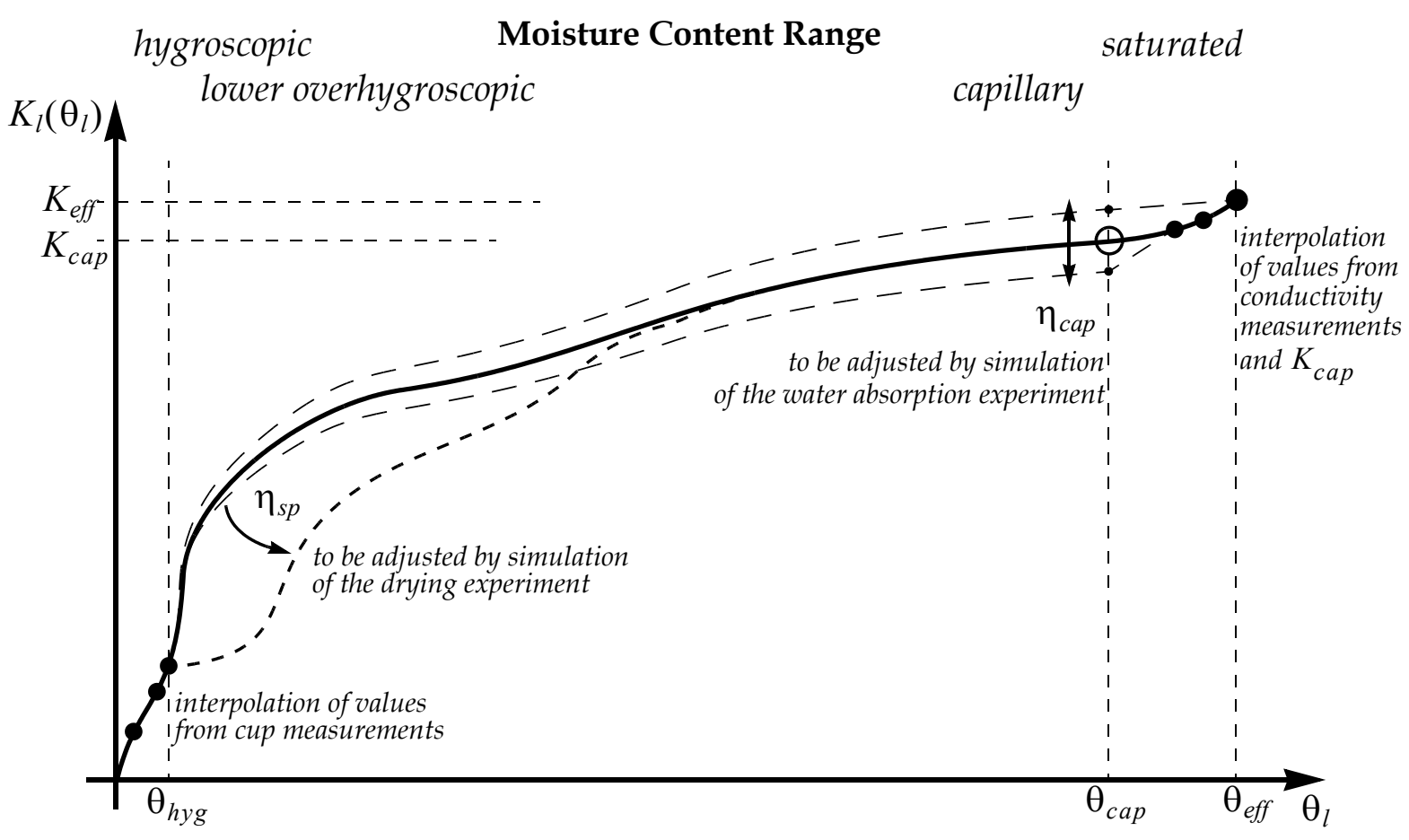

Figure 5.6: Calibration of the liquid water conductivity function. Data in the hygroscopic moisture range is derived from cup measurements, data in the saturated moisture range from permeameter or infiltrometer measurements. The slope in between is derived from the pore structure and can be calibrated adjusting $\eta_{s p}$ and $\eta_{\text {cap }}$ by the help of numerical simulation and data of water uptake and drying experiments.

$$
K_{l}\left(\theta_{l}\right)=\left\{\begin{array}{lll}
K_{l, \text { cap }}\left(\theta_{l}\right) & \text { if } & \left(\theta_{l} \leq \theta_{\text {cap }}\right) \\
K_{l, \text { sat }}\left(\theta_{l}\right) & \text { if } & \left(\theta_{l}>\theta_{\text {cap }}\right)
\end{array}\right\} \quad \text { liquid water conductivity }
$$

If none of the conductivity data in the saturated moisture content range is available, the conductivity derived by the pore model can be used for the whole moisture range up to effective saturation $\theta_{\text {eff }}$. But then the value $K_{\text {eff }}$ is to be estimated. Within a study reported by Weier (2002), the relation between the saturated conductivity $K_{\text {eff }}$ and the water uptake coefficient $A_{w}$ was investigated. Unfortunately, a well-defined relation could not be found. However, if one follows the argumentation given above concerning the water absorption experiment and its corresponding moisture content range, a relation between the water uptake coefficient $A_{w}$ and the liquid water conductivity at capillary saturation $K_{l}\left(\theta_{\text {cap }}\right)$ appears more likely. Based on the dependence supposed by Weier (2002), measured water uptake coefficients were compared with the adjusted conductivity data at capillary saturation for different materials proving a general empirical relation in the form of eq. (5.20).

$$
K_{l}\left(\theta_{c a p}\right)=10^{-8} \cdot \eta_{A w} \cdot A_{w}^{2}
$$


For the investigated materials the coefficient $\eta_{A w}$ varied between $\eta_{A w} \approx 0.95 \ldots 16$. Certainly, this is more than one order of magnitude. However, with regard to the influences on moisture transport which were observed during the experiments reported in Chapter 3, the relation (5.20) is still subjected to considerable uncertainties. Circumventing these uncertainties, a slightly different conductivity function is probable to be obtained during calibration which, in turn, will lead to different values for $\eta_{A w}$, too. Therefore, as an initial estimate in case no measured conductivity value is available, eq. (5.20) with $\eta_{A w}=1$ is absolutely useable.

Calibration of the liquid water conductivity function can be done by inverse modelling, i.e. by simulation of experiments under adjustment of the internal modelling parameters $\eta_{c a p}$ and $\eta_{s p}$. The global scaling factor $\eta_{c a p}$ is to be adjusted using water absorption data. The parameter of the serial-parallel model $\eta_{s p}$ influencing the liquid water conductivity in the lower overhygroscopic moisture range can be adjusted on the basis of drying data, see also Fig. 5.6.

The vapour diffusivity, which is specified in eq. (5.17), is influenced by $\eta_{s p}$, too. However, in comparison with the liquid water conductivity, the influence is here of minor importance, as shown in Fig. 5.7. Note that the abscissa is of normal scale and not, as the conductivity in Fig. 5.6, in logarithmic scale.

The vapour diffusivity is defined for moisture contents between dry and porosity, as vapour diffusion can occur in any air-filled pore. With an increasing moisture content, however, the vapour diffusivity decreases, which is in accordance to the reduction of pore space available for vapour diffusion.

Since the maximum of the vapour diffusivity is moved to unreliable high moisture contents for increasing values of $\eta_{s p}$, this value is set fix to $\eta_{s p}=1$ for vapour diffusion. This is an inconsistency of the model. However, no discontinuity arises in the description of moisture transport in general, as the liquid water conductivity in the hygroscopic moisture range is always determined as the difference between measured moisture transport according to the wet-cup data and the vapour transport according to the vapour transport function. The task was to choose between a consistent model delivering an unreliable slope of the

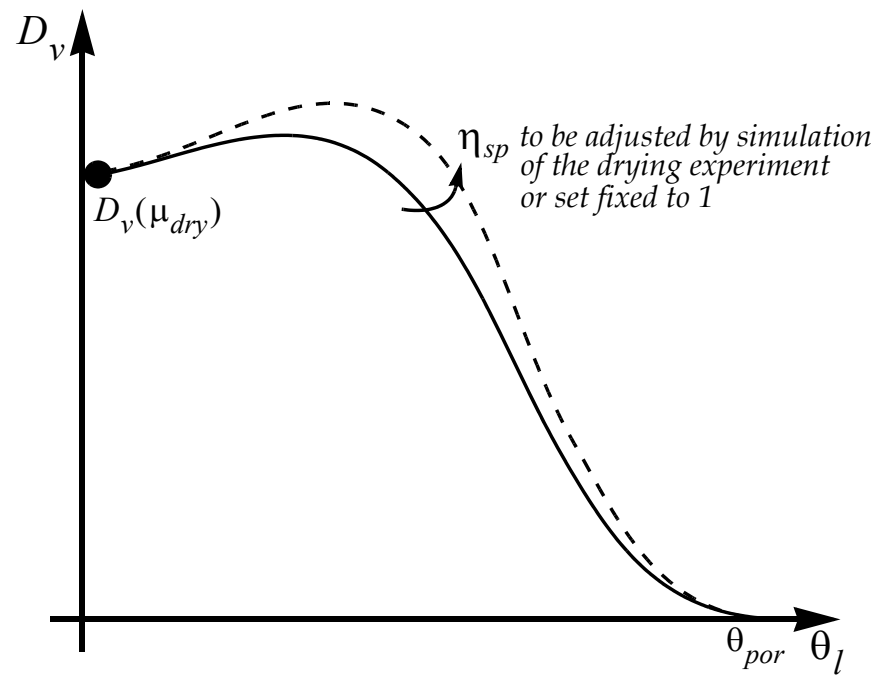

Figure 5.7: $\quad$ Vapour diffusivity in dependence to the water content and the parameter $\eta_{s p}$ from the serial-parallel model. vapour diffusion function or a small inconsistency for the gain of a reliable vapour diffusivity. And the choice has been made in favour of a reliable vapour diffusivity. The fixed serial-parallel model parameter for vapour diffusion may therefore be considered as a blemish of the model. 


\subsubsection{Heat conduction}

The heat transport depends on the material, its pore structure and on the degree of saturation, i.e. on the moisture content. The thermal conductivity of the dry material accounts for the first two dependencies. For the latter, a similar serial-parallel model can be derived as described under paragraph 5.1.2.2 Mechanistical approach: serial-parallel model. However, since the commonly used linear increase in dependence to the moisture content according to eq. (5.21) gives good results for most application cases, this simplified approach was applied here, too.

$$
\lambda\left(\theta_{l}\right)=\lambda_{R}+\lambda_{w} \cdot \theta_{l}
$$

In eq. (5.21), $\lambda_{R}$ is the calculation value of the thermal conductivity of the material and $\lambda_{w}$ is the thermal conductivity of water with $\lambda_{w}=0.56 \mathrm{~W} / \mathrm{mK}$. Determination of the thermal conductivity is described in paragraph 3.1.2 Thermal properties. The temperature dependency of the thermal conductivity is not taken into account due to the little influence it has within the relevant temperature range in Building Physics. In addition for most materials this influence is not determined.

\subsubsection{Concluding the developments in material modelling}

The developed material model is a conductivity approach providing a functional description for moisture storage and a derivation procedure for the phase separated moisture transport functions. Liquid transport is expressed by a conductivity function associated with the capillary pressure gradient. The driving force of vapour transport is the vapour pressure gradient which a vapour diffusivity is assigned to.

The central material property is the moisture storage function for which a functional description is given. It consists of a sum of weighted cumulative distribution functions of the Gauss type and is to be adjusted to measured adsorption and desorption data.

The liquid water conductivity can be derived from the moisture storage function applying the bundle of tubes model according to Burdine (1953). However, some modifications, as e.g. a coupling with a mechanistical approach, were introduced enabling calibration of the conductivity within the whole moisture content range.

The same mechanistical approach provides also a description for the moisture dependency of vapour transport. The resulting vapour diffusivity can be adjusted by vapour diffusion measurements. The moisture dependency of the heat conduction is also taken into account.

The introduced material model covers the whole moisture content range from dry to effective saturation. The chosen description of moisture storage has a high flexibility in adjustment to measured data and forms the basis of the liquid transport function. Liquid and vapour transport functions can be calibrated by the help of measured data and inverse modelling providing a general material model with enough flexibility to be adjusted and calibrated on the basis of experimental data. 


\subsection{Methodological Developments for the Drying Experiment}

The drying experiment is considered to be very important for the hygric material characterisation since it reveals information about the moisture transport properties within the lower overhygroscopic moisture range. It is an integral transport experiment which does not require expensive equipment to be performed. Together with the water uptake experiment, two complementary moisture transport investigation methods are available enabling material model calibration for the whole overhygroscopic moisture range.

However, the drying is much more dependent on the boundary and transfer conditions than the water uptake. Therefore, if the measured data is to be used for inverse modelling, all conditions have to be known, i.e. measured. At present, there exists neither any standardisation for the experimental investigation nor a clear and possibly simple material parameter which can be derived from it. Therefore, the following paragraphs investigate the drying process and its governing influences. On that basis, requirements on a drying device will be formulated and the developed apparatus will be shown. Finally, the definition of a drying coefficient, based on the first attempts made by Krus et al. (2007), will be discussed on the basis of own measurement and simulation studies.

\subsubsection{Basics of the drying process}

The drying process is identified by the phase transition of liquid water into water vapour. The moisture stored within a material is given to the surrounding air by evaporation. Depending on the moisture content and the moisture transport properties of the material, two processes can be distinguished. For the first, typical at high moisture contents, the water is conducted to the surface in its liquid phase where evaporation proceeds. The second one, which is distinctive for lower moisture contents, is characterised by evaporation occurring already inside the material and causing a
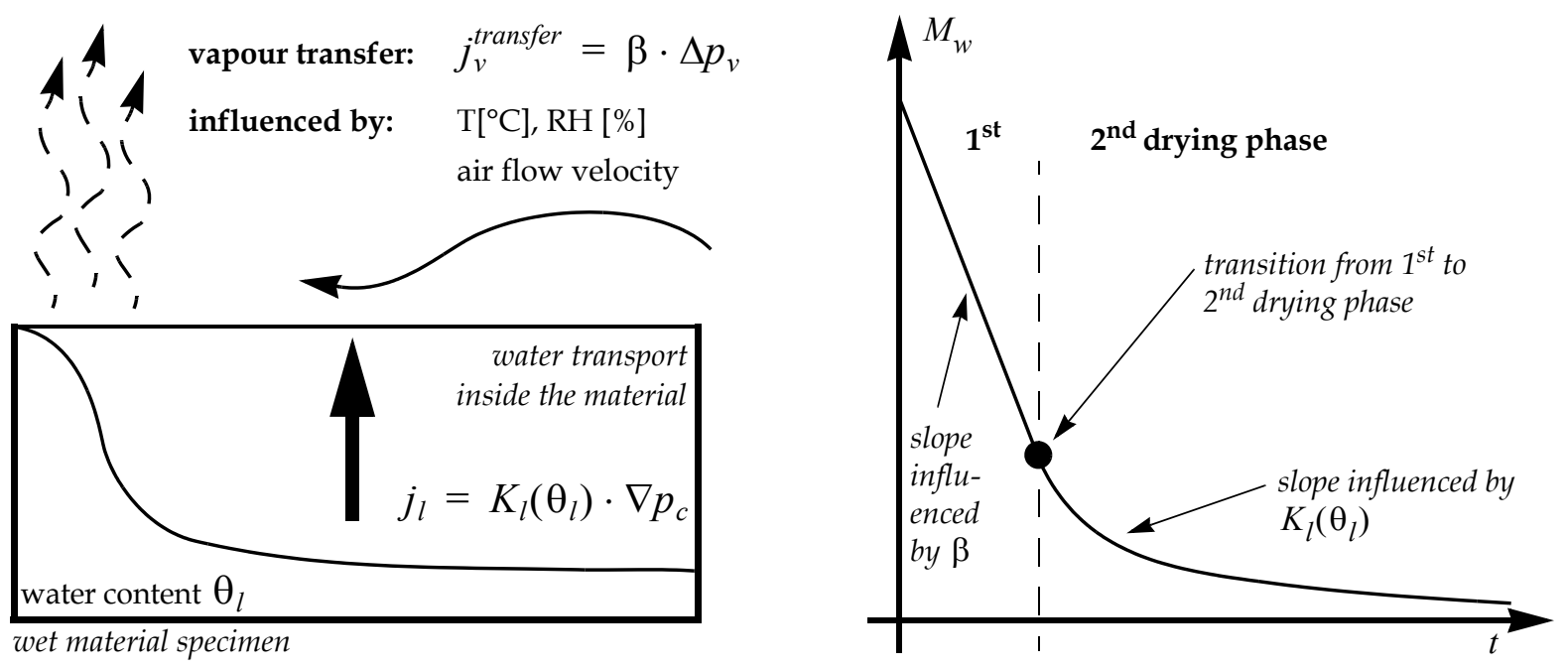

Figure 5.8: The drying process: boundary conditions and material properties influencing the drying process (left) and example integral drying curve with a distinction of the two drying phases indicating its influencing parameters (right). 
vapour diffusion flow out of the material. For a drying process starting at a high degree of saturation, both transport mechanisms are normally passed through one after another.

Following these two processes, Krischer (1956) distinguished two important drying phases. Assuming constant boundary conditions, the first drying phase is characterized by a constant drying rate, i.e. by a linear weight loss in time, see Fig. 5.8 at the right. In this phase, the evaporation rate is limited by the boundary conditions. During the second drying phase, the process slows down depending on the material properties of the examined specimen.

Hence, three parameters influence the drying behaviour of a building material in particular. These are the climatic conditions of the drying environment (temperature and relative humidity) and the transfer conditions at the drying surface, which can be summarized as the boundary conditions, and the transport properties of the material, see Fig. 5.8. Within the following two subsections, these influences will be elaborated in detail.

Besides the previous influences, the phase change of liquid water to water vapour leads to a cooling effect at the material surface. This results in a three dimensional heat transport. The drying process is therefore a multi-dimensional coupled heat and moisture transport problem.

\subsubsection{Vapour transfer - Influence of boundary conditions}

Climatic and transfer conditions control the amount of moisture which can be given off the material to the environment. The vapour pressure $p_{v}$ can be calculated according to eq. (5.22) with the relative humidity $\varphi$, the temperature $T$ and the saturation vapour pressure $p_{s}(T)$. The saturation vapour pressure $p_{s}(T)$ is a non-linear function of the temperature which is given by different authors. A commonly used relation is e.g. given in DIN 4108-3.

$$
p_{v}(T, \varphi)=\varphi \cdot p_{s}(T) \quad \text { vapour pressure }
$$

The vapour pressure difference $\Delta p_{v}$ between the material surface and the environment causes a vapour transport in the direction of lower pressures, i.e. out of the material. The transfer vapour mass flow density $j_{v}^{\text {transfer }}$ and the vapour pressure difference $\Delta p_{v}$ are associated by the vapour transfer coefficient $\beta$ according to eq. (5.23). The vapour transfer coefficient $\beta$ is dependent on the air flow conditions and the surface texture of the sample (Worch (2004)). According to Worch (2002), the surface texture can lead to significantly larger evaporation rates for the same air velocity above the sample compared with a pure water surface.

$$
\begin{array}{lr}
j_{v}^{\text {transfer }}=\beta \cdot \Delta p_{v} & \text { vapour mass flow density } \\
\text { with } \Delta p_{v}=p_{v}\left(T_{s}, \varphi_{s}\right)-p_{v}\left(T_{e}, \varphi_{e}\right) & e \ldots \text { environment, s ... surface } \\
j_{Q}^{\text {transfer }}=\alpha \cdot \Delta T & \text { heat flow density }
\end{array}
$$


The vapour pressure difference $\Delta p_{v}$ is the evaporation force. According to eq. (5.22), the vapour pressure depends on the temperature and the relative humidity. High surface temperatures and high humidities cause a high vapour pressure at the material surface whereas low air humidities cause a low vapour pressure in the surrounding air. The evaporation rate is raised by increasing the vapour pressure difference $\Delta p_{v}$.

The factor $\beta$ in eq. (5.23) is the vapour transfer coefficient representing a phenomenological transfer parameter which summarizes thermodynamical and fluid-mechanical effects for practical purposes. Analogous to the heat transfer coefficient $\alpha$ in eq. (5.24), it is influenced by the air flow velocity along the evaporation surface and by the surface texture.

\subsubsection{Moisture inside the material - Influence of transport properties}

The transport properties of the material limit the amount of water that is conducted to the evaporation surface. Generally, the liquid water transport is much more efficient than the vapour transport. A material with a high liquid water conductivity $K_{l}\left(\theta_{l}\right)$ will therefore dry out much faster than one with a low conductivity. In this case, the first drying phase is very long indicating that the process is only limited by the boundary and transfer conditions.

For a material with a low liquid water conductivity though, the transition from the first to the second drying phase is quickly reached, where less water can be conducted to the surface as could evaporate there due to the boundary and transfer conditions. The possible mass flow density $j_{v}^{\text {transfer }}$ is then bigger than the actual water flow $j_{l}$ to the surface. The moisture front moves into the material slowing the drying process down. See again Fig. 5.8 for an illustration of these processes.

\subsubsection{Drying apparatus - Concept and design}

In view of these influences, it becomes important to perform the experiments under defined and reproducible conditions when measured drying data is to be applied to hygrothermal material characterisation. Information concerning the material behaviour is revealed during the second drying phase only. A short first drying phase is hence desirable. For this reason, a special device was developed on the basis of the following considerations concerning the drying process and its utilisation for inverse modelling.

\subsubsection{Influences and considerations}

The main unknown variable of the drying process is the vapour transfer coefficient $\beta$. Under some assumptions however, the vapour transfer coefficient can be determined from the measured drying data in a simplified way which is sufficient for the data evaluation required for inverse modelling.

The moisture release of a material sample is described by the vapour diffusion flux density $j_{v}$ in $\left[\mathrm{kg} / \mathrm{m}^{2} \mathrm{~s}\right]$. It can be measured gravimetrically as the mass change versus time related to the sample surface and as a mean value of the corresponding measurement interval. Since the vapour pressure depends on the temperature and the relative 
humidity, see eq. (5.22), these two parameters should be known for the measurement environment. For the vapour pressure at the material surface, the relative humidity there can be assumed to have a value close to $100 \%$ during the first drying phase. Thus, the surface temperature remains as another parameter to be measured in order to determine the vapour pressure difference $\Delta p_{v}$.

The developing surface temperature profile due to the cooling effect is influenced by different factors. One of them is the sample shape: round specimens will have a radial symmetric temperature profile which will be evaluated much easier than those of rectangular specimens. Another factor is the environment whose climatic conditions should be kept constant for that purpose. The less the temperature profile is disturbed, the more meaningful it is. The use of larger specimens is hence recommended as the influence of boundary effects will decrease with increasing sample diameter.

If all these data are known, the vapour transfer coefficient $\beta$ can be determined in a simplified way. One may assume the surface temperature to be constant during the first drying phase after an initial period for its development. A measured temperature distribution at the surface can be averaged, otherwise the temperature of the surface centre may be chosen leading to slightly increased values for $\beta$. Bednar $\mathcal{E}$ Dreyer (2003) indicated however, that the resulting error is less than $5 \%$. Then ultimately, the vapour transfer coefficient can be calculated from the parameters measured during the first drying phase according eq. (5.25).

$$
\beta=\frac{j_{v}^{\text {transfer }}}{p_{v}\left(T_{s}, \varphi_{s}\right)-p_{v}\left(T_{e}, \varphi_{e}\right)} \quad \text { vapour transfer coefficient }
$$

To determine the vapour transfer coefficient as precisely as possible and gain meaningful drying data to be used for inverse modelling, the following considerations concerning the drying experiment were concluded:

Sample shape:

- The advantage of round specimens is that all state variables behave radial symmetric. They can be measured as well as simulated more reliably since radial symmetric discretisation and simulation is possible. Therefore the use of round specimens is suggested.

- Thermal insulation of the specimens helps to reduce the influence of boundary disturbance. On the other hand, simulation is complicated by that because another unknown variable is added. Furthermore a thermal insulation of the material specimen is laborious and not urgently needed. For those reasons, a thermal insulation of the specimens is not recommended.

Boundary conditions: The vapour flux according to eq. (5.23) consists of two factors whose influence on the drying process should be taken into consideration.

- The vapour transfer coefficient, depending on the air flow conditions, can be calculated form the boundary conditions according to eq. (5.25) under the mentioned simplification (first drying phase, constant vapour flux and constant boundary conditions). The assumption of 
$100 \%$ relative humidity at the sample surface is only valid as long as the material is able to conduct more moisture to the surface than evaporates due to the transfer conditions. This characterizes the first drying phase. Its end can be seen in both, the deviance from the linear drying course versus time and the rising surface temperature whereof the latter one is more sensitive.

- The other factor in eq. (5.23) is the vapour pressure gradient as the driving force which can be influenced by the climatic conditions of temperature and relative humidity. It is recommended to keep this conditions constant as fluctuations in humidity and temperature actually influence the vapour flux via the vapour pressure gradient. As eq. (5.25) is typically evaluated with mean values of the gravimetric measurement interval, these fluctuations may be wrongly interpreted into the transfer coefficient.

\subsubsection{Development of a drying apparatus}

The following specific demands on a drying measurement apparatus can be derived from the stated considerations about the drying behaviour. They are primarily a consequence of the requirements on reproducibility and controllability of the measurement conditions:

- air flow conditions: possibly constant, adjustable and measurable,

- $\quad$ temperature and relative humidity: possibly constant and measurable,

- $\quad$ surface temperature: measurable for each specimen,

- $\quad$ possibility to use a variable number of specimens.

A reproducible air flow condition at each sample position could be achieved with a flow channel. The air flow velocity could be controlled by ventilators at the in- and the outflow of the channel. Temperature and relative humidity should be measured within

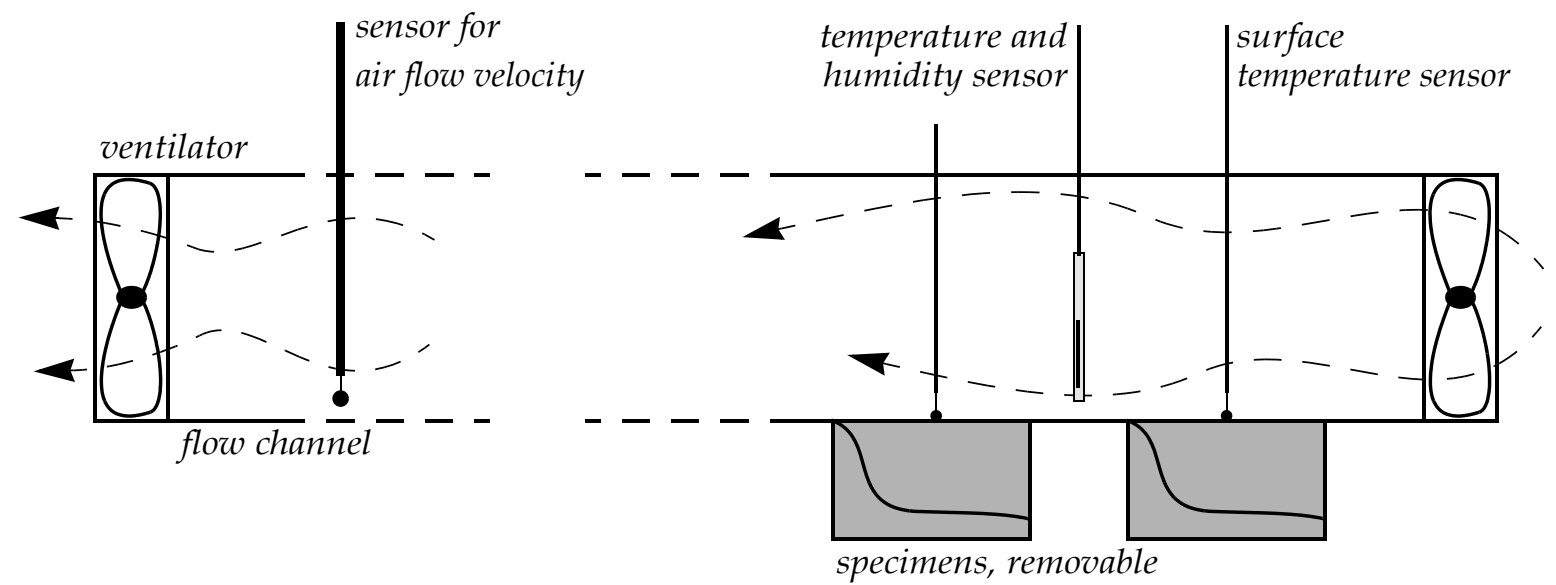

Figure 5.9: $\quad$ Scheme of the drying apparatus which is designed on the basis of the discussed influences. 



Figure 5.10: Pictures of the developed drying apparatus: view of the whole device (a), sensor for boundary temperature and humidity $(b)$, detail of a specimen with a surface temperature sensor (c), round material samples $(d)$, view inside the flow channel with sensors for surface temperature and air velocity (e), and drawer with adjustable inlets and template for the specimens (f).

the channel and possibly at more than one position. The evaporation surface should be overflown in the channel and the surface temperature had to be measured in the surface centre. Finally the specimens should be removable without sustainably disturbing the measurement course. All these requirements are summarized in a scheme shown in Fig. 5.9. 
On the basis of these requirements a flow channel according to Fig. 5.9 was designed containing the following elements: an air flow channel with assessable ventilators, adjustable sensors for the surface temperature at each specimen, sensors to measure the boundary conditions (temperature, relative humidity and air flow velocity) and removable drawers for the material samples.

\subsubsection{Realisation of the drying apparatus}

The drying apparatus was built as a prototype made of alloy, PVC and perspex forming an air flow channel. It consists of an alloy frame covered by perspex panels, a set of three ventilators at each end of the channel and the holders for four drawers at the bottom. The drawers are divided into three cells where each contains a height adjustable insert to hold the specimen. The top of the drawer is covered by a template for the specimens.

The installed measurement technology consists of three times four temperature sensors being height adjustable to the surface centre of each specimen, and two coupled temperature and relative humidity sensors (see paragraph 3.2.2.2 Moisture potential measurement) at different positions. Furthermore sensors measuring the air flow velocity can be inserted. The following pictures of Fig. 5.10 give an overview of the apparatus and the installed measurement technique.

\subsubsection{Derivation of a drying coefficient}

To ensure a high quality for advanced material characterization, the availability of drying and water uptake data sets is a prerequisite. As discussed above, the boundary conditions under which the experiments were carried out must also be known for that purpose. On the other hand, for a rough approximation of material properties, both, building industry and design practise, ask for possibly simple material parameters. The vapour diffusion resistance factor $\mu$ giving a measure for the vapour permeability of the material, or the water uptake coefficient $A_{w}$ giving a measure for the liquid transport properties at high moisture contents during imbibition, are such parameters. For lower moisture contents, however, a single moisture transport coefficient is still missing. The drying experiment reveals information about the moisture transport properties in this moisture range. For this reason, some first attempts have been made recently to derive a simple material parameter from the measured drying data.

\subsubsection{Attempts to standardize drying data}

Due to the manifold dependencies of the drying process, a simple single parameter as the slope of the drying curve during the first drying rate or the duration of this phase would be partly or solely dependent on the drying conditions and would thus not contain the desired information about the material behaviour. Recently, two attempts have been made to standardize the drying data, and in both the author was involved. The first study aims at the reproduction of integral drying data by the help of an analytic function containing one or more parameters whereas the second is an approach to define a single value drying parameter. 


\section{Analytic expression to describe the drying course}

Jurk et al. (2004) investigated five different approaches to describe the integral drying curve with an analytic expression. The objective was a simplified and standardized storage of drying data in data bases restricting the experiments to defined climatic conditions as well as sample dimensions. Under such restricted conditions, the drying course of three different materials - two types of brick and a calcium silicate - was approximated by two exponential functions and three combinations of a linear and an exponential function, each with a different number of adjustable coefficients.

The study showed that only a combination of a linear function describing the first drying phase and an exponential function as introduced by Sander et al. (2000) with two parameters describing the second drying phase is able to fairly represent the measured drying data. All other approaches showed unacceptable agreement. The approach is given in eq. (5.26) and (5.27) below. There, $\theta_{0}$ is the moisture content at the beginning of the experiment, $\theta_{1}$ and $t_{1}$ are the moisture content and the drying time at the end of the first drying phase, respectively. $\theta_{e}$ is the moisture content at the end of the experiment, and $\eta_{a}$ and $\eta_{b}$ are adjustable parameters influencing the shape of the curve describing the second drying phase.

$$
\begin{array}{ll}
\theta_{l}(t)=\theta_{0}+\frac{\theta_{1}-\theta_{0}}{t_{1}} \cdot t & \text { for } t \leq t_{1} \\
\theta_{l}(t)=\theta_{e}+\left(\theta_{1}-\theta_{e}\right) \cdot \exp \left[-\eta_{a} \cdot\left(t-t_{1}\right)\right]^{\eta_{b}} & \text { for } t \geq t_{1}
\end{array}
$$

Both equations take the same value $\theta_{1}$ at $t_{1}$. If the first derivatives of eq. (5.26) and (5.27) are set equal for $t=t_{1}$, both parameters $\eta_{a}$ and $\eta_{b}$ vanish. This impedes to reduce the number of parameters by forcing continuity in the slope at $t_{1}$ for both functions. The condition of continuity is hence not fulfilled for the given approach. Moreover, the functional flexibility is still limited for the second drying phase as can be seen in the example of Fig. 5.11 comparing measured drying data of the brick with the adjusted function. The

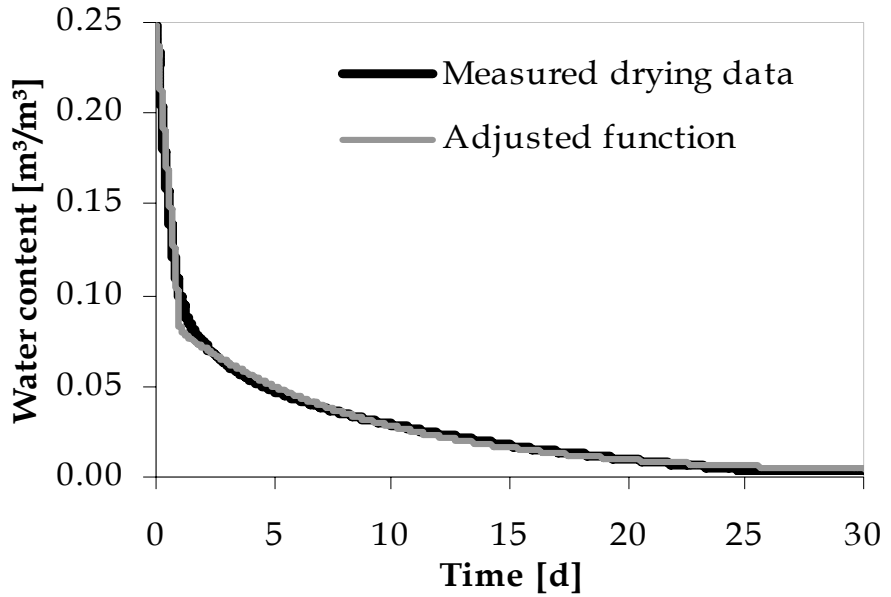

Figure 5.11: Comparison of measured drying data with adjusted function according to eq. (5.26) and (5.27) for the investigated brick. measured soft bend at the end of the first drying phase can not be reproduced. The transition between first and second drying phase, however, is a very sensitive and hence important data range which needs to be reproduced correctly. 
Since the investigations revealed that no simple functional expression could be found describing the general drying course with enough flexibility, these attempts have been abandoned. For storage in data bases, a normalized set of measured data points in combination with the climatic boundary conditions appears to be the most reliable alternative. Further on, it was focused on the definition of a drying coefficient which is supposed to be as easily used for practical purposes as the water uptake coefficient.

\section{Single number drying coefficient}

The main problems in defining a drying coefficient as a single value parameter are the manifold dependencies on the sample dimensions as well as on the initial, boundary and transfer conditions. The discussion on a drying coefficient was initiated in the frame of a national project ${ }^{1}$ aiming on hygrothermal material data collection and evaluation. Some first ideas were summarized by Krus et al. (2007) relating the duration of the first drying phase to the square-root of the sample height for an experiment under defined drying conditions.

Assuming the applied material functions ${ }^{2}$ represent the material's drying behaviour correctly, Krus used one-dimensional simulation studies to investigate the influences of the drying process. The simulations were started at capillary saturation under constant drying conditions of $50 \%$ relative humidity and a temperature of $23^{\circ} \mathrm{C}$. For the vapour transfer, the heat and mass analogy (Lewis-relation) was applied which is given in eq. (5.28) below. The investigated materials were a lime-sand brick and an aerated concrete with varying sample heights between $0.07 \mathrm{~m}$ and $0.5 \mathrm{~m}$.

Setting all climatic conditions constant, the only varied parameter was the height of the simulated specimen. As a criterion for the drying behaviour, the duration of the first drying phase was chosen. The obtained results indicated that this duration shows a square-root dependence on the sample height. Therefore, Krus et al. (2007) suggested to define the drying coefficient as the slope of the linear curve which meets the data points of the end of the first drying phase for a material of different sample heights in a square-root scale of the sample thickness. Fig. 5.12 displays this relation schematically following the simulation results shown by Krus et al. (2007) for the two materials.

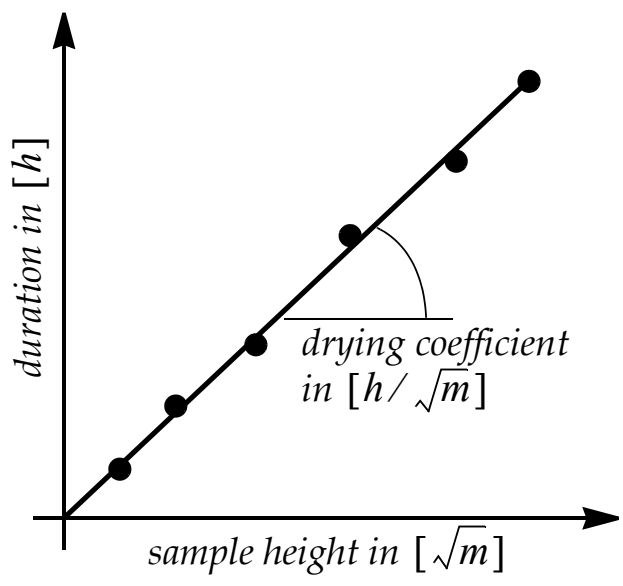

Figure 5.12: Duration of the first drying phase versus square-root of sample height.

1. The national project MASEA was funded by the German Ministry of Economy and Technology. The project aim was to develop a hygrothermal material data base with main emphasis on materials from the building stock. It is available under http:/ / www.masea-ensan.de

2. Krus did not specify the applied material model, but it is assumed that he used the material model according to Krus $\mathcal{E}$ Holm (1999) for his investigations. This model was discussed in detail under paragraph 2.2.1.4 Diffusivity approach according to Krus and Holm. 
Krus et al. (2007) further on discussed the challenge of keeping all external measurement conditions, especially the transfer conditions, constant on the basis of two variants. The first is the use of an apparatus as developed here, allowing to adjust these conditions and measure them at the same time. Krus, however, considers this to be too elaborate. The other variant he suggested was to install a vapour diffusion resistance at the sample surface to ensure equal conditions for all specimens under normal laboratory conditions. But the square-root of sample height relation could not be achieved by such a set-up.

Hence, there is still a number of problems remaining in both, measurement and drying coefficient definition. Krus et al. (2007) suggest to measure the drying behaviour under any possible conditions which should be preferably known. Then the obtained data should be applied for the approximation of the moisture transport function to be used in numerical simulation. The drying coefficient as defined above should then be derived by the help of numerical simulation of the drying experiment under the defined and constant conditions.

\subsubsection{Discussion on the proposed drying coefficient}

The discussion of the proposed drying coefficient starts with an illustration of the proposal to install a vapour retarder at the evaporation surface to keep the transfer conditions constant. Subsequently, results from own studies are presented which show another dependency between the first drying phase duration and the sample height. Possible reasons for these deviations will be discussed on the basis of an investigation of the different influencing parameters. An analytic solution of the drying problem will finally prove the hypotheses which were already indicated by a systematic simulation study before some conclusions on the drying coefficient will be drawn.

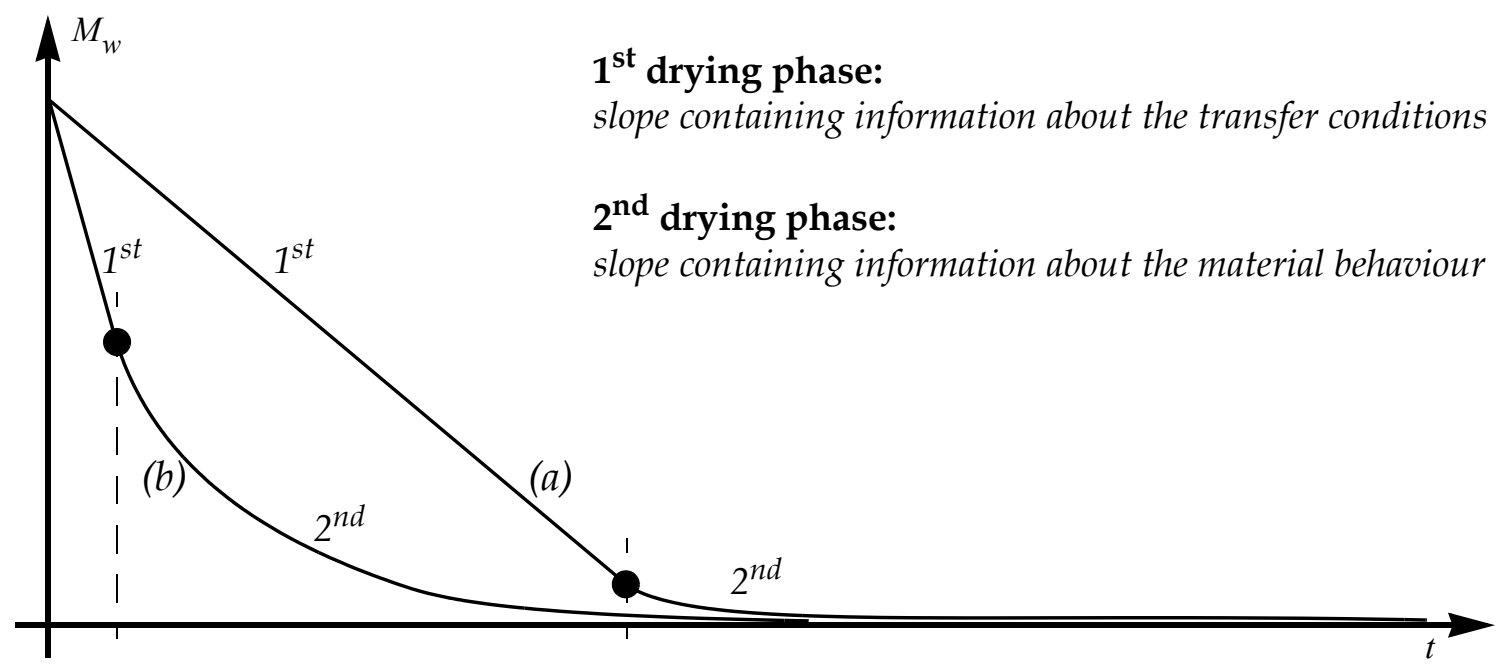

Figure 5.13: Illustration of the measurement effect, if the vapour transfer at the drying surface would be reduced (case (a)): the drying experiment would reveal almost no information about the material behaviour. 


\section{Drying measurement with an attached vapour retarder}

The idea of Krus to attach a vapour retarder to the evaporation surface in order to eliminate the influence of the transfer conditions on the drying process is seen counterproductive. Doing so, the drying rate is extremely slowed down and the first drying phase is largely extended, as shown in Fig. 5.13. In such case (case (a) in Fig. 5.13), the experiment would not reveal any information about the material behaviour anymore, since the drying is only limited by the attached vapour retarder. It would not make any difference whether the drying of a pot of water or of a concrete is measured (if both start at the same moisture content). For that reason, this idea would turn the whole experiment into absurdity since the reason to perform it is to reveal information about the moisture transport properties of the material. It is hence not recommended to perform drying experiments under conditions of strongly reduced vapour transfer.

\section{Evidence from own studies}

Based on the proposed drying coefficient definition, own studies are carried out on an experimental as well as on a numerical simulation basis. Since the simulations for the four building materials introduced in Chapter 4 shows the same results as the performed measurements, only a selection of the experimental data will be discussed here.

The experimentally investigated materials are a ceramic brick and a calcium silicate insulation material. Cylindric specimens are prepared with a diameter of either $53 \mathrm{~mm}$ or $88 \mathrm{~mm}$. The experiments are carried out with the drying device described under paragraph 5.2.2 Drying apparatus - Concept and design above under laboratory conditions of $23 \pm 1{ }^{\circ} \mathrm{C}$ and $40 \pm 5 \% \mathrm{RH}$. There, different vapour transfer rates are adjusted which are summarized in two vapour transfer coefficient groups, indicated in the graphics with $\beta$ small and $\beta$ large. The initial moisture content is adjusted to effective saturation, which is $\theta_{\text {eff }}=0.91 \mathrm{~m}^{3} / \mathrm{m}^{3}$ for the calcium silicate and $\theta_{\text {eff }}=0.21 \mathrm{~m}^{3} / \mathrm{m}^{3}$ for the ceramic brick. To avoid uncertainties due to material variability, the experiments are carried out with the large specimens fist. Then these specimens are cut in smaller cylinders that are saturated and dried, again.
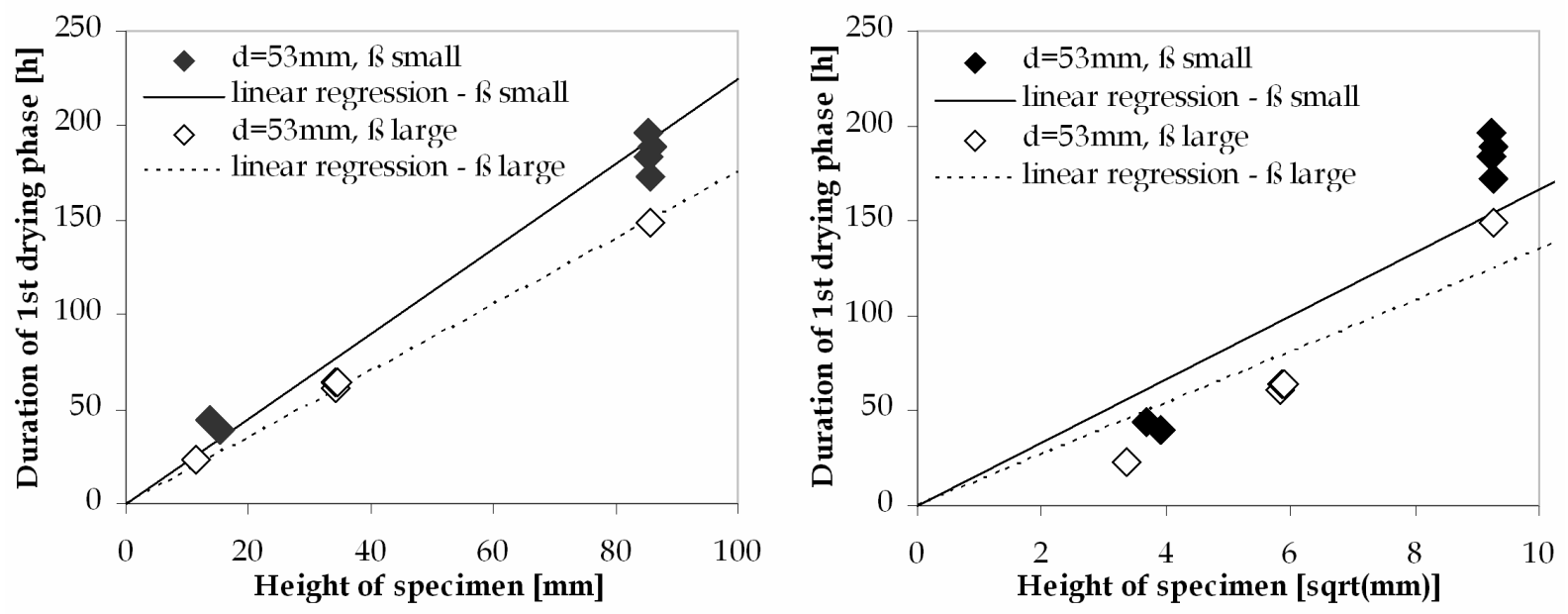

Figure 5.14: Duration of the first drying phase for different sample heights. Measured drying data of calcium silicate samples with a diameter of $53 \mathrm{~mm}$ under low and high vapour transfer conditions at the drying surface. The data shows a linear dependence of the duration of the first drying phase on the sample thickness. 

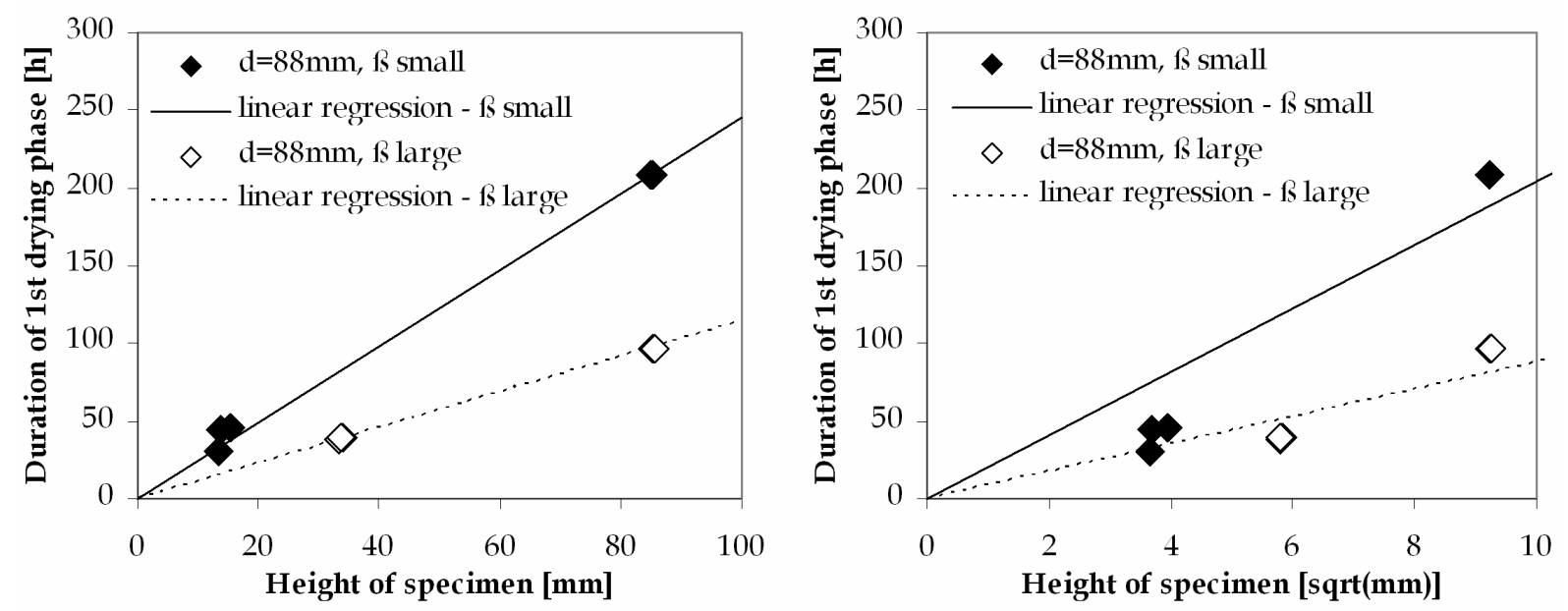

Figure 5.15: Duration of the first drying phase for different sample heights. Measured drying data of calcium silicate samples with a diameter of $88 \mathrm{~mm}$ under low and high vapour transfer conditions at the drying surface. This data also shows a linear dependence of the duration of the first drying phase on the sample thickness.

From the drying curves, the end of the first drying phase is detected by the help of its first derivative. In Fig. 5.14, 5.15 and 5.16, these times are plotted versus the height of the investigated specimens in a normal scale (left hand side) and a square-root of height scale (right hand side). It can be seen that for all varied conditions, a linear dependence between the end of the first drying phase and the sample height is obtained. The simulations, which are performed in many variations show the same dependency. The obtained measurement and simulation results hence reveal a different dependency than those reported by Krus et al. (2007). This will be the subject of the following discussion.
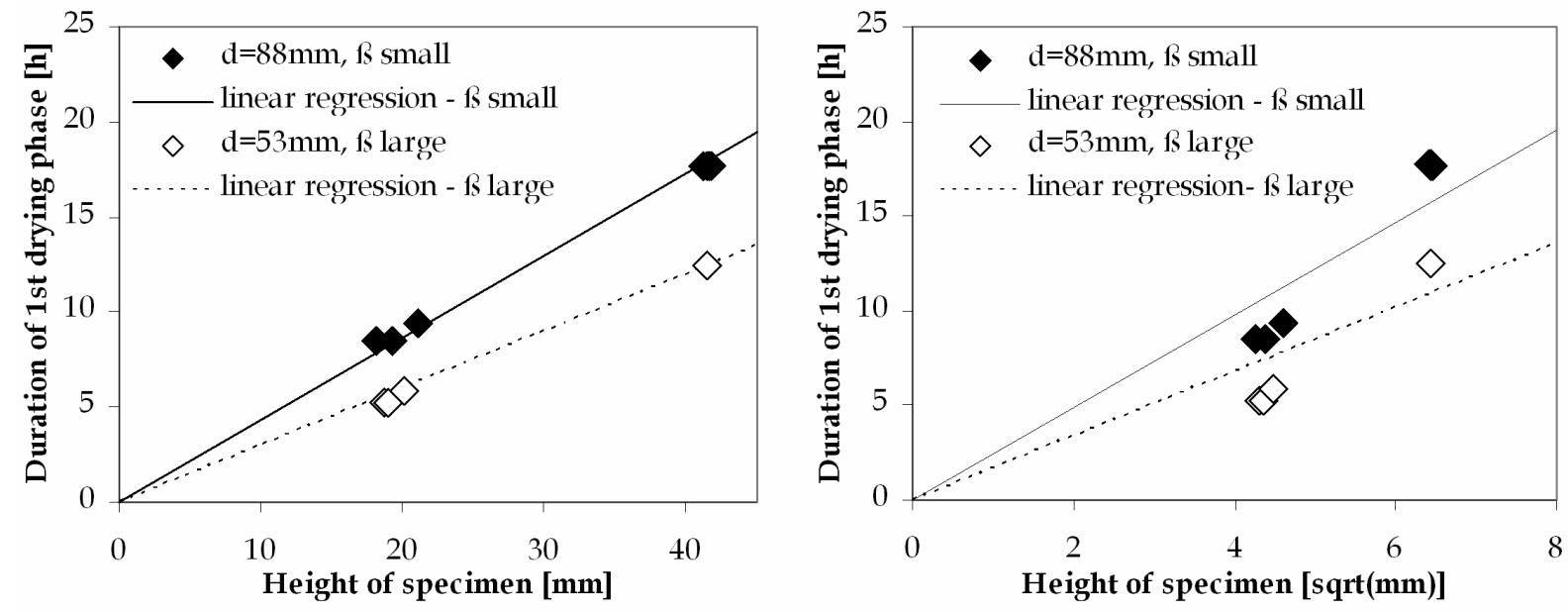

Figure 5.16: Duration of the first drying phase for different sample heights. Measured drying data of ceramic brick samples with different diameters (d) and under low and high vapour transfer conditions ( $\beta$ ) at the drying surface. Sample thickness in $[\mathrm{mm}]$ at the left and in square root scale [sqrt $(\mathrm{mm})]$ at the right. These measured data show a linear dependence of the first drying phase duration on the sample thickness, too. 


\section{Discussion of the drying coefficient proposed by Krus}

The basis of the investigations performed by Krus are results from numerical simulations of the drying behaviour of two materials. Without further studies on a larger variety of building materials and without experimental proof, this approach is seen problematic. It implies that these particular simulations are able to correctly reproduce all governing processes which occur during the drying and represent the general material behaviour at the same time. From the authors point of view, this is not unreservedly valid for a number of simulation aspects:

1. Validity of the heat and mass transfer analogy

2. Quality of material functions used for simulations

3. Lateral heat flux (one-dimensional simulations)

4. Initial moisture content

5. Investigated sample thickness

Special emphasis is placed on the following discussion of these aspects providing a profound basis for both, the evaluation of the disclosed differences in the dependency of the first drying phase duration as well as a further judgement of the influences on the drying process. For the latter one, a summary is given in Tab. 5.1.

1. Validity of the heat and mass transfer analogy: The vapour transfer rate has a strong influence on the drying time as well as on the mean material moisture content at the end of the first drying phase. One can see from Fig. 5.17, that this moisture content decreases with decreasing vapour transfer rates. Thus, that the larger the transfer rate, the higher is the moisture content at the end of the first drying phase, and the more information can be revealed concerning the moisture transport properties of the material. From the point of view of material characterization, a high vapour transfer rate is hence desirable.

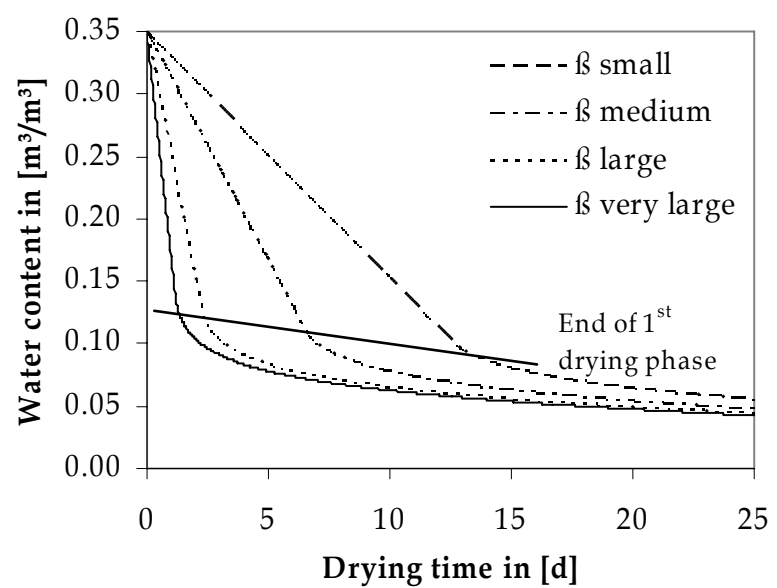

Figure 5.17: Influence of the vapour transfer coefficient $\beta$ on the drying behaviour of the brick.

To account for the vapour transfer within numerical simulation, two frequently used options exist. The first is to determine the vapour transfer coefficient $\beta$ from the measured drying rate and the boundary and surface temperatures and relative humidities during the first drying phase, as proposed here. Then, the heat transfer coefficient $\alpha$ can be adjusted during simulation by following not only the measured drying behaviour, but also the measured course of the surface temperature.

The second option, which is normally applied when no data of the surface temperature is available, is to use the heat and mass transfer analogy (5.28) to couple both transfer coefficients and adjust them by numerical simulation according to the drying course. The relation (5.28), often called Lewis-relation, is derived from the boundary layer theory, as e.g. given by Baehr $\mathcal{E}$ Stephan (2003). Le is the dimensionless Lewis-number 
which the authors give with $L e=0.87$ for vapour. $m$ is a dimensionless parameter given with $m=1 / 3$.

$$
\beta=\frac{\alpha}{R_{v} \cdot T \cdot c_{p, a} \cdot \rho_{a}} \cdot L e^{m-1}
$$

The Lewis-relation is though strictly valid only under laminar flow conditions at the surface. Such conditions do not necessarily occur during drying experiments. The comparison of measured and simulated surface temperatures during the performed drying experiments showed that the surface temperature is a very sensitive parameter of the experiment. For many different measured drying cases it was not possible to follow both, the moisture content and the surface temperature when the heat and mass transfer analogy (5.28) was applied. Therefore, the first option to adjust both transfer coefficients separately appears to be more reliable when enough data is available.

Krus used the heat and mass transfer analogy for both, the adjustment of the moisture transport functions and the subsequent simulation studies. The adjusted transport function may therefore be subjected to errors as the transfer conditions may not be applied correctly. However, the overall influence on the drying experiment and its evaluation is of less importance when using the Lewis-relation since the same assumptions are used for any evaluation.

\section{Quality of material functions} used for simulations: The quality of the applied material functions is an often disputed problem whenever numerical simulations are carried out. Unfortunately, Krus did not entirely prove the ability of his adjusted and applied moisture transport function to reproduce the measured drying course correctly. Due to the chosen plots, where smoothed curves were used for the measured data, it is very difficult to estimate the actual approximation quality. In Fig. 5.18, an example of the typical deviation between measured and simulated drying curves is given. The figure was created based on those shown by Krus et al. (2007).

In addition, the conditions, under which the experiments used to adjust the transport function were carried out, are not

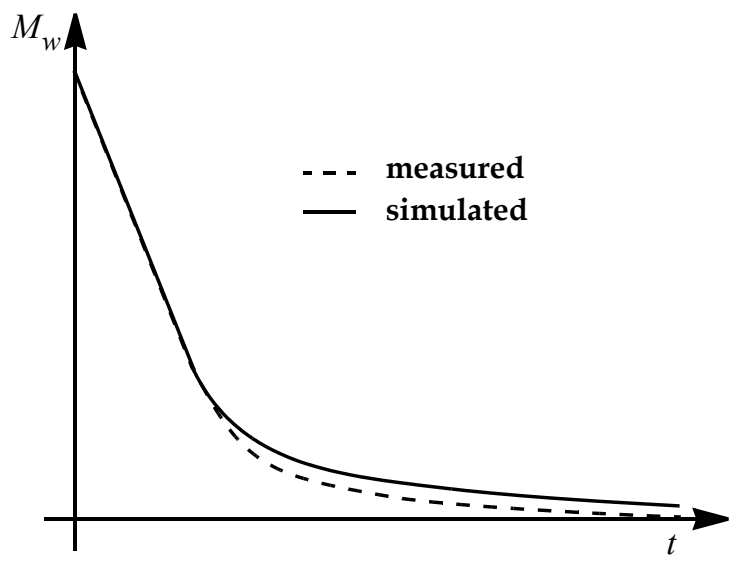

Figure 5.18: Quality illustration of the adjusted material functions used by Krus for his investigations. The figure shows a qualitative comparison as it is to be found in Krus et al. (2007). specified. This makes a reproduction most difficult. Finally, the applied material model itself is not named, but the author considers it to be the one according to Krus $\mathcal{E}$ Holm (1999), here described in paragraph 2.2.1.4 Diffusivity approach according to Krus and Holm. 
3. Lateral heat flux (one-dimensional simulations): The lateral heat flux at the drying surface, which will occur due to the surface cooling, leads to a temperature increase and, thus, increases the evaporation rate. A thermal insulation could cushion this problem partly but would add another unknown variable to the system. It is therefore recommended to use cylindric specimen which can be handled by the numerical simulation program by radial symmetry accounting for the lateral heat flux.

One-dimensional simulations do though not allow to take the lateral heat flux into account. As a consequence, the actual surface temperature will be higher in dependence on the sample diameter than for the one-dimensional case. Fig. 5.19 illustrates the influence of the lateral heat flux for samples with a diameter of $5 \mathrm{~cm}$ and $10 \mathrm{~cm}$ and for the one-dimensional case. The figure shows radial-symmetric three-dimensional simulation results carried out with the DELPHIN program (Grunewald (1997), Nicolai (2006)) for the brick introduced in Chapter 4. It is obvious that this influence is significant and needs to be taken into account. This effect is the more pronounced, the longer the first drying phase of the material lasts. As the first drying phase is not only dependent on the moisture transport properties of the material but also on the sample height, the influence of lateral heat transport increases with the sample height. The results shown in Fig. 5.19 belong to specimens with a height of $10 \mathrm{~cm}$.

One-dimensional simulations, as done by Krus, disregard this influence. As shown above, this significantly affects the duration of the first drying phase. When comparing the end of the first drying phase of one material with different sample heights, this might be of less importance since the same error occurs for all calculations. For a comparison with measured drying data though, as either done for an experimental proof of the proposed coefficient or done during the approximation of the material functions, the influence has to be taken into account when further results are not to be subjected to errors. According to Bednar (2002), a thermal insulation of the material specimen during the drying experiment reduces this influence and is hence to be preferred in case no radial symmetric simulation is possible. Generally though, a thermal insulation introduces another unknown variable to the system.

4. Initial moisture content: The initial moisture content plays an important role for the drying time, as can be seen in Fig. 5.20 for the building brick introduced in Chapter 4 . The author recommends to start the drying experiment always at effective saturation, since only in this case different materials can be compared. Starting an experiment at capillary saturation is seen problematic, see also the discussion in para- 
graph 3.1.5 Water uptake experiment and in section Capillary moisture content range of paragraph 5.1.2.1 Moisture content regions. Capillary saturation is hardly adjustable. As it marks a mean moisture content at the moment where the moisture front reaches the top of the specimen during a water uptake experiment, an equal moisture distribution will not be obtained. Moreover, for materials which do not strictly follow the squareroot of time behaviour, the capillary moisture content itself is hardly being defined. It thus can be concluded, that the initial moisture content of the drying experiment is very important for two reasons, the drying time and the reproducibility of the experiment.

The duration of the first drying phase is the proposed criterion for the drying coefficient. For a numerical simulation study on the drying behaviour, any clearly defined moisture content would be suitable. However, when both, simulation and experimental results should be applicable and - above that - experimental proof is required, the same initial conditions should be adjustable for both, simulation and experiment. This is not the case for the capillary saturation chosen by Krus. The results on which he based his drying coefficient definition are hence neither verified experimentally nor are they meaningful by means of a

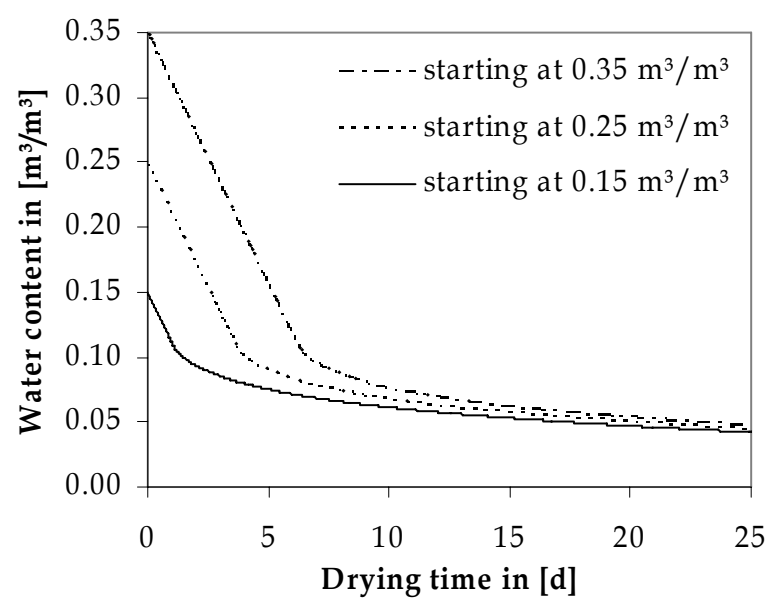

Figure 5.20: Drying curves of the building brick starting from different initial moisture contents. real material property since the capillary saturation is a more or less arbitrary moisture content.

5. Investigated sample thickness: The sample thicknesses which were simulated appear to be another aspect for which the drawn conclusions of Krus may not be generally valid. As mentioned above, he applied sample heights between $7 \mathrm{~cm}$ and $50 \mathrm{~cm}$ for his simulations. For experimental investigations, typical sample heights lie between $1 \mathrm{~cm}$ and $10 \mathrm{~cm}$ depending on the material and its properties. Performing a drying experiment for a calcium silicate, it is reasonable to use sample heights of up to $10 \mathrm{~cm}$. Due to the high capillary conductivity, such high specimen would reveal more information about the material properties whereas small specimens would only show a very short second drying phase. However, for a concrete having a very low capillary conductivity, such high specimens would require many months to dry which is not acceptable for a normal material investigation. Sample heights of up to $50 \mathrm{~cm}$ are therefore not useable at all. Moreover, for many building materials, such heights are not even available. A material parameter study based on numerical simulation should thus be related to the laboratory practise.

\section{Reasons for the disclosed differences}

Since the influences of the lateral heat flux, the sample diameter and the vapour transfer conditions need to be taken into account when comparing measured and simulated drying curves, but do not change the general material behaviour, these aspects may be 
excluded from the list of possible reasons for the occurred two different dependencies of the first drying phase duration on the sample height. This leaves the applied material functions, the initial moisture content and the sample height itself on the list.

The approximation quality of the material functions is crucial for any calculation where the results need to be correct. The general material behaviour though does not change when the quality is not very high. Simulations with a stepwise increased or decreased liquid water conductivity (for both, the whole function or only for the lower moisture content range) yield different results but do still show a linear dependence between first drying phase duration and sample height. Therefore, the approximation quality may also be excluded from the list of possible reasons.

The end of the first drying phase is characterized by the deviation of the drying rate from an almost linear behaviour. This is due to the fact that at a certain moment the moisture transport properties of the material are not anymore able to conduct as much moisture to the surface as could evaporate there due to the transfer conditions. Inside the material, the end of the first drying phase coincides with the formation of a distinct moisture profile. While a developing moisture profile is quickly equilibrated during the first drying phase due to the moisture transport properties, this is not the case within the second drying phase anymore.

This leads to the question, whether the calculated moisture profiles do influence the results or not. Since the model applied by Krus is based on the diffusion equation (2.16), whereas the model applied here uses the Richards-equation (2.15) with (2.2), the calculated moisture profiles may be different depending on the moisture storage function. A comparison of moisture profiles calculated with both approaches for the drying of the aerated concrete introduced in Chapter 4 is given in Fig. 5.21 showing significant differences. However, although also the corresponding drying curves are slightly different, the general dependency is the same for both approaches. Different transport equations therefore yield different moisture profiles but do not explain the different obtained relations of first drying phase duration and sample height.

Finally, the initial moisture content and the chosen height of the material specimens may be responsible for the observed differences. Further simulations were performed
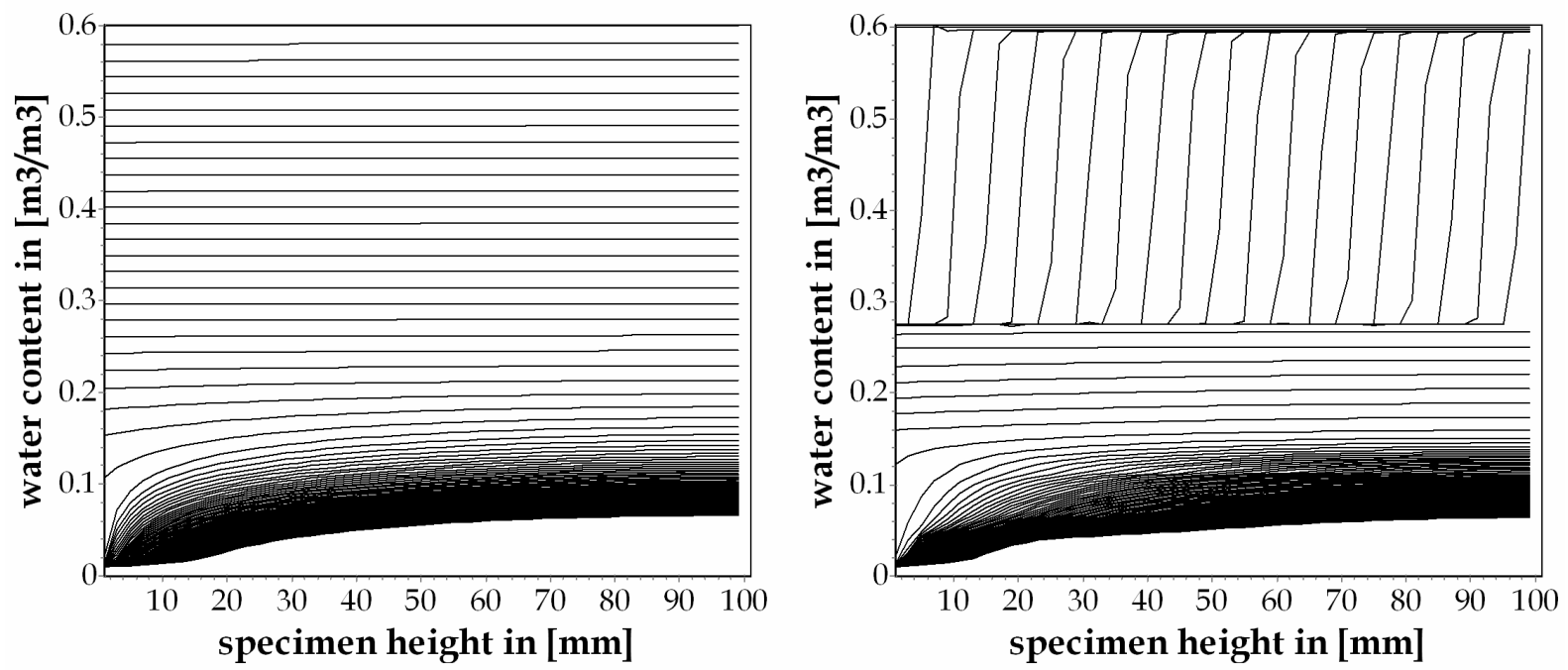

Figure 5.21: Moisture profiles of aerated concrete during a simulated drying process using the diffusion equation (left) and the Richards-equation (right). 

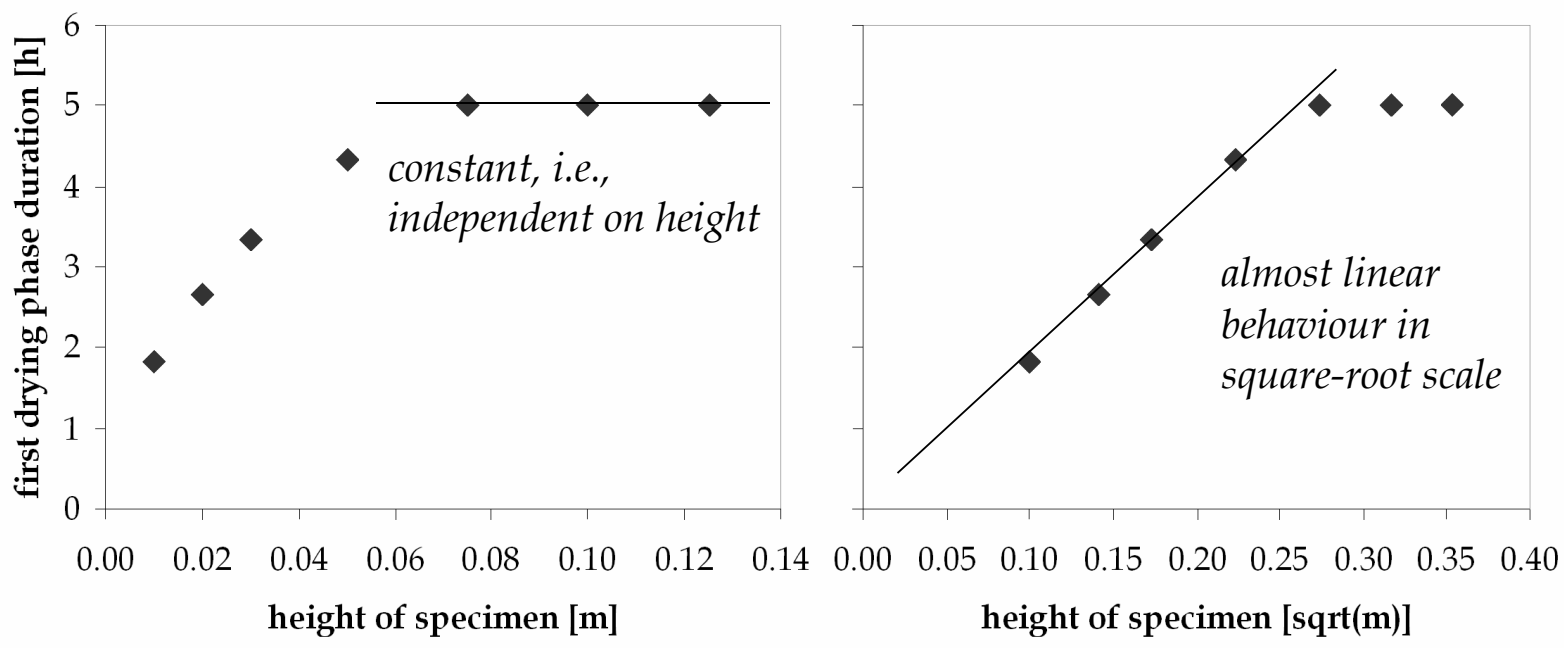

Figure 5.22: Calculated first drying phase duration of aerated concrete versus specimen height in normal (left) and square-root scale (right). The simulations were started at a moisture content of $0.15 \mathrm{~m}^{3} / \mathrm{m}^{3}$.

investigating different initial moisture contents and different sample heights for the four building materials. For the aerated concrete with an initial moisture content of $\theta_{\text {init }}=0.15 \mathrm{~m}^{3} / \mathrm{m}^{3}$, the results are displayed in Fig. 5.22. Under these conditions, the first drying phase duration deviates from a linear dependence on the sample height. For specimens higher than a certain level, the first drying phase becomes even independent on the height. When starting with an increased moisture content, e.g. with $\theta_{\text {init }}=0.18 \mathrm{~m}^{3} / \mathrm{m}^{3}$, this kind of behaviour moves to increased sample heights whereas for small heights a linear dependency appears.

With these findings, the square-root dependence of the first drying phase duration, which was observed by Krus, can be explained with the development of a distinct moisture profile inside the material and with the initial conditions as well as the sample heights that were chosen for the calculations. The moisture propagation inside a
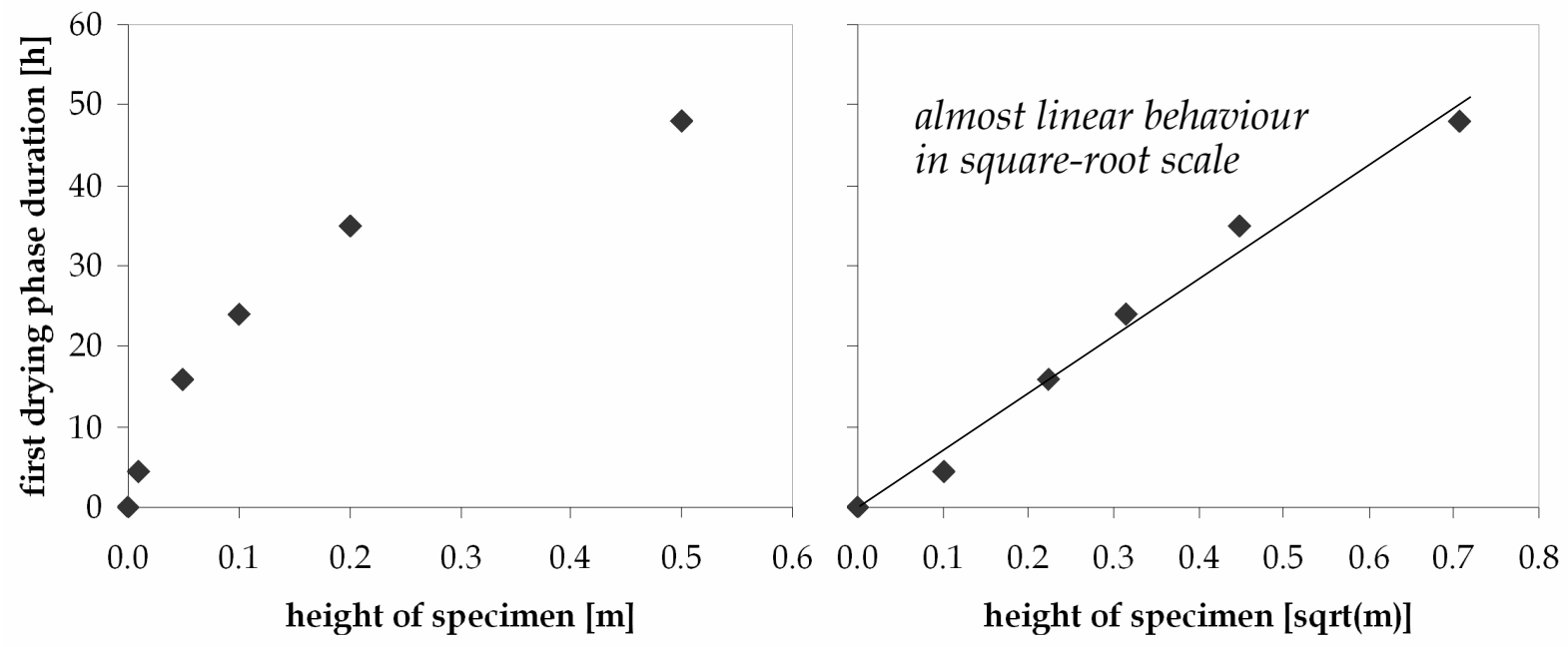

Figure 5.23: Calculated first drying phase duration of aerated concrete versus specimen height in normal (left) and square-root scale (right). The simulations were started at a moisture content of $0.22 \mathrm{~m}^{3} / \mathrm{m}^{3}$. 
material is dependent on the square-root of time as normally witnessed during a water absorption experiment. The development of a moisture profile may hence also depend on the square-root of time. In the beginning of a drying process, no distinct moisture profile develops until a certain level of moisture content and coinciding moisture transport properties is reached. Then, a distinct moisture profile forms. When the initial conditions of a drying process are close to those at which the moisture profile forms, the duration of the first drying phase is mainly influenced by the development of the moisture profile. Slightly higher initial moisture contents extent the first drying phase duration in dependence to the sample height. The material behaviour observed from the simulations performed by Krus are therefore seen as a combination of somewhat higher initial moisture contents compared with the one at which the moisture profile develops and the selection of sample heights. Fig. 5.23 strengthens this hypothesis where simulation results are shown for the aerated concrete starting at an initial moisture content of $\theta_{\text {init }}=0.22 \mathrm{~m}^{3} / \mathrm{m}^{3}$ and for extended sample heights. The quality of the linear dependence shown in the right figure corresponds with the one published by Krus et al. (2007).

\section{Analytic solution of the drying problem}

For additional proof, the drying problem was solved analytically by the help of the diffusion equation. Depending on the initial and the boundary conditions, there exists a large number of analytic solutions for the differential equation for heat transport, and due to the analogy with the diffusion, also for the diffusion equation. This equation, $a$ combination of Fick's first law (2.8) and the mass conservation balance equation (2.2), can be written according to eq. (5.29) implying that $D_{l}$ is independent of $x$.

$$
\frac{\partial \theta_{l}}{\partial t}=D_{l} \cdot \frac{\partial^{2} \theta_{l}}{\partial x^{2}}
$$

It is assumed that the water diffusivity of the material is $D_{l}=$ const, i.e., independent of $x$ and $\theta_{l}$, and that the material forms a plate of unlimited extension except for its thickness in direction of $x$. The thickness is $2 L$ where $x=0$ in the middle of the plate. The plate has the initial moisture content of $\theta_{l}(t=0, x)=\theta_{0}$ and the constant boundary condition of $j_{l}(t>0, x= \pm L)=j_{\text {trans }}$. The whole configuration is shown in Fig. 5.24.

According to Tautz (1971), Baehr E Stephan (2003) and Häupl (2007a), the following solutions of eq. (5.29) can be found: eq. (5.30) for small times and eq. (5.31) for large times.

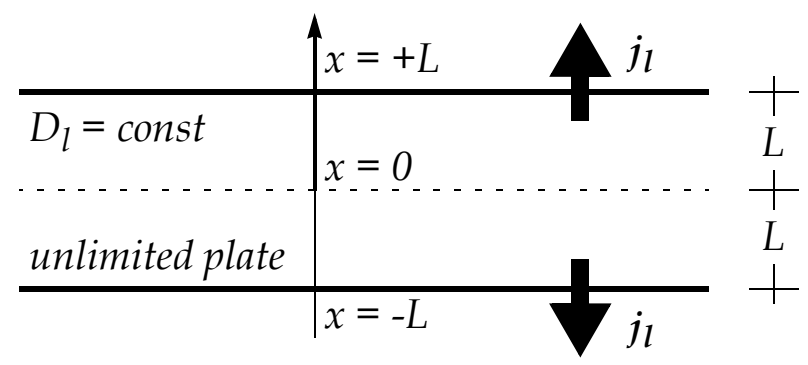

initial condition:

$\theta_{l}(t=0, x)=\theta_{0}$

boundary condition:

$j_{l}(t>0, x= \pm L)=j_{\text {trans }}$

Figure 5.24: System configuration for the analytic solution of the drying problem. 


$$
\begin{aligned}
& \theta_{l}(t, x)= \\
& \theta_{0}-2\left(\left(\frac{j_{\text {trans }}}{D_{l} \cdot \rho_{l}} \cdot \sqrt{D_{l} \cdot t}\right) \cdot \sum_{n=1}^{\infty}\left(F\left(\frac{(2 n+1) \cdot L+x}{\left(2 \cdot \sqrt{D_{l} \cdot t}\right)}\right)+F\left(\frac{(2 n+1) \cdot L-x}{\left(2 \cdot \sqrt{D_{l} \cdot t}\right)}\right)\right)\right)
\end{aligned}
$$

$$
\text { with } F(\alpha)=-\alpha \cdot \operatorname{erfc}(\alpha)+\frac{1}{\sqrt{\pi}} \cdot \exp \left(-\alpha^{2}\right)
$$

$$
\begin{aligned}
& \theta_{l}(t, x)=\theta_{0}-1\left(( \frac { j _ { \text { trans } } } { D _ { l } \cdot \rho _ { l } } \cdot L ) \cdot \left[\left(\frac{D_{l} \cdot t}{L^{2}}+\frac{x^{2}}{2 L^{2}}-\frac{1}{6}\right)\right.\right. \\
& \left.\left.+\left(\frac{-2}{\pi^{2}} \cdot \sum_{n=1}^{\infty}\left(\frac{(-1)^{n}}{n^{2}} \cdot \cos \left(n \pi \frac{x}{L}\right) \cdot \exp \left(-n^{2} \cdot \pi^{2} \cdot \frac{D_{l} \cdot t}{L^{2}}\right)\right)\right)\right]\right)
\end{aligned}
$$

For $0 \leq x \leq L$, a graphical illustration of these solutions is displayed in Fig. 5.25. For this as well as for the following evaluation of these equations, the water diffusivity was set to $D_{l}=5 \cdot 10^{-9} \cdot \mathrm{m}^{2} / \mathrm{s}$, the boundary water flux to $j_{\text {trans }}=1 \cdot 10^{-4} \cdot \mathrm{kg} /\left(\mathrm{m}^{2} \mathrm{~s}\right)$ and the initial moisture content to $\theta_{0}=1$. If one now defines a criterion for the end of the first drying phase, for instance the moment when the moisture content at the boundary becomes zero, $\theta_{l}(t, x)$ can be evaluated for varying $L$ at $x=L$ yielding the boundary moisture content in dependence of $L$.
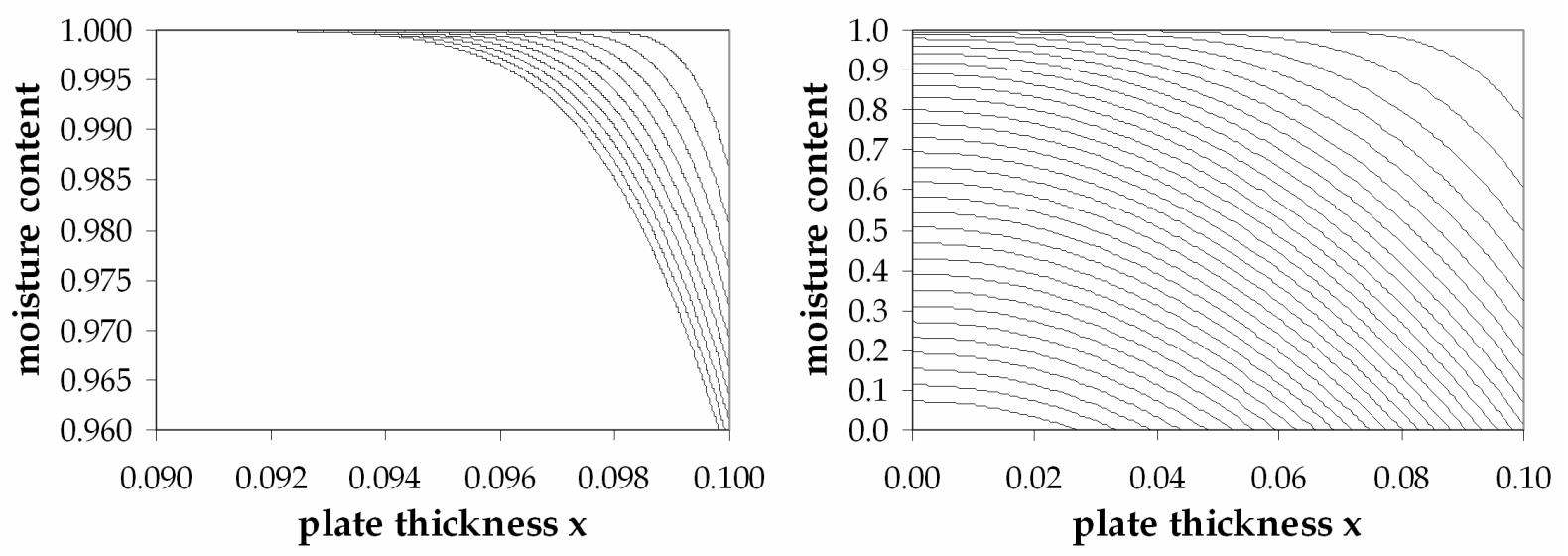

Figure 5.25: Analytic solution of the drying problem with a constant boundary water flux according to eq. (5.30) for small times (left) and eq. (5.31) for large times (right). 
For values of $5 \mathrm{~mm} \leq L \leq 80 \mathrm{~cm}$, the moisture content at $x=L$ versus time is shown in Fig. 5.26 at the left hand side. A linear increase in $L$ was chosen for this plot. With increasing $L$, the difference in the moisture content at the edge of the plate is decreasing until a certain threshold is reached, where the moisture content at the boundary becomes independent of the sample height. The right chart in Fig. 5.26 shows this even more pronounced. There, the time when the moisture content at the boundary equals zero was plotted versus the specimen height (data points). For small heights, a linear dependence is observed. With increasing heights, the dependence becomes smaller and vanishes completely for heights above a certain threshold. This threshold is dependent on the material properties as well as on the initial and boundary conditions. The continuous line in this chart is a linear combination of the $F(\alpha)$ function given in eq. (5.30) which is adjusted to the data points to give an idea of the functional relation for the observed behaviour.

It is obvious that the analytic solution proves the material behaviour which was already observed during previous simulations, compare Fig. 5.22 and Fig. 5.26 (right). A simple relation between the first drying phase duration and the specimen height, neither a linear, nor a square-root dependency, does thus not generally exist. According to the results presented here, the simulations on which Krus based his proposal represented only a special frame of combinations of material properties, boundary conditions and initial conditions. Within this frame, a non-linear relation is obtained. Above it, the first drying phase duration is independent of the specimen height and below it, a linear relation exists. Hence, it can be concluded, that the drying coefficient definition proposed by Krus et al. (2007) is not suitable to represent the drying behaviour of a building material and is, thus, as a drying coefficient not expedient.
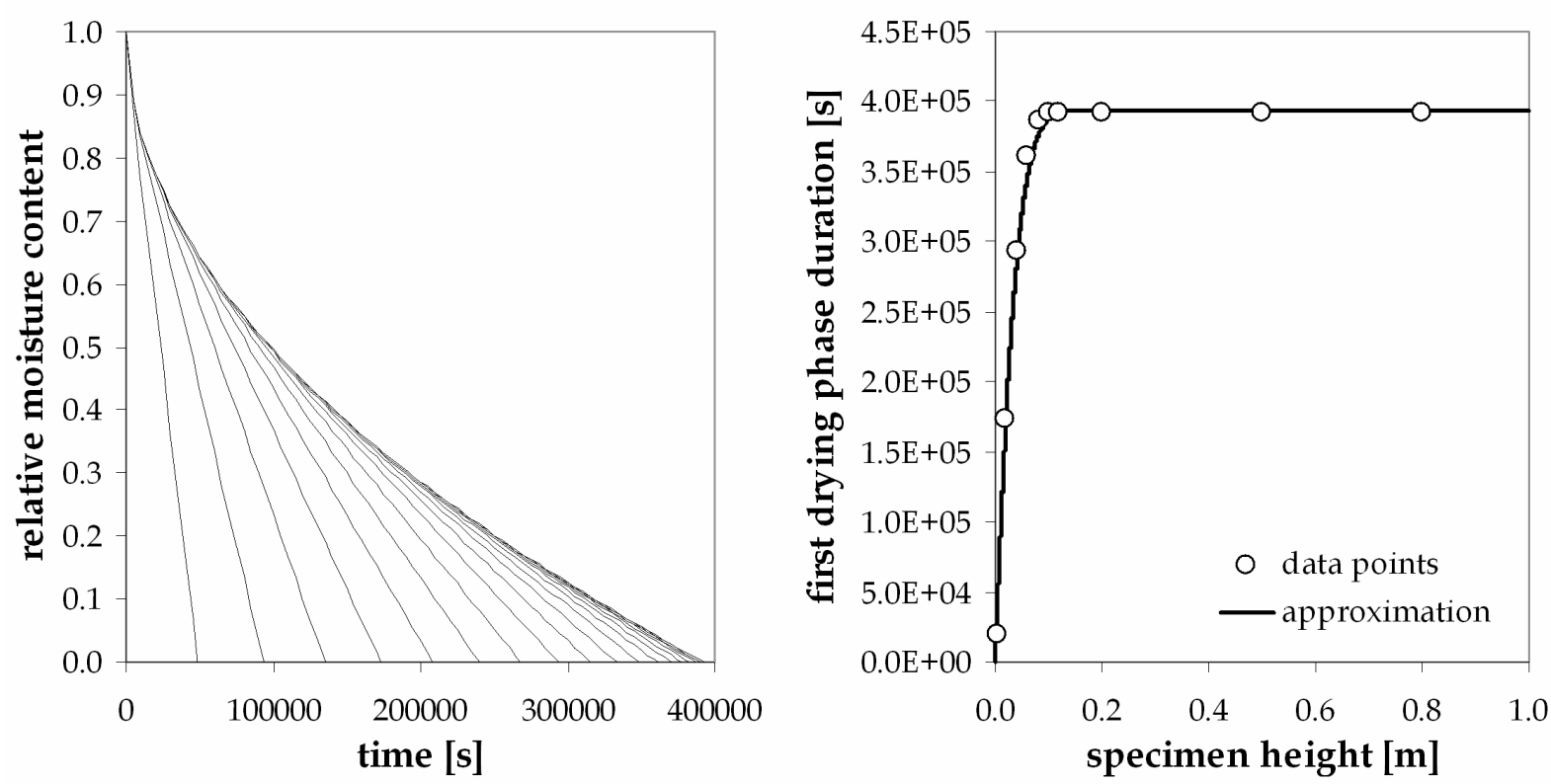

Figure 5.26: Analytic solution of the drying problem: boundary moisture content versus time for different specimen heights (left) and first drying phase duration versus specimen height (right). 


\subsubsection{Concluding the drying coefficient}

Two ways to standardize drying data are presented. The first is an attempt to describe the drying course by an analytic expression as a combination of a linear and an exponential function. The flexibility of such an expression is though not high enough to represent the measured drying data adequately. Therefore, the attempt is abandoned. The second way is the definition of a single value drying coefficient proposed by Krus et al. (2007) as the slope of the assumed square-root dependence of first drying phase duration versus specimen height.

The parameters influencing the drying process are investigated by a comprehensive simulation study. On that bases, the influences are evaluated for their consideration during drying measurement and simulation, which is summarized in Tab. 5.1. Subsequently, it is proven by experimental data, by numerical simulation and by an analytic solution of the diffusion equation for the drying problem, that a distinct or unique relation between the sample height and the first drying phase duration does not exist. It can be concluded, that the drying coefficient based on the definition of Krus et al. (2007) is not expedient.

Table 5.1: Parameters influencing the drying process.

\begin{tabular}{|c|c|c|}
\hline Parameter & Influence & Significance \\
\hline $\begin{array}{l}\text { Material properties, } \\
\text { especially the water } \\
\text { transport function }\end{array}$ & $\begin{array}{l}\text { strong influence on the drying, } \\
\text { responsible for the first drying phase } \\
\text { duration as well as for the drying } \\
\text { course of the second drying phase }\end{array}$ & $\begin{array}{l}\text { very significant, the actual reason } \\
\text { why drying experiments are per- } \\
\text { formed }\end{array}$ \\
\hline $\begin{array}{l}\text { Boundary tempera- } \\
\text { ture and relative } \\
\text { humidity }\end{array}$ & $\begin{array}{l}\text { influence on the vapour pressure dif- } \\
\text { ference and hence on the evaporation } \\
\text { rate }\end{array}$ & $\begin{array}{l}\text { significant during the first drying } \\
\text { phase, important parameter of the } \\
\text { drying process which needs to be } \\
\text { monitored }\end{array}$ \\
\hline Surface temperature & $\begin{array}{l}\text { determines the vapour pressure at the } \\
\text { surface and by this the evaporation } \\
\text { rate, } \\
\text { very sensitive parameter of the dry- } \\
\text { ing process }\end{array}$ & $\begin{array}{l}\text { When measured, the surface tempera- } \\
\text { ture can be used to calculate the } \\
\text { vapour transfer coefficient and to } \\
\text { determine the end of the first drying } \\
\text { phase }\end{array}$ \\
\hline $\begin{array}{l}\text { Air flow velocity } \\
\text { (vapour transfer } \\
\text { conditions) }\end{array}$ & $\begin{array}{l}\text { influence on the evaporation rate, } \\
\text { limiting factor during the first drying } \\
\text { phase }\end{array}$ & $\begin{array}{l}\text { very important parameter of the first } \\
\text { drying phase, should be kept con- } \\
\text { stant, can be determined indirectly }\end{array}$ \\
\hline $\begin{array}{l}\text { Initial moisture } \\
\text { content }\end{array}$ & $\begin{array}{l}\text { influences the duration of the whole } \\
\text { drying process }\end{array}$ & $\begin{array}{l}\text { very important parameter, especially } \\
\text { concerning reproducibility and com- } \\
\text { parability, should be always adjusted } \\
\text { to effective saturation. }\end{array}$ \\
\hline $\begin{array}{l}\text { Sample diameter / } \\
\text { lateral heat flux }\end{array}$ & $\begin{array}{l}\text { influences the surface temperature } \\
\text { and by this the evaporation rate }\end{array}$ & $\begin{array}{l}\text { Important when comparing measured } \\
\text { and simulated material behaviour, } \\
\text { should be therefore consistent, is in } \\
\text { general of less significance }\end{array}$ \\
\hline Sample height & $\begin{array}{l}\text { influences the duration of the drying } \\
\text { process }\end{array}$ & $\begin{array}{l}\text { very important parameter of the dry- } \\
\text { ing process }\end{array}$ \\
\hline
\end{tabular}


Still, the general attempt to define a single value drying parameter is considered to be important for a broad acceptance, especially in building practise. Therefore, further approaches should be developed on the basis of the recent results. Due to the numerous dependencies, the idea to use the numerical simulation for relating the drying coefficient to standard conditions is seen as the most meaningful opportunity. The influence of the boundary conditions, the transfer conditions and the sample diameter can be eliminated leaving only the sample height, the initial moisture content and the material properties itself to be combined within one parameter. The author's proposal on such a drying parameter is, hence, to relate the duration of the whole drying process to the sample height and the open porosity or effective saturation. However, this is not tested, yet, and requires further research studies.

\subsubsection{Conclusions on the drying experiment}

The drying process is influenced by the liquid transport properties of a material within the lower overhygroscopic moisture range. The drying experiment is thus very suitable to gain information about these material properties. For that reason, the drying process is introduced and examined with regard to its governing influences. In particular, the drying process is strongly influenced by the boundary and the transfer conditions which have to be monitored in order to correctly evaluate and interpret the drying data. Based on this examination, an apparatus has been developed, designed and built to measure the drying behaviour of building materials under defined and reproducible conditions.

In a second step, recent attempts to standardize drying data are evaluated and discussed. Special emphasis is placed on the definition of a single value drying coefficient which should be able to give a general measure of the material's drying behaviour. The definition found in literature which relates the first drying phase duration to the sample height can not be approved by own experimental as well as simulation studies. Subsequent investigations indicate that such a general dependency does not exist which is finally proved by an analytic solution of the drying problem. The proposed definition is considered to be not expedient. An overview on the influences on the drying experiment is given and further ideas on a single value drying coefficient are drawn leaving their investigation to be subject of further research. 


\subsection{Instantaneous profile measurements}

Hygrothermal material model validation requires data from a series of experiments which are not used as input to the model. For that reason, instantaneous profile measurements are performed for the four building materials introduced in Chapter 4. For both, the hygroscopic and the overhygroscopic moisture content range, a special measurement procedure is developed. Starting from the principle set-up according to Plagge (1991) and Plagge et al. (2006a), special devices are designed to apply the different boundary conditions in a defined way. Within both moisture content ranges, a series of ad- and desorption cycles is performed. The particular chronology of applied boundary conditions is established by the help of a simulation study.

The following two paragraphs will introduce these particular set-ups including the measurement schedule for both moisture content ranges, respectively. The subsequent paragraph is dedicated to the evaluation of TDR data. Since the output quantity of a TDR measurement is the apparent dielectric constant versus time, these data have to be recalculated into moisture contents. Different available approaches will be introduced and evaluated with respect to their input data requirements and their accuracy. Finally, conclusions on the instantaneous profile measurements will be drawn.

\subsubsection{Measurement set-up for the hygroscopic moisture range}

For the hygroscopic moisture content range, time domain reflectometry (TDR) sensors are applied to measure the moisture content whereas the moisture potential is measured with coupled temperature - relative humidity sensors. A brief introduction of these sensors is given in paragraph 3.2.2 Sensor technology.

The material samples with a length and a height of $10 \mathrm{~cm}$ each and a depth of $5 \mathrm{~cm}$ are laterally sealed with epoxy and perspex plates. Into the plates, holes are drilled and threads are cut for a precise sensor installation and fixation. The top of the specimens is sealed with an adhesive aluminium foil whereas the bottom side is left open for the boundary conditions. Since air pressure build-up significantly influences the water transport behaviour of a building material, as proven e.g. by Descamps (1997), the aluminium foil is perforated with a needle in order to maintain a pressure equilibration with the environment. The prepared and preconditioned material specimens are placed on specially designed containers for the exposure to the boundary conditions. These containers have an opening at the top side with the same dimensions as the material samples, on which these are sealed. Inside the container, a vessel with varying aqueous salt solutions is placed to adjust constant boundary conditions. The containers have an opening at the front side, through which the vessel can be removed for monitoring the absorbed or released moisture mass or for a change in boundary condition. A small outlet at the top side enables the installation of a temperature - relative humidity sensor inside the container to monitor these applied conditions. Fig. 5.28 shows a picture of the measurement set-up for the hygroscopic moisture content range as it is constructed. 


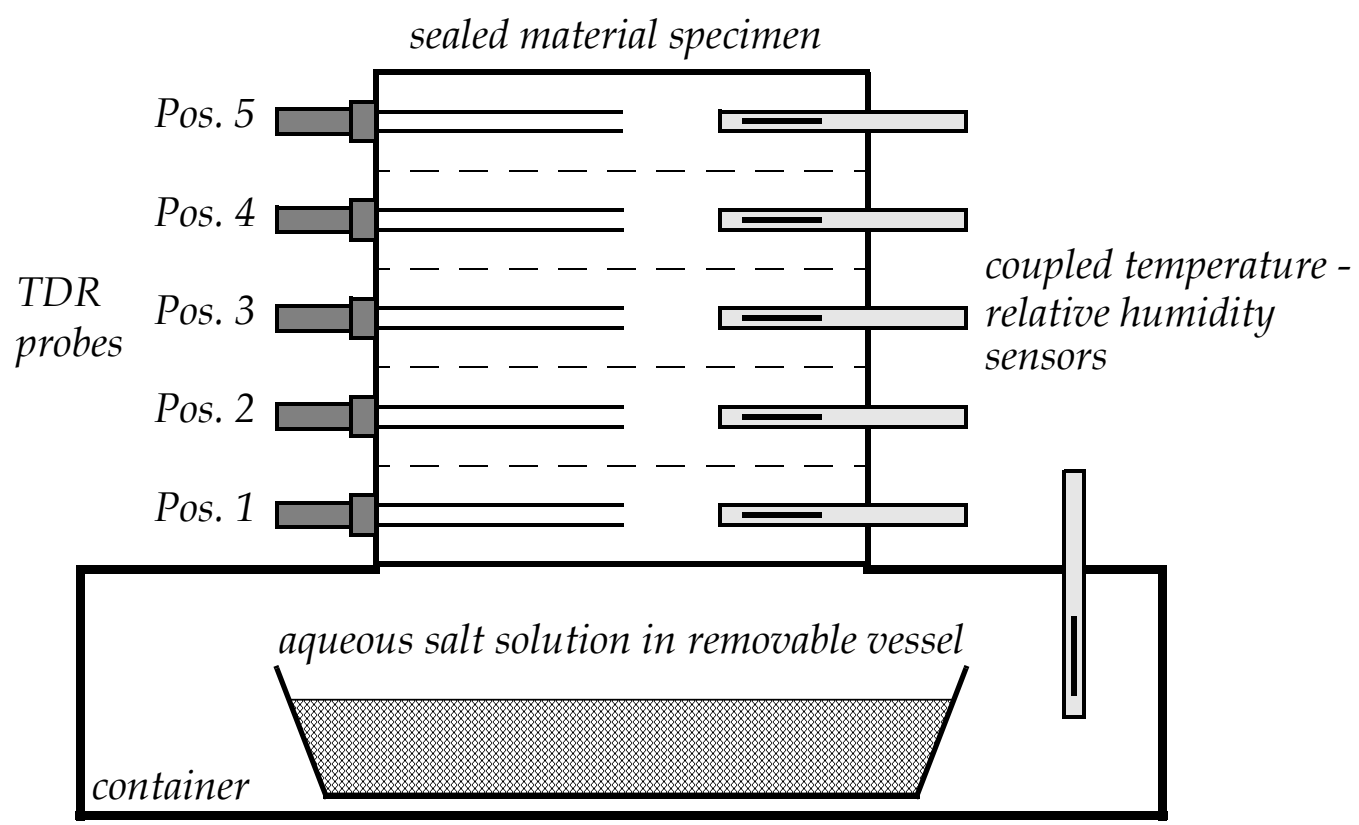

Figure 5.27: IPM set-up for the hygroscopic moisture content range.

Table 5.2: Schedule of boundary conditions for the instantaneous profile measurement experiments within the hygroscopic moisture content range.

\begin{tabular}{lccc}
\hline \multicolumn{1}{c}{ Measurement step } & Duration & Relative humidity & Salt-solution \\
\hline initial equilibration & $14 d$ & $32.9 \%$ & $\mathrm{MgCl}_{2}$ \\
\hline stepwise increase (1) & $14 d$ & $58.2 \%$ & $\mathrm{NaBr}$ \\
stepwise increase (2) & $14 d$ & $84.7 \%$ & $\mathrm{KCl}$ \\
stepwise increase (3) & $14 d$ & $97.4 \%$ & $\mathrm{~K}_{2} \mathrm{SO}_{4}$ \\
\hline stepwise decrease (1) & $14 d$ & $84.7 \%$ & $\mathrm{KCl}$ \\
stepwise decrease (2) & $14 d$ & $58.2 \%$ & $\mathrm{NaBr}$ \\
stepwise decrease (3) & $21 d$ & $32.9 \%$ & $\mathrm{MgCl}_{2}$ \\
\hline large step increase & $35 d$ & $97.4 \%$ & $\mathrm{~K}_{2} \mathrm{SO}_{4}$ \\
\hline large step decrease & $35 d$ & $32.9 \%$ & $\mathrm{MgCl}_{2}$ \\
\hline
\end{tabular}

The sealed samples are preconditioned under laboratory conditions, i.e., at $23^{\circ} \mathrm{C}$ and $50 \%$ relative humidity for a duration of several weeks. After the installation, the containers with the material samples are placed inside a climatic chamber to guarantee a constant temperature of $20^{\circ} \mathrm{C}$ for the whole experiment. The measurement schedule is designed in two cycles of adsorption and desorption processes scanning the whole hygroscopic moisture content range. The schedule is shown in Tab. 5.2. For the hygroscopic moisture range, it is decided to have only one measurement schedule for all investigated materials. Measurement values were taken every 15 minutes for the temperature - relative humidity sensors and every 30 minutes for the TDR sensors. 


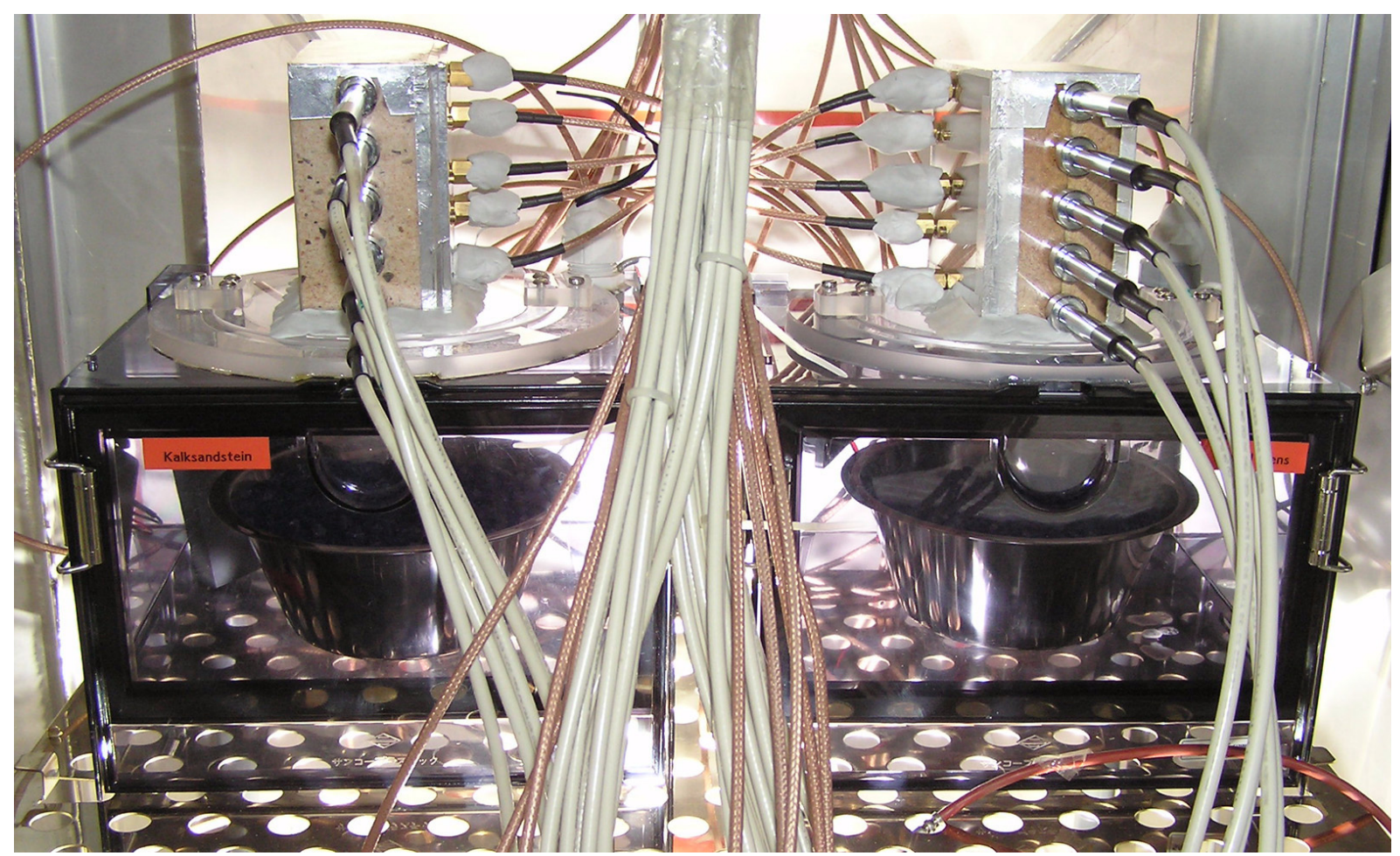

Figure 5.28: Picture of the IPM set-up for the hygroscopic moisture content range.

\subsubsection{Measurement set-up for the overhygroscopic moisture range}

The installed TDR sensors are also used to measure the moisture content within the overhygroscopic range. The moisture potential can not be measured by relative humidity sensors in this moisture range. Two general options are possible: miniaturized tensiometers or psychrometers, as introduced in paragraph 3.2.2 Sensor technology. Tensiometers can only be used for capillary pressure measurement in a range of 0 to $0.095 \mathrm{MPa}$. A measurement cycle scanning the whole moisture content range from dry to wet and back is hence not possible with tensiometers. And since psychrometer sensors are not available for this study, the overhygroscopic experiments are conducted with the installed TDR sensors, only. Values were generally measured with an interval of 30 minutes.

The planned measurement procedure consists of three types of boundary conditions which are applied to the open surface of the material specimens. These are:

- no-pressure water contact - water uptake experiment,

- vapour transfer - drying experiment,

- no flow - distribution. 

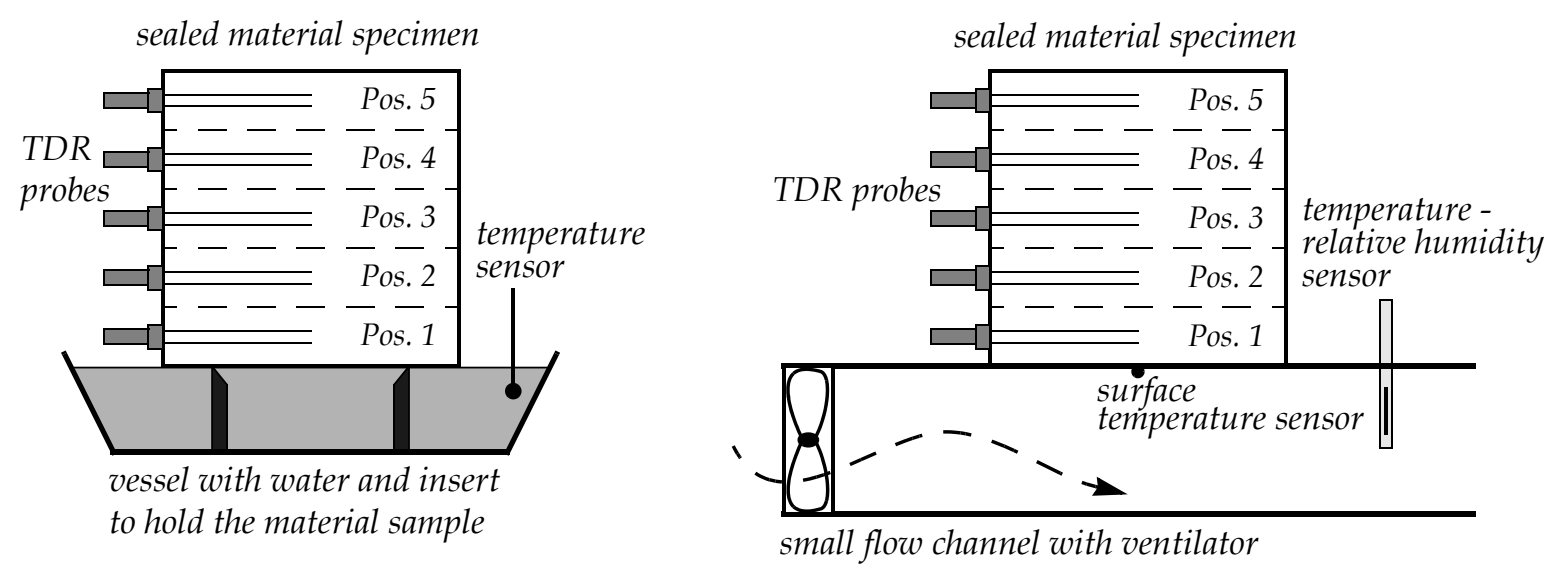

Figure 5.29: Designed set-up for water uptake (left) and drying (right) for the instantaneous profile measurements within the overhygroscopic moisture content range.

For the water contact and the vapour transfer boundary conditions, corresponding setups are designed according to Fig. 5.29. For the water contact, this is a water-filled vessel with a special insert on which the material sample can be placed. The water level is adjusted according to the height of the insert enabling an almost no-pressure boundary condition, and the water temperature is measured. For the vapour transfer boundary condition, a small and simplified drying device has been developed. It consists of a small flow channel with the same width as the material specimens (i.e., $10 \mathrm{~cm}$ ). The inflow is controlled with two ventilators. The boundary conditions of such a drying is measured with a coupled temperature and relative humidity sensor. For the surface temperature measurement, a small temperature probe is attached to the evaporation surface. In Fig. 5.30, two pictures of these set-ups are shown. The no-flow boundary condition is provided by sealing the open side of the specimens with an adhesive aluminium foil. For the next measurement step, this foil is removed again.

The vapour transfer coefficient has been determined in advance. For that purpose, saturated sample duplicates are dried with this equipment for each material. The air flow velocity is adjusted, boundary temperature, boundary relative humidity and surface temperature are measured and the gravimetrically derived vapour transfer flow can be related to these conditions according to eq. (5.25). For the actual measurements, the
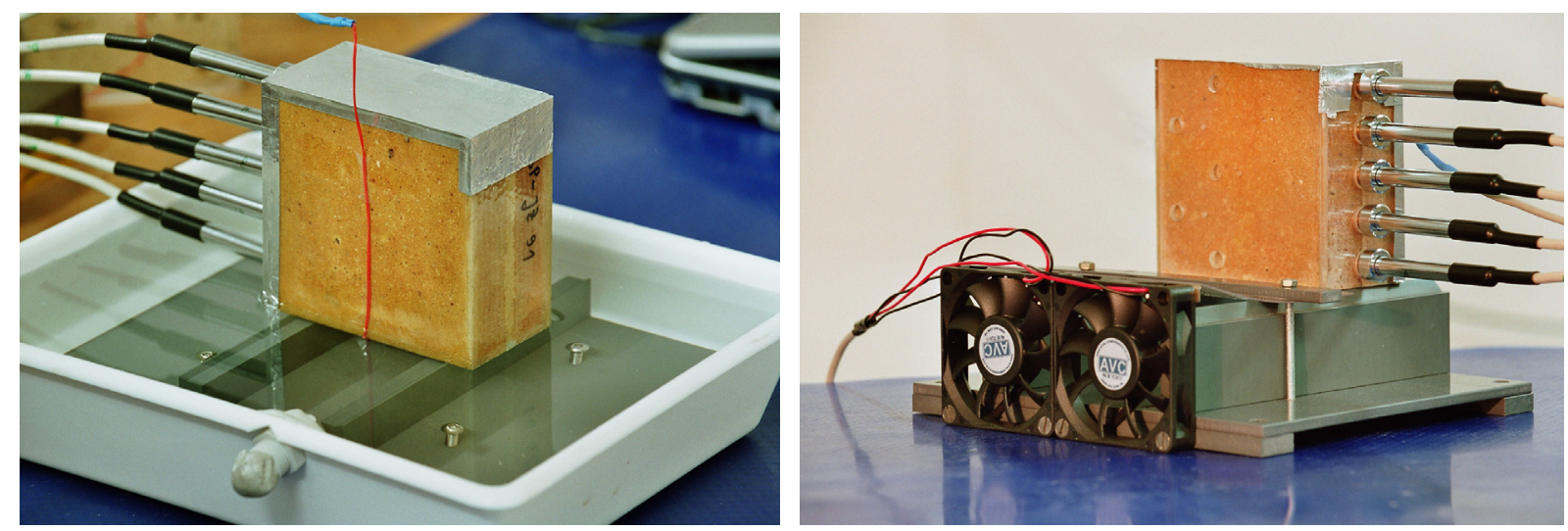

Figure 5.30: Pictures of the developed IPM set-up for the overhygroscopic moisture range: water uptake (left) and drying (right). 
same air flow velocity is adjusted providing approximately the same vapour transfer conditions as for the tested cases.

Table 5.3: Boundary condition schedule for the overhygroscopic IPM experiments, brick and calcium silicate at the left and aerated concrete and lime-sand brick at the right.

\begin{tabular}{lc}
\hline \multicolumn{1}{c}{ Boundary condition } & Duration \\
\hline water contact - water uptake & $15 \mathrm{~min}$ \\
no-flow - distribution & $7 \mathrm{~d}$ \\
vapour transfer - drying & $7 \mathrm{~d}$ \\
water contact - water uptake & $15 \mathrm{~min}$ \\
no-flow - distribution & $14 d$ \\
water contact - water uptake & $15 \mathrm{~min}$ \\
no-flow - distribution & $14 d$ \\
vapour transfer - drying & $33 \mathrm{~d}$ \\
\hline
\end{tabular}

\begin{tabular}{lc}
\hline \multicolumn{1}{c}{ Boundary condition } & Duration \\
\hline water contact - water uptake & $15 \mathrm{~h}$ \\
no-flow - distribution & $14 \mathrm{~d}$ \\
vapour transfer - drying & $21 \mathrm{~d}$ \\
water contact - water uptake & $15 \mathrm{~h}$ \\
no-flow - distribution & $14 \mathrm{~d}$ \\
water contact - water uptake & $15 \mathrm{~h}$ \\
no-flow - distribution & $27 \mathrm{~d}$ \\
vapour transfer-drying & $100 \mathrm{~d}$ \\
\hline
\end{tabular}

The measurement schedule is adjusted by the assistance of a simulation study and according to the moisture transport properties of the examined materials. In consequence, two different time schedules are developed which are listed in Tab. 5.3. As the building brick and the calcium silicate have a rather high liquid water conductivity, the scheduled times for each boundary condition are relatively short, see the left side of Tab. 5.3. The right schedule, adjusted for the aerated concrete and the lime-sand brick whose liquid water conductivities are of a lower order of magnitude, provides therefore significantly longer time intervals for each boundary condition. Before the experiments start, all specimens were preconditioned at laboratory conditions for several weeks.

\subsubsection{TDR calibration and data evaluation}

As already indicated in paragraph 3.2.2.1 Moisture content measurement, the measurement with the time domain reflectometry technology requires both, a system calibration and a special measurement data evaluation. The following section will briefly highlight the background and the procedure of TDR calibration. Subsequently, different models to derive the moisture content from the measured dielectric properties will be introduced and discussed.

\subsubsection{TDR system configuration and calibration}

The actually measured quantity of a TDR probe is the propagation time of an electromagnetic pulse. Inside a medium, part of this pulse is reflected depending on the dielectric properties of the medium. For most building materials, the dielectric properties are significantly influenced by the moisture content, which is the reason why the TDR technology can be used for its measurement. However, the reflected signal of the electromagnetic pulse is also dependent on the whole system configuration since any impedance change, e.g. at the cable connection, causes a reflection. 
It is hence important, that only that part of the reflected signal is evaluated, which belongs to the propagation within the TDR probe. As this propagation does depend on both, the system configuration and the geometry of the TDR probe, a calibration is required once the measurement configuration has been installed.

For the calibration, the measured signal is evaluated for two defined media, one with a low and one with a high dielectric constant $\varepsilon$. It is recommended to use two liquids since there an optimal contact with the TDR probe can be achieved, see Fig. 5.31. Thus, for a high dielectric constant, distilled water was chosen with $\varepsilon_{\mathrm{H}_{2} \mathrm{O}}=80.141$. For a low dielectric constant, benzene with $\varepsilon_{C_{6} H_{6}}=2.284$ was used. Both $\varepsilon$-values were given by Weast (1979) for a reference temperature of $20^{\circ} \mathrm{C}$. The calibration data, i.e., the time interval which is evaluated for the actual measurement, is stored in a data file for each probe which is read whenever the probe is called.

This briefly sketched calibration procedure ensures that the propagation time of the electromagnetic

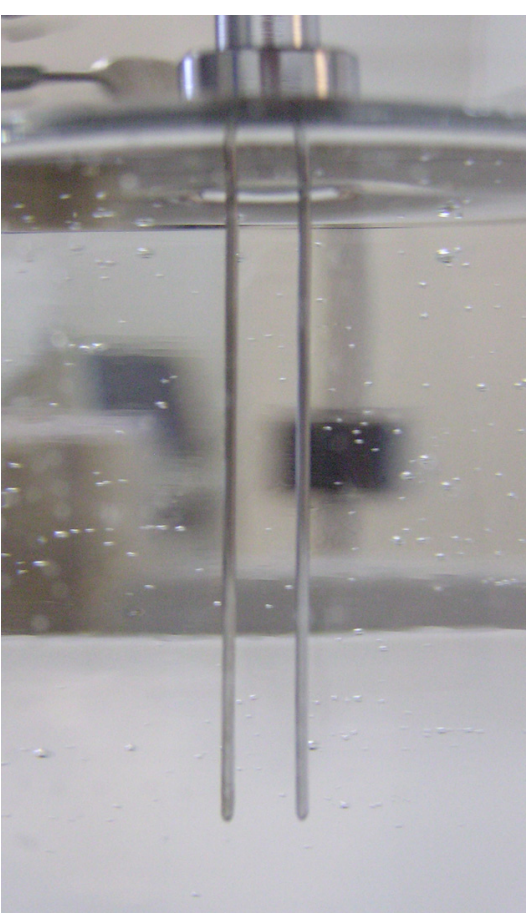

Figure 5.31: TDR calibration with distilled water. pulse is evaluated only for the length of the TDR probe enabling a precise measurement of the apparent dielectric constant $\varepsilon_{a}$ of the medium in which the probe was installed. How the moisture content can be derived from this apparent dielectric constant is subject of the next section. For a more detailed elaboration on the method of time domain reflectometry, the reader may be referred to Plagge (2003) and Sobczuk E Plagge (2007).

\subsubsection{TDR data evaluation}

The interpreted propagation time of the electromagnetic pulse delivers the actual output quantity of the TDR measurement, the apparent dielectric constant $\varepsilon_{a}$. The apparent dielectric constant comprises the dielectric properties of the solid, the liquid and the gaseous phase of the medium where the TDR probe is installed in. Due to the high dielectric constant of water, $\varepsilon_{a}$ is a distinct function of the moisture content. For the relation between moisture content and apparent dielectric constant $\theta_{l}\left(\varepsilon_{a}\right)$, a number of different approaches can be found in literature. They can be divided into two classes. The first class provides functional relationships with enough flexibility to fit the measured data points, but without a particular physical meaning. They are regarded as empirical models with a varying number of adjustable fitting parameters. Approaches of the second class are derived from dielectric mixing models, which relate the apparent dielectric constant of a medium to the volume fractions and dielectric constants of its constituents. They differ by the implied geometrical assumptions and the number of considered constituents.

For the present TDR data evaluation, one empirical, two semi-empirical and one pure dielectric mixing models were implemented. The choice was made with respect to the different input data requirements as well as to their flexibility providing the basis for 
a broad range of application cases. These models will be briefly introduced and discussed within the following paragraphs.

\section{Empirical approach}

There exists a large number of different mathematical expressions which are suitable to describe the moisture content - apparent dielectric constant relation. Plagge (2003) and Sobczuk E Plagge (2007) specify different own approaches as well as some found in literature. Frequently, these expressions are adjusted for a special type of soil or material enabling their application without a distinct knowledge of the particular material as it is often the case for field studies in soil physics.

A more general functional description which also contains the bulk density $\rho_{b}$ of the investigated material is given by Malicki et al. (1994) according to eq. (5.32). It is supposed applicable for both, mineral and organic soils and materials.

$$
\theta_{l}\left(\varepsilon_{a}\right)=\frac{\sqrt{\varepsilon_{a}}-0.819+0.168 \rho_{b}-0.159 \rho_{b}^{2}}{7.17+1.18 \rho_{b}}
$$

In case no reference data is available and the information about the investigated material is very limited, the given approach provides a simple and useful relation for the evaluation of measured TDR data. However, its flexibility is very limited as no further adjustment is possible. It is therefore recommended to apply one of the following mixed-dielectric approaches in case more input data is available.

\section{Dielectric mixing approaches}

The first model introduced here traces back to the modelling works of De Loor (1965) and De Loor (1968), who developed a mixed-component approach to describe the macroscopic dielectric behaviour of soils. He treats the solid material matrix as granules of a distinct shape, in which the other components are embedded in a random but oriented way. This approach has been further developed by Dobson et al. (1985) who assume these inclusions to be disc-shaped, yielding eq. (5.33). There, the apparent dielectric constant $\varepsilon_{a}$ is related to the dielectric constants of the solid material matrix $\varepsilon_{m}$, the gaseous phase $\varepsilon_{g}$, and the liquid phase divided in free water $\varepsilon_{l}$ and bound water $\varepsilon_{b w}$ depending on the porosity $\theta_{p o r}$ and the volume fractions of bound and total moisture $\theta_{b w}$ and $\theta_{l}$. To express the moisture content $\theta_{l}$ in dependence on $\varepsilon_{a}$, eq. (5.33) is rearranged to eq. (5.34).

$$
\varepsilon_{a}=\frac{3 \varepsilon_{m}+2\left(\theta_{l}-\theta_{b w}\right)\left(\varepsilon_{l}-\varepsilon_{m}\right)+2 \theta_{b w}\left(\varepsilon_{l}-\varepsilon_{m}\right)+2\left(\theta_{p o r}-\theta_{l}\right)\left(\varepsilon_{g}-\varepsilon_{m}\right)}{3+\left(\theta_{l}-\theta_{b w}\right)\left(\frac{\varepsilon_{m}}{\varepsilon_{l}}-1\right)+\theta_{b w}\left(\frac{\varepsilon_{m}}{\varepsilon_{b w}}-1\right)+\left(\theta_{p o r}-\theta_{l}\right)\left(\frac{\varepsilon_{m}}{\varepsilon_{b w}}-1\right)}
$$




$$
\begin{gathered}
\theta_{l}\left(\varepsilon_{a}\right)=\frac{3\left(\varepsilon_{m}-\varepsilon_{a}\right)+\theta_{b w} X_{b w}-\theta_{p o r} \cdot\left[\varepsilon_{a}\left(\frac{\varepsilon_{m}}{\varepsilon_{b w}}-1\right)-2\left(\varepsilon_{g}-\varepsilon_{m}\right)\right]}{\varepsilon_{a}\left(\frac{\varepsilon_{m}}{\varepsilon_{l}}-1\right)-2\left(\varepsilon_{l}-\varepsilon_{m}\right)-\varepsilon_{a}\left(\frac{\varepsilon_{m}}{\varepsilon_{b w}}-1\right)+2\left(\varepsilon_{g}-\varepsilon_{m}\right)} \\
\text { with } X_{b w}=\left[2\left(\varepsilon_{b w}-\varepsilon_{m}\right)-\varepsilon_{a}\left(\frac{\varepsilon_{m}}{\varepsilon_{b w}}-1\right)+\varepsilon_{a}\left(\frac{\varepsilon_{m}}{\varepsilon_{l}}-1\right)-2\left(\varepsilon_{l}-\varepsilon_{m}\right)\right]
\end{gathered}
$$

This four-component mixing model allows to express the relation of the dielectric properties in a porous medium on a physical basis. No further adjustable parameter and thus no special reference data is required. Only the porous medium itself has to be known very well since not only the dielectric constant of its solid matrix, but also the porosity and the fraction of the bound water are required.

As bound water, normally the first one or two molecular water layers are regarded, which are absorbed at the inner surfaces of the material. Since the inner binding forces are very strong, these first absorbed water layers exhibit explicitly different dielectric properties than free water. The inner surface area is therefore another required input parameter. Its determination will be elaborated within the next section subsequent to the mixed-dielectric approaches.

On the other hand, the model does not possess a high flexibility. If measured reference data on the relation of apparent dielectric constant and moisture content is available, there exists no further adjustment option. The approach is appropriate when a model with a physical basis should be applied (especially for clayey soils for which the model was derived), the porous material is well known and no further reference data is available. If reference data is available, the application of one of the following semi-empirical models is recommended due to their enlarged flexibility.

These semi-empirical dielectric mixing models are also based on the approach to relate the apparent dielectric constant of a medium to the dielectric numbers of its constituents and their corresponding volume fractions. Different assumptions about the geometrical arrangement of the constituents lead to different relations, as e.g. summarized by Tinga et al. (1973). For a layered system of two phases, Brown (1956) formulates a simple relation in dependence on the orientation within the electric field. A generalized and extended form of this relation is given by Roth et al. (1990) for a three-phase system according to eq. (5.35).

$$
\begin{gathered}
\varepsilon_{a}=\left[\theta_{l} \varepsilon_{l}^{\eta_{3}}+\left(1-\theta_{\text {por }}\right) \varepsilon_{m}^{\eta_{3}}+\left(\theta_{\text {por }}-\theta_{l}\right) \varepsilon_{g}^{\eta_{3}}\right]^{\frac{1}{\eta_{3}}} \\
\theta_{l}\left(\varepsilon_{a}\right)=\frac{\varepsilon_{a}^{\eta_{3}}-\left(1-\theta_{\text {por }}\right) \varepsilon_{m}^{\eta_{3}}-\theta_{\text {por }} \varepsilon_{g}^{\eta_{3}}}{\varepsilon_{l}^{\eta_{3}}-\varepsilon_{g}^{\eta_{3}}}
\end{gathered}
$$


The parameter $\eta_{3}$ accounts for the orientation of the layered system. Brown (1956) specifies $\eta_{3}=1$ if the electric field is parallel to the layering and $\eta_{3}=-1$ if the field is perpendicular to the layering. According to Birchak et al. (1974), $\eta_{3}=0.5$ for an isotropic medium. Dobson et al. (1985), however, who extended the approach to four phases, as given in eq. (5.37), found $\eta_{4}=0.65$ for different soil types.

$$
\begin{gathered}
\varepsilon_{a}=\left[\left(\theta_{l}-\theta_{b w}\right) \varepsilon_{l}^{\eta_{4}}+\theta_{b w} \varepsilon_{b w}^{\eta_{4}}+\left(1-\theta_{p o r}\right) \varepsilon_{m}^{\eta_{4}}+\left(\theta_{p o r}-\theta_{l}\right) \varepsilon_{g}^{\eta_{4}}\right]^{\frac{1}{\eta_{4}}} \\
\theta_{l}\left(\varepsilon_{a}\right)=\frac{\varepsilon_{a}^{\eta_{4}}+\theta_{b w} \varepsilon_{l}^{\eta_{p}}-\theta_{b w} \varepsilon_{b w}^{\eta_{4}}-\left(1-\theta_{p o r}\right) \varepsilon_{m}^{\eta_{4}}-\theta_{p o r} \varepsilon_{g}^{\eta_{4}}}{\varepsilon_{l}^{\eta_{4}}-\varepsilon_{g}^{\eta_{4}}}
\end{gathered}
$$

Therefore, $\eta$ is regarded as a fitting parameter of the semi-empirical model here. The rearrangement of both forms yields eq. (5.36) and (5.38), respectively. They have to be adjusted to measured reference data, where the given values are used as initial estimates.

The semi-empirical mixing model in the form of either eq. (5.35) or eq. (5.37) is a very flexible approach. It can be adjusted specifically to the investigated material. In case enough material information is provided and reference data for the moisture content apparent dielectric constant relation is available, this model yields the best approximation. The four-phase approach of eq. (5.37) and (5.38) is therefore applied for the evaluation of the TDR data obtained from the IPM measurements.

\section{Bound water fraction and inner surface area}

Two of the dielectric mixing models distinguish between free and bound water enabling to account for the different dielectric behaviour of water molecules which are bound to surfaces or in chemical structures. The following digression appears therefore appropriate to disclose a way how the bound water fraction can be related to the inner surface area and how this inner surface area can be determined from vapour adsorption data.

Water molecules which are not totally flexible under an external force, as e.g. an electric field, exhibit a different dielectric behaviour. They are therefore regarded as bound water. Typical examples are water molecules which are chemically bound, as it is the case during hydration processes, or the first few water molecules which are absorbed due to Van-der-Waals forces at hydrophilic material surfaces. A practical approach to account for the bound water fraction $\theta_{b w}$ during TDR data evaluation is to regard it as an adjustable number of molecular layers of water $n_{m l w}$ which are absorbed the inner material surfaces according to eq. (5.39). The inner surface area $A_{i s}$ of the material must hence be known for that purpose. The monolayer thickness of water molecules is given with $\delta_{H_{2} \mathrm{O}}=3.04 \cdot 10^{-10} \mathrm{~m}$.

$$
\theta_{b w}=n_{m l w} \cdot \delta_{H_{2} O} \cdot A_{i s}
$$


The inner surface area can be determined with nitrogen adsorption experiments, which require special equipment. However, for the present study, measured water adsorption isotherms are available. It is therefore self suggested to use this data to determine the inner surface area. Three models are found in literature which are evaluated for this purpose. One is the well-known BET method according to Brunauer et al. (1938), which has been standardized for inner surface area measurement in DIN ISO 9277. The other two methods have been developed by Adolphs (1994). They will be sketched as follows.

The principle idea is to relate the adsorbed particle number to the energy status of the material which is typically described by the relative pressure $p / p_{0}$. As the relevant binding forces, i.e., Van-der-Waals forces, decrease strongly with increasing inner surface area occupation by the adsorbate, a complete monolayer will establish before the molecules start to adsorb in a second or a third layer. This fact is used to determine the inner surface area of a material by adsorption measurements within a certain range of $p / p_{0}$.

\section{BET-method according to Brunauer et al. (1938)}

According to the BET-method, the adsorbed particle number $n_{a d s}$ follows a linear relation to the relative pressure in the range between $p / p_{0}=0.05 \ldots 0.30$.

$$
\frac{p / p_{0}}{n_{\text {ads }}\left(1-p / p_{0}\right)}=\frac{1}{n_{\text {mono }} C_{B E T}}+\frac{C_{B E T}-1}{n_{\text {mono }} C_{B E T}} \cdot p / p_{0}
$$

With a linear regression to measured adsorption data in this range, the unknown parameters $C_{B E T}$ and $n_{\text {mono }}$ of eq. (5.40) can be determined, see Fig. 5.32 at the left. Then, the inner surface area can be derived with the knowledge of the monolayer capacity $n_{\text {mono }}$ and the area requirements of an adsorbed molecule $A_{\text {mol }}$ according to eq. (5.41). There, $N_{A}=6.022 \cdot 10^{23} \mathrm{~mol}^{-1}$ is the Avogadro constant and $\rho_{\text {ads }} / \rho_{\text {mat }}$ is the density ratio of adsorbate and adsorbent agent. For water, the required area of one molecule is given with $A_{m o l, H_{2} O}=10.6 \cdot 10^{-20} \mathrm{~m}^{2}$.

$$
A_{\text {is }}=n_{\text {mono }} \cdot N_{A} \cdot A_{m o l} \cdot \frac{\rho_{a d s}}{\rho_{m a t}}
$$

\section{Gibbs free energy methods according to Adolphs (1994)}

Basis of the methods developed by Adolphs (1994) and Adolphs E Setzer (1996) is the Gibbs free energy $G=H-T S$. The finite change in free energy is related to the chemical potential $\mu$ and to the number of adsorbed particles $n_{a d s}$ according to eq. (5.42). The change in chemical potential $\Delta \mu$ is expressed in relation to the relative pressure $p / p_{0}$ following eq. (5.43) under the assumption of an ideal gas.

$$
\begin{array}{lr}
\Delta G=n_{\text {ads }} \cdot \Delta \mu & \text { change in free enthalpy } \\
\Delta \mu=R \cdot T \cdot \ln \left(p / p_{0}\right) & \text { change in chemical potential }
\end{array}
$$



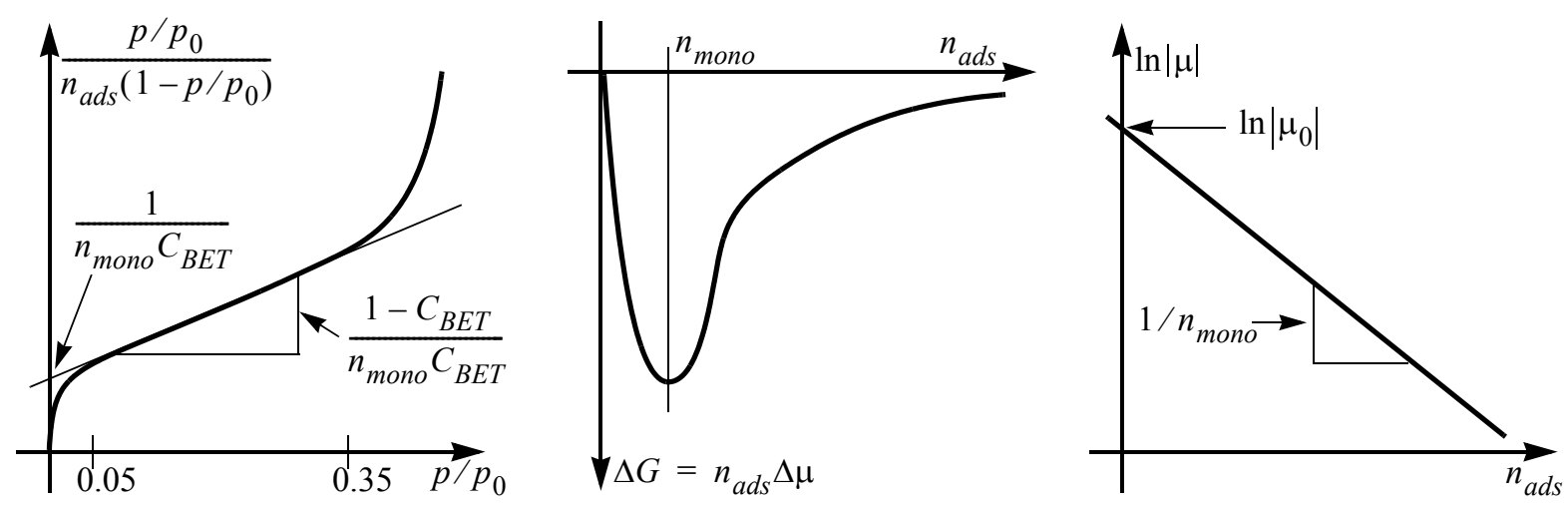

Figure 5.32: Illustration of the monolayer capacity determination according to the three introduced methods: BET method (left), GMM (middle) and GIM (right). The figures follow the illustrations of Adolphs (1994).

The change of free energy is dependent on the number of adsorbed particles as well as on the interaction between adsorbent and adsorbate. With increasing number of adsorbed particles, this interaction will increase. When the first molecular layer is completed though, the particles of a second layer will mainly interact with the particles of the first layer, and so on. The interaction with the adsorbent will hence become weaker. Since thermodynamic systems tend to reach the lowest level of free energy, the mono layer will be completed first before a second molecular layer will be adsorbed. The change in the Gibbs free energy possesses therefore a minimum at the monolayer capacity.

Adolphs uses this fact to determine the monolayer capacity. He called it Gibbs Minimum Method (GMM). According to this method, the Gibbs free energy ${ }^{1}$ is plotted versus the number of adsorbed particles. At the position where $\Delta G \rightarrow \min$, the mono layer capacity $n_{\text {mono }}$ can be determined. An illustration of this method is given in Fig. 5.32 in the middle. With the monolayer capacity, the inner surface area can be calculated again applying eq. (5.41).

If, as shown by Adolphs (1994), the monolayer capacity is to be found at the minimum of the Gibbs free energy, the total derivative of $\Delta G$ must disappear, yielding eq. (5.44). After integration and rearrangement into a linearized form, eq. (5.45) can be written as the basis if the second method which is called Gibbs integral method (GIM) by Adolphs (1994).

$$
\begin{aligned}
& \frac{1}{n_{\text {mono }}} d n_{a d s}=-\frac{1}{\Delta \mu} d \Delta \mu \\
& \ln |\Delta \mu|=-\frac{1}{n_{\text {mono }}} \cdot n_{a d s}+\ln \left|\Delta \mu_{0}\right|
\end{aligned}
$$

1. In Adolphs (1994), $\Delta G$ is called Gibbs free energy, whereas in Adolphs $\mathcal{E}$ Setzer (1996) the term excess surface work with the symbol $\Phi$ is used. Since both methods are related to the Gibbs free energy also by their name, it was stuck to this previous naming here. 
If $\ln |\Delta \mu|$ is plotted versus $n_{a d s}$ according to eq. (5.45), one can see that the monolayer capacity $n_{\text {mоnо }}$ can be determined from the reciprocal of the slope, and $\Delta \mu_{0}$ from the intersection with the ordinate, see also Fig. 5.32 at the right. $\Delta \mu_{0}$ is called the onset potential, as it is the minimum potential for adsorption processes to occur.

The main advantage of the two methods developed by Adolphs (1994) is their validity and applicability for a largely extended relative pressure range. In Adolphs $\mathcal{E}$ Setzer (1996) an example is given with almost perfect agreement in a range of $0<p / p_{0} \leq 0.95$ between measured and calculated adsorption data. The methods GMM and GIM are therefore particularly suitable for the inner surface area determination in the present case, where only limited vapour adsorption data is available and where the application of the BET-method is often not appropriate due to the limited relative pressure range.

For the TDR data evaluation, a computer program has been developed allowing to chose between the introduced models, i.e. the empirical approach according to Malicki et al. (1994), the two semi-empirical models according to Tinga et al. (1973), Birchak et al. (1974) and Roth et al. (1990) and the dielectric mixing model according to De Loor (1968) and Dobson et al. (1985). To account for the fraction of bound water exhibiting significantly different dielectric properties than free water, the introduced approaches to determine the inner surface are from measured adsorption data are also implemented. The program as well as the particular steps of data evaluation will be introduced in paragraph 6.3 Implementation of TDR data evaluation.

\subsubsection{Conclusions on instantaneous profile measurements}

For the four building materials introduced in Chapter 4, instantaneous profile measurements are performed. The developed measurement cycles consisting of a series of ad- and desorption processes, as well as the designed devices for a defined application of all scheduled boundary conditions are introduced for both, the hygroscopic and the overhygroscopic moisture content range. The moisture content is generally measured by the help of TDR probes. The moisture potential is measured by coupled relative humidity and temperature sensors within the hygroscopic moisture range. In the overhygroscopic moisture range, no moisture potential is measured.

The moisture content measurement with the TDR technology requires special procedures for both, calibration and data evaluation. The performed calibration of the measurement system configuration is explained. Subsequently, four approaches to derive the moisture content from the output quantity of the TDR measurement, the apparent dielectric constant, are introduced. Since two of these approaches allow to account for the bound water content, a short digression on its determination is added relating the bound water fraction to the first few molecular water layers which are adsorbed at the inner surface area of a material. This inner surface area can be determined from adsorption measurements by different methods, that are also introduced. 


\subsection{Concluding Developments and Utilisation}

Combined with a stringent assessment of the methods found, the requirements on an improved material model have been formulated. A material model meeting these defined requirements is successfully developed and introduced. It consists of a functional description of moisture storage to be adjusted to measured sorption and retention data and a procedure how to derive the transport function from this pore structure related data. The definitions of liquid water conductivity and vapour diffusivity possess enough flexibility to be calibrated on the basis of different moisture transport experiments. The proposed model therefore forms the basis of the investigations reported in the following chapters.

Methodological developments are accomplished for the drying experiment, where the influencing parameters are studied and assessed first. This is summarized in Tab. 5.1. A special device is developed and presented enabling drying measurement under defined and reproducible conditions which is a key issue if using its data for calibration of hygrothermal material modelling. Finally, an attempt to define a single value drying coefficient found in literature is introduced. On the basis of a comprehensive measurement and simulation study, however, this definition is challenged and ultimately disproved by an analytic solution of the drying problem. The drying experiment has thus become more clear and more defined. A deep insight is given and many questions are answered, although there is still a need for further investigations, especially regarding the derivation of a simple material parameter for which some prospects are given, too.

Within this chapter, the third focus is placed on the instantaneous profile measurement technique and its application for moisture transport experiments under transient conditions. The particularly designed set-ups for both moisture content ranges are introduced. Measurement schedules are proposed consisting of distinct adsorption and desorption cycles which are adjusted to the properties of the investigated materials. Subsequently, it is emphasized on the special requirements of moisture content measurement with TDR. The calibration procedure of the set-up configuration is briefly sketched. Finally, four approaches to derive the moisture content from the measured apparent dielectric constant are elaborated having different requirements on provided material and reference data. Since two of these models account for the fraction of bound water which can be related to the inner surface area, special emphasis is placed on its derivation from vapour sorption data. Both, the instantaneous profile measurements and their data evaluation can be performed in a well defined and sophisticated manner. 
CHAPTER 6

\section{IMPLEMENTATION AND APPLICATION}

To apply and validate the developed material modelling, two very laborious tasks have to be completed. One is the application of the material model to the measured basic data of the investigated building materials. The other is the evaluation of measured TDR data. To facilitate these tasks as well as to provide a basis for further applicability of the conducted developments, the corresponding models were implemented into two computer programs.

Within this chapter, these developed programs will be briefly introduced by their structural components. In a second step, the particular application will be presented. For the material model, the adjusted and calibrated material functions will be shown for each material. For the TDR data evaluation, only an example of the applied model functions and the reference data will be given whereas the whole set of measured data will be shown and discussed in Chapter 7.

\subsection{Implementation of the material model}

The material model was implemented into a computer program to facilitate both, the adjustment procedure and the data exchange between the laboratory and the simulation program. The developed program was called MATGEN, which is an abbreviation of input MATerial data GENeration for simulation programs. Within this paragraph, the different program components will be introduced first. Subsequently, the adjustment and calibration procedure will be sketched from an application-oriented point of view. 


\subsubsection{Program components}

In Fig. 6.1, a scheme of the general program structure is given. MATGEN consists of a number of processing steps which have to be completed one after another, and of data handling and additional options supporting data analysis and data exchange with the simulation program. These different program components are elaborated as follows.

\section{Input material data}

The material model forms the link between laboratory and simulation program. The basic input of the MATGEN program is therefore a laboratory data sheet containing all measured basic material properties. For the purpose of measured material data storage and evaluation, a special Excel template was developed at the Institute of Building Climatology ${ }^{1}$ consisting of one summary sheet and a number of storage and evaluation sheets corresponding to the different basic experiments as introduced in Chapter 3. Within the summary sheet, information concerning the material and its producer and all basic material properties as density, porosity, moisture storage data, vapour diffusion resistance values, water uptake coefficient and capillary saturation, liquid water conductivity data, thermal conductivity and heat capacity are stored. This summary page is read by the MATGEN program providing all material properties for further account.

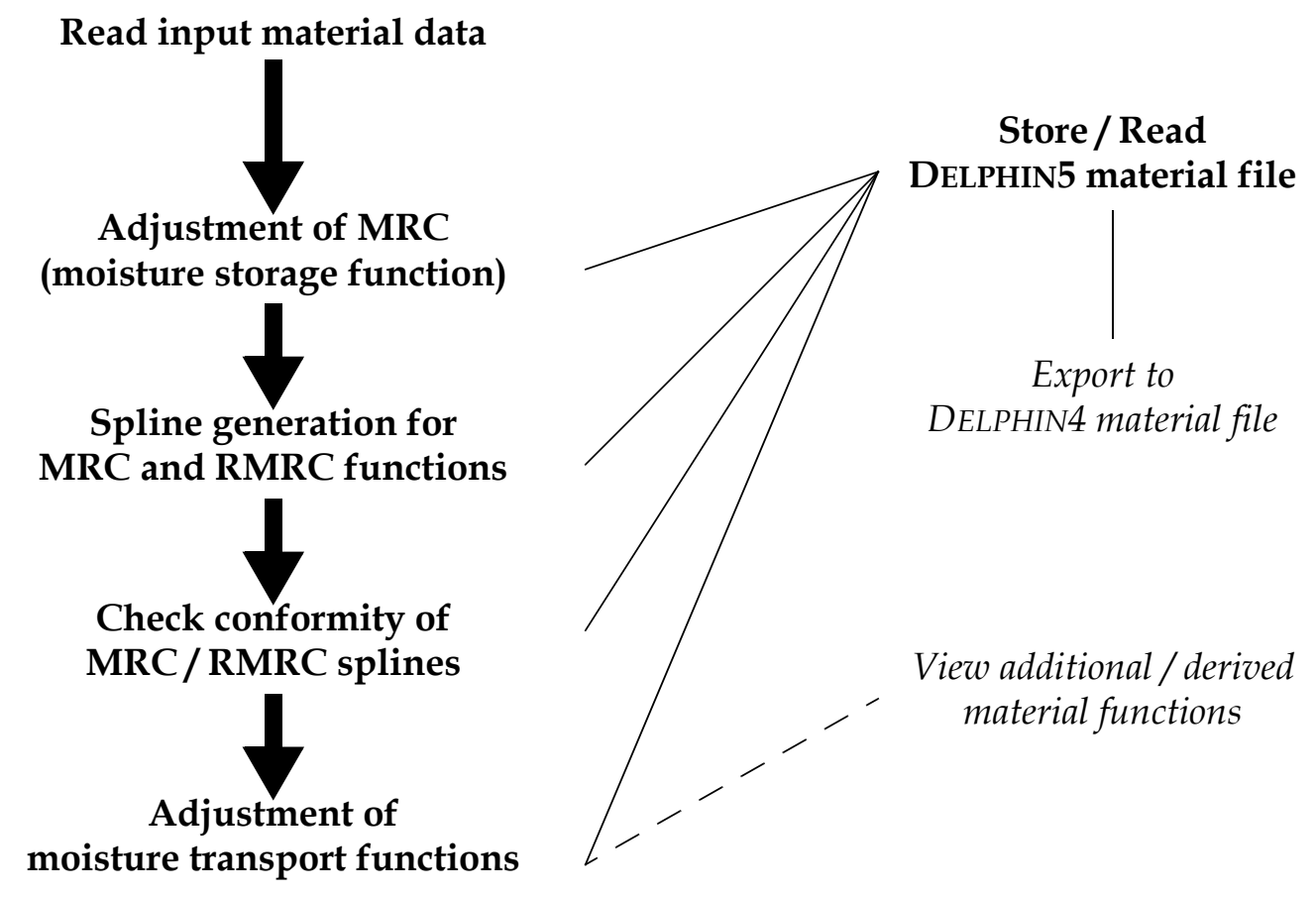

Figure 6.1: $\quad$ Structure of the developed program to apply the material model to measured basic material data. The lines indicate main interaction paths.

1. The laboratory Excel template was developed by Rudolf Plagge, Frank Meißner and by the author during the years 1997-2007 at the Institute of Building Climatology, Dresden University of Technology. 


\section{Adjustment of the moisture storage characteristic}

The first step in applying the material model to measured input data is the moisture storage characteristic (MRC) adjustment. The parameters of the multimodal function given in eq. (5.1) have to be adjusted to the measured sorption and retention values for both, the adsorption and the desorption moisture storage characteristic. With a specified number of modalities, the program allows to calculate initial values for the functional parameters as well as to use a numerical parameter optimization routine. These were implemented according to Franke (2007), who used the Levenberg-Marquardt algorithm for the optimization. Further adjustment by the user is possible via a parameter table and a graphical illustration of the moisture storage data versus capillary pressure or relative humidity in a logarithmic and a normal scale.

\section{Spline generation for MRC and RMRC}

As a second step, data points for linear spline interpolation are calculated for the adjusted moisture retention functions as well as for their reverse functions (RMRC). A special algorithm developed by Nicolai (2005) is used providing linear spline data which enable a high performance use in numerical simulation in such a way that the more data points are set the stronger the slope change of the particular function is.

Since the reverse moisture retention function is used to calculate the liquid water conductivity function, see eq. (5.3), the interpolated data points for this function are specified by this routine, too. A limiting number of data points as well as the accuracy requirements for these splines can be adjusted by the user.

\section{MRC and RMRC conformity check}

Once the spline generation is fulfilled, a check option can be used. Two aspects are controlled by this option. First, a check for double abscissa values is run which is required due to the limited accuracy in the storage of numerical values in a file. Normally, this check becomes important when a large number of data points was chosen for the spline generation and when the adjusted material data was stored and read again from a file. The double values can be deleted automatically once they were detected. And second, the conversion accuracy is controlled and the mean deviation of original and approximated value is displayed. Then the user may decide whether increasing the limiting number of data points for a further refinement of the spline data or not.

\section{Adjustment of the moisture transport functions}

Based on the RMRC spline data, the wet-cup vapour diffusion data and the conductivity measurements in the saturated moisture content range, the liquid water conductivity as well as the vapour diffusivity are calculated as elaborated in paragraph 5.1.2 Liquid water and water vapour transport. The contained parameters $\eta_{c a p}$ and $\eta_{s p}$ have to be adjusted by the user. This is done in interaction with the numerical simulation of the water uptake and the drying experiment. For that purpose, the material data must be stored and included into the simulation program. First, the water uptake experiment should be simulated as it gives an indication of the order of magnitude for the liquid water conductivity within the capillary moisture content range, adjusting $\eta_{c a p}$. Once 
$\eta_{\text {cap }}$ has been determined, the drying experiment indicates the liquid water conductivity within the lower overhygroscopic moisture range. By this, $\eta_{s p}$ can be adjusted, see also paragraph 5.1.2.3 Calibration of liquid and vapour transport coefficients.

In case this adjustment procedure does not deliver acceptable results, an additional menu is available allowing to adjust the upper integration boundary of eq. (5.3), to modify the limit of the relative conductivity function and to add further conductivity values to the hygroscopic liquid water conductivity.

Changing the upper integration boundary may become important for materials with a large pore fraction at low capillary pressures, i.e. with a large capillary radius. Since these pores would dominate the whole relative conductivity function leading to an underestimation of the conductivity in the lower moisture content range, see also Fig. 6.2 , it is recommended to truncate them partly from the conductivity evaluation by adjusting $p C_{l i m}$.

For materials with a large pore volume but a very low capillary moisture content, the relative conductivity derived from the pore structure is applied for a very short moisture range only because its upper limit is the capillary moisture content. For such materials,
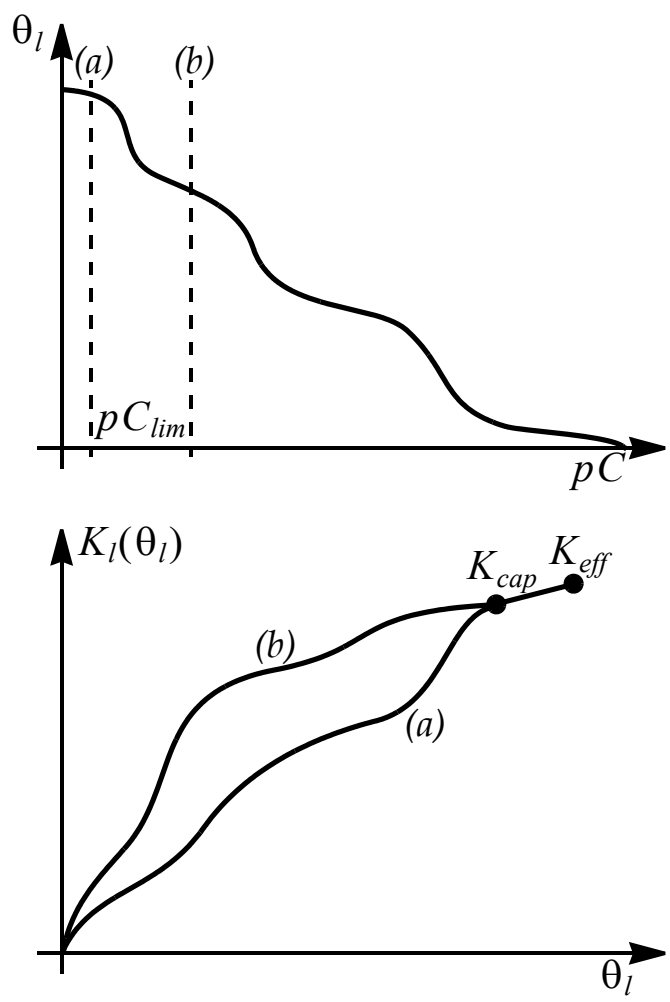

Figure 6.2: Influence of the limiting capillary pressure $p C_{\text {lim }}$ on the liquid water conductivity function. the capillary moisture content (which has only this meaning within the model) can be set to a higher moisture content value enabling the application of the bundle of tubes model for a larger pore size range.

Finally, for cases where it is not possible to follow the drying course correctly when adjusting the parameter $\eta_{s p}$ only, additional conductivity values can be defined in the lower moisture content range. For most building materials, however, the adjustment of $\eta_{c a p}$ and $\eta_{s p}$ by the help of water uptake and drying experiment simulation should be expedient.

\section{Data storage}

After each adjustment step it is possible to save the material data. The material data storage was designed in the style of the DELPHIN5 program according to Nicolai (2006). The stored material data file is thus a material input file of the DELPHIN5 simulation program. All modelling options and parameters are stored in specially encoded strings enabling to continue with the adjustment procedure if it was interrupted. To avoid an error message when using the simulation program, it is recommended to save the material data and read it again once the spline generation has been completed, and use apply the conformity check option. 
As it is possible with DELPHIN5 not to include the material file into the project but assign a link to a corresponding file, the optimization procedure for the two parameters $\eta_{c a p}$ and $\eta_{s p}$ is significantly simplified. Once the simulation projects are created, the changed material data has only to be saved and another simulation can be run.

To use the applied material model also for numerical simulations with the DELPHIN4 program according to Grunewald (1997) and Grunewald \& Fechner (2006), it is possible to export the data to a corresponding material input file. In that file though, the modelling information is not saved and it is thus not possible to read a DELPHIN4 material file.

\section{Derived material functions}

An additional menu was designed to view additional and derived material functions as the pore-volume distribution or the liquid or moisture diffusivities. Moreover, it is possible to display the different function in dependence to different quantities, as e.g. the liquid water conductivity versus moisture content, versus capillary pressure or versus capillary radius.

\section{Further program options}

It is certainly possible to include other material models into the MATGEN program. Either a complete model, as e.g. a simplified diffusivity approach which would account for cases where very limited input data is available, or another functional approach to describe the moisture storage function, as the one according to Van Genuchten (1980) and Durner (1994) or the one according to Häupl E Fechner (2003b) and Häupl (2007b), could be implemented. Moreover, the material data export could also be implemented to support other simulation programs. ${ }^{1}$

\subsubsection{Adjustment and calibration procedure}

The adjustment and calibration procedure to apply the material model to measured data consists of two main components. The first is the model itself and the procedure which has to be followed for its application. This is what the MATGEN program does which was introduced in paragraph 6.1.1 Program components above. The second component is the comparison of measured and simulated material behaviour for the water uptake and the drying experiment. As both experiments contain information about the moisture transport properties in different moisture content ranges, the modelling parameters $\eta_{c a p}$ and $\eta_{s p}$ can be adjusted by their help. However, this is an iterative process between the adjustment procedure and the simulation. Fig. 6.3 shows a process scheme for the whole procedure indicating the different dependencies and interactions of model application, parameter adjustment and numerical simulation of the two transport experiments.

1. As this was not the task for the current project to include different material models, the basic structure of data handling was designed to be extended only, but the corresponding extensions were not implemented, yet. If there is a special interest for it, please feel invited to contact the author. 


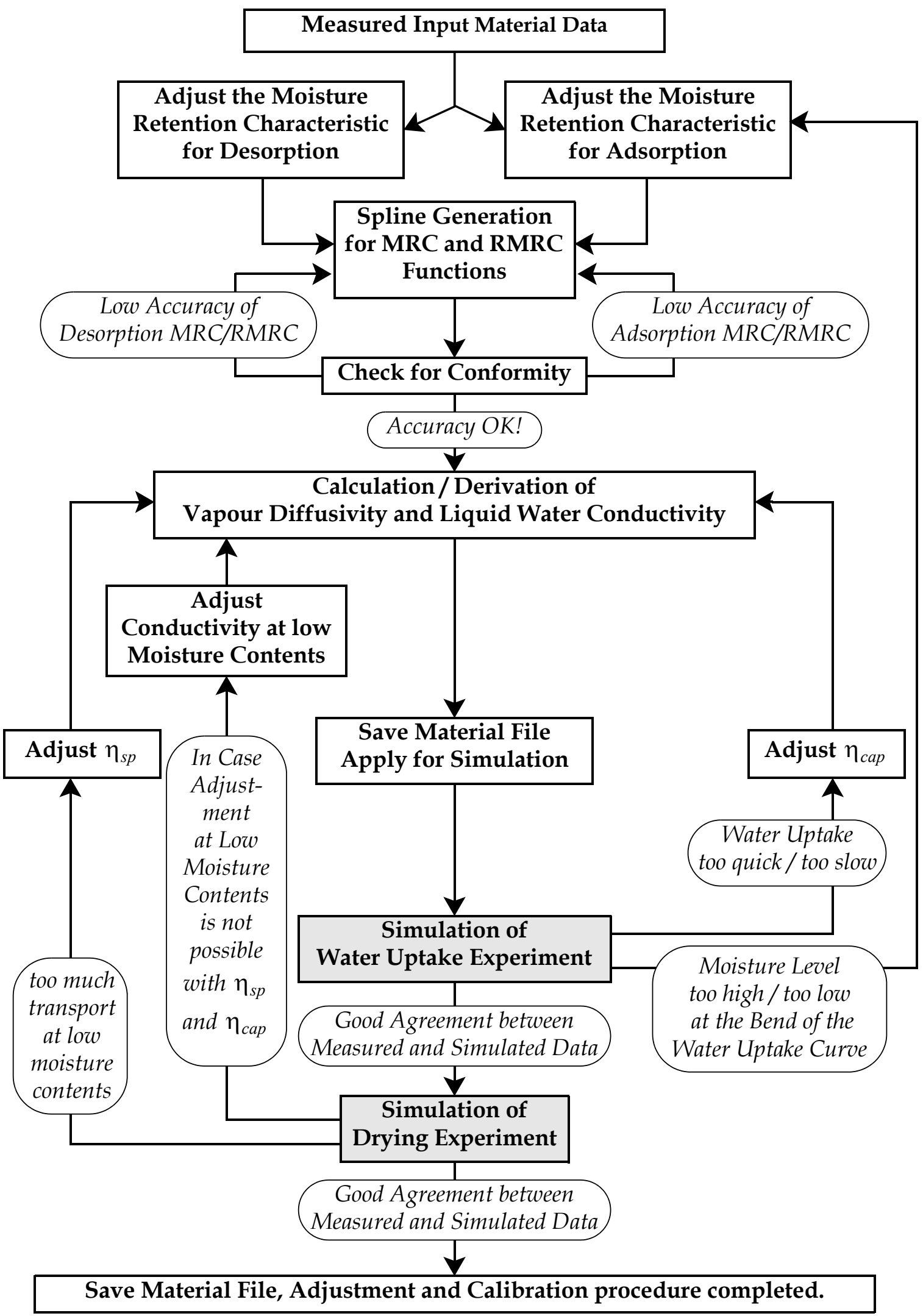

Figure 6.3: Scheme of the adjustment and calibration procedure when applying the material model to measured data. The calibration is an iterative process between adjustment of the modelling parameters and the comparison of measured and simulated water uptake and drying courses. 
It would be preferable to have one large algorithm which includes all the processes summarized in Fig. 6.3. This is, however, from the author's point of view not practically applicable for a number of reasons.

The first problem is the measurement data storage. One data file would be required where all information is stored in a strictly defined way. This is already difficult for a summary of basic material parameters. For all storage and transport experiments though, it would imply that all materials behave in the same way and no special evaluation regarding the specific material behaviour is required. This patiently does not coincide with the laboratory practise.

The second problem is the choice of the particular material samples for the simulation. Normally, the experiments are carried out for a number of material samples. For the simulation, one sample is chosen, its geometry data is applied, its boundary conditions are selected and the measured course is compared with the simulation results. This choice depends on different conditions as the geometry itself, the quality of measurement data, the boundary conditions etc. Many of these criteria belong to the measurement history and are therefore difficult to be implemented into the data evaluation.

The third problem is the choice of relevant measurement curves for the comparison with the simulation results. Sometimes, the experimental conditions are different for all examined samples and only the particular curve which belongs to the chosen specimen can be used for the evaluation. Sometimes, however, many samples of similar dimensions are prepared and measured delivering a number of curves indicating the variety of the material. In such a case, more than one measurement course should be used for the evaluation.

Finally, the fourth problem is the interpretation of a deviation in the measured and simulated material behaviour. As the water uptake and drying experiments can only indicate the moisture transport properties due to the complex interaction of moisture potential gradients, moisture potential - moisture content relation and moisture transport properties, there exist no clear criteria of what should be changed to what extent in dependence to what deviation. The experience and the interpretation of the person who is doing the calibration is required here which can be hardly implemented into a large algorithm.

Despite these data handling and interpretation criteria problems, the implementation of the scheme shown in Fig. 6.3 would require the coupling of the material data storage and evaluation (e.g. Excel), the material model adjustment program (e.g. MATGEN), the coupled heat and moisture simulation program (e.g. DELPHIN) and the evaluation of measured and simulated courses. This is, from the author's point of view, due to the mentioned problems and the large amount of input data this would require, extremely laborious and therefore not reasonable. The material model application remains therefore the iterative process described above which has to be accomplished by the user.

\subsection{Application of the material model}

The implemented material model was applied to the four building materials according to the procedure illustrated in Fig. 6.3. The adjusted material functions and the comparison of measured and simulated experiments will be elaborated here. 


\subsubsection{Building brick}

For the building brick, desorption moisture storage data was available for the whole moisture content range. Adsorption data could though only be measured within the hygroscopic, i.e., the relative humidity range. Fig. 6.4 shows the desorption and the adsorption moisture storage function adjusted to the measured data. The data is displayed as moisture content versus relative humidity (left) and versus capillary pressure (right). The building brick has a very low hygroscopic moisture capacity. For a better illustration, the moisture content was hence plotted in a logarithmic scale.

Within the overhygroscopic moisture range, the adsorption moisture storage function was adjusted on the basis of two criteria. The first criterion is the general pore structure which was tried to be kept according to the desorption characteristic. The second criterion is the water uptake behaviour, in particular the bend of the water uptake curve and the corresponding moisture content level. The simulation was conducted applying a no-pressure boundary condition. The boundary moisture content as well as the mean moisture content at the bend of the water uptake curve is therefore determined by the adsorption moisture storage function which was adjusted in that way.

In Fig. 6.5 at the left, the pore volume distribution is displayed for the building brick. It was derived from the desorption moisture storage function according to eq. (5.2) where the capillary pressure was replaced by the capillary radius following the Laplace equation (2.14) and the assumption of $\gamma=0^{\circ}$ for the contact angle. At the right side of Fig. 6.5, the liquid water conductivity function is shown as it was derived and adjusted during the calibration procedure. The total moisture conductivity as the sum of vapour and liquid water conductivity is also shown there.

Within the lower overhygroscopic moisture content range, the liquid water conductivity function starts from a low conductivity level but increases rapidly. In the capillary and the saturated moisture content range, the building brick has a rather high liquid water conductivity. This course of the conductivity function is also indicated by the two calibration transport experiments shown in Fig. 6.6. For the water uptake experi-
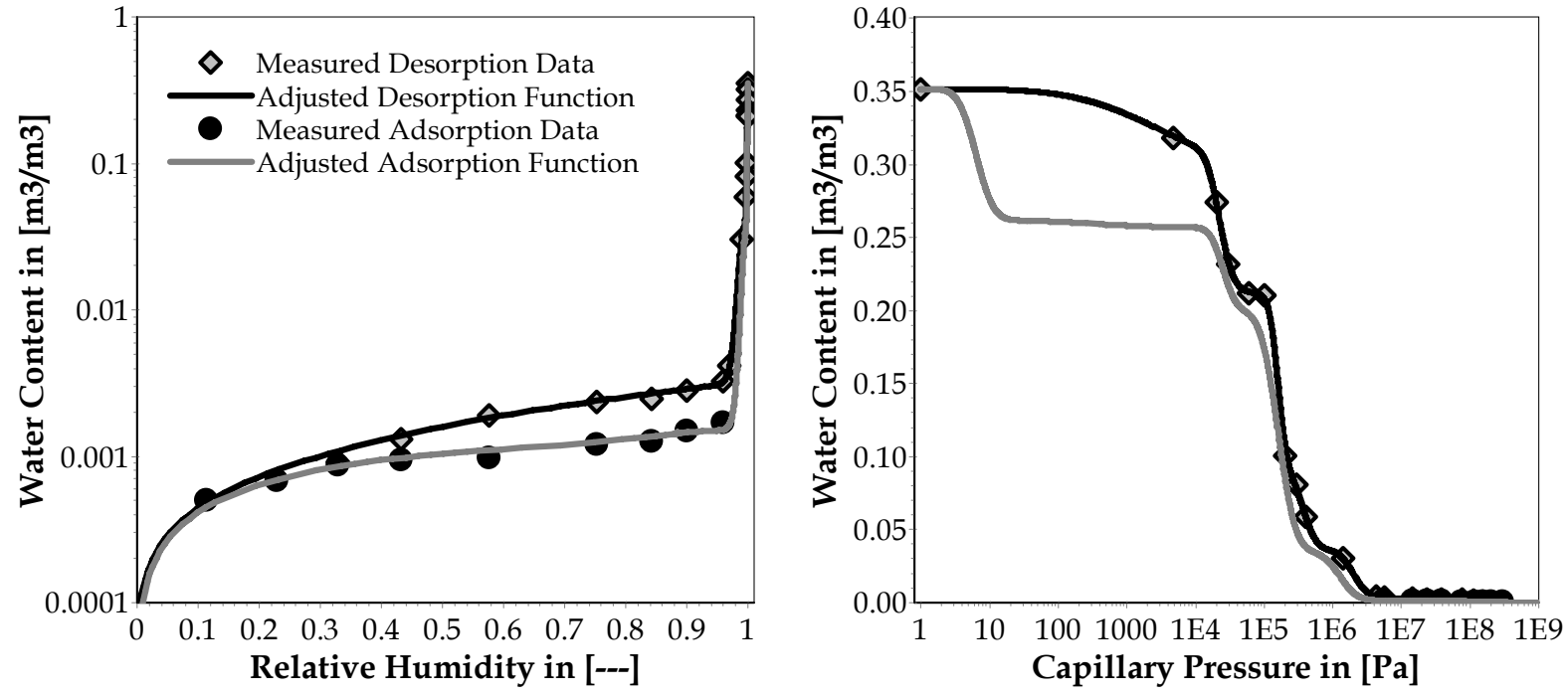

Figure 6.4: Desorption and adsorption moisture storage function of the building brick: sorption isotherm (left) and moisture retention characteristic (right). 

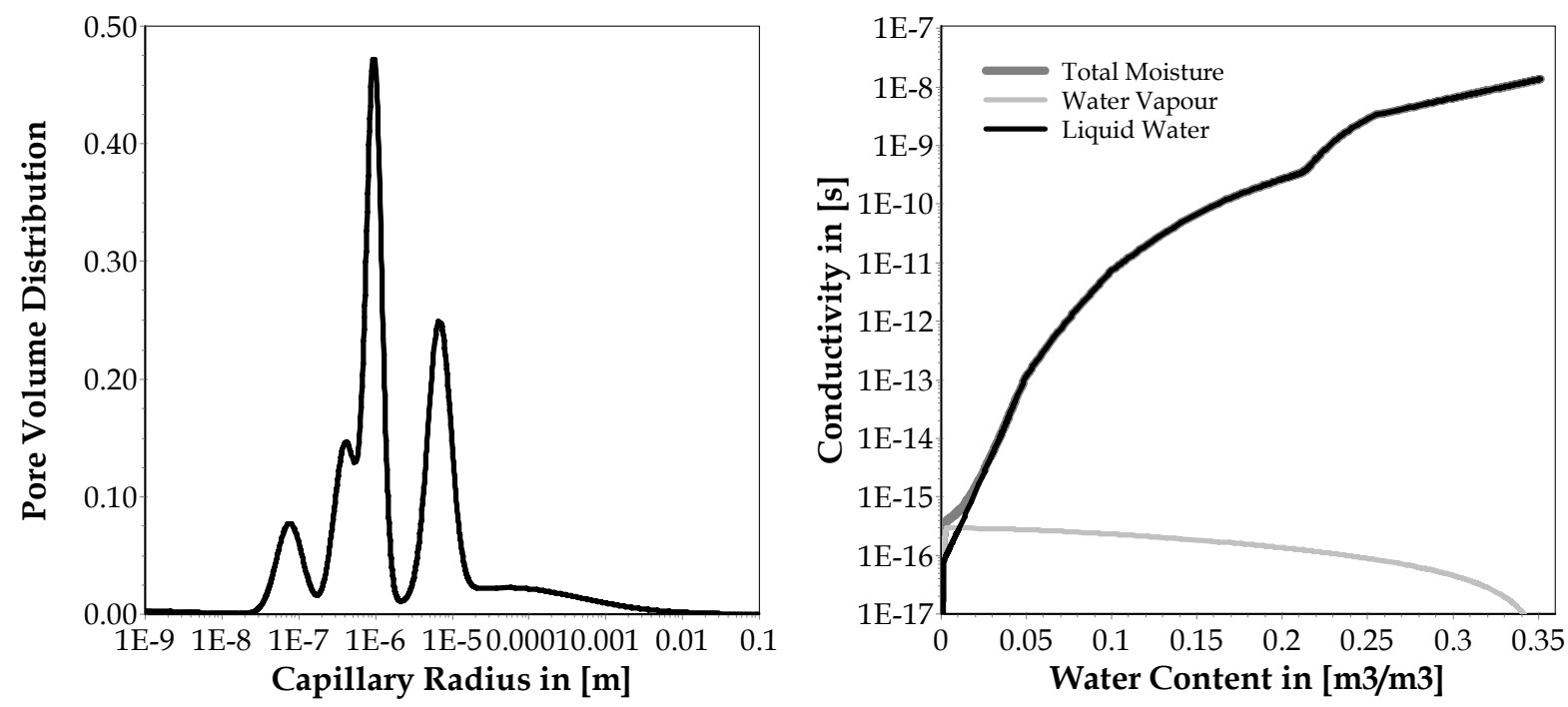

Figure 6.5: Building brick: pore volume distribution (left) and moisture conductivity function as the sum of liquid and vapour conductivity (right).

ment, where measured and simulated courses are displayed at the left, the low hygroscopic moisture capacity and the high capillary conductivity lead to a quick transport process expressed by the steep slope of the water uptake curve. For the drying, which is shown at the right of Fig. 6.6, the same combination leads to a long first drying phase. The low conductivity within the lower overhygroscopic moisture content range, however, leads to a distinct second drying phase and is also responsible for the corresponding drying duration.

As Fig. 6.6 shows, the agreement between measured and simulated results is very good (all simulations were done applying the DELPHIN Program according to Nicolai (2006)). The building brick is, though, a rather convenient material with regard to the material model calibration.
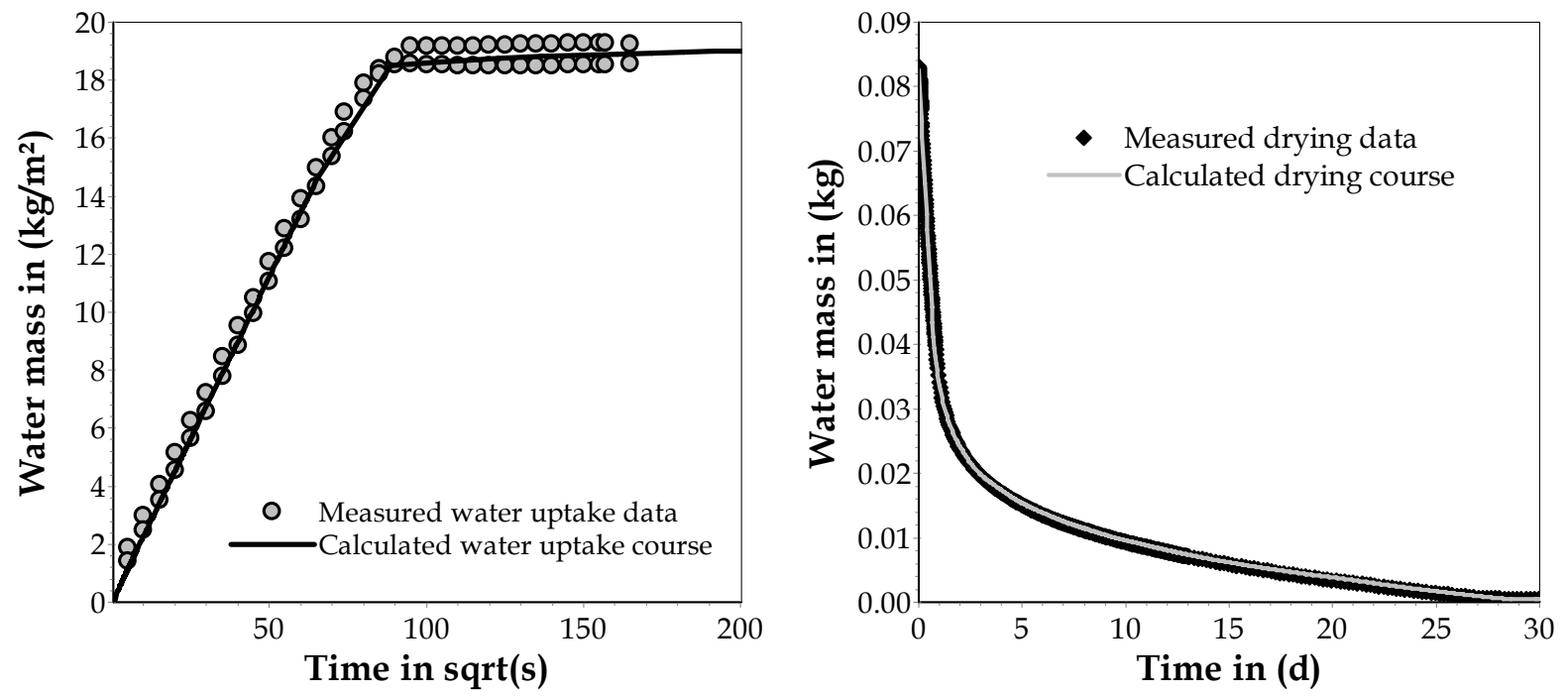

Figure 6.6: Calibration of the building brick: comparison of measured and simulated courses for water uptake (left) and for drying (right). The drying data was measured with an automatic balance device. 


\subsubsection{Calcium silicate}

The moisture storage function of the calcium silicate is given in Fig. 6.7 as the sorption isotherm versus relative humidity (left) and moisture retention curve versus capillary pressure (right). Again, adsorption data is only available for the hygroscopic region whereas desorption data could be measured for the whole moisture content range. The adjustment was hence performed in the same way as for the building brick. The calcium silicate has a very high porosity which is assigned to the mid-pore range. Thus, the moisture capacity is very high for capillary pressures around $1 \mathrm{MPa}$. In contrast to this, the hygroscopic moisture capacity is almost negligible.

In Fig. 6.8 at the left, the pore volume distribution derived from the desorption moisture storage function is displayed for the calcium silicate accentuating the distinct pore maxima within the mid-pore range.

The moisture transport functions of the calcium silicate are shown in Fig. 6.8 at the right. The liquid water conductivity, the vapour conductivity and the total moisture conductivity are displayed there. The liquid water conductivity quickly increases for low moisture contents passing over into a plateau before a second steep slope leads to very high conductivity values within the saturated moisture content range. The calcium silicate is thus a material with most pronounced moisture transport properties which becomes also visible when viewing the water uptake and the drying data displayed in Fig. 6.9.

There, measured and simulated water uptake data are shown at the left. The steep slope of the water uptake curve indicates the discussed course of the liquid water conductivity. Due to the high porosity, the amount of absorbed water is formidable.

At the right side of Fig. 6.9, the measured and simulated drying courses are compared. Again due to both, the high porosity and the distinct liquid transport properties, the first drying phase is very long. According to the pronounced plateau and the lower conductivity values for low moisture contents, a clear second drying phase follows.
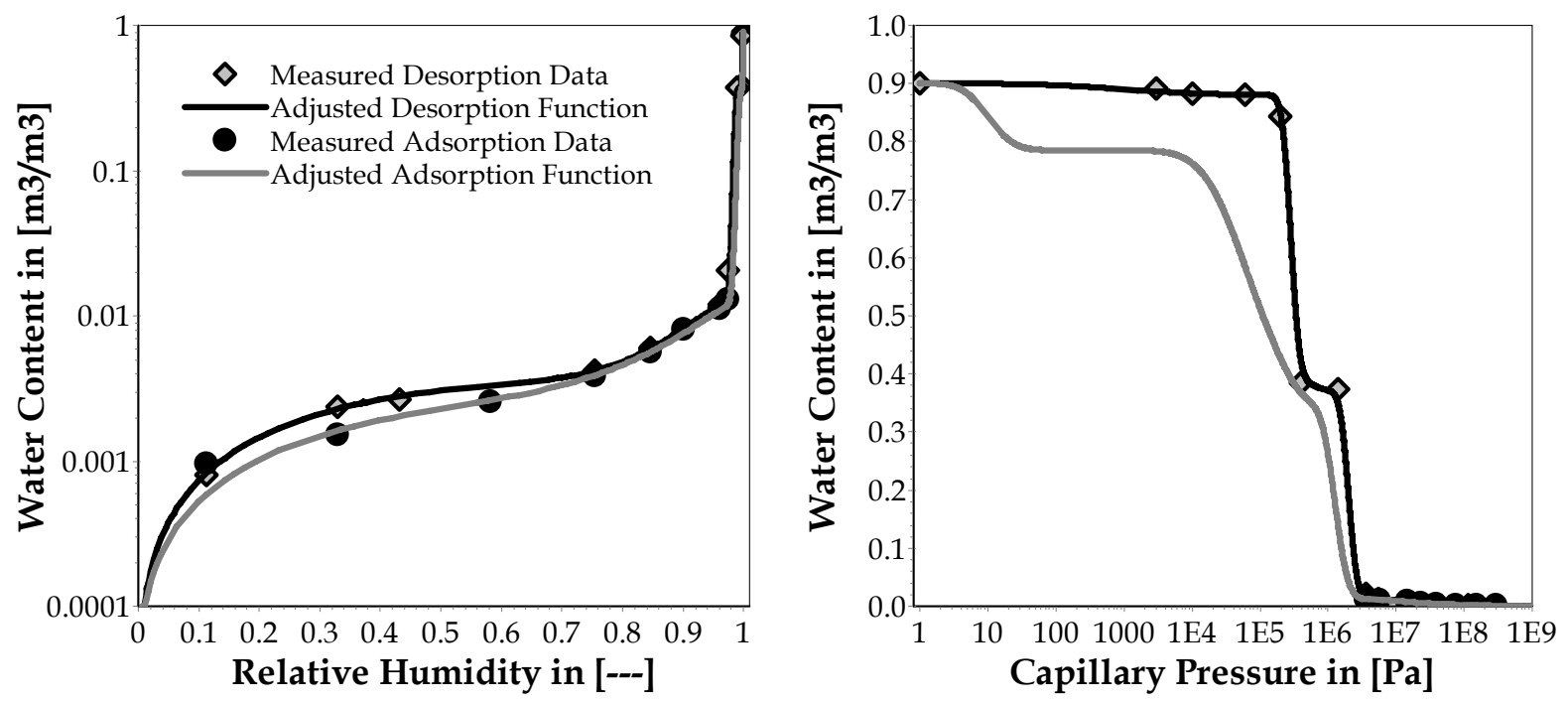

Figure 6.7: Desorption and adsorption moisture storage function of the calcium silicate: sorption isotherm (left) and moisture retention characteristic (right). 

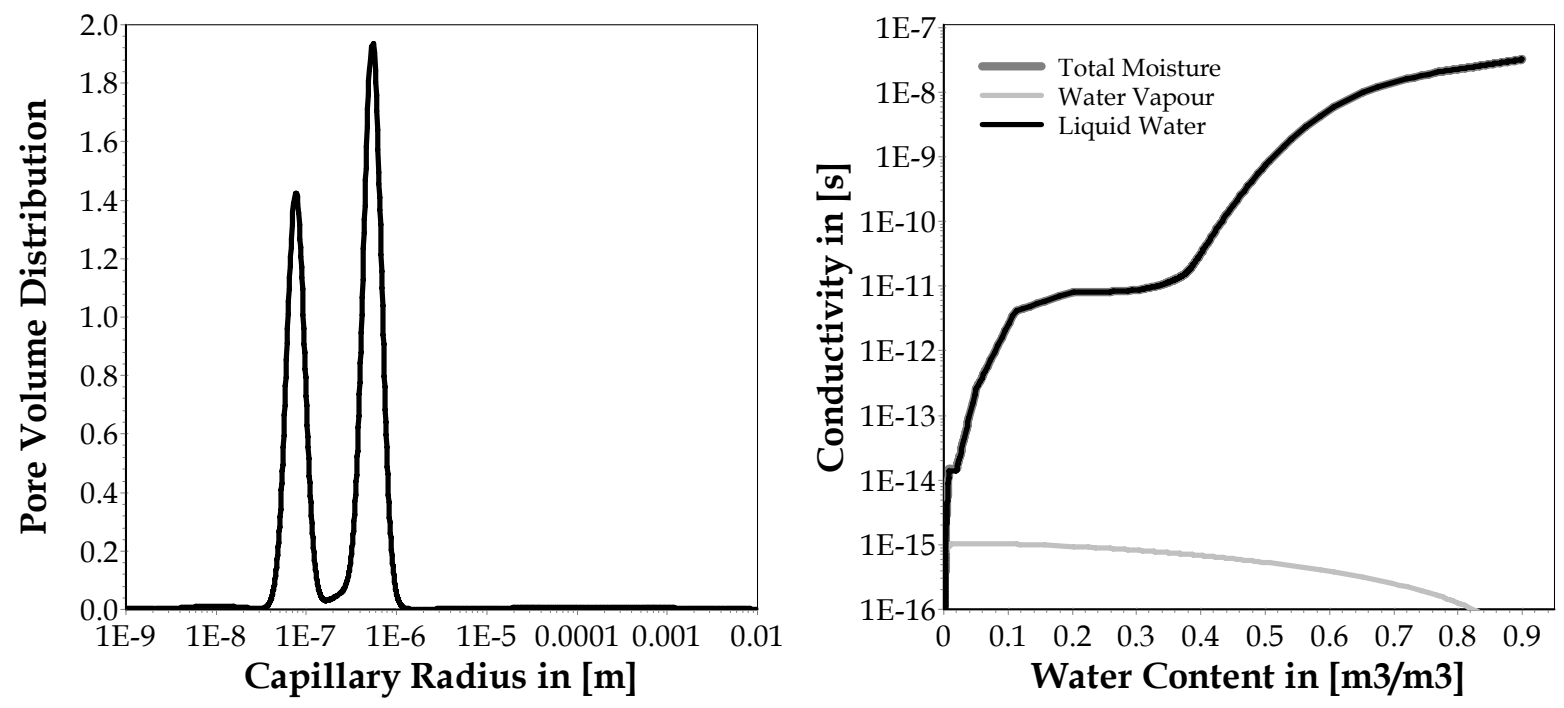

Figure 6.8: Calcium silicate: pore volume distribution (left) and liquid water conductivity function as the sum of liquid and vapour conductivity function (right).

However, the total drying duration, especially in combination with the high initial moisture contents and the high material specimens, is very short.

The agreement of measured and simulated water uptake and drying behaviour is almost perfect. The calcium silicate is a rather simple material with respect to the adjustment and calibration procedure. However, the moisture transport properties are often overestimated within the lower overhygroscopic moisture range. Therefore, the drying experiment performed for high material specimens and with high vapour exchange rates is very important to reveal enough information for an accurate calibration.
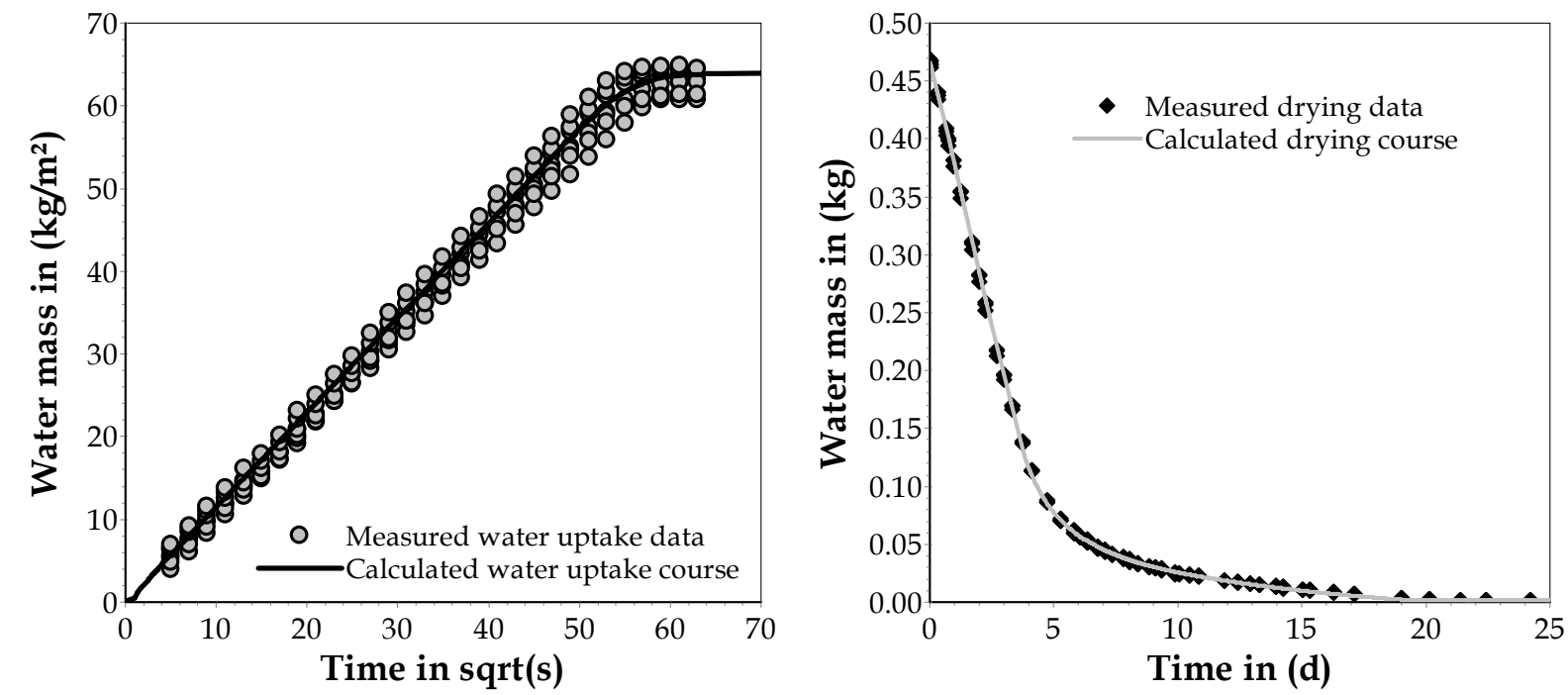

Figure 6.9: Calibration of the calcium silicate: comparison of measured and simulated courses for water uptake (left) and for drying (right). 


\subsubsection{Aerated concrete}

The desorption and adsorption moisture storage data which was measured for the aerated concrete is given in Fig. 6.10 together with the adjusted curves. The aerated concrete possesses a clearly distinguished pore structure providing pores of almost all sizes. This leads to the plateaus of the moisture retention curve passing over into each other, as shown at the right of Fig. 6.10, as well as to the significant moisture capacity within the hygroscopic moisture range, as shown in the sorption isotherm displayed at the left of Fig. 6.10.

In Fig. 6.11 at the left, the pore volume distribution versus capillary radius is given. The gradual pore structure becomes visible there, too. As it was done for the other materials, this pore volume distribution function was also derived from the desorption moisture storage function.

The moisture transport functions of the aerated concrete are displayed at the right of Fig. 6.11. The liquid and vapour conductivity as well as the total moisture conductivity are shown in the right chart. The liquid water conductivity, taking relatively high values already in the hygroscopic moisture content range, exhibits then a slow but rather constant increase over a large range of moisture contents. For high moisture contents of $\theta_{l}>0.25 \mathrm{~m}^{3} / \mathrm{m}^{3}$, the conductivity takes high values. However, these high conductivity values are assigned to the very large pores, as can be seen in Fig. 6.10 at the right, which are normally not water filled. For lower moisture contents, the moisture transport is much less pronounced.

Both, the moisture transport and moisture storage behaviour of the aerated concrete, become indirectly visible when looking at the water uptake and the drying data. The comparison of measured and simulated courses is shown in Fig. 6.12, at the left for the water uptake experiment and at the right for the drying. The duration of the water uptake experiment is rather long, the slope quite low. The first drying phase is relatively short, the second drying phase is very pronounced. However, due to the distinct
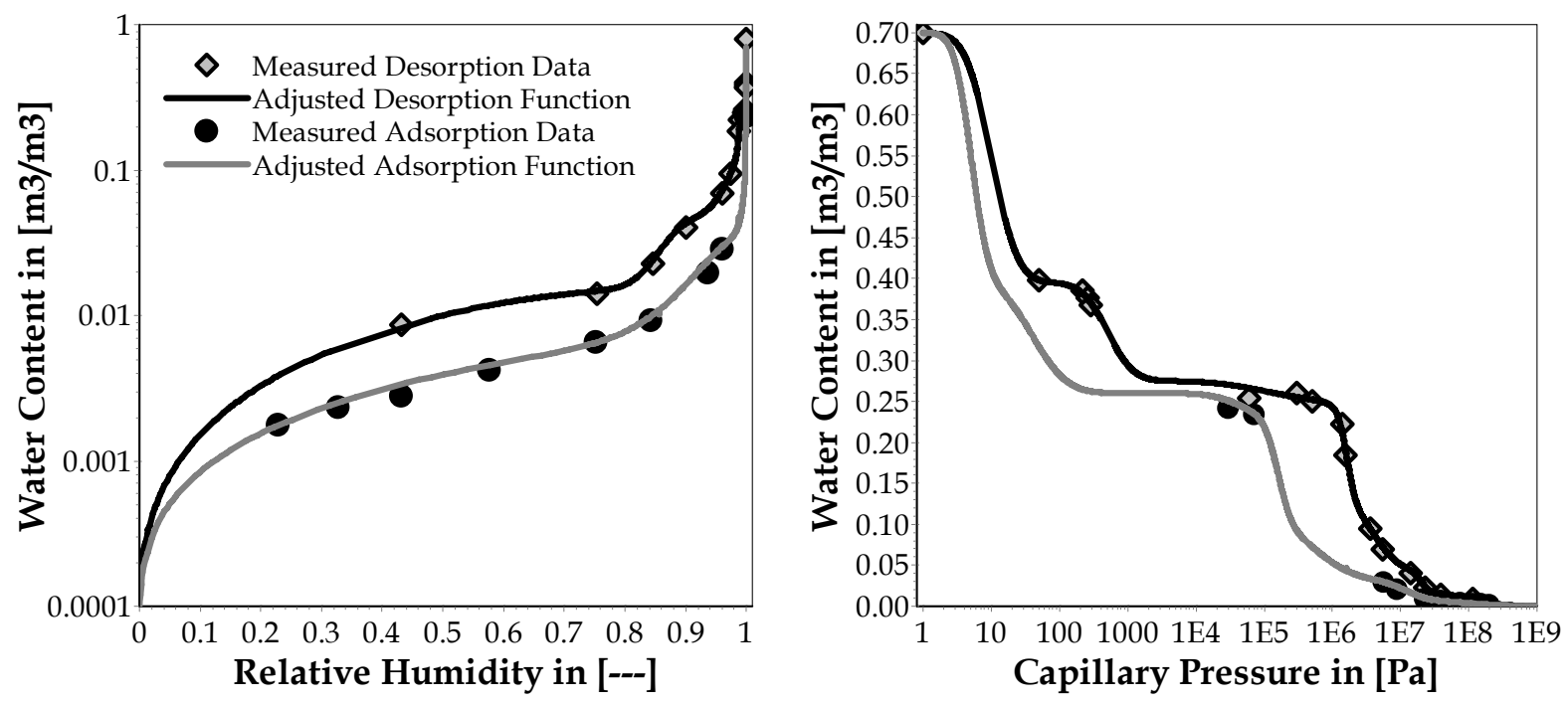

Figure 6.10: Desorption and adsorption moisture storage function of the aerated concrete: sorption isotherm (left) and moisture retention characteristic (right). 

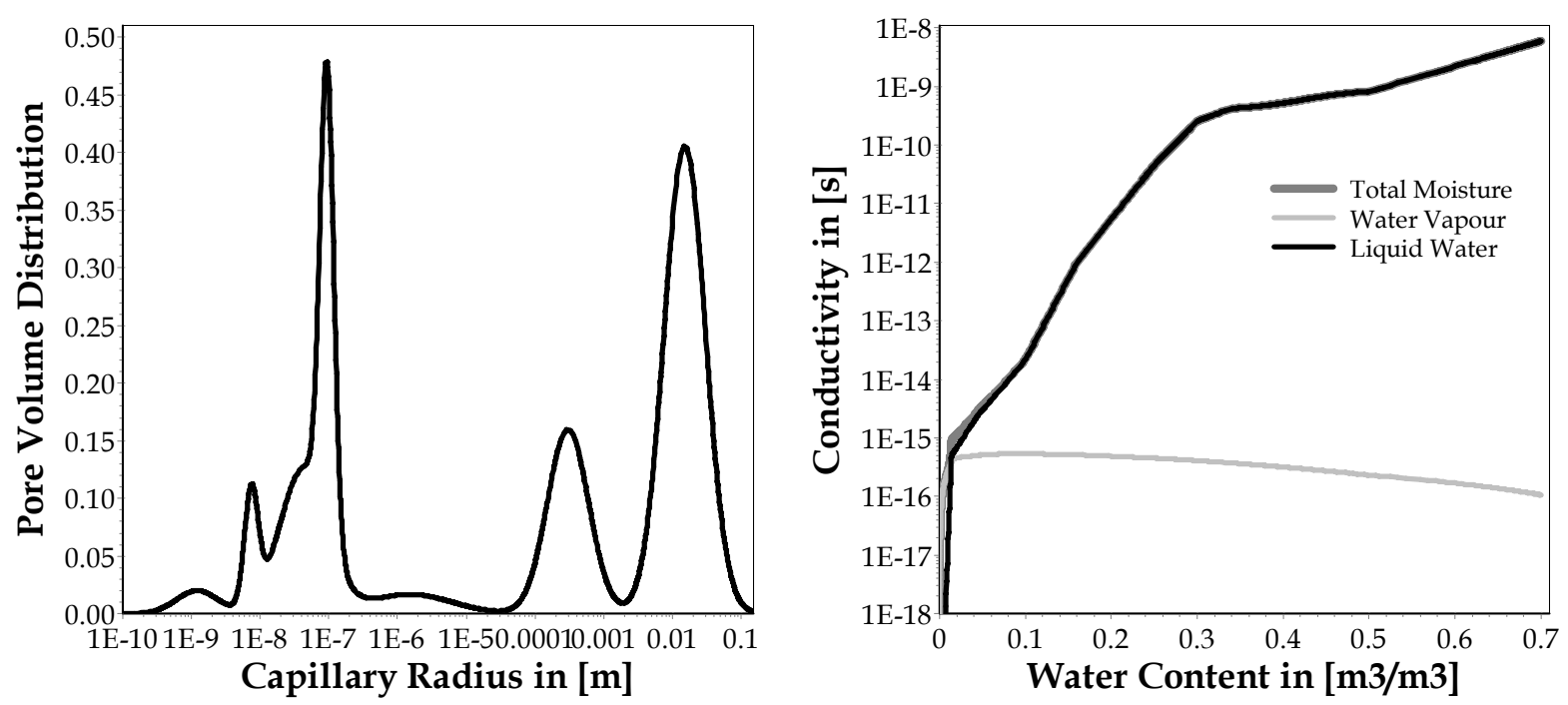

Figure 6.11: Aerated concrete: pore volume distribution (left) and liquid water conductivity function as the sum of liquid and vapour conductivity function (right).

moisture transport in the hygroscopic and the lower overhygroscopic moisture content range, the drying within the second phase proceeds quickly leading to a comparatively short total drying duration.

The agreement between measurement and simulation is very good. However, it has to be stated, that the aerated concrete is a very difficult material for hygrothermal characterization due to the large variety in material properties and the strong process dependencies which will be discussed in detail within Chapter 7.
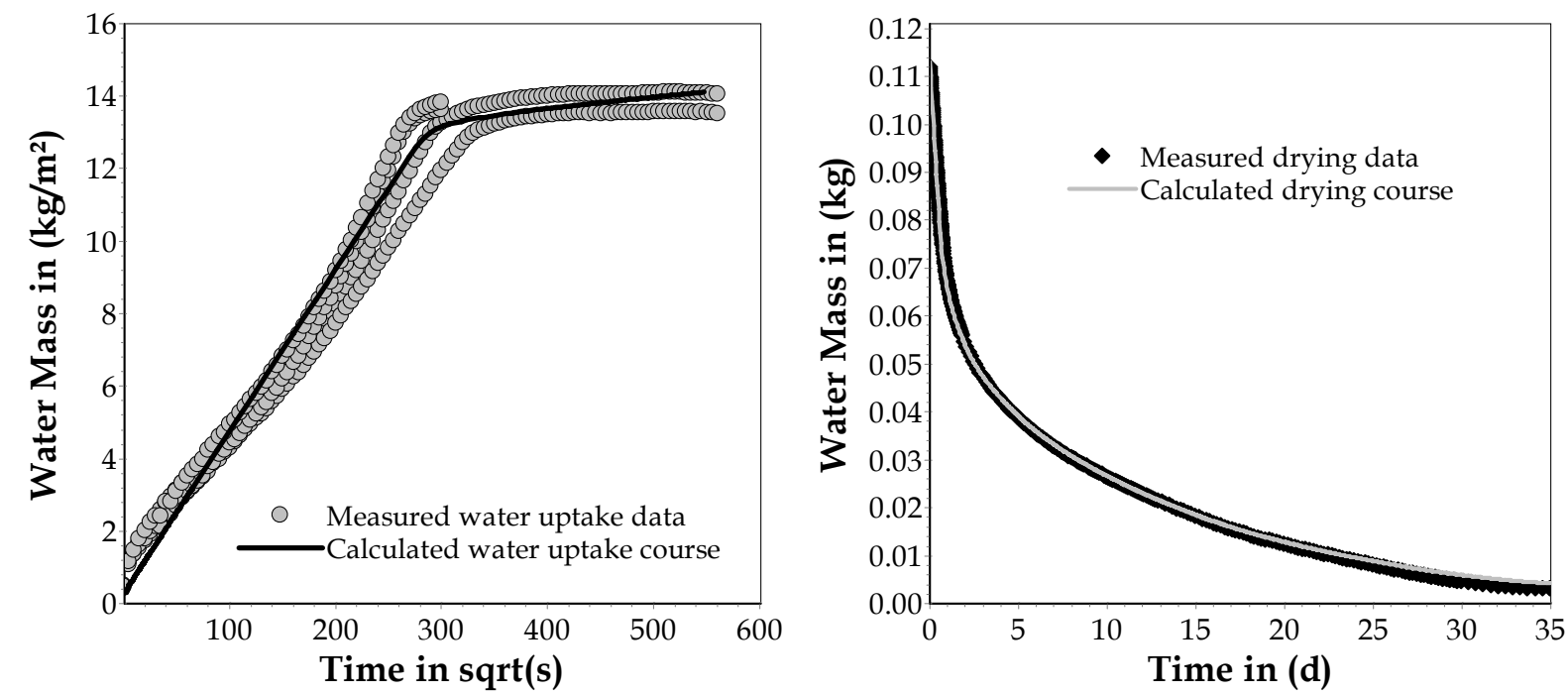

Figure 6.12: Calibration of the aerated concrete: comparison of measured and simulated courses for water uptake (left) and for drying (right). The drying data was measured with an automatic balance device. 


\subsubsection{Lime-sand brick}

Fig. 6.13 shows the adsorption and desorption moisture storage data of the lime-sand brick. Again, the sorption isotherm is displayed at the left and the moisture retention curve at the right. The lime-sand brick exhibits a significant moisture capacity within the hygroscopic moisture content range. It comprises more than $20 \%$ of the pore volume.

The pore volume distribution of the lime-sand brick forms therefore two sharp pore maxima superimposed with one very wide pore maximum (or a number of different small pore maxima passing into each other). Fig. 6.14 shows the pore volume distribution at the left as is was derived from the desorption moisture storage function.

The liquid water conductivity, the vapour conductivity and the total moisture conductivity are given in Fig. 6.14 at the right. The high hygroscopicity of the lime-sand brick becomes visible here, too. The vapour transport contributes significantly to the total moisture conductivity within the hygroscopic moisture range. The vapour transport function itself is rather low though. The liquid water conductivity increases constantly but slowly. Within the saturated moisture range, it still remains one to two orders of magnitude lower than the conductivity of the other materials. The lime-sand brick is therefore a material with a high moisture capacity but with low moisture transport properties.

The data of the two calibration experiments is displayed in Fig. 6.15. At the left, the water uptake data is shown forming a slowly increasing slope. At the right of Fig. 6.15, the drying course is given. Both characteristics become visible there, again. The total drying time is very long whereas the first drying phase is existent but rather short. The combination of the water stored within a wide range of pores and a significantly decreasing liquid water conductivity for the corresponding moisture contents is responsible for this behaviour.
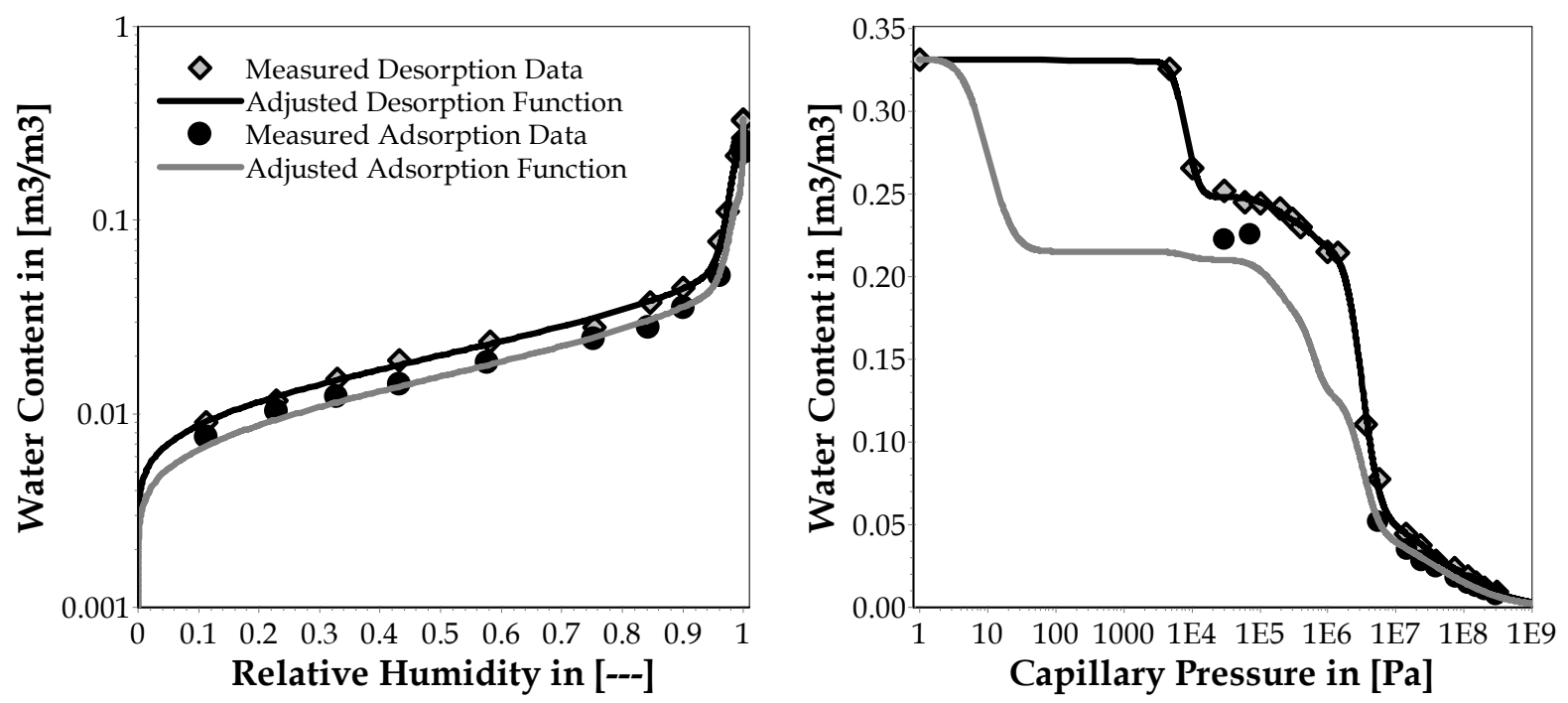

Figure 6.13: Desorption and adsorption moisture storage function of the lime-sand brick: sorption isotherm (left) and moisture retention characteristic (right). 

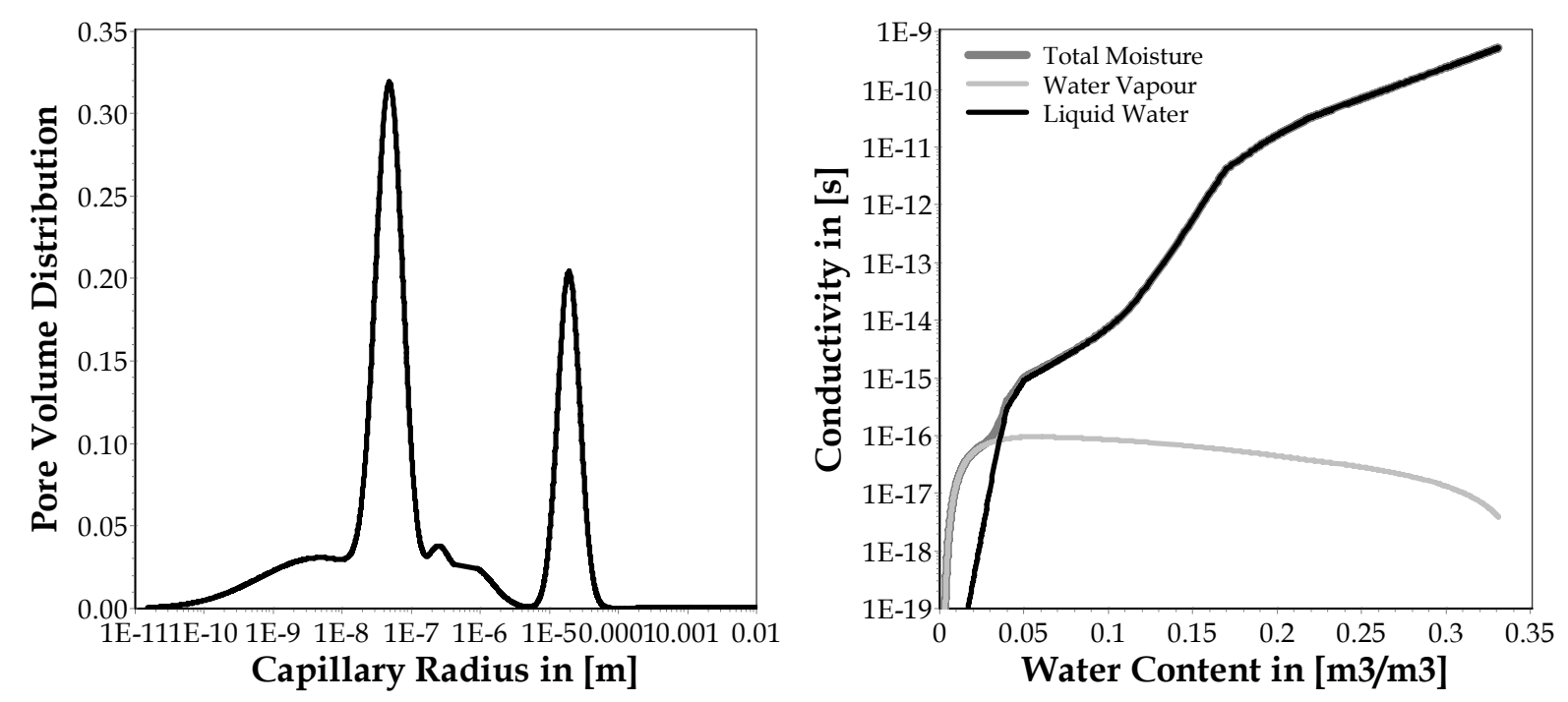

Figure 6.14: Lime-sand brick: pore volume distribution (left) and liquid water conductivity function as the sum of liquid and vapour conductivity function (right).

The agreement between measured and simulated material behaviour is very good after the calibration procedure. However, also the lime-sand brick is a rather difficult material due to the wide pore ranges contributing to both, the water uptake and the drying moisture transport.
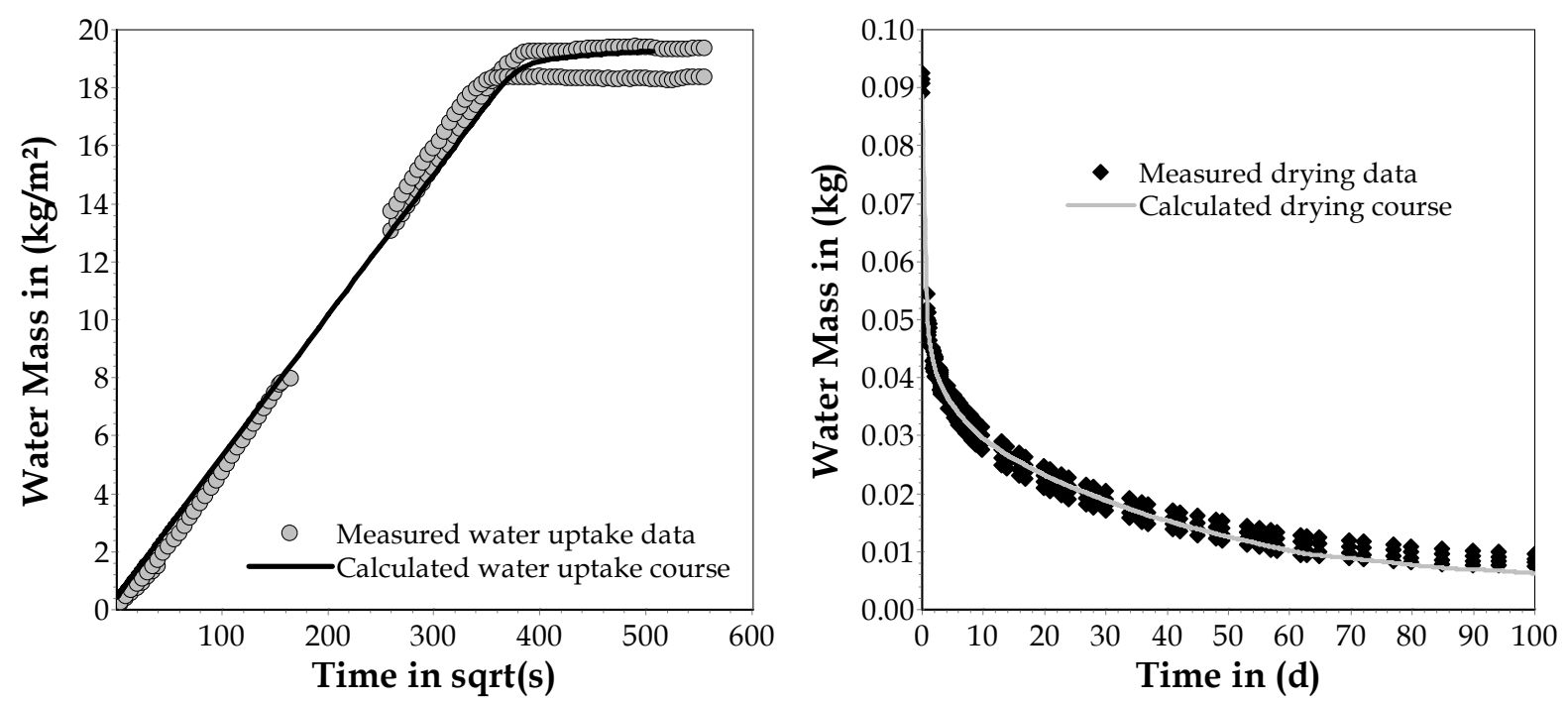

Figure 6.15: Calibration of the lime-sand brick: comparison of measured and simulated courses for water uptake (left) and for drying (right). 


\subsection{Implementation of TDR data evaluation}

To simplify the evaluation of measured TDR data, a second program was developed called TDR-EVALUATION. The main program tasks are the data handling of measured apparent dielectric constant and electric conductivity data having an inexpedient output format, and the further conversion of this measurement data according to different evaluation models. As the electric conductivity is not the topic of the present work, the corresponding models and program features are ignored here. An overview of the different water content related program components is given in Fig. 6.16. These will be sketched as follows.

\section{Reading of measurement data files}

The TDR device used for the instantaneous profile measurements writes the measured data into two files. One file contains the apparent dielectric constant data and the other the apparent electric conductivity data. During the measurement, each sensor channel is stepwise queried leading to an unfavourable output format which consists of a list of increasing time values to which the channel number and the measured value are assigned. As the first TDR evaluation step, the reading of such TDR output files is required. Both, dielectric constant and electric conductivity files can be read which are tested whether they belong to each other or not.

\section{Selection of measurement channels for evaluation}

Due to the unfavourable measurement output format, the second step after input data reading is to select the particular channels which are supposed to be evaluated. For the case of the performed measurements, the five sensors belonging to one material specimen had to be selected out of the twenty channels. In addition, the name for the particular measurement interval can be edited and a time correction can be carried out here.

\section{Derivation of the water content}

The four models ${ }^{1}$ introduced in paragraph 5.3.3.2 TDR data evaluation were implemented to derive the water content from measured apparent dielectric constant data. For the application of these models, the required parameters, in particular the dielectric constants of bound water, free water, the solid matrix and air, have to be supplied. For the estimation of the bound water fraction, the three methods BET, GMM and GIM ${ }^{2}$ were implemented to be evaluated on the basis of measured sorption data. Finally, the reference data for the material specific relation of water content and apparent dielectric constant can be entered.

1. The four models establish a relation between the water content and the apparent dielectric constant. They comprise one empirical approach and three dielectric mixing models requiring a different amount of input data. For more details, please refer to paragraph 5.3.3.2 TDR data evaluation on page 130 et seqq.

2. Concerning the methods BET, GMM and GIM, please refer to section Bound water fraction and inner surface area under paragraph 5.3.3.2 TDR data evaluation on page 133 et seqq. 
Read Input Data

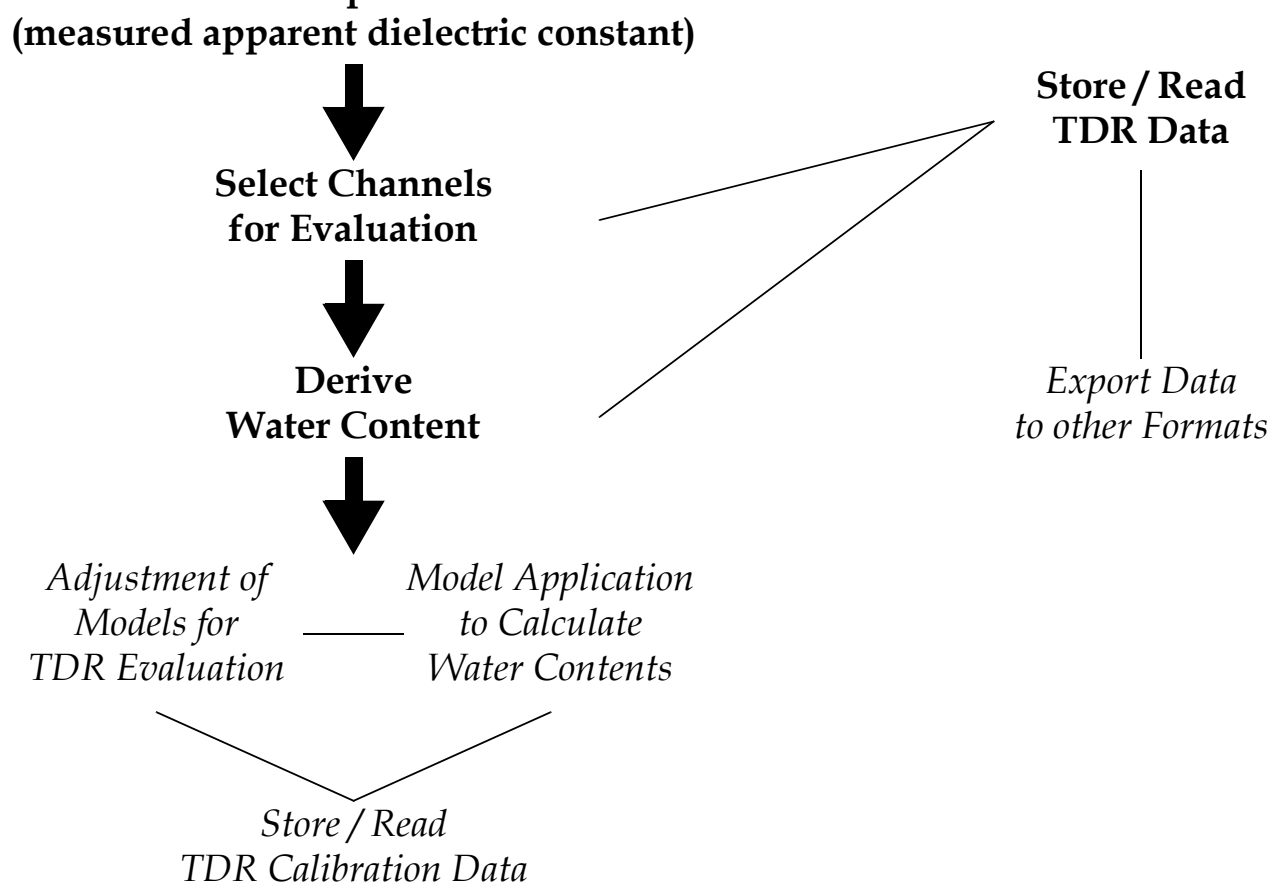

Figure 6.16: Structure of the developed TDR-evaluation program. The lines indicate main interaction paths.

Depending on the available input and reference data sets, one of the four models can be selected. In case of one of the semi-empiric dielectric mixing models, the free parameter can be adjusted to the reference data. At the same time, the model is applied to the selected TDR data delivering the water content versus time for each channel.

The data belonging to the evaluation models, i.e. the reference data as well as the input and modelling parameters for all models, can be saved and read. Although this is not a calibration in its strict sense, this data is called calibration data. It is hence possible to create a calibration data set for each investigated material which can be easily read. This significantly simplifies the TDR data evaluation procedure. For the investigated building materials introduced in Chapter 4, such files were created, too.

\section{Data storage and export}

The output data file of the TDR-EVALUATION is a text file. This text file contains all supplied modelling data and a check which evaluation model was chosen. Then, for all selected channels, the input apparent dielectric constant data as well as the derived water content data is stored. If the file is saved before applying one of the evaluation models, it contains only the input data in a sorted form.

For further graphical evaluation, the data can be exported either in a single data file for each channel or for all channels together in different data arrangements. The options here could be extended easily. Currently, they are adapted to the requirements of the laboratory at the Institute of Building Climatology where the post-processing tool according to Fechner (2006) is applied. 


\subsection{Application of the TDR models}

Within this paragraph, an example will be given for the TDR data evaluation for which one of the lime-sand brick measurements was chosen. The developed program TDR-EVALUATION was applied on the basis of the measured reference data as well as the basic properties of the material.

In a first step, the five channels whose sensors were installed in the lime-sand brick are selected from the TDR output file. These data is transferred to the evaluation routine. Here, depending on the available material and reference data, the evaluation model has to be chosen. Since the lime-sand brick was investigated very intensively, reference data for the apparent dielectric constant - water content relation as well as all other required material data is available. Therefore, the four-phase dielectric mixing model according to eq. (5.38) could be applied.

To feed the model, the following properties have to be known: the dielectric constants of the gaseous phase $\varepsilon_{g}$, of the solid material matrix $\varepsilon_{m}$ and of the free and bound water $\varepsilon_{l}$ and $\varepsilon_{b w}$, the porosity $\theta_{p o r}$ and the bound water content $\theta_{b w}$. To determine $\theta_{b w}$, the inner surface area $A_{i s}$ of the material and the number of molecular water layers $n_{m l w}$ which are regarded as bound water are required. Moreover, since the inner surface area is typically written in $\left[m_{\text {surface area }}^{2} / g_{\text {material }}\right]$, the density is also needed for the conversion to $\left[m_{\text {surface area }}^{2} / m_{\text {material }}^{3}\right]$ as required in eq. (5.39). Finally, the model parameter $\eta_{4}$ has to be adjusted according to the reference data. All measured, derived and adjusted parameters are given in Tab. 6.1.

To determine the inner surface area of the lime-sand brick, the introduced adsorption models were applied to measured adsorption data. A comparison of the relevant graphical illustrations is given in Fig. $6.17^{1}$. At the left, the mono layer capacity determination according to the BET method is shown. For the lime-sand brick, this method appears to be suitable for relative humidities up to 0.5. Due to the few single measurement values, the Gibbs Minimum Method is of less applicability as it requires more data points for very low relative humidities to determine the actual minimum, as illustrated in the middle graphic. The Gibbs Integral Method, shown at the right of Fig. 6.17, delivers
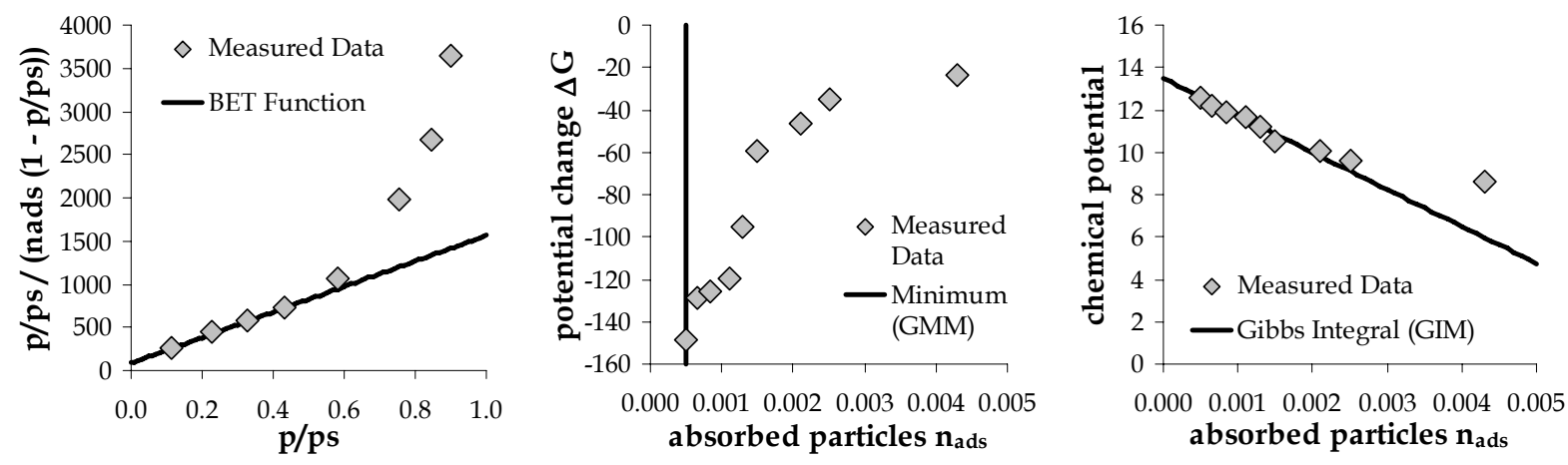

Figure 6.17: Comparison of the different models to derive the inner surface area from measured adsorption data. BET method left, Gibbs Minimum Method in the middle and Gibbs Integral Method at the right.

1. Compare also Fig. 5.32 under paragraph 5.3.3.2 TDR data evaluation on page 135. 
the most reliable results as it is valid for a relative humidity range up to 0.9 . Therefore, the inner surface area was applied as determined by this method, although the values derived by the other methods are - for this material - of the same order of magnitude:

$$
\begin{aligned}
& \text { - } A_{i s, B E T}=19.59 \cdot \mathrm{m}^{2} / \mathrm{g} \\
& \text { - } A_{i s, G M M}=17.70 \cdot \mathrm{m}^{2} / \mathrm{g} \\
& \text { - } A_{i s, G I M}=20.18 \cdot \mathrm{m}^{2} / \mathrm{g}
\end{aligned}
$$

The dielectric constant of the solid matrix $\varepsilon_{s}$ was derived from the reference data. The parameter $\eta_{4}$ as well as the number of molecular water layers $n_{m l w}$ to be regarded as bound water was adjusted to fit the curve to the reference data. A comparison of the adjusted model function with this data is given in Fig. 6.18.

One can see that the reference data of the apparent dielectric constant - water content relation does not strictly follow the functional course the model assumes. However, with respect to the material variation, the adjusted curve represents the measured data quite well, especially for the hygroscopic moisture content range comprising moisture contents up to $\theta_{l}=0.7 \cdot \mathrm{m}^{3} / \mathrm{m}^{3}$. This curve was then used to derive the water content from the measured TDR data of the lime-sand brick.

In the same way, the four-phase dielectric mixing model was applied to the other materials. Based on the adsorption data, the inner surface area and hence the bound water fraction was determined. The modelling parameters $\eta_{4}$ and $n_{m l w}$ were then adjusted according to the measured reference data.

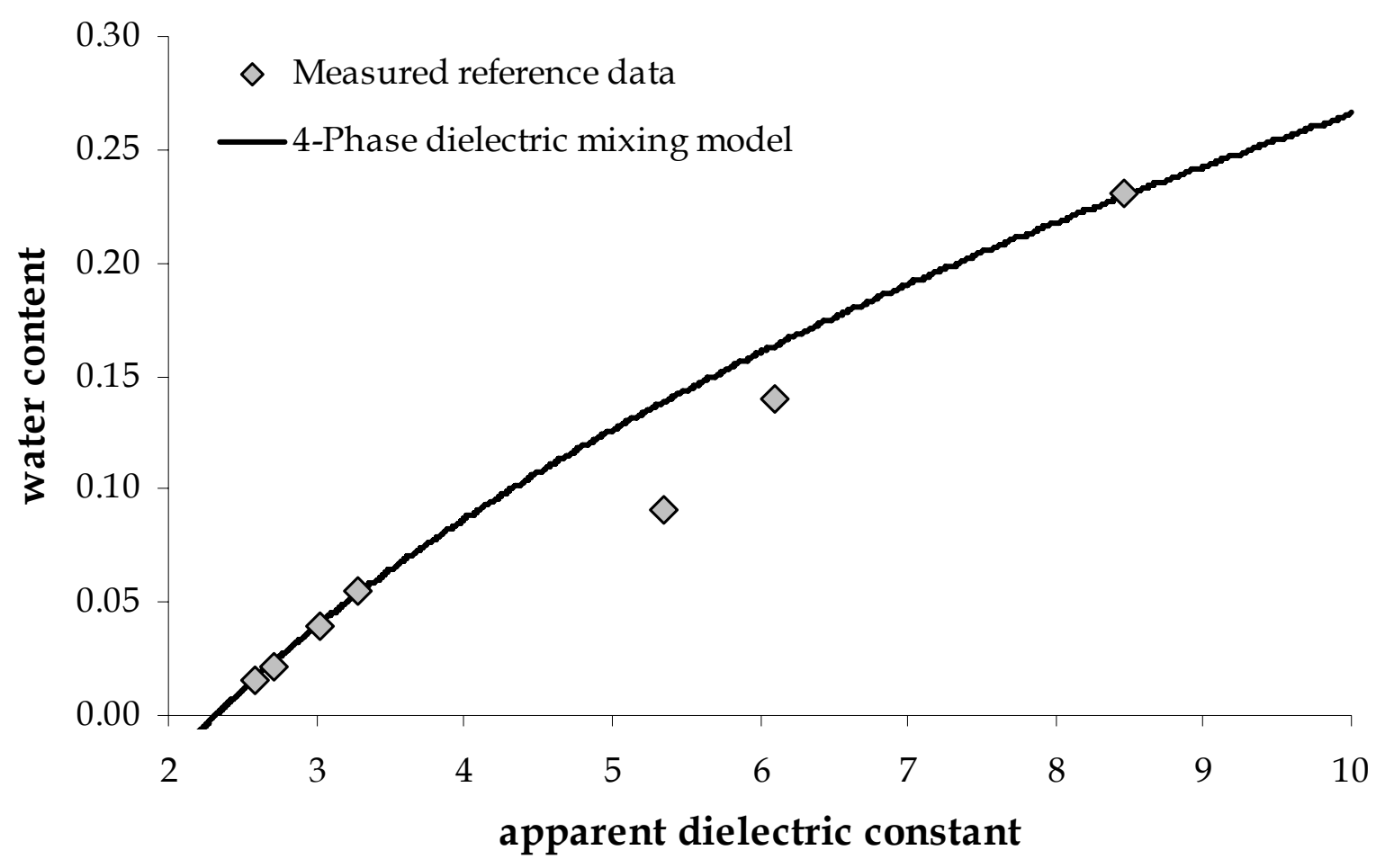

Figure 6.18: Relation between water content and apparent dielectric constant for the limesand brick. Comparison of measured reference data with the adjusted 4-phase dielectric mixing approach. 
An illustration of the other TDR evaluation models is given in Fig. 6.19 .

As far as required by the model, the same parameters were used as given in Tab. 6.1. The empirical as well as the physical model following eq. (5.32) and (5.34) are too inflexible to follow the reference data. Obviously, the structure of the lime-sand brick does not correspond with the geometrical configuration which

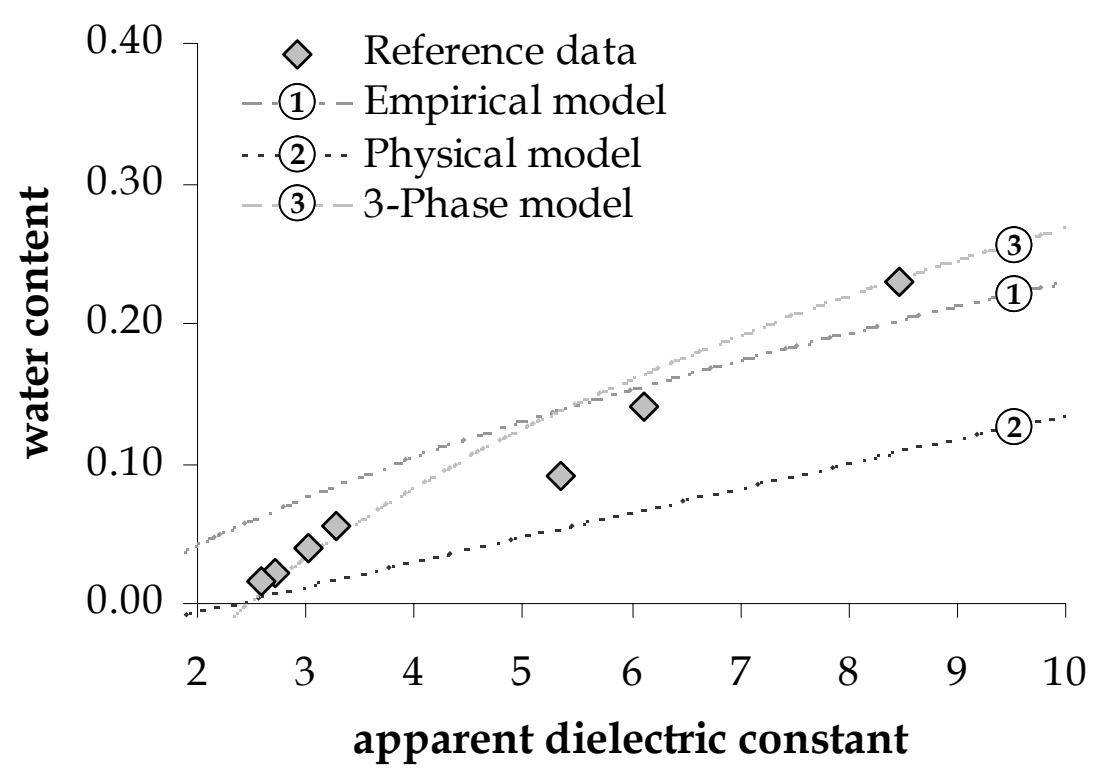
was assumed during

Figure 6.19: Comparison of the empirical and the other dielectric mixing models with the measured reference data the model derivation.

The three-phase dielectric mixing model according to eq. (5.36) follows the measured course with almost the same agreement as the four-phase model. Small deviations, however, occur especially for lower moisture contents. This is due to the small shift caused by the account of the bound water which is only included in the four phase models. The parameter $\eta_{3}$ was adjusted to $\eta_{3}=0.23$ for the curve shown in Fig. 6.19.

Finally, a derived water content profile is shown in Fig. 6.20. The measured data belongs to the lime-sand brick during a hygroscopic adsorption from 33\% to $97 \%$ relative humidity. The evaluation was done with the four-phase dielectric mixing model according to eq. (5.38) and Fig. 6.18 with the parameters given in Tab. 6.1.

Table 6.1: Parameters for the TDR-evaluation of the lime-sand brick.

\begin{tabular}{lcclcc}
\hline Parameter & Value & Unit & Parameter & Value & Unit \\
\hline gaseous phase $\varepsilon_{g}$ & 1.00 & {$[---]$} & density $\rho_{b}$ & 1813.5 & {$\left[\mathrm{~kg} / \mathrm{m}^{3}\right]$} \\
solid phase $\varepsilon_{m}$ & 3.75 & {$[---]$} & porosity $\theta_{\text {por }}$ & 0.3436 & {$\left[\mathrm{~m}^{3} / \mathrm{m}^{3}\right]$} \\
free water $\varepsilon_{l}$ & 80.00 & {$[---]$} & inner surface area $A_{i s}$ & 20.184 & {$\left[\mathrm{~m}^{2} / \mathrm{g}\right]$} \\
bound water $\varepsilon_{b w}$ & 6.20 & {$[---]$} & number of water layers $n_{m l w}$ & 1.5 & {$[---]$} \\
model parameter $\eta_{4}$ & 0.28 & {$[---]$} & bound water fraction $\theta_{b w}$ & 0.01647 & {$\left[\mathrm{~m}^{3} / \mathrm{m}^{3}\right]$} \\
\hline
\end{tabular}




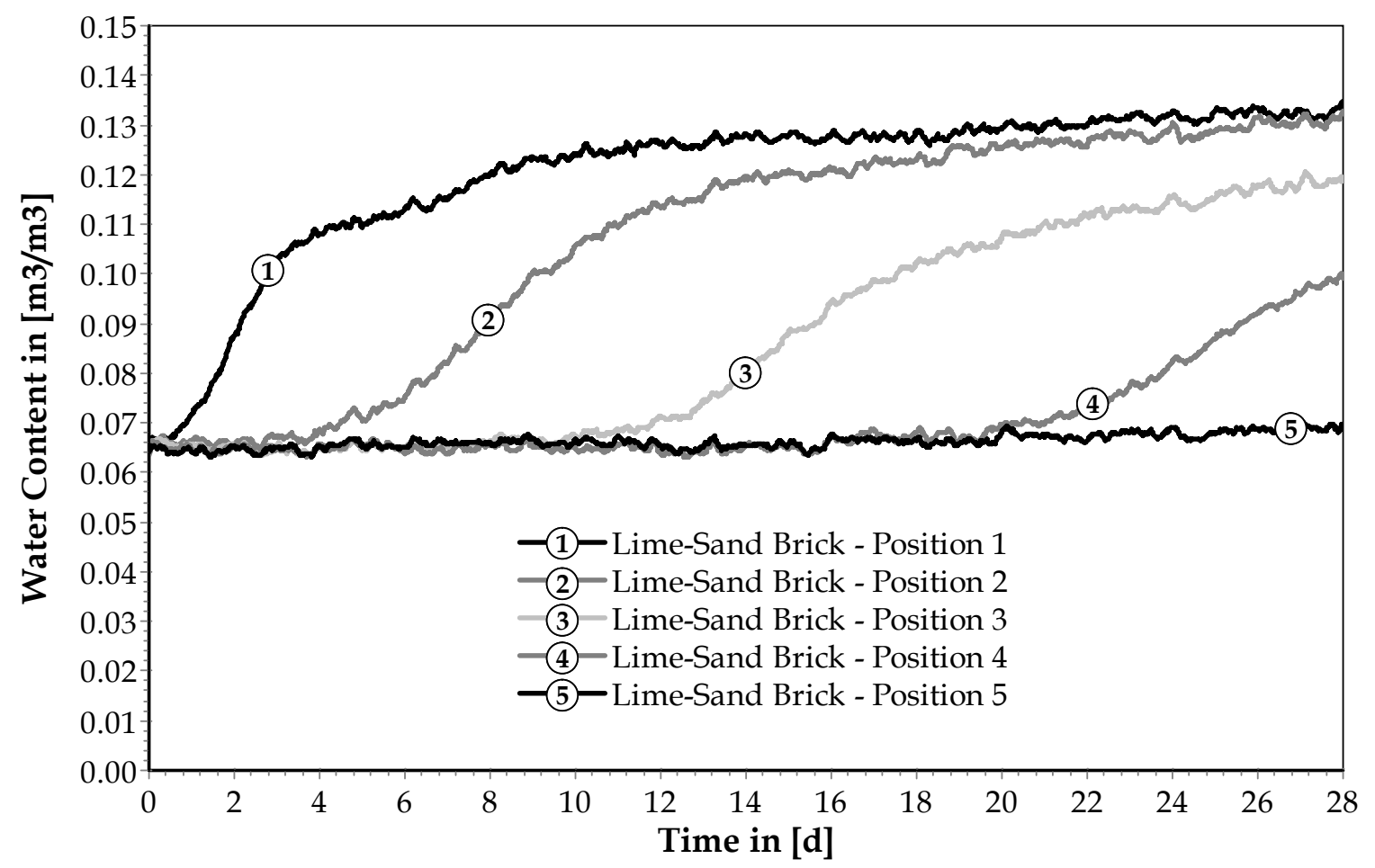

Figure 6.20: Evaluated water content profile measured with TDR sensors inside the limesand brick during an adsorption process from $33 \%$ to $97 \%$ relative humidity. Position 1 was exposed to the boundary condition - see also Fig. 5.27 on page 126 for the measurement set-up.

\subsection{Conclusions}

Within the first part of this chapter, the developed computer program to apply the material model to the measured basic material data was introduced in its basic components. Subsequently, a process scheme showing the dependencies of the calibration procedure as an interaction of numerical simulation, model data application and data evaluation was given highlighting its complexity. Ultimately, the adjusted moisture storage and transport functions were discussed for all four building material and the calibration quality was proved by the comparison of measured and simulated water uptake and drying curves.

The second part of this chapter focused on the other developed computer program dealing with the evaluation of measured TDR data. The program was briefly introduced in its basic structure. Then the application of the implemented models was exemplarily demonstrated for a data-set of the lime-sand brick.

With these developments and their application to either the measured basic material properties or the instantaneous profile measurements, the final prerequisite steps approaching the material model validation were accomplished. 


\section{CHAPTER 7}

\section{SIMULATION AND VALIDATION}

Within this chapter, the two widely elaborated and strengthened fields of experimental investigation and material modelling are combined opening new perspectives on both, the actual quality of material modelling within the frame of numerical simulation, and the driving forces of moisture transport with their dependencies on the state, the process and its history.

The large number of conducted IPM experiments is evaluated. The material model is adjusted and calibrated by the help of the basic hygrothermal properties. Applying the measured initial and boundary conditions, all experiments are simulated using the DELPHIN5 program according to Nicolai (2006). Ultimately, measured and simulated results are compared enabling a fundamental model validation.

Due to the large amount of data the following paragraphs will present only the most meaningful data-sets comprising a representative selection of measured and simulated experiments. A distinction is made between the hygroscopic and the overhygroscopic moisture content range with the corresponding experiments. A single treatment of the investigated building materials appears though to be of less significance as similar tendencies were witnessed. Therefore, the discussion follows the general observations on the broad but explicitly no further distinguished experimental basis.

\subsection{Hygroscopic moisture transport}

The measured data comprises profiles of water content, temperature and relative humidity versus time for the IPM experiments within the hygroscopic moisture content range. Despite the intended simulation of these experiments on the basis of the 
adjusted material functions and the measured initial and boundary conditions, it is reasonable to visualise the transient sorption isotherms. The moisture content data is therefore plotted versus the relative humidity data for the conducted experiments. Their discussion forms the first subsection followed by the simulation results. The assessment of the material model with regard to hygroscopic moisture transport processes will conclude this paragraph.

\subsubsection{Transient moisture storage data}

The first data shown and discussed belongs to the investigated calcium silicate. In Fig. 7.1, the transient moisture storage data of an adsorption process from 33\% to $97 \%$ relative humidity is displayed (large step increase according to Tab. 5.2 on page 126). The single data points belong to the five compartments into which the material sample is subdivided according to the installed TDR and temperature - relative humidity sensors. Position 1 indicates the bottom compartment which is exposed to the boundary conditions whereas position 5 refers to the top section. See paragraph 5.3.1 Measurement set-up for the hygroscopic moisture range and especially Fig. 5.27 on page 126 for the measurement configuration.

The continuous lines in all these figures represent the adjusted adsorption and desorption moisture storage functions according to the applied material model and the measured sorption and retention data. Due to the fact that these data are obtained from static equilibrium measurement methods, the displayed curves are regarded as static equilibrium adsorption and desorption functions.

The measured data points in Fig. 7.1 show a significant deviation from the static moisture storage curves. Generally, the transient data takes lower moisture content values for a given relative humidity than the adjusted functions. Especially positions 1 and 2 follow the strong increase in applied boundary relative humidity with increasing moisture contents. However, the corresponding relative humidity inside the material seems to be shifted to higher values.

In Fig. 7.2, data of the same material specimen but for the subsequent desorption process from $97 \%$ to $33 \%$ relative humidity is given (large step decrease according to Tab. 5.2 on page 126). Again the data points indicate the different measurement positions, the solid lines represent the static ad-and desorption data. Here, the transient data takes generally higher moisture content values than during the adsorption. Especially position 1 exhibits a very interesting behaviour. While during the adsorption, the relative humidity increased rapidly first before the moisture content increased slowly, too, the opposite occurs during the desorption. The relative humidity decreases much faster than the moisture content.

The second compartment (position 2) shows a similar, but much less pronounced behaviour. Here, the followed transient curves are much closer to the static ones than those of position 1. The other compartments follow in principle the same path for both depicted experiments with slightly higher moisture content values for the desorption.

The measured data of the adsorption - desorption cycle are shown in Fig. 7.3, at the left for position 1 and at the right for position 2. The comprised hysteresis loop is much more pronounced for position 1 than for position 2. In both cases though, this loop is larger than the hysteresis measured under static conditions. Position 1 is exposed to the 
boundary conditions. Therefore, the largest gradients occur within this compartment. The observed material behaviour indicates, that the step-size of the applied boundary condition and the size of the arising moisture potential gradients influence the moisture content - moisture potential relation which is followed under transient conditions.

Looking at Fig. 7.4 underlines this observation. It shows the hysteresis loop measured during an adsorption - desorption cycle from $85 \%$ to $97 \%$ to $85 \%$ relative humidity (stepwise increase (3) and stepwise decrease (1) according to Tab. 5.2 on page 126). The loop of position 1 is given in the left chart, the one of position 2 is shown on the right hand side. Two observations can be made from these charts. First, the moisture level of the measured courses is generally lower than the static storage functions. Only the transient desorption branch of position 1 intersects with the static curves. And second, the hysteresis loop is much less pronounced compared with the large step cycle of 33\%97\%-33\%. At position 2 (right chart in Fig. 7.4), there is almost no hysteretic behaviour cognizable. At position 1 (left chart in Fig. 7.4), it is existent and still more pronounced than the static hysteresis, but the strong deviations observed in Fig. 7.3 during the large step cycle did not occur during the smaller steps shown in Fig. 7.4.

These first results reveal that the flow states occurring under transient conditions do not follow the measured equilibrium curves. This means that additionally to the state and the process history, also the process itself, i.e. the size of the occurring moisture potential gradients, influence the moisture transport behaviour of a building material. This is a rather new observation in Building Physics which has not been a research topic, yet. In Soil Science, authors of previous studies obtained similar results which gave first evidence on these so called "dynamic effects". The discussion in paragraph 7.1.3 Concluding hygroscopic assessment will give a brief review of dynamic effects in literature. 


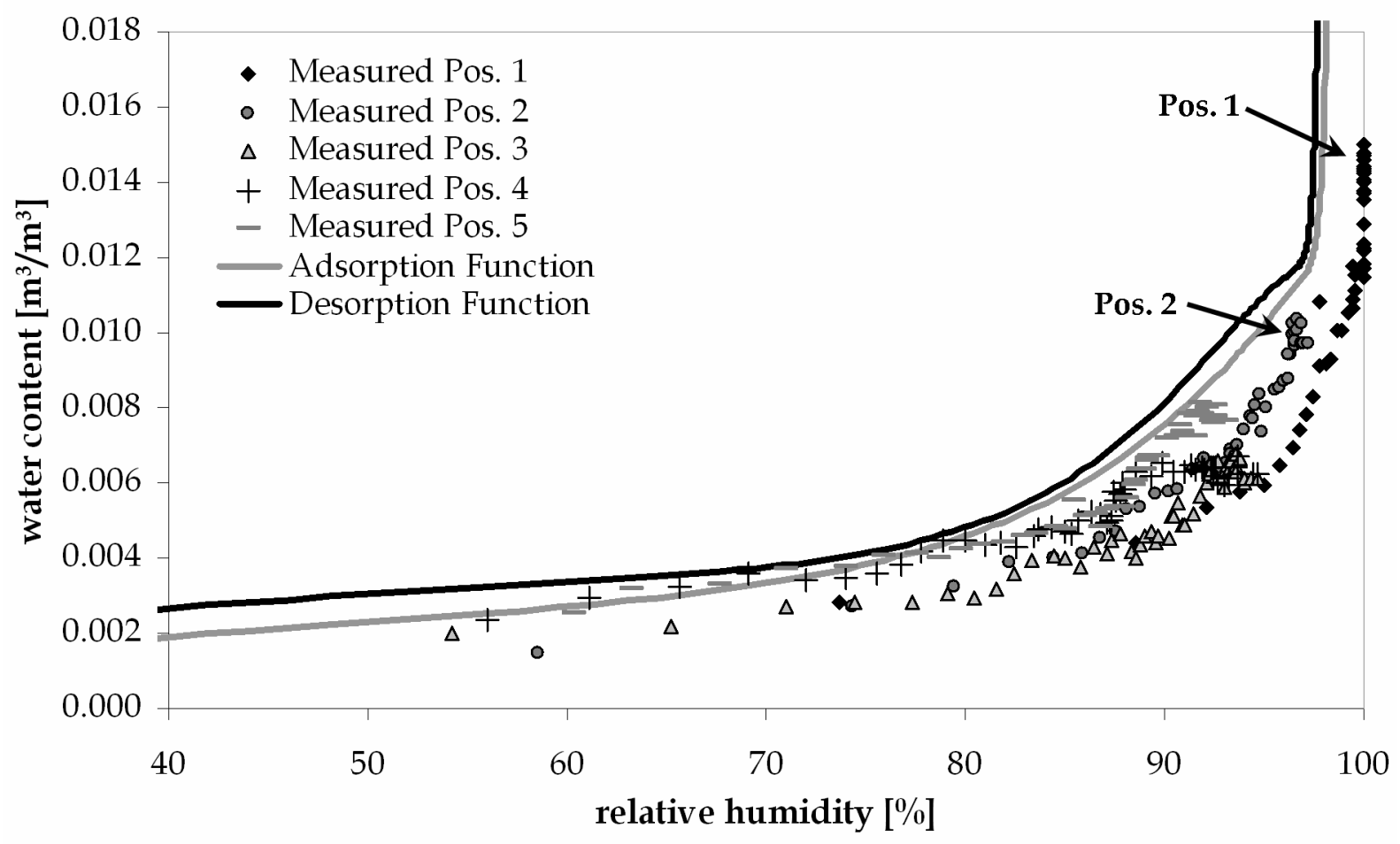

Figure 7.1: $\quad$ Transient moisture storage data of the calcium silicate during adsorption from $33 \%$ to 97\% relative humidity. The continuous lines represent the adjusted adand desorption functions shown and discussed in Chapter 6.

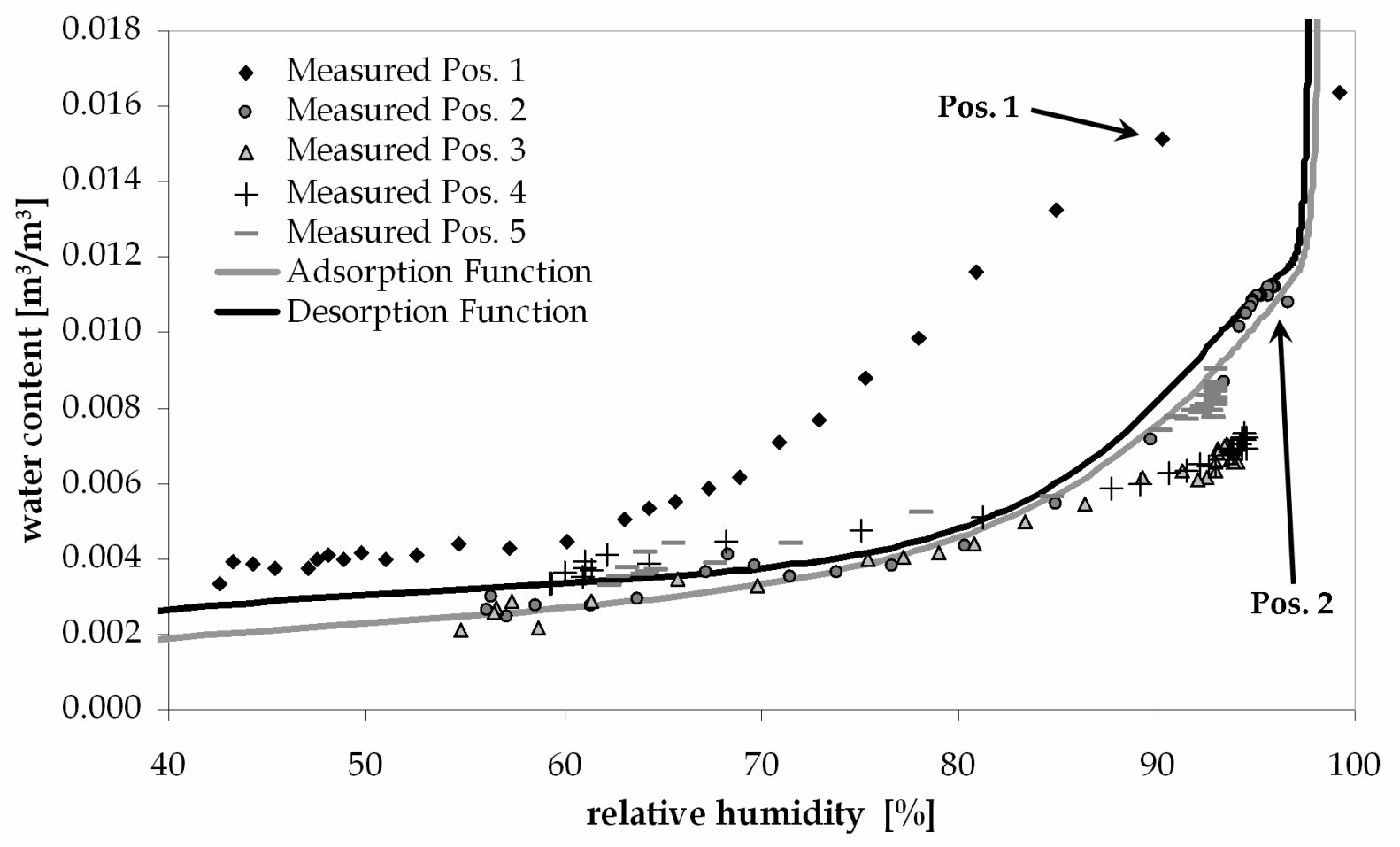

Figure 7.2: Transient moisture storage data of the calcium silicate during desorption from $97 \%$ to $33 \%$ relative humidity. The continuous lines represent the adjusted adand desorption functions shown and discussed in Chapter 6. 

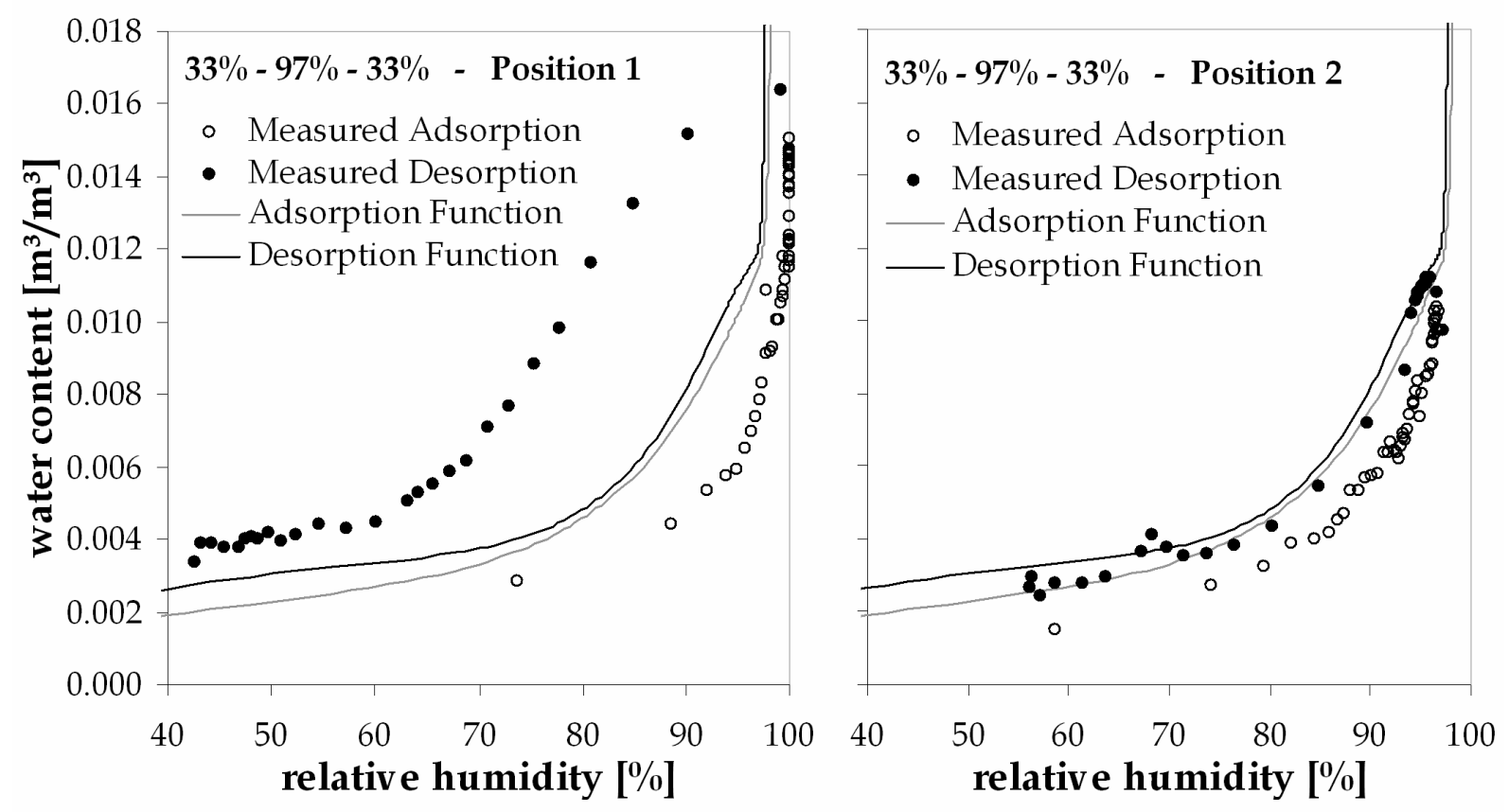

Figure 7.3: Dynamic hysteresis loop during a cycle of 33\%-97\%-33\% relative humidity for the calcium silicate. The left chart shows position 1 which is exposed to the boundary condition, the right chart shows position 2.
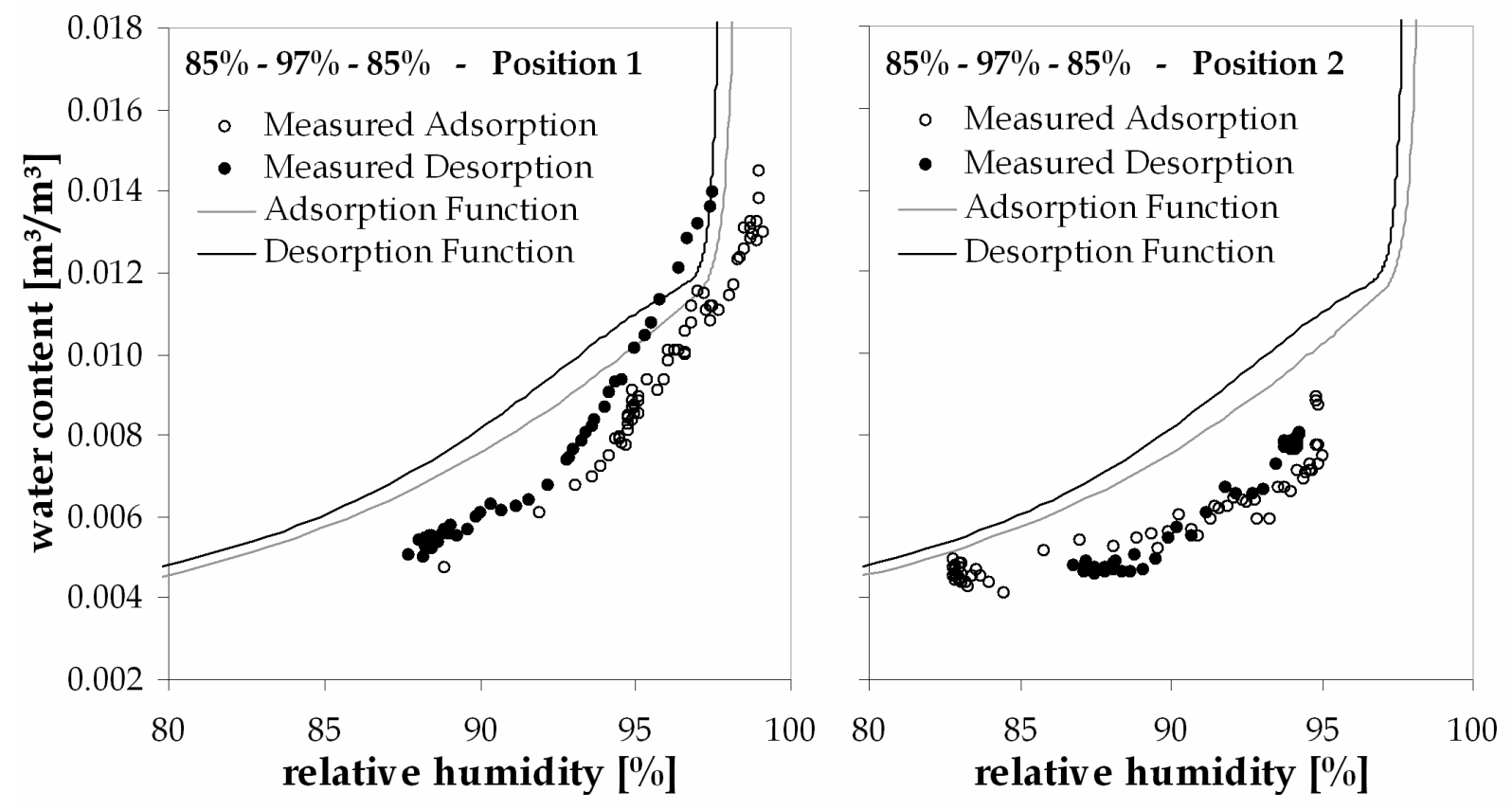

Figure 7.4: Dynamic hysteresis loop during a cycle of $85 \%-97 \%-85 \%$ relative humidity for the calcium silicate. The left chart shows position 1 which is exposed to the boundary condition, the right chart shows position 2. 


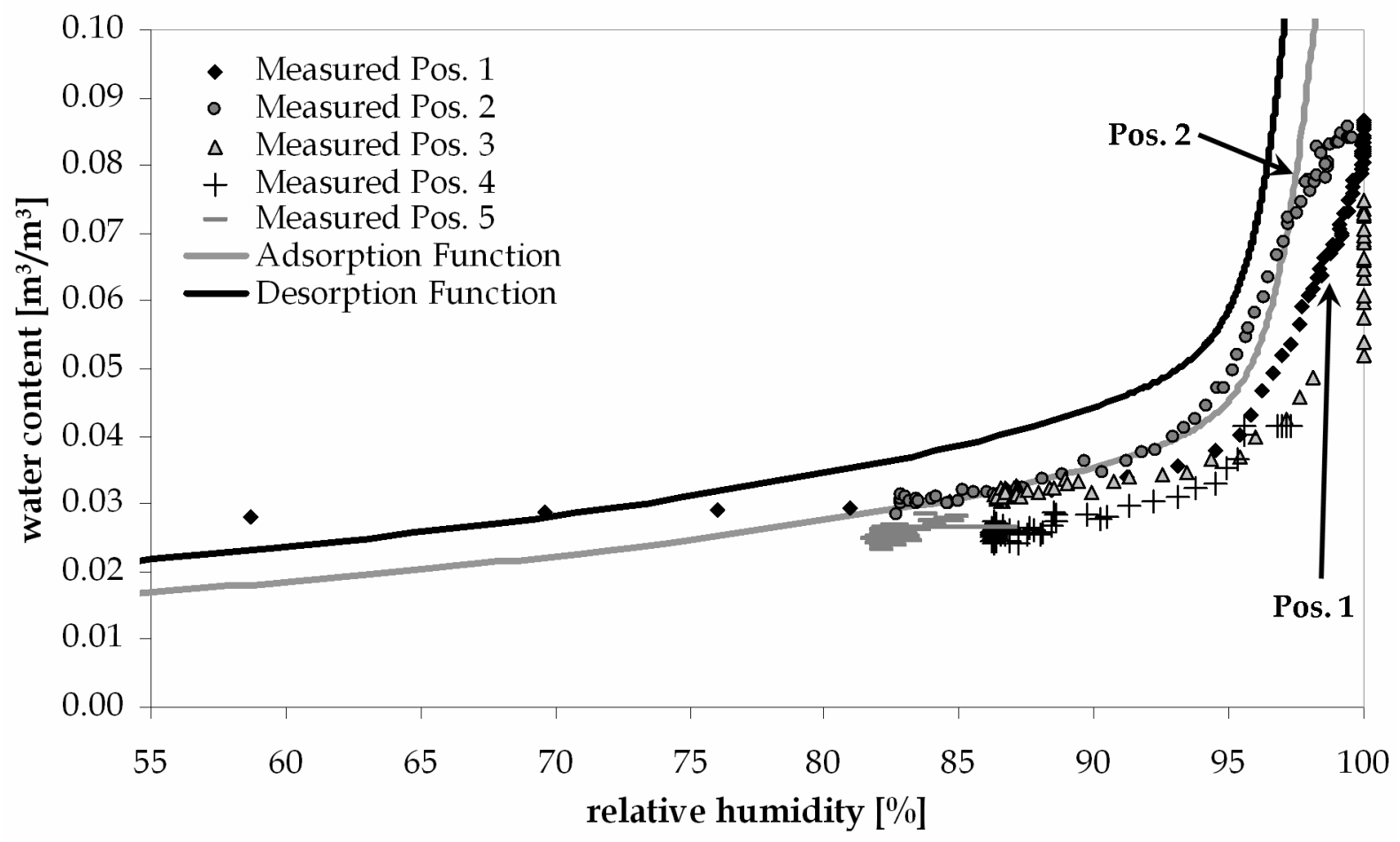

Figure 7.5: Transient moisture storage data of the lime-sand brick during adsorption from $33 \%$ to $97 \%$ relative humidity.

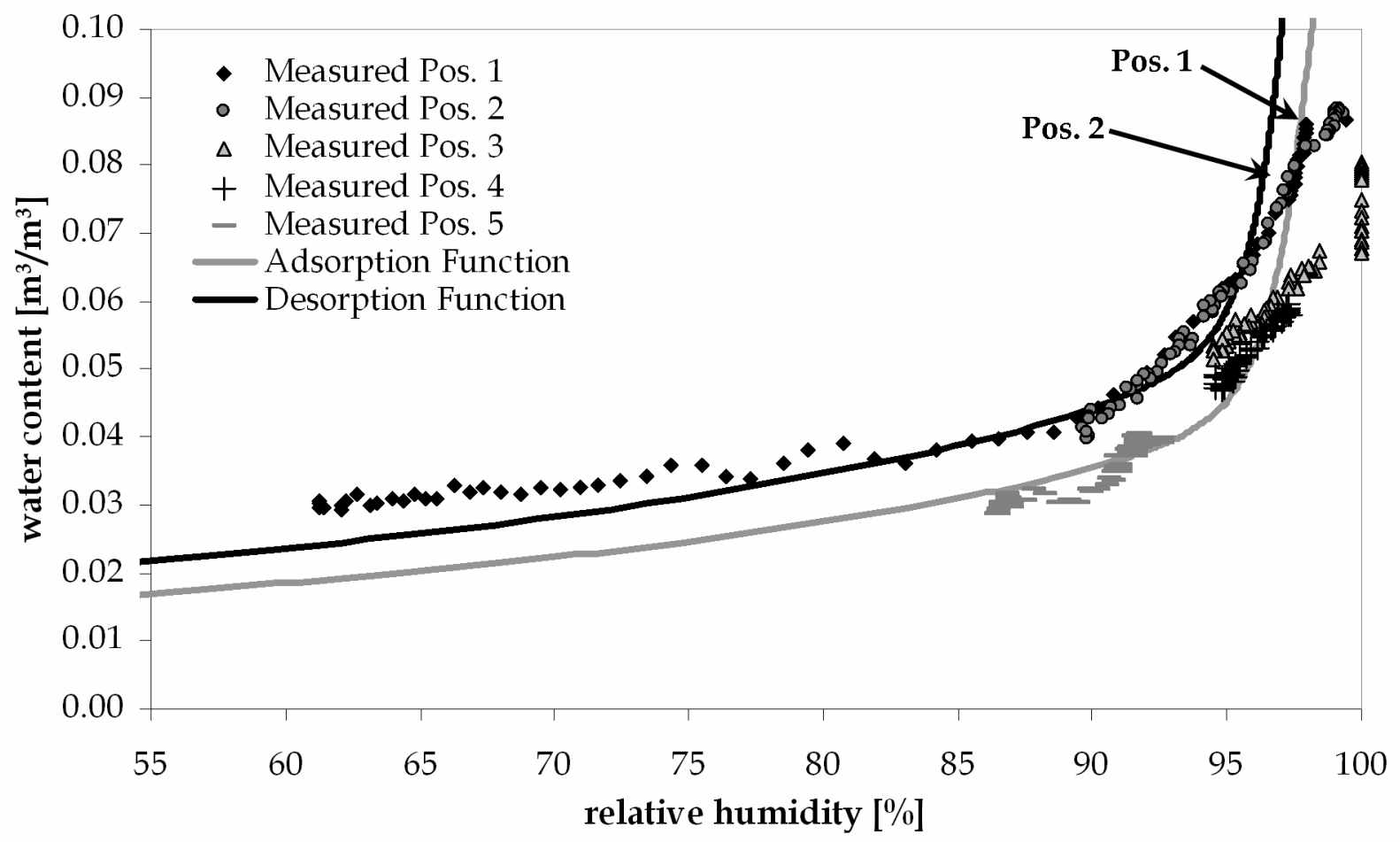

Figure 7.6: $\quad$ Transient moisture storage data of the lime-sand brick during the subsequent desorption from $97 \%$ to $33 \%$ relative humidity. 

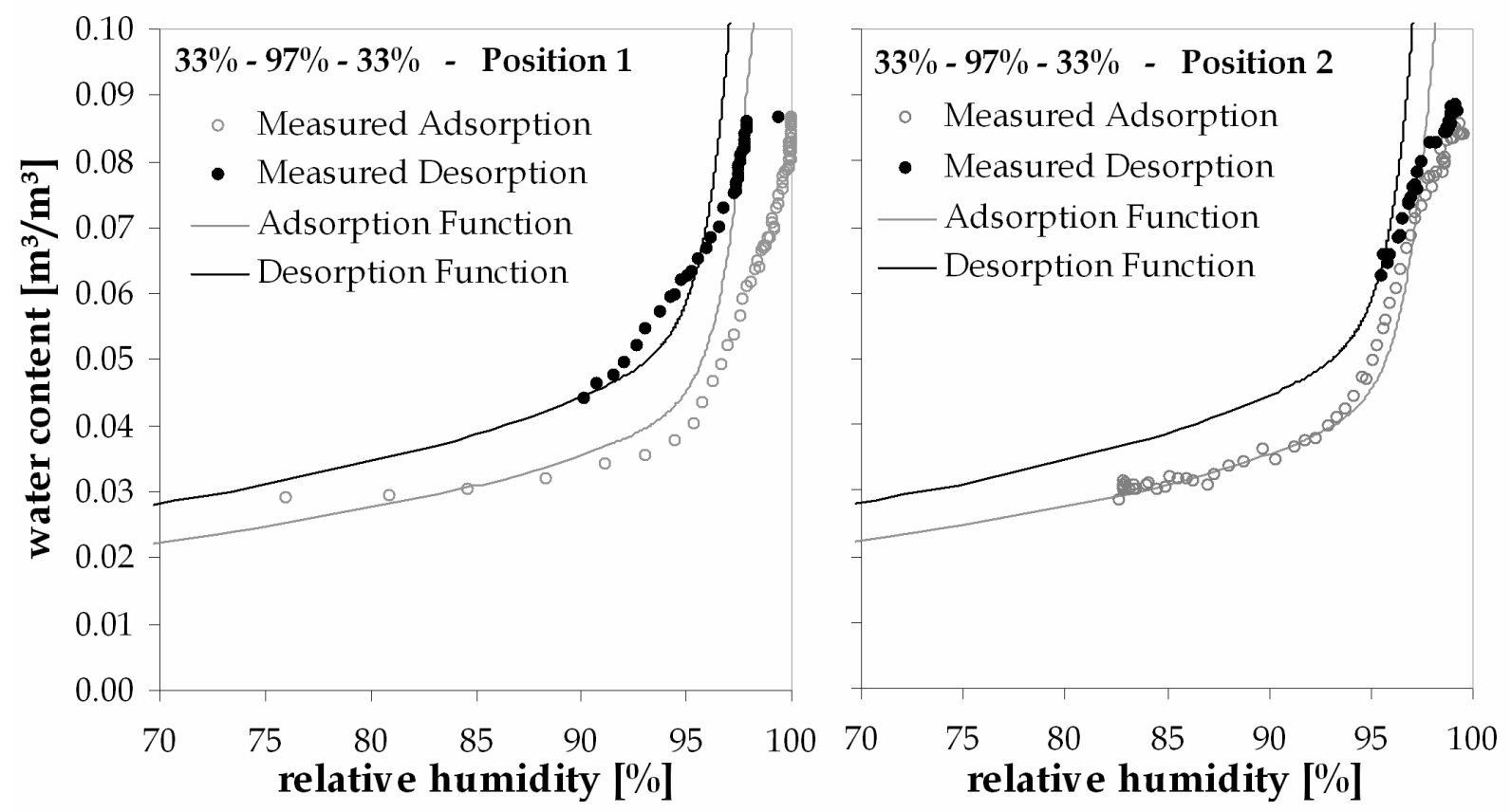

Figure 7.7: Dynamic hysteresis loop during a cycle of 33\%-97\%-33\% relative humidity for the lime-sand brick. The left chart shows position 1, which is exposed to the boundary condition, the right chart shows position 2.
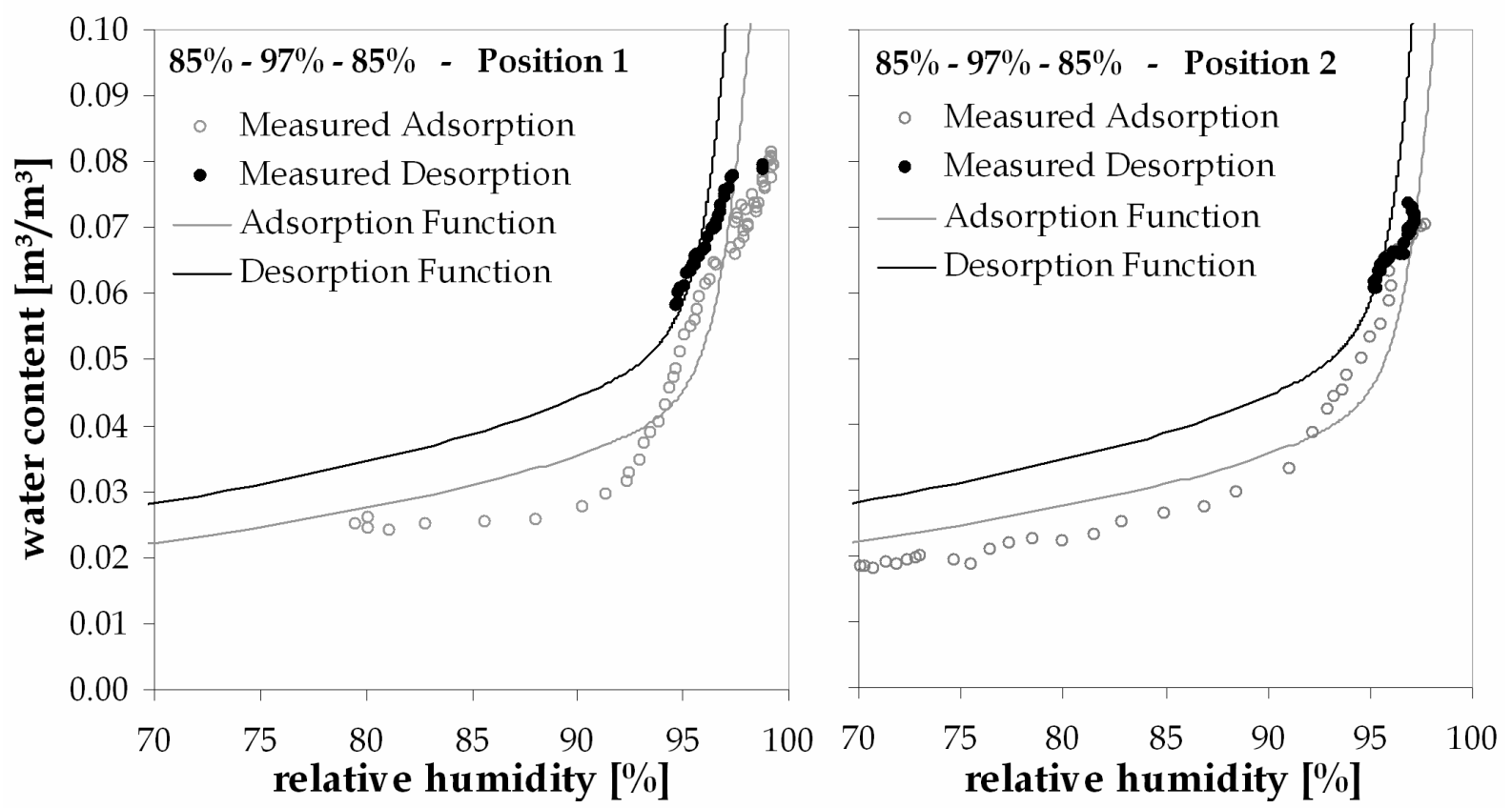

Figure 7.8: Dynamic hysteresis loop during a cycle of $85 \%-97 \%-85 \%$ relative humidity for the lime-sand brick. The left chart shows position 1 which is exposed to the boundary condition, the right chart shows position 2. 


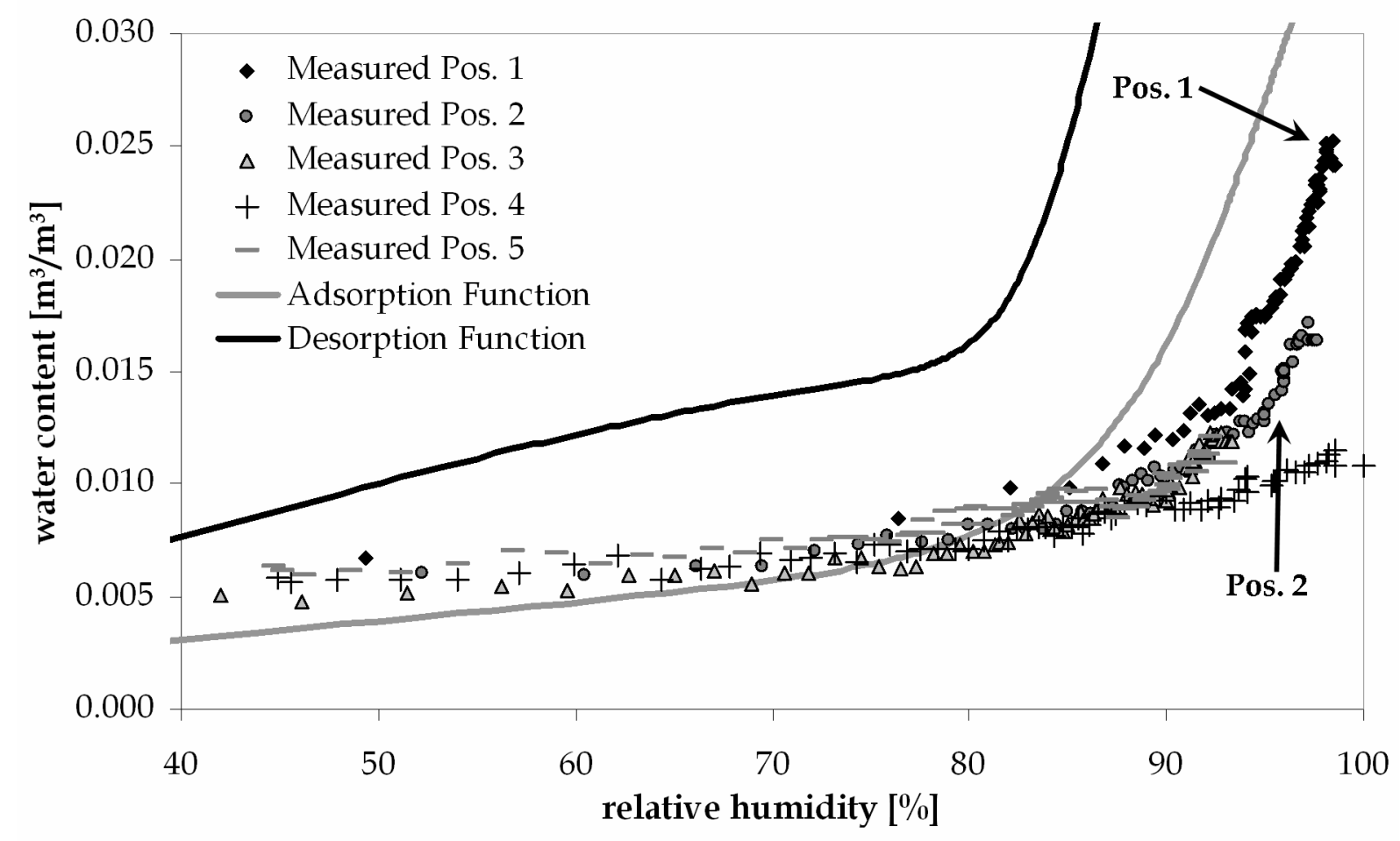

Figure 7.9: Transient moisture storage data of the aerated concrete during adsorption from $33 \%$ to $97 \%$ relative humidity.

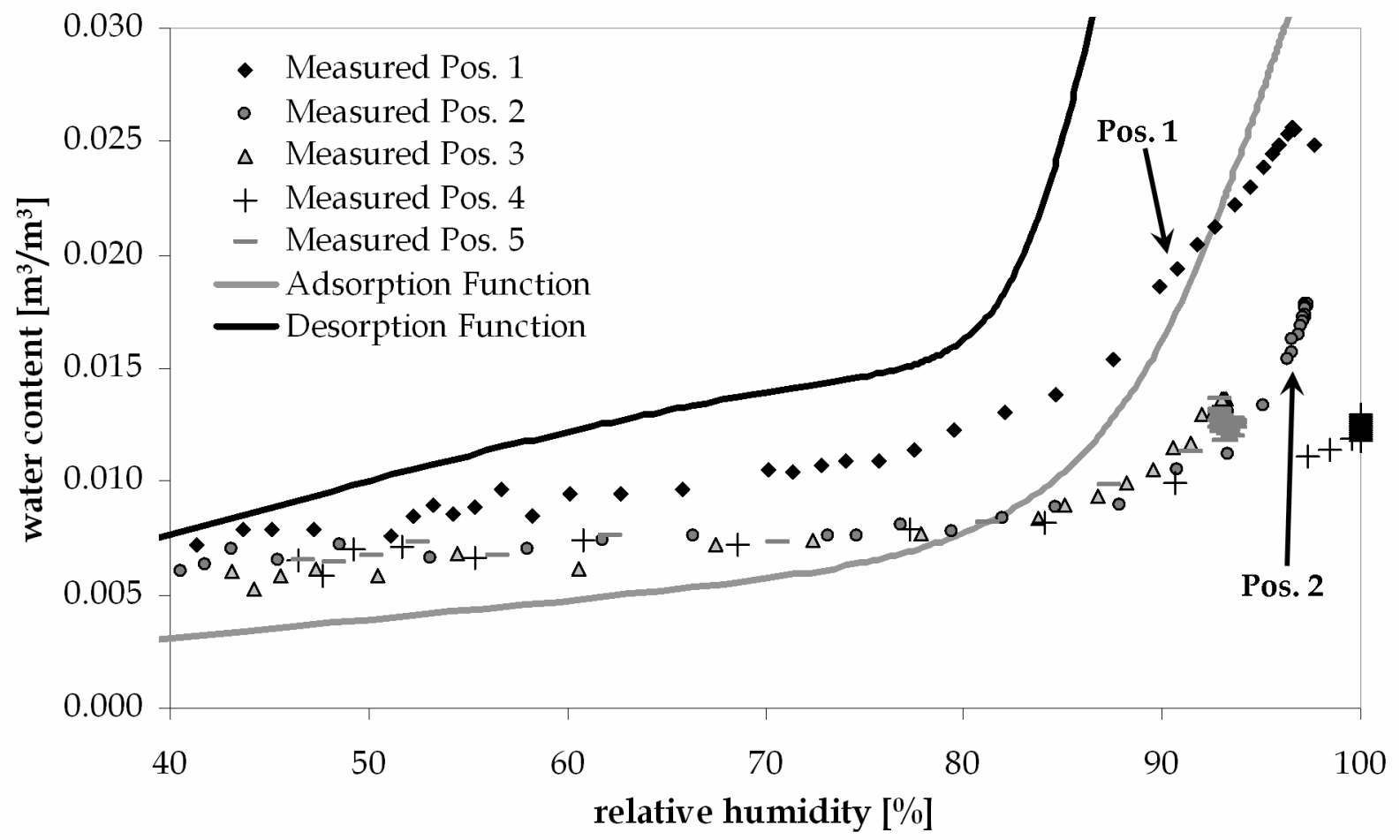

Figure 7.10: Transient moisture storage data of the aerated concrete during the subsequent desorption from $97 \%$ to $33 \%$ relative humidity. 

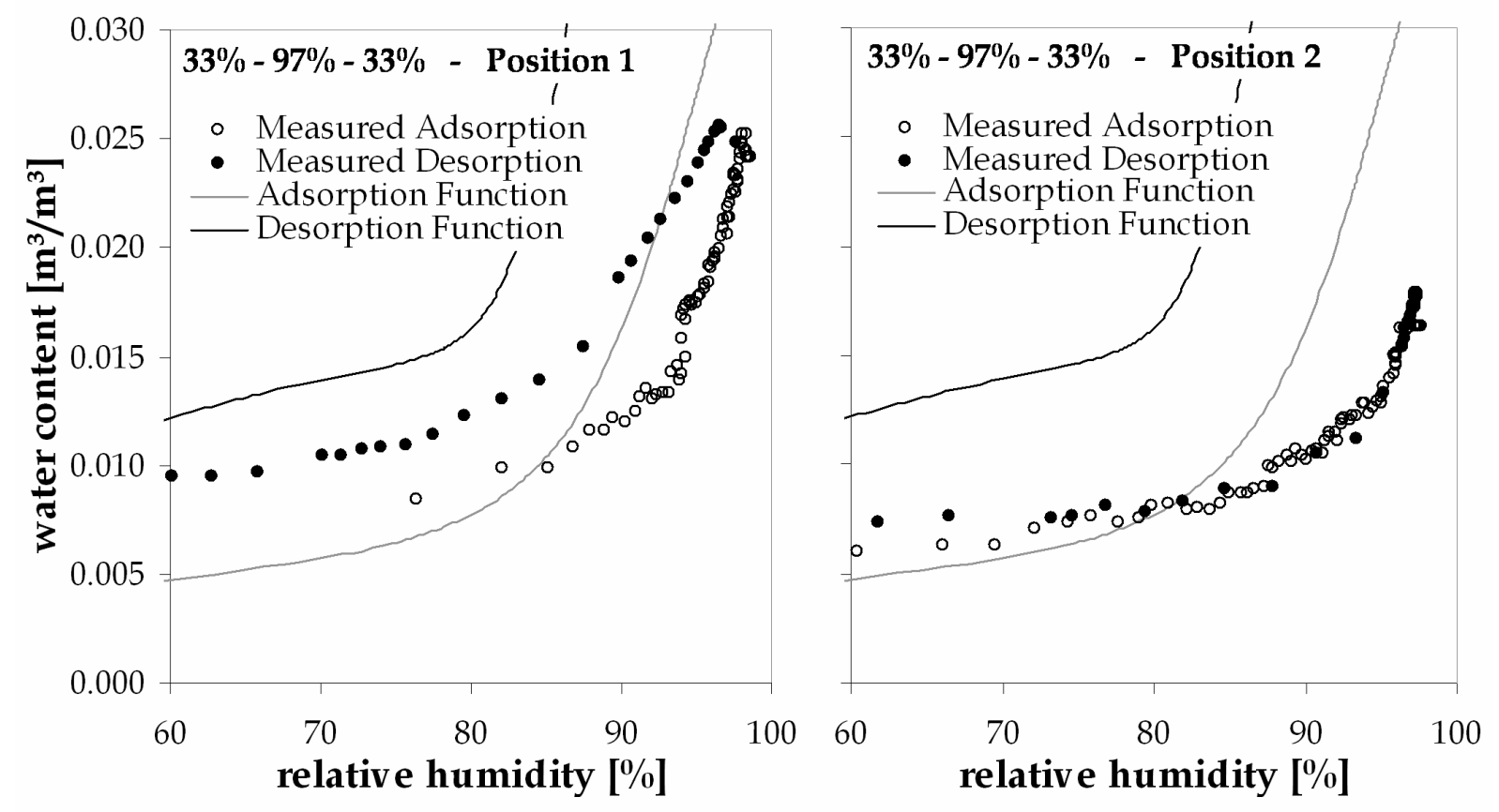

Figure 7.11: Dynamic hysteresis loop during a cycle of 33\%-97\%-33\% relative humidity for the aerated concrete. The left chart shows position 1, which is exposed to the boundary condition, the right chart shows position 2.
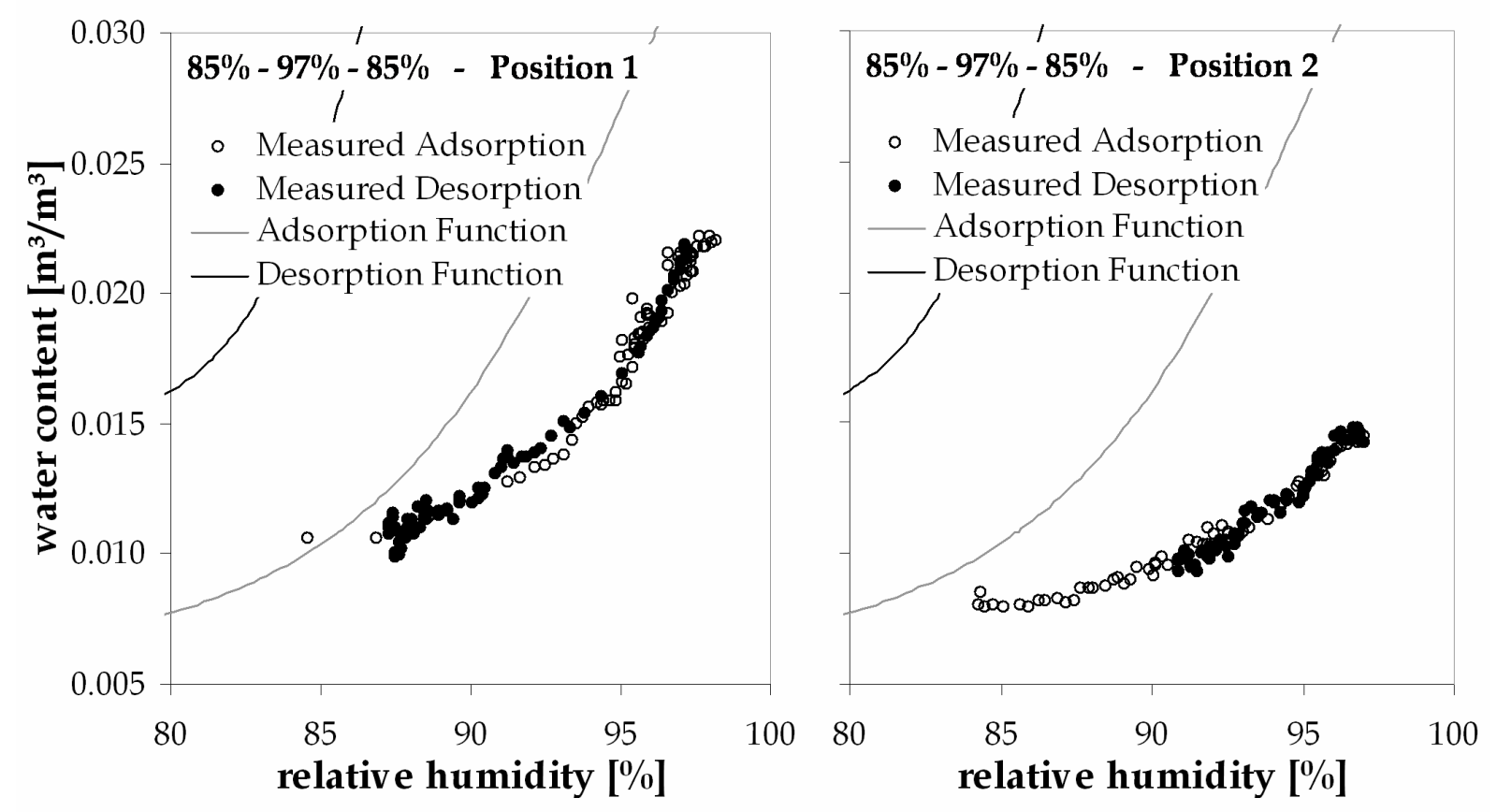

Figure 7.12: Dynamic hysteresis loop during a cycle of $85 \%-97 \%-85 \%$ relative humidity for the aerated concrete. The left chart shows position 1 which is exposed to the boundary condition, the right chart shows position 2. 
The experiments carried out for the building brick are less clear than those of the calcium silicate. Due to the very low sorption isotherm which starts to strongly increase not until relative humidities above $97 \%$, the differences in moisture content during the applied relative humidity steps are very small (see Tab.5.2 on page 126). For most measurement steps, they vary within the precision range of the employed TDR sensors. This low hygroscopic moisture capacity causes the vapour to condensate inside the relative humidity sensors when the high boundary humidity is applied. The sensors report values of $100 \%$ relative humidity then, making part of the large step data of position 1 infeasible.

Thus, the data to be shown here is the hysteresis loop obtained for position 2 of the building brick during the large step adsorption - desorption cycle. It is given in Fig. 7.13 together with the static adsorption and desorption functions. The hysteresis is visible but less pronounced than the one obtained from static experiments. Dynamic effects are not visible within the second compartment.

By means of Fig. 7.13, one of the advantages of the applied TDR technology becomes vividly apparent. The building brick provides a very low

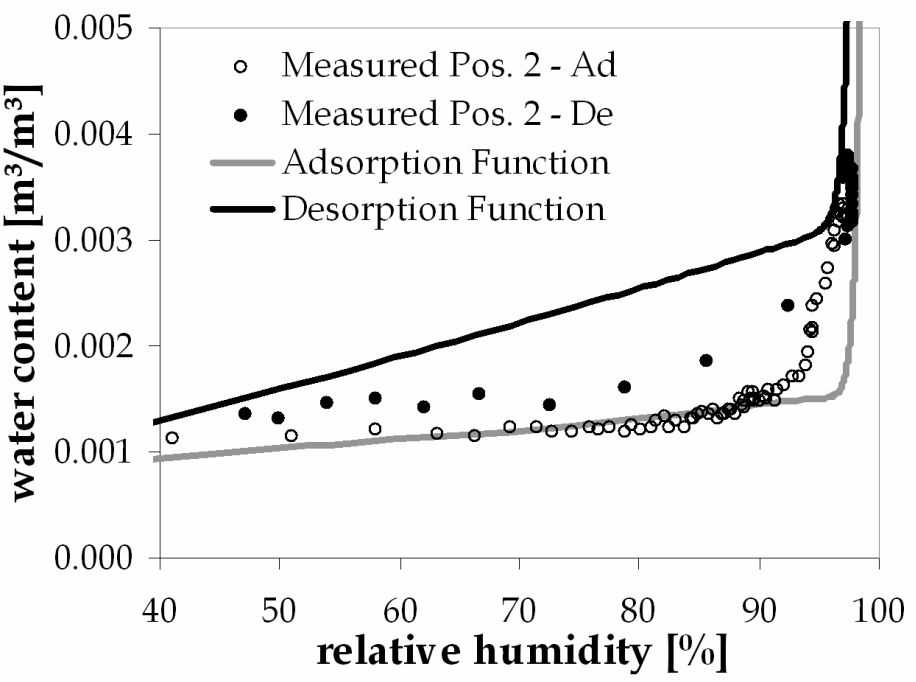

Figure 7.13: Dynamichysteresis loop of the building brick, position 2, during ad-and desorption from $33 \%$ to $97 \%$ to $33 \%$ relative humidity. hygroscopic moisture storage function. The water content changes due to the applied boundary humidity step are therefore very small. Nevertheless, it is possible to detect even these small differences with the TDR sensors. This technology is therefore very suitable for this kind of research where a high measurement precision is required.

The lime-sand brick and the aerated concrete are materials with very low moisture conducting properties in contrast to the calcium silicate or the building brick. The implications of the single experimental steps superimpose each other therefore much more for these materials. In Fig. 7.5, the transient moisture storage data of the limesand brick is given during the large step adsorption and in Fig. 7.6 for the large step desorption experiment.

Fig. 7.5 shows, that the relative humidity of the bottom compartment (pos. 1) is quickly increasing whereas the moisture content remains rather constant up to humidities of $85 \%$. Here both, moisture content and relative humidity, rise, partly following the static adsorption curve. However, this rather constant increase of both of them continues beyond the static adsorption curve leading to lower moisture content values than this adsorption curve would provide. The other compartments show a similar behaviour taking lower moisture content values than the static adsorption curve. Only position 2 behaves slightly different. Here, the moisture content starts to rise at a lower 
relative humidity leading to transient moisture storage data which touches the static adsorption function. This different behaviour is presumed to be due to the process history as the experiment did not start at equilibrium conditions (see schedule in Tab. 5.2 on page 126).

The subsequent desorption process, displayed in Fig. 7.6, shows the opposite behaviour. At all positions - although with varying significance - the relative humidity decreases first before the water content follows. The corresponding hysteresis loops are given in Fig. 7.7 for position 1 and 2. The hysteresis loops of the same positions during the adsorption - desorption cycle of $85 \%-97 \%-85 \%$ relative humidity are shown in Fig. 7.8. The comparison reveals again, that the difference between the transient adsorption and desorption data is much more pronounced if larger gradients are applied. In Fig. 7.7, the difference between both branches is already much smaller for position 2 than for position 1. Looking at the small steps in Fig. 7.8 discloses, that the hysteresis is almost vanishing for position 2 whereas it is relatively small for position 1. The same material behaviour has already been observed for the calcium silicate.

In Fig. 7.9, the data of the large step adsorption and in Fig. 7.10 the following desorption of the aerated concrete are given. Again, a shifting of the transient moisture storage data in direction of higher relative humidity values can be observed for all compartments. Due to the moisture transport properties of the aerated concrete, only positions 1 and 2 denote a distinct moisture content increase. However, both positions do also remain below the static adsorption curve. The subsequent desorption process caused most compartments to follow the same branch as during the adsorption. Only position 1 moves on a different path taking higher moisture content values for decreasing relative humidities.

This becomes clearer when looking at Fig. 7.11 where the adsorption - desorption branches during the large step experiments for the first two compartments are compared. The left chart shows position 1. A distinct difference between transient adsorption and desorption is visible. For higher relative humidities, the adsorption branch remains below the static curve. The transient desorption branch intersects with the static adsorption curve but remains below the static desorption function. The dynamic effect which is most pronounced for the calcium silicate appears to be much less intensive for the aerated concrete. Moreover, the general hysteresis which is distinctive for the static curves does not occur within the other compartments during the transient experiments. The right chart in Fig. 7.11 shows the two branches for position 2 taking almost the same values for both, adsorption and desorption.

Another comparison of aerated concrete ad- and desorption branches is given in Fig. 7.12. This data belongs to the boundary condition cycle of $85 \%-97 \%-85 \%$ relative humidity. Here also two observations can be made. The first is, that the transient moisture storage data generally remains below the static adsorption function during these experiments for both positions and for both, adsorption and desorption branches. The second is, that there occurs no hysteresis between the measured transient adsorption and the desorption courses. In both positions, the desorption follows the same path as the previous adsorption. This is even more astonishing as the primary hysteresis which is measured by the static methods is very pronounced for the aerated concrete.

From the experimental results shown and discussed here, the following conclusions can be drawn: All performed experiments within the hygroscopic moisture content range show that building materials under transient conditions do not strictly 
follow along the equilibrium stages determined by static methods. Dynamic effects are visible in all experiments. However, the influence of these effects is different for different materials. A narrow pore-size distribution and a high moisture conductivity leads to very large deviations from the static behaviour as it is the case for the calcium silicate. It seems that such materials are more susceptible to dynamic effects than materials with a wider pore-size distribution and a lower moisture conductivity. This kind of material behaviour has also been observed by Wanna-Etyem (1982) for soils with different texture where fine-textured materials are less susceptible to dynamic effects.

However, the yet shown experiments comprise only the hygroscopic moisture content range. The building brick and the calcium silicate have a very low hygroscopic moisture capacity. The applied relative humidity steps could therefore barely be buffered and caused thus a stronger moisture transport into the material. A faster moisture transport process is though much more susceptible to transient effects. In contrast to this, the aerated concrete and the lime-sand brick provide a high hygroscopic moisture capacity. The applied humidity steps cause a distinct ad- or desorption of moisture inside the material slowing the moisture transport processes down. The equilibrium stages are therefore hardly achieved, not even for position 1, and the conditions of previous measurement steps superimpose the whole material behaviour. Therefore, the above given thesis on susceptibility to dynamic effects may be discussed at the end of this chapter, again, when data of the whole range of moisture contents is shown and analysed.

It is very interesting, that this kind of material behaviour previously observed only for much higher moisture contents does also apply for moisture transport phenomena within the hygroscopic moisture content range. Besides the results presented by Plagge et al. (2006b), this is the first evidence on dynamic effects occurring already at such low moisture contents. Moreover, these results prove their presence in both, adsorption and desorption processes whereas most literature on transient effects refers to desorption processes only. The presented data may therefore be very valuable for further research beyond the scope of the current study.

As it is focused on material model validation to reveal limits and options of the developed material modelling here, the data are evaluated by means of their current reproducibility by numerical simulation. These calculations are based on the transport theory briefly sketched in Chapter 2 and the material model introduced in Chapter 5. The following paragraph will present and discuss the simulation results for these hygroscopic moisture transport experiments.

\subsubsection{Hygroscopic simulation results}

The obtained experimental data indicate that the simulation results will hardly follow the measured courses of relative humidity and water content. Neither the static hysteresis, nor the dynamic influences are included into the transport theory of the simulation program. The calculated material behaviour will hence follow along the chosen static moisture storage functions typically leading to higher moisture contents compared with the measured data. But before discussing the simulation results, some details concerning the simulation specifications are given. 
The modelled material specimens have a width of $5 \mathrm{~cm}$, a height of $10 \mathrm{~cm}$ and a depth of $10 \mathrm{~cm}$. Width and height are discretised with a regular mesh allowing to calculate heat and moisture transport in two dimensions. The initial conditions are assigned according to the measured quantities for each position. The assignments thus follow the five compartments with a height of $2 \mathrm{~cm}$ each. The same is done for the output specifications. However, since the applied sensors have diameters of less than $1 \mathrm{~cm}$, mean output values are taken from a height of $1 \mathrm{~cm}$ at the centre of each compartment. An illustration scheme is given in Fig. 7.14.

For the initial conditions, the problem exists that either relative humidity or moisture content data can be allocated. The other quantity is then determined according to the moisture storage function during the calculations. Hence, only one of these two quantities can be assigned correctly as the measurement data revealed that the transient data does not follow the static moisture storage functions. Since the water content is the state variable which is of higher interest than the relative humidity, it is decided to apply the measured water content as initial moisture state for each position. Consequently, for some simulations, this leads to totally different initial relative humidities than the measurements provided.

As boundary conditions, the measured temperature of the climatic chamber is assigned to all sides of the material sample except the bottom side. There, the measured temperature and relative humidity inside the desiccator box is allocated. The heat transfer coefficients are adjusted by following the measured temperature profiles. For the vapour transfer, the heat and mass transfer analogy according to eq. (5.28) is applied.

To account for the hysteresis of the moisture storage function, the static adsorption and desorption curves are applied in dependence on the occurring process. This means, that the adsorption moisture storage function is assigned if an adsorption process is simulated and the desorption moisture storage function is assigned for the simulation of a desorption process. As already stated above, there is no hysteresis module implemented into the simulation program, yet. It is hence not possible to model the

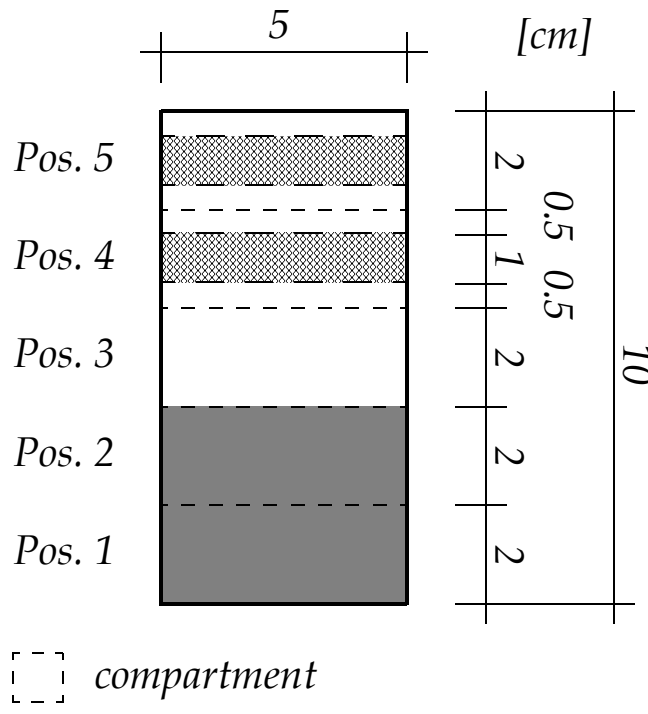

[-] initial condition assignment

single position output assignment

Figure 7.14: Geometry and assignments for simulation. transition from one process to the other. The impact of this simplification becomes clearly visible from the comparison of measured and simulated material behaviour.

Out of the investigated materials, the building brick and the calcium silicate possess similar moisture properties within the hygroscopic moisture content range. Both materials have a low hygroscopic moisture capacity. The sorption isotherm forms its steep increase not until very high values of relative humidity. And both materials have rather high moisture transport properties. For this reason, both materials show also similar measurement and simulation results. 
Thus, for the following discussion, the simulation results of the calcium silicate are chosen to be shown. The building brick exhibits the same kind of material behaviour during the simulations. For the building brick, the fluxes are very small due to the small water content differences within the hygroscopic moisture content range. The visualisation of the calcium silicate data provides therefore a more vivid data basis which is preferred for the discussion.

Fig. 7.15 shows the comparison of measured and calculated moisture content profiles for the large step adsorption of the calcium silicate. The simulation results deviate from the measurement in three aspects. First, the water absorption of the calculation proceeds much faster than during the experiment. Second, the maximum moisture content occurring in position 1 is lower for the simulation than for the experiment. And third, the distinct moisture profiles which develop during the experiment equilibrate quickly during the simulation.

Comparing these results with those presented in Fig. 7.1 and Fig. 7.3 clarifies the calculated material behaviour. The discussion may be started with the first observed deviation. The simulation has to follow along the applied adsorption moisture storage function. This means, as soon as the relative humidity increases, the moisture content must increase, too. Both are coupled via the transport properties which means, that both, relative humidity and moisture content can only rise as quickly as the moisture transport properties allow. Since these properties are rather high, a quick moisture transport proceeds. Looking at the measured dynamic moisture storage function shows though, that during the experiment, the relative humidity inside the first compartment increases much faster than the moisture content. This results in a relation which is somehow shifted to higher relative humidity values staying below the static adsorption function, see Fig. 7.1. The moisture potential gradient between the boundary and the first compartment is therefore significantly reduced leading to a reduced moisture transport into the material.

For the simulation, however, this is not the case. As the relative humidity inside the first compartment increases slower than during the measurement, the potential gradient remains higher and, by that, also the resulting moisture transport rates. When applying the static adsorption moisture storage function, the transport rates must hence be higher than actually measured.

The second aspect, that a maximum moisture content is reached at position 1 during the experiment which can not be achieved during the simulation, is due to the boundary relative humidity. The calculated water absorption stops as soon as the equilibrium with the boundary condition is reached, i.e. as soon as the equilibrium moisture content according to the adsorption moisture storage function is attained. This equilibrium moisture content is obviously lower than the one according to the measurement conditions. As can be seen at the adsorption moisture storage function (see Fig. 7.1 or Fig. 6.7), a very small change in relative humidity will cause a drastic change in moisture content. The range around $97 \%$ of relative humidity is thus very sensitive to small changes. When looking at the precision of the applied relative humidity sensors ${ }^{1}$, it is likely that a small measurement error occurred although the sensors were calibrated at three different relative humidities before and after the IPM experiments.

1. The producer specifies the precision with $\pm 2 \%$ and the reproducibility with $\pm 1 \%$ for the whole measurement range. Refer to paragraph 3.2.2.2 Moisture potential measurement on page 80 et seqq. and to the final discussion in paragraph 7.3.2 Discussion. 
Ultimately, the moisture profiles are the third significant difference between measured and calculated material behaviour. This is again recognized to be due to the applied moisture storage function. As the relation between potential and state variable is assumed to be unique by the simulation, an equilibrating potential does automatically result into an equilibrating state variable. For the treated example, the simulation accesses only the one static adsorption moisture storage function. Assigned differences as they come from the initial conditions, or implied differences as they result from the boundary conditions equilibrate in dependence on the transport properties of the material. For a material as the calcium silicate which has high moisture transport properties, these differences must equilibrate quickly. The finally reached moisture content is corresponding to the boundary relative humidity according to the applied moisture storage characteristic.

The integral water mass inside the calcium silicate during the large step adsorption from $33 \%$ to $97 \%$ relative humidity is displayed in Fig. 7.16 . The consequences of the discussed influences which led to the deviations between measured and simulated moisture content profiles become visible here, too. The calculated water mass increases much faster than the measured one. This is due to the higher transport rates implied by the higher potential gradients according to the static storage function. And the totally absorbed moisture mass is higher according to the simulation. The reason for this is that the calculated moisture transport processes continue until equilibrium is reached. As one moisture storage function is valid for all compartments, this equilibration process is quickly completed as becomes visible at the bend of the integral water mass curve in Fig. 7.16 or at the coincidence of the moisture profiles in Fig. 7.15, both after around 4 days.

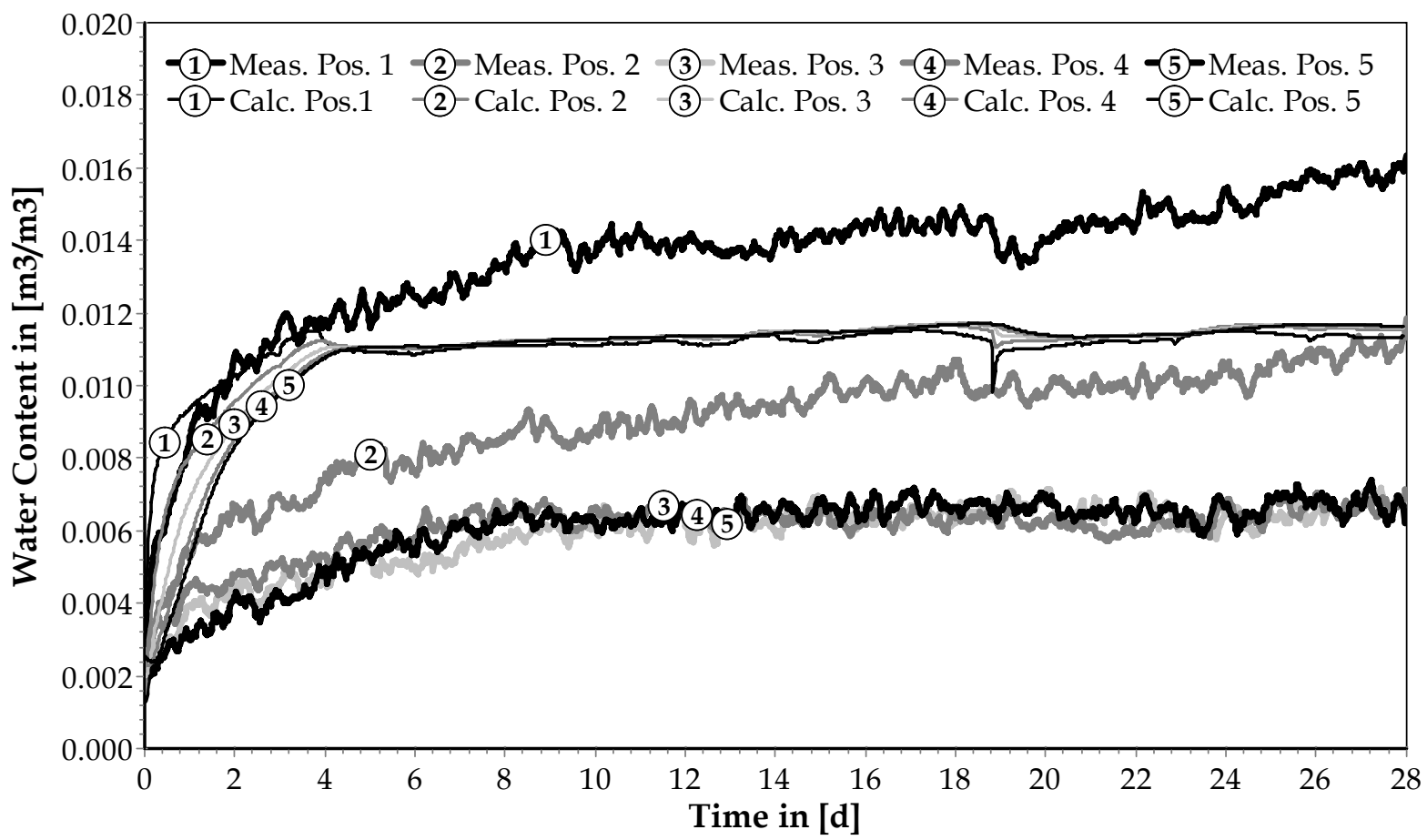

Figure 7.15: Measured and calculated data of the large step adsorption from 33\% to $97 \%$ for the calcium silicate. Thick lines indicate the measurement whereas thin lines indicate the simulation results. 
Summarizing the hygroscopic adsorption, it has become evident, that the simulation based on the static adsorption moisture storage function overestimates both, the moisture transport rates and the totally absorbed moisture mass. The reasons for this behaviour stem from the applied moisture storage function which differs from the ones obtained by the transient measurements. Moreover, only one moisture storage function is applied - the static adsorption function. The experiments revealed though, that different moisture potential - moisture content relations occur for all compartments.

The distinct deviations between measured and calculated material behaviour are connected to the particular range of

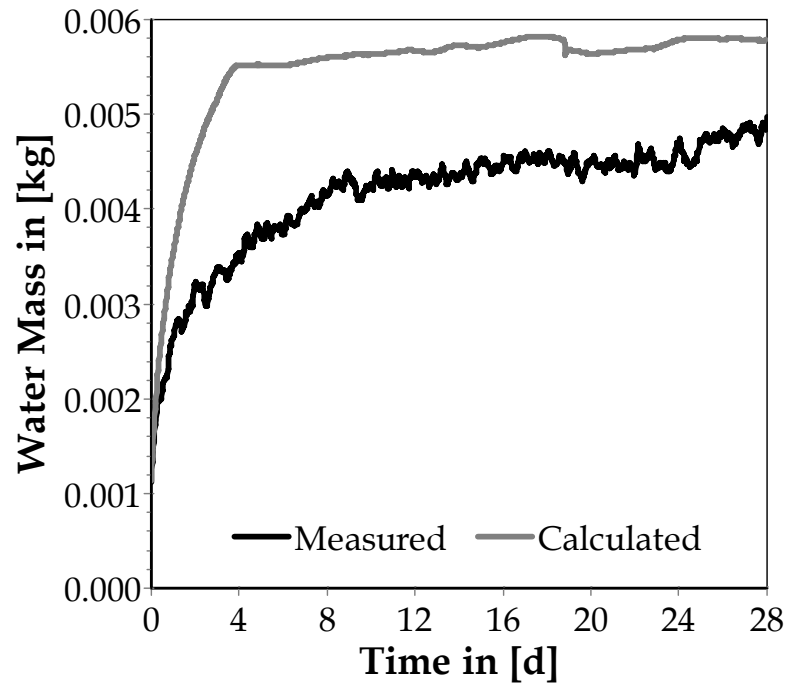

Figure 7.16: Integral water mass inside the calcium silicate during the large step adsorption. applied boundary relative humidity and to the total step size. As long as the applied humidity step does not exceed the critical relative humidity at which the moisture storage function starts to increase rapidly, the total differences between experiment and simulation are much less pronounced. The simulation of the stepwise increases ${ }^{1}$ shows that the tendency of overestimation still remains but the general impact, i.e. the total difference between measured and calculated moisture mass is small. The dynamic effects which lead to the different moisture potential and thus to the different transport rates are less pronounced for smaller moisture potential, i.e. relative humidity steps.

The large step desorption from $97 \%$ to $33 \%$ relative humidity of the calcium silicate is chosen to be the desorption example. The comparison of measured and calculated material behaviour is given in Fig. 7.17. Again three aspects become visible from this chart. The first two have already been observed during the adsorption. These are the faster transport rates and the vanishing moisture profile of the calculated data. The third aspect is not very pronounced here, though still interesting. It is the fact, that during the experiment, the desorption process starts immediately in all positions although with different characteristics. During the simulation, the desorption starts only in positions 1 and 2. The upper three compartments, especially position 3 and 4, exhibit a moisture increase first before the desorption process initiates there, too.

At the first moment, the faster desorption at positions 1 and 2 is surprising. During the adsorption experiment, the relative humidity increases first and quickly before the moisture content follows - as the measurement results of Fig. 7.1 and Fig. 7.3 reveal. This leads to a reduction of the moisture potential gradient, and by that to a reduction of the moisture transport force. The consequence is a reduced moisture transport rate. The simulation though, which strictly follows the static adsorption curve, does not include this dynamic effect. The calculated adsorption moisture transport rates are therefore higher than the measured ones, see Fig. 7.15 again.

1. Refer to Tab. 5.2 on page 126 for the applied hygroscopic boundary condition steps. 


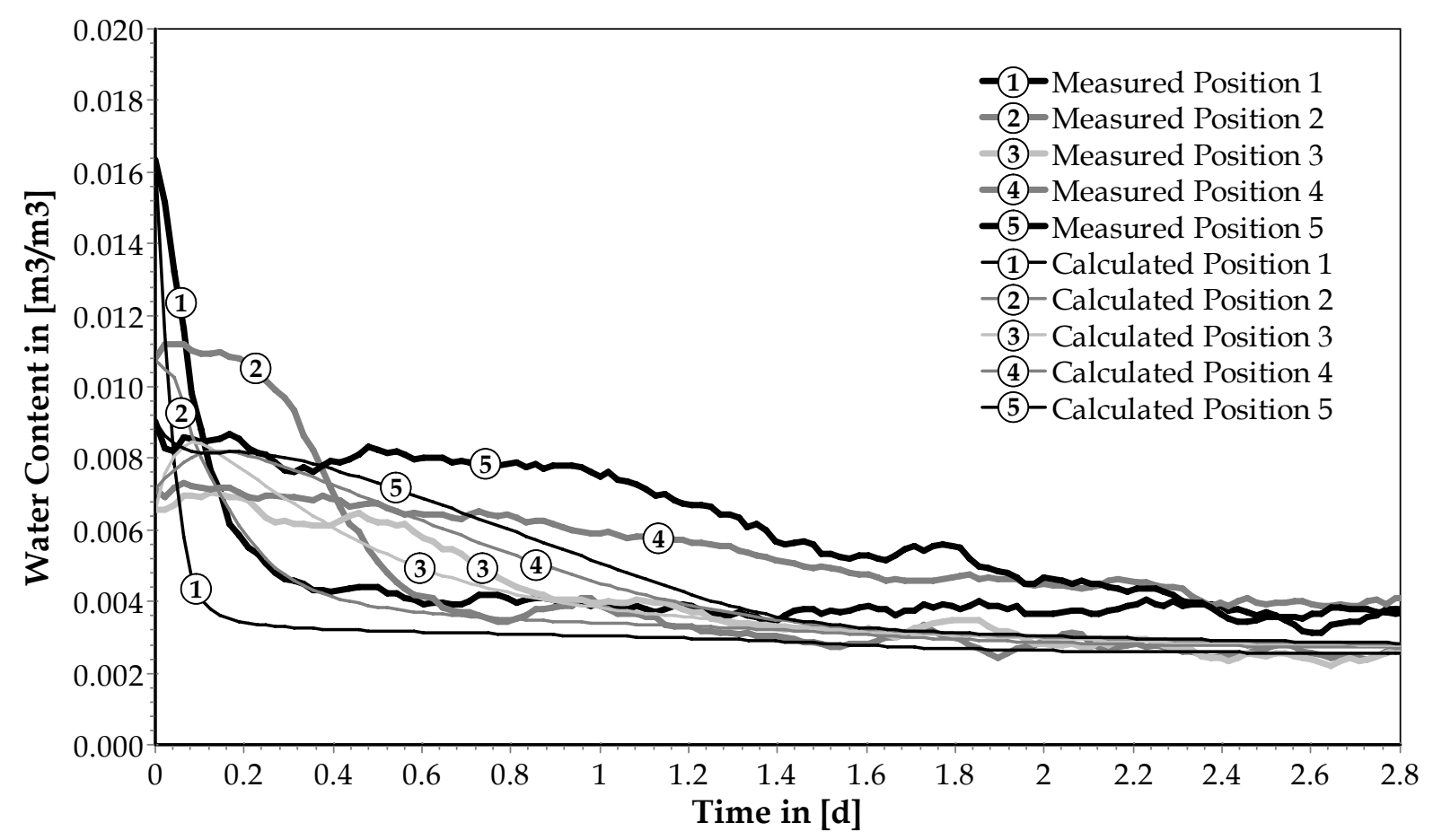

Figure 7.17: Measured and calculated data of the large step desorption from 97\% to 33\% for the calcium silicate. Thick lines indicate the measurement whereas thin lines indicate the simulation results. Note that only the first 2.8 days are displayed.

In contrast to these observations, the desorption process exhibits the opposite effect - as the results given in Fig. 7.2 and Fig. 7.3 underline. The relative humidity at position 1 decreases very quickly before the moisture content follows this trend. The moisture transport force inside the material is thus increased during the experiment. The simulation, which is done by assigning the static desorption moisture storage characteristic to all compartments, has to follow this desorption function strictly. It is hence expected that the calculated transport rates underestimate the actual material behaviour. When looking at the data pictured in Fig. 7.17, this is obviously not the case. Therefore, additional information as the integral water mass versus time course and the relative humidity distribution are consulted as follows.

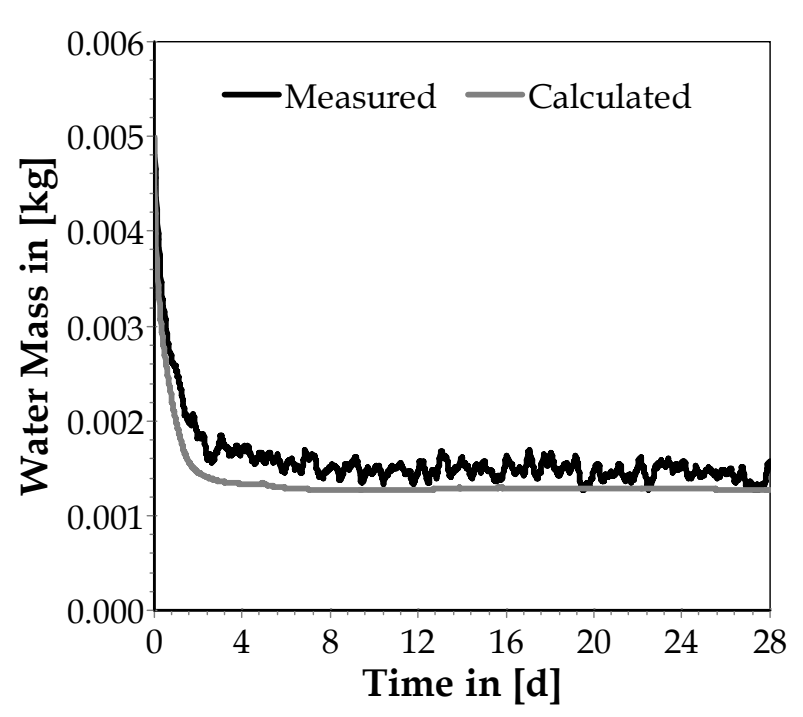

Figure 7.18: Integral water mass inside the calcium silicate during the large step desorption. 


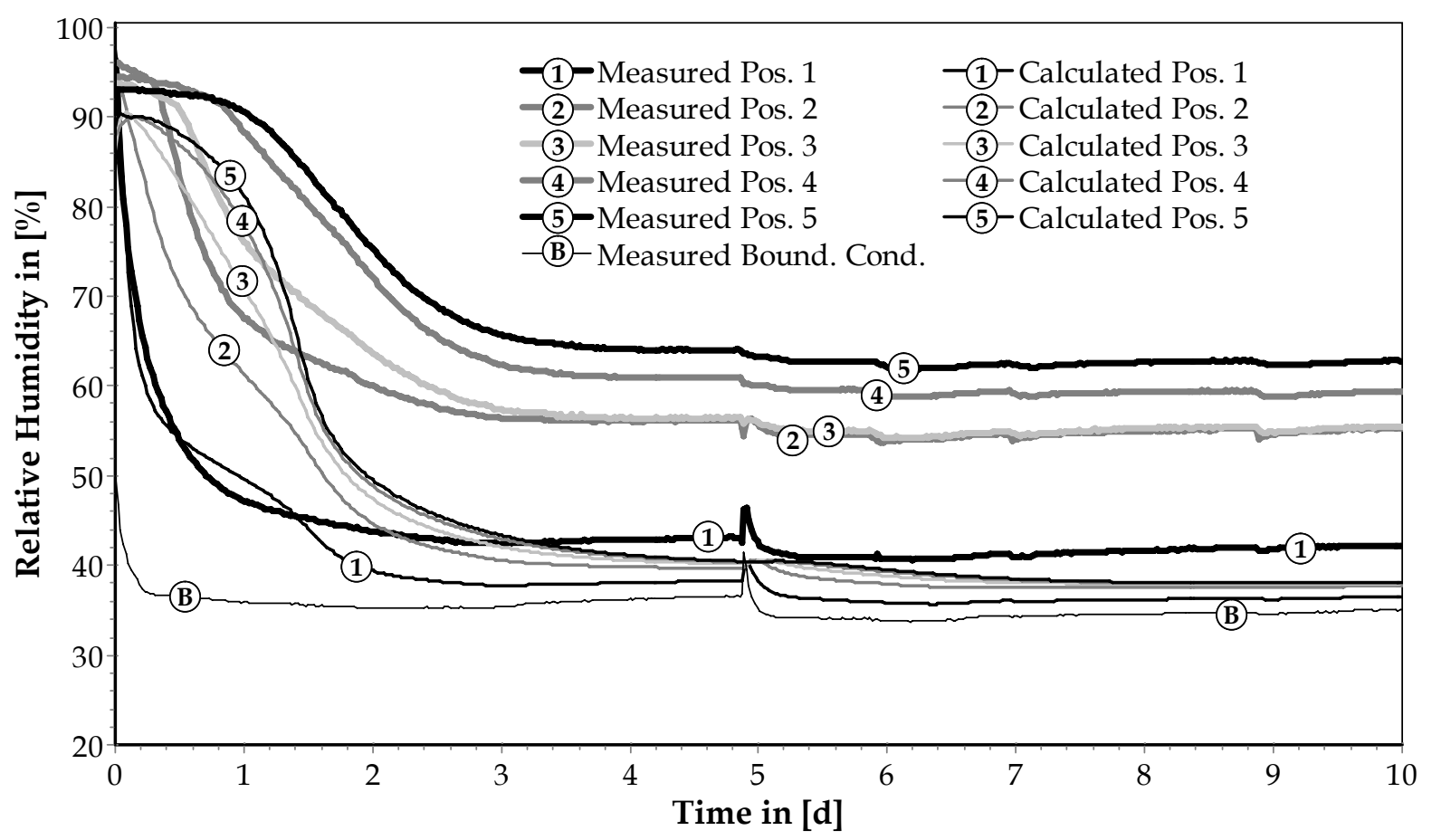

Figure 7.19: Measured and calculated relative humidity data of the large step desorption from 97\% to $33 \%$ for the calcium silicate. Thick lines indicate the measurement whereas thin lines indicate the simulation results.

When comparing the courses of measured and calculated integral moisture mass as shown in Fig. 7.18, it becomes visible, that these courses do not differ as strongly as expected from the compared moisture content profiles. But still, the simulation overestimates the transport rates leading to a faster moisture mass decrease than during the experiment. The general impact on the integral moisture content changes appears to be less pronounced, but the tendency of overestimation remains.

For further comprehension, the discussion may include also the relative humidity profiles. The comparison of measured and calculated relative humidity courses during the large step desorption of the calcium silicate is shown in Fig. 7.19. One can see, that the calculated relative humidity at position 1 follows the measured course quite well. The relative humidities of the other compartments decrease faster during the simulation than during the measurements. However, another very interesting material behaviour becomes apparent. The measured relative humidity profiles do neither equilibrate within the shown duration of 10 days, nor do they within 40 days. A difference of almost $10 \%$ of relative humidity remains already between the boundary condition and the measurement position of the first compartment. Within the upper compartments, an equilibrium relative humidity establishes which is $30 \%$ higher than the applied boundary relative humidity.

During the experiment, a distinct decrease of relative humidity occurs only within the first compartment. The other compartments follow with a clear delay and establish an explicit profile between $60 \%$ and $70 \%$ of relative humidity. The same material behaviour is also observed during the other stepwise decrease experiments. During desorption from high to low relative humidities, a profile establishes which does not 
equilibrate with the boundary condition. The profile is the more pronounced the lower the applied boundary humidity is. The humidity difference between the upper compartments and the boundary conditions is $10 \%$ to $15 \%$ for a desorption from $85 \%$ to $58 \%$ of relative humidity whereas it is $20 \%$ to $30 \%$ for the desorption from $58 \%$ to $33 \%$ of relative humidity. The large step desorption from $97 \%$ to $33 \%$ which is shown in Fig. 7.19 causes a profile of the same order of magnitude.

During the adsorption processes though, this behaviour has not been observed. At all adsorption steps, the relative humidity inside the material specimen approaches the boundary condition. Moreover, the experiments performed on the building brick show exactly the same phenomena, i.e. equilibrating relative humidity profiles during adsorption and not equilibrating relative humidity profiles during desorption processes.

It is concluded, that for materials with a low hygroscopic moisture capacity, small differences in relative humidity do not equilibrate completely. The actual transport rates inside the material reduce at a stage where the simulation based on a static moisture storage function will still exhibit a considerable moisture transport. The higher transport rates occurring during the calculation are hence due to the equilibrating relative humidity and the implied transport processes. The simulation approaches only one equilibrium stage which is defined by the moisture storage function. Therefore, all positions will attain this level during the calculation and no moisture profile will remain. The duration is only depending on the moisture transport properties within the treated moisture content range which are rather high for the calcium silicate. The measurements, however, show, that different equilibrium stages exist in dependence on the process and probably also on the process history which do not smooth out.

Another interesting observation can be made by the comparison of measured and calculated desorption data shown in Fig. 7.17. A redistribution of moisture occurs in the upper compartments at the beginning of the simulation which is not visible in the measured data. The change in boundary humidity and the corresponding switch from an adsorption to a desorption process does apparently affect the whole material sample. As the simulation defines only one equilibrium stage for all compartments, the initial moisture content profile is compensated in the upper compartments while the desorption proceeds at the boundary. This is understood as another indication that there exist many different stages of equilibrium. One static moisture storage function is not able to correctly reproduce this material behaviour.

For the calcium silicate and the building brick, the following observations on the moisture transport behaviour within the hygroscopic moisture content range can be made by means of the given simulation results:

- Observations during the adsorption processes:

- The calculated adsorption proceeds too fast.

- The simulation equilibrates all moisture profiles in dependence on the boundary condition.

- The totally absorbed moisture mass is sensitive to the slope of the moisture storage function in the range of the boundary relative humidity. 
- Observations during the desorption processes:

- The calculated desorption proceeds too fast.

- The simulation equilibrates all moisture content and moisture potential profiles in dependence on the boundary condition.

- The distinctly measured profiles of relative humidity can not be reproduced by the simulation.

From these observations, the following is concluded. There exist many different equilibrium stages depending on the process and its history. These stages can not be reproduced by one single moisture storage function which is the reason why the calculated water content profiles do not correspond with the measured ones. The non-equilibrium stages which occur during transient processes are not included into the transport theory. The moisture potential gradients are always calculated on the basis of the static moisture storage function. This is recognized to be the main reason why the transport rates are not correctly simulated since the transport function is directly determined by the basic steady state data from vapour diffusion measurements within the hygroscopic moisture content range.

However, the simulation reproduces the general tendencies correctly. Deviations between measured and calculated integral water masses remain, but they should not be over-interpreted for two reasons. First, the absolute moisture contents are very low which is the reason why small differences become more pronounced. For instance, the absolute difference between measured and calculated water absorption during the large step from $33 \%$ to $97 \%$ relative humidity is around $1 \mathrm{~g}$ of water for a specimen with dimensions of $10 \times 10 \times 5 \mathrm{~cm}^{3}$. And second, the data revealed a number of effects which are not yet included into the transport theory. Bearing this in mind, the obtained simulation results are fairly acceptable.

Although the lime-sand brick and the aerated concrete are not as similar as the building brick and the calcium silicate by means of their hygroscopic moisture capacity and their moisture transport properties, they are close enough to be combined to a second material group for the data evaluation. It was hence intended to show the results of only one of them being representative for this material group. However, their simulation performance appeared to be totally different. For this reason, the simulation results of both building materials are shown and discussed as follows.

First, the results of the lime-sand brick are presented. Due to the highest content of information, again the large step experiments are chosen to be presented and discussed. Fig. 7.20 shows the moisture content profiles during the large step adsorption from $33 \%$ to $97 \%$ relative humidity for the lime-sand brick. As before, the thick lines represent the measured data whereas thin lines show the calculated profiles. Position 1 corresponds with the bottom compartment which is exposed to the boundary condition, position 5 belongs to the upper compartment of the material specimen.

The comparison of measured and calculated water contents reveals two main deviations: the calculated moisture transport rates are apparently much lower than those occurring during the experiment and the maximum moisture content reached during the simulation is higher than the measured one. At position 1, the general tendency is reproduced quite well. However, the absolute moisture content at the end of the exper- 


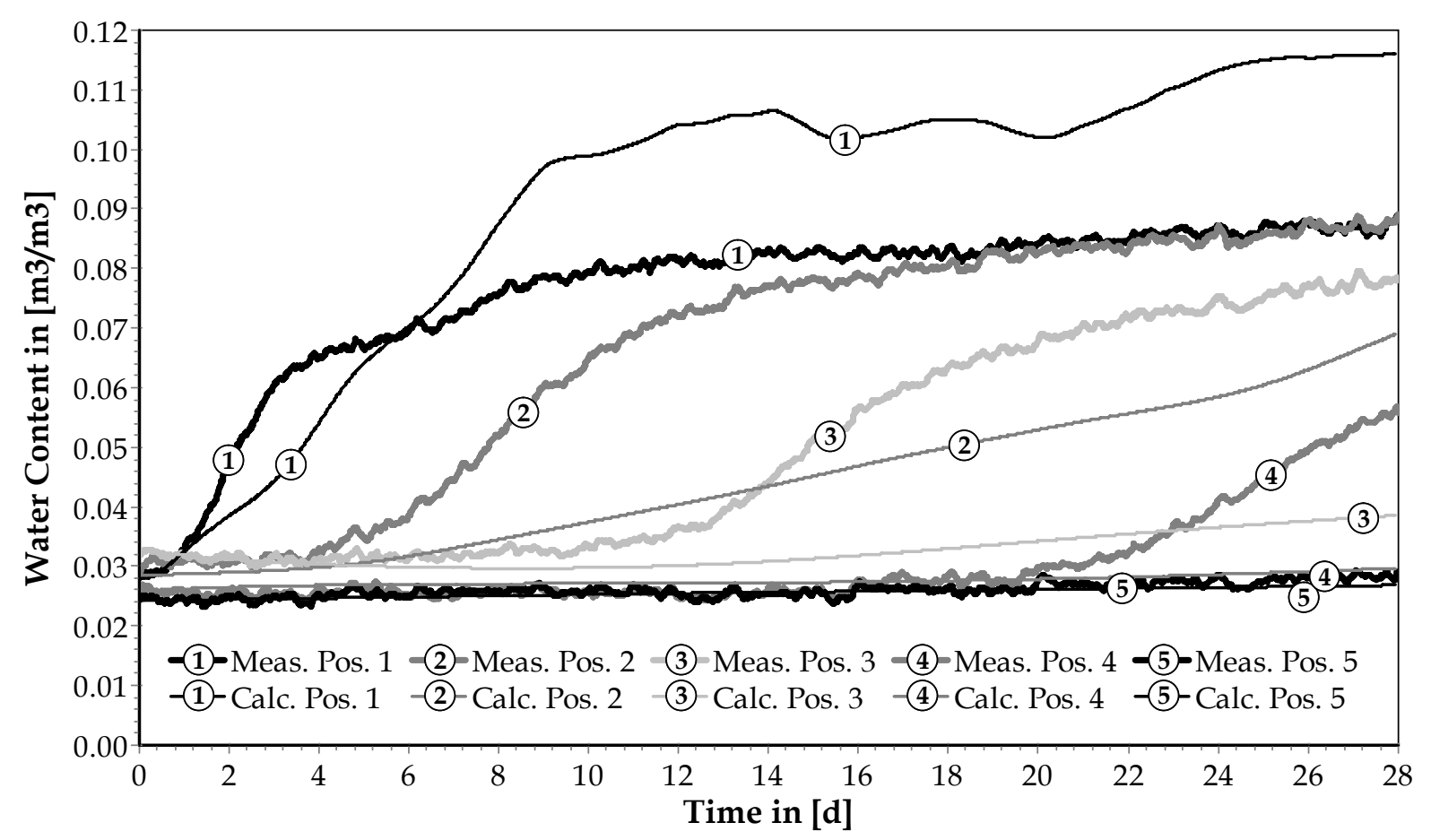

Figure 7.20: Measured and calculated water content data of the large step adsorption from $33 \%$ to $97 \%$ of relative humidity for the lime-sand brick. Thick lines indicate the measurement whereas thin lines indicate the simulation results.

iment is higher during the simulation than during the measurement. The reason for this deviation stems from the equilibrium moisture content of the applied adsorption moisture storage function which corresponds with the boundary relative humidity. This phenomenon has already been observed for the calcium silicate and the building brick. A small change in boundary humidity causes a large change in the equilibrium moisture content due to the steep slope of the moisture storage function in this humidity range. The high moisture content in position 1 at the end of the experiment is thus a consequence of the applied moisture storage function and the boundary relative humidity data.

The deviating moisture profiles are though difficult to explain. The difference between static and transient moisture storage data, as shown for this experiment in Fig. 7.6, would partly explain a faster calculated moisture transport. Since here the opposite behaviour appears, the moisture potential can not be the reason. The moisture transport properties applied for the calculation do hence not represent those of the investigated material specimen. This means in particular, that in the moisture content range of $0.02 \ldots 0.1 \mathrm{~m}^{3} / \mathrm{m}^{3}$, the applied liquid water conductivity is too low. On the other hand, the first compartment follows the measured course with rather good agreement. The simulation reproduces therefore the vapour transfer and the moisture transport within the first compartment quite well.

The integral water mass curves according to the experiment and the simulation are shown in Fig. 7.21. One can see in the left chart, that these curves do not deviate as significantly as the water content profiles do. Within the first 10 days of adsorption, the calculation follows the measured course. Then the simulation starts to exhibit a constantly lower mass increase. Nevertheless, both curves differ with around $5 \mathrm{~g}$ of 

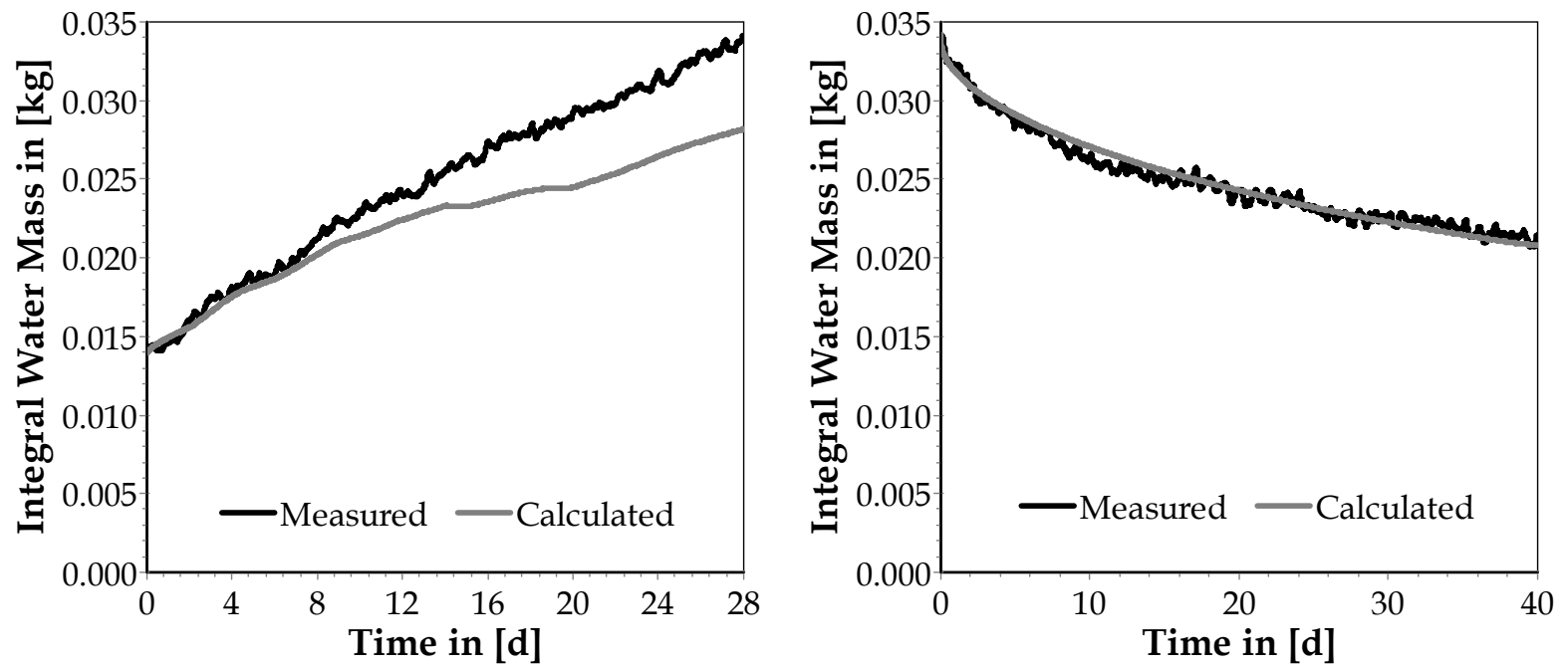

Figure 7.21: Integral water mass inside the lime-sand brick during the large step adsorption (left chart) and during the large step desorption (right chart).

adsorbed water at the end of the experiment after 28 days. The adjusted moisture transport functions may hence underestimate the actual transport rates. Before further discussing this observation, the simulation of the large step desorption from $97 \%$ to $33 \%$ relative humidity is supposed to be evaluated.

The integral water mass curves for the large step desorption process are given in the right chart of Fig. 7.21. The calculated desorption follows the measured course almost perfectly. In contrast to the adsorption, the simulation is able to reproduce the integral water mass curve for the whole experiment duration here.

When looking at the comparison of measured and calculated moisture content profiles though, the agreement between the integral curves appears to be the sum of over- and underestimating the moisture transport rates within the material specimen. Fig. 7.22 shows the moisture content profiles for the large step desorption of the lime-sand brick where the calculated water content deviates in both directions from the measured data.

The measured water content at position 1 exhibits a fast decrease at the beginning of the experiment which is passing over into a rather constant slope until the 10th day. Here, another smooth bend reduces the slope of the curve which remains until the end of measurement. The calculated water content course looks rather different. The initial decrease starts with a small delay but stays much longer leading to a significantly lower moisture content than the measured position 1 . Between the second and the fourth day, the water content curve follows a smooth bend. It decreases until the end of the experiment with a further decreasing slope. The taken moisture content values stay below the measured data.

Except for position 5, the measured desorption process begins immediately in all compartments as soon as the low boundary humidity is applied. The absolute desorption rate is different, it decreases with increasing distance from the bottom. The calculated water contents of positions 2 and 3 show the same tendency, but much less pronounced. Within the second compartment, it takes around five days until the distinct desorption starts. At position 3, this behaviour is even more delayed and smoothed. The calculated curves of both, position 2 and 3, intersect with the measured data. 


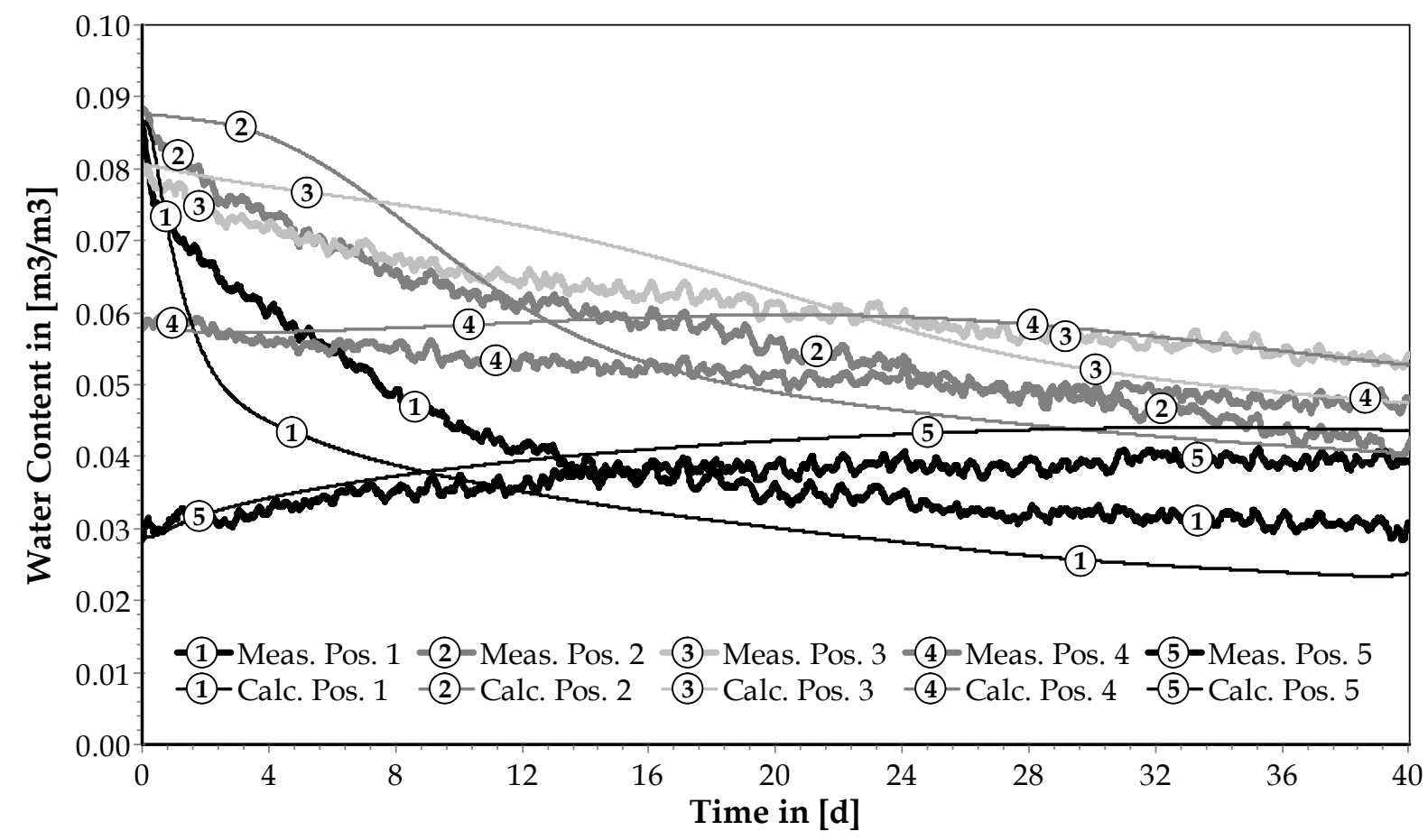

Figure 7.22: Measured and calculated water content data of the large step desorption from 97\% to 33\% of relative humidity for the lime-sand brick. Thick lines indicate the measurement whereas thin lines indicate the simulation results.

The measurement of the upper compartment shows, that an adsorption still proceeds there. The inner compartments desorb in both directions. The simulation slightly overestimates this effect for position 5 . However, for position 4 , the same behaviour occurs during the calculation. Although a desorption process starts there after 20 days, the moisture content is generally overestimated for position 4 by the simulation.

The calculated fast desorption at the bottom compartment is obviously compensated by redistribution processes within the other compartments leading to a satisfying agreement between measured and calculated integral moisture content. The actual moisture profiles and the local transport rates inside the lime-sand brick can not be reproduced correctly by the simulation. And this although the calibration in Fig. 6.15 shows an almost perfect agreement between measurement and simulation.

Summarizing the performance of the lime-sand brick within the hygroscopic moisture content range, it can be stated that it is generally not possible to follow the measured moisture profiles in detail. The integral water content can be reproduced for around 8 days during the adsorption and for the whole measurement period during the desorption process. The observed deviations can not be explained by the non-uniqueness of the moisture storage characteristic alone. In fact, the liquid water conductivity is underestimated within the corresponding range of moisture contents. This may be partly an effect of the calibration procedure where unsteady state experiments are simulated on the basis static moisture storage data to adjust the moisture transport function. The material variability may be another reason for the observed deviations. 
The aerated concrete, although possessing similar material properties by means of hygroscopic moisture capacity and moisture transport, behaves significantly different than the lime-sand brick. Fig. 7.23 shows the comparison of measured and simulated water content data during the large step adsorption. The calculation generally follows the measured curves but overestimates the transport rates. The water content level at the end of the experiment is higher for the simulation than for the measurement. This is again due to the equilibrium moisture content of the applied adsorption moisture storage function which corresponds with the boundary relative humidity.

The higher calculated transport rates can either be due to the moisture transport function or to the deviating moisture storage data. When comparing the measured moisture storage data with the static curves, see Fig. 7.9, it becomes apparent that a calculation based on the static adsorption curve would yield different results. The measured transient adsorption curves take lower moisture content values at higher relative humidities compared to the static curve. Thus, the moisture potential equilibrates faster during the experiment leading to smaller gradients and, by that, to smaller transport rates. The simulation though, strictly following the static adorption curve, provides a more pronounced relative humidity profile until the equilibrium with the boundary condition is reached in all compartments. The calculated transport rates must therefore be higher than the measured ones if the observed dynamic effects occur.

In Fig. 7.24, the integral water mass curves of the large step adsorption (left hand side) and the large step desorption (right hand side) are given. The left chart shows that the difference between measured and simulated integral moisture mass is rather small

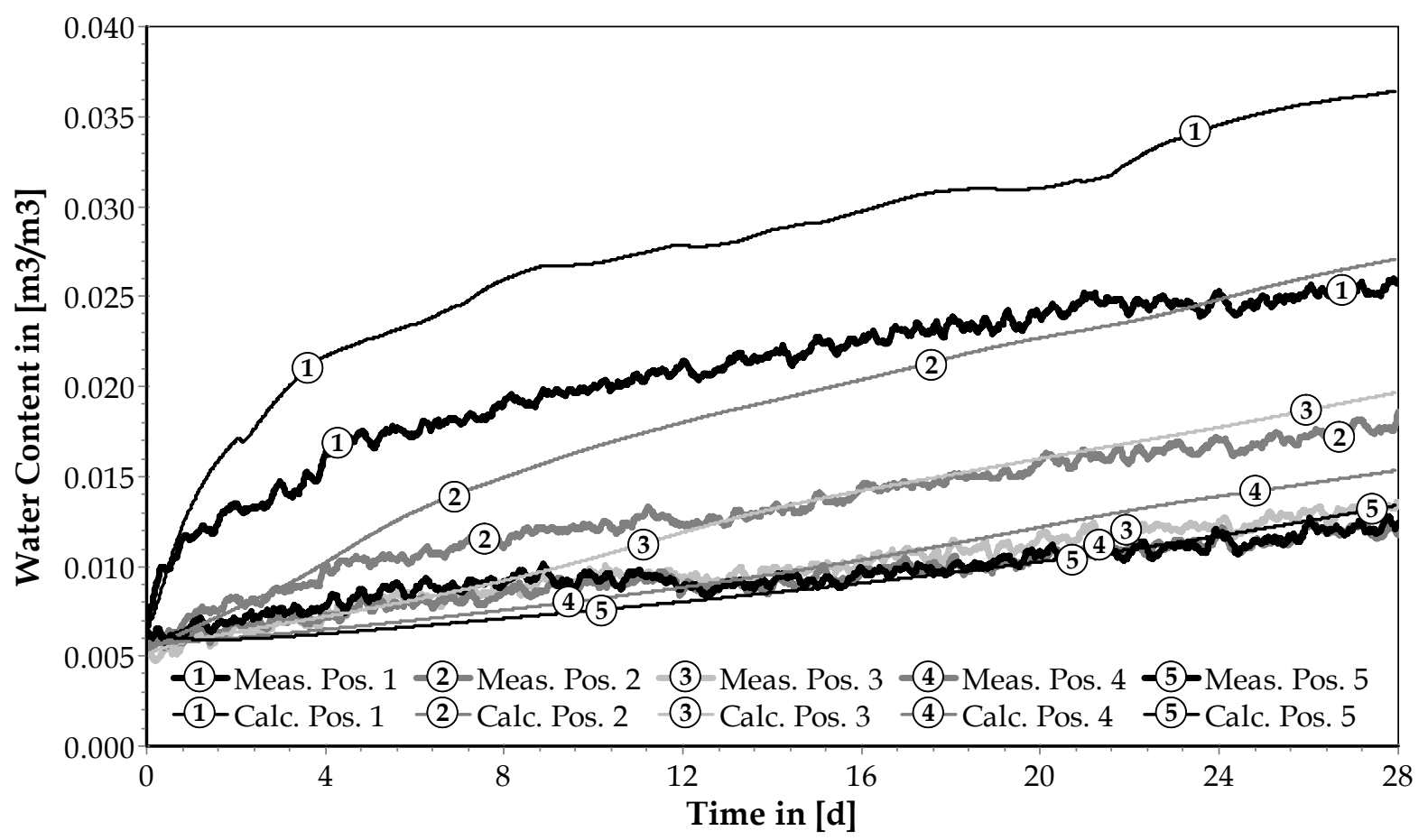

Figure 7.23: Measured and calculated water content data of the large step adsorption from $33 \%$ to $97 \%$ of relative humidity for the aerated concrete. Thick lines indicate the measurement whereas thin lines indicate the simulation results. 

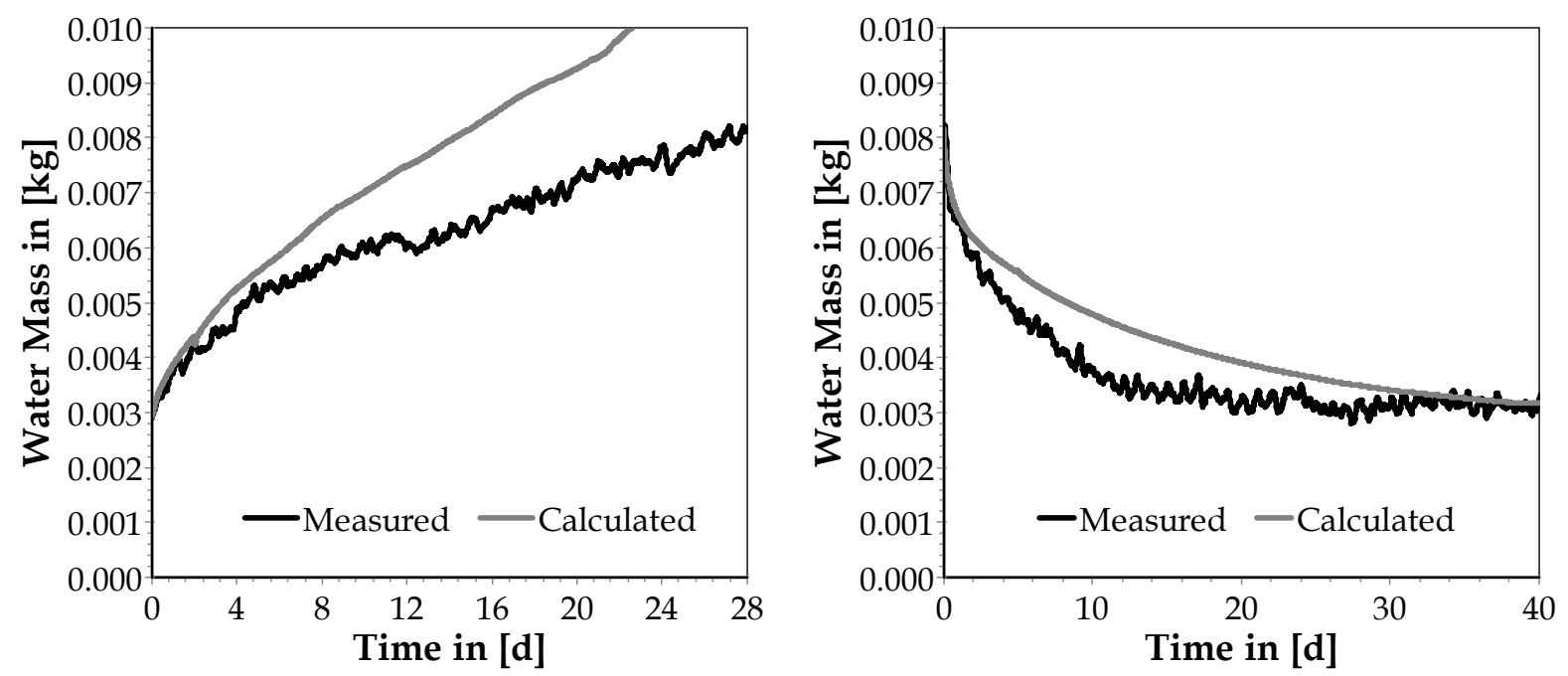

Figure 7.24: Integral water mass inside the aerated concrete during the large step adsorption (left) and the large step desorption (right).

within the first 4 days, but it increases with increasing duration. This underlines the given explanation as the moisture potential gradients are high in the beginning of the experiment for both, measurement and simulation. The equilibration proceeds though much faster in the experiment which can not be reproduced by the simulation.

At the right hand side of Fig. 7.24, the integral desorption curves are shown. The corresponding moisture content profiles are displayed in Fig. 7.25. Here, the opposite effect appears. The calculated integral moisture mass curve follows the measured one

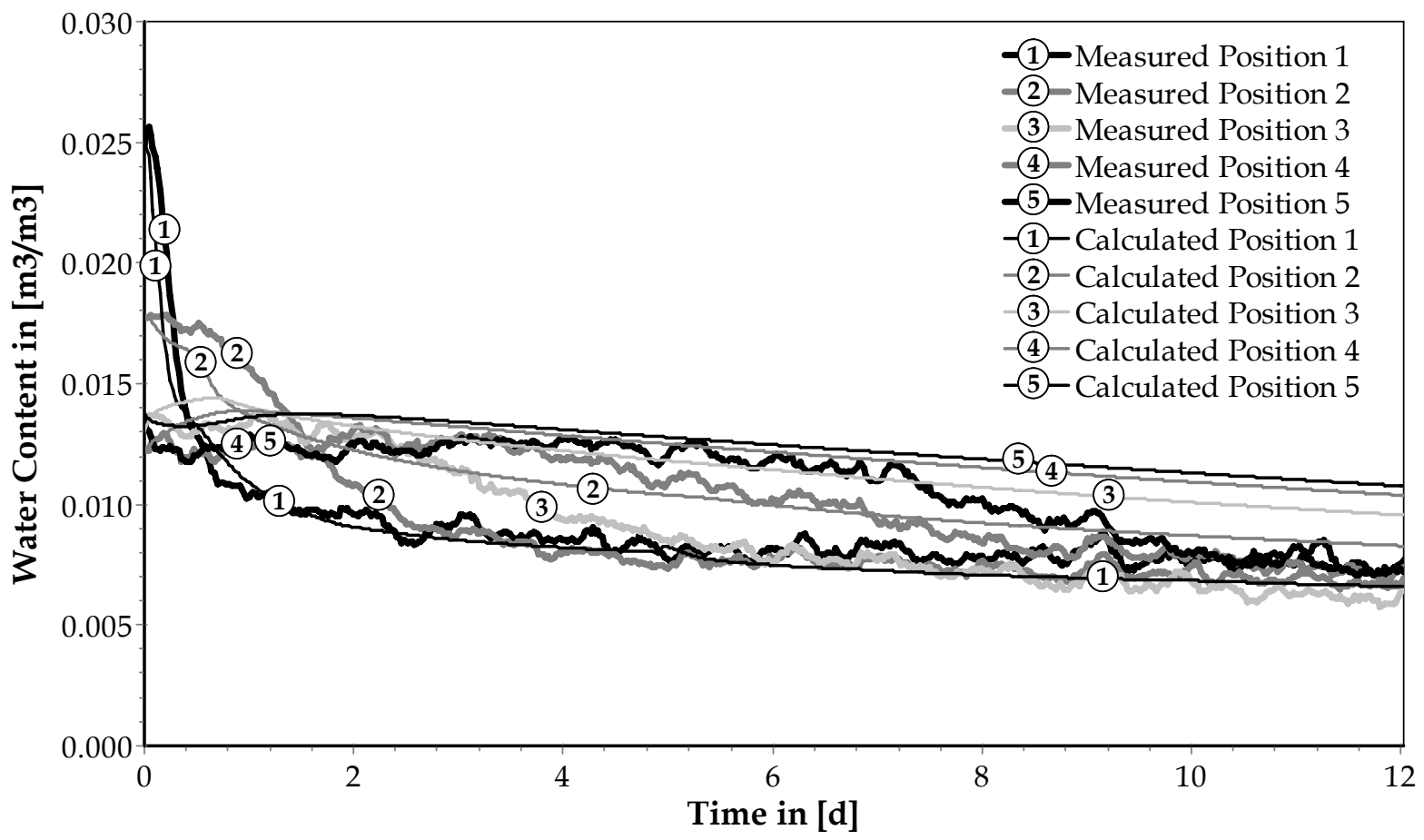

Figure 7.25: Measured and calculated water content data of the large step desorption from $97 \%$ to $33 \%$ of relative humidity for the aerated concrete. Thick lines indicate the measurement whereas thin lines indicate the simulation results. 
again for the first couple of days. Then it deviates, underestimating the actual transport rates. In Fig. 7.25, the water content within the first compartment corresponds well between measurement and simulation. At position 2, the calculated moisture content decrease is overestimated within the first two days whereas it is underestimated thereafter. The other compartments exhibit an increasing water content within the same period before they start to desorb. In general, this desorption proceeds much slower than the measured profiles indicate. After twelve days, which is the maximum of the time label in Fig. 7.25, the measured moisture profiles are in equilibrium. In Fig. 7.24, the simulation reaches this stage not before the 30th day.

Since both experiments comprise the same range of moisture contents and relative humidities and since the simulation of one process overestimates and of the other underestimates the transport rates, the moisture transport functions can not be the reason of the deviations. In fact, these deviations are an effect of the applied moisture storage functions which do not correspond with those measured during the transient experiments.

In contrast to the lime-sand brick, the dynamic effects can be clearly identified as the reason for the observed deviations between measurement and simulation of the aerated concrete. The shifted moisture storage characteristics lead to quickly decreasing moisture potential gradients for the adsorption and to increasing gradients for the desorption. Applying the static curves must hence overestimate the transport rates during adsorption processes and underestimate them during desorption processes. This can be particularly found at the given comparison between measurement and simulation of the aerated concrete. Although the general transport tendencies are followed, the total transport rates and thus also the developing moisture profiles can not be reproduced correctly by the simulation.

\subsubsection{Concluding hygroscopic assessment}

The measured transient moisture storage data provides evidence of the nonuniqueness of the moisture potential - moisture content relation. Under transient conditions, the moisture potential reacts quickly to the applied boundary condition leading to significant deviations in the potential - state variable configuration compared with the static case. The presented measurement results therewith confirm the observations of previous authors which are briefly reviewed as follows.

It was already witnessed by Topp et al. (1967) and by Smiles et al. (1971), that the size of the applied moisture potential gradient significantly influences the relation between moisture potential and moisture content under transient conditions. Both author consortia used a combination of gamma ray attenuation and tensiometers to perform instantaneous profile measurements on a sandy soil. Their results indicate, that the higher moisture potential gradients are imposed on the transient experiments, the larger is the deviation from the static moisture potential - moisture content relation. For small and constant, i.e. steady state, gradients though, the transient curves are very close to the static ones. Fig. 7.26 shows a reproduction of Figure 2 according to Topp et al. (1967) where these effects become clearly visible.

Stauffer (1977), who performed instantaneous drainage experiments on fine-textured sandy soils, confirmed the results of Topp et al. (1967). Stauffer has found that the capil- 


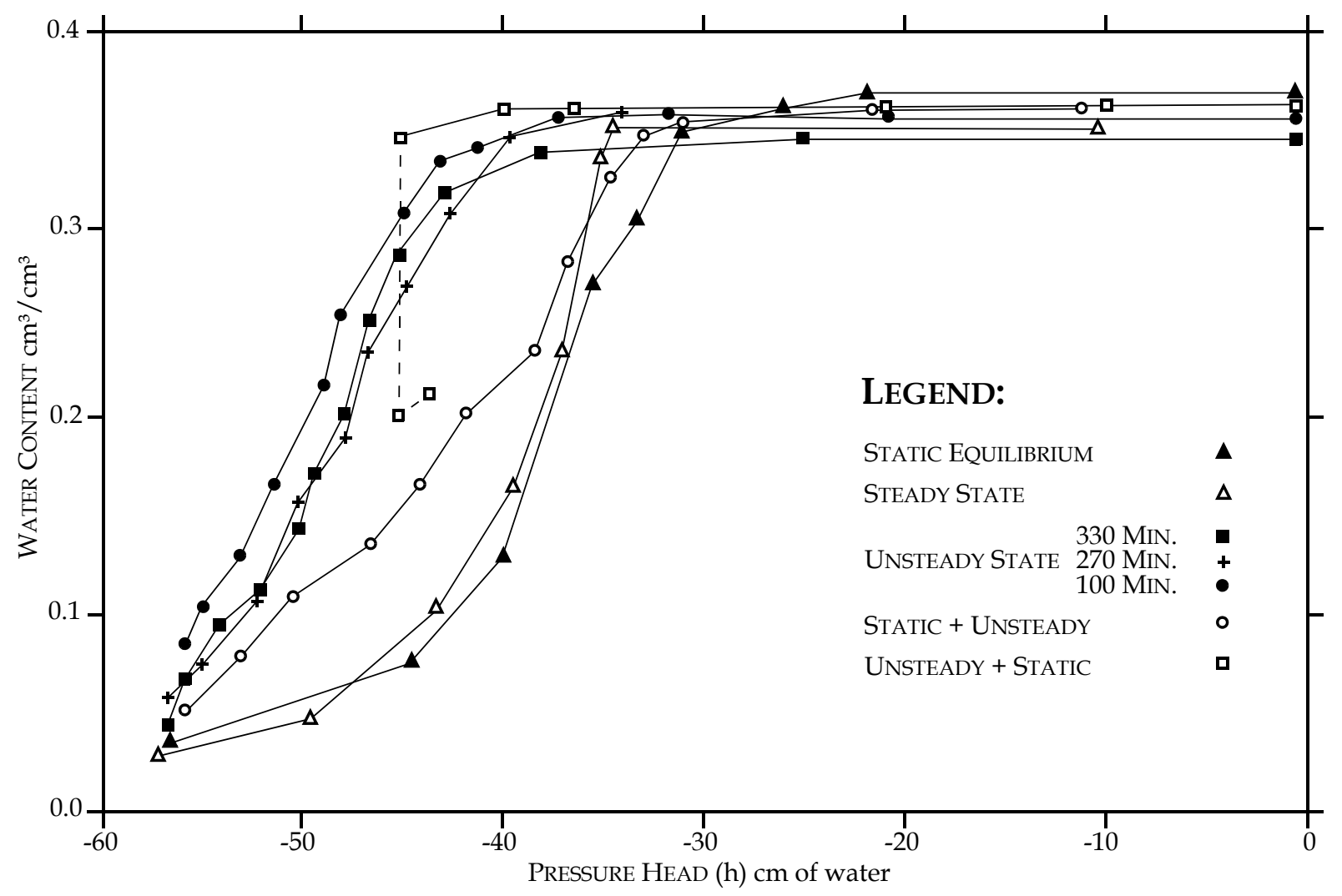

Figure 7.26: Water content versus pressure head data of a sand according to Topp et al. (1967). Comparison of drainage measurements under static, steady state and unsteady state conditions visualizing the influences of the process dynamics.

lary pressure - water content relation is dependent on the flow state. The same water content is assigned to higher capillary pressures under transient conditions than for the static case. He has formulated a generalized form of the capillary pressure - water content relation and has developed a first approach to account for this dynamic behaviour. In a final discussion, Stauffer (1977) relates these dynamic effects to hydrodynamic changes in the meniscus curvature and to the relation of main drainage direction and pore orientation.

A comprehensive experimental study on dynamic effects for both, wetting and drainage, was conducted by Wanna-Etyem (1982). He investigated four soils with varying grain size and texture. For all experiments, dynamic effects are observed. Their significance is dependent on the type of soil indicating that fine-textured soils are less affected than coarse textured soils. Probably as the first, Wanna-Etyem (1982) was able to prove the flow rate dependence to apply also for wetting processes.

Further experimental evidence is provided by Plagge (1991), Malicki et al. (1992) and Sobczuk et al. (1992), and later by Plagge et al. (1999), who investigated fine-textured soils with a new developed IPM technology based on TDR sensors for water content and tensiometers for moisture potential measurement. The performed drainage experiments exhibit a flow rate dependence of the moisture content - moisture potential relation under transient conditions. The stronger the gradient is, the larger is the shift of the moisture potential in direction of higher capillary pressures. 
Fig. 7.27 shows a reproduction of Fig. 2 according to Plagge et al. (1999) where transient water potential data is plotted versus the water content for a fine-textured soil. The measurement was conducted with a similar IPM setup as used for the present study. The water content was measured with TDR probes whereas the moisture potential was measured with tensiometers. The saturated specimens were drained either by a forced evaporation or by an applied suction via a ceramic plate providing various gradients in different moisture ranges. The sensor positions indicate the distance from the boundary condition. This means $1 \mathrm{~cm}$ for position $1,5 \mathrm{~cm}$ for position 5 and $7 \mathrm{~cm}$ for position 7 . The dynamic effects are clearly recognizable in Fig. 7.27, too. The measured data of position 1 provides the highest water contents for a given moisture potential. At this position, also the highest moisture potential gradients occur. The higher the gradient, the more moisture is retained in the material.

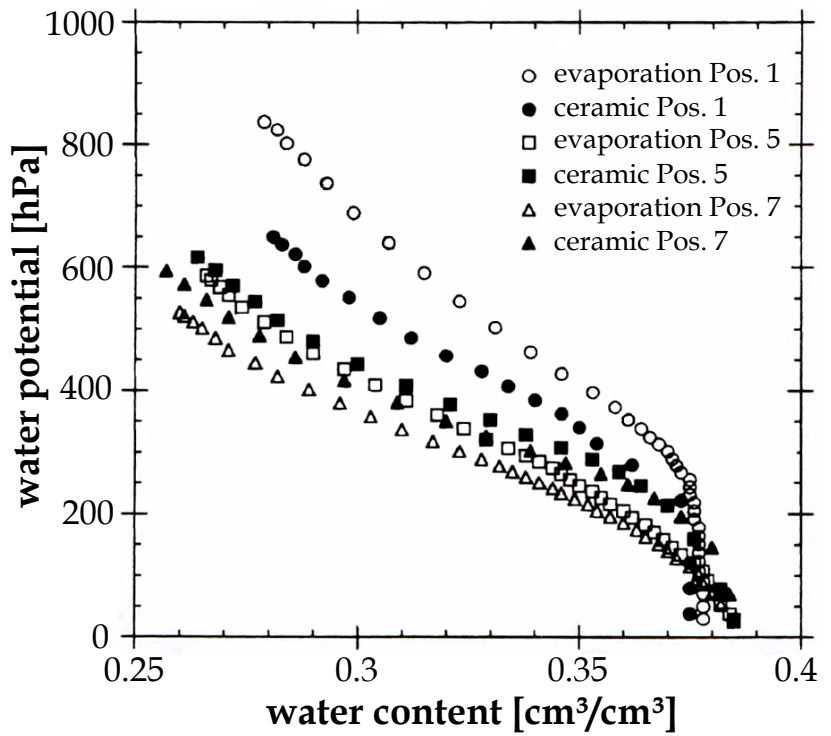

Figure 7.27: Transient capillary pressure versus water content data of a fine-textured soil according to Plagge, Renger \& Häupl (1999). The position numbering indicates the distance from the boundary condition in [cm]. Position 1 which is exposed to the highest moisture potential gradients provides the highest moisture contents for a given potential.

The IPM technology developed by Plagge (1991) was also applied to a building material, as reported by Plagge et al. (2006a), Plagge et al. (2006b), Scheffler et al. (2006) and Scheffler et al. (2007-b). They examined a calcium silicate insulation material similar to the one investigated here in a series of adsorption and desorption experiments. Their results revealed the existence of dynamic influences on the moisture content - moisture potential relation to occur not only for high degrees of saturation, but also within the hygroscopic moisture content range. Moreover, dynamic behaviour was observed during adsorption and desorption processes.

The review shows, that dynamic effects, as they became visible by means of the presented hygroscopic data, have also been observed by other authors in previous studies. However, most of these authors are related to Soil Sciences whereas in Building Physics, the dynamic influences have not yet been the subject of a broad discussion. This discussion has started now with the presented measurement and simulation results.

The comparison between measurement and simulation revealed a number of deviations. These regard the absolute values of absorbed or desorbed moisture as well as the particular water content profiles developing inside the specimens. Not all investigated building materials exhibited the same behaviour though. In fact, different effects superimpose depending on the material and its hygric properties. These are discussed above and may be summarized as follows. 
The calcium silicate and the building brick are materials with a low hygroscopic moisture capacity and high moisture transport properties. Their performance is similar and is hence discussed for both of them on the basis of the calcium silicate data. Two effects are clearly observed: the process dynamics and the not equilibrating relative humidity profiles during hygroscopic desorption. The process dynamics have already become visible in the dynamic moisture storage curves. Disregarding them during the simulations leads to higher transport rates for the adsorption and to lower transport rates for the desorption process. The effect of not equilibrating relative humidity profiles superimposes the desorption as the simulation approaches the assigned equilibrium condition for both, relative humidity and water content. The moisture potential differences are therefore larger during the simulation than during the measurements resulting in higher transport rates for the simulation. The desorption process can hence be reproduced quite well for these materials.

The similarity of the hygric storage and transport characteristic between the aerated concrete and the lime-sand brick does not lead to similar simulation results. The performance of the lime-sand brick is different. The integral water absorption can be reproduced for the first couple of days. Then, the simulation starts to underestimate the absorbed amount of moisture which is in contrast to the observed dynamic moisture storage data and the effects this would imply. The calculated desorption follows the measured course with satisfying agreement. For both processes, however, the calculated moisture profiles do not correspond with the measured data.

The material behaviour of the lime-sand brick is much more complex than for the other materials. Due to the low moisture transport properties, the actual equilibrium stages are never reached. The hygric performance of the lime-sand brick is a complex superposition of non-equilibrium stages that influence each other. The transport function is adjusted during the calibration procedure. This procedure is based on two unsteady state flow experiments comprising one configuration of transient states which do hardly correspond with those passed during the hygroscopic ad-and desorption experiments. The mixture of different static, steady state and unsteady state data for the adjustment procedure is hence another reason for the observed deviations.

The calculations performed for the aerated concrete visualize the dynamic influences most distinctively. The simulation overestimates the moisture transport during the adsorption and underestimates it during the desorption experiments. Beyond these deviations, the calculated water content profiles are rather close to the measured ones. The measured effect of not equilibrating relative humidity profiles is much less pronounced for the aerated concrete which is the reason why the dynamic effects are not as intensively superimposed as for the brick or the calcium silicate.

The simulation results can ultimately be generalized as follows. On the one hand, it is observed that the general tendencies of moisture transport are followed correctly. On the other hand, the moisture profiles in general and the absolute values of absorbed or desorbed moisture for many cases can not be reproduced with satisfying agreement. The influence of the moisture storage function is very significant since most deviations between measurement and simulation are a consequence of deviating moisture potential - water content relationships under transient conditions. In addition, for materials with low moisture transport properties, the process history sustainably influences this relationship. The other reason for observed differences is the remaining of not equilibrating relative humidities which occur especially during desorption processes of the materials with very low hygroscopic moisture capacities. 
For the assessment of the applied material model, the distinction between material modelling and transport theory which is made at the very beginning of Chapter 2 may be highlighted, again. Task of material modelling is to derive storage and transport functions from measured basic material data which can be used for numerical simulation. However, material modelling is always dependent on the transport theory into which it is embedded. Simplifications at the transport theory level affect also the quality which can be achieved by the material model. This is very important since the presented measurement as well as simulation data clearly show the impact of such simplifications on the transport theory level. Neither the influence of the process history, nor the implications of its dynamics are included there. The simulation applies only one moisture storage function for any process.

The discussion reveals that the non-uniqueness of the moisture storage characteristic is the main reason for the observed deviations between measurement and simulation. Within the frame of the current configuration, it is the transport theory which limits the precision of hygrothermal calculations. It is very difficult to assess the material model detached from the transport theory and, in addition, it is not reasonable. However, it is one of the current objectives to develop such an assessment. Based on the presented hygroscopic experiments, the following conclusions on the material model are therefore drawn.

The material model itself is very suitable to describe moisture transport processes within the hygroscopic moisture content range since it combines the information of a number of different types of experiments as sorption, vapour diffusion, water uptake and drying measurements. Moreover, the material model is open for further developments on the transport theory level. The current model provides a stable basis for the material behaviour description despite the observed deviations between measurement and simulation. However, before continuing this discussion, the results for the overhygroscopic moisture content range are to be shown and discussed. This is the subject of the following paragraph.

\subsection{Overhygroscopic moisture transport}

The experiments within the overhygroscopic moisture content range consist of three components: water uptake, equilibration and drying. In paragraph 5.3.2 Measurement set-up for the overhygroscopic moisture range on page 127 et seqq., the measurement schedule as well as the experimental set-up are introduced. Only the water content is measured for these experiments. Transient moisture storage data as it is presented for the hygroscopic moisture content range can not be evaluated here. However, the comparison of measured and calculated water content profiles opens a new and interesting perspective on the moisture transport and its dependencies.

For this paragraph, a further subdivision is not conducted as it appears to be of less importance to discuss the measured results detached from the simulation. Two interesting effects which become visible at the measured water content profiles are highlighted within the frame of the general discussion.

The simulations are performed in the same way as before. Initial and boundary conditions as well as the output specifications are assigned as described at the beginning of 
paragraph 7.1.2 Hygroscopic simulation results and illustrated in Fig. 7.14. The material data is assigned according to the measured data. This means, if an adsorption process occurs within one compartment, the adsorption moisture storage function is assigned and if a desorption proceeds, the desorption moisture storage function is used for the simulation. For those experiments where a water uptake is followed by a distribution period, the material data is partly reassigned at the end of the water uptake period.

In contrast to the hygroscopic experiments, the overhygroscopic results of one material group are very similar. The presented results are therefore reduced to two materials: the building brick for the one and the aerated concrete for the other material group. Out of the experimental runs of each material, one water uptake and subsequent distribution period and one drying period are selected for the building brick. These are discussed as follows.

Fig. 7.28 shows measured and calculated water content profiles of the building brick. For the first 20 minutes, water contact is applied to the bottom side of the specimen. Then, this side is also sealed and the material is left with a no-flow boundary conditions at all sides. During the simulation of the water uptake phase, the adsorption function is assigned to all compartments. Afterwards, the desorption function is assigned to compartments 1 and 2 according to the material behaviour of the measured data.

The comparison of measured and calculated profiles in Fig. 7.28 reveals three main differences. The first is the total amount of absorbed water, the second is the signal velocity with which the water front moves into the sample, and the third is the developing moisture profile.

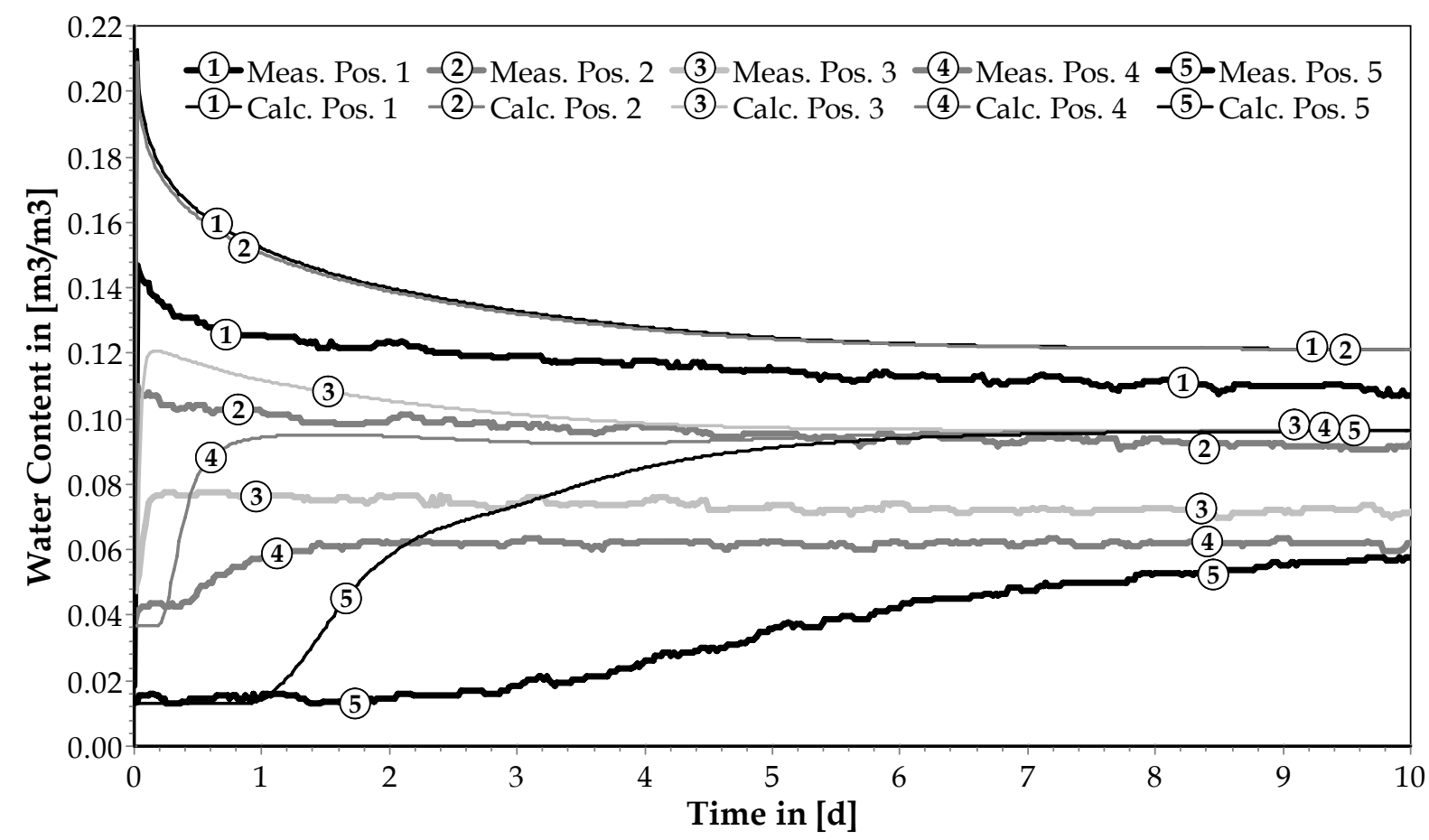

Figure 7.28: Measured and calculated water content data of the second water uptake and subsequent equilibration period for the building brick. Thick lines indicate the measurement whereas thin lines indicate the simulation results. 
Starting with the latter observation, it becomes apparent that the measured data forms a distinct moisture profile with different remaining water contents in all compartments. During the simulation though, the developing profiles equilibrate to only two different moisture contents at the end of the experiment. As only two different moisture storage functions are applied, namely the adsorption characteristic for compartments 3, 4 and 5 and the desorption characteristic for compartments 1 and 2, only two different water contents can remain as soon as the capillary pressures have equilibrated. This deviation from the measured data is a consequence of the applied moisture storage functions which define the equilibrium states for ad- and desorption processes. The measured data shows, that the conditions inside the brick do not necessarily approach one of these states.

The second aspect is the signal velocity, i.e. the velocity with which the moisture front moves into the material sample. The calculated water content profiles react much faster than the measured ones, as can be seen at positions 3, 4 and 5 in Fig. 7.28. This effect is closely connected to the third observation that the calculated water uptake is higher than the measured. The transport rates are overestimated by the simulation which becomes even more apparent when looking at the integral water content versus time curve in Fig. 7.29.

Here, only twelve hours are depicted where water contact is applied during the first 20 minutes. The calculated water absorption proceeds clearly too fast leading to an absorbed water mass which is almost twice as high as during the measurement.

There exist two possible explanations for this calculated material behaviour. On the one hand, air entrapment can be the reason for the reduced water absorption during the measurements. And on the other hand, dynamic effects can be their cause.

It was shown by different authors that the entrapment of air can significantly reduce the amount of absorbed water during a water absorption experiment, as already discussed under paragraph

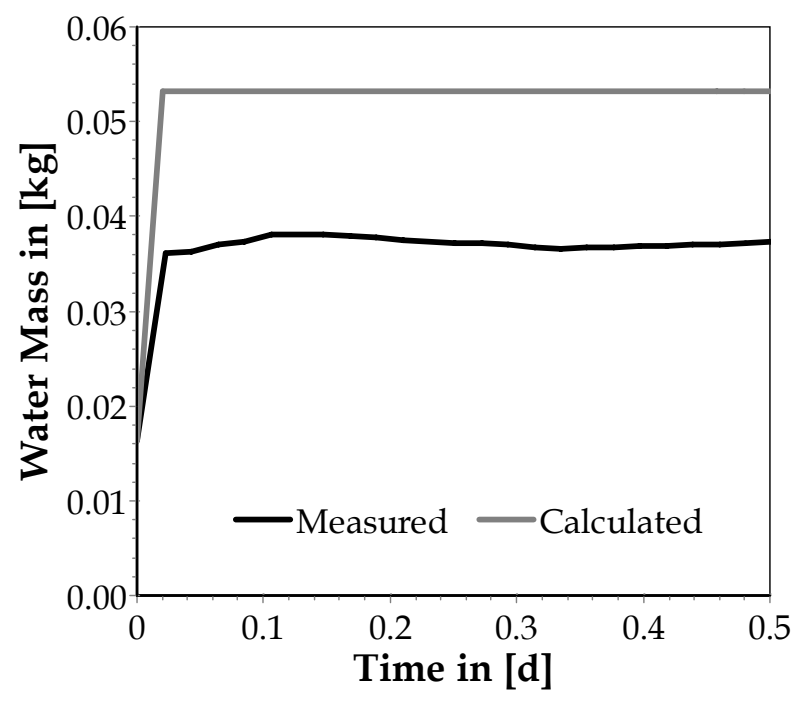

Figure 7.29: Integral water mass inside the building brick during the second water uptake and subsequent distribution period. 2.1.4 Remarks on heat, air and moisture transport as well as under paragraph 3.1.5 Water uptake experiment. According to Schultze et al. (1999), a significant portion of the dynamic effects they have observed are caused by non-negligible air flow resistances. The top sealing of the specimens which are investigated for the present study is therefore pierced to allow air pressure equilibration. However, the experiments alternate between water uptake, distribution and drying processes. The data shown in Fig. 7.28 and Fig. 7.29 belong to an experiment which starts after previous water uptake, distribution and drying periods. During the first water absorption, the air pressure can surely equilibrate with the atmosphere due to the pierced sealing. However, for the presented experiment, a considerable amount of water is already inside the specimen. 
Air entrapment can therefore occur between the water profile which has already established during the previous experiments and the penetrating water front of the current one. As a consequence, the absorption process is slowed down during the experiment and the simulation which does not include the air mass balance overestimates the absorbed amount of water.

If air entrapment is the reason for this overestimation, the initial water uptake would have to provide different results. This is not the case. In fact, the simulated water absorption is overestimated by the same amount of water for all three water uptake experiments and no difference is observed between the initial and the other absorption processes. The air flow resistance may hence not be the reason for the witnessed differences between measurement and simulation. Instead, dynamic influences are their actual cause.

According to the general effects the process dynamics imply, the water transport rates are reduced during adsorption and increased during desorption processes. The simulation which does not include these effects must overestimate adsorption and underestimate desorption processes. The obtained water uptake results perfectly correspond with this explanation.

The integral water mass curves for the final drying experiment of the building brick are given in Fig. 7.30. Here, the opposite behaviour can be observed. The measured water mass decreases much faster than the calculated over a large period of time. Not until approximately the 20th day, the calculated curve corresponds with the measured one. The observed drying behaviour therefore supports the given explanation that dynamic effects are the reason for the deviations between measured and calculated material behaviour.

Fig. 7.31 shows the water content profiles of the building brick drying. Except for position 2, all calculated water content profiles differ significantly from the measured data. Posi-

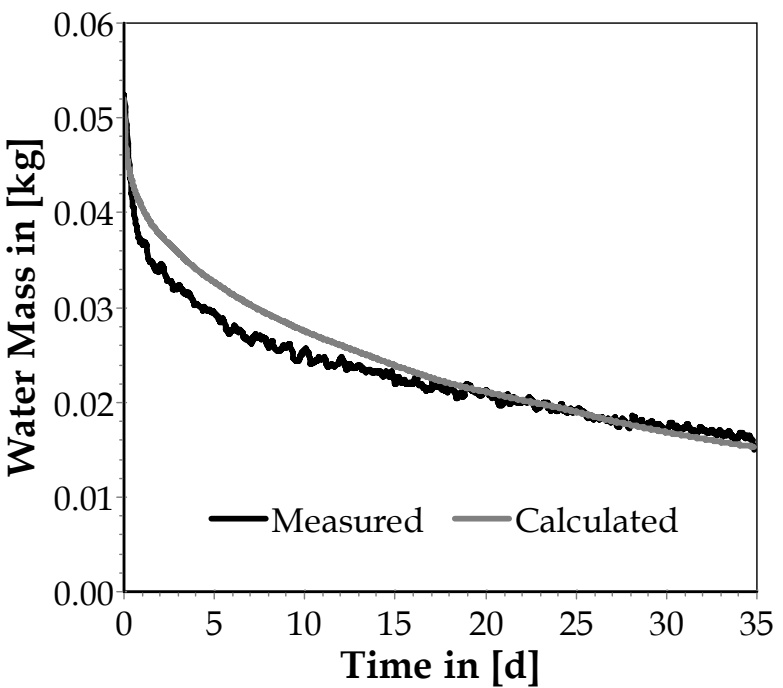

Figure 7.30: Integral water mass inside the building brick during the final drying period. tion 1 exhibits a drying which is faster during the simulation than during the measurement. In addition, the calculated remaining water content is significantly lower than the measured. The calculated water content curves of positions 3, 4 and 5 increase during the first two days before they start to decrease. The measured data start to decrease immediately at the beginning of the experiment. Moreover, the distinct water content profile which establishes in the range of $0.06 \ldots 0.02 \mathrm{~m}^{3} / \mathrm{m}^{3}$ is not such clearly reproduced by the simulation, although a similar behaviour is discernible at lower water contents.

The calculated redistribution process at the beginning of the experiment causing a water content increase for positions 3,4 and 5 is a consequence of the assigned moisture storage function. The experiment reaches an equilibrium stage where a distinct 
water content profile remains inside the material specimen. A further distribution does not occur. The changed boundary condition into evaporation at the bottom surface can therefore imply a desorption process for the whole specimen. During the simulation, this is different. There, only one equilibrium criterion, i.e. one moisture storage function, is applied. As this is the same for all compartments, the assigned initial moisture contents cause a non-equilibrium condition by means of moisture potential which is superimposed by the implied drying process. The initial redistribution within the upper compartments is thus a consequence of the circumstance that the simulation implies only one criterion for the hygric equilibrium.

Although position 1 is drying too fast, the water transport inside the brick is underestimated for a large range of time. Bearing in mind the hygroscopic data, two effects interact here, too. One is the process dynamics which is already discussed above and is seen as the main reason for the deviating moisture transport rates. The other is the remaining relative humidity profile which is most pronounced for the desorption of the calcium silicate and the building brick. Unfortunately, no moisture potential data is available for the present experiment. But it is likely that the same behaviour occurs during this desorption process, too. The different water contents in position 1 at the end of the experiment indicate that another system state is reached here than the one the simulation assumes, i.e. the equilibrium with the boundary relative humidity according to the desorption moisture storage function. For this reason, the calculated drying ends at a lower water content than the measurements provide. Moreover, the moisture transport rates which are underestimated for the first 20 days are overestimated by the simulation at the end of the experiment due to the higher moisture potential gradients that occur when no relative humidity profile remains. This becomes visible in Fig. 7.30, too.

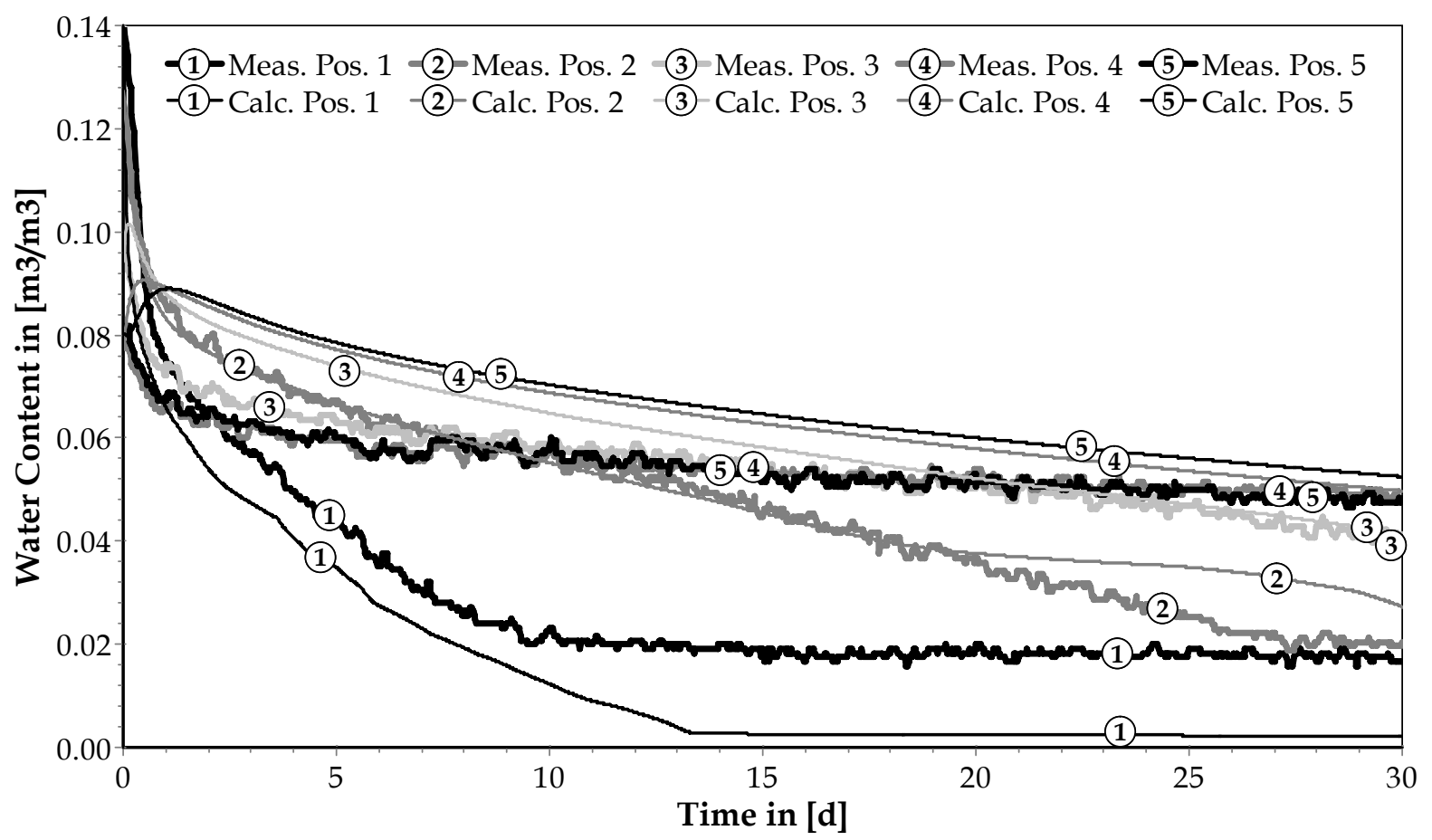

Figure 7.31: Measured and calculated water content data of the final drying for the building brick. Thick lines indicate the measurement whereas thin lines indicate the simulation results. 
The overhygroscopic experiments of the building brick revealed that the current combination of transport and material modelling is able to generally follow the measured data, especially for the drying process. It is, however, not able to correctly describe the different moisture transport processes in detail. The main reason of the observed deviations between measurement and simulation is the incorrectly described moisture potential - water content relation which appears to be significantly influenced by the process dynamics. The consequence is a number of different equilibrium states and thus also different induced transport rates. But before continuing the discussion on the material model, the results of the aerated concrete are to be shown as follows.

The measured data obtained during the first water uptake experiment and the subsequent distribution period of the aerated concrete is compared with the corresponding simulation results in Fig. 7.32. Here, the signal velocity appears to be rather well expressed by the calculation. However, the simulation overestimates the total absorbed water mass. In Fig. 7.33 at the left hand side, the respective integral water mass curves are displayed confirming this observation. The measured water content profiles are not reproduced by the simulation. Instead, two different water contents are approached at the end of the experiment which correspond with the water content values of the assigned moisture storage characteristics. For positions 1, 2 and 3, this is the desorption moisture storage function whereas it is the adsorption curve for positions 4 and 5 .

Additionally, two interesting phenomena can be observed here which are even more pronounced during the third water uptake experiment of the aerated concrete shown in Fig. 7.33 at the right hand side and in Fig. 7.34. The first is the peak appearing in the

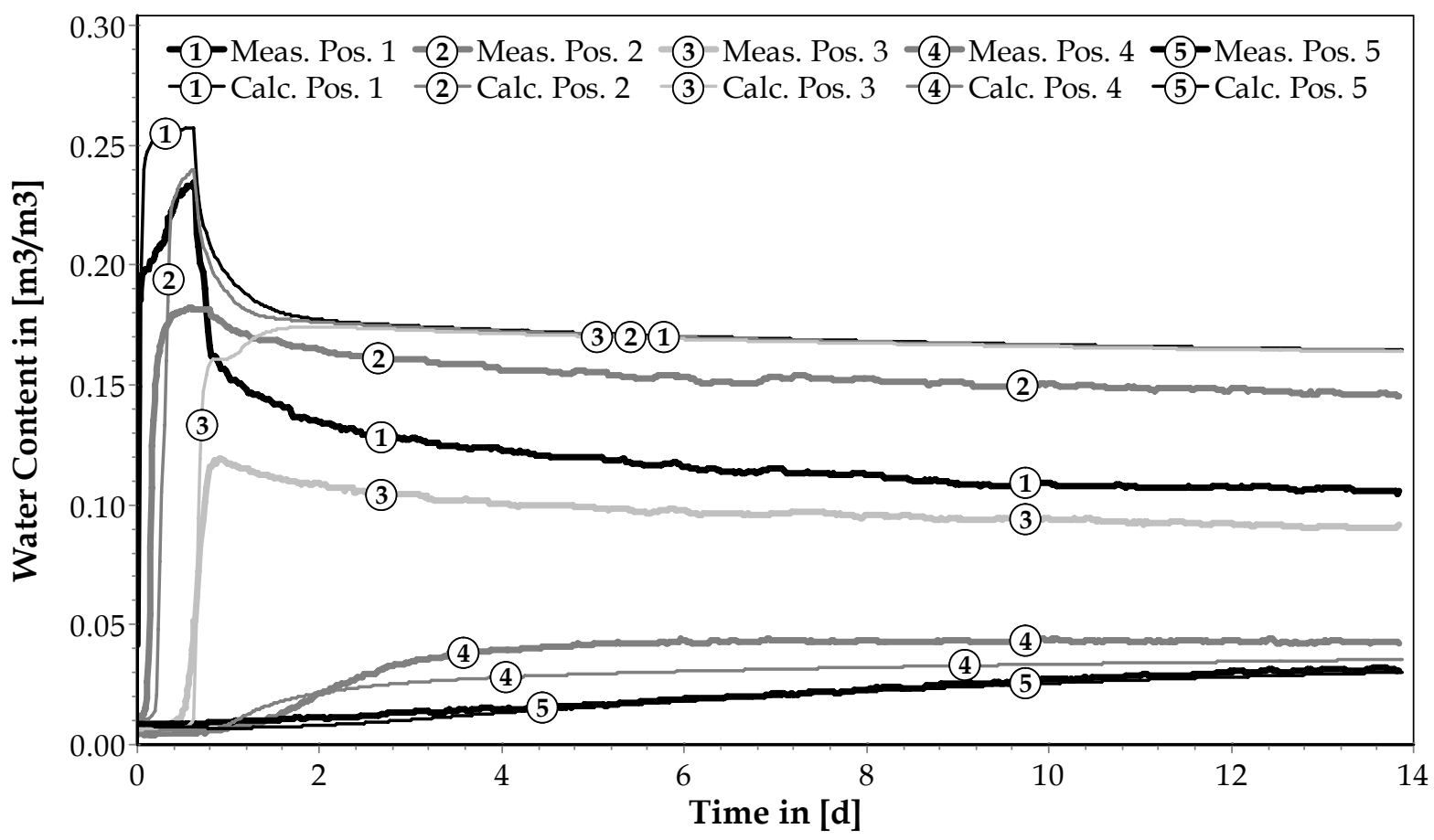

Figure 7.32: Measured and calculated water content data of the initial water uptake and the subsequent distribution period for the aerated concrete. Thick lines indicate the measurement whereas thin lines indicate the simulation results. 

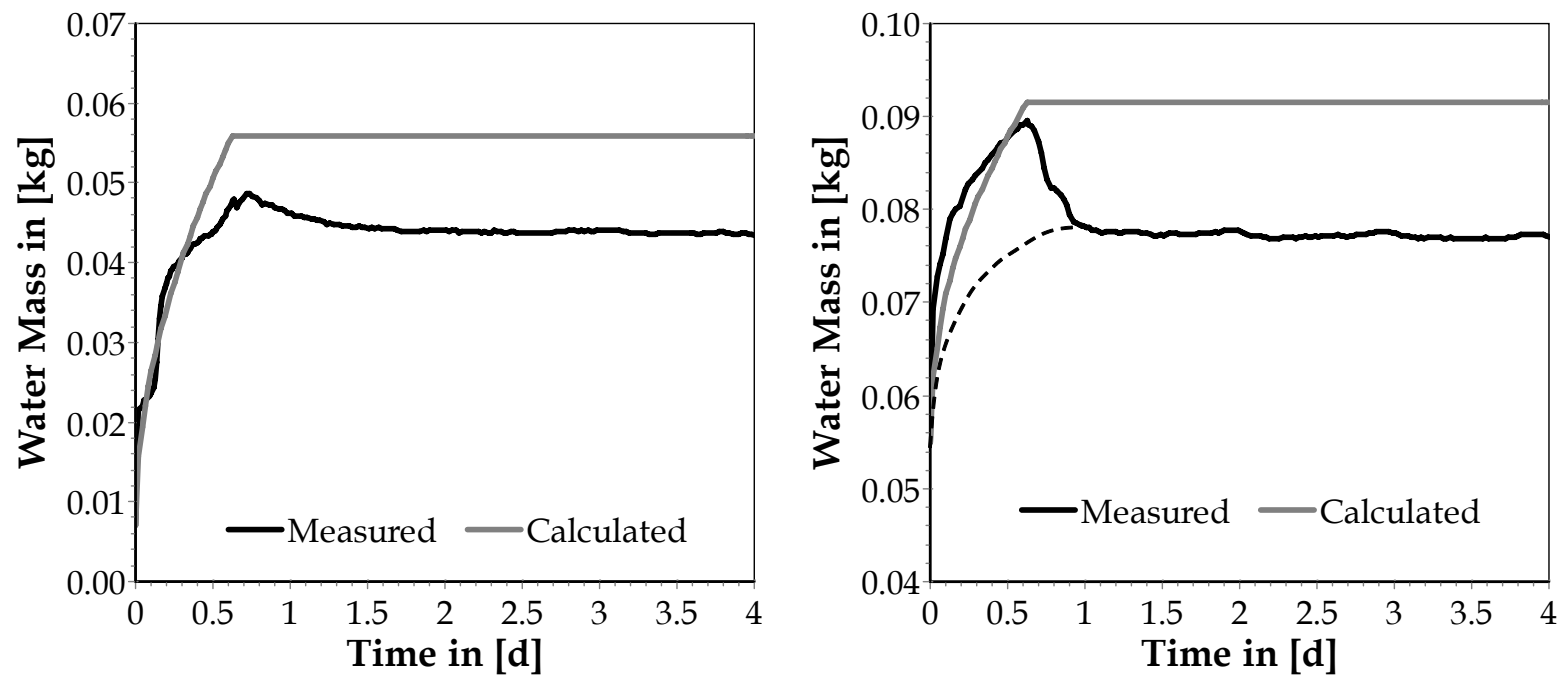

Figure 7.33: Integral water mass inside the aerated concrete during the initial water uptake (left) and the third water uptake (right), each with the subsequent distribution period. Note that the ordinate scales are different.

integral water uptake curve, see Fig. 7.33, and the second is the course of the water content within the first compartment, see Fig. 7.34.

The strong water absorption within the first compartment and the corresponding peak of the integral water uptake curve is a measurement artefact. At higher water contents, water accumulates in the large pores around the lower TDR probe which significantly

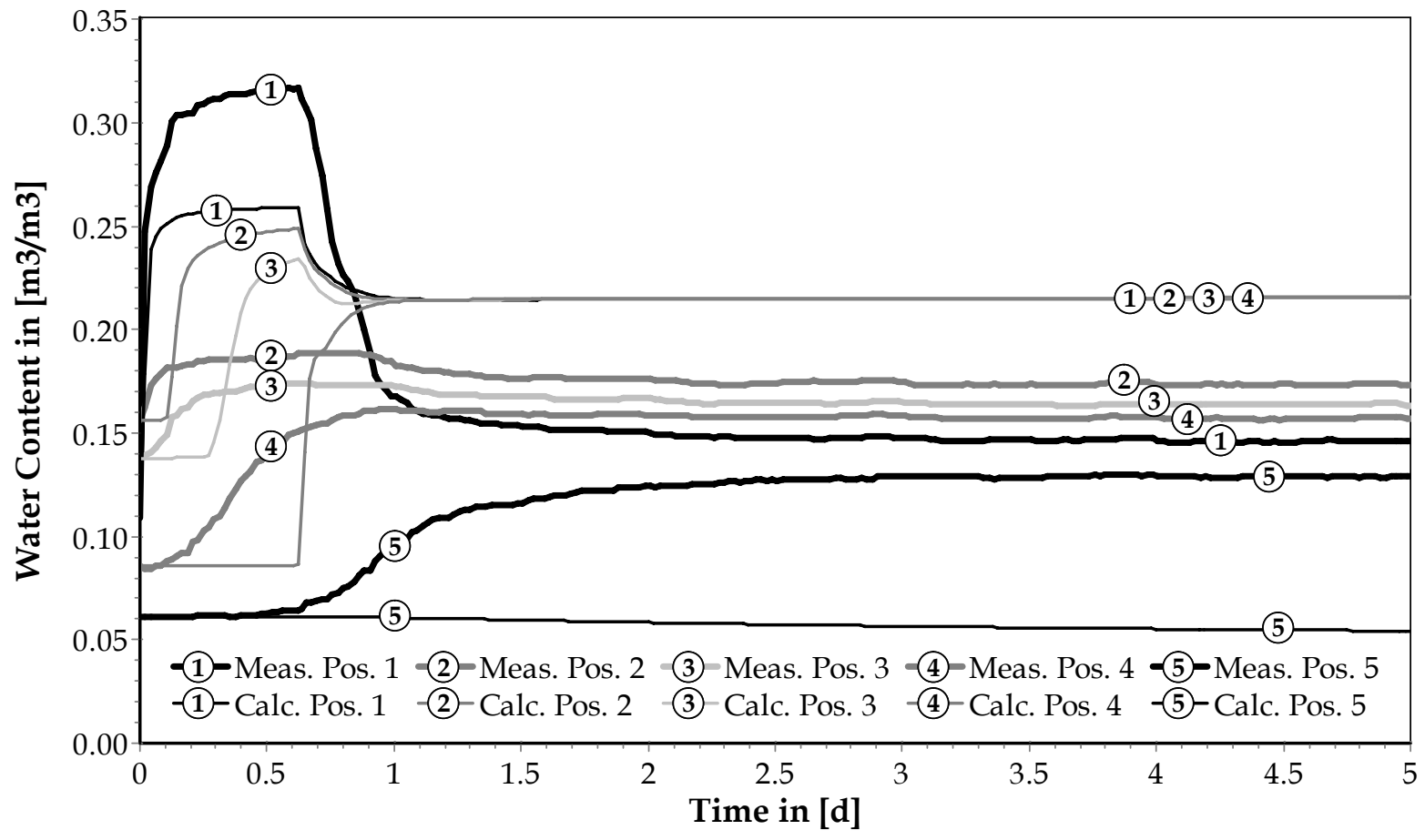

Figure 7.34: Measured and calculated water content data of the third water uptake and the subsequent distribution period for the aerated concrete. Thick lines indicate the measurement whereas thin lines indicate the simulation results. 
distorts the measurement signal. The probe interprets this signal as such a high dielectric constant which normally corresponds with a much higher water content. As soon as the water contact is removed from the bottom surface, this water is absorbed by the smaller pores and the measurement signal is interpreted correctly, again. The dashed line in the right chart of Fig. 7.33 would therefore be the actual integral water mass curve for the first day.

Initially, it was presumed that this effect could be a consequence of a leaky sealing. However, after a rather short equilibration time subsequent to the end of the water uptake phase, the integral water mass remains constant for all experiments. The hypothesis of a leaky sealing is hence refuted. Moreover, the observed effect exhibits a varying significance depending on the material as well as on the water content level. For the aerated concrete at high water contents, it is most pronounced but it occurs also during the lime-sand brick as well as the building brick measurements.

The second interesting phenomenon is the course of the water content curve of the bottom compartment during the distribution period subsequent to the water uptake. In Fig. 7.32, the measured water content of position 1 takes significantly lower values than position 2. The data of Fig. 7.34 shows this effect even more pronounced. Here, the water content of position 1 decreases below positions 2, 3 and 4. Although less distinctive, the same material behaviour is observed for the lime-sand brick, too.

This phenomenon is another indication for the complex interactions between moisture content, moisture potential and moisture transport which are not yet totally understood. During the transient water absorption, the capillary pressure within the first compartment decreases significantly and the water potential - water content relation follows neither of the static curves. Instead, different adsorption scanning curves may have established within each compartment as illustrated for positions 1 and 2 in Fig. 7.35. During the subsequent redistribution process, each compartment follows another type of desorption scanning curve. By that, the equilibration of the capillary pressure

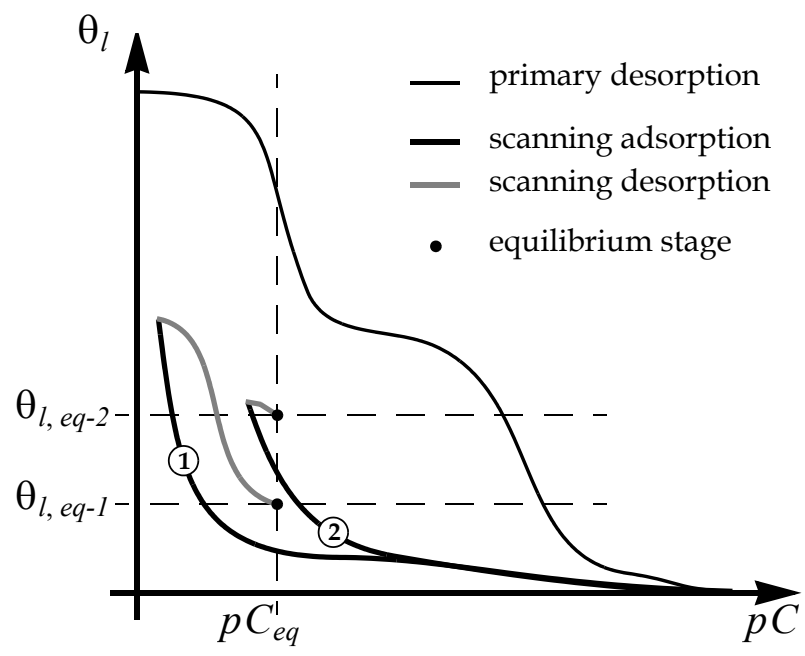

Figure 7.35: Possible scanning curve configuration leading to lower water contents in position 1 than in position 2. leads to the observed water content profile as indicated in Fig. 7.35.

Since this effect is experienced for the aerated concrete and the lime-sand brick only, it appears that materials with a wide pore-size distribution and low liquid transport properties are much more susceptible. But as the effect is another consequence of the process dynamics, this kind of classification is not very valuable since the only implied conclusion is that the process dynamics affect the material behaviour differently in dependence on their own storage and transport properties. 
Besides these two effects, it may be noted that the calculated water uptake proceeds too fast leading to a larger amount of absorbed water than measured during the experiment. The distinct water content profiles can not be reproduced correctly by the simulation, especially not the effect of a lower water content in position 1 at the end of the distribution period. The primary reason for the observed deviations between measurement and simulation is the complex interaction between water content and water potential depending on the process and its history. As these dependencies are yet not included on the transport theory level, the simulation can only follow the general tendencies but not the particular profiles.

The comparison of measured and calculated integral water mass during the final drying period of the aerated concrete is given in Fig. 7.36. The simulation follows the measured course at the beginning. After around five days, the calculated curve starts to deviate from the measurements underestimating the drying and the corresponding moisture transport rates. Around the 30th day of drying, a smooth bend of the measured drying curve indicates that the drying process is slowed down. This bend is not observed in the calculated curve. As a consequence, both curves meet again around the 60th day of drying. Thereafter, both follow more or less the same drying path until the equilibrium with the drying environ-

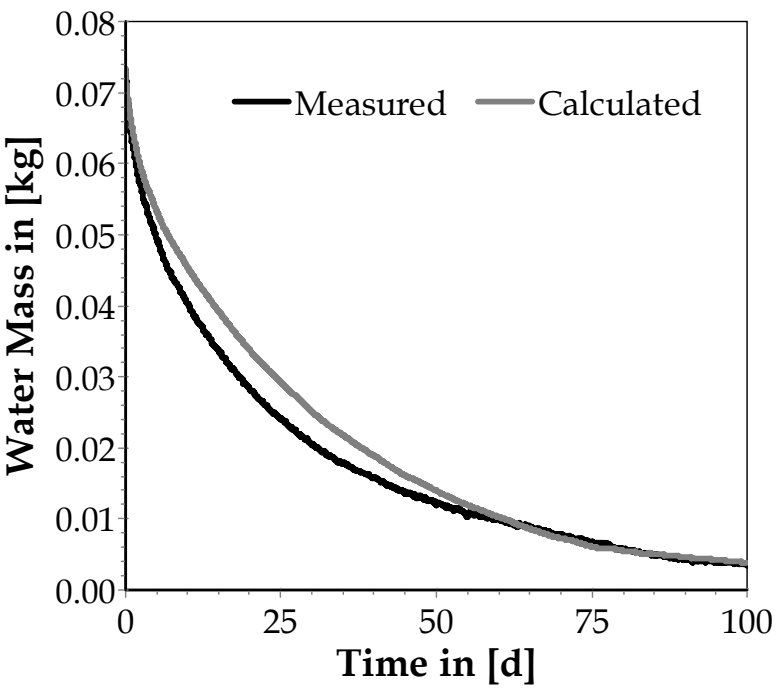

Figure 7.36: Integral water mass inside the aerated concrete during the final drying period. ment is reached after circa 100 days.

The respective water content profiles are presented in Fig. 7.37. To improve the legibility of the chart, only 80 days are displayed there. Although measured and calculated integral drying curves are rather close, the calculated water content profiles look perspicuously different than the measured ones. At position 1 , the simulation shows a faster water content decrease than the measurement. In position 2 , the same occurs. At positions 3, 4 and 5, the calculated water content data deviates from the measured data in the other direction. At the beginning of the experiment, the upper compartments exhibit a redistribution first before they start to participate in the general desorption process. This desorption proceeds too slowly leading to the underestimation which has been observed at the integral drying curve. At the end of the experiment, the simulated water content profiles meet or even intersect with their measured counterparts.

Except for the redistribution process within the upper compartments at the beginning of the experiment, the simulation generally reproduces the measured material behaviour quite well. However, the particular water content profiles exhibit deviations of varying significance. The calculated redistribution at the beginning is again a consequence of the applied moisture storage function. The experiment reaches an equilibrium stage during the previous distribution period. When the drying starts, the previous transport processes are subsided and the desorption affects the whole material specimen. For the simulation, the measured initial water contents are assigned to 


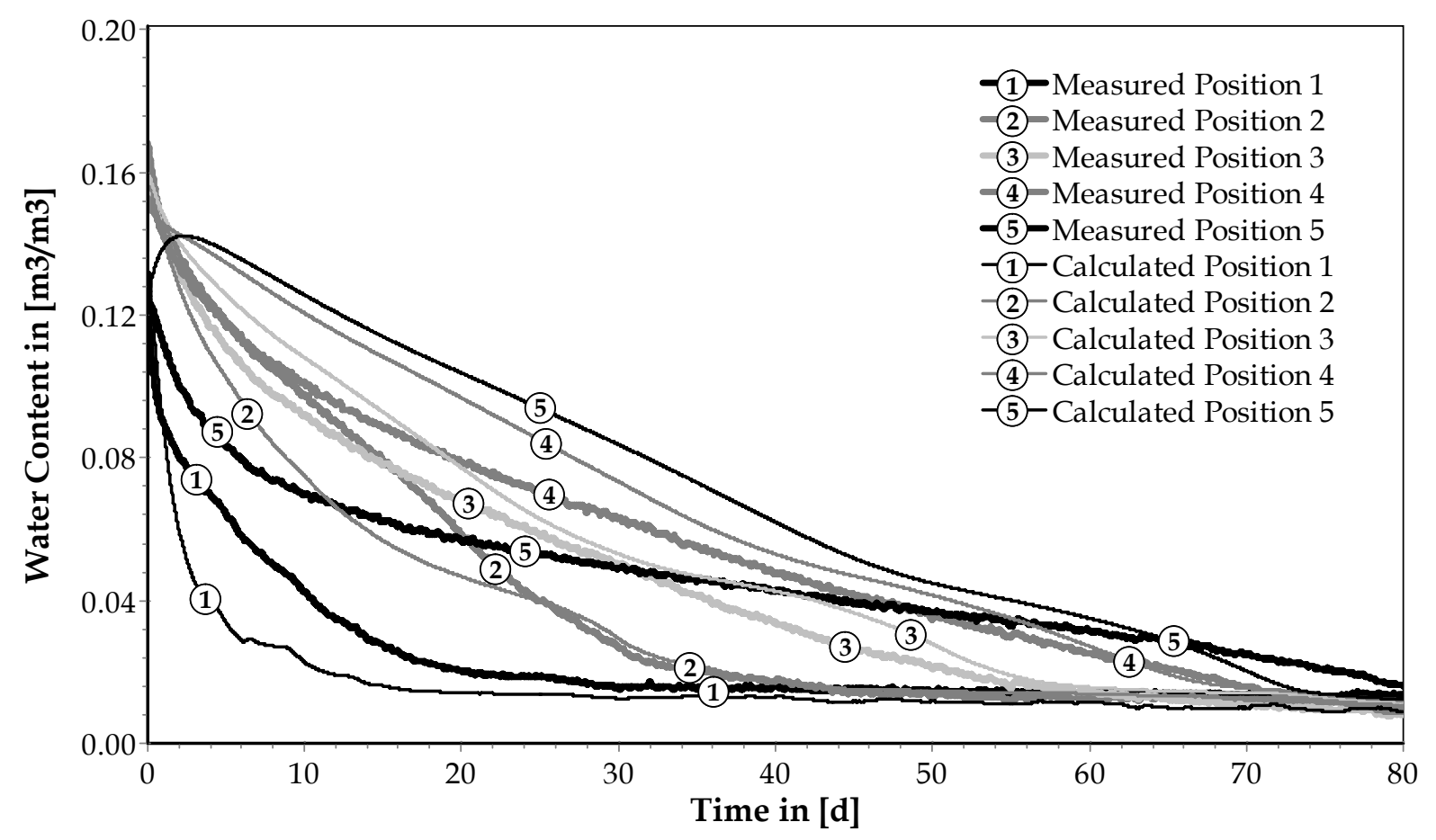

Figure 7.37: Measured and calculated water content data of the final drying period for the aerated concrete. Thick lines indicate the measurement whereas thin lines indicate the simulation results.

the corresponding compartments. These water contents do though not define an equilibrium stage according to the applied desorption moisture storage function causing the initial redistribution process.

The other deviations are difficult to assign to a single cause. It is the author's opinion that the differentiated water content - water potential states at the beginning and their change and development during the process are responsible for the measured water content data. The deviation cause is therefore seen to be a combination of insufficiently considered process history and dynamic changes in the water potential - water content relation.

The presented results of the aerated concrete are summarized as follows. The simulation results follow the general tendencies during the measured moisture transport processes. The particular water content profiles can though not be correctly reproduced, neither for the adsorption nor for the desorption processes. Moreover, the integral curves differ from the measured data in such a way that the simulation overestimates the moisture transport during the water absorption and underestimates it during the drying. The deviations are attributed to the process dynamics which cause the water content - water potential relation inside the material to follow along different paths than the moisture storage function measured under static conditions would imply.

The consequences of this dynamic behaviour are, on the one hand, changed moisture potential gradients and thus changed moisture transport forces inside the material, and, on the other hand, numerous different equilibrium stages which are attained. The simulation which applies either the static adsorption or the static desorption moisture 
storage function must therefore fail twice to follow the measured data. First, the transport rates are either overestimated during an adsorption or underestimated during a desorption process. And second, the water content profiles at the end of a water absorption experiment must vanish. The calculation exhibits a moisture transport as long as the moisture potential equilibrium is not reached. When this equilibrium is reached though, the same water content occurs in all compartments where the same moisture storage function is assigned. Moreover, if a simulation is started with the measured water content distribution as initial condition, a redistribution process will start if this distribution does not display an equilibrium state according to the applied moisture storage function.

The data of the aerated concrete as well as the lime-sand brick generally obey to this given explanation. For some experiments of the lime-sand brick, these dynamic effects are even more pronounced than for the aerated concrete which is reflected in larger deviations between measurement and calculation. These becomes visible in both, the water content profiles and the integral curves. Before the final discussion will attempt to distinguish the different influences, the observed material behaviour of all materials within the overhygroscopic moisture content range may be summarized as follows.

The comparison of measured and calculated water content profiles generally reveals that the process history as well as the process dynamics significantly influence the moisture transport within the overhygroscopic moisture content range, too. All materials appear to be susceptible to these influences although the developing moisture profiles differ for different materials in dependence on their particular properties. Basically, the following deviations between measured and simulated data are observed by means of the investigated materials:

- Observations during adsorption and distribution:

- The calculated water absorption is generally too fast, the totally absorbed amount of water is overestimated.

- The distinctly measured water content profiles do not develop during simulation. Instead, the equilibrium water content is reached for all compartments according to the assigned moisture storage function (either adsorption or desorption).

- During the distribution period, the water content within the first compartment of the lime-sand brick and the aerated concrete falls below the water content of the next compartments. This effect can not be reproduced by the simulation.

- Observations during desorption:

- The calculated desorption is generally too slow, the drying is underestimated over a large period of time.

- At the beginning of the desorption experiments, the simulation exhibits an additional redistribution of moisture which does not occur during the measurements.

- The calculated water content profiles go partly below and partly above their measured counterparts. 
Although the basic tendencies can be followed by the simulation, a number of significant deviations between measurement and calculation occurs also within the overhygroscopic moisture content range. In general, the calculation overestimates water absorption processes and underestimates the desorption processes. The distinct water content profiles confirm these deviations between measurement and simulation. Furthermore, the attained water content profiles at the end of the distribution periods can not be reproduced by the simulation and, as a consequence, the application of these profiles as initial conditions of the subsequent drying causes a redistribution which does not occur during the measurements.

However, the observed deviations can not be attributed to the material model. Instead they are a consequence of simplifying assumptions made on the transport theory level, in particular the assumption of local thermodynamic equilibrium ${ }^{1}$. The material model itself provides an expedient basis for a precise and flexible description of general hydrophilic material behaviour. From the presented experimental data, clear deficiencies of the model can not be discovered. The material model is therefore seen to be very suitable to describe overhygroscopic moisture transport processes. A final statement is though supposed to be derived from the general discussion on all presented data of both moisture content ranges which is the subject of the following concluding paragraph.

\subsection{Discussion and Conclusions}

The comprehensive IPM study carried out on the four building materials provides a strong and reliable experimental basis to investigate whole range moisture transport processes. Their simulation and subsequent comparison with the measured data challenges both, the transport theory and the material model. A number of interesting effects are observed which are to be summarized and discussed here. Entering this concluding discussion, a summary on the observed material behaviour and the deviations between measurement and simulation is given first. Subsequently, the single aspects are discussed. Finally, a concluding assessment of the applied material model is derived.

\subsubsection{Observation summary}

Within the hygroscopic moisture content range, experimental adsorption and desorption cycles are performed. The water content is measured by TDR sensors whereas the water potential is measured by coupled temperature and relative humidity sensors. From this data, transient water content - water potential curves are derived and compared with the static adsorption and desorption functions. This comparison revealed that the water content - water potential relation does not follow the static curves under transient conditions. The difference between static and transient data as well as the corresponding hysteresis loops are the more pronounced, the higher moisture potential gradients are implied. Moreover, this dynamic effect which has previ-

1. Refer to the list of assumptions given in paragraph 2.1.1 Transport processes on page 18 et seqq. 
ously been witnessed mainly during desorption processes and for much higher degrees of saturation become clearly visible for the hygroscopic moisture transport and for the adsorption, too.

The observed effects are confirmed by the simulation of the hygroscopic experiments. The transient processes can not be correctly reproduced during the calculation based on static moisture storage data. The simulation study reveals that different effects influence the material behaviour. Except for the lime-sand brick, the calculated adsorption is generally overestimated. During the hygroscopic desorption of the building brick and the calcium silicate, a distinctive relative humidity profile remains inside the specimens which does not equilibrate. During simulation, these profiles equilibrate implying a moisture transport to proceed until the equilibrium is reached. As a consequence, the desorption is also overestimated for these two materials. Inside the aerated concrete and the lime-sand brick, the remaining relative humidity profiles during desorption are visible but much less pronounced. The calculated desorption is hence rather close to the measurement for the lime-sand brick. The aerated concrete underestimates the desorption rates. However, it must be pointed out that the calculated water content profiles differ from the measured ones in all hygroscopic experiments, albeit with varying significance.

Within the overhygroscopic moisture content range, only water content data is measured. Therefore, transient moisture storage data as obtained for the hygroscopic moisture content range can not be derived here. The measured water content profiles are compared to calculated data revealing a number of deviations. Basically three effects are observed. The first is a general overestimation of moisture transport during water absorption. The calculated water uptake proceeds too fast and the absorbed water mass is larger than measured. The second is a different moisture transport behaviour during the distribution periods. The distinctly measured water content profiles vanish during simulation. The same equilibrium is attained for all compartments according to the assigned moisture storage function whereas the measured data reveals that different equilibrium stages occur. Ultimately, the third effect is a generally underestimated moisture transport during desorption processes. Especially during the mid range of the drying process, the calculated water mass as well as the particular water content profiles often deviate significantly from the measured data.

Summarizing these observations, adsorption processes are frequently overestimated whereas desorption processes are frequently underestimated by the simulation. Especially within the hygroscopic moisture content range, this behaviour is superimposed by other influences. Here, the process history is most distinctive for materials with a high hygroscopic moisture capacity but low moisture conducting properties whereas the not equilibrating humidity profiles are most distinctive for materials with a low moisture capacity but high moisture conducting properties. In addition, all experimental data confirm the existence of many different equilibrium stages depending on both, the process and its history. These different stages, i.e. the distinct water content profiles, can often not be reproduced by the simulation.

\subsubsection{Discussion}

The observed deviations are generally understood to be the consequence of the significant changes in the water content - water potential relation which occur under 
transient conditions. On these changes, considerable experimental evidence is found in literature and confirmed by the own measurement results. However, a number of other factors could have influenced the presented results, too. These are measurement errors of the applied sensors, adjustment and calibration errors during data evaluation, limits of the material model as well as insufficiently adjusted material functions, and material variability. This is accounted for as follows.

\section{Measurement and data evaluation}

Before the experiments started, the coupled temperature - relative humidity sensors have been calibrated. Three defined constant climates adjusted by aqueous salt solutions are used for this purpose. The corresponding relative humidities are 32\%, 52\% and $90 \%$. On this basis, a linear calibration curve has been assigned to all sensors. After the experiments had been completed, another reference measurement has been performed on these three constant climates investigating the absolute values as well as the response behaviour of all the sensors, again. To determine whether the observed nonequilibrating relative humidity profiles are due to measurement errors or not, the sensors are placed into the high relative humidity climate first before the lower steps follow. In all cases, the output value of the sensors is close to the correct value and clearly within the specified tolerance range. Moreover, for any sensor, the duration to display the new value correctly is less than one minute.

On that account, a measurement error of the coupled temperature - relative humidity sensors can be excluded. The non-equilibrating relative humidity profiles as well as the transient moisture storage data must be regarded as effectively measured material behaviour.

The performed TDR calibration as well as the data evaluation procedure is described in paragraph 5.3.3 TDR calibration and data evaluation. Based on this calibration, the TDR sensors measure the apparent dielectric constant correctly. For the translation of apparent dielectric constant into water content data, the most flexible four phase dielectric mixing model has been selected. The model is adjusted to the measured reference data of each material before it is applied to the measured TDR data. The adjustment is shown in Fig. 6.18 for the lime-sand brick. Not all reference values are met by the curve which is also the case for the other materials. Small deviations between actual and derived absolute water content could therefore have occurred. However, these deviations are small and, from the author's point of view, negligible. As they are distinctly present in the apparent dielectric constant data, the relative changes as well as the temporal evolution of water content profiles is measured correctly.

Measurement errors of the TDR sensors can thus be excluded, too. Small deviations during the data evaluation may have occurred leading to slightly over- or underestimated absolute water content values. But they can not be regarded as significant. In fact, all effects are already visible in the apparent dielectric constant data which is measured correctly.

The measurement errors have generally been minimized and the obtained data can therefore be regarded as appropriate. The observed material behaviour can thus not be explained with inappropriate measurement data or measurement errors. 


\section{Material model and adjustment procedure}

The developed material model combines the data of many different basic experiments as sorption and retention, vapour diffusion, water uptake, and drying. It comprises the whole range of moisture contents from dry to saturation and it is not restricted to a fixed amount of input data. With an increasing number of measured parameters, the quality of the adjusted material functions increases, too. In addition, the model is not confined to the current state of transport modelling. Further developments on the transport theory level would directly benefit the simulation quality without requiring an adaptation of the material model. For this reason, the adjustment procedure already allows to include the static adsorption function although currently only one moisture storage function is considered, yet.

Besides this high degree of flexibility which the model contains, the calibration results presented in Chapter 6 are rather convincing. The moisture retention curves are precisely adjusted to the measured sorption and retention data. For all materials, the calculated water uptake and drying courses correspond perfectly with their measured counterparts. On that account, the observed deviations between measured and calculated water content profiles appear to be not due to a material model deficiency or an insufficient adjustment procedure.

One restriction of the material model is, however, still implied. Since the numerical simulation is also applied for the adjustment and calibration procedure, current limits in transport modelling affect also the adjusted moisture transport functions. If dynamic effects or the process history influence the material during one of the calibration experiments, these effects must be included into the transport theory to allow an unaltered adjustment. Otherwise, the dynamic effects as well as implications of the process history are interpreted into the moisture storage or moisture transport functions.

For the present material model calibration, this is the case. The static desorption moisture storage function is the basis of all desorption calculations as well as of the derived liquid water conductivity. The static adsorption function can not be measured in such a high resolution as the desorption function. For those capillary pressure ranges where no measured data is available, the adsorption function is adjusted by the help of the water uptake experiment. This experiment is though significantly influenced by the process dynamics. In the consequence, the adjusted adsorption function is a mixture of static and dynamic data. Recent measurements according to Plagge (2007) indicate, that the static ad- and desorption curves are much closer than the adjustment by the help of the water uptake experiment would imply. See e.g. the right chart in Fig. 6.13 on page 152 where two measured adsorption values are depicted which are not met by the adjusted adsorption function. If the adsorption function would meet them, the calculated water uptake would proceed too fast and the bend in the water uptake curve would form at higher water contents.

The same effect applies for the liquid water conductivity. Under transient conditions, the changed water content - water potential relation leads to different moisture potential gradients. These imply changed flux rates which depend on the process. As witnessed, adsorption processes are slowed down whereas desorption processes are accelerated by the process dynamics. The adjustment and calibration procedure of the material model uses the measured static desorption curve to calculate the drying and the mixed static - dynamic adsorption curve to calculate the water uptake experiment. 
The appearing dynamic effects are thus also implied into the liquid water conductivity as the actually higher transport rates due to higher moisture potential gradients during the drying can only be reproduced by the simulation with an increased liquid water conductivity function for the corresponding range of water contents. At the same time, the reduced moisture transport during the water absorption leads to a reduced liquid water conductivity at high water contents. For some materials where the important water content range for the water uptake experiment coincides with the one for the drying experiment, this results in the problem that either the water uptake or the drying experiment can be calculated correctly. In paragraph 6.2 Application of the material model, these materials are regarded as "difficult materials" by means of adjustment and calibration.

The discussion shows that the material model is dependent on the transport theory and that unconsidered effects are consequently implied into the material functions. The applied material functions are based on static, steady state and unsteady state data. The calibration procedure on the current basis of transport modelling delivers therefore material functions which contain dynamic effects. But as it is the character of dynamics, every process has a different history and different initial and boundary conditions. Consequently, every process is different and material functions which include some dynamic effects are not able to correctly simulate a process under different conditions. Although the water uptake and the drying experiments are correctly reproduced during the calibration procedure, it is not possible to adequately follow the adand desorption experiments which were measured by TDR, not even the integral courses.

The material data is hence subjected to errors stemming from the dependencies on the process and its history which are currently not included into the transport modelling. As soon as these effects are considered, the material model is able to provide improved material functions without further changes. The material functions which are altered by the dynamic effects are though not a convincing general explanation of the observed deviations between measured and calculated water content profiles. Instead, this discussion supports the perception that the process history and the process dynamics need to be included into the transport theory to provide a fundamental and sophisticated description of hygrothermal material behaviour. However, part of the deviations, especially for the lime-sand brick, may also be a consequence of the adjustment and calibration procedure in its current frame of transport modelling.

\section{Material variability}

The transport theory as well as the developed material model imply that the material properties are homogeneous. In contrast to this, most building materials exhibit distinct heterogeneities which influence their hygrothermal performance. Part of the observed deviations between measured and calculated water content profiles may therefore be a consequence of such variability in material properties.

In addition, most basic experiments are conducted with a number of repetitions allowing to estimate the material variability for each parameter. Adjustment and calibration can include this information to provide a representative set of material data for the use in numerical simulation. Although heterogeneous material behaviour can not be included within one set of material data, the use of such a representative data set accounts in average for most heterogeneous influences. 
There exist approaches to describe the material behaviour on a micro or meso scale in order to account for material heterogeneities. Roels (2000) for instance developed such a model which is introduced in paragraph 2.2.2.2 Network models. Unfortunately, network models are rather complex. They require a high amount of input data which can not be provided by basic experiments only. The applicability of these models is hence restricted to special cases whereas other approaches are required for a broad and general material modelling.

Heterogeneities and material variability form thus a problem general material models have to cope with. Out of the investigated building materials, the most susceptible one is the lime-sand brick. The other materials can be regarded as rather homogeneous since their basic material properties exhibit a very low variance. In conclusion, part of the smaller deviations - especially of the lime-sand brick - can probably be regarded as a consequence of inhomogeneous material properties. The general trends and, by that, most of the observed deviations go though far beyond the order of magnitude of measured material variability. The witnessed process dependency and the respective large deviations between measurement and simulation can therefore not be explained by material variability.

\subsubsection{Concluding assessment}

The discussion above clarifies three aspects which could influence the obtained results. The first is, that the measurement errors are negligible. The second is, that the material model is dependent on the moisture transport modelling which means, that effects which are not included at the transport theory level influence the derived material data since the simulation is applied for their calibration. And thirdly, the material variability has an influence when comparing measurement and simulation results but the variance obtained from the basic material property determination was such less pronounced that the deviations between measured and calculated IPM data can not be assigned to the material variability.

The cause of the observed deviations is therefore clearly attributed to the process history and the process dynamics which are not considered during simulation. The effects of this non-consideration are that neither different equilibrium stages nor the shifted water content - water potential relation can be correctly reproduced by the simulation. In the consequence, the distinctly developing water content profiles equilibrate during the calculations as well as the changed moisture potential gradients and the corresponding moisture transport rates are either over- or underestimated in dependence to the process. Within the presented data, these two effects are observed as the most pronounced deviations between measured and calculated water content profiles.

The developed material model generally meets the requirements on a wide applicability to hydrophilic building materials, on a high flexibility and on the adjustability for the whole range of possible moisture contents. Especially the opportunity to use a different amount of input data allows its application for cases where limited data is available and a rough approximation is required only as well as for cases where very detailed material data is available and a sophisticated and precise approximation is desired. 
The developed material model is not restricted to the current state of heat and moisture transport modelling. Developments regarding solute transport as well as the dependency on the process and its history would immediately benefit the whole simulation without requiring further developments on the level of material modelling. However, the material model is dependent on the transport theory since effects which are not included are partly implied into the material functions during the calibration procedure where simulation is applied. The current state of transport modelling thus limits the quality of the derived material functions.

In consequence of both, the current state of transport modelling which does not include the process history and its dynamics as well as the material data which partly implies these effects due to the calibration procedure, the quality of hygrothermal simulation results is limited. The transport tendencies are generally and the integral moisture contents frequently correctly reproduced. The water content profiles, however, can not be calculated correctly, yet.

But as the reasons for these limits stem from the currently implemented moisture transport modelling, and as the material model is not limited to this current level, further developments by means of the process history and the process dynamics will immediately improve both, the calibration quality of the material model and the general output quality of simulation results. The developed material model is therefore understood to be verified and validated. It is widely applicable due to its high flexibility and, at the same time, it meets the requirements of a sophisticated modelling on a material structural basis.

Besides disclosing limits and options of the developed material modelling, the presented measurement and simulation results point out further modelling demands on the moisture transport theory level. The observed deviations are attributed to the limits this current transport theory level implies, in particular the deviations which occur in the water content profiles, and the deviations occurring if large moisture potential gradients are implied. The presented data is therefore not restricted to serve for the elaborated purpose of the present study only, but also as the basis of further research in the field of dynamic moisture transport modelling. 


\section{CHAPTER 8}

\section{SUMMARY, CONCLUSIONS AND FUTURE PROSPECTS}

Material modelling is an important component within the hygrothermal description of building materials and components under transient climatic conditions. To enable numerical simulation on a broad, general and still sophisticated level, a flexible material model is required which is not only applicable to a varying amount of basic material data, but also widely adjustable and not limited by means of the current state of heat and moisture transport modelling.

Within the present study, developments on material modelling, basic experiments and instantaneous profile measurements (IPM) have been carried out. Based on the structure of the material and the basic experimental data, the developed material model has been adjusted. A comprehensive instantaneous profile measurement study has provided valuable data to evaluate limits and options of this material model from which a fundamental validation as well as future research demands have been derived.

In a first paragraph of this chapter, a summary of the whole study is provided. The second paragraph gives a résumé of derived results and drawn conclusions. Finally, the author's recommendations on future research topics are presented and discussed.

\subsection{Content summary}

Based on a comprehensive review on hygrothermal material modelling as well as on experimental methods, developments have been accomplished in three major fields. These are material modelling, basic experiments for material property determination, and instantaneous profile measurements for moisture storage and transport investigations under transient conditions. 
Within the context of the current state of heat and moisture transport modelling, a material model has been developed. It satisfies the Richards-equation and is based on a bundle of tubes model which is combined with a mechanistical approach accounting for serial and parallel structured moisture transport. In its principle, the material model is based on moisture storage data for which a multimodal functional description is defined. From this moisture storage function, the relative liquid water conductivity is derived via the bundle of tubes model. It is scaled to measured conductivity data at saturation and combined with hygroscopic conductivity data derived from vapour diffusion measurements. The scaling as well as the coupling with the mechanistical serial - parallel model provides two modelling parameters. By their help, the liquid water conductivity function is adjusted and calibrated by inverse modelling based on water uptake and drying data. By that, a very flexible conductivity model which is generally applicable to hydrophilic building materials is now available.

Methodological developments have been accomplished for the drying experiment and for the instantaneous profile measurement principle. The drying experiment is very important for material modelling since it reveals moisture transport information for the lower moisture content range and is thus complementary to the water uptake experiment. However, although the drying is much more dependent on its boundary conditions than the water absorption, very few literature on the drying and its applicability in material modelling is available. A comprehensive study has therefore been conducted: a drying device has been designed and built, the influencing parameters have been investigated and classified, and recent attempts to standardize drying data and derive a single number drying coefficient have been evaluated and partly refuted. By that, the set of basic experimental methods provides now a comprehensive, fundamental and sophisticated basis for material modelling and material model calibration.

The instantaneous profile measurement technique has been further developed and applied to four building materials. Special devices have been designed and a series of experiments has been conducted in both moisture content ranges. Profiles of water content and relative humidity have been measured within the hygroscopic and water content profiles have been measured within the overhygroscopic moisture content range. Based on dielectric models found in literature, the methods of TDR data evaluation have been extended, implemented into computer code and applied to the measured IPM data. By that, a substantial set of transient moisture transport data is now available enabling a fundamental material model validation as well as serving as an experimental basis of further research on moisture transport process modelling.

The material model has been implemented into a computer program to facilitate its application to basic experimental data. With it, the model has been applied and adjusted to the four building materials. Based on these material data as well as on the measured initial and boundary conditions, the IPM experiments have been simulated by the aid of the DELPHIN program. Measurement and simulation results have been evaluated and compared. Finally, the limits and options of the developed material modelling as well as of the transport theory into which the material model is embedded have been revealed and discussed. 


\subsection{Results and conclusions}

Within the scope of the conducted design, development and implementation work, the following achievements are available now:

- with regard to material modelling:

- a sophisticated and flexible material model for quantification of moisture storage and transport which is generally applicable to hydrophilic building materials,

- a computer program to apply this model to basic material data,

- a clear specification of the adjustment and calibration procedure,

- a complete set of adjusted and calibrated material functions for the four investigated building materials;

- with regard to the drying experiment:

- a specific and detailed definition of requirements on drying data with special regard to material model calibration,

- a drying apparatus and its design specifications,

- a table giving a clear and qualitative assessment of parameters which influence the drying with regard to material modelling and drying parameter derivation;

- with regard to the instantaneous profile measurement technique:

- different IPM devices for whole range moisture transport investigations and their design specifications,

- a computer program enabling the application of different models to derive the water content from measured TDR data;

- $\quad$ with regard to transient moisture transport data:

- transient moisture storage data of the four investigated building materials within the hygroscopic moisture content range,

- a comprehensive data set of moisture profiles on these materials in both, hygroscopic and overhygroscopic moisture content ranges

On this basis, the material model has been challenged. The experiments have been recalculated applying the adjusted material functions and the measured initial and boundary conditions. The simulation results have been compared with the experimental data. By that, a number of effects and influences have been revealed which limit the precision of hygrothermal building component simulations. These have been classified according to their occurrence, related to their cause and attributed to either the level of material modelling or the level of general moisture transport modelling within a certain moisture content range. This is summarized in Tab. 8.1 below. 
Table 8.1: Effects causing deviations between measured and calculated material behaviour.

\begin{tabular}{|c|c|c|c|}
\hline Effect measurement & Effect simulation & Cause & Attributed to \\
\hline $\begin{array}{l}\text { particular not equili- } \\
\text { brating relative humid- } \\
\text { ity profiles during } \\
\text { hygroscopic desorption } \\
\text { processes }\end{array}$ & $\begin{array}{l}\text { equilibration of relative } \\
\text { humidity and water con- } \\
\text { tent profiles, } \\
\text { overestimated moisture } \\
\text { transport at desorption } \\
\text { processes }\end{array}$ & $\begin{array}{l}\text { binding forces at the } \\
\text { inner surface area of the } \\
\text { material, } \\
\text { limited validity of the } \\
\text { Kelvin-equation at such } \\
\text { low moisture contents }\end{array}$ & $\begin{array}{l}\text { hygroscopic moisture } \\
\text { transport modelling, } \\
\text { materials with a low } \\
\text { hygroscopic moisture } \\
\text { capacity }\end{array}$ \\
\hline $\begin{array}{l}\text { not equilibrating water } \\
\text { content profiles (adsorp- } \\
\text { tion and desorption) }\end{array}$ & $\begin{array}{l}\text { equilibration of all water } \\
\text { content profiles depend- } \\
\text { ing on the applied mois- } \\
\text { ture storage functions }\end{array}$ & $\begin{array}{l}\text { inside the material, dif- } \\
\text { ferent equilibrium stages } \\
\text { occur depending on the } \\
\text { process and its history, } \\
\text { during simulation, only } \\
\text { equilibrium stages } \\
\text { according to the applied } \\
\text { moisture storage func- } \\
\text { tions can occur }\end{array}$ & $\begin{array}{l}\text { general hygroscopic and } \\
\text { overhygroscopic mois- } \\
\text { ture transport model- } \\
\text { ling, } \\
\text { the process history is } \\
\text { more important for } \\
\text { materials with low mois- } \\
\text { ture transport properties }\end{array}$ \\
\hline $\begin{array}{l}\text { shifted transient mois- } \\
\text { ture storage data } \\
\text { depending on the size of } \\
\text { the applied potential } \\
\text { gradients, } \\
\text { particular ad- and des- } \\
\text { orption moisture profiles }\end{array}$ & $\begin{array}{l}\text { overestimated moisture } \\
\text { transport at adsorption } \\
\text { processes, } \\
\text { underestimated mois- } \\
\text { ture transport at desorp- } \\
\text { tion processes }\end{array}$ & $\begin{array}{l}\text { process dynamics lead- } \\
\text { ing to a changed water } \\
\text { content - water potential } \\
\text { relation in dependence } \\
\text { on the size of the implied } \\
\text { moisture potential gra- } \\
\text { dients }\end{array}$ & $\begin{array}{l}\text { general hygroscopic and } \\
\text { overhygroscopic mois- } \\
\text { ture transport model- } \\
\text { ling, } \\
\text { partly implied into the } \\
\text { material data due to the } \\
\text { applied simulation dur- } \\
\text { ing calibration }\end{array}$ \\
\hline $\begin{array}{l}\text { during equilibration } \\
\text { after a water absorption, } \\
\text { the contact element does } \\
\text { not apply to the general } \\
\text { water content profile but } \\
\text { remains with a lower } \\
\text { water content }\end{array}$ & $\begin{array}{l}\text { this behaviour is not } \\
\text { reproduced, } \\
\text { the water content pro- } \\
\text { files equilibrate accord- } \\
\text { ing to the applied } \\
\text { moisture storage charac- } \\
\text { teristics }\end{array}$ & $\begin{array}{l}\text { process dynamics in } \\
\text { combination with a } \\
\text { gradual course of the } \\
\text { moisture storage func- } \\
\text { tion }\end{array}$ & $\begin{array}{l}\text { overhygroscopic mois- } \\
\text { ture transport model- } \\
\text { ling, } \\
\text { materials with a wide } \\
\text { pore size distribution are } \\
\text { susceptible }\end{array}$ \\
\hline $\begin{array}{l}\text { particular water con- } \\
\text { tent profiles during des- } \\
\text { orption processes, } \\
\text { measured profiles start } \\
\text { at equilibrium condi- } \\
\text { tions, almost no super- } \\
\text { position with previous } \\
\text { processes occurs }\end{array}$ & $\begin{array}{l}\text { distinct initial redistri- } \\
\text { bution of moisture, } \\
\text { pronounced superposi- } \\
\text { tion of desorption and } \\
\text { redistribution }\end{array}$ & $\begin{array}{l}\text { process history, the } \\
\text { material has reached an } \\
\text { equilibrium stage which } \\
\text { does not correspond } \\
\text { with the static storage } \\
\text { functions, assigning the } \\
\text { measured water content } \\
\text { as initial condition } \\
\text { causes a non-equilib- } \\
\text { rium and thus, mois- } \\
\text { ture transport }\end{array}$ & $\begin{array}{l}\text { general hygroscopic and } \\
\text { overhygroscopic mois- } \\
\text { ture transport model- } \\
\text { ling, } \\
\text { the consideration of the } \\
\text { process history and the } \\
\text { possibility of different } \\
\text { process dependent equi- } \\
\text { librium stages is } \\
\text { required }\end{array}$ \\
\hline
\end{tabular}


Most of the observed deviations between measured and simulated material behaviour are due to the limiting assumptions made on the transport theory level. The developed material model though meets the initially defined requirements on flexibility, adjustability and precision. The observed deviations are not a consequence of limits in material modelling. Moreover, further developments on the moisture transport theory level automatically benefit the material model without requiring further adjustment or improvement of the model. The developed material model hence provides a strong and sustainable basis for a high quality description of hygrothermal material behaviour.

\subsection{Future prospects}

Providing many valuable answers, this study also opens a number of new questions which may be the subject of future research. From the author's point of view, such research should aim at different levels of theoretical and experimental moisture transport treatment. Following these levels according to the present study, future research may primarily regard three aspects. These are:

1. drying - derivation of a reasonable drying coefficient which is applicable for building industry and practise,

2. instantaneous profile measurements - determination of additional transient moisture storage data in the unsaturated overhygroscopic moisture content range,

3. moisture transport modelling - consideration of the process history and the process dynamics on the moisture transport theory level.

4. moisture transport and material modelling - development of modelling approaches accounting for the temporal variability of material structures and properties

With regard to the first aspect, the importance of drying data for material modelling has been pointed out and the influencing parameters have been quantified. However, a simple but reliable drying coefficient which could be used also in building practise has not been derived, yet. Future research may hence follow the proposed approaches to derive such a drying coefficient from measured data. Moreover, based on the present study, the drying experiment should be standardized to guarantee a high data quality for a broad application within material modelling.

As a second research subject, further experimental data by means of transient moisture storage are still desirable. Comprehensive data on transient moisture storage are available for high degrees of saturation, especially in Soil Science literature. The present study provides such data for the hygroscopic moisture content range. However, the intermediate range of moisture contents lacks extensive transient moisture content moisture potential data, mainly due to the limited applicability of most moisture potential sensors. As this moisture content range is of highest interest for moisture transport processes in Building Physics, further research may regard the development and improvement of moisture potential measurement techniques in the range of $95 \%$ to $100 \%$ relative humidity. Moreover, this technology may be applied to building 
materials during IPM experiments, e.g. in a similar way as done within the present study, to provide an extensive and reliable experimental basis for further moisture transport modelling.

As a third aspect, future research should focus on modelling the dependencies of moisture transport processes on the process history and the process dynamics, and on their consideration within moisture transport modelling. As first approaches exist here, the research may be based on the works of e.g. Stauffer (1977), Beliaev \& Hassanizadeh (2001), Hassanizadeh et al. (2002), Dahle et al. (2005), Manthey (2006) and Porter et al. (2006) who generally assign the dynamic effects to the capillary pressure definition.

$$
p_{g}-p_{l}=p_{c}=f(S)
$$

The general form of eq. (8.1), stating that the capillary pressure $p_{c}$ is a function of saturation $f(S)$ only, needs to be extended by a term accounting for the process dependency. The capillary pressure is therefore not only a function of saturation $S$ but also of the saturation change $\partial S / \partial t$ as indicated in eq. (8.2).

$$
p_{g}-p_{l}=p_{c}=f\left(S, \frac{\partial S}{\partial t}\right)
$$

Available modelling approaches, as proposed by the cited authors, should be evaluated with regard to their generality and their applicability. A suitable model should be developed and implemented into the existing structure of moisture transport modelling. Ultimately, the sensitivity and susceptibility of certain materials, structures and cases on dynamic influences should be evaluated. Such differentiation would allow to estimate the importance to consider dynamic influences for certain cases as well as the expected errors if disregarding them.

Finally as a fourth aspect, the temporal variability of the material structure and its impact on the hygrothermal material properties should be included into either material and transport modelling. Many building materials are susceptible to physical or chemical changes which often affect the hygrothermal material properties. Accounting for these effects would additionally improve the quality of hygrothermal building component simulations, e.g. with regard to wood and wood based materials or composite materials containing cellulose additives. 


\section{LIST OF SYMBOLS AND ABBREVIATIONS}

\section{List of arabic symbols}

\begin{tabular}{|c|c|c|}
\hline Symbol & Unit & Description \\
\hline$A_{\text {is }}$ & {$\left[m^{2} / g\right]$} & Specific inner surface area \\
\hline$A_{w}$ & {$\left[\mathrm{~kg} / \mathrm{m}^{2} \sqrt{\mathrm{s}}\right]$} & Water uptake coefficient versus sqrt(sec) \\
\hline \multirow[t]{2}{*}{$c$} & {$[\mathrm{~m} / \mathrm{s}]$} & $\begin{array}{l}\text { Propagation velocity of an electromagnetic pulse in para- } \\
\text { graph 3.2.2.1 Moisture content measurement, }\end{array}$ \\
\hline & & $c_{0}$ is the speed of light in vacuum. \\
\hline$c_{\alpha}$ & {$[J / k g K]$} & Specific heat capacity of phase $\alpha$ (at constant volume) \\
\hline$c_{g}^{H_{g}}$ & {$[\mathrm{~J} / \mathrm{kgK}]$} & Specific heat capacity of the gaseous phase \\
\hline$c_{l}^{H_{l}}$ & {$[\mathrm{~J} / \mathrm{kgK}]$} & Specific heat capacity of the liquid phase \\
\hline$c_{v}^{H_{v}}$ & {$[J / k g K]$} & Specific heat capacity of vapour \\
\hline$c_{p, a}$ & {$[J / k g K]$} & Specific isobaric heat capacity of air \\
\hline$D_{v}$ & {$\left[m^{2} / s\right]$} & Diffusivity of component $v$ \\
\hline$D_{l}$ & {$\left[m^{2} / s\right]$} & Liquid water diffusivity \\
\hline$D_{v}$ & {$\left[m^{2} / s\right]$} & Vapour diffusivity \\
\hline
\end{tabular}




\begin{tabular}{|c|c|c|}
\hline Symbol & Unit & Description \\
\hline$D_{v, \text { air }}$ & {$\left[m^{2} / s\right]$} & Vapour diffusivity in free air \\
\hline$D_{v, m a t}$ & {$\left[m^{2} / s\right]$} & Vapour diffusivity in a material \\
\hline$G$ & {$[J]$} & Gibbs free energy \\
\hline$g_{k}$ & {$\left[m / s^{2}\right]$} & Gravity constant in direction of $k$ \\
\hline$H_{\alpha}$ & {$[J]$} & Enthalpy of phase $\alpha$ \\
\hline$h$ & {$[m]$} & Capillary head, $h=p_{c} / \rho_{l} g$ \\
\hline$I_{\alpha}$ & {$[\mathrm{kg} \cdot \mathrm{m} / \mathrm{s}]$} & Momentum of phase $\alpha$ (extensive quantity) \\
\hline$j_{k}$ & {$\left[\mathrm{~kg} / \mathrm{m}^{2} \mathrm{~s}\right]$} & Flux in direction of $k$ \\
\hline$j_{k, d i f f}^{M_{v}}$ & {$\left[\mathrm{~kg} / \mathrm{m}^{2} \mathrm{~s}\right]$} & Diffusive vapour flux in direction of $k$ \\
\hline$j \stackrel{Q}{i, d i f f}$ & {$\left[W / m^{2} s\right]$} & Diffusive energy flow in direction of $k$ (heat conduction) \\
\hline$K_{\text {cap }}$ & {$[s]$} & Liquid water conductivity at capillary saturation \\
\hline$K_{e f f}$ & {$[s]$} & Liquid water conductivity at effective saturation \\
\hline$K_{g}$ & {$[s]$} & Advection coefficient (permeability) of the gaseous phase \\
\hline$K_{l}$ & {$[s]$} & Liquid water conductivity \\
\hline$L$ & {$[m]$} & Length or thickness \\
\hline Le & {$[---]$} & Lewis number \\
\hline$l_{T D R}$ & {$[m]$} & Length of the TDR probe \\
\hline$M_{\alpha}$ & {$[k g]$} & Mass of phase $\alpha$ (extensive quantity) \\
\hline$N_{A}$ & {$[1 / \mathrm{mol}]$} & Avogadro constant: $N_{A}=6.022 \cdot 10^{23} \mathrm{~mol}^{-1}$ \\
\hline$m_{\alpha}$ & {$\left[\mathrm{kg} / \mathrm{m}^{3}\right]$} & Volume specific mass (density) of phase $\alpha$ \\
\hline$m_{a}$ & {$\left[\mathrm{~kg} / \mathrm{m}^{3}\right]$} & Volume specific mass of air \\
\hline$m_{g}$ & {$\left[\mathrm{~kg} / \mathrm{m}^{3}\right]$} & Volume specific mass of the gaseous phase (air + vapour) \\
\hline$m_{v}$ & {$\left[\mathrm{~kg} / \mathrm{m}^{3}\right]$} & Volume specific mass of water vapour \\
\hline$m_{w}$ & {$\left[\mathrm{~kg} / \mathrm{m}^{3}\right]$} & Volume specific mass of liquid water \\
\hline$m_{w+v}$ & {$\left[\mathrm{~kg} / \mathrm{m}^{3}\right]$} & Volume specific mass of water (liquid and gaseous phase) \\
\hline$n_{a d s}$ & {$[\mathrm{~mol} / \mathrm{g}]$} & Number of adsorbed particles \\
\hline$n_{m l w}$ & {$[---]$} & Number of molecular layers of water \\
\hline
\end{tabular}




\begin{tabular}{|c|c|c|}
\hline Symbol & Unit & Description \\
\hline$n_{\text {mono }}$ & {$[\mathrm{mol} / \mathrm{g}]$} & $\begin{array}{l}\text { Number of particles forming a monolayer at the inner sur- } \\
\text { faces, } n_{\text {mono }} \text { is also called monolayer capacity }\end{array}$ \\
\hline$p_{c}$ & {$[P a]$} & Capillary pressure \\
\hline$p C$ & {$[\log (P a)]$} & Logarithmic capillary pressure: $p C=\log \left(-p_{c}\right)$ \\
\hline$p_{g}$ & {$[P a]$} & Gas pressure \\
\hline$p_{l}$ & {$[P a]$} & Liquid pressure \\
\hline$p_{s}$ & {$[P a]$} & Saturation vapour pressure \\
\hline$p_{v}$ & {$[P a]$} & Vapour pressure \\
\hline$R$ & {$[\mathrm{~J} / \mathrm{kgK}]$} & Gas constant \\
\hline$R_{v}$ & {$[\mathrm{~J} / \mathrm{kgK}]$} & Gas constant of water vapour \\
\hline$r$ & {$[m]$} & Capillary radius \\
\hline$S_{\alpha}$ & {$[J / K]$} & Entropy phase $\alpha$ (extensive quantity) \\
\hline$T$ & {$[K]$} & Absolute (Kelvin-)temperature \\
\hline$t$ & {$[s]$} & Time \\
\hline$U_{\alpha}$ & {$[J]$} & Inner energy phase $\alpha$ (extensive quantity) \\
\hline$u_{\alpha}$ & {$\left[J / m^{3}\right]$} & Volume specific inner energy of phase $\alpha$ \\
\hline$v_{k}^{M_{g}}$ & {$[m / s]$} & Mass velocity of the gaseous phase in direction of $k$ \\
\hline$v_{k}^{M_{l}}$ & {$[m / s]$} & Mass velocity of the liquid phase in direction of $k$ \\
\hline$x_{k}$ & {$[m]$} & Spatial coordinate in direction of $k$ \\
\hline $\overrightarrow{Y_{\alpha}}$ & & State variable vector of phase $\alpha$ \\
\hline
\end{tabular}

\section{List of greek symbols}

\begin{tabular}{lll}
\hline Symbol & Unit & Description \\
\hline$\alpha$ & {$\left[\mathrm{W} / \mathrm{m}^{2} \mathrm{~K}\right]$} & Heat transfer coefficient \\
$\beta$ & {$[\mathrm{s} / \mathrm{m}]$} & Vapour transfer coefficient \\
$\gamma$ & {$[\mathrm{Rad}]$} & Contact angle between solid and liquid phases \\
$\varepsilon_{a}$ & {$[---]$} & Apparent dielectric constant \\
\hline
\end{tabular}




\begin{tabular}{|c|c|c|}
\hline Symbol & Unit & Description \\
\hline$\varepsilon_{b w}$ & {$[---]$} & Dielectric constant of bound water \\
\hline$\varepsilon_{g}$ & {$[---]$} & Dielectric constant of the gaseous phase \\
\hline$\varepsilon_{l}$ & {$[---]$} & Dielectric constant of the liquid phase \\
\hline$\varepsilon_{m}$ & {$[---]$} & Dielectric constant of the material matrix (solid phase) \\
\hline$\eta$ & {$[---]$} & $\begin{array}{l}\text { General symbol for an adjustable modelling parameter, nor- } \\
\text { mally with an index specifying the model }\end{array}$ \\
\hline$\Theta$ & {$[---]$} & Relative water content: $\Theta=\theta_{l} / \theta_{\text {por }}$ \\
\hline$\theta_{b w}$ & {$\left[m^{3} / m^{3}\right]$} & Volumetric content of the bound water fraction \\
\hline$\theta_{c a p}$ & {$\left[m^{3} / m^{3}\right]$} & Water content at capillary saturation \\
\hline$\theta_{\text {eff }}$ & {$\left[m^{3} / m^{3}\right]$} & Water content at effective saturation \\
\hline$\theta_{\text {por }}$ & {$\left[m^{3} / m^{3}\right]$} & Porosity \\
\hline$\theta_{l}$ & {$\left[m^{3} / m^{3}\right]$} & Volumetric water content \\
\hline$\lambda$ & {$[W / m K]$} & Thermal conductivity \\
\hline$\lambda$ & {$[m / \sqrt{s}]$} & $\begin{array}{l}\text { Boltzmann variable in paragraph 2.2.1 Diffusivity } \\
\text { approaches, } \lambda=x / \sqrt{t}\end{array}$ \\
\hline$\mu$ & {$[\mathrm{J} / \mathrm{mol}]$} & Chemical potential \\
\hline$\mu$ & {$[---]$} & Vapour diffusion resistance factor \\
\hline$\rho_{a}$ & {$\left[\mathrm{~kg} / \mathrm{m}^{3}\right]$} & Bulk density of air \\
\hline$\rho_{b}$ & {$\left[\mathrm{~kg} / \mathrm{m}^{3}\right]$} & Bulk density (mass / volume of bulk material) \\
\hline$\rho_{g}$ & {$\left[\mathrm{~kg} / \mathrm{m}^{3}\right]$} & Bulk density of the gaseous phase \\
\hline$\rho_{m}$ & {$\left[\mathrm{~kg} / \mathrm{m}^{3}\right]$} & Matrix density (mass / volume of solid matrix) \\
\hline$\sigma_{\alpha}^{M_{\alpha}}$ & {$\left[\mathrm{kg} / \mathrm{m}^{3} \mathrm{~s}\right]$} & Source production rate of $M_{\alpha}$ in phase $\alpha$ \\
\hline$\sigma_{l}^{M_{w+v}}$ & {$\left[\mathrm{~kg} / \mathrm{m}^{3} \mathrm{~s}\right]$} & Water source production rate in the liquid phase \\
\hline$\sigma_{u}^{Q}$ & {$\left[W / m^{3} s\right]$} & Heat source production rate of inner energy \\
\hline$\sigma_{l g}$ & {$[N / m]$} & Tension of the liquid-gaseous interface, i.e. surface tension \\
\hline$\sigma_{s l}$ & {$[N / m]$} & Tension of the solid-liquid interface \\
\hline$\sigma_{s g}$ & {$[N / m]$} & Tension of the solid-gaseous interface \\
\hline$\varphi$ & {$[---]$} & Relative humidity \\
\hline
\end{tabular}




\section{List of indices}

\begin{tabular}{ll}
\hline Subscript & Description \\
\hline$a$ & Air (Chapter 2) \\
$a$ & Apparent (paragraph 5.3.3 TDR calibration and data evaluation) \\
$e$ & Environment \\
$g$ & Gaseous phase \\
$k$ & Spatial direction index $(x, y, z)$ \\
$l$ & Liquid phase \\
$m$ & Solid phase (material matrix) \\
$s$ & Surface \\
$v$ & Water vapour \\
$w$ & Liquid water \\
\hline$\alpha$ & Phase index \\
$v$ & Component index \\
\hline
\end{tabular}

\begin{tabular}{ll}
\hline Superscript & Description \\
\hline$H_{\alpha}$ & Enthalpy of phase $\alpha$ the main symbol is related to \\
$M_{\alpha}$ & Mass of phase $\alpha$ the main symbol is related to \\
$Q$ & Reduced heat (i.e. heat conduction) \\
\hline
\end{tabular}




\section{List of proper names and abbreviations}

\begin{tabular}{ll}
\hline Name & Description \\
\hline DELPHIN & $\begin{array}{l}\text { Numerical simulation program for coupled heat, air, moisture and salt transport } \\
\text { developed at the Institute of Building Climatology, Dresden University of Technol- } \\
\text { ogy. The program was previously known as DIM. The current main DELPHIN ver- } \\
\text { sion has index number 4. In cooperation with the Syracuse University, NY, a new } \\
\text { version has been developed with index number 5. This program is now available } \\
\text { under the name CHAMPS which stands for coupled heat, air, moisture, pollutant } \\
\text { and salt transport. } \\
\text { For more details concerning the program, refer to Grunewald (1997), Funk et al. } \\
\text { (2003), Grunewald \& Fechner (2006) and Nicolai (2006). }\end{array}$ \\
\hline
\end{tabular}

\begin{tabular}{ll}
\hline Abbreviation & Description \\
\hline BET & Adsorption theory according to Brunauer et al. (1938), named according to the ini- \\
& tials of the three authors Brunauer, Emmet and Teller. \\
& For more details, see section BET-method according to Brunauer et al. (1938) in \\
& paragraph 5.3.3.2 TDR data evaluation. \\
& Gibbs integral method according to Adolphs (1994). \\
FIM & For more details, see section Gibbs free energy methods according to Adolphs \\
& (1994) in paragraph 5.3.3.2 TDR data evaluation. \\
Gibbs minimum method according to Adolphs (1994). \\
For more details see section Gibbs free energy methods according to Adolphs \\
(1994) in paragraph 5.3.3.2 TDR data evaluation. \\
IPMstantaneous profile method - technique to measure one or more quantities at dif- \\
& ferent sample positions versus time during a transient process. \\
& For more details, see paragraph 3.2 Instantaneous profile measurements and para- \\
& graph 5.3 Instantaneous profile measurements. \\
Moisture retention characteristic - moisture storage function. The term is derived \\
from the pressure plate experiments where the water is retained in the sample \\
against an outside overpressure. It sometimes comprises only the wet range of stor- \\
age data, here however the term MRC is used as a synonym for moisture storage \\
function. \\
Reversed moisture retention characteristic - accounts for the inverse (or reverse) of \\
the moisture storage function. \\
Time domain reflectometry - measurement principle to derive water content and \\
electric conductivity data. \\
For more details, see paragraph 3.2.2 Sensor technology. \\
TDR
\end{tabular}




\section{REFERENCES}

ADAN (1994): Adan, O.C.G.; "On the fungal defacement of interior finishes" Ph.D. thesis, Eindhoven University of Technology, the Nederlands, 1994.

ADOLPHS (1994): Adolphs, J.; "Thermodynamische Beschreibung der Sorption“ Dissertation Universität Gesamthochschule Essen, Forschungsbericht aus dem Bauwesen Heft 61, 1994.

AdOLPHS \& SETZER (1996): Adolphs, J. \& Setzer, M. J.; "A Model to Describe Adsorption Isotherms" Journal of Colloid and Interface Science Vol. 180: 70-76, 1996.

AdOLPHS ET AL. (2002): Adolphs, J., Setzer, M. J., Heine, P.; „Changes in pore structure and mercury contact angle of hardened cement paste depending on relative humidity" Materials and Structures, Vol. 35: 477-486, 2002.

ARFIDSON \& CUNNINGHAM (2000): Arfidson, J. \& Cunningham, M.J.; ,'A transient technique for determining diffusion coefficients in hygroscopic materials" Building and Environment, Vol. 35: 239-249, 2000.

AtKins \& De Paula (2006): Atkins, P. W. \& de Paula, J.; „Physikalische Chemie“ Wiley-VCH, 4. vollst. überarb. Auflage, 2006.

BAEHR \& STEPHAN (2003): Baehr \& Stephan, K.; „Wärme- und Stoffübertragung“ $3^{\text {rd }}$ Issue, Springer, 2003.

BEAR \& BACHMAT (1991): Bear, J. \& Bachmat, Y.; „Introduction to Modeling of Transport Phenomena in Porous Media“ Kluwer Academic Publishers, Dordrecht 1991.

BEDNAR (2000): Bednar, T.; "Beurteilung des feuchte- und wärmetechnischen Verhaltens von Bauteilen und Gebäuden - Weiterentwicklung der Meß- und Rechenverfahren“ Dissertation, TU Wien, 2000.

BEDNAR (2002): Bednar, T.; "Approximation of liquid moisture transport coefficient of porous building materials by suction and drying experiments. Demands on determination of drying curve" $6^{\text {th }}$ Symposium on Building Physics in the Nordic Countries, 493-500, Trondheim 2002.

BEDNAR \& DREYER (2003): Bednar, T. \& Dreyer, J.; „,Determination of moisture surface transfer coefficients under transient conditions" ${ }^{\text {nd }}$ International Building Physics/Science Conference, Leuven 2003, published in Research in Building Physics - Carmeliet, Hens \& Vermeir (eds),

Balkema, Swets \& Zeitlinger Publishers, Lisse 2003. 
BeliaeV \& HassanizadeH (2001): Beliaev, A. Y. \& Hassanizadeh, S. M.; "A Theoretical Model of Hysteresis and Dynamic Effects in the Capillary Relation for Two-phase Flow in Porous Media" Transport in Porous Media Vol. 43: 487-510, 2001.

BIRchAK ET AL. (1974): Birchak, J. R., Gardner, C. G., Hipp, J. E., Victor, J. M.; "High dielectric constant microwave probes for sensing soil moisture" Proc IEEE 62: 933-98, 1974.

Bomberg eT AL. (2005): Bomberg, M., Pazera, M., Plagge, R.; "Analysis of Selected Water Absorption Coefficient Measurements" Journal of Thermal Envelope and Building Science 28: 227-243, 2005.

BoumA (1977): Bouma, J.; "Soil survey and the study of water in unsaturated soil“ Soil Survey Papers No. 13, Soil Survey Institute, Wageningen, the Nederlands, 1977.

BROKEN (1998): Broken H. J. P., "Moisture transport in brick masonry: the grey area between bricks", Ph.D. thesis, Eindhoven University of Technology, the Nederlands, 1998.

BROWN (1956): Brown, W. F.; „Dielectrics“ in Encyclopedia of Physics, Vol. 17, Berlin, Springer 1956.

BRUNAUER ET AL. (1938): Brunauer, S., Emmet, P. H., Teller, E.; "Adsorption of Gases in Multimolecular Layers" Journal of American Chemical Society Vol. 60: 309-319, 1938.

BurCH ET AL. (1992): Burch, D.M., Thomas, W.C. \& Fanney, A.H.; „Water Vapor Permeability Measurement of Common Building Materials" ASHRAE Transactions, V. 98, Pt. 2, 1992.

BURDINE (1953): Burdinde, N. T.; „Relative permeability caculations from pore-size distribution data“ Trans. AIME 198: 71-78, 1953.

CARMeliet ET AL. (1999): Carmeliet, J., Descamps, F., Houvenaghel, G.; "A Mulstiscale Network Model for Simulating Moisture Transfer Properties in Porous Media" Transport in Porous Media Vol. 35: 67-88, 1999.

CARMELIET \& RoElS (2001): Carmeliet, J. \& Roels, S.; "Determination of Isothermal Moisture Transport Properties of Porous Building Material“ Journal of Thermal Envelope and Building Science, Vol. 24: 183-210, 2001.

CARMEliet \& Roels (2002): Carmeliet, J. \& Roels, S.; „Determination of the Moisture Capacity of Porous Building Materials" Journal of Thermal Envelope and Building Science, Vol. 25:, 209-237, 2002.

CARMeliet ET Al. (2004): Carmeliet, J., Hens, H., Roels, S., Adan, O., Brocken, H., Cerny, R., Pavlik, Z., Hall, C., Kumaran, K., Pel, L.; „Determination of liquid water diffusivity from transient moisture transfer experiments" Journal of Thermal Envelope \& Building Science, Vol. 27, No. 4, 2004.

CARMELIET \& JANSSEN (2007): Carmeliet, J. \& Janssen, H.; „Determination of the moisture diffusivity and permeability of porous building materials" submitted and accepted to be published by ASHRAE, 2007.

CAssel \& KLute (1986): Cassel, D.K. \& Klute, A.; „Water Potential: Tensiometry“ In: Klute, A. (ed.). Methods of soil analysis. Part I. Physical and mineralogical methods. 2nd edition, No.9, Agronomy Series, Am. Soc. Agron., Soil Sci. Soc. Am. Madison. p. 563-596, 1986.

Clusius \& WALdMANN (1942): Clusius K. \& Waldmann L.; "An overlooked kinetic effect in gases" Naturwissenschaften 30: 711-711, 1942.

DAHLE ET AL. (2005): Dahle, H.K., Celia, M.A., Hassanizadeh, S.M.; „Bundle-of-Tubes Model for Calculating Dynamic Effects in the Capillary-Pressure-Saturation Relationship" Transport in Porous Media Vol. 58: 5-22, 2005.

DESCAMPS (1997): Descamps, F.; „Continuum and discrete modelling of isothermal water and air transfer in porous media", Ph.D. Thesis, Catholic University Leuven. Leuven, Belgium 1997.

Dobson et AL. (1985): Dobson, M.C.; Ulaby, F.T.; Hallikainen, M.T.; El-Rayes, M.A.; „Microwave Dielectric Behavior of Wet Soil-Part II: Dielectric Mixing Models“ IEEE Transactions on Geoscience and Remote Sensing, Volume GE-23, Issue 1: 35-46, 1985.

DuFOUR (1873): Dufour L.; "Diffusion of gases through porous walls and the accompanying changes of temperature" J. Chem. Soc. Vol 26: 835-835, 1873.

Dullien (1979): Dullien, F. A. L.; „Porous Media - Fluid Transport and Pore Structure“ Academic Press, New York, USA, 1979.

DURNER (1992): Durner, W.; "Predicting the Unsaturated Hydraulic Conductivity using Multi-Porosity Water Retention Curves" Proceedings of the International Workshop on Indirect Methods for Estimating the Hydraulic Properties of Unsaturated Soils, Riverside, California, 
October 1989, edited by M. Th. van Genuchten, F. J. Leij and L. J. Lund, University of California, 1992.

DURNER (1994): Durner, W.; "Hydraulic conductivity estimation for soils with heterogeneous pore structure" Water Resources Research, Vol. 30, No 2: 211-223, 1994.

ENDERBY (1955): Enderby, A. J.; „The Domain Model of Hysteresis“ Trans. Faraday Soc. 51: 835-848, 1955.

ENDERBY (1956): Enderby, A. J.; „The Domain Model of Hysteresis: 2“ Trans. Faraday Soc. 52: 106-120, 1956.

EVERETT (1950): Everett, D. H.; "The Thermodynamics of Adsorption, Part II - Thermodynamics of Monolayers in Solids" Trans. Faraday Soc. 46: 1950.

Everett \& WhitTon (1952): Everett, D. H. \& Whitton, W. I., „A general approach to hysteresis“ Trans. Faraday Soc. 48: 749-757, 1952.

EvereTt \& SMITH (1954): Everett, D. H., \& Smith, F. W.; „A general approach to hysteresis: 2“ Trans. Faraday Soc. 50: 187-197, 1954.

Everetr (1954): Everett, D. H.; „A General Approach to Hysteresis: 3“ Trans. Faraday Soc. 50: 1077-1096, 1954.

EVERETT (1955): Everett, D. H.; „A general approach to hysteresis: 4“ Trans. Faraday Soc. 51: 1551-1557, 1955.

EsPINOSA (2004): Espinosa, R. M.; „Sorptionsisothermen von Zementstein und Mörtel“ Dissertation Technische Universität Hamburg-Harburg 2004.

FATT (1956): Fatt, I.; „, The network model of porous media: I. - III.“ Trans. AIME Petrol. Div., 207: 144-177, 1956.

FECHNER (2006): Fechner, H.; "Postprocessing for DELPHIN outputs" Program for data evaluation and visualisation, developed at the Institute of Building Climatology, Dresden University of Technology. Personal communication and program supply, 2006.

FECHNER (2007): Fechner, H.; „Numerische Simulation der Raumluftfeuchte unter Berücksichtigung der Pufferwirkung feuchtespeichernder Materialien" Proceedings to the $12^{\text {th }}$ Symposium for Building Physics, Dresden University at Technology, Institute of Building Climatology, Vol. 2, 764-771, Dresden 2007.

FICK (1855): Fick, A.; „Über Diffusion“ Poggendorf’s Annalen der Physik, 94: 59-86, 1855.

FITZ \& KRUS (2004): Fitz, C. \& Krus, M.; „Normenwirrwarr bei der Bestimmung von feuchtetechnischen Kennwerten" IBP Mitteilung 441, 2004.

FRANKE (2007): Franke, C.; „Numerische Behandlung semilinearer Quadratmittelprobleme aus der Bauphysik" Master Thesis at Dresden University of Technology, Institute of Numerical Mathematics, 2007.

FreUdENBERGER (1989): Freudenberger, A.; „Möglichkeiten der Feuchtemessung bei verfahrenstechnischen Trocknungsprozessen" Chemie Ingenieur Technik Vol. 61, No. 12: 953-957, 1989.

FUNK ET AL. (2003): Funk, M., Grunewald, J., Deckelmann, G., Kaps, C., Steiger, M.; Abschlussbericht der 1. Förderperiode des DFG-Forschungprojektes „Modellierung, SoftwareImplementierung und Verifikation des Feuchte- und Salztransportes, der Salzkristallisation und schädigung in kapillar-porösen Mauerwerksbaustoffen " im Schwerpunktprogramm SPP 1122 der DFG, April 2003.

FUNK \& GRUNEWALD (2005): Funk, M. \& Grunewald, J.; „Balance equations for heat, air, moisture and salt simulation" unpublished manuscript of Max Funk, EMPA Dübendorf, Switzerland 2005.

FUNK (2006): Funk, M.; "Hysteresis der Feuchtespeicherung: Modellierung und Anwendungsperspektiven“ Presentation at the ETH Zurich, December 2006.

FUNK \& GHAZI WAKILI (2007): Funk, M. \& Ghazi Wakili, K.; „Driving potentials of heat an mass transport in porous building materials: A comparison between general linear, thermodynamic and micromechanical approaches" Transport in Porous Media, online available at www.springerlink.com, doi: 10.1007/s11242-007-9150-3, 2007.

GALBRAITH (1992): Galbraith, G. H.; "Heat and mass transfer within porous building materials", Ph.D. thesis at the Department of Mechanical Engineering, University of Strathclyde, Glasgow, 1992.

GALbRAITH ET AL. (1997): Galbraith, G.H., McLean, R.C. \& Kelly, D.; "Moisture permeability measurements under varying barometric pressure" Building Research and Information, Vol. 25, No. 6: 348-353, 1997. 
GALbRAITH ET AL. (1998): Galbraith, G.H., McLean, R.C., Gillespie, I., Guo, J., Kelly, D.; „,Nonisothermal moisture diffusion in porous building materials" Building Research \& Information, Vol. 26 (6): 330-339, 1998.

GALbRAITH ET AL. (2003): Galbraith, G.H., Kelly, D.J., McLean, R.C.; „Alternative methods for measuring moisture transfer coefficients of building materials" $2^{\text {nd }}$ International Building Physics / Science Conference, Leuven 2003, published in Research in Building Physics - Carmeliet, Hens \& Vermeir (eds), Balkema, Swets \& Zeitlinger Publishers, Lisse 2003.

GERKE \& VAN GENUCHTEN (1993): Gerke, H. H. \& Van Genuchten, T. M.; „A Dual-Porosity Model for Simulating the Preferential Movement of Water and Solutes in Structured Porous Media" Water Resources Research, Vol. 29, No. 2: 305-319, 1993.

GNOTH ET AL. (2007): Gnoth, S., Hansel F., Fechner, H., Häupl, P.; „Hygrothermische Bauteilsimulation unter berücksichtigung der Konvektion " Proceedings to the $12^{\text {th }}$ Symposium for Building Physics, Dresden University at Technology, Institute of Building Climatology, Vol. 1, 414421, Dresden 2007.

GrAU \& Rode (2005): Grau, K. \& Rode, C.; „Whole Building Ham Simulation With A Multizone Air Flow Model" Working paper A41-T1-Dk-05-6 for IEA Annex 41 Meeting, October 26-28, Trondheim 2005.

GRUNEWALD (1997): Grunewald, J.; „,Diffusiver und konvektiver Stoff- und Energietransport in kapillarporösen Baustoffen", Dissertation, TU Dresden, Fakultät Architektur, Institut für Bauklimatik, 1997.

GRUNEWALD \& HÄUPL (2002): Grunewald, J. \& Häupl, P., „,Ein Modell zur Beschreibung der feuchteabhängigen Dampfleitfähigkeit kapillarporöser Materialien", Proceedings to the $11^{\text {th }}$ Symposium for Building Physics, Dresden University at Technology, Institute of Building Climatology, Vol.1: 286-294, Dresden 2002.

GRUNEWALD \& HÄUPL (2003): Grunewald, J. \& Häupl, P., „Gekoppelter Feuchte-, Luft-, Salz- und Wärmetransport in porösen Baustoffen", Bauphysik Kalender 2003: 377 - 434, Ernst \& Sohn Berlin 2003.

GRUNEWALD \& BoMberg (2003): Grunewald, J. \& Bomberg, M.; "An Engineering Approximation of Material Characteristics for Input to Heat, Air and Moisture Transport Simulations", Journal of Thermal Envelope \& Building Science, Vol. 26 No. 4, April 2003.

GRUNEWALD ET AL. (2003): Grunewald, J., Funk, M., Scheffler, G., Sladek, V., Sladek, J.; „,Development of insulation materials with specially designed properties for building renovation, Workpackage 1 modelling, software development and simulation" Abschlussbericht des INSUMAT Projektes, Workpackage 1, Eigenverlag der TU Dresden, Dezember 2003.

GRUNEWALD \& FECHNER (2006): Grunewald, J. \& Fechner, H.; „DELPHIN 4 - Simulation program for coupled heat, air, moisture and salt transport in capillary porous building materials", http:/ / bauklimatik-dresden.de/delphin/delphin_description.html, 2006.

GRUNEWALD \& NiCOLAI (2007): Grunewald, J. \& Nicolai, A.; „Effekte der Luftströmung auf das hygrothermische Verhalten von leichten Umfassungskonstruktionen" Bauphysik Vol. 29 No. 01: 48-54, 2007.

GURR (1962): Gurr, C.G.; "Use of gamma rays in measuring water content and permeability in unsaturated columns of soil" Soil Science Vol. 94: 224-229, 1962.

HAINES (1930): Haines, W. B.; "Studies in the physical properties of soil. V. The hysteresis effect in capillary properties, and the modes of moisture distribution associated therewith" Journal of Agricultural Science Vol. 20: 97-116, 1930.

HANSEN (1986): Hansen, K. K.; „Sorption Isotherms. A Catalogue“ Building Materials Laboratory, Danish Technical University, Technical Report 162/86, 1986.

HANSEN ET AL. (2001): Hansen, K.K., Rode, C., Hansen, E.J. de Place, Padfield, T., Kristiansen, F.H. "Experimental Investigation of the Hygrothermal Performance of Insulation Materials" Proceedings from the Thermal Performance of the Exterior Envelopes of Whole Buildings VIII Conference, ASHRAE 2001.

HANSEN \& HANSEN (2002): Hansen, M. H. \& Hansen, E. J. de Place, „Determination of liquid diffusivity using single point moisture content measurements and Boltzmann transformation" Proceedings to 6th Symposium on Building Physics in Nordic Countries, Trondheim 2002.

Hassanizadeh \& Gray (1997): Hassanizadeh, S. M. \& Gray, W. G., "Recent advances in theories of twophase flow in porous media" contribution to "Fluid Transport in Porous Media" in Advances in Fluid Mechanics, Vol. 13, Computational Mechanics Publications, Southampton 1997. 
HAssanizAdeH ET AL. (2002): Hassanizadeh, S. M., Celia M. A., Dahle, H. K.; „Dynamic Effect in the Capillary Pressure-Saturation Relationship and its Impacts on Unsaturated Flow" Vadose Zone Journal Vol. 1: 38-57, 2002.

HÄUPL (1987): Häupl, P.; „Feuchtetransport in Baustoffen und Bauwerksteilen. Teil 1: Mathematische Modellierung des gekoppelten Feuchte- und Wärmetransportes in kapillarporösen Stoffen, Teil 2: Analytische Lösungen des gekoppelten, partiellen, nichtlinearen Transportgleichungssystems für thermisch-hygrische Anfangs- und Randbedingungen" Dissertation Technische Universität Dresden, 1987.

HÄUPL ET AL. (1989): Häupl, P., Stopp, H., Strangfeld, P.; „Messverfahren und Messanordnung zur gleichzeitigen Bestimmung der Kapillarwasserleitfähigkeit und der gravimetrischen Feuchteleitfähigkeit von kapillarporösen Stoffen" Patentschrift DD 290951 A5, ISSN 0433-6461, 1989.

HÄUPL ET AL. (1999): Häupl, P., Fechner, H., Neue, J., Stopp, H., „,Bestimmung der Parameter der Kapillarwasserleitfähigkeit aus dem Wasseraufnahmekoeffizienten" Proceedings to the $10^{\text {th }}$ Symposium for Building Physics, Dresden University of Technology, Institute of Building Climatology, Vol. 2: 483-493, 1999.

HÄUPL ET AL. (2001): Häupl, P., Plagge, R., Fechner, H.; „Hygrische Materialfunktionen von porösen Baustoffen" Gesundheitsingenieur 122, Heft 6: 305-316, 2001.

HÄUPL ET AL. (2003): Häupl, P., Fechner, H., Jurk, K., Stopp, H., Finkenstein, C., „Entwicklung leistungsfähiger Wärmedämmsysteme mit wirksamem physikalischen Feuchteschutz", Projektabschlussbericht Band II, Institut für Bauklimatik, Fakultät Architektur, TU Dresden, April 2003.

HÄUPL \& FECHNER (2003A): Häupl, P. \& Fechner, H., „Hygric Material Properties of Porous Building Materials", Journal of Thermal Envelope \& Building Science, Vol. 26 No. 3, January 2003, Sage Publications London, Thousand Oaks, New Delhi 2003.

HÄUPL \& FECHNER (2003B): Häupl, P. \& Fechner, H.; „Hygric material properties of porous building materials" $2^{\text {nd }}$ International Building Physics/Science Conference, Leuven 2003, published in Research in Building Physics - Carmeliet, Hens \& Vermeir (eds), Balkema, Swets \& Zeitlinger Publishers, Lisse 2003.

HÄUPL (2006): Häupl, P.; „Bauphysik in math cad - Klima, Wärme, Feuchte, Schall“ Skript zur Vorlesung Bauphysik, Institut für Bauklimatik, Technische Universität Dresden, 2006.

HÄUPL (2007A): Häupl, P.; "Analytic solution of the diffusion equation for an infinite plate during the drying process" Unpublished conversation, Institute of Building Climatology, Dresden University of Technology, 2007.

HÄUPL (2007B): Häupl, P.; „Improved closed form equation for a bundle of tubes model“ Unpublished conversation, Institute of Building Climatology, Dresden University of Technology, 2007.

Holm (2001): Holm, A.; „Ermittlung der Genauigkeit von instationären Bauteilberechnungen mittels eines stochastischen Konzeptes", Dissertation, Universität Stuttgart, Fakultät Bauingenieur- und Vermessungswesen, 2001.

IPPISCH ET AL. (2006): Ippisch, O., Vogel, H.-J., Bastian, P.; „, Validity limits for the van Genuchten-Mualem model and implications for parameter estimation and numerical simulation" Advances in Water Resources, Vol. 29, No. 12: 1780-1789, 2006.

JANSSEN (2002): Janssen, H.; "The influence of soil moisture transfer on building heat loss via the ground" Ph.D. Thesis, Catholic University Leuven. Leuven, Belgium 2002.

JANSSEN ET AL. (2007): Janssen, H., Derluyn, H., Carmeliet, J.; "Moisture transfer through mortar joints: interface resistances or hygric propertiy changes?" Proceedings to the $12^{\text {th }}$ Symposium for Building Physics, Dresden University at Technology, Institute of Building Climatology, Vol. 2, 808-815, Dresden 2007.

JAYNES (1985): Jaynes, D. B.; „Comparison of Soil-Water Hysteresis Models“ Journal of Hydrology, Vol. 75 : 287-299, 1984/1985.

JAYNES (1992): Jaynes, D. B.; "Estimating hysteresis in the soil water retention function" Proceedings of the International Workshop on Indirect Methods for Estimating the Hydraulic Properties of Unsaturated Soils, Riverside, California, October 1989, edited by M. Th. van Genuchten, F. J. Leij and L. J. Lund, University of California, 1992.

JOHANNESSON \& JANZ (2002): Johannesson, B. \& Janz, M.; „Test of Four Different Experimental Methods to Determine Sorption Isotherms" Journal of Materials in Civil Engineering, Vol. 14, No. 6: 471-477, 2002. 
JURK ET AL. (2004): Jurk, K., Scheffler, G., Grunewald, J., Plagge, R.; „Durchführung und Analyse von Trocknungsexperimenten von Baustoffen - Methoden, Funktionen und Parameter" Conribution to the Feuchtetag 2004 in Berlin, Proc. 229-236, www.dgzfp.de, 2004.

Kaluarachchi \& PARKer (1987): Kaluarachchi, J. J. \& Parker, J. C.; „Effects of Hysteresis with Air Entrapment on Water Flow in the Unsaturated Zone" Water Resources Research, Vol. 23, No. 10: 1967-1976, 1987.

KIEßL (1983): Kieß1, K.; „Kapillarer und dampfförmiger Feuchtetransport in mehrschichtigen Bauteilen Rechnerische Erfassung und bauphysikalische Anwendung." Dissertation, Universität Gesamthochschule Essen, 1983.

KLUTE (1972): Klute, A.; "The determination of the hydraulic conductivity and diffusivity of unsaturated soils" Soil Science, Vol. 113, No. 4: 264-276, 1972.

KLUTE (1986): Klute, A.; „Water Retention: Laboratory Methods“ In: Klute, A. (ed.). Methods of soil analysis. Part I. Physical and mineralogical methods. 2nd edition, No.9, Agronomy Series, Am. Soc. Agron., Soil Sci. Soc. Am. Madison. p. 635-662, 1986.

KLUTE \& DiRKSEN (1986): Klute, A. \& Dirksen, C.; „Hydraulic conductivity and diffusivity laboratory methods" In: Klute, A. (ed.). Methods of soil analysis. Part I. Physical and mineralogical methods. 2nd edition, No.9, Agronomy Series, Am. Soc. Agron., Soil Sci. Soc. Am. Madison. p. 687-734, 1986.

KRISCHER (1940): Krischer, O.; „Wärme-, Flüssigkeits- und Dampfbewegung bei der Trocknung poriger Stoffe" Z. VDI-Beiheft Verfahrenstechnik. 1, 17-25, 1940.

KRISCHER (1942): Krischer, O.; "Der Wärme- und Stoffaustausch in Trocknungsgut" VDI-Forschungsheft 415, 1-22, 1942.

KRISCHER (1956): Krischer, O.; „Die wissenschaftlichen Grundlagen der Trocknungstechnik. “ SpringerVerlag, 1956, here referred to as: Krischer, O. \& Kast, W.; "Die wissenschaftlichen Grundlagen der Trocknungstechnik." 3. Auflage. Springer-Verlag, Berlin/Heidelberg, 494 S, 1992.

KRUS (1995): Krus, M.; „Feuchtetransport- und Speicherkoeffizienten poröser mineralischer Baustoffe. Theoretische Grundlagen und neue Meßtechniken." Dissertation Universität Stuttgart 1995.

Krus \& Holm (1999): Krus, M. \& Holm, A.; „Approximationsverfahren für die Bestimmung feuchtetechnischer Materialkennwerte" Proceedings to the $10^{\text {th }}$ Symposium for Building Physics, Dresden University of Technology, Institute of Building Climatology, Vol. 2: 423432, 1999.

KRUS ET AL. (2007): Krus, M., Lenz, K., Plagge, R., Scheffler, G.; „Ein Trocknungskoeffizient als neuer hygrothermischer Standardmaterialkennwert" $12^{\text {th }}$ Symposium for Building Physics, Proc. Vol. 1: 283-293, Dresden University of Technology, Institue of Building Climatology, March 2007.

Kumaran (1999): Kumaran, M. K.; „Moisture Diffusivity of Building Materials from Water Absorption Measurements" Journal of Thermal Envelope and Building Science, Vol. 22: 349-355, 1999.

KÜNZEL (1994): Künzel, H. M.; „Verfahren zur ein- und zweidimensionalen Berechnung des gekoppelten Wärme- und Feuchtetransports in Bauteilen mit einfachen Kennwerten", Dissertation, Universität Stuttgart, Fakultät Bauingenieur- und Vermessungswesen, 1994.

KUPFER ET AL. (2004): Kupfer, K., Trinks, E., Schäfer, T.; „TDR-Sensoren zur Kontrolle von Deponieabsichtungen in Salzbergwerken" Contribution to the Feuchtetag 2004 in Berlin, Proc. 91-98, www.dgfzp.de, 2004.

LANGMUIR (1918): Langmuir, I.; ",The Adsorption of Gases on Plane Surfaces of Glass, Mica and Platinum" Journal of American Chemical Society, Vol. 40(9): 1361-1403, 1918.

LIN \& COHEN (1982): Lin, C. \& Cohen, M. H.; "Quantitative methods for microgeometric modelling“ Journal of Applied Physics Vol. 53: 4152-4165, 1982.

DE LOOR (1965): De Loor, G. P.; „Dielectric properties of heterogeneous mixtures with a polar constituent” Applied Scientific Research, Vol. 11, No. 1: 310-320, 1965.

DE LOOR (1968): De Loor, G. P.; "Dielectric properties of heterogeneous mixtures containing water" Journal of Microwave Power Vol. 3: 67-73, 1968.

LUDWIG (1856): Ludwig, C.; "Diffusion zwischen ungleich erwärmten Orten gleich zusammengesetzter Lösungen" Sitz. ber. Akad. Wiss. Wien Math.-naturew. Kl. 20 (1856), 539. 
LUTZ ET AL. (2002): Lutz, Jenisch, Klopfer, Freymuth, Krampf, Petzold; „Lehrbuch der Bauphysik“ B. G. Teubner, 5. Auflage, 2002.

Malicki ET AL. (1992): Malicki, M. A., Plagge, R., Renger, M., Walczak, R. T.; „Application of timedomain reflectometry (TDR) soil moisture miniprobe for the determination of unsaturated soil water characteristics from undisturbed soil cores" Irrigation Sci. Vol. 13: 65-72, 1992.

MALICKI ET AL. (1994): Malicki, M. A., Plagge, R., Roth, C. H.; "Reduction of matrix on TDR soil moisture readings and its elimination" Symposium on Time Domain Reflectometry in Environmental, Infrastructure and Mining Applications, Evanston (Chicago), USA. Unitest States Department of Interior Bureau of Mines, Special Publication SP19 94: 105-114, 1994.

MANTHEY (2006): Manthey, S.; „Two-phase flow processes with dynamic effects in porous media - parameter estimation and simulation" Ph.D. Thesis at the Institute of Water Structures, University of Stuttgart, 2006.

Moropoulou ET AL. (2005): Moropoulou, A., Karoglou, M., Giakoumaki, A., Krokida, M.K., Maroulis, Z.B., Saravacos, G.D.; „Drying kinetics of some building materials“ Brazilian Journal of Chemical Engineering, Vol. 22, No. 2: 203-208, 2005.

MuAlem (1974): Mualem, Y.; "A conceptual model of hysteresis“ Water Resources Research Vol. 10: 514$520,1974$.

MuALEM (1976A): Mualem, Y.; "A new model for predicting the hydraulic conductivity of unsaturated porous media" Water Resources Research Vol. 12: 513-522, 1976.

MuALEM (1976B): Mualem, Y.; "Hysteretical models for prediction of the hydraulic conductivity of unsaturated porous media" Water Resources Research Vol. 12: 1248-1254, 1976.

MuAlem (1984): Mualem, Y.; „A modified dependent-domain theory of hysteresis“ Soil Science Vol. 137: 283-291, 1984.

MuAlem (1992): Mualem, Y.; „Modeling the Hydraulic Conductivity of Unsaturated Porous Media“ Proceedings of the International Workshop on Indirect Methods for Estimating the Hydraulic Properties of Unsaturated Soils, Riverside, California, October 1989, edited by M. Th. van Genuchten, F. J. Leij and L. J. Lund, University of California, 1992.

MuKhOPADHYAYA ET AL. (2002): Mukhopadhyaya, P., Kumaran, K., Normandin, N, Goudreau, P.; "Effect of Surface Temperature on Water Absorption Coefficient of Building Materials" Journal of Thermal Envelope and Building Science, Vol. 26, No. 2: 179-195, 2002.

NiCOLAI (2005): Nicolai, A.; "Iterative refinement algorithm for generation of spline data“ Personal communication and code supply, Syracuse University, Syracuse NY, 2005.

NicOlaI (2006): Nicolai, A.; "Delphin 5 - Coupled heat, air, moisture, pollutant and salt simulation software", http:/ / www.bauklimatik-dresden.de/delphin5, August 2006.

NicOlAI \& ZHANG (2006): Nicolai, A. \& Zhang, J. S.; „An extendible material database for indoor air quality and hygrothermal research and applications." $3^{\text {rd }}$ International Building Physics/Science Conference, Montreal 2006, published in Research in Building Physics and Building Engineering - Fazio, Ge, Rao \& Desmarais (eds), Balkema, Taylor \& Francis Group, London.

OJANEN ET AL. (2002): Ojanen, T., Salonvaara, M., Simonson, C.J.; „Integration of simplified drying tests and numerical simulation in moisture performance analysis of the building envelope" $6^{\text {th }}$ Symposium on Building Physics in the Nordic Countries, 151-158, Trondheim 2002.

Padfield ET Al. (2002): Padfield, T., Peuhkuri, R., Rode, C., Hansen, K.K.; „Non-Isothermal Vapour Transmission through Porous Insulation. Part 1: The Climatic Chamber" 6 th Symposium on Building Physics in the Nordic Countries, 413-419, Trondheim 2002.

PARlANGe (1976): Parlange, J.-Y.; "Capillary hysteresis and the relationship between drying and wetting curves" Water Resources Research Vol. 12: 224-228, 1976.

PAYATAKes \& DiAs (1984): Payatakes, A. C. \& Dias, M. M.; „Immiscible microdisplacement and ganglin dynamics in porous media" Rev. Chem. Engng. 85-174, 1984.

PECK (1965A): Peck, A.J.; „Moisture profile development and air compression during water uptake by bounded porous bodies: 2. Horizontal columns" Soil Science, Vol. 99, No. 5: 327-334, 1965.

Реск (1965в): Peck, A.J.; „Moisture profile development and air compression during water uptake by bounded porous bodies: 3. Vertical columns" Soil Science, Vol. 100, No. 1: 44-51, 1965. 
Pedersen (1989): Pedersen, C. R.; „MATCH - A Computer Program for Transient Calculation of Combined Heat and Moisture Transfer." CIB W-40 - Reducing Moisture-Related Damage in the World's Buildings, Victoria, Canada, 11-14 September 1989.

Pedersen (1990): Pedersen, C. R.; „Combined moisture and heat transfer in building constructions" Ph.D. thesis at the Thermal Insulation Laboratory, Danish Technical University, 1990.

Pel (1995): Pel, L.; „Moisture transport in building materials" Ph.D. thesis at Eindhoven University of Technology, 1995.

Pel ET AL. (1996): Pel, L., Brocken, H., Kopinga, K.; „Determination of moisture diffusivity in porous media using moisture concentration profiles" International Journal of Heat and Mass Transfer, Vol. 39: 1273-1280, 1996.

PhiliP \& De Vries (1957): Philip, J. R. \& De Vries, D. A.; „Moisture movement in porous materials under temperature gradients" Trans. American Geophysical Union, Vol 38: 222-232, 1957.

Plagge (1991): Plagge R.; ,Bestimmung der ungesättigten hydraulischen Leitfähigkeit im Boden“ Dissertation, Bodenökologie und Bodengenese, Heft 3, 1991.

Plagge ET AL. (1999): Plagge, R., Häupl, P., Renger, M.; „Transient effects on the hydraulic properties of porous media" Proceedings of the International Workshop on Characterization and Measurement of the Hydraulic Properties of Unsaturated Porous Media, Riverside, California, 1999, edited by M. Th. van Genuchten et al., p. 905-912, 1999.

Plagge (2001): Plagge, R.; "Development and design of a cup diffusion measurement device“ unpublished, Institute of Building Climatology, Dresden University of Technology, 2001.

Plagge ET AL. (2002): Plagge, R., Häupl, P., Grunewald, J., Fechner, H.; „Functional description of water storage and transfer in capillary porous building materials" Proceedings to the $11^{\text {th }}$ Symposium for Building Physics, Dresden University at Technology, Institute of Building Climatology, Vol.1, 338-350, Dresden 2002.

Plagge (2003): Plagge, R.; „Bestimmung von Materialfeuchte und Salzgehalt in kappilar porösen Materialien mit TDR" Colloquium and Workshop Innovative Feuchtemessung in Forschung und Praxis, Soil Moisture Group at the University of Karlsruhe, July 2003.

Plagge et Al. (2003): Plagge, R., Cerny, R., Mathiasovsky, P.; „Development of insulation Materials with specially designed properties for building renovation, Workpackage 3 - Laboratory Measurements" Final report of the INSUMAT project, Workpackage 3, Self publishing at TU Dresden, 2003.

Plagge ET AL. (2005): Plagge, R., Scheffler, G., Grunewald, J.; „A Automatic measurement of the water uptake behavior for building materials" $7^{\text {th }}$ Symposium on Building Physics in the Nordic Countries, Reykjavik June 2005. Vol. 1, 15-22.

Plagge ET AL. (2006A): Plagge, R., Scheffler, G., Grunewald, J.; „Measurement of water retention and moisture conductivity at transient conditions " $3^{\text {rd }}$ International Building Physics/Science Conference, Montreal August 2006, published in Research in Building Physics and Building Engineering - Fazio, Ge, Rao \& Desmarais (eds), Balkema, Taylor \& Francis Group, London.

Plagge ET AL. (2006B): Plagge, R., Scheffler, G., Grunewald, J. \& Funk, M.; „,On the Hysteresis in Moisture Storage and Conductivity measured by the Instantaneous Profile Method", Journal of Building Physics, Volume 29, No. 3, January 2006.

PLAGGE (2007): Plagge, R.; „Whole water content range adsorption data of building materials measured at the Institute of Building Climatology" Recent measurement results and personal communication, Institute of Building Climatology, Dresden University of Technology, unpublished, 2007.

Plagge ET AL. (2007): Plagge, R., Scheffler, G., Nicolai, A.; "Experimental methods to derive hygrothermal material functions for numerical simulation tools" submitted to Buildings X Conference, Clearwater, Florida 2007.

PORTER ET AL. (2006): Porter, M. L., Schaap, M. G., Wildenschild, D.; „Capillary pressure-saturation curves: toward simulating dynamic effects with the lattice-boltzmann method" in P. Binning, P. Engesgaard, H. Dahle, G. Pinder and W. G. Gray, Computational methods in water resources XVI, Proc., Technical University of Denmark, June 2006.

Poulovassilis (1962): Poulovassilis, A.; „Hysteresis of Pore Water, an application of independent domains“ Soil Science Vol. 93: 405-412, 1962. 
Poulovassilis (1969): Poulovassilis, A.; "Hysteresis of Pore Water in Granular Porous Bodies“ Soil Science Vol. 109, No. 1: 5-12, 1969.

Poulovassilis (1970): Poulovassilis, A.; „The Effect of the Entrapped Air on the Hysteresis Curves of a Porous Body and on its Hydraulic Conductivity“ Soil Science Vol. 109, No. 3: 154-162, 1970.

Poulovassilis \& Tzimas (1975): Poulovassilis, A. \& Tzimas, E.; „, The Hysteresis in the Relationship between Hydraulic Conductivity and Soil Water Content" Soil Science Vol. 120: 327-331, 1975.

QUENARD \& SALLE (1992): Quenard, D. \& Salle, H.; ,W Water vapour adsorption and transfer in cement-based materials: a network simulation" Materials and Structures, Vol. 25: 515-522, 1992.

QueNARD ET AL. (1998): Quenard, D.A., Xu, K., Künzel, H.M., Bentz, D.P., Martys, N.S.; "Microstructure and transport properties of porous building materials" Materials and Structures, Vol. 31: 317-324, 1998.

RAWLINS \& GARDNER (1963): Rawlins, S.L. \& Gardner, W.H., "A test of the validity of the diffusion equation for unsaturated flow of soil water" Soil Sci. Soc. Am. Proc., Vol. 27: 507-511, 1963.

RAWlins \& CAMPBEll (1986): Rawlins, S.L. \& Campbell, G.S.; „Water Potential: Thermocouple Psychrometry" In: Klute, A. (ed.). Methods of soil analysis. Part I. Physical and mineralogical methods. 2nd edition, No.9, Agronomy Series, Am. Soc. Agron., Soil Sci. Soc. Am. Madison. p. 597-618, 1986.

ReNARD \& MARsiLy (1997): Renard, Ph. \& de Marsily, G.; „Calculating equivalent permeability: a review" Advances in Water Resources, Vol. 20, No. 5-6: 253-278, 1997.

RichaRds \& Fireman (1943): Richards, L.A. \& Fireman, M.; „Pressure-Plate Apparatus for Measuring Moisture Sorption and Transmission by Soils" Soil Science, Vol. 56, No. 6: 395-404, 1943.

RichARDS \& WEEKS (1953): Richards, L.A. \& Weeks, L.V.; "Capillary conductivity values from moisture yield and tension measurements on soil columns" Soil Sci. Soc. Am. Proc., Vol. 17: 206-209, 1953.

RICHARDS ET AL. (1992): Richards, R.F., Burch, D.M. \& Thomas, W.C.; „Water Vapor Sorption Measurements of Common Building Materials“ ASHRAE Transactions V. 98, Pt. 2, 1992.

Rode ET AL. (2002): Rode, C., Grau, K., Sørensen, L. C., Christoffersen, L. D.; „Application of a Computer Model for Integrated Hygrothermal Building Analysis" Proceedings to the $11^{\text {th }}$ Symposium for Building Physics, Dresden University at Technology, Institute of Building Climatology, Vol.2, 652-660, Dresden 2002.

RoEls (2000): Roels, S.; "Modelling unsaturated moisture transport in heterogeneous limestone“ Ph.D. Thesis at Katholic University of Leuven 2000.

Roels ET AL. (2000): Roels, S., Carmeliet, J., Hens, H.; "Microscopic analysis of imbibition processes in oolitic limestone“ Geophysical Research Letters, Vol. 27, No. 21: 3533-3536, 2000.

RoEls ET AL. (2001): Roels, S., Elsen, J., Carmeliet, J., Hens, H.; „'Characterisation of pore structure by combining mercury porosimtetry and micrography" Materials and Structures, Vol. 34: 76-82, 2001.

Roels ET AL. (2003A): Roels, S., Carmeliet, J., Hens, H.; "Modelling Unsaturated Moisture Transport in Heterogeneous Limestone - Part 1: A Mesoscopic Approach" Transport in Porous Media Vol. 52: 333-350, 2003.

Roels ET AL. (2003B): Roels, S., Carmeliet, J., Hens, H.; „Modelling Unsaturated Moisture Transport in Heterogeneous Limestone - Part 2: Macroscopic Simulations" Transport in Porous Media Vol. 52: 351-369, 2003.

Roels ET AL. (2003C): Roels, S., Vandersteen, K., Carmeliet, J.; „Measureing and simulating moisture uptake in a fractured porous medium" Advances in Water Resources Vol. 26: 237-246, 2003.

Roels ET AL. (2004): Roels, S., Carmeliet, J., Hens, H., Brocken, H., Hall, C., Plagge, R., Cerny, R., Pavlik, Z., Kumaran, K.; "A comparison of different techniques to quantify moisture content profiles in porous building materials" Journal of Thermal Envelope \& Building Science, Vol. 27, No. 4, 2004.

Roth ET AL. (1989): Roth, K., Flühler, H., Attinger, W.; „TDR. Eine Methode zur Messung des volumetrischen Wassergehaltes" Bull. Bodenkdl. Ges. d. Schweiz, Vol. 13: 117-122, 1989.

Rotн ET AL. (1990): Roth, K., Schulin, R., Flühler, H., Attinger, W.; „Calibration of Time Domain Reflectometry for Water Content Measurement Using a Composite Dielectric Approach" Water Resources Research, Vol. 26, No. 10: 2267-2273, 1990. 
SANDER ET AL. (2000): Sander, A., Kardum, J.P., Skansi, D.; „Transport properties in drying of solids“ Poster Session on drying, P1.110, $14^{\text {th }}$ International Congress of Chemical and Process Engineering, Prague, August 2000.

SASIC KAlAGASIDIS (2004): Sasic Kalagasidis, A.; "HAM-Tools - An Integrated Simulation Tool for Heat, Air and Moisture Transfer Analyses in Building Physics." Ph.D. Thesis at Chalmers University of Technology, Sweden 2004.

SCHEFFLER ET AL. (2004): Scheffler, G., Grunewald, J., Häupl, P.; „Calibration of an engineering model of hygrothermal material characteristics" CIB W40, Glasgow, September 2004.

SCHEFFler \& Plagge (2005A): Scheffler, G. \& Plagge, R.; „Defined drying behaviour measurement for building materials" $7^{\text {th }}$ Symposium on Building Physics in the Nordic Countries, Reykjavik June 2005.Vol. 1, 23-30.

SCHEFfler ET AL. (2006): Scheffler, G., Plagge, R., Grunewald, J., Häupl, P.; „Evaluation of instantaneous profile measurements indicating dependencies of moisture transport on hysteresis and dynamics" $3^{\text {rd }}$ International Building Physics/Science Conference, Montreal 2006, published in Research in Building Physics and Building Engineering - Balkema Publishers, 129-138, 2006.

SCHEFFler ET AL. (2007-A): Scheffler, G., Grunewald, J., Plagge, R.; „Evaluation of Functional Approaches to Describe the Moisture Diffusivity of Building Materials" Journal of ASTM International (JAI), Vol. 4, No. 2, 2007.

SCHefFleR ET AL. (2007-B): Scheffler, G., Plagge, R., Häupl, P.; "Hygrische Materialmodellierung und ihre Abhängigkeit vom Prozess und der Prozessdynamik" Bauphysik Vol. 29 No. 03: 164-177, 2007.

SCHIRMER (1938): Schirmer, R.; „Die Diffusionszahl von Wasserdampf-Luftgemischen und die Verdampfungsgeschwindigkeit." 2. VDI Beiheft Verfahrenstechnik 170-177, 1938.

SCHUltze ET AL. (1999): Schultze, B., Ippisch, O., Huwe, B., Durner, W.; „Dynamic nonequilibrium during unsaturated water flow" Proceedings of the International Workshop on Characterization and Measurement of the Hydraulic Properties of Unsaturated Porous Media, Riverside, California, 1999, edited by M. Th. van Genuchten et al., p. 877-892, 1999.

SIMUNEK ET AL. (1999): Simunek, J., Kodesova, R., Gribb, M. M., Van Genuchten, M. T.; „Estimating hysteresis in the soil water retention function from cone permeameter experiments" Water Resources Research, Vol. 35, No. 5: 1329-1345, 1999.

SLAWINSKI ET AL. (2006): Slawinski, C., Walczak, R. T., Skierucha, W.; "Error analysis of water conductivity coefficient measurement by instantaneous profiles method" International Agrophysics Vol. 20: 55-61, 2006.

SMiles ET AL. (1971): Smiles, D. E., Vauchaud, G., Vauclin, M.; „A test of the uniqueness of soil moisture characteristic during transient, nonhysteretic flow of water in a rigid soil" Soil Sci. Soc. Amer. Proc., Vol. 35: 534-539,1971.

SobczUK ET AL. (1992): Sobczuk, H. A., Plagge, R., Walczak, R. T., Roth, C. H.; „Laboratory equipment and calculation procedure to rapidly determine hysteresis of some soil hydrophysical properties under nonsteady flow conditions" Z. Pflanzenernähr. Bodenk., 1555, 157-163, 1992.

SobczUK \& Plagge (2007): Sobczuk, H. \& Plagge, R.; „Time domain reflectometry method in environmental measurements" Polskiej Akademii Nauk, Vol. 39, ISBN 83-89293-51-X, Lublin 2007.

SORET (1880): Soret, C.; "Influence de la température sur la distribution des sels dans leurs solutions" C.R. Acad. Sci.,Paris, Vol. 91, No. 5: 289-291, 1880.

SORET (1881): Soret, C.; „Sur L'etat d'équilibre que prend au point de vue de sa concentration une dissolution saaline primitivement homogéne dont deux parties sont portées a des températures differentes." Ann. Chim. Phys., Vol. 22: 293-297, 1881.

STAUFFER (1977): Stauffer, F.; „Einfluss der kapillaren Zone auf instationäre Drainagevorgange“ Ph.D. Thesis at ETH Zurich, Diss. ETH Nr. 5931, 1977.

TAUTz (1971): Tautz, H.; "Wärmeleitung und Temperaturausgleich“ Akademie-Verlag Berlin, 1971.

Time (1998): Time, B.; "Hygroscopic Moisture Transport in Wood“ Ph.D. thesis at the Department of Building and Construction Engineering, Norwegian University of Scienc and Technology, Trondheim, 1998.

Tinga ET AL. (1973): Tinga, W. R., Voss, W. A. G., Blossey, D. F.; „Generalized approach to multiphase dielectric mixture theory“ Journal of Applied Physics, Vol. 44, No. 9, 1973. 
TopP ET AL. (1967): Topp, G. C., Klute, A., Peters, D. B.; „Comparison of water content-pressure head data obtained by equilibrium, steady-state, and unsteady-state methods" Soil Sci. Soc. Amer. Proc., Vol. 31: 312-314, 1967.

TOUMA \& VAUCLIN (1986): Touma, J. \& Vaulcin, M.; „Experimental and numerical analysis of two-phase infiltration in a partially saturated soil" Transport in Porous Media, Vol. 1: 27-55, 1986.

TUller \& OR (2002): Tuller, M. \& Or, D.; „Unsaturated Hydraulic Conductivity of Structured Porous Media: A Review of Liquid Configuration-Base Models" Vadose Zone Journal, Vol. 1: 14-37, 2002.

TzIMAS (1979): Tzimas, E.; "The Measurement of Soil-Water Hysteretic Relationships on a Soil Monolith“ Journal of Soil Science, Vol. 30: 529-534, 1979.

VANDERsteEN ET AL. (2003): Vandersteen, K., Carmeliet, J., Feyen, J.; „A Network Modeling Approach to Derive Unsaturated Hydraulic Properties of a Rough-Walled Fracture" Transport in Porous Media Vol. 50: 197-221, 2003.

VAN Genuchten (1980): Van Genuchten, M. T.; "A Cosed-form Equation for Predicting the Hydraulic Conductivity of Unsaturated Soils" Soil Sci. Soc. Amer. J., Vol. 44: 892-898, 1980.

VAN GeNuchten \& LeIJ (1992): Van Genuchten, T. M. \& Leij, F. J.; „On Estimating the Hydraulic Properties of Unsaturated Soils" Proceedings of the International Workshop on Indirect Methods for Estimating the Hydraulic Properties of Unsaturated Soils, Riverside, California, October 1989, edited by M. Th. van Genuchten, F. J. Leij and L. J. Lund, University of California, 1992.

Viaene eT Al. (1994): Viaene, P., Vereecken, H., Diels, J., Feyen, J.; "A Statistical Analysis of six Hysteresis Models for the Moisture Retention Characteristic" Soil Scienece, Vol. 157, No. 6: 345$355,1994$.

DE VRIES (1958): De Vries, D. A.; "Simultaneous transfer of heat and moisture in porous media“ Transactions of the American Geophysical Union, Vol. 39: 909-916, 1958.

DE VRIES (1987): De Vries, D. A.; "The theory of heat and moisture transfer in porous media revisited" International Journal of Heat and Mass Transfer, Vol. 30, No. 7: 1343-1350, 1987.

WANNA-ETYEM (1982): Wanna-Etyem, C.; "Static and dynamic water content-pressure head relations of porous media" Ph.D. Thesis at Colorado State University, 1982.

WEAST (1979): Weast R.C.; "CRC Handbook of Chemistry and Physics“ Boca Raton, Florida: CRC Press, 1979.

WEEKS \& RICHARDS (1967): Weeks, L.V. \& Richards, S.J.; „Soil water properties computed from transient flow data" Soil Sci. Soc. Amer. Proc., Vol. 31: 721-725, 1967.

WEIER (2002): Weier, H.; „Abhängigkeit zwischen Wasseraufnahmekoeffizienten und Sättigungsleitfähigkeit” Internal report at the Institute of Building Climatology, Dresden University of Technology, 2002.

DE WIT \& VAN SCHINDEL (1993): de Wit, M. \& van Schindel, J.; „Estimation of the Moisture Diffusivity" IEA Annex XXVI Report, T1-NL-93/04, 1993.

WHITAKER (1969): Whitaker, S.; "Advances in theory of fluid motion in porous media" Industrial and Engineering Chemistry, 61: 14-28, 1969.

WHITAKER (1977): Whitaker, S.; "Simultaneous heat, mass and momentum transfer in porous media: A theory of drying porous media" Advances in Heat Transfer, Vol. 13: 119-200, 1977.

WhitAKer (1986): Whitaker, S.; "Flow in Porous Media I: A Theoretical Derivation of Darcy's Law" Transport in Porous Media, Vol. 1: 3-25, 1986.

WILSON \& LUTHIN (1963): Wilson, L.G. \& Luthin, J.N.; "Effect of air flow ahead of the wetting front on infiltration" Soil Science, Vol. 96: 136-143, 1963.

WORCH (2002): Worch, A.; „Überlegungen und Versuche zur Erfassung des Wasserdampfübergangs an Bauteiloberflächen" Dissertation Universität Dortmund 2002, Shaker Verlag Aachen.

WORCH (2004): Worch, A.; "The Behaviour of Vapour Transfer on Building Material Surfaces: The Vapour Transfer Resistance" Journal of Thermal Envelope \& Building Science, Volume 28 No. 2 2004.

XU ET AL. (1997A): Xu, K., Daian, J.-F., Quenard, D.; „Multiscale Structures to Describe Porous Media Part I: Theoretical Background and Invasion by Fluids" Transport in Porous Media, Vol. 26: 51-73, 1997. 
XU ET AL. (1997в): [1]Xu, K., Daian, J.-F., Quenard, D.; „Multiscale Structures to Describe Porous Media Part II: Transport Properties and Application to Test Materials" Transport in Porous Media, Vol. 26: 319-338, 1997.

Youngs \& PECK (1964): Youngs, E.G. \& Peck, A.J.; "Moisture profile development and air compression during water uptake by bounded porous bodies: 1. Theoretical introduction" Soil Science, Vol. 98: 290-294, 1964.

\section{Standards and regulations}

DIN 4108-3: DIN 4108 Teil 3 Wärmeschutz und Energie-Einsparung in Gebäuden, Teil 3: Klimabedingter Feuchteschutz, Anforderungen, Berechnungsverfahren und Hinweise für Planung und Ausführung, DIN Deutsches Institut für Normung e.V., Berlin, Juli 2001

DIN EN 1936: DIN EN 1936 Prüfverfahren für Naturstein - Bestimmung der Reindichte, der Rohdichte, der offenen Porosität und der Gesamtporosität, DIN Deutsches Institut für Normung e.V., Berlin, Februar 2007.

DIN ISO 11274: DIN ISO 11274 Bodenbeschaffenheit - Bestimmung des Wasserrückhaltevermögens Laborverfahren, DIN Deutsches Institut für Normung e.V., Berlin, Januar 2001.

DIN ISO 11275: DIN ISO 11275 Bodenbeschaffenheit - Bestimmung der ungesättigten hydraulischen Leitfähigkeit und des Wasserrückhaltevermögens - Evaporationsverfahren nach Wind, DIN Deutsches Institut für Normung e.V., Berlin, Januar 2005.

DIN ISO 9277: DIN ISO 9277 Bestimmung der spezifischen Oberfläche von Feststoffen durch Gasadsorption nach dem BET-Verfahren, DIN Deutsches Institut für Normung e.V., Berlin, Mai 2003.

DIN EN ISO 12571: DIN EN ISO 12571 Wärme- und feuchtetechnisches Verhalten von Baustoffen und Bauprodukten - Bestimmung der hygroskopischen Sorptionseigenschaften, DIN Deutsches Institut für Normung e.V., Berlin, April 2000.

DIN EN ISO 12572: DIN EN ISO 12572 Wärme- und feuchtetechnisches Verhalten von Baustoffen und Bauprodukten - Bestimmung der Wasserdampfdurchlässigkeit, DIN Deutsches Institut für Normung e.V., Berlin, September 2001.

DIN EN ISO 12664: DIN EN ISO 12664 Wärme- und feuchtetechnisches Verhalten von Baustoffen und Bauprodukten - Bestimmung des Wärmedurchlasswiderstandes nach dem Verfahren mit dem Plattengerät und dem Wärmestrommessplatten-Gerät - Trockene und feuchte Produkte mit mittlerem und niedrigem Wärmedurchlasswiderstand, DIN Deutsches Institut für Normung e.V., Berlin, Mai 2001.

DIN EN ISO 15148: DIN EN ISO 15148 Wärme- und feuchtetechnisches Verhalten von Baustoffen und Bauprodukten - Bestimmung des Wasseraufnahmekoeffizienten bei teilweisem Eintauchen, DIN Deutsches Institut für Normung e.V., Berlin, März 2003.

WÄRMELEITRECHENWRL (1999): WärmeleitRechenWRL: Verfahren zur Festlegung eines alternativen Rechenwertes $\lambda_{R}$ der Wärmeleitfähigkeit für Mauerwerk im Rahmen des Übereinstimmungsnachweises, Deutsches Institut für Bautechnik Berlin, Ernst \& Sohn Verlag für Architektur und technische Wissenschaften GmbH \& Co. KG, 1999.

ISO 8302 (1991): ISO 8302: Thermal insulation - Determination of steady-state thermal resistance and related properties - Guarded hot plate apparatus. 1991.

All presented pictures were taken and all graphics and illustrations have been drawn by Gregor A. Scheffler. Some illustrations are based on graphics of other authors. In such cases, this is indicated in the figure caption or in the text where the figure is referenced. 
